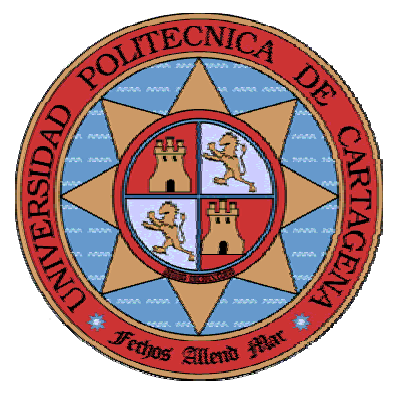

UNIVERSIDAD POLITÉCNICA DE CARTAGENA

DEPARTAMENTO DE FÍSICA APLICADA

Grupo de Simulación por Redes

\title{
SOLUCIÓN DE PROBLEMAS INVERSOS EN CONDUCCIÓN DE CALOR MEDIANTE EL MÉTODO DE SIMULACIÓN POR REDES
}

\section{TESIS DOCTORAL}

Joaquín Zueco Jordán

Cartagena, 2003 
D. Francisco Alhama López y D. Carlos F. González Fernández, Profesores Doctores del Área de Física Aplicada en el Departamento de Física Aplicada.

\begin{abstract}
AUTORIZAN:
La presentación de la Tesis Doctoral titulada "Solución de problemas inversos en conducción de calor mediante el Método de simulación por redes", realizada por D. Joaquín Zueco Jordán, bajo nuestra dirección y supervisión, en el Departamento de Física aplicada, y que presenta para la obtención de doctor por la Universidad Politécnica de Cartagena.
\end{abstract}

En Cartagena, a 3 de marzo de 2003

LOS DIRECTORES DE LA TESIS

Fdo: Dr. D. Francisco Alhama López Fdo: Dr. D. Carlos F. González Fernández 
Carlos F. González Fernández, Director del Departamento de Física Aplicada

INFORMA:

Que la Tesis Doctoral titulada "Solución de problemas inversos en conducción de calor mediante el Método de simulación por redes" ha sido realizada por D. Joaquín Zueco Jordán, bajo la dirección y supervisión de D. Francisco Alhama López y D. Carlos F. González Fernández y que el Departamento ha dado su conformidad para que sea presentada ante la Comisión General de Doctorado.

En Cartagena, a 3 de marzo de 2003

EL DIRECTOR DEL DEPARTAMENTO

Fdo: Carlos F. González Fernández 
Agradezco a los directores de esta tesis, Francisco Alhama y Carlos F. González la ayuda que me han prestado en todo momento; sus consejos, ideas y total disposición han sido decisivos no sólo para llevar a buen fin este trabajo, sino también para aumentar mi ilusión por la Universidad. Sin su ayuda no hubiera sido posible conseguir este objetivo. 
A mi mujer y a mi futuro hijo 


\section{Índice}

\section{CAPITULO I: PRESENTACIÓN}

I.1. Introducción 2

I.2. Antecedentes. El método de simulación por redes 4

$\begin{array}{ll}\text { I.3. Objetivos y desarrollo } & 6\end{array}$

I.4. Perspectivas 8

I.5. Referencias 8

\section{CAPITULO II: FUNDAMENTOS TEÓRICOS Y METODOLÓGICOS}

$\begin{array}{ll}\text { II.1. Introducción } & 14\end{array}$

$\begin{array}{ll}\text { II.2. La transmisión del calor por conducción } & 15\end{array}$

$\begin{array}{ll}\text { II.2.1. Condiciones iniciales y de contorno } & 19\end{array}$

II.3. El problema inverso en conducción del calor 20

II.3.1. Estimación de función y estimación de parámetros 26

II.3.1.1. Dificultad del problema inverso. Sensibilidad a los errores de medida 28

II.3.1.2. Coeficientes de sensibilidad 28

II.3.2. Tipos de problemas inversos $\quad 29$

II.3.2.1. Problemas inversos estacionarios y transitorios 29

II.3.2.2. Problemas lineales y no lineales $\quad 30$

II.3.2.3. Problema inverso según la naturaleza de los procesos 32

II.3.3. Métodos para la resolución de problemas inversos 33

II.4. El método de simulación por redes (MESIR) 38

$\begin{array}{ll}\text { II.4.1. Descripción } & 38\end{array}$

II.4.2. El MESIR como método numérico 42

II.4.3. Simulación 43

II.4.4. Ventajas del MESIR 45

II.5. Referencias 46 


\section{CAPITULO III: PROTOCOLOS DE RESOLUCIÓN}

III.1. Planteamiento de los problemas inversos $\quad 54$

III.1.1. Modelo matemático $\quad 54$

III.1.2. Obtención de las temperaturas de entrada 55

III.1.3. Planteamiento 59

III.2 Estimación de funciones dependientes del tiempo 63

III.2.1. Especificación del funcional 63

III.2.2. Procedimiento computacional 63

III.3 Estimación de funciones dependientes de la temperatura 65

III.3.1. Especificación del funcional $\quad 65$

$\begin{array}{ll}\text { III.3.2. Procedimiento computacional } & 67\end{array}$

$\begin{array}{lll}\text { III.4 Estimación simultánea de dos funciones ambas dependientes de la temperatura } & 69\end{array}$

III.4.1. Especificación del funcional 69

III.4.2. Procedimiento computacional 70

III.5 Estimación de parámetros de funciones lineales 72

III.5.1. Especificación del funcional $\quad 72$

$\begin{array}{ll}\text { III.5.2. Procedimiento computacional } & 72\end{array}$

III.6 Algoritmo general de resolución $\quad 74$

III.7 Referencias

$\begin{array}{ll}\text { Contribuciones y conclusiones } & 77\end{array}$

\section{CAPITULO IV: MODELOS EN RED}

$\begin{array}{lr}\text { IV.1. Problema directo. Modelos en red } & 80\end{array}$

IV.2. Problema inverso. Modelos en red 90

IV.2.1. Estimación de magnitudes constantes $\quad 92$

IV.2.1.1. Estimación de propiedades termofísicas (conductividad y calor específico) $\quad 92$

IV.2.1.2. Estimación de condiciones de contorno de primera y segunda clase 93

IV.2.1.3. Estimación de otros parámetros 93

IV.2.2. Estimación de funciones $\quad 94$

IV.2.2.1. Estimación del calor específico dependiente de la temperatura

IV.2.2.2. Estimación de la conductividad térmica variable 
con la temperatura $\quad 94$

IV.2.2.3. Estimación de flujos incidentes (condición de contorno)

IV.2.2.4. Estimación de focos térmicos (condición de contorno de temperatura en función del tiempo) 95

IV.2.2.5. Estimación de otras funciones

IV.2.2.6. Estimación simultánea del calor especifico y de la conductividad térmica

IV.2.3. Estimación de funciones lineales 99

$\begin{array}{lr}\text { IV.3. Referencias } & 101\end{array}$

$\begin{array}{lr}\text { Contribuciones y conclusiones } & 101\end{array}$

\section{CAPÍTULO V: ESTIMACIÓN DE FLUJOS INCIDENTES. ESTIMACIÓN DE TEMPERATURAS DE CONTORNO}

V.1. Estimación del flujo de calor en la superficie de un sólido 105

V.1.1. Introducción $\quad 105$

V.1.2. Estimación del flujo de calor en medios con características térmicas $\begin{array}{ll}\text { constantes } & 108\end{array}$

V.1.3. Estimación del flujo de calor en medios con características térmicas dependientes de la temperatura $\quad 123$

$\begin{array}{lr}\text { V.2. Estimación de la temperatura } & 129\end{array}$

$\begin{array}{ll}\text { V.2.1. Introducción } & 129\end{array}$

V.2.2. Estimación de temperatura en un medio sometido a convección forzada 130

$\begin{array}{lr}\text { V.3. Referencias } & 135\end{array}$

$\begin{array}{ll}\text { Contribuciones y conclusiones } & 137\end{array}$

\section{CAPITULO VI: GENERACIÓN INTERNA DE CALOR, COEFICIENTE DE CONVECCIÓN, EMISIVIDAD Y RESISTENCIA TÉRMICA DE CONTACTO; SU ESTIMACIÓN}

$\begin{array}{lr}\text { VI.1. Generación interna de calor } & 140\end{array}$

VI.1.1. Introducción 140

VI.1.2. Aplicaciones $\quad 141$

VI.2. Coeficiente de convección 144

VI.2.1. Introducción 144 
$\begin{array}{ll}\text { VI.2.2. Aplicaciones } & 145\end{array}$

$\begin{array}{ll}\text { VI.3. Emisividad } & 147\end{array}$

VI.4. Resistencia térmica de contacto 151

$\begin{array}{ll}\text { VI.5. Referencias } & 156\end{array}$

$\begin{array}{ll}\text { Contribuciones y conclusiones } & 159\end{array}$

\section{CAPITULO VII: ESTIMACIÓN DEL CALOR ESPECÍFICO Y DE LA} CONDUCTIVIDAD TÉRMICA DEPENDIENTES DE LA TEMPERATURA

$\begin{array}{ll}\text { VII.1. Introducción } & 162\end{array}$

$\begin{array}{ll}\text { VII.2. Estimación del calor específico } & 166\end{array}$

VII.2.1. Dependencia de la temperatura. Convección forzada débil 167

VII.2.2. Dependencia de la temperatura. Convección natural 179

VII.2.3. Aplicación 183

VII.3. Estimación de la conductividad térmica 190

$\begin{array}{ll}\text { VII.3.1. Estimación de función } & 190\end{array}$

$\begin{array}{ll}\text { VII.3.2. Estimación de parámetros } & 198\end{array}$

VII.4. Estimación simultanea del calor especifico y de la conductividad térmica $\quad 199$

VII.4.3. Aplicación 203

VII.5. Referencias 205

$\begin{array}{ll}\text { Contribuciones y conclusiones } & 208\end{array}$

\section{CAPITULO VIII: CONTRIBUCIONES Y CONCLUSIONES}

$\begin{array}{ll}\text { Contribuciones y conclusiones } & 211\end{array}$

\section{ANEXO: SOFTWARE DESARROLLADO}

A.1. Estimación de flujos incidentes y focos térmicos 216

A.2. Estimación simultánea del calor específico y de la conductividad térmica 230 


\title{
Simbología
}

\author{
A área, $\mathrm{m}^{2}$ \\ C capacidad de almacenamiento de calor $\left(c_{\mathrm{e}} \rho\right), \mathrm{Jm}^{-3} \mathrm{C}^{-1}$ \\ capacidad, $\mathrm{F}$ \\ $c_{e}$ \\ calor específico, $\mathrm{Jkg}^{-1}{ }^{\circ} \mathrm{C}^{-1}$ \\ $\mathrm{c}_{\text {mean }}$ \\ $\mathrm{D}, \mathrm{d}$ \\ calor específico medio, $\mathrm{Jkg}^{-1}{ }^{\circ} \mathrm{C}^{-1}$ \\ E \\ constantes \\ F \\ fuente de tensión controlada por tensión \\ funcional \\ fuente de corriente controlada por corriente \\ $\mathrm{f}$ \\ frecuencia, $1 / \mathrm{s}$ \\ relativo a un sensor de medida \\ G \\ fuente de corriente controlada por tensión \\ $\mathrm{G}_{\mathrm{r}}$ \\ número adimensional Grashof \\ $\mathrm{g}_{\mathrm{gen}}$ \\ generación interna de calor, $\mathrm{Wm}^{-3}$ \\ $\mathrm{H}$ \\ fuente de tensión controlada por corriente \\ coeficiente convectivo, $\mathrm{Wm}^{-2}{ }^{\circ} \mathrm{C}^{-1}$ \\ entalpía específica, $\mathrm{kJkg}^{-1}$ \\ $h$ \\ $\mathrm{h}_{\mathrm{i}}$ \\ I \\ $\mathrm{K}, \mathrm{K}_{1}, \mathrm{~K}_{2}$ \\ $\mathbf{J}$ \\ factores de escala según tipo de coordenadas \\ fuente de corriente temporal \\ factores de reducción en la estimación \\ flujo de calor, $\mathrm{W}$ \\ densidad de flujo de calor, $\mathrm{Wm}^{-2}$ \\ correspondencia con la temperatura medida, $(1 \leq \mathrm{j} \leq \mathrm{n})$ \\ $\mathrm{j}_{\text {est }}$ \\ densidad de flujo de calor estimado, $\mathrm{Wm}^{-2}$ \\ densidad de flujo de calor incidente, $\mathrm{Wm}^{-2}$ \\ densidad de flujo de calor inicial, $\mathrm{Wm}^{-2}$ \\ $\mathrm{j}_{\text {ini }}$ \\ densidad de flujo de calor constante, $\mathrm{Wm}^{-2}$ \\ $\mathrm{j}_{\mathrm{o}}$ \\ $\mathrm{k}$ \\ L \\ $\mathrm{N}$ \\ conductividad térmica, $\mathrm{Wm}^{-1}{ }^{\mathrm{o}} \mathrm{C}^{-1}$ \\ longitud axial, $\mathrm{m}$ \\ número total de celdas \\ medidas de temperatura medidas en un punto $f$ \\ vector normal a la superficie \\ n \\ $\mathrm{N}_{\mathrm{u}}$ \\ $\mathrm{P}$ \\ número adimensional Nusselt \\ número total de puntos de medidas \\ $P_{r}$ \\ número adimensional Prandtl \\ $\mathrm{q}_{\mathrm{i}}$ \\ tipo de coordenadas \\ r \\ número de términos del funcional \\ coordenada radial y cilíndrica, $m$ \\ $r_{z}$ \\ número de térmicos del funcional para el tramo $\mathrm{z}$ \\ $\mathrm{r}_{\mathrm{z}, \mathrm{f}}$ \\ $\mathrm{R}$ \\ número de térmicos del funcional para el tramo $\mathrm{z}$ y punto de medida $\mathrm{f}$ \\ $r_{c}$ \\ resistencia térmica, ${ }^{\circ} \mathrm{C} \mathrm{W}^{-1}$ \\ resistencia térmica de contacto, ${ }^{\circ} \mathrm{C} \mathrm{W}^{-1}$ \\ $\mathrm{R}_{\mathrm{a}} \quad$ número adimensional Rayleigh, $\left(\mathrm{P}_{\mathrm{r}} \mathrm{G}_{\mathrm{r}}\right)$ \\ S \\ error cuadrático medio, $\left[\left(\zeta_{\text {est }}-\zeta_{\text {exa }}\right)^{2} / Z\right]^{1 / 2}$ \\ S coeficiente de sensibilidad \\ t tiempo, $\mathrm{s}$
}




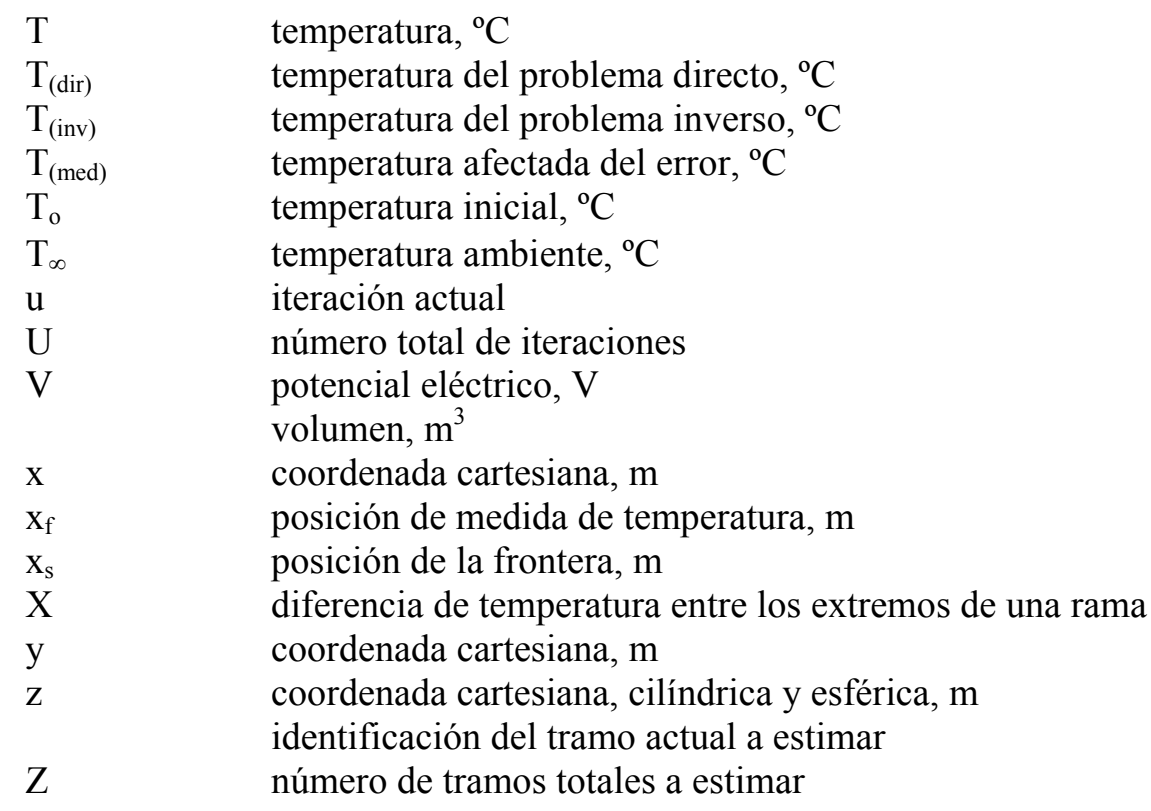

\section{Letras griegas}

$\begin{array}{ll}\nabla \mathrm{T} & \text { gradiente térmico, }{ }^{\circ} \mathrm{C} \mathrm{m}^{-1} \\ \alpha & \text { difusividad térmica, } \mathrm{m}^{2} \mathrm{~s}^{-1} \\ \Delta & \text { operador laplaciana } \\ \Delta \mathrm{c}_{\mathrm{e}} & \text { variación del calor específico }\left[\mathrm{c}_{\mathrm{e}}(\mathrm{T})-\mathrm{c}_{\text {mean }}\right], \mathrm{Jkg}^{-1}{ }^{\circ} \mathrm{C}^{-1} \\ \Delta \mathrm{t} & \text { intervalo de tiempo entre medidas, } \mathrm{s} \\ \Delta \mathrm{T}_{\mathrm{a}} & \text { intervalo de estimación de temperaturas, }{ }^{\circ} \mathrm{C} \\ \Delta \zeta, \Delta \psi & \text { intervalos de paso en estimación de funciones } \\ \Delta \rho & \text { intervalo de paso en estimación de parámetros } \\ \Delta \mathrm{x} & \text { espesor de la celda en la dirección axial, } \mathrm{m} \\ \Delta \mathrm{r} & \text { espesor de la celda en la dirección radial, } \mathrm{m} \\ \delta & \text { criterio de convergencia } \\ \varepsilon & \text { error en las medidas } \\ \varepsilon & \text { emisividad } \\ \eta & \text { variable dependiente o independiente en estimación de parámetros } \\ \varphi & \text { coordenada esférica, rad } \\ \theta & \text { coordenada cilíndrica y esférica, rad } \\ \rho & \text { densidad, kgm } \\ \rho_{\mathrm{i}}^{-3} & \text { parámetros a estimar en estimación de parámetros, }(1 \leq \mathrm{i} \leq \mathrm{I}) \\ \sigma & \text { desviación típica de los errores de las temperaturas medidas } \\ \sigma_{\mathrm{b}} & \text { constante de Boltzmann } \\ \nu & \text { pendiente en estimación de parámetros } \\ \omega & \text { números aleatorios de distribución N }(0,1) \\ \omega & \text { frecuencia angular, rad s } \\ \psi & \text { funciones a estimar } \\ \psi & \end{array}$

\section{Subíndices}

$\begin{array}{ll}\text { alm } & \text { almacenada } \\ \text { con } & \text { relativo a la convección } \\ \text { ent } & \text { entrada } \\ \text { est } & \text { valor estimado }\end{array}$




$\begin{array}{ll}\text { exa } & \text { valor exacto } \\ \mathrm{f} & \text { relativo al punto de medida } \\ \mathrm{i} & \text { relativo a la celda } \mathrm{i} \\ \text { max } & \text { valor máximo } \\ \text { mean } & \text { valor medio } \\ \text { min } & \text { valor mínimo } \\ \mathrm{rad} & \text { relativo a la radiación } \\ \text { sal } & \text { salida } \\ \infty & \text { ambiente lejos de la superficie }\end{array}$





\section{Capítulo I}

\section{Presentación}

I.1. Introducción

I.2. Antecedentes. El método de simulación por redes

I.3. Objetivos y desarrollo

I.4. Perspectivas

I.5. Referencias 


\section{Capítulo I}

\section{Presentación}

\section{I.1. INTRODUCCIÓN}

La conducción del calor, ya sea en medios con propiedades térmicas constantes o dependientes de la temperatura, es aspecto físico de gran relevancia tanto desde el punto teórico como también técnico, en problemas relacionados con la industria metalúrgica, la soldadura, estudio de aleaciones y plásticos y tratamiento y fabricación de materiales en general, etc.

Si se conocen las características térmicas de un medio sólido, su geometría, las condiciones iniciales y las condiciones de frontera, el campo térmico en el mismo y las densidades de flujo a través de cualquier sección quedan determinadas. Esto es lo que se llama problema directo cuya solución (única) puede obtenerse por medio de métodos analíticos o numéricos. El problema directo es el problema clásico.

En el problema inverso se conoce "parte de la solución" y lo que hay que determinar, por así decirlo, es "una parte del planteamiento"; ejemplo, cuando se trata de determinar características térmicas de un medio o parámetros de cualquier índole que intervienen en un problema, tales como condiciones de contorno, geometría, etc. En general el problema inverso puede tener muchas soluciones por lo que se habla de él como un problema de planteamiento incompleto (ill-posed problem).

Los problemas inversos, que surgen en situaciones donde es extremadamente difícil realizar mediciones, se pueden encontrar en diversas ramas de la ciencia y tecnología, y es especialmente durante las pasadas dos décadas la cuando la metodología para obtener soluciones a estos problemas se ha desarrollado muy intensamente. Los campos de aplicación del problema inverso son innumerables. En este sentido podemos establecer la siguiente 
clasificación general que indica el campo de la ingeniería objeto de estudio: (i) energía nuclear (pruebas de componentes de reactores nucleares), (ii) automoción (calentamiento periódico de las cámaras de combustión de los motores de automoción), (iii) fabricación (solidificación de aleaciones, vidrio, etc), (iv) termometría (calorimetría indirecta), (v) cambio de fase (estudio de las curvas de ebullición), etc.

La determinación de parámetros característicos en un proceso transitorio de transferencia de calor a partir de medidas de temperaturas realizadas en un punto (o en varios) es lo que se conoce como problema inverso de trasferencia del calor (IHCP, inverse heat conduction problem). Por el contrario, el problema clásico o tradicional es la determinación de la distribución de temperatura y flujos de calor en el interior de cuerpos sólidos cuando los parámetros característicos antes citados son conocidos; este es el problema directo de transferencia de calor (DHCP, direct heat conduction problem). Los parámetros o variables de interés más comunes en el IHCP pueden ser: las temperaturas y/o flujos de calor en la superficie, las condiciones de contorno e iniciales (o parte de ellas), la generación de calor interna, la resistencia térmica de contacto entre sólidos, etc. El IHCP puede utilizarse, además, para resolver problemas de radiación, cambio de fase y otros procesos asociados con estudios de transferencia de calor.

Para las solución del problema inverso se precisa de un conjunto de temperaturas o "medidas experimentales" tomadas a intervalos de tiempo regulares en una determinada posición (o varias) del medio, generalmente proporcionadas mediante instrumentos de medida afectados de su error o precisión (termómetros, termopares, etc). Para simular estas medidas, evitando el trabajo de laboratorio, se ha recurrido a modificar los valores obtenidos de la solución (mediante el MESIR) del problema directo, afectándolos de un cierto error aleatorio. Este es un procedimiento común en la literatura científica cuando se trata de verificar los métodos de solución del IHPC.

Por otro lado, es práctica general en estos problemas la definición, mediante mínimos cuadrados, de un funcional que compara iterativamente los resultados parciales del IHCP con las medidas experimentales anteriormente aludidas. Los resultados parciales que hacen mínimo este funcional se retienen para elaborar la solución definitiva del IHCP.

El libro clásico y actual, por excelencia, que aborda extensamente la temática del problema inverso es Inverse Heat Conduction [Beck y col., 1985]. No obstante existen textos especializados en transmisión del calor que incluyen algún capítulo sobre este tema [Necati y Özisik, 1993]. Su interés es indudable y se soporta tanto en las reuniones internacionales específicas [Eurotherm] como en las revistas científicas especializadas en esta temática (Inverse Problem e Inverse Problem Engineering) y otras muchas dentro del campo de transmisión de 
calor que publican frecuentemente artículos relacionados con el problema inverso (International Journal Heat Mass Transfer, Journal Heat Transfer-T ASME, Numerical Heat Transfer, Heat Mass Transfer, etc).

El MESIR aplicado al IPC aúna el potencial existente en la analogía termo-eléctrica con la potencia de los modernos ordenadores y con la posibilidad de desarrollar programas en cualquier lenguaje de programación (Visual C, Visual Basic, Fortram, etc.) que generen, por un lado los ficheros ejecutables en el software de simulación de circuitos y por otro permitan ejecutar la solución completa del IHPC de forma automática sin intervención del usuario. La combinación del MESIR (que permite el diseño de complicadas redes equivalentes del proceso en estudio) con las posibilidades de los programas actuales de resolución de circuitos, que

i) trabajan con dispositivos ideales

ii) disponen de amplias librerías de componentes

iii) aportan soluciones con errores tan pequeños como se soliciten

iv) requieren u tiempo de ejecución relativamente pequeño

permiten abordar con éxito los tipos de problemas inversos de transmisión de calor estudiados en esta memoria que, prácticamente, cubren todo el espectro de interés.

El software para la programación no requiere más que un pequeño número de reglas en los tipos de problemas que nos ocupan.

El presente trabajo aplica el MESIR a procesos inversos de transferencia de calor, en particular a la obtención de flujos de calor, temperaturas superficiales, flujos de generación interna de calor, coeficientes convectivos, propiedades termofísicas dependientes de la temperatura, resistencia térmica de contacto, emisividades, etc. Se enmarca así dentro de la línea de investigación que utiliza como base el MESIR para la simulación digital de las respuestas del sistema y que ya ha sido aplicada en otros campos, como en el de difusión de membranas [Horno y col., 1990], en procesos electroquímicos [González-Fernández y col., 1995], en la determinación de propiedades de coloides [López-García y col., 1996], en procesos no lineales de conducción del calor [Alhama, 1999], así como en sistemas con aletas [Alarcón, 2001].

\section{I.2. ANTECEDENTES. EL MÉTODO DE SIMULACIÓN POR REDES}

Diferentes métodos numéricos se han empleado hasta la fecha para abordar problemas directos de conducción no lineal. Entre estos podemos citar: (i) métodos en diferencias finitas en problemas estacionarios [Crank-Nicolson, 1947; Crandall, 1956; Farnia y Beck, 1977; Wood, 1977, y Orivuri, 1979] y transitorios, [Collatz, 1960]; (ii) métodos de elementos finitos en 
problemas estacionarios [Suzuki y col., 1977; Thornton, y Wieting, 1978; Suzuki y Maeda, 1978; Meric, 1979, y Lin, 1979] y transitorios [Wellford y Ayer, 1980; Varoglu y Liam Finn, 1980, y Chung, 1981]; (iii) métodos iterativos específicos [Peaceman y Rachford, 1955; Stone, 1968; Ames, 1977, y Patankar, 1978] y (iv) métodos de autovalores [Palmieri y Rathjem, 1978].

Con respecto a la solución de problemas inversos se emplean básicamente los mismos métodos numéricos citados anteriormente: el método de diferencias finitas empleado por Beck [1970 y 1982], Beck y Wolf [1965], Willians y Curry [1977], el método de elementos finitos usado por Bass [1980] y por Orlande y Ozisik [1993], el de volúmenes de control usado por AlKhalidy [1998] y Kurpisz [1991] y el método de elementos de contorno aplicado por Maillet y col. [1991]

La mayoría de los métodos anteriores requieren una formación matématica considerable y modificaciones sustanciales en el software al cambiar cualquier dependencia paramétrica o condición de contorno.

El MESIR [Horno, 2002] es una técnica para la solución numérica de problemas cuyo modelo matemático puede contener una o más ecuaciones en derivadas parciales, ecuaciones constitutivas entre variables, condiciones iniciales, de contorno y otra específicas. A partir de este modelo se obtiene un grafo equivalente (modelo en red) al proceso cuya simulación se lleva a cabo mediante un software adecuado, en este trabajo se ha utilizado Pspice [Pspice, 1994; Nagel, 1975 y 1977, y Vladimirescu, 1994].

El MESIR presenta diferencias notables respecto de los métodos numéricos clásicos. Desde el punto de vista conceptual supone la sustitución de un complicado sistema de ecuaciones diferenciales en diferencias finitas (que ya no es necesario manipular matemáticamente), por un circuito eléctrico equivalente de cuya solución se encarga Pspice. El modelo en red permite visualizar la interconexión entre flujos y fuerzas y relacionar los procesos físicos locales con la evolución de las variables en los componentes eléctricos que simulan el medio. En cuanto a la reticulación sólo requiere una división de la variable espacial, como en los llamados métodos de líneas [Berezin y Zhidkov, 1965, y Rukos, 1978]. Por otro lado no es preciso considerar (a efectos de programación adicional) las leyes de conservación del flujo eléctrico y de unicidad de la temperatura en cualquier punto del medio, en tanto que éstas están contenidas implícitamente en los teoremas de circuitos eléctricos $\left(1^{\mathrm{a}}\right.$ y $2^{\mathrm{a}}$ ley de Kirchhoff) que a su vez son asumidas por el programa de simulación (Pspice advierte al usuario cuando alguna de estas reglas no ha sido respetada en el diseño del modelo).

Cabe señalar la ventaja que supone un buen conocimiento de la teoría de circuitos a la hora de implementar el modelo; no obstante es preciso poco esfuerzo para familiarizarse con 
este aspecto del método, ya que son bastante reducidas las agrupaciones de términos de las expresiones matemáticas que se convierten en elementos individuales o partes del circuito (del orden de cuatro o cinco elementos).

En cuanto a la manipulación y elaboración del programa podemos afirmar que las dificultades son mínimas. La presentación en forma esquemática bajo Windows (tanto para PC o estación de trabajo) permite implementar directamente el modelo (componentes eléctricos y sus conexiones), mientras que la elaboración a través de un fichero de texto requiere un mayor esfuerzo de programación. En ambos casos se dispone de una ayuda continua al usuario que advierte de los posibles errores en la elaboración del programa.

\section{I.3. OBJETIVOS Y DESARROLLO}

El objetivo principal de esta memoria es el estudio de problemas inversos lineales y principalmente no lineales en transferencia de calor: (i) estimación de diferentes flujos aplicados a la superficie de un sólido con propiedades termofísicas constantes o dependientes de la temperatura, (ii) estimación de temperaturas superficiales, (iii) estimación de la generación interna de calor, del coeficiente de convección, emisividad superficial y resistencia térmica de contacto entre sólidos y (iv) estimación de propiedades térmicas (calor específico y conductividad térmica) dependientes de la temperatura. Un segundo objetivo es proponer al MESIR como método de resolución de este tipo de problemas, proporcionando resultados suficientemente precisos (con márgenes de error propios del campo de la ingeniería térmica). El tercer objetivo es, analizar la incidencia en los resultados de diferentes parámetros que afectan a la resolución de este tipo de problemas, errores en las medidas, punto de medida, número de medidas, etc.

El desarrollo de este trabajo comienza en el Capítulo II, donde se presentan los fundamentos teóricos y metodológicos de la ciencia de transmisión de calor por conducción, así como los fundamentos básicos del problema inverso en transferencia de calor, enumerándose los tipos de problemas inversos que existen en el campo de la conducción de calor. Dentro de este capítulo se recoge una síntesis de los conceptos que utiliza el MESIR (método numérico empleado tanto para la solución del problema directo como del inverso).

En el Capítulo III se describe, en primer lugar, los tipos de problemas inversos abordados mediante sus correspondientes modelos matemáticos, incidiendo en la descripción de los múltiples parámetros que intervienen en ellos. A continuación se desarrollan los protocolos de resolución de los problemas de estimación de función y de estimación de parámetros, tanto 
para el caso de variables (incógnita) dependientes de la temperatura como de variables dependientes del tiempo. Se definen los funcionales típicos de estos problemas así como los algoritmos de programación diseñados en esta tesis para ejecutar de forma automática los cálculos numéricos requeridos por el método de simulación por redes. Finalmente se aborda el problema de la estimación simultánea de dos variables y la descripción del procedimiento propuesto para este caso.

El capítulo IV presenta los modelos en red (celda elemental y condiciones de contorno) de los diferentes tipos de problemas inversos abordados en esta memoria, que incluyen modelos para estimación de funciones (lineales o no), estimación de parámetros y estimación simultánea de propiedades térmicas.

En el capítulo V se obtienen estimaciones de flujos incidentes y temperaturas, como condición de contorno, usando los procedimientos y modelos descritos en los capítulos anteriores. Se analiza un amplio espectro de formas de onda que incluyen el flujo incidente en escalón y el triangular, propuesto por Beck [Beck, 1985] para verificar la bondad del método. La influencia en las estimaciones de los diferentes parámetros del problema es analizada en detalle aportando en todos los casos los errores obtenidos gracias al conocimiento de la solución exacta por la forma de plantear el problema.

En el capítulo VI se presentan estimaciones de cuatro magnitudes térmicas de interés en transmisión de calor utilizando diferentes modelos matemáticos. Se trata de la estimación de: generación interna de calor, coeficiente de convección, emisividad superficial y resistencia térmica de contacto. Se estudia la influencia, en los resultados, de diversos parámetros tales como el número de términos del funcional, los errores en las medidas, la posición del punto de medida, el número de medidas, etc.

El capítulo VII abarca las estimaciones de las propiedades térmicas (calor especifico y conductividad térmica), por separado y simultáneamente. Se presentan aplicaciones a productos concretos, estudiando la incidencia de los principales parámetros del problema en la estimación.

El capítulo VIII contiene las aportaciones y conclusiones de esta memnoria.

La descripción de cada uno de los símbolos utilizados a lo largo del texto se recoge al principio del trabajo bajo el epígrafe "Simbología".

Por último, en el apéndice se muestran los códigos fuentes de los programas realizados para algunos de los casos estudiados. 


\section{I.4. PERSPECTIVAS}

En el presente trabajo se aborda la resolución de problemas inversos empleando para ello el MESIR, quedando demostrado la potencialidad de dicho método. En un futuro puede emplearse toda la experiencia acumulada hasta el momento para abordar diversos problemas inversos, estimación de condiciones iniciales, problemas bidimensionales y tridimensionales, introducción en los problemas inversos en transferencia de calor por radiación para la estimación de los parámetros radiativos, inclusión de medidas experimentales reales, problemas inversos donde alguna propiedad varíe a lo largo del tiempo en forma no totalmente definida, etc.

Otras líneas de trabajo dentro del problema inverso son los medios heterogéneos (por ejemplo en la estimación de la resistencia térmica de contacto en procesos de fusión de compuestos), conducción en aletas (ya estudiada como problema directo, [Alarcón y col., 2001]), conducción en medios anisótropos, siendo otro objetivo el incluir estudios más complejos como conducción en fluidos.

Otra acción a abordar en el futuro es el desarrollo de una interface programa-usuario para que el empleo de todos los algoritmos de cálculo diseñados sean de fácil manejo para el usuario.

\section{I.5. REFERENCIAS}

Alarcón García, M., "Transporte de calor en sistemas con aletas. Coeficientes de rendimiento y red de transferencia". Tesis Doctoral. Universidad Politécnica de Cartagena (2001)

Alarcón, M., Alhama, F. y González-Fernández, C.F. “Transient conduction in a fin-wall assembly with harmonic excitation. Network thermal admitance". I. J. Thermal Sci., 41, 386-395 (2002)

Alhama López, F., "Estudio de respuestas térmicas transitorias en procesos no lineales de conducción de calor mediante el método de simulación por redes". Tesis doctoral. Universidad de Murcia (1999)

Al-Khalidy, N., "On the solution of parabolic and hyperbolic inverse heat conduction problems", Int. J. Heat Mass Transf., 41, 3731-3740 (1998) 
Ames, W.F., "Numerical methods for partial differential equations". Academic, 113. New York (1977)

Bass, B.R., "Applications of the finite elements to the inverse heat conduction problem using Beck's second method", J. Eng. Ind., 102, 168-176 (1980)

Beck, J. V. y Wolf, H., "The nonlinear inverse heat conduction problem", ASME Paper, 62HT-46 (1965)

Beck, J.V., "Nonlinear Estimation Applied to the Nonlinear Heat Conduction Problem", Int. J. Heat Mass Transf., 13, 703-716 (1970)

Beck, J. V., Litkouhi B. y St.Clair.Jr. C.R., "Effective sequential solution of the nonlinear inverse heat conduction problem", Numer. Heat Transfer, 5, 275-286. (1982)

Beck, J, Blacwell, B, y Clair Jr. C. "Inverse heat conduction (III-posed problems)“, John Wiley \& Sons, Inc, New York (1985)

Berezin, I.S. y Zhidkov, N.P., “Computing methods II’. Pergamon, Oxford (1965)

Chung, K.S., "The fourth-dimension concept in the finite element analysis of transient heat transfer problems", Int. J. Num. Methods Eng., 17, 315-325 (1981)

Collatz, L., "The numerical treatment of differential equations". Springer-Verlag, 396-405, Berlin (1960)

Crandall, S.H., "Engineering analysis". McGraw-Hill, New York (1956)

Crank J. y Nicolson, P., "A practical method for numerical evaluation of solutions of partial differential equations of the heat-conduction pipe". Proc. Camb. Philos. Soc., 189-191. Wiley-Interscience, New York (1947)

Farnia, I. y Beck, J.V., "Numerical solution of transient heat conduction equation for heattreatable alloys whose thermal properties change with time and temperature", J. Heat Transfer, 99, 471-478 (1977)

González-Fernández, C.F., García Hernández, M. y Horno, J., “Computer simulation of a square scheme with reversible and irreversible charge transfer by network method", J. Electr. Chem., 395, 39-44 (1995)

Horno, J., González-Caballero, A., Hayas, A. and González-Fernández, C.F., "The effect of previous convective flux on the nonstationary through membranes". J. Membr. Sci., 48, 6777 (1990) 
Horno J., Ed. Network Simulation Method, Research Signpost. Trivandrum. Kerala (India), (2002)

Kurpisz K., "Numerical solution of one case inverse heat conduction problems", J. Heat Tran., $113,280-286(1991)$

Lin, S.H., "Transient heat conduction in a composite slab with variable thermal conductivity", Int. J. Num. Meth. Eng., 14, 1726-1731 (1979)

López-García, J.J., Moya, A.A., Horno, J., Delgado, A. y González-Caballero, F., “A network model of the electrical double layer around a colloid particle", J. Colloid Interface Sci., 183, 124-130 (1996)

Maillet, D., Degiovanni, A. y Pasquetti R., "Inverse heat conduction applied to the measurement of heat transfer coefficient on a cylinder: Comparison between an analytical and a boundary element technique”, J. Heat Transfer, 113, 549-557 (1991)

Meric, R.A., "Finite element analysis of optimal heating of a slab with temperature dependent thermal conductivity", Int. J. Heat Mass Transf., 22, 1347-1353 (1979)

Nagel, L. W., "SPICE2: A computer program to simulate semiconductor circuits". University of California, Electronics Res. Lab., ERL-M520, Berkeley, CA (1975)

Nagel, L. W., "SPICE (Simulation program with integrated circuit emphasis)". University of California, Electronics Res. Lab., ERL-M380, Berkeley, CA (1977)

Necati, M y Özisik, “Heat conduction”, John Wiley \& Sons, Inc, New York, cap. 14 (1993)

Orivuri, S., "Efficient method for solution of nonlinear heat conduction problems", Int. J. Num. Meth. Eng., 14, 1461-1476 (1979)

Orlande, H.R.B y Özisik, M.N., "Inverse problem of estimating interface conductance between periodically contacting surfaces", J. Therm. Heat Transf., 7, 2, 319-325 (1993)

Palmieri, J.V. y Rathjem, K.A., "CAVE 3-A General transient heat transfer computer code utilizing eigenvectors and eigenvalues", NASA Contract. Rept. 145290 (1978)

Patankar, S.V., "A numerical method for conduction in composite materials, flow in irregular geometries and conjugate heat transfer", Proc. $6^{\circ}$ Int. Tran. Conf. Toronto, 3, 297 (1978)

Peaceman, D.W. y Rachford, H.H., "The numerical solution of parabolic and elliptic differential equations", J. Soc. Ind. Appl. Math., 3, 28 (1955)

Pspice 6.0. Microsim Corporation, 20 Fairbanks, Irvine, California 92718 (1994) 
Rukos, E.A., "Continuous elements in the finite element method". Int. J. Num. Meth. Eng., 12, 11-33 (1978)

Seminar Eurotherm 68, "Inverse problem and experimental design in thermal and mechanical engineering", Poitiers (Francia) (2001)

Stone, H.L., "Iterative solution of implicit approximations of multi-dimensional partial differential equations", SIAM J. Num. Anal., 5, 530 (1968)

Suzuki, M. y Maeda, S., "Nonlinear diffusion problems with variable diffusivity and timedependent flux boundary conditions”, Int. J. Heat Mass Transf., 21, 653-654 (1978)

Suzuki, M., Matsomoto, S. y Maeda, S., "New analytical method for a non-linear diffusion problem", Int. J. Heat Mass Transf., 20, 883-889 (1977)

Thornton, E.A. y Wieting, A.R., "A finite element thermal analysis procedure for several temperature-dependent parameters", J. Heat Transfer, 100, 551-553 (1978)

Varoglu, E. y Liam Finn, W.D., "Finite elements incorporating characteristic for onedimensional diffusion-convection equation", J. Comp. Phys., 34, 371-389 (1980)

Vladimirescu, A., The spice book. John Wiley \& Sons, Inc., New York (1994)

Wellford, L.C. Jr. y Ayer, R.M., "A finite element free boundary formulation for the problem of multiphase heat conduction", Int. J. Num. Meth. Eng., 11, 933-943 (1980)

Willians, S.D., y Curry, D.M., "An analytical experimental study for surface heat flux determination", J. Spacecraft Rockets, 14, 632-637 (1977)

Wood, W.L., "Control of Crack-Nicolson noise in the numerical solution of the heat conduction equatons", Int. J. Num. Meth. Eng., 11, 1059-1065 (1977) 


\section{Capítulo II}

\section{Fundamentos teóricos y metodológicos}

II.1. Introducción

II.2. La transmisión del calor por conducción

II.2.1. Condiciones iniciales y de contorno

II.3. El problema inverso en conducción del calor

II.3.1. Estimación de función y estimación de parámetros

II.3.1.1. Dificultad del problema inverso. Sensibilidad a los errores de medida

II.3.1.2. Coeficientes de sensibilidad

II.3.2. Tipos de problemas inversos

II.3.2.1. Problemas inversos estacionarios y transitorios

II.3.2.2. Problemas lineales y no lineales

II.3.2.3. Problema inverso según la naturaleza de los procesos

II.3.3. Métodos para la resolución de problemas inversos

II.4. El método de simulación por redes (MESIR)

II.4.1. Descripción

II.4.2. El MESIR como método numérico

II.4.3. Simulación

II.4.4. Ventajas del MESIR

II.5. Referencias 


\section{Capítulo II}

\section{Fundamentos teóricos y metodológicos}

\section{II.1. INTRODUCCIÓN}

En este capítulo se presenta, en primer lugar, una síntesis de la teoría de la conducción del calor de Fourier, modo fundamental de transmisión de calor tal en los medios sólidos. La ecuación diferencial en derivadas parciales para este tipo de proceso de transporte, obtenida bajo la hipótesis de Fourier que asume una relación de proporcionalidad entre las variables densidad de flujo calorífico j $\left(\mathrm{Wm}^{-2}\right)$ y gradiente térmico $\nabla \mathrm{T}\left({ }^{\mathrm{o}} \mathrm{C}\right)$, tiene solución analítica, $\mathrm{T}=\mathrm{T}(\mathrm{r}, \mathrm{t})$, únicamente bajo ciertas hipótesis.

En segundo lugar se presenta el concepto de problema inverso restringido al caso de transmisión de calor por conducción, su planteamiento, los diversos tipos de problemas que tienen interés en la actualidad dentro de este campo, los métodos, procedimientos y algoritmos computacionales propuestos por diferentes investigadores para encontrar soluciones a estos problemas y los criterios para la evaluación de estos métodos.

Por último se describen los fundamentos del Método de simulación por redes (MESIR), herramienta de cálculo numérico para la solución de los diferentes problemas estudiados en esta Memoria. En la aplicación del MESIR podemos distinguir dos etapas bien diferenciadas: por un lado la obtención del modelo en red, o sea, el diseño del circuito eléctrico cuyas ecuaciones en diferencias finitas son formalmente equivalentes a las deducidas del modelo matemático, incluyendo los componentes asociados a las condiciones de contorno, y por otro, la simulación o solución numérica del modelo en red por medio de un software de computación adecuado. Pspice [1994] es el paquete informático que se utiliza en este trabajo y a él dedicaremos un apartado específico bajo el epígrafe “simulación”. 


\section{II.2. LA TRANSMISIÓN DEL CALOR POR CONDUCCIÓN}

La teoría de conducción de calor en medios sólidos puede encontrarse en numerosos libros de texto clásicos y modernos [Mc Adams, 1954; Ozisik, 1997; Hewitt y col., 1994; Mills, 1995; Incropera y Dewitt, 1996, etc]. El objetivo principal del estudio de los procesos de conducción de calor es conocer la distribución transitoria y/o estacionaria de temperaturas $\mathrm{T}(\mathrm{x}, \mathrm{y}, \mathrm{z}, \mathrm{t})$, es decir el campo térmico y, a partir de éste, deducir la densidad de flujo de calor, $\mathrm{j}(\mathrm{t})$, en cualquier sección del medio material. La teoría de conducción puede extenderse a sólidos orgánicos, como ciertos productos alimenticios, en los cuales los procesos de convección son despreciables. La conducción de calor a nivel microscópico se explica merced al intercambio de energía cinética entre las partículas del sólido, que fluye desde las zonas de mayor temperatura hacia las zonas de menor temperatura. Tal descripción, sin embargo, no es necesaria desde un planteamiento macroscópico en el que el medio material se considera como un continuo en términos matemáticos de forma que cualquier elemento de volumen del mismo (dV) es una cantidad infinitesimal respecto de las medidas globales del medio pero contiene un elevado número de partículas elementales (átomos o moléculas).

La descripción analítica del proceso de conducción en un medio continuo parte del balance energético que tiene lugar en un volumen infinitesimal (elemento de volumen o celda), $d \mathrm{~V}$. En ausencia de fuentes y sumideros en el medio, dicho balance puede escribirse en la forma

$$
\mathbf{J}_{\mathrm{ent}}-\mathbf{J}_{\mathrm{sal}}=\mathbf{J}_{\mathrm{alm}}
$$

donde $\mathbf{J}_{\mathrm{ent}}$ es calor por unidad de tiempo (W) que penetra hacia el interior de la celda por su superficie exterior, $\mathbf{J}_{\text {sal }}$ es el calor por unidad de tiempo que sale de la misma y $\mathbf{J}_{\text {alm }}$ es la energía térmica almacenada en su interior, también por unidad de tiempo. Con auxilio del operador divergencia la ecuación (II.1) puede escribirse

$$
\rho c_{\mathrm{e}}(\partial \mathrm{T} / \partial \mathrm{t})+\nabla \cdot \mathrm{j}=0
$$

donde $\rho$ es la densidad del sólido $\left(\mathrm{kg} / \mathrm{m}^{3}\right)$ y c e el calor específico del mismo $\left(\mathrm{J} \mathrm{kg}^{-1} \mathrm{~K}^{-1}\right)$. Ahora, por medio de la ecuación de Fourier

$$
\mathrm{j}=-\mathrm{k} \nabla \mathrm{T}
$$

donde $\mathrm{k}$ es la conductividad térmica y $\nabla \mathrm{T}$ el gradiente de temperaturas, la ecuación (II.2) 
conduce a $\rho c_{\mathrm{e}}(\partial \mathrm{T} / \partial \mathrm{t})+\nabla \cdot(-\mathrm{k} \nabla \mathrm{T})=0$

que es la ecuación general de conducción del calor para cualquier tipo de medios. Si el sólido es homogéneo e isótropo, su conductividad térmica no depende de la posición ni de la dirección espacial con lo que k es un escalar. En este caso la ecuación anterior se reduce a

$$
\rho c_{\mathrm{e}}(\partial \mathrm{T} / \partial \mathrm{t})-\mathrm{k} \Delta \mathrm{T}=0
$$

o bien

$$
(1 / \alpha)(\partial \mathrm{T} / \partial \mathrm{t})-\Delta \mathrm{T}=0
$$

donde $\Delta$ es el operador laplaciana y $\alpha\left(\mathrm{m}^{2} \mathrm{~s}^{-1}\right)$ es la llamada difusividad térmica del sólido, $\alpha=$ $\mathrm{k} / \mathrm{\rho c}_{\mathrm{e}}$. Cuanto mayor sea la difusividad menor es la duración del proceso transitorio de difusión de calor.

En la mayor parte de los problemas estudiados en esta Memoria, tanto la conductividad como el calor específico son parámetros dependientes de la temperatura por lo que la ecuación (II.4) es no lineal y, en general, carece de solución analítica exacta, requiriendo para su solución el concurso de métodos numéricos o analíticos aproximados.

Por medio de la expresión generalizada de la divergencia en coordenadas curvilíneas ortogonales

$$
\nabla \cdot \bar{A}=\left(h_{1} h_{2} h_{3}\right)^{-1} \sum_{i} \frac{\partial}{\partial q_{i}}\left(h_{j} h_{k} A_{i}\right), i \neq j \neq k
$$

donde $\mathrm{q}_{\mathrm{i}}$ son las coordenadas y $\mathrm{h}_{\mathrm{i}}$ los factores de escala, podemos particularizar la ecuación de conducción para los diferentes sistemas de coordenadas.

\section{Expresión general en coordenadas cilíndricas}

Para el elemento de volumen en coordenadas cilíndricas, representado en la figura II.1, tenemos

$$
\begin{array}{lll}
\mathrm{q}_{1}=\mathrm{r} ; & \mathrm{q}_{2}=\theta ; & \mathrm{q}_{3}=\mathrm{z} \\
\mathrm{h}_{1}=1 ; & \mathrm{h}_{2}=\mathrm{r} ; & \mathrm{h}_{3}=1
\end{array}
$$

de modo que la ecuación (II.2) se transforma en

$$
\rho c_{\mathrm{e}}(\partial \mathrm{T} / \partial \mathrm{t})+\mathrm{r}^{-1}\left[\partial\left(\mathrm{rj} \mathrm{j}_{\mathrm{r}}\right) / \partial \mathrm{r}\right]+\left[\partial \mathrm{j}_{\theta} /(\mathrm{r} \partial \theta)\right]+\left[\partial \mathrm{j}_{\mathrm{z}} / \partial \mathrm{z}\right]=0
$$




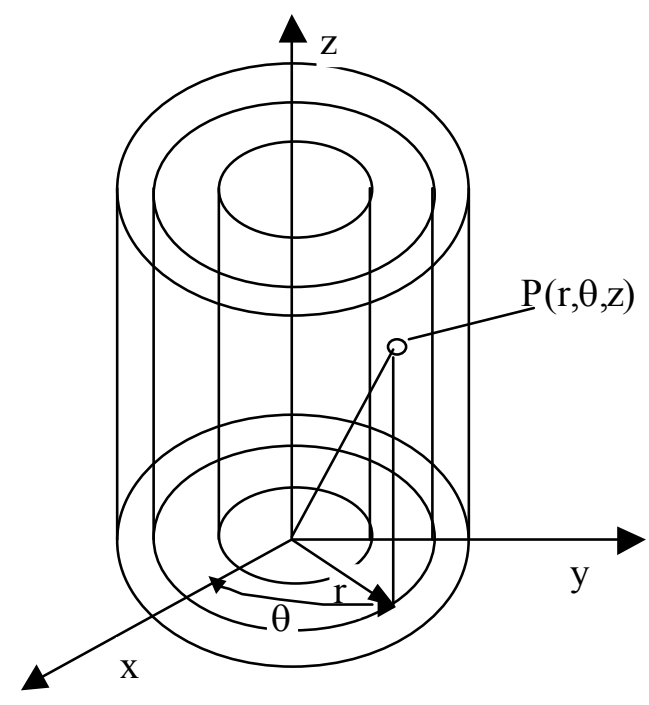

Figura II.1. Elemento diferencial en coordenadas cilíndricas

Las densidades de flujo de calor son:

$\mathrm{j}_{\mathrm{r}}=\mathrm{J}_{\mathrm{r}} / \mathrm{A}_{\mathrm{r}}=-\mathrm{k}_{\mathrm{r}}(\partial \mathrm{T} / \partial \mathrm{r}) ; \mathrm{j}_{\theta}=\mathrm{J}_{\theta} / \mathrm{A}_{\theta}=-\mathrm{k}_{\theta}(\partial \mathrm{T} / \mathrm{r} \partial \theta) ; \mathrm{j}_{\mathrm{z}}=\mathrm{J}_{\mathrm{z}} / \mathrm{A}_{\mathrm{z}}=-\mathrm{k}_{\mathrm{z}}(\partial \mathrm{T} / \partial \mathrm{z})$

(II.10)

donde A es el área transversal, perpendicular a la dirección del flujo térmico (de acuerdo con la dirección que indica el subíndice) y $J$ es el flujo térmico total, $J=j A(W)$, en dicha dirección. Sustituyendo, en la expresión del operador, las áreas elementales para esta geometría,

$$
\mathrm{dA}_{\mathrm{r}}=\mathrm{rd} \mathrm{d} \mathrm{dz} ; \quad \mathrm{dA}_{\theta}=\mathrm{drdz} ; \mathrm{dA}_{\mathrm{z}}=\mathrm{rd} \mathrm{d} \mathrm{dr}
$$

la ecuación (II.5) se transforma en

$$
-\rho c_{e}(\partial T / \partial t)=k_{r}\left[\left(\partial^{2} T / \partial r^{2}\right)+(1 / r) \partial T / \partial r\right]+k_{z}\left(\partial^{2} T / \partial z^{2}\right)+k_{\theta}\left(1 / r^{2}\right)\left(\partial^{2} T / \partial \theta^{2}\right)
$$

que en un medio isótropo se reduce a

$$
-(\partial \mathrm{T} / \partial \mathrm{t})=\alpha\left[\left(\partial^{2} \mathrm{~T} / \partial \mathrm{r}^{2}\right)+(1 / \mathrm{r})(\partial \mathrm{T} / \partial \mathrm{r})+\left(\partial^{2} \mathrm{~T} / \partial \mathrm{z}^{2}\right)+\left(1 / \mathrm{r}^{2}\right)\left(\partial^{2} \mathrm{~T} / \partial \theta^{2}\right)\right.
$$

Para el caso de conducción transitoria unidimensional (radial), (II.9) puede escribirse en la forma

$$
\rho c_{\mathrm{e}}(\partial \mathrm{T} / \partial \mathrm{t})+(1 / \mathrm{r})\left[\partial\left(\mathrm{r} \cdot \mathrm{J}_{\mathrm{r}} / 2 \pi \cdot \mathrm{r} \cdot \mathrm{z}\right) / \partial \mathrm{r}\right]=\rho \cdot \mathrm{c}_{\mathrm{e}} \cdot(\partial \mathrm{T} / \partial \mathrm{t})+(1 / 2 \pi \cdot \mathrm{r} \cdot \mathrm{z})\left[\partial \mathrm{J}_{\mathrm{r}} / \partial \mathrm{r}\right]=0
$$

o bien

$$
-(\partial \mathrm{T} / \partial \mathrm{t})=\alpha\left[(2 / \mathrm{r})(\partial \mathrm{T} / \partial \mathrm{r})+\left(\partial^{2} \mathrm{~T} / \partial \mathrm{r}^{2}\right)\right]
$$




\section{Expresión general en coordenadas esféricas}

Sea el elemento diferencial de volumen representado en la figura II.2. Por medio de las expresiones

$$
\begin{array}{lll}
\mathrm{q}_{1}=\mathrm{r} ; & \mathrm{q}_{2}=\varphi ; & \mathrm{q}_{3}=\theta ; \\
\mathrm{h}_{1}=1 ; & \mathrm{h}_{2}=\mathrm{r} \operatorname{sen} \varphi ; & \mathrm{h}_{3}=\mathrm{r} ;
\end{array}
$$

la ecuación (II.5) se transforma en

$\rho c_{\mathrm{e}}(\partial \mathrm{T} / \partial \mathrm{t})=1 / \mathrm{r}^{2}\left[\partial\left(\mathrm{r}^{2} \mathrm{j}_{\mathrm{r}}\right) / \partial \mathrm{r}\right]+(1 / \mathrm{r} \operatorname{sen} \varphi)\left[\partial\left(\operatorname{sen} \varphi \mathrm{j}_{\varphi}\right) / \partial \varphi\right]+(1 / \mathrm{r} \operatorname{sen} \varphi)\left[\partial \mathrm{j}_{\theta} / \partial \theta\right]$

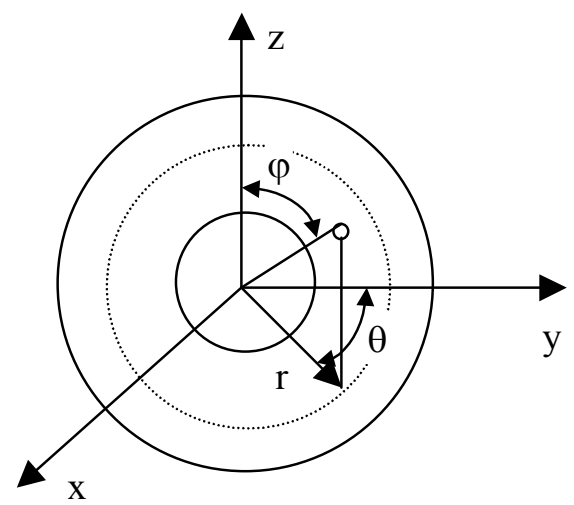

Figura II.2. Elemento de volumen en coordenadas esféricas

y las densidades de flujo de calor son:

$$
\begin{aligned}
& \mathrm{j}_{\mathrm{r}}=\mathrm{J}_{\mathrm{r}} / \mathrm{A}_{\mathrm{r}}=-\mathrm{k}_{\mathrm{r}}(\partial \mathrm{T} / \partial \mathrm{r}) \\
& \mathrm{j}_{\varphi}=\mathrm{J}_{\varphi} / \mathrm{A}_{\varphi}=-\mathrm{k}_{\varphi}(\partial \mathrm{T} / \mathrm{r} \partial \varphi) \\
& \mathrm{j}_{\theta}=\mathrm{J}_{\theta} / \mathrm{A}_{\theta}=-\mathrm{k}_{\theta}(\partial \mathrm{T} / \mathrm{r} \operatorname{sen} \varphi \partial \theta)
\end{aligned}
$$

donde A y J tienen el significado ya mencionado anteriormente. Sustituyendo las áreas elementales

$$
A_{r}=r \cdot d \varphi \cdot r \cdot \operatorname{sen} \varphi \cdot d \theta ; A_{\varphi}=d r \cdot r \cdot \operatorname{sen} \varphi \cdot d \theta ; A_{\theta}=d r \cdot r \cdot d \varphi
$$

en la expresión del operador, obtenemos

$$
\begin{aligned}
& \rho c_{\mathrm{e}}(\partial \mathrm{T} / \partial \mathrm{t})=\mathrm{k}_{\mathrm{r}}\left[(2 / \mathrm{r})(\partial \mathrm{T} / \partial \mathrm{r})+\left(\partial^{2} \mathrm{~T} / \partial \mathrm{r}^{2}\right)\right]+ \\
& +\left(\mathrm{k}_{\varphi} / \mathrm{r}\right)\left[\operatorname{cotg} \varphi(\partial \mathrm{T} / \partial \varphi)+\left(\partial^{2} \mathrm{~T} / \partial \varphi^{2}\right)\right]+\left(\mathrm{k}_{\theta} / \mathrm{r}^{2} \operatorname{sen}^{2} \varphi\right)\left(\partial^{2} \mathrm{~T} / \partial \theta^{2}\right)
\end{aligned}
$$


que para el caso de un medio isótropo se reduce a

$$
\begin{aligned}
& (1 / \alpha)(\partial \mathrm{T} / \partial \mathrm{t})=\left(1 / \mathrm{r}^{2}\right)\left[(\partial / \partial \mathrm{r})\left(\mathrm{r}^{2} \partial \mathrm{T} / \partial \mathrm{r}\right)\right]+\left(1 / \mathrm{r}^{2} \operatorname{sen} \varphi\right)[(\partial / \partial \varphi)(\operatorname{sen} \varphi(\partial \mathrm{T} / \partial \varphi)]+ \\
& +\left(1 / \mathrm{r}^{2} \operatorname{sen}^{2} \varphi\right)\left(\partial^{2} \mathrm{~T} / \partial \theta^{2}\right)=\Delta \mathrm{T}
\end{aligned}
$$

y en conducción transitoria unidimensional (radial)

$$
-(\partial \mathrm{T} / \partial \mathrm{t})=\alpha\left[(2 / \mathrm{r})(\partial \mathrm{T} / \partial \mathrm{r})+\left(\partial^{2} \mathrm{~T} / \partial \mathrm{r}^{2}\right)\right]
$$

o bien

$$
\rho c_{e}(\partial T / \partial t)+\left(1 / r^{2}\right)\left[\partial\left(r^{2} \cdot J_{r} / 4 \pi \cdot r^{2}\right) / \partial r\right]=\rho \cdot c_{e} \cdot(\partial T / \partial t)+\left(1 / 4 \pi \cdot r^{2}\right)\left[\partial J_{r} / \partial r\right]=0
$$

\section{II.2.1. Condiciones iniciales y de contorno}

Para la solución de las ecuaciones diferenciales anteriores, (II.5) ó (II.6), (II.14) ó (II.15), (II.23) ó (II.24), se requiere la especificación de las condiciones de contorno y, en su caso, de las condiciones en la frontera de separación entre distintos medios, además de las condiciones iníciales. Las condiciones de frontera, que informan del valor de la temperatura o del flujo de calor en la superficie exterior del sólido, se expresan matemáticamente mediante ecuaciones diferenciales o algebraicas cuyos argumentos son las variables dependientes, temperatura y flujo de calor. Por otro lado, las condiciones iniciales dan la información del campo de temperaturas en todo el medio en el instante $\mathrm{t}=0$. Estos valores iniciales de temperatura son fácilmente incorporados al modelo en red asignando un potencial inicial a los condensadores asociados con el calor específico.

En problemas de conducción de calor es habitual clasificar las condiciones de contorno de acuerdo con los siguientes tipos:

- Especificación de la temperatura (condición de frontera de primera clase). En este caso se especificará cuál es la temperatura en la superficie límite considerada, pudiendo ser una función de la posición o del tiempo, o ser constante. Si la temperatura es nula se habla de condición de contorno de primera clase homogénea. Esta condición suele expresarse en la forma

$$
\mathrm{T}_{\text {frontera }}=\mathrm{T}(\mathrm{t})
$$

- Especificación del flujo de calor (condición de frontera de segunda clase). La distribución o el valor del flujo de calor a través de la superficie límite puede ser especificada como una función del tiempo o un valor constante. Si $\mathbf{n}$ es el vector normal a la superficie A, esta condición límite suele ser escrita en la forma 


$$
\mathrm{j}_{\text {frontera }}=-\mathrm{k}(\partial \mathrm{T} / \partial \mathbf{n})_{\text {sólido }}=\mathrm{j}(\mathrm{t})
$$

- Condición de contorno convectiva (condición de frontera de tercera clase). Si existe una transferencia convectiva de calor entre la superficie límite (cuya temperatura es $T_{\text {frontera }}$ ) y un medio fluido cuya temperatura lejos de la frontera es $T_{\text {con }}$, esta condición se escribe en la forma (ley de enfriamiento de Newton)

$$
\mathrm{j}_{\text {frontera }}=\mathrm{j}_{\text {con }}=-\mathrm{k}(\partial \mathrm{T} / \partial \mathbf{n})_{\text {sólido }}=\mathrm{h}\left[\mathrm{T}_{\text {frontera }}(\mathrm{t})-\mathrm{T}_{\text {con }}\right]
$$

donde $\mathrm{h}$ es el coeficiente de transferencia de calor o coeficiente de convección. La temperatura del fluido $\mathrm{T}_{\text {con }}$ puede ser constante o función de la posición y/o del tiempo. Si la temperatura de referencia es cero, $\mathrm{T}_{\infty}=0$, la condición de contorno se denomina de tercera clase homogénea. Por otro lado si h tiende a infinito la condición de tercera clase se transforma en la de primera clase.

- Condición de contorno de radiación. Si existe una transferencia de calor por radiación entre la superficie límite y un medio externo cuya temperatura de referencia para la radiación es $\mathrm{T}_{\text {rad }}$ esta condición se escribe en la forma

$$
\mathrm{j}_{\text {frontera }}=\mathrm{j}_{\mathrm{rad}}=-\mathrm{k}(\partial \mathrm{T} / \partial \mathrm{n})_{\text {sólido }}=\sigma_{\mathrm{b}} \varepsilon\left[\mathrm{T}_{\text {frontera }}^{4}(\mathrm{t})-\mathrm{T}_{\mathrm{rad}}{ }^{4}\right]
$$

donde $\varepsilon$ es la emisividad de la superficie y $\sigma_{\mathrm{b}}$ es la constante de Stefan-Boltzmann. Se trata de una condición fuertemente no lineal al involucrar la cuarta potencia de la temperatura.

\section{II.3. EL PROBLEMA INVERSO EN CONDUCCIÓN DEL CALOR}

Cuando se conoce el flujo térmico o la temperatura en la superficie exterior de un sólido (generalmente función del tiempo) y sus características termofísicas, entonces es posible encontrar la distribución de temperaturas en su interior. Esto es lo que se conoce como problema directo (DHCP, direct heat conduction problem). La mayor parte de los problemas de transmisión de calor son problemas de este tipo. Los problemas directos se definen, en general, mediante modelos matemáticos, es decir, por un conjunto de: i) ecuaciones diferenciales, ii) ecuaciones que definen ciertos parámetros en función de las variables dependientes o independientes y iii) ecuaciones que definen las condiciones de frontera y las condiciones iniciales. La solución analítica exacta del problema directo sólo es posible en determinados 
casos frecuentemente alejados de situaciones reales. En general, la no linealidad inherente a los problemas reales de interés científico o técnico requiere el concurso de métodos numéricos o analíticos aproximados para encontrar soluciones. Podemos decir, no obstante, que estos problemas están bien planteados (well-posed) en tanto que su solución existe y es única.

En otros muchos procesos de transferencia de calor, estacionarios o no estacionarios, el flujo incidente o el campo térmico transitorio en el sólido debe ser estimado a partir de ciertas medidas de temperatura tomadas en una o varias localizaciones del mismo. Esto es lo que se conoce como problema inverso. La palabra "estimación” tiene aquí un significado literal ya que no es posible asegurar la solución exacta del problema en ningún caso y ello especialmente debido a que los errores están presentes siempre, de algún modo, en las mencionadas medidas de temperatura expresadas por medio de un número finito (aunque sea alto) de cifras significativas.

En las décadas (1965-85), el problema de la determinación del flujo térmico incidente a partir de medidas de temperatura efectuadas en el interior del sólido es lo que vino en llamarse el problema inverso en conducción de calor (IHCP, inverse heat conduction problem). Naturalmente, existen otros muchos tipos de problemas inversos en conducción de calor pero, históricamente, la denominación IHCP ha hecho referencia a ese problema concreto. En la práctica, el problema directo aparece principalmente en aplicaciones de diseño, mientras que el problema inverso surge en análisis de datos experimentales.

Los problemas inversos se pueden encontrar en diversas ramas de la ciencia y de la ingeniería, en particular en los campos de la ingeniería química, la geofísica, la aeronáutica, la medicina, en metalurgia, procesos mecánicos de fabricación, etc., y es especialmente durante las pasadas dos décadas cuando se ha desarrollado intensamente metodologías para resolver estos tipos de problemas. En el campo de la transmisión de calor se plantean problemas inversos relacionados con procesos de control industrial, tecnología nuclear, procesos de fundición y ablación, de congelación y descongelación, etc. En la actualidad existen tanto encuentros de trabajo de carácter internacional, por ejemplo los organizados por Eurotherm [Seminar 68, Poitiers, 2001], como revistas científicas especializadas dedicadas al estudio de estos problemas tales como las revistas internacionales Inverse Problems e Inverse Problems in Engineering. Por otro lado, es frecuente encontrar secciones especiales en congresos internacionales dedicadas al problema inverso [International Heat Conduction Conference 12, 2002; $3^{\text {nd }}$ European Thermal Sciences Conference, 2000 y Congreso Iberoamericano de Ingeniería Mecánica, 2001] así como continuos trabajos en diferentes revistas internacionales de interés en transferencia de calor (Journal of Heat Transfer-T ASME, International Journal of Heat and Mass Transfer, Heat 
Transfer Engineering, Numerical Heat Transfer, International Journal of Thermal Sciences, Heat Mass Transfer, etc).

Como hemos apuntado, en los problemas inversos de conducción de calor se conoce parte de lo que habitualmente es la solución del problema directo asociado, a saber, un conjunto de valores de temperatura y/o flujos en un punto o sección del medio, respectivamente. Este conjunto de datos, junto con la ecuación de conducción de Fourier, forman parte del enunciado del problema. Cualquiera de las ecuaciones o parámetros restantes, que integrarían el modelo matemático del problema directo asociado, puede ser la incógnita en el problema inverso. Por ejemplo, resulta extremadamente difícil la medida directa del flujo de calor en ciertos lugares tales como la superficie de una pared en contacto con el fuego, la superficie interior de una cámara de combustión, la pared del cilindro de un motor de combustión interna alternativo, etc; en tales situaciones, el flujo de calor puede obtenerse de manera indirecta a partir de las medidas de temperatura en uno o varios puntos del sistema conociendo las propiedades termo-físicas del medio. En los problemas inversos de conducción de calor las incógnitas pueden ser: las condiciones de contorno, las condiciones iniciales, el valor de la generación interna de calor, las propiedades termofísicas del medio, etc.

En contraposición con el problema clásico, podemos decir que el IHCP es un problema mal planteado desde un punto de vista formal (ill-posed problem), matemáticamente hablando, en tanto que existen muchas soluciones al mismo; es decir se trata de problemas que no satisfacen los requisitos generales de existencia, unicidad y estabilidad bajo pequeños cambios de los datos de entrada.

Conseguir una solución convergente y precisa en estos problemas es una tarea extremadamente difícil y muchos métodos numéricos clásicos que aportan soluciones a algunos tipos de problemas inversos sencillos no son capaces de proporcionar soluciones en otros más complejos. En este sentido Beck y col. en su libro "Inverse Heat Conduction, Ill-posed problem" [1985] propone comprobar la eficiencia del método propuesto resolviendo el problema de la determinación directa de flujos de calor (condición de contorno de segunda clase). Aunque la obtención de la temperatura en la frontera, en lugar del flujo de calor, permitiría conocer este último de forma indirecta mediante la determinación del gradiente térmico, la validación del método se refiere a la obtención directa de la condición de contorno de segunda clase (la cual es más difícil de obtener que el campo térmico) sin una evaluación previa del campo térmico. Textualmente "... the following seemingly contradictory statement can be made: the heat flux is more difficult to calculate accurately than the surface temperature" [Beck y col., 1985]. 
En las figuras II.3, II.4 y II.5 se representan simbólicamente tres ejemplos de problemas inversos (la línea continua es la solución exacta): estimación de un flujo de calor en la frontera $j(t)$, estimación del calor específico en función de la temperatura $c_{e}(T)$, y estimación del coeficiente de convección $\mathrm{h}(\mathrm{T})$, respectivamente. Es frecuente encontrar también, en la literatura científica, la estimación de este coeficiente en función del tiempo, h(t).

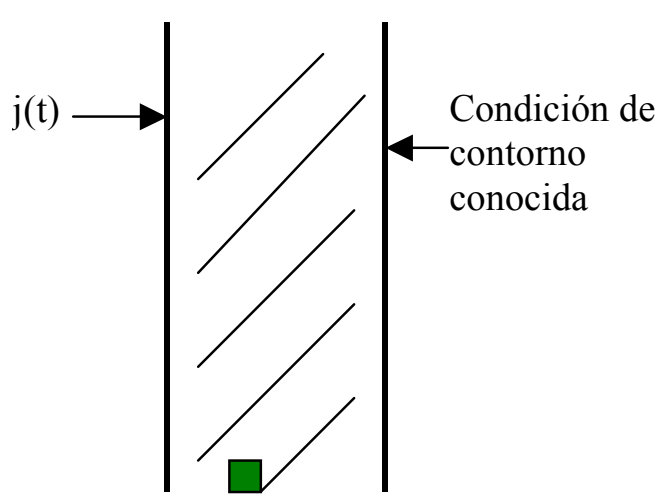

Punto de medida

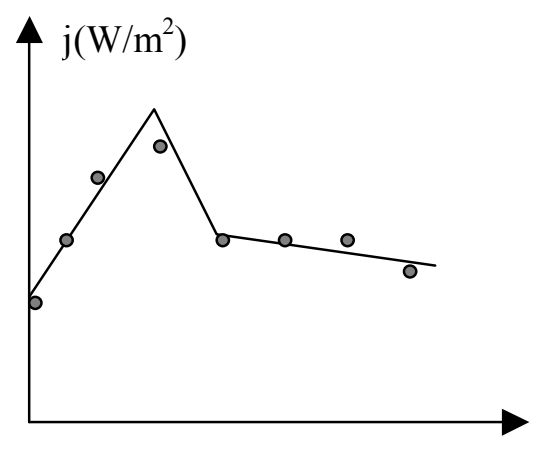

$\mathrm{t}(\mathrm{s})$

Solución del flujo de calor

Figura II.3. Estimación de un flujo de calor, j(t)

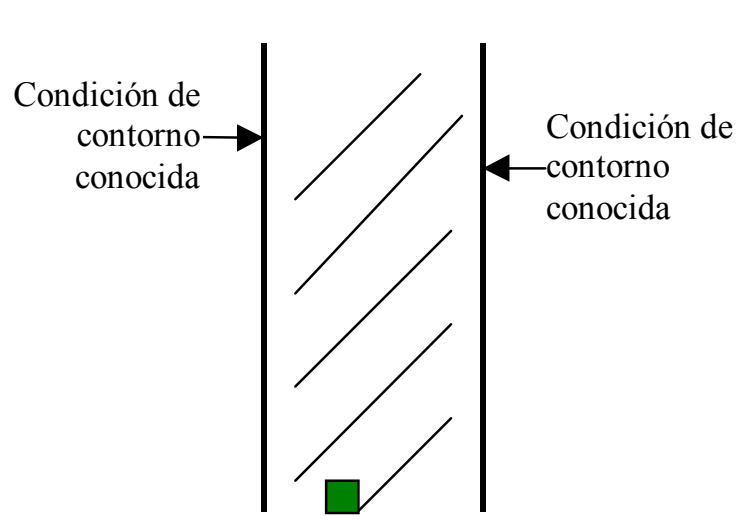

Punto de medida

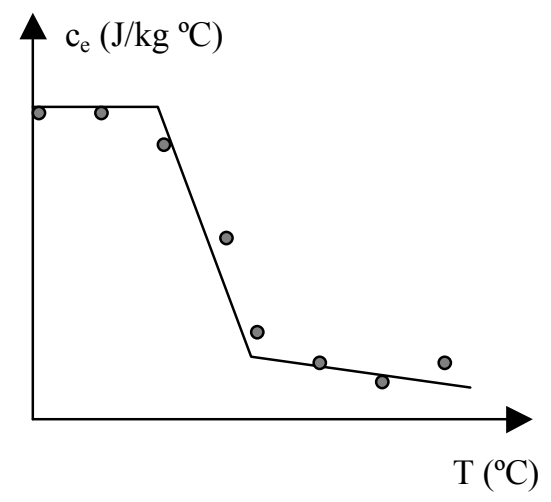

Estimación del calor específico

Figura II.4. Estimación del calor específico, $\mathrm{c}_{\mathrm{e}}(\mathrm{T})$ 


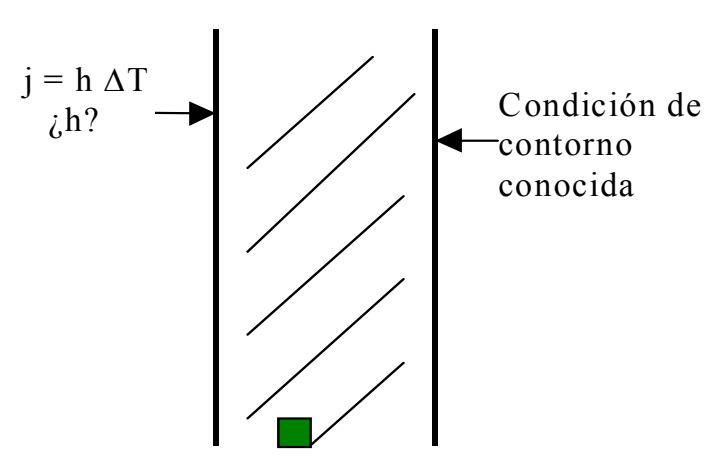

Punto de medida

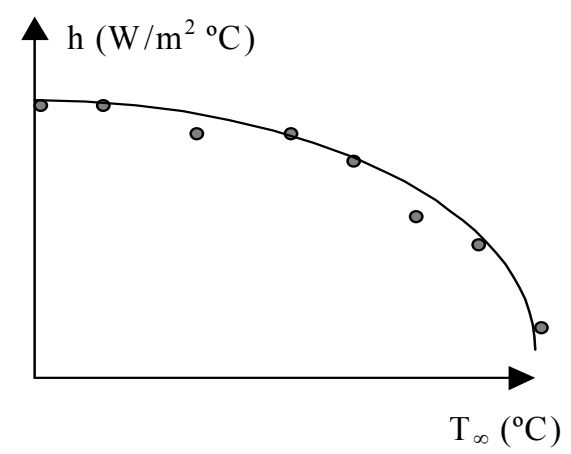

Estimación del coeficiente de convección

Figura II.5. Estimación del coeficiente de convección convectivo, h(T)

Mientras que el modelo matemático del DHCP, en un medio 1D con propiedades físicas constantes, siendo $\mathrm{j}_{\mathrm{o}}(\mathrm{t}), \mathrm{T}_{\mathrm{o}}(\mathrm{t})$ y $\mathrm{f}(\mathrm{x})$ y $\rho, \mathrm{c}_{\mathrm{e}}(\mathrm{T})$ y $\mathrm{k}(\mathrm{T})$ funciones y parámetros conocidos, es de la forma:

\section{Modelo matemático de un problema directo}

$$
\begin{array}{ll}
\rho c_{\mathrm{e}}(\partial \mathrm{T} / \partial \mathrm{t})=\nabla(\mathrm{k} \nabla \mathrm{T}) & 0<\mathrm{x}<\mathrm{L}, \mathrm{t}>0 \\
\mathrm{j}\left(\mathrm{x}_{\mathrm{s}}, \mathrm{t}\right)=\mathrm{j}_{\mathrm{o}}(\mathrm{t}) \text { o } \mathrm{T}\left(\mathrm{x}_{\mathrm{s}}, \mathrm{t}\right)=\mathrm{T}_{\mathrm{o}}(\mathrm{t}) & \left(\text { condiciones de } 1^{\mathrm{a}} \mathrm{o} 2^{\mathrm{a}} \text { clase }\right) \\
\mathrm{T}(\mathrm{x}, 0)=\mathrm{f}(\mathrm{x}) & (\text { condición inicial) }
\end{array}
$$

donde $\mathrm{x}_{\mathrm{s}}$ designa la posición de la frontera, para el IHCP donde la incógnita es la condición de contorno de $2^{\mathrm{a}}$ clase, bajo las mismas hipótesis, el planteamiento matemático conduce al siguiente modelo

\section{Modelo matemático de un problema inverso}

$$
\begin{array}{ll}
\rho \mathrm{c}_{\mathrm{e}}(\partial \mathrm{T} / \partial \mathrm{t})=\nabla(\mathrm{k} \nabla \mathrm{T}) & 0<\mathrm{x}<\mathrm{L}, \mathrm{t}>0 \\
\mathrm{~T}(\mathrm{x}, 0)=\mathrm{f}(\mathrm{x}) & (\text { condición inicial) } \\
\mathrm{T}\left(\mathrm{x}_{\mathrm{f}}, \mathrm{t}_{\mathrm{j}}\right) \equiv \mathrm{T}_{\mathrm{j}} & \mathrm{t}=\mathrm{t}_{\mathrm{j}}(\mathrm{j}=1,2, \ldots \mathrm{n}) \text { (condición adicional) }
\end{array}
$$


donde $\mathrm{T}\left(\mathrm{x}_{\mathrm{f}}, \mathrm{t}_{\mathrm{j}}\right) \equiv \mathrm{T}_{\mathrm{j}}$ es un conjunto de medidas de temperatura en la posición $\mathrm{x}_{\mathrm{f}}$ en los instantes $\mathrm{t}=\mathrm{t}_{\mathrm{j}}(\mathrm{j}=1,2, \ldots \ldots \mathrm{n})$.

Los primeros trabajos sobre problemas inversos estaban orientados hacia el campo de investigación militar, en particular hacia la industria de armamento y la industria aeroespacial. Entre estos trabajos pioneros cabe mencionar los de Mirsepassi [1959] y Stolz [1960], quienes estimaron los flujos de calor en enfriamiento de cuerpos simples con métodos numéricos y gráficos. Por esas mismas fechas fue traducido un trabajo de Shumakov [1957], sobre el problema inverso en medios semi-infinitos. Beck y algunos colaboradores iniciaron trabajos similares en esos años y desarrollaron los conceptos fundamentales que permitieron refinar los resultados iniciales de Stolz y Mirsepassi (v. referencias bibliográficas en Beck y col., 1985, Cap. 1). Antes de 1980 ya se habían publicado cerca de 300 trabajos relacionados con el problema inverso en general y se había trabajado en casos de gran interés técnico tales como el estudio térmico de componentes de reactores nucleares bajo prueba, calentamiento periódico en cámaras de motores de combustión interna alternativos, solidificación del vidrio, calorimetría indirecta para uso en laboratorio, estudio de curvas transitorias en ebullición, etc. Una detallada referencia bibliográfica de los trabajos más importantes en este campo puede encontrarse en el libro de Beck anteriormente citado.

Desde entonces hasta la fecha son innumerables los trabajos que se han publicado sobre problema inverso en transmisión de calor, en sus diversas modalidades. La complejidad de los mismos ha ido creciendo hasta llegar a planteamientos fuertemente no lineales y de cálculo simultáneo de varios parámetros. Algunos de los trabajos de reciente publicación en este campo son Huang y Özisik [1991], Yang [1999], Lorion y col. [2001] y Jarny y col. [2001].

Conviene hacer mención en este punto al problema inverso fuertemente no lineal de conducción-radiación (IHCRP) [Ruperti y col., 1995]. Se trata de estimar cualquiera de las propiedades térmicas de un sólido semitransparente a partir de las medidas transitorias de temperatura en su interior. La ecuación de la energía contiene un término fuente asociado a la radiación. Sin embargo, en el problema inverso de radiación (IRP) se estima el perfil de temperaturas u otra condición de contorno (incluso las propiedades de radiación del medio) a partir de la medida de la radiación externa [Li y Yang, 1997]. Estos materiales semitransparentes son ampliamente utilizados en la construcción, instalaciones de energía solar y sistemas de alta temperatura.

Una de las dificultades para la solución del problema inverso es su extremada sensibilidad a los errores en las mediciones. Como hemos mencionado, parte de los datos conocidos en el problema inverso son una serie de medidas experimentales que, por su propia 
naturaleza, no son exactas; entre éstas están las medidas de temperatura, las de posición de los sensores, las del tamaño del sólido, etc. En la mayor parte de los problemas la principal fuente de error o incertidumbre está en la medida de temperaturas por lo que, en aras de simplificar los cálculos, el resto de las medidas y parámetros del problema se consideran no afectados de error. Además, la influencia en las medidas de la propia presencia del sensor, su calibración, las pérdidas por conducción y convección, etc. se consideran despreciables hasta el límite de que los errores puedan considerarse aleatorios y describirse en términos estadísticos. Las hipótesis, generalmente aceptadas, que permiten esta descripción pueden encontrarse en el Capítulo 1, p. 10 de texto de Beck y col. [1985]. Algunas de ellas serán comentadas a lo largo de esta Memoria.

Para llegar a la solución se procede a sucesivos tanteos hasta conseguir que los resultados de la comparación entre los datos experimentales (medidas) con los que proporciona la solución parcial del problema inverso, obtenidos por medio de una expresión matemática a la que llamamos funcional, sean aceptables dentro de un cierto rango.

\section{II.3.1. Estimación de función y estimación de parámetros}

Como se ha mencionado anteriormente, el IHCP esta asociado a la determinación del flujo de calor en la frontera del medio. En este sentido la denominación "estimación de función" (function estimation) está igualmente asociada a la estimación concreta de dicho flujo y no a otras funciones desconocidas dependientes del tiempo, de la posición o de la propia variable dependiente temperatura. En este sentido, el problema inverso de la estimación del calor especifico función de la temperatura, a pesar de que obviamente se trata de la estimación de una función desconocida no se ha clasificado históricamente como un problema de estimación de función sino como un problema de estimación de parámetros. Lo mismo ocurre con las estimaciones de la conductividad térmica $\mathrm{k}(\mathrm{T})$, el coeficiente de convección en convección natural $\mathrm{h}(\mathrm{T})$, y otras muchas variables incógnitas en problemas inversos. Esta "inadecuada" nomenclatura, adoptada hasta mediados de la década 80-90, se justifica en parte por la importancia del problema clásico IHCP, especialmente en el campo de la ingeniería aeroespacial. Uno de los problemas más interesantes en este sentido era la determinación de las condiciones de flujo térmico incidente en la superficie externa de un transbordador espacial que re-entra en la atmósfera terrestre; condiciones muy afectadas por la decisión de los técnicos de control o de los pilotos de la nave. Puede apreciarse que en este sentido no se trata del mismo problema que la determinación de las características termofísicas de un medio, las cuales no pueden ser manipuladas en modo alguno. A partir de aquí seguiremos la nomenclatura ya extendida en la literatura científica incluyendo dentro de la denominación "estimación de 
funciones" los problemas inversos en los que se trata de estimar una magnitud variable de la que no se conoce a priori información matemática sobre el tipo de dependencia. Por otro lado, como "estimación de parámetros" se considera la evaluación de las constantes que intervienen en la forma funcional de la dependencia de una cierta magnitud (expresión matemática definida en función de uno o más parámetros).

Los problemas de estimación de parámetros, abordados fundamentalmente desde una perspectiva estadística, no suelen ser tan complejos como los de estimación de funciones (especialmente si se trata de un pequeño número de parámetros), cuyas dificultades principales están asociadas al cálculo numérico [Beck, 1977; Beck y Arnold, 1977]. Para estimación del flujo incidente del IHCP clásico se requieren cientos de valores discretos de dicho flujo para definir adecuadamente una solución mientras que sólo unos pocos valores de la conductividad térmica podrían especificar suficientemente este parámetro cuando es independiente de la temperatura. En consecuencia, el tiempo de computación de los problemas de estimación de función es muy superior al tiempo de computación de los problemas de estimación de parámetros.

Al estimar una magnitud cuyo tipo de dependencia se ignora, la función desconocida se reproduce por medio de un número finito de tramos (problemas de minimización dimensional infinita) [Silva-Neto y Özisik, 1992; Huang y col., 1995]. Si, por el contrario, se dispone de información previa de la forma de la función buscada, cuya expresión matemática contendrá un cierto número de parámetros, hablamos de un problema inverso de estimación de parámetros (problemas de minimización dimensional finita) [Beck, 1977; Huang y Ozisik, 1991].

Los ejemplo de las figuras II.3 a II.5 corresponden a estimación de funciones. En la figura II.6 se presenta explícitamente un caso de estimación de parámetros ya que el flujo de calor incidente depende del tiempo de acuerdo con la expresión $\mathrm{j}(\mathrm{t})=\mathrm{k}_{0}+\mathrm{k}_{1} \mathrm{t}$. Los parámetros a estimar son sólo dos $\mathrm{k}_{0} \mathrm{y} \mathrm{k}_{1}$ por lo que se trata de un problema relativamente sencillo, cuya solución podría alcanzarse con pocas iteraciones.

Todos resultarían ser problemas de estimación de parámetros si la forma de las curvas $j(t), c_{e}(T), h\left(T_{\infty}\right)$, fueran, a priori, conocidas. 


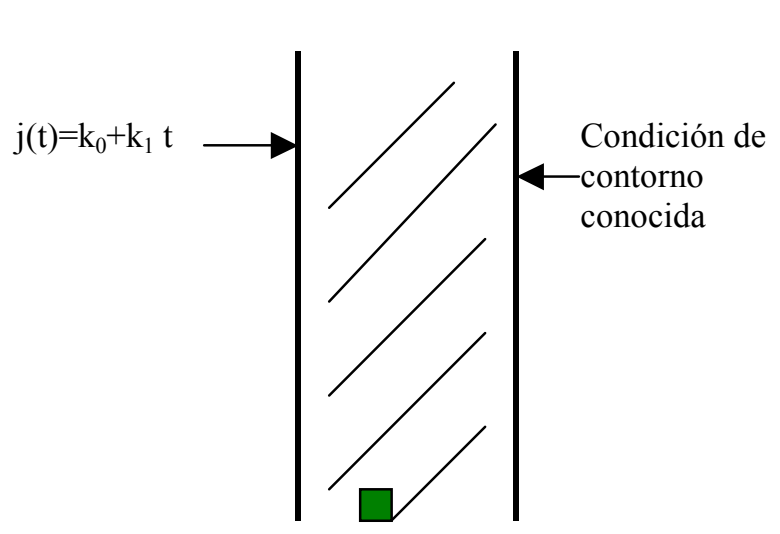

Punto de medida

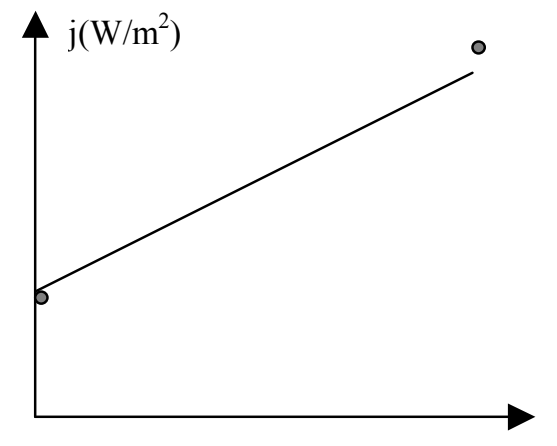

$\mathrm{t}(\mathrm{s})$

Flujo de calor estimado mediante parámetros

Figura II.6. Estimación de los parámetros que definen un flujo de calor incidente

\section{II.3.1.1. Dificultad del problema inverso. Sensibilidad a los errores de medida}

La obtención de una solución convergente en los problemas inversos de transmisión de calor y, en general, en cualquier tipo de problema inverso, es muy difícil debido entre otras cosas a que dicha solución es extremadamente sensible a los errores de las medidas y al número de éstas. Por ejemplo, para un problema inverso 1D de estimación de un flujo de calor j(t), figura II.3, si se aumenta la cantidad de información (es decir, el número de medidas experimentales) es necesario reducir el tiempo de paso entre los valores discretos de la curva $\mathrm{j}_{\mathrm{i}}(\mathrm{t})$ estimada. Sin embargo, el uso de pequeños tiempos de paso frecuentemente introduce inestabilidades en la solución del IHCP a menos que se empleen restricciones específicas. Así, mientras que en el problema directo las dificultades asociadas con la estabilidad o la falta de convergencia en la solución se corrigen aumentando la reticulación temporal (disminuyendo el intervalo de tiempo de cálculo), en el problema inverso, las dificultades para alcanzar una solución crecen al disminuir este tiempo, es decir, al aumentar el número de medidas experimentales.

\section{II.3.1.2. Coeficientes de sensibilidad}

El coeficiente de sensibilidad se define como la primera derivada de la variable que se mide (por ejemplo, la temperatura) con respecto al parámetro cuyo valor o dependencia se desea calcular (por ejemplo el flujo de calor en la frontera, $\mathrm{j}$ ). Si dichos coeficientes son pequeños o están correlacionados el problema numérico de la estimación del parámetro es difícil por ser muy sensible a los errores en las medidas. En la estimación de funciones o de parámetros es importante conocer los valores de los coeficientes de sensibilidad para tener una idea de las dificultades de la estimación (zonas de difícil estimación, etc) y también incluso mejorar el 
diseño experimental. Información sobre los coeficientes de sensibilidad puede encontrarse en la literatura científica [Beck y col., 1985; Necati y Ozisik, 1993; Woodbury y Ke, 2000] o elaborarse mediante la solución numérica del problema concreto.

En concreto, en el caso de estimar un flujo de calor dependiente del tiempo, el coeficiente de sensibilidad es la primera derivada de la temperatura $T$ en el punto de medida, $\mathrm{x}_{\mathrm{f}}$, $(\mathrm{f}=1,2, \ldots . \mathrm{P})$, siendo $\mathrm{P}$ el número total de puntos de medida, $\mathrm{y}$ en los instantes $\mathrm{t}_{\mathrm{j}}(\mathrm{j}=1,2, \ldots . . \mathrm{n})$, respecto al flujo de calor $\mathrm{j}$ [Beck y col., 1985]. Si llamamos $\mathrm{S}_{\mathrm{f}}\left(\mathrm{x}_{\mathrm{f}}, \mathrm{t}_{\mathrm{j}}\right)$ al coeficiente de sensibilidad podemos escribir

$$
\mathrm{S}_{\mathrm{f}}\left(\mathrm{x}_{\mathrm{f}}, \mathrm{t}_{\mathrm{j}}\right) \equiv \partial \mathrm{T}\left(\mathrm{x}_{\mathrm{f}}, \mathrm{t}_{\mathrm{j}}\right) / \partial \mathrm{j}
$$

Para que una estimación sea óptima, los sensores de medida deben colocarse en posiciones donde las lecturas sean más sensibles a los cambios del parámetro desconocido. Esto equivale a decir que la variación de temperatura entre dos medidas de temperatura consecutivas debe ser lo mayor posible; en particular, mayor que los errores de medida.

Para establecer la mejor localización de los sensores, intervalo de tiempo entre medidas y demás parámetros importantes, es necesario hacer una evaluación, bien numérica o experimental, para optimizar los coeficientes de sensibilidad para cada uno de estos parámetros. Si no se consiguen coeficientes de sensibilidad apreciables hay que elegir otro tipo de parámetros. Los coeficientes de sensibilidad dependen sólo de las condiciones de contorno y de la geometría del problema considerado.

\section{II.3.2. Tipos de problemas inversos}

Los problemas inversos en conducción del calor se pueden clasificar según diversos criterios:

\section{II.3.2.1. Problemas inversos estacionarios y transitorios}

Los problemas estacionarios son los más simples puesto que su solución sólo exige conocer la conductividad térmica del medio, $\mathrm{k}$, y no es preciso disponer de una historia de temperaturas [Martin y Dulikravich, 1996; Al-Najem y col., 1998; Throne y Olson, 2001]. Cuando se quiere estimar la conductividad térmica en un medio en situación estacionaria, es necesario recurrir a dos métodos: i) el método directo, donde la conductividad puede ser obtenida directamente aplicando la ley de Fourier. Normalmente el error obtenido en la estimación es considerable al realizarlo experimentalmente, ya que existen pérdidas de calor difíciles de medir, Laubitz [1969], y ii) el método indirecto, más complicado, en donde es 
necesario obtener la solución inversa de la ecuación de transferencia del calor, asumiendo la existencia de no linealidades importantes. Algunos trabajos en este campo son los de Sawaf y col. [1995], Huang y Chin [2000], Martin, y Dulikravich [2000] y Chantasiriwan [2002]

El problema inverso de transmisión del calor transitorio se puede dividir a su vez en dos categorías: aquellos que permiten ser resueltos mediante formulación agrupada (lumped capacity model), en los que la distribución de temperaturas es prácticamente la misma en todo el medio [Beck, 1985], y aquellos a los que no puede aplicarse dicho modelo (la mayor parte de los problemas entran dentro de esta última categoría). En todos los problemas transitorios, el conjunto de medidas de temperatura forma parte de los datos de entrada. En la formulación agrupada el único parámetro térmico de interés es la capacidad térmica volumétrica (calor específico por unidad de volumen), $\rho c_{\mathrm{e}}$, pues la conductividad puede tomarse infinita para esta hipótesis. En el caso de la formulación distribuida es preciso conocer también la conductividad térmica y su dependencia con la temperatura.

\section{II.3.2.2. Problemas lineales y no lineales}

Esta clasificación es estrictamente matemática. Las causas de no linealidad están principalmente en las dependencias de las características térmicas del medio con la temperatura y/o en ciertas condiciones de frontera, aunque existen otras. Las características térmicas pueden ser función de la posición sin afectar la linealidad del problema. La linealidad, si existe, es una propiedad importante ya que permite la superposición y generalmente elimina la necesidad de iterar para buscar la solución. Si el IHCP lineal es tratado como si fuera no lineal se consume excesivo tiempo de computación.

Un camino para determinar la linealidad en un problema de estimación consiste en inspeccionar los coeficientes de sensibilidad. Si los coeficientes de sensibilidad no son funciones de los parámetros a estimar, entonces el problema de estimación es lineal, en caso contrario es no lineal. Para aclarar este punto mencionamos un ejemplo. Sea una placa 1D de longitud $\mathrm{L}$ expuesta por una de sus superficies laterales a un flujo de calor constante $\mathrm{j}_{\mathrm{o}} \mathrm{y}$ en el otro extremo a condición adiabática. Se definen los parámetros adimensionales siguientes:

$$
\mathrm{T}_{\mathrm{O}}=\frac{\mathrm{T}-\mathrm{T}_{\infty}}{\mathrm{j}_{\mathrm{O}} \mathrm{L} / \mathrm{k}} ; \mathrm{t}_{\mathrm{o}}=\frac{\alpha \mathrm{t}}{\mathrm{L}^{2}} ; \mathrm{x}_{\mathrm{o}}=\frac{\mathrm{x}}{\mathrm{L}}
$$

La solución del campo térmico transitorio viene dada por: 


$$
\mathrm{T}_{\mathrm{o}}\left(\mathrm{x}_{\mathrm{o}}, \mathrm{t}_{\mathrm{o}}\right)=\mathrm{t}_{\mathrm{o}}+\frac{1}{3}-\mathrm{x}_{\mathrm{o}}+\frac{1}{2} \mathrm{x}_{\mathrm{o}}^{2}-\frac{2}{\pi^{2}} \sum_{\mathrm{m}=1}^{\infty} \frac{1}{\mathrm{~m}^{2}} \mathrm{e}^{-\mathrm{n}^{2} \pi^{2} \mathrm{t}_{\mathrm{o}} \cos \left(\mathrm{n} \pi \mathrm{t}_{\mathrm{o}}\right)}
$$

Para tiempos pequeños, la solución para $\mathrm{x}_{\mathrm{o}}=0$ (o sea en el extremo donde se aplica el flujo de calor) es:

$$
\mathrm{T}_{\mathrm{o}}\left(0, \mathrm{t}_{\mathrm{o}}\right)=2\left(\frac{\mathrm{t}_{\mathrm{o}}}{\pi}\right)^{1 / 2} \quad \text { para } \mathrm{t}_{\mathrm{o}}<0.3
$$

que volviendo a las variables dimensionales nos proporciona la expresión

$$
\mathrm{T}(0, \mathrm{t})=\mathrm{T}_{\infty}+\frac{2 \mathrm{j}_{\mathrm{O}} \mathrm{L}}{\mathrm{k}}\left(\frac{\alpha \mathrm{t}}{\pi \mathrm{L}^{2}}\right)^{1 / 2} \quad \text { para } \mathrm{t}_{\mathrm{o}}<0.3
$$

Si derivamos la temperatura respecto a $\mathrm{j}_{\mathrm{o}}$ (coeficiente de sensibilidad) se obtiene:

$$
\frac{\partial T(0, t)}{\partial \mathrm{j}_{\mathrm{o}}}=2 \frac{\mathrm{L}}{\mathrm{k}}\left(\frac{\alpha \mathrm{t}}{\pi \mathrm{L}^{2}}\right)^{1 / 2} \quad \operatorname{para~}_{\mathrm{o}}<0.3
$$

expresión que resulta independiente de $\mathrm{j}_{\mathrm{o}}$, por lo tanto el problema de estimación es lineal. A la misma conclusión se hubiera llegado estudiando el modelo matemático.

Si de lo que se trata es de estimar el parámetro $\alpha$, tomando la derivada de $\mathrm{T}$ con respecto a dicho parámetro se obtiene:

$$
\frac{\partial \mathrm{T}(0, \mathrm{t})}{\partial \alpha}=\frac{\mathrm{j}_{\mathrm{o}} \mathrm{L}}{\mathrm{k}}\left(\frac{\mathrm{t}}{\pi \alpha \mathrm{L}^{2}}\right)^{1 / 2} \quad \operatorname{para~}_{\mathrm{o}}<0.3
$$

Dado que este resultado es función del parámetro estimado, $\alpha$, se trata de un problema no lineal.

\section{II.3.2.3. Problema inverso según la naturaleza de los procesos}


Una clasificación inicial del problema inverso hace referencia precisamente al parámetro o ecuación que se busca. Así el problema inverso de conducción de calor puede referirse a:

i) estimación de condiciones de contorno en una o ambas fronteras (temperaturas y/o flujos de calor). Este es el caso de paredes de hornos y tuberías, superficies exteriores de aeronaves, etc. Los datos conocidos son las temperaturas o flujos de calor en puntos interiores del sólido o en uno de sus extremos,

ii) estimación de condiciones iniciales (campo térmico del medio en $\mathrm{t}=0$ ),

iii) estimación de propiedades térmicas del medio (conductividad, calor específico, calores de fusión, la emisividad superficial...) dependientes o no de la temperatura y de la posición. Caso de materiales compuestos (composites), alimentos y otros medios homogéneos y heterogéneos,

iv) estimación de coeficientes térmicos asociados a la frontera de separación entre medios, tales como el coeficiente de convección (dependiente o no de la temperatura y de la posición), conductancia de contacto, etc.,

v) estimación de fuentes o sumideros de calor, asociados con reacciones exotérmicas o endotérmicas, en el interior de los medios,

vi) estimación de parte de la solución cuando se conoce otra parte de la misma, es decir determinar la respuesta transitoria entre $0<\mathrm{t}<\mathrm{t}_{1}$ conocida la respuesta entre $\mathrm{t}_{1}<\mathrm{t}<\mathrm{t}_{2}$, o bien determinar la respuesta espacial en $0<\mathrm{x}<$ $\mathrm{x}_{1}$ a partir de los datos en $\mathrm{x}_{1}<\mathrm{x}<\mathrm{x}_{2}$,

vii) estimación de geometrías en medios multicapa,

viii) otros tipos de problemas: estimación de movimientos de frontera en problemas de frontera móvil, estimación de distribución de heterogeneidades, determinación de parámetros en mecanismos en ingeniería, obtención de las permeabilidades absoluta y relativa en medios porosos, determinación de propiedades ortotrópicas, problemas de optimización y control, etc. 


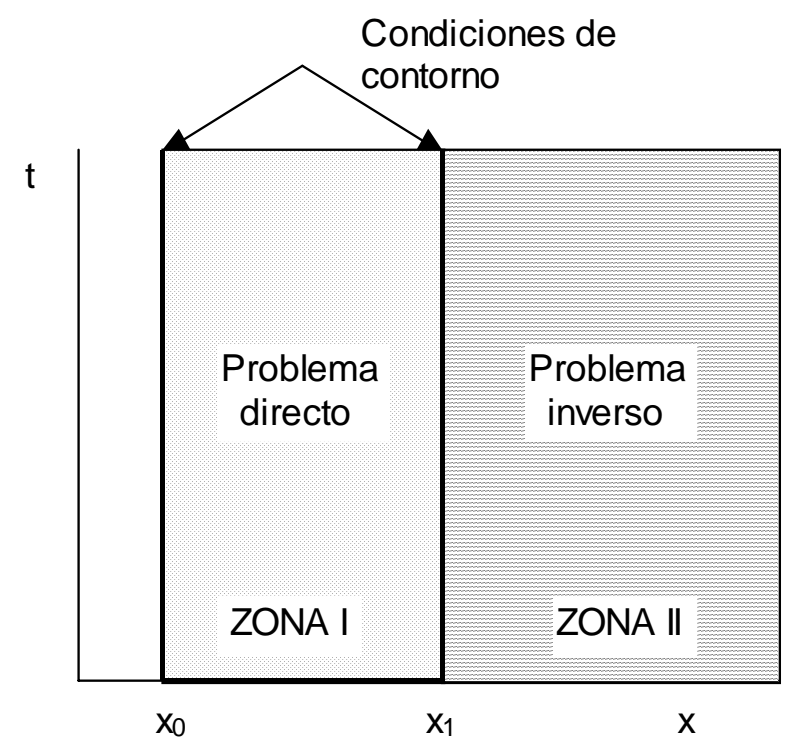

Figura II.7. Problema inverso de estimación de una condición de contorno

El problema de estimación de una condición de contorno (caso i) es el más antiguo de los conocidos. La figura II.7 ilustra por medio de un diagrama x-t la diferencia entre el problema directo y el inverso en función de los datos conocidos. Las condiciones de contorno (flujos de calor o temperaturas) están especificadas en los puntos $\mathrm{x}_{0} \mathrm{y} \mathrm{x}_{1}$, y las condiciones iniciales están representadas en $\mathrm{t}=0$. Si las condiciones a determinar están en un punto de la zona $\mathrm{I}$, el problema es de tipo directo, por el contrario para puntos dentro de la zona II el problema es de tipo inverso, ya que en el primer caso las condiciones de contorno están especificadas, mientras en el segundo caso es necesario estimarlas para posteriormente obtener la distribución de temperaturas en todos los puntos interiores de la zona II. Los trabajos más antiguos relacionados con este tipo de problemas son Stoltz [1960], Burggraf [1964] y Weber [1981].

\section{II.3.3. Métodos para la resolución de problemas inversos}

Como es conocido, para resolver la ecuación de conducción se emplean métodos analíticos o semi-analíticos, tales como el Método de Transformadas de Laplace u otros métodos integrales, el Método de las funciones de Green, uso del Teorema de Duhamel, etc., los cuales requieren un profundo conocimiento en matemáticas, y son aplicables estrictamente a problemas lineales [Stolz, 1960; Beck, 1963, 1968 y 1979; Sparrow y col., 1964; Murio, 1981; Grysa y col. 1981]. Muchas de las soluciones aportadas por estos métodos son desarrollos serie cuya convergencia es lenta, lo cual es un inconveniente añadido. Técnicas de solución exactas fueron propuestas por Stolz [1960], Burggraf [1964], Langford [1967], e Imber y Khan [1972]. Los métodos numéricos son generalmente preferibles por su generalidad y precisión aceptable 
así como por permitir abordar problemas no lineales. El método de diferencias finitas (FDM) ha sido usado por el propio Beck [1970 y 1982], Beck y Wolf [1965], Willians y Curry [1977] y otros autores; en estos casos el objetivo fue la determinación de flujos de calor incidentes. Yang [1997] utilizó el FDM para estimar la generación interna de calor en un medio unidimensional. Por otro lado, el método de elementos finitos (FEM) fue usado por Bass [1980], Orlande y Ozisik [1993] y Hsu y col [1992], y el de volúmenes de control (CVM), similar al FDM, fue usado por Al-Khalidy [1998] que además utilizó un filtro digital para suavizar los errores en las medidas de temperaturas y Kurpisz [1991]. El método de elementos de contorno (BEM), fue aplicado por Maillet y col. [1991] para obtener la variación del coeficiente convectivo en función del ángulo de giro de un cilindro que trabaja en situación estacionaria.

En cuanto a los métodos de resolución del problema inverso podemos citar principalmente el método de especificación de la función [Beck, 1982; Beck y col., 1985], referido inicialmente a la estimación de un flujo de calor dependiente del tiempo, aplicable a problemas lineales y no lineales, donde existen dos posibilidades, i) estimación simultánea de todos los parámetros que definen la función problema, para el intervalo de tiempo completo (estimación para el dominio completo), ii) estimación de los parámetros secuencialmente, también denominado método de especificación de función secuencial (SFSM), [Tikhonov y Arsenin, 1977; Chantasiriwan, 1999], donde la función se estima por segmentos, obtenidos progresivamente, comenzando con aquellos correspondientes con menores tiempos. El método de estimación secuencial es más eficiente desde el punto de vista computacional.

Los conceptos básicos en el procedimiento de especificación de la función secuencial, que han servido de base para el desarrollado de esta tesis doctoral, son los siguientes:

a) Obtención de la función por tramos.

b) Determinación de las medidas de temperatura, obtenidas experimentalmente o del problema directo afectándolas de error.

c) En cada tramo temporal de estimación se fija un valor de la función buscada y se van obteniendo las temperaturas calculadas T(inv).

d) Definición del funcional, mediante mínimos cuadrados, para evaluar el grado de acercamiento en la estimación. Este funcional esta basado en la suma de los cuadrados de la diferencia entre las temperaturas calculadas, T(inv), y las medidas por el sensor, $\mathrm{T}(\mathrm{med})$,

$$
\mathrm{F}=\sum[\mathrm{T}(\mathrm{inv})-\mathrm{T}(\mathrm{med})]^{2}
$$


e) La estimación de cada valor de la función se corresponde con el mínimo valor del funcional.

De acuerdo con la descripción del método de especificación de función secuencial [Beck y col., 1985], como se ha mencionado, los tramos de la variable estimada para reproducir la función son constantes, figura II.8. En la presente memoria se adoptan tramos inclinados permitiendo modificar tanto el ángulo de inclinación como la longitud de la trama (por separado), de forma que la solución es una función continua a tramos (piece-wise function). Esta adopción permite alcanzar soluciones del IHCP más precisas.

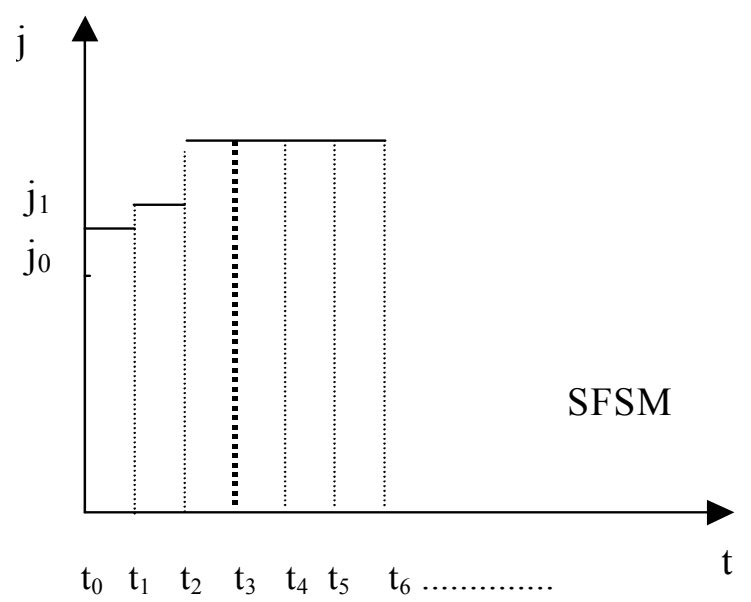

Figura II.8. Método de especificación de función secuencial

Otro método empleado para la resolución de problemas inversos, valido también para problemas no lineales, es el método de regularización iterativo. En él se modifica el funcional a minimizar por mínimos cuadrados añadiendo diversos términos, para intentar reducir las fluctuaciones que se cometen en la estimación de la función desconocida. Muchos investigadores han estudiado diferentes esquemas de regularización [Alifanov y Artyukhin, 1975; Beck y col., 1985]. Al igual que el SFSM el procedimiento se puede realizar en el dominio completo de tiempo (caso más general) o de forma secuencial. Como ejemplo de esquema de regularización citamos el desarrollado por Necati y Ozisik [1993].

$$
\mathrm{F}(\mathrm{z})=\sum[\mathrm{T}(\text { inv })-\mathrm{T}(\mathrm{med})]^{2}+\alpha \sum \mathrm{j}_{\mathrm{o}}^{2}
$$

donde $\alpha$ es el factor de regularización. 
Otro método para conseguir la estabilización de la solución de un problema inverso es el empleo de filtros digitales [Al-Khalidy, 1998; Hills, y Hensel, 1986], consistentes en sustituir las temperaturas de trabajo por otras. Si se conoce la estadística en los errores de los aparatos de medida, Hamming [1983] propone el prefiltro digital definido en la ecuación II.44, donde se sustituye la temperatura medida $\mathrm{T}$ (med) por el valor suministrado por el filtro, $\mathrm{T}^{\prime}$ (med), para posteriormente aplicar el algoritmo de resolución. También es posible filtrar el parámetro buscado obtenido en la resolución del problema inverso.

$$
\mathrm{T}^{\prime}(\mathrm{med})_{\mathrm{t}}=\frac{\mathrm{T}(\mathrm{med})_{\mathrm{t}-1}+2 \mathrm{~T}(\mathrm{med})_{\mathrm{t}}+\mathrm{T}(\mathrm{med})_{\mathrm{t}+1}}{4}
$$

Otros métodos derivados de los anteriores son: el método de gradiente conjugado [Silva-Neto y Özisik, 1992; Chen y col., 2001; Huang y Yan, 1995], el método de DavidonFletcher-Powell [Tervola, 1989], el método de Monte-Carlo [Haji-Sheikh y Buckingham, 1993].

Como proceso de comparación la mayoría de investigadores emplean el método de mínimos cuadrados, con lo que tienen que trabajar con un modelo no lineal. Sin embargo en la literatura existen algunos trabajos donde es posible sustituir el empleo de mínimos cuadrados por algoritmos que trabajan en un dominio lineal [Yang, 1997]. Posteriormente, después de la fase de análisis suele venir una fase de optimización, donde, a su vez, se proponen diferentes algoritmos de optimización.

El tiempo de dominio utilizado en el IHCP puede también ser utilizado para clasificar los métodos de solución. Tres tiempos de dominio pueden considerarse:

- Sólo en tiempo real

- En tiempo real más unas pocas medidas en tiempos futuros y

- El dominio completo de tiempo

El primer caso es muy intuitivo pero los algoritmos basados en él son con frecuencia extremadamente sensibles a los errores de las medidas. Los algoritmos usados en el segundo caso, denominados de tipo "secuencial", permiten reducir considerablemente la sensibilidad a los errores y usar tiempos de muestreo menores lo que, a su vez, permite encontrar mejores soluciones. El procedimiento del completo dominio del tiempo es también muy potente porque se puede adoptar tiempos de paso muy pequeños, pero no tan computacionalmente eficiente como lo es el uso de sólo unas pocas temperaturas futuras.

Como ya se ha dicho anteriormente, no existe una solución única para el IHCP. Al utilizar datos afectados de error, el error en los resultados es generalmente mayor que el error en 
los citados datos y la solución puede ser oscilante y, en consecuencia, no válida. El mejor algoritmo de resolución es aquel que presente una sensibilidad menor a los errores. Hay muchos métodos para la estabilización de los resultados. Beck y col. [1985] reconocieron que la información sobre temperaturas futuras permite estabilizar los resultados numéricos. Se recomienda usar muchas temperaturas futuras cuando el intervalo de tiempo entre medidas sea pequeño.

Para la evaluación de los procedimientos de resolución de problemas inversos, se citan los siguientes criterios extraídos de Beck y col. [1985]:

a) Si las medidas realizadas por los sensores tienen una precisión muy alta las funciones estimadas deberían ser bastante aproximadas a su valor exacto.

b) El método debe ser, en lo posible, insensible a los errores en las medidas.

c) El método debe ser estable para pequeños tiempos de paso o intervalos. Esto permite extraer la mayor información posible de las medidas.

d) Se debe permitir la medida desde uno o más sensores.

e) Se debe de permitir flujos de calor aplicados a la superficie que cambien bruscamente con el tiempo.

f) El conocimiento preciso del tiempo de comienzo de la aplicación del flujo de calor en la superficie no es necesario.

g) Se debe de poder aplicar a sólidos tipo "composite".

h) El método debe admitir propiedades térmicas dependientes de la temperatura.

i) Debe ser capaz de estimar conductancias de contacto entre materiales.

j) Debe ser fácil de programar.

k) El tiempo de computación debe ser moderado.

1) El usuario no debe poseer una alta habilidad en procedimientos matemáticos para usar el método o adaptarlo a otras geometrías.

m) El método debe ser capaz de permitir varios sistemas de coordenadas.

n) El método debe tener una base estadística y asumir las hipótesis corrientes relacionados con los errores en las medidas.

El método propuesto en esta memoria, que en mayor o menor medida satisface los requisitos anteriores, en unión con el MESIR como herramienta numérica, permite incluso la estimación simultánea de dos funciones (por ejemplo la estimación de la conductividad térmica y del calor específico, ambas dependientes de la temperatura). En la misma se resuelven problemas de estimación de función, tanto para estimar condiciones de contorno como parámetros térmicos dependientes de la temperatura, y estimación de parámetros, para el caso de parámetros constantes. 


\section{II.4. EL MÉTODO DE SIMULACIÓN POR REDES (MESIR)}

\section{II.4.1. Descripción}

El Método de simulación por redes (MESIR) es una técnica de cálculo numérico para la solución de problemas que pueden definirse mediante un conjunto de ecuaciones o modelo matemático [González-Fernández, 2002]. Su aplicación se extiende desde procesos de transporte en general, estacionarios o transitorios (tales como procesos de difusión de masa y/o de calor con o sin generación interna, problemas de
El método de simulación por redes.

¿En qué consiste?

¿Cuáles son sus objetivos?

¿Para qué se utiliza?

¿Cuales son sus ventajas? fluidos, etc.) hasta problemas técnicos de cualquier tipo. Numerosos trabajos, fruto de dicha aplicación, han sido publicados en los campos de difusión en membranas [Horno y col, 1990], procesos electroquímicos [González-Fernández y col., 1995] y transmisión de calor [Alhama y col., 1997; González Fernández y col., 1998a y 1998b; Alhama y Campo, 2001; Alhama y González-Fernández, 2002a y 2002b; Alarcón y col., 2002a y 2002b].

Partiendo de las ecuaciones diferenciales que integran el modelo matemático, el procedimiento consiste, en primer lugar, en elaborar un "modelo en red" completo o circuito eléctrico equivalente al proceso, incluyendo las condiciones iniciales y de contorno, y en segundo lugar, en simular dicho proceso obteniendo la solución numérica mediante un programa adecuado de resolución de circuitos eléctricos. La equivalencia formal entre las ecuaciones en diferencias finitas obtenidas por discretización de la o las variables espaciales del proceso y las ecuaciones Kirchhoff aplicadas al modelo en red, constituye la base de esta técnica ya que los errores introducidos por el software de resolución de circuitos son prácticamente nulos [Alhama, 1999].

El modelo en red completo está constituido por una agrupación de "celdas elementales", eléctricamente conectadas entre sí, que se corresponden a elementos de volumen del medio más una serie de dispositivos complementarios asociados a las condiciones de contorno. La equivalencia general establecida entre las variables térmicas (temperatura, T, y densidad de flujo de calor, j) y las variables eléctricas (potencial eléctrico, V, y corriente eléctrica, J) es

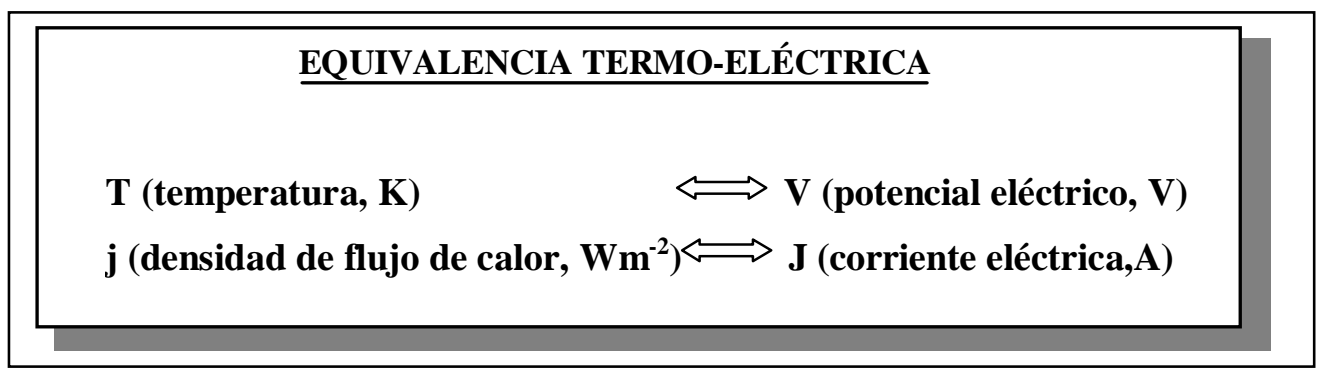


¿Cómo se elabora el modelo en red de la celda elemental? La técnica consiste en reticular espacialmente el medio en elementos de volumen o celdas elementales. Como resultado de discretizar las ecuaciones en derivadas parciales del modelo matemático se obtiene un conjunto de ecuaciones diferenciales en diferencias finitas (el tiempo permanece como una variable continua) que constituyen el punto de partida para el diseño del modelo en red de la celda elemental. Usando la anterior equivalencia entre variables, cada uno de los sumandos de la ecuación diferencial se constituye en una corriente eléctrica que, de acuerdo con su expresión particular, se implementa en el modelo por medio de lo que llamamos una monopuerta. Éstas están eléctricamente conectadas a los nudos del circuito de forma que el conjunto de sumandos de la mencionada ecuación satisface la ley de corrientes de Kirchhoff que en definitiva es la ley de conservación de la energía en el proceso térmico. Dado que esta ley se satisface automáticamente en el circuito, no es preciso ninguna consideración añadida para la solución del problema que no esté ya asumida por el software de simulación. Las fronteras de celdas elementales contiguas se conectan eléctricamente de acuerdo con la geometría del medio de forma que su potencial es el mismo. De esta forma la variable potencial eléctrico satisface la ley de voltajes de Kirchhoff o ley de unicidad del potencial lo cual también es asumido por el software seleccionado con la consiguiente simplificación para el programador.

Como en cualquier otro método numérico, aumentando el número de celdas disminuye el error de la solución. De acuerdo con los resultados establecidos por Alhama [1999], utilizando un número de celdas superior a 50-60 en procesos transitorios 1-D, los errores se reducen a valores del orden del $0.1 \%$ tanto en problemas lineales como no lineales, valor más que aceptable en problemas de ingeniería en general.

En el caso de los procesos de transmisión de calor, la posibilidad de elaborar modelos en red representativos de los mismos, es decir, el hecho de que admitan redes eléctricas equivalentes, supone no sólo la equivalencia matemática sino, también, la equivalencia física entre las variables características de unos y otros procesos. Además esta equivalencia física permite, en casos muy concretos, determinar cualitativa y cuantitativamente ciertas magnitudes asociadas a la red que pueden jugar un papel, en la descripción del fenómeno de transporte, similar al correspondiente en el transporte de carga eléctrica, como es el caso de la admitancia térmica [Alarcón, 2001]. En este sentido, los modelos en red, sin la implementación de las condiciones de contorno se constituyen en auténticas redes de transferencia que contienen toda la información del sistema. Dado que la solución de estos modelos es inmediata con un adecuado software y que la obtención de esta red de transferencia no requiere el concurso de complicados procesos de manipulación matemática, el MESIR se convierte en una potente herramienta al proporcionar de una forma sencilla y compacta toda la información del sistema. 
Los dispositivos o componentes característicos que representan el modelo en red, llamados monopuertas, se obtienen de las relaciones constitutivas que ligan las variables densidad de calor (flujo) y temperatura (potencial), o sus derivadas, las cuales como hemos señalado anteriormente satisfacen leyes de conservación y unicidad, respectivamente. Las diferentes relaciones constitutivas que aparecen en los problemas estudiados en este trabajo dan lugar a los siguientes tipos de monopuertas:

i) Monopuertas pasivas. En función de la relación expresa existente entre las variables de flujo de calor y temperatura que obedecen a la ley de corrientes de Kirchhoff, LCK, y a la ley de voltajes de Kirchhoff, LVK, las monopuertas pasivas tienen nombre específicos:

Monopuerta resistiva. Es un elemento de circuito asociado a una relación entre las derivadas temporales de las variables flujo de calor que circula por una rama, J, y la diferencia de temperatura entre los extremos de dicha rama, X, mediante una función independiente del tiempo que llamaremos resistencia, $\mathrm{R}$, que puede depender o no del flujo de calor o de la temperatura:

$$
\mathrm{dX}(\mathrm{t}) / \mathrm{dt}=\operatorname{RdJ}(\mathrm{t}) / \mathrm{dt}
$$

Por tanto,

$$
\mathrm{R}=\mathrm{dX}(\mathrm{t}) / \mathrm{dJ}(\mathrm{t})
$$

Una monopuerta resistiva es lineal cuando la relación entre las variables $\mathrm{X}(\mathrm{t})$ y $\mathrm{J}(\mathrm{t})$ lo es, es decir $\mathrm{X}(\mathrm{t})=\mathrm{R} J(\mathrm{t})$; naturalmente $\mathrm{R}$ es una constante en este caso. Su acción es instantánea, no importa cual sea su estado anterior, en este sentido carecen de memoria. En su analogía física representan efectos disipativos, fricciones, efectos viscosos, energías de reacción, etc., y desde el punto de vista termodinámico son elementos generadores de entropía. Las monopuertas resistivas no lineales se definen a través de las funciones que las caracterizan, $\mathrm{J}(\mathrm{t})=\mathrm{F}_{\mathrm{R}}^{-1}(\mathrm{X})$. Constituyen, en definitiva, fuentes controladas de corriente.

La traducción al modelo en red es una resistencia eléctrica de valor $\mathrm{R}$ ohmios para el caso lineal o una fuente controlada de corriente para el caso no lineal.

Monopuerta capacitiva. Es un elemento de circuito asociado a una relación entre la variable flujo de calor y la derivada temporal de la variable temperatura de una misma rama, mediante una función no dependiente del tiempo que designaremos como capacidad, $\mathrm{C}$, 


$$
J(t)=C d X(t) / d t
$$

En estas monopuertas se produce algún tipo de almacenamiento, sin pérdidas (no hay disipación energética), y su estado, que no cambia instantáneamente, tiene en cuenta todas las operaciones llevadas a cabo en el pasado (se trata de elementos con memoria).

La traducción al modelo en red es un condensador eléctrico de valor C faradios.

Los procesos de almacenamiento y disipación de energía, bajo la hipótesis de continuidad en el medio, se originan en todos los puntos del sistema. Los elementos $\mathrm{R}$ y $\mathrm{C}$ se identifican sin embargo con regiones pequeñas pero finitas del medio y sus conexiones con las otras puertas se realizan con enlaces ideales de energía, es decir, con conductores de resistencia nula. El que cada elemento pueda ser caracterizado por un par de variables conjugadas con una única ecuación constitutiva entre ellas es una hipótesis básica en el MESIR que deriva de la teoría de redes. Físicamente equivale a decir que es posible elegir un elemento de volumen lo suficientemente pequeño como para que su tiempo de relajación interna sea mucho menor que el del sistema global, pero suficientemente grande como para que las fluctuaciones de las variables que describe el sistema en él sean despreciables.

ii) Monopuertas activas. En éstas se produce una aportación o extracción de energía al sistema. Cabe distinguir:

Fuentes constantes. Son monopuertas definidas de acuerdo con las expresiones $\mathrm{Fj}(\mathrm{J})=0$ y $\operatorname{Fx}(X)=0$, según se trate de fuentes de flujo o de fuerza, respectivamente. Tienen asignado un sentido (o signo) que indica la dirección en que fluye la energía. Eléctricamente se corresponden con pilas o generadores de corriente constante.

Fuentes controladas. Se trata de monopuertas especiales asociadas a relaciones constitutivas entre variables, conjugadas o no, expresadas mediante cualquier función que no contiene explícitamente el tiempo. Se trata de elementos, de entradas múltiples con una única salida que corresponde a un flujo o una fuerza que depende funcionalmente de otros flujos o fuerzas de distintas ramas y nudos del mismo o diferente circuito. Estas fuentes van a permitir especificar acoplamientos energéticos de distinto tipo.

Existen cuatro tipos de fuentes controladas por una sola variable

$$
\mathrm{X}=\mathrm{E}(\mathrm{Xc})
$$




$$
\begin{aligned}
& X=H(J c) \\
& J=G(J c) \\
& J=F(X c)
\end{aligned}
$$

según se trate de

i) fuentes de tensión controladas por tensión,

ii) fuentes de tensión controladas por corriente,

iii) fuentes de corriente controladas por corriente y

iv) fuentes de corriente controladas por tensión, respectivamente.

E, H, G y F designan funciones arbitrarias de la variable de control (subíndice c). La acción de control puede ser ejercida por una o más variables y las funciones de control pueden ser complejas.

Aunque la monopuerta puede especificarse arbitrariamente, su implementación como elemento de circuito puede no ser posible en tanto no esté contenida, en las librerías del software elegido. En este sentido, la teoría de circuitos permite, mediante circuitos auxiliares independientes que se incorporan al propio modelo y se simulan al mismo tiempo, resolver prácticamente todos los casos de diseño de la red eléctrica que se necesiten para cualquier tipo de fuente controlada por complejos que sean. Ejemplos de esta aplicación se presentan en este trabajo. Para terminar conviene indicar que es posible la implementación tanto de fuentes controladas simultáneamente por variables de tensión y corriente como de fuentes controladas por variables de tensión y corriente más variables temporales.

\section{II.4.2. El MESIR como método numérico}

En el Método de simulación por redes, el punto de partida es siempre el modelo matemático del proceso o problema, esto es, el conjunto de ecuaciones en derivadas parciales (EDP) espacio-temporales cuya discretización de la variable espacial conduce a un conjunto de ecuaciones diferenciales en diferencias finitas del cual se obtiene tanto el modelo en red de la celda elemental, que por acoplamientos entre sí reproduce el modelo en red del medio completo, como los dispositivos o componentes que implementan las condiciones de contorno. Ésta es la única manipulación directa que se hace de las ecuaciones.

Dado que teoremas de conservación y unicidad de las variables flujo de corriente y potencial eléctrico, respectivamente (leyes de Kirchhoff), se satisfacen en los circuitos, algunas de las ecuaciones que habitualmente forman parte del modelo matemático no necesitan ser consideradas para el diseño del modelo en red. Tal ocurre con la conservación de flujo calorífico 
en la frontera de distintos medios y la unicidad en el valor de la temperatura en la misma.

El modelo en red es el formato dado al modelo matemático para poder ser utilizado como fichero de entrada en el programa de resolución de circuitos eléctricos, en concreto en PSPICE2 [Pspice, 1994], el software elegido en esta memoria para la resolución numérica del problema. A continuación exponemos las diferencias de estrategias más notables de este método, en comparación con otros métodos numéricos más o menos clásicos.

Cuando en una ecuación en derivadas parciales se hace una doble reticulación, espacial y temporal, se reemplazan de hecho las derivadas parciales por aproximaciones finitas que conducen a un conjunto de ecuaciones algebraicas. Para la solución numérica de éstas se utiliza un software adecuado, generalmente un software de lenguaje propiamente matemático. Este procedimiento es la base de los bien conocidos métodos numéricos de diferencias finitas, elementos finitos y volúmenes finitos para la solución de las EDP. Como ya se ha comentado, la elaboración del modelo en red pasa por la reticulación espacial, pero no temporal. Esta es una diferencia esencial entre el MESIR y los métodos más clásicos. Conviene mencionar que tanto el método de líneas (MOL) [Liskovets, 1965; Schiesser, 1991] como el método de líneas transversal (TMOL) [Rothe, 1930; Liskovets, 1965; Rektorys, 1982], siguen estrategias similares a las del MESIR. En el primer caso (MOL), se retícula la variable espacial para resolver el sistema de ecuaciones diferenciales en diferencias finitas, con el tiempo como variable continua, con ayuda de un adecuado software matemático; en el segundo caso se retícula la variable tiempo y se mantiene como variable continua la variable espacial, recurriendo también a un software matemático adecuado para resolver el sistema de ecuaciones diferenciales en diferencias finitas resultante.

\section{II.4.3. Simulación}

En el proceso de simulación, el modelo en red o circuito equivalente es introducido en el ordenador como un fichero que puede ser simbólico (fichero esquemático con la simbología típica de los circuitos eléctricos) o de texto (cuya elaboración utiliza muy pocas reglas, sencillas y muy intuitivas). El software Pspice asocia a cada uno de los componentes del modelo en red una ecuación (o conjunto de ellas) matemática que relaciona las variables flujo y fuerza, generalmente una ecuación diferencial. El conjunto de todas estas ecuaciones se resuelve utilizando las más sofisticadas técnicas numéricas. Pspice es un software continuamente actualizado en su doble vertiente; por un lado incorporando sin cesar nuevos dispositivos eléctricos dentro de sus librerías de componentes, y por otro, integrando nuevos y refinados procesos de cálculo en sus programas y rutinas de análisis numérico. 
El resultado de la simulación sería equivalente al obtenido experimentalmente construyendo físicamente el circuito en el laboratorio, pero con un margen de error prácticamente despreciable y pudiendo obtener datos sobre el comportamiento del circuito más allá de los límites que virtualmente se pueden obtener con medidas reales. Más aún, la simulación permite la "parametrización", es decir, la modificación controlada mediante rutinas de programación de cualquiera de los parámetros del circuito (que se corresponden por ejemplo a las características térmicas del medio, a los diferentes coeficientes o parámetros del proceso, a las condiciones de contorno, etc.). De esta forma se puede observar las tendencias en la respuesta ante variaciones de cualquier parámetro y determinar valores de comportamientos límite; todo ello sin alterar el modelo en red.

El conjunto de tareas que componen el proceso de simulación puede ser agrupado en los siguientes tópicos (o algoritmos de computación),

i) formulación de las ecuaciones,

ii) solución de ecuaciones lineales,

iii) solución de ecuaciones no lineales,

iv) integración numérica

PSPICE es miembro de la familia de programas de simulación de circuitos Pspice2 [Pspice, 1994; Nagel, 1977]; mucho más potente y rápido que sus predecesores fue desarrollado en la Universidad de California en los años setenta utilizando algoritmos numéricos más refinados con formatos de entrada-salida idénticos. El uso extendido de PSPICE da fe de su capacidad para el tratamiento de una extensa variedad de problemas en simulación de circuitos [Horno y col., 1993; González-Fernández y col., 1995; Alhama y col., 1997], resolviendo:

i) respuesta en corriente continua,

ii) respuesta transitoria y análisis de Fourier en el dominio de la frecuencia, y

iii) análisis de pequeña señal en corriente alterna y distorsión.

De estas posibilidades nuestro interés se ciñe al estudio de transitorios y estacionarios.

En el análisis de continua Pspice determina el punto de trabajo, es decir, los valores de polarización de sus componentes en ausencia de excitaciones alternas. Para este cálculo se elimina la acción de los condensadores y bobinas, los primeros quedan como circuitos abiertos y las bobinas se cortocircuitan. Para el análisis transitorio Pspice parte del intervalo de tiempo $(0, \mathrm{t})$ solicitado, que puede ser menor o mayor que la duración del transitorio, y facilita los datos alternativamente en forma de listados numéricos o mediante gráficos. Si los resultados se quieren en forma tabular el usuario debe indicar el instante inicial, el final, el paso temporal y el número de variables listadas; si se solicitan en forma gráfica una simple sentencia de programa 
permite organizarlos y almacenarlos para ser utilizados con ese propósito en cada momento.

Los algoritmos utilizados en Pspice, que se documentan en la tesis de Nagel [1977], son el resultado de implementaciones, modificaciones y comparaciones cuidadosas de los métodos numéricos existentes en el contexto especial de la simulación de circuitos. El objeto de la tesis es seleccionar los métodos de simulación de circuitos más exactos y eficaces, con la mínima interacción por parte del usuario.

Como se ha mencionado anteriormente, la introducción del modelo en red en Pspice puede hacerse de dos maneras: (i) directamente mediante una representación gráfica del mismo, en donde aparecen los componentes clásicos, de acuerdo con la simbología al uso en teoría de circuitos, y sus interconexiones y (ii) mediante un fichero elaborado con un editor de texto en el que se especifican los nombres de los componentes eléctricos, sus valores, sus nudos de conexión, etc. La sintaxis de entrada, en este último caso, no requiere especiales disposiciones ordenadas de datos y su estilo puede catalogarse más bien como libre. Por otro lado, el software Pspice dispone de una razonable fuente de datos que se adjudican por omisión a los componentes del circuito cuando éstos no se especifican en detalle. También realiza un buen número de chequeos para asegurar que el circuito ha sido introducido correctamente (tanto si se programa con editor como si se hace a través de esquemas) advirtiendo al programador de posibles errores de todo tipo mediante mensajes previos a la ejecución.

En definitiva, un usuario principiante necesita especificar un número mínimo de parámetros y controles de simulación para extraer unos resultados de simulación aceptables.

\section{II.4.4. Ventajas del MESIR}

La familiaridad con el uso y comprensión de la teoría de circuitos eléctricos es una ventaja apreciable, sin duda, a la hora de diseñar el modelo en red, o dicho de otra forma, una formación escasa en el campo de la teoría de circuitos puede dar lugar a alguna dificultad para el programador. Sin embargo, y particularmente en el caso de problemas lineales, este tipo de dificultades es fácilmente subsanable ya que son muy pocos los componentes que contienen estos circuitos. Para problemas no lineales también son pocos los componentes que entran en juego (un problema fuertemente no lineal como el cambio de fase, por ejemplo, requiere tan solo el concurso de seis componentes en el modelo de la celda elemental); ello es debido a que la mayor parte de las no linealidades se implementan por medio de las llamadas fuentes controladas de las que existen 4 tipos diferentes que cubren todo el espectro de necesidades. En definitiva, unos cuantos ejemplos podrían ser suficientes para resolver la mayor parte de los problemas que surgen en la fase de elaboración del modelo. 
Entre las ventajas del MESIR podemos citar:

- La única manipulación matemática que se requiere es la conversión de las ecuaciones en derivadas parciales del modelo en ecuaciones diferenciales en diferencias finitas para la variable espacial. El tiempo permanece como variable continua. En este sentido no es necesario el uso del software matemático (común en la mayor parte los métodos numéricos) que se requiere para la resolución del conjunto de ecuaciones algebraicas obtenidas de las ecuaciones en diferencias finitas.

- No es preciso ocuparse de los problemas de convergencia. PSpice asigna automáticamente valores al intervalo de tiempo, $\Delta \mathrm{t}$ (función del grado de precisión exigido a la solución), derivados del compromiso entre el error y el tiempo de computación.

- Los componentes eléctricos del modelo permiten en cierto modelo una visualización directa del proceso de transmisión de calor, permitiendo el acceso a ciertas magnitudes de interés tales como energía acumulada, diferencias de temperatura entre dos puntos cualesquiera, etc.

- La implementación de componentes o circuitos auxiliares permite también obtener magnitudes no directas tales como calores totales (o integrados).

- Pspice dispone de un programa llamado Probe con gran número de opciones que da acceso directo a los resultados de la simulación y los representa en forma gráfica.

- Los tiempos de computación para tipos de problemas idénticos son comparables a los de otras técnicas numéricas comunes.

- Las amplias librerías de dispositivos eléctricos que dispone Pspice permite simular en la práctica cualquier tipo de proceso, lineal o no lineal, por complejo que sea.

\section{II.5. REFERENCIAS}

Alarcón García, M., "Transporte de calor en sistemas con aletas. Coeficientes de rendimiento y red de transferencia". Tesis Doctoral. Universidad Politécnica de Cartagena (2001) 
Alarcón, M., Alhama, F. y González-Fernández, C.F., "Time dependent heat transfer in a finwall assembly. New performance coefficient: Thermal reverse admittance “. Int. J. Therm. Sci., 41, 386-395 (2002a)

Alarcón, M., Alhama, F. y González-Fernández, C.F., “ Transient heat conduction in a fin-wall assembly under harmonic excitation. Network thermal admittance”. Heat Transfer Eng., 23, 31-43 (2002b)

Alhama López, F., "Estudio de respuestas térmicas transitorias en procesos no lineales de conducción de calor mediante el Método de simulación por redes”. Tesis Doctoral. Universidad de Murcia (1999)

Alhama, F., López-Sánchez, J. F. y González-Fernández, C. F., "Heat conduction through a multilayered wall with variable boundary conditions”. Energy, 22, 797-803 (1997)

Alhama, F. y González-Fernández, C.F., "Network simulation method for solving phase change heat transfer problems with variable thermal properties". Heat Mass Transfer, 38, 327-339 (2002a)

Alhama, F. y González-Fernández, C. F., "Transient thermal behaviour of phase-change processes in solid foods with variable thermal properties". J. Food Eng., 54(4), 331-336, (2002b)

Alhama, F. y Campo, A., "The connection between distributed and lumped models for asymmetric cooling of long slabs by heat convection”. Int. Commun. Heat Mass Transf., 28 (1), 127-137 (2001)

Alifanov O.M., y Artyukhin, F.A. "Regularized numerical solution of nonlinear inverse heatconduction problem", J. Eng. Phy. 29, 934-938 (1975)

Al-Khalidy, N., "On the solution of parabolic and hyperbolic inverse heat conduction problems", Heat Mass Transfer, 41, 3731-3740 (1998)

Al-Najem, N.M., Osman, A.M., El- Refaee, M.M. y Khanafer, K.M., "Two dimensional steadystate inverse heat conduction problems", Int. Commun. Heat Mass Transf., 25, 4, 541-550 (1998)

Bass, B.R., "Applications of the finite elements to the inverse heat conduction problem using Beck's second method", J. Eng. Ind., 102, 168-176 (1980)

Beck, J.V., " Calculation of surface heat flux from an internal temperature history", ASME Paper 62-HT-46 (1963) 
Beck, J.V., "Determination of optimun, transient experiments for thermal contact conductance", Int. J. Heat Mass Transf., 12, 621-633 (1968)

Beck, J. V. y Wolf, H., "The nonlinear inverse heat conduction problem”, ASME Paper, 62HT-46 (1965)

Beck, J.V. y Arnold, K.J., "Parameter estimation in engineering and science". Wiley. New York (1977)

Beck, J. V., Litkouhi B. y St.Clair.Jr. C.R., "Effective sequential solution of the nonlinear inverse heat conduction problem", Numer. Heat Transfer-A, 5, 275-286. (1982)

Beck, J. V., Blackwell, B. y St.Clair.Jr. C.R., "Inverse heat conduction”, New York, John Wiley \& Sons. Inc. (1985)

Beck, J.V., "Nonlinear Estimation Applied to the Nonlinear Heat Conduction Problem", Int. J. Heat Mass Transf., 13, 703-716 (1970)

Beck, J.V., "Criteria for comparasion of methods of solution of the inverse heat conduction problem", Nucl. Eng. Des., 53, 11-22, (1979)

Beck, J.V., “Sequential estimation of thermal parameters”, J. Heat Transfer, 19, 314-321 (1977)

Burggraf, O.R. "An exact solution of the inverse problem in heat conduction theory and pubblications", J. Heat Transfer, 86C, 373-382 (1964)

Chantasiriwan, S., "Inverse heat conduction problem of determining time-dependent heat transfer coefficient", Int. J. Heat Mass Transf., 42, 4275-4285 (1999)

Chantasiriwan, S., "Steady-state determination of temperature-dependent thermal conductivity", Int. Commun. Heat Mass Transf., 29, 6, 811-819 (2002)

Chen, U.C., Chang, W.J. y Hsu, J.C., "Two-dimensional inverse problem in estimating heat flux of pin fins", Int. Commun. Heat Mass Transf., 28, 6, 793-801 (2001)

Congreso Iberoamericano de Ingeniería Mecánica, "Termodinámica y transferencia de calor", Mérida (Venezuela) (2002)

González-Fernández, C. F., “Applications of the network simulation method to transport processes", en "Network Simulation Method", Ed. J. Horno, Research Singpost, Trivandrum, India (2002) 
González-Fernández, C. F., García Hernández, M. T. y Horno, J., “Computer simulation of a square scheme with reversible and irreversible charge transfer by the network method". J. Electroanal Chem., 395, 39-44 (1995)

González-Fernández, C. F., Alhama, F. y López Sánchez, J. F., “Application of the network method to heat conduction processes with polynomial and potential-exponentially varying thermal properties”. Numer. Heat Transfer-A., 33, 549-559 (1998a)

González-Fernández, C. F., Alhama, F. y Alarcón, M. y López Sánchez, J. F., “Digital simulation on transient heat conduction with polynomial variable thermal conductivity and specific heat”. Comput. Phys. Commun., 109, 1-6 (1998b)

González-Fernández, C.F. y Alhama, F., "Heat Transfer and the Network Simulation Method", en "Network Simulation Method", Ed. J. Horno, Research Singpost, Trivandrum, India (2002)

Grysa, K., Cialkowski, M.J., y Kaminski, H., "An inverse temperature field problem of the theory of thermal stresses", Nucl. Eng. Des., 64, 169-184 (1981)

Hamming, R.W. "Digital filters", $2^{\text {nd }}$ ed., Prentice-Hall, Englewood Cliffs, NJ (1983)

Haji-Sheikh, A y Buckingham, F.P., "Multidimensional inverse heat conduction using the Monte Carlo method", J. Heat Transfer, 115, 26-33 (1993)

Hewitt, G.F., Shires,G.L. y Bott, T.R., “Process heat transfer”, CRC Press, Inc. (1994)

Hills, R.G. y Hensel, E.C. Jr., "One-dimensional nonlinear inverse heat conduction technique", Numer. Heat Transfer, 10, 369-393 (1986)

Horno, J., González Caballero, A., Hayas, A. y González-Fernández, C. F., “The effect of previous convective flux on the nonstationary diffusion through membranes”. J. Membrane Sci., 48, 67-77 (1990)

Horno, J., García Hernández, M. T. y González-Fernández, C. F., "Digital simulation of electrochemical processes by network approach”. J. Electroanal Chem., 352, $83-97$ (1993)

Hsu, T.R., Sun, N.S., Chen, G.G. y Gong, Z.L., "Finite element formulation for twodimensional inverse heat conduction analysis", J. Heat Transfer, 114, 553-557 (1992)

Huang C.H. y Özisik, M.N."Direct integration approach for simultaneously estimating temperature dependent thermal conductivity and heat capacity", Numer. Heat Transfer-A, 20, 95-110 (1991) 
Huang C.H., Yan J.Y. y Chen H.T., "Function estimation in predicting temperature-dependent thermal conductivity without internal measurements", J. Thermophys Heat Transf., 9, 4, 667-673 (1995)

Huang, C.H. y Yan, J.Y., "An inverse problem in simultaneously measuring temperaturedependent thermal conductivity and heat capacity", Int. J. Heat Mass Transf., 38, 18, 34333441 (1995)

Huang C.H. y Chin S.C., "A two-dimensional inverse problem in imaging the thermal conductivity of a non-homogeneous medium", Int. J. Heat Mass Transf., 43(22), 4061-4071 (2000)

Imber, M. y Khan, J., "Prediction of transient temperature distributions with embedded thermocouples", AIAA J. 10, 784-789 (1972)

Incropera, F.P. y Dewitt, "Fundamentals of heat and mass transfer”, 3rd. ed., Wiley, New York (1996)

International Heat Conduction Conference 12, Grenoble (2002)

Jarny, Y., Lecointe, Y. y Ahssaï ni, A. "Inverse free convection problem estimation of a surface heat flux", Proc. Eurotherm 68, 151-157 (2001)

Kurpisz K., "Numerical solution of one case inverse heat conduction problems", J. Heat Transfer, 113, 280-286 (1991)

Langford, D., "New analytic solutions of the one-dimensional heat equations for temperature and heat flow rate both prescribed at the same fixed boundary (with applications to the change problem)" Q. Appl. Math. 24, 315-322 (1967)

Laubitz, M.J., "Measurement of the thermal conductivity of solids at high temperature by using steady-state linear and quasi-linear heat flow”, R.P. Tye, Thermal conductivity, 1, cap. 3, Academic Press, London (1969)

Li, H.Y. y Yang, C.Y., "A genetic algorithm for inverse radiation problems", Int. J. Heat Mass Transf., 40, 545-1549 (1997)

Liskovets, O.A., “The method of lines (Review)", Differential equations, 1, 1308-1323 (1965)

Lorion, R., Chabriat, J.P., Bessafi, M. y Lan-Sun-Luk, J.D. “Genetic algorithm for thermal properties distribution estimation in IHCP ", Proc. Eurotherm Seminar 68, Poitiers, 351$358(2001)$ 
Maillet, D., Degiovanni, A. y Pasquetti R., "Inverse heat conduction applied to the measurement of heat transfer coefficient on a cylinder: Comparison between an analytical and a boundary element technique", J. Heat Transfer, 113, 549-557 (1991)

Martin, T.J. y Dulikravich, G.S., "Inverse determination of boundary conditions and sources in steady heat conduction with heat generation", J. Heat Transfer, 118, 546-554 (1996)

Martin, T.J. y Dulikravich, G.S., "Inverse determination of temperature-dependent thermal conductivity using steady surface data on arbitrary objects ", J. Heat Transfer, 122, 450459 (2000)

Mc Adams, W.H., "Heat transmission”. 3rd ed., New York, McGraw Hill (1954)

Mills, A.F., "Heat and mass transfer". Richard D. Irwin, Inc., Chicago (1995)

Mirsepassi, T.J., "Heat transfer charts for time-variable boundary conditions", Brit. Chem. Eng., 4, 130-136 (1959)

Nagel, L. W., "SPICE, a computer program to simulate semiconductor circuits". Cap. 4,5,6, Memo UCB/ERL M520, University of California, Berkeley, CA. (1977)

Necati, M. y Ozisik, M.N., "Heat conduction". cap. 14, John Wiley \& Sons, Inc. New York (1993)

Orlande, H.R.B y Özisik, M.N., "Inverse problem of estimating interface conductance between periodically contacting surfaces", J. Therm. Heat Transf., 7, 2, 319-325 (1993)

Ozisik, M.N., “Basic heat transfer”, Mc-Graw-Hill, Nueva York (1997)

Pspice 6.0, Microsim Corporation, 20 Fairbanks, Irvine, California 92718. (1994)

Rektorys, K. "The method of discretization in time for partial differential equations", D. Reidel Publishers, Dordrecht. The Netherlands (1982)

Rothe, E. "Zweidimensionale parabolische Randwertaufgaben als Grenzfall eindimensionaler Randwertaufgaben", Math. Ann., 102, 650-660 (1930)

Ruperti, N.J. Jr., Raynaud, M. y Sacadura, J.F., "A method for the solution of the coupled inverse heat conduction-radiation problem", Int. J. Heat Mass Transf., 118, 10-17 (1995)

Schiesser, W.E. "The numerical method of lines. Integration of partial differential equations". Academic Press, Inc. (1991)

Seminar Eurotherm 68, "Inverse problem and experimental design in thermal and mechanical engineering", Poitiers (Francia) (2001) 
Sawaf, B., Özisik, M.N. y Jarny, Y., "An inverse analysis to estimate linearly temperature dependent thermal conductivity components and heat capacity of an orthotropic medium", Int. J. Heat Mass Transf., 28 (16), 3005-3010 (1995)

Shumakov, N. V. "A method for the experimental study of the process of heating a solid body". Soviet-Technical Physics (translated by American Institute of Physics), 2, 771 (1957)

Silva-Neto A.J. y Özisik, M.N., "Two-dimensional inverse heat conduction problem of estimating the time-varying strength of a line heat source", J. Appl. Phys., 71 (11) 5357$5362(1992)$

Sparrow, E. M., Haji-Sheikh, A. y Lundgren, T. S., "The inverse problem in heat conduction". J. Appl. Mech., 32 (3), 369-375 (1964)

Stolz, G. Jr., "Numerical solutions to an inverse problem of heat conduction for simple shapes", J. Heat Transfer, 82C, 20-60 (1960)

Tervola, P. "A method to determine the thermal conductivity from measured temperature profiles", Int. J. Heat Mass Transf., 32, 8, 1425-1430 (1989)

Throne, R. y Olson, L., "The steady inverse heat conduction problem: A comparison of methods with parameter selection”, J. Heat Transf., 123, 633-644 (2001)

Tikhonov, A.N. y Arsenin, V.Y., "Solutions of III-posed problemas", V.H. Winston \& Sons, Wahington, D.C. (1977)

Weber, C.F., "Analysis and solution of ill-posed inverse heat conduction problem", Int. J. Heat Mass Transf., 24, 11, 783-1792 (1981)

Willians, S.D., y Curry, D.M., "An analytical experimental study for surface heat flux determination", J. Spacecraft Rockets, 14, 632-637 (1977)

Woodbury Keith, A. y Ke Quanpeng, "An inverse algorithm for direct determination of heat transfer coefficients", Proc. of NHTC'00, Pittsburgh, 1-7 (2000)

Yang Ching-Yu, "Non-iterative solution of inverse heat conduction problems in one dimension", Commun. Numer. Meth. Eng., 13, 419-427 (1997)

Yang, Ching-Yu, "Estimation of the temperature dependent thermal conductivity in inverse heat conduction problem", Appl. Math. Model., 23, 469-478 (1999) 


\section{Capítulo III}

\section{Protocolos de resolución}

III.1. Planteamiento de los problemas inversos

III.1.1. Modelo matemático

III.1.2. Obtención de las temperaturas de entrada

III.1.3. Planteamiento

III.2 Estimación de funciones dependientes del tiempo

III.2.1. Especificación del funcional

III.2.2. Procedimiento computacional

III.3 Estimación de funciones dependientes de la temperatura

III.3.1. Especificación del funcional

III.3.2. Procedimiento computacional

III.4 Estimación simultánea de dos funciones ambas dependientes de la temperatura

III.4.1. Especificación del funcional

III.4.2. Procedimiento computacional

III.5 Estimación de parámetros de funciones lineales

III.5.1. Especificación del funcional

III.5.2. Procedimiento computacional

III.6 Algoritmo general de resolución

III.7 Referencias

Contribuciones y conclusiones 


\section{Capítulo III}

\section{Protocolos de resolución}

\section{III.1. PLANTEAMIENTO DEL PROBLEMA INVERSO}

Enunciado general del problema:

"Partiendo de un proceso transitorio o estacionario regido por la ecuación de difusión térmica y conociendo, excepto el parámetro o función a determinar, los parámetros geométricos, las características térmicas del medio, y los datos asociados con las condiciones iniciales y de frontera, se trata de estimar la solución para el parámetro o función desconocida mediante un conjunto de temperaturas medidas a intervalos de tiempo regulares en algún punto o puntos del medio".

El parámetro o función incógnita puede ser, en principio, bien cualquiera de las características termofísicas del medio (o más de una de dichas características), bien una parte o la totalidad de las condiciones de contorno, o de las condiciones iniciales. Eventualmente pueden seleccionarse intervalos irregulares de tiempo para las medidas, si fuera conveniente, sin necesidad de modificar los protocolos propuestos.

\section{III.1.1. Modelo matemático}

La solución de los tipos de problemas inversos de conducción de calor estudiados en esta Memoria, sean lineales o no lineales, parte de la formulación de los mismos mediante un modelo matemático bien definido y completo. Dicho modelo está integrado por: 
(i) La ecuación diferencial en derivadas parciales representativa del comportamiento físico del proceso que liga la variable (dependiente) temperatura con las variables (independientes) posición y tiempo. Para los problemas estudiados en esta Memoria, dicha ecuación es la de conducción del calor, resultado de combinar la ecuación de Fourier y la de conservación de la energía térmica en un elemento diferencial del medio.

(ii) Un conjunto de ecuaciones que definen la totalidad o parte de las condiciones de frontera en las superficies exteriores del sólido.

(iii) La ecuación o ecuaciones que recogen las condiciones iniciales.

(iv) Las ecuaciones de dependencia de las características termofísicas con la temperatura, (o con la temperatura y la posición en medios heterogéneos).

(v) Un conjunto de $\mathrm{n}$ temperaturas a intervalos de tiempo regulares (o irregulares) en un determinado punto (o puntos) del sólido, $\mathrm{x}_{\mathrm{f}}$.

El siguiente cuadro muestra el conjunto de ecuaciones mencionado:

$$
\begin{aligned}
& \rho c_{e}(\partial T / \partial t)=\nabla(k \nabla T)+g_{g e n} \\
& T(x, t=0) \equiv T_{0}(x) \\
& j(0, t) \equiv j(t) o T(0, t) \equiv T(t) \\
& j(L, t)=h\left[T(L, t)-T_{c o n}\right] \text { o j(L,t) }=0 \\
& T\left(x=x_{f}, t=t_{1}, t_{2}, t_{3}, \ldots t_{n}\right) \equiv T\left(\text { med, } x_{f}, t_{1}\right), T\left(\operatorname{med}, x_{f}, t_{2}\right), \ldots, T\left(\operatorname{med}, x_{f}, t_{n}\right)
\end{aligned}
$$

La ecuación (III.1) es aplicable a cualquier tipo de geometría aunque en esta memoria sólo se estudian problemas de geometrías 1-D. La ecuación (III.2) es la condición inicial. Las ecuaciones (III.3) representan condiciones de contorno y pueden darse indistintamente en cada una de las superficies exteriores del medio. Por último, la ecuación (III.4) es el conjunto de medidas experimentales.

\section{III.1.2. Obtención de las temperaturas de entrada}

Las temperaturas de entrada son las "medidas experimentales" tomadas por medio de instrumentos de medida tales como termómetros, termopares, termoresistencias, etc., los cuales están afectados de un error que consideraremos aleatorio. En ocasiones y con objeto de simplificar el tratamiento se admite que los errores satisfacen ciertas hipótesis: i) los errores son 
aditivos, esto es, la medida es el resultado de sumar algebraicamente al valor exacto un cierto error; ii) La media de los errores es cero; iii) la desviación típica (o la varianza) es constante; iv) los errores no están correlacionados (covarianza nula entre errores), y v). Los errores tienen una distribución normal o gaussiana [Beck, 1985].

Con objeto de evitar el montaje experimental se ha recurrido con carácter general a obtener las medidas experimentales alterando los resultados (exactos) del problema directo por medio de un error aleatorio de valor máximo prefijado, $\varepsilon$. Los resultados del problema directo se han obtenido, a su vez, aplicando el Método de Simulación por Redes. En la práctica la solución del problema directo mediante el MESIR proporciona resultados prácticamente idénticos a los valores analíticos escogiendo un número de celdas suficientemente alto [Alhama, 1999]; esta aproximación es aún mayor en el caso de problemas lineales.

El planteamiento anterior (de resolver el problema directo) implica, obviamente, conocer a priori la solución del problema inverso. Efectivamente, se trata de resolver un problema inverso cuya solución exacta conocemos a priori; esto permite conocer las desviaciones de la solución respecto del valor exacto y poder comprobar la bondad del método propuesto. Esta manera de proceder es común en la literatura científica para verificar la idoneidad de los diferentes métodos numéricos que presentan diferentes autores.

En resumen se plantea un problema directo (su modelo matemático) cuya solución numérica cuasi-exacta se obtiene aplicando el MESIR. El campo térmico obtenido se manipula para obtener el conjunto de datos térmicos de entrada para el problema inverso. A continuación se plantea el problema inverso: a partir de estos datos y suponiendo desconocido una parte del enunciado del problema directo se procede a su determinación y se evalúa la bondad del método por comparación con la solución exacta.

Para la generación del error se han seguido dos procedimientos. En el primero se aplica un error aleatorio a la solución del problema directo con el único requisito añadido de un valor límite, es decir, la medida experimental (la ya afectada de error) está dentro de unos límites porcentuales de su valor exacto (solución del problema directo). Se trata de un procedimiento en el que el valor del error aplicado tiene la misma probabilidad para cualquiera de los valores permitidos dentro de su rango. Para un mismo valor límite del error (y siempre dentro de unos rangos pequeños), dos tandas distintas de números aleatorios pueden dar lugar a valores muy diferentes de la dispersión de las medidas experimentales. La utilización de este procedimiento puede estar justificada cuando el número de medidas experimentales es muy reducido, y va a permitir también, considerar la influencia que sobre la solución tiene el tipo de distribución del error. 
En el segundo procedimiento el error aleatorio satisface las hipótesis mencionadas al principio de este epígrafe. Se trata de un procedimiento más acorde con la distribución real de errores en las medidas por lo que su uso es más frecuente en la literatura científica.

La información generada por los sensores colocados en puntos estratégicos del medio es limitada. Por un lado las localizaciones de los sensores son discretas, es decir hay sólo un número finito de sensores, de aquí que la variación de la temperatura en la coordenada espacial es bastante incompleta. Por otro lado, las medidas de cada sensor corresponden a intervalos de tiempo discretos y no continuos. En general podemos afirmar que (paradójicamente) cuanto mayor sea el número de medidas en un punto (es decir cuanto menor sea el intervalo de tiempo entre medidas) mayor es la dificultad en estimar la solución debido a los problemas de convergencia (el intervalo de tiempo entre las componentes de la función a reproducir disminuye también al aumentar el número de medidas).

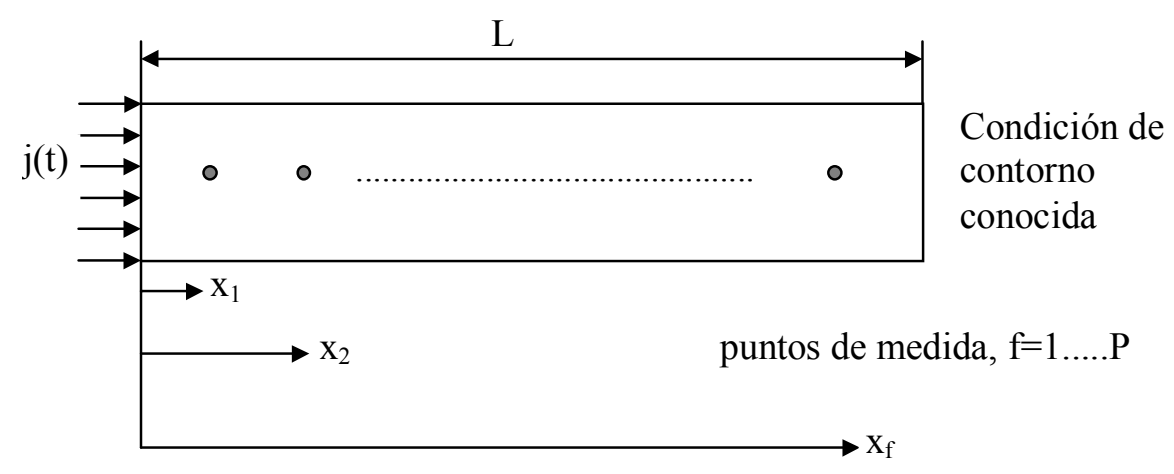

Figura III.1. Diferentes puntos de medida en un medio unidimensional

\section{Primer procedimiento}

Si llamamos al conjunto de $\mathrm{n}$ valores de la solución del problema directo $\mathrm{T}\left(\mathrm{dir}, \mathrm{x}_{\mathrm{f}}, \mathrm{t}_{\mathrm{j}}\right)=$ $\mathrm{T}\left(\operatorname{dir}, \mathrm{x}_{\mathrm{f}}, \mathrm{t}_{1}\right), \mathrm{T}\left(\operatorname{dir}, \mathrm{x}_{\mathrm{f}}, \mathrm{t}_{2}\right), \mathrm{T}\left(\operatorname{dir}, \mathrm{x}_{\mathrm{f}}, \mathrm{t}_{3}\right), \ldots, \mathrm{T}\left(\operatorname{dir}, \mathrm{x}_{\mathrm{f}}, \mathrm{t}_{\mathrm{j}}\right), \ldots, \mathrm{T}\left(\operatorname{dir}, \mathrm{x}_{\mathrm{f}}, \mathrm{t}_{\mathrm{n}}\right), 1 \leq \mathrm{j} \leq \mathrm{n}$, la serie de temperaturas de la ecuación (III.4) es el resultado de multiplicar cada uno de los valores anteriores por un número comprendido entre $1+|\varepsilon|$ y $1-|\varepsilon|$,

$$
\mathrm{T}\left(\operatorname{med}, \mathrm{x}_{\mathrm{f}}, \mathrm{t}_{\mathrm{j}}, \varepsilon\right)=\mathrm{T}\left(\mathrm{dir}, \mathrm{x}_{\mathrm{f}}, \mathrm{t}_{\mathrm{j}}\right)\left(1+\varepsilon_{\mathrm{j}}\right), 1 \leq \mathrm{j} \leq \mathrm{n}
$$

por lo que se ha incluido un nuevo parámetro $\varepsilon$ en la expresión de la temperatura, $\mathrm{T}\left(\mathrm{med}, \mathrm{x}_{\mathrm{f}}, \mathrm{t}_{\mathrm{j}}\right.$, $\varepsilon)$ para especificar en cada serie el valor máximo de error. $\varepsilon_{\mathrm{j}}$ es el valor concreto del número aleatorio correspondiente a la medida $\mathrm{t}_{\mathrm{j}},+|\varepsilon| \geq \varepsilon_{\mathrm{j}} \geq-|\varepsilon|$. Por lo tanto la serie $\mathrm{T}\left(\mathrm{med}, \mathrm{x}_{\mathrm{f}}, \mathrm{t}_{1}, \varepsilon\right)$, $\mathrm{T}\left(\mathrm{med}, \mathrm{x}_{\mathrm{f}}, \mathrm{t}_{\mathrm{n}}, \varepsilon\right)$, representa el conjunto total de $\mathrm{n}$ medidas de temperatura 
“obtenidas" por el sensor en un punto f. Siendo P el número total de puntos de medida, existirán $\mathrm{P}$ series de $\mathrm{n}$ temperaturas.

En ausencia de hipótesis sobre el error, la probabilidad de cualquier número aleatorio dentro del intervalo $[|\varepsilon|,-|\varepsilon|]$ es la misma por lo que los datos estadísticos de dispersión correspondientes a cada serie (si se quieren conocer) pueden determinarse una vez obtenido cada uno de los conjuntos de valores $\varepsilon_{j}$.

\section{Segundo procedimiento}

En cuanto al segundo procedimiento el conjunto de temperaturas viene dado por,

$$
\mathrm{T}\left(\operatorname{med}, \mathrm{x}_{\mathrm{f}}, \mathrm{t}_{\mathrm{j}}, \varepsilon\right)=\mathrm{T}\left(\operatorname{dir}, \mathrm{x}_{\mathrm{f}}, \mathrm{t}_{\mathrm{j}}\right)+\varepsilon_{\mathrm{j}}=\mathrm{T}\left(\operatorname{dir}, \mathrm{x}_{\mathrm{f}}, \mathrm{t}_{\mathrm{j}}\right)+\omega_{\mathrm{j}} \sigma
$$

Los errores $\varepsilon_{\mathrm{j}}=\omega_{\mathrm{j}} \sigma$ se obtienen a partir de los números aleatorios $\omega_{\mathrm{j}}$ a los que se exige que obedezcan a una distribución normal tipificada, esto es, de media cero y desviación típica 1 , $\mathrm{N}(0,1)$. $\sigma$ es una constante, de modo que el producto $\omega_{\mathrm{j}} \sigma$, es decir $\varepsilon_{\mathrm{j}}$ tiene también una distribución normal de media cero y desviación típica $\sigma$. Con ello se puede fijar a voluntad la desviación típica de la distribución de los errores $\varepsilon_{\mathrm{j}}$. Para generar el conjunto de números aleatorios se emplea el programa de estadística Statistics [1998].

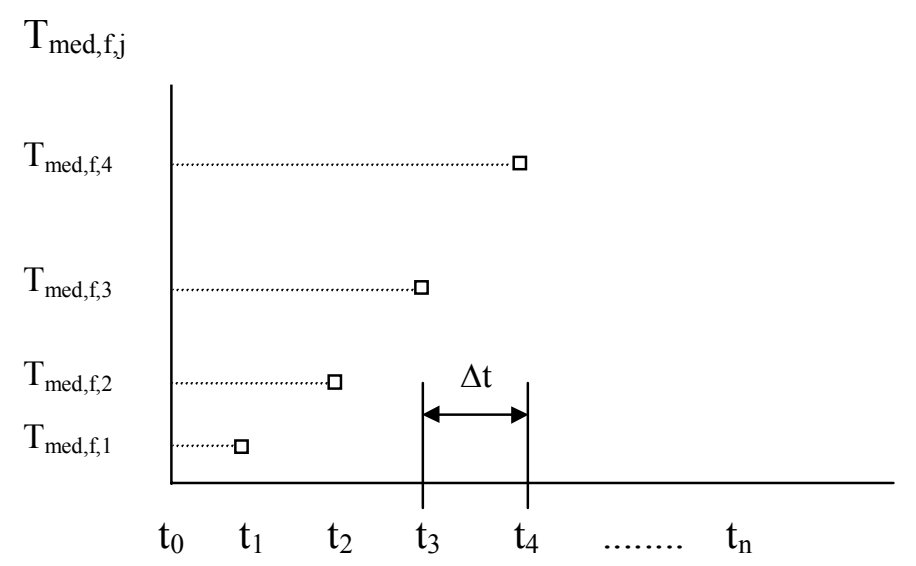

Figura III.2. Medidas de temperatura para la localización $\mathrm{x}_{\mathrm{f}} \mathrm{y}$ tiempos $\mathrm{t}_{\mathrm{j}}$

En la figura III.2 se ilustra la medida de temperaturas $T\left(m e d, x_{f}, t_{1, \ldots n}, \varepsilon\right)$ en un punto $x_{f}$ a intervalos de tiempo de medida $\Delta \mathrm{t}=\mathrm{t}_{\mathrm{j}}-\mathrm{t}_{\mathrm{j}-1}, \mathrm{y}$ afectadas del error $\varepsilon$. 


\section{III.1.3. Planteamiento}

Con la especificación completa del problema inverso, y una vez establecido el modelo en red, es el momento de comenzar el proceso inverso de búsqueda de la función a estimar (que

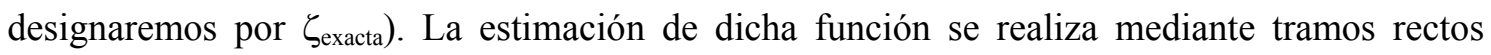
inclinados, unidos por los puntos $\zeta_{1}, \zeta_{2, \ldots . . .} \zeta_{Z}$, cuyo valor es el que se corresponde al mínimo de un funcional, $\mathrm{F}$, que a continuación se especificará, siendo $\mathrm{Z}$ es el número de tramos a emplear. La estimación inversa por tanto consiste en obtener estos puntos.

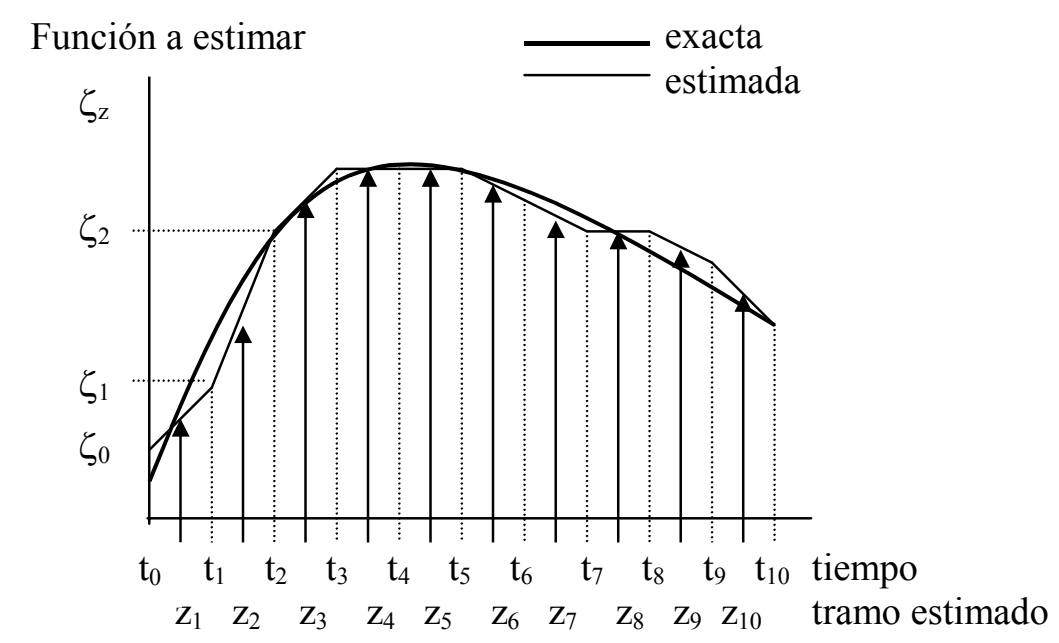

Figura III.3. Ilustración de la función a estimar por tramos rectos

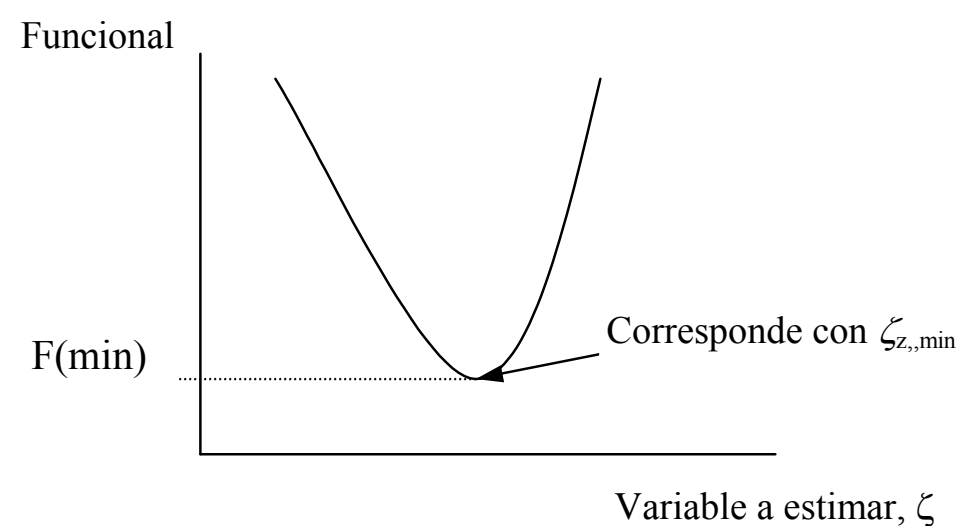

Figura III.4. Ilustración en la obtención del mínimo valor del funcional

El funcional, F, característico en este tipo de problemas viene dado por la suma de las desviaciones cuadráticas entre el campo de temperaturas "suministrado" por los sensores, 
$\mathrm{T}(\mathrm{med})$, y el campo térmico obtenido por aplicación del MESIR para un valor de prueba de la variable $\zeta$ en estudio, T(inv), en cada intervalo temporal.

$$
\mathrm{F}\left[\mathrm{x}_{\mathrm{f}}, \zeta, \mathrm{t}, \varepsilon_{\mathrm{j}}, \Delta \mathrm{t}, \mathrm{z}\right]=\sum_{\mathrm{j}}\left[\mathrm{T}\left(\operatorname{inv}, \mathrm{x}_{\mathrm{f}}, \mathrm{t}_{\mathrm{j}}, \zeta\right)-\mathrm{T}\left(\operatorname{med}, \mathrm{x}_{\mathrm{f}}, \mathrm{t}_{\mathrm{j}}, \varepsilon\right)\right]^{2}
$$

Aquel valor de $\zeta$ para el que T(inv) es tal que hace mínimo el funcional $\mathrm{F}$, es el valor $\zeta_{z, \text { min }}$ elegido para definir el punto del tramo correspondiente.

En el cálculo del valor $\zeta_{z}$ correspondiente al tiempo $t_{z}$, se emplearan $r$ tiempos, desde $t_{z}$ hasta $\mathrm{t}_{\mathrm{z}+\mathrm{r}-1}$, por lo tanto, $\mathrm{r}$ temperaturas correspondientes a dichos tiempos. El número $\mathrm{r}$ se ha elegido según el tipo de problema a considerar. En general el uso de varias temperaturas en cada intervalo temporal, proporciona una gran estabilidad y reduce sustancialmente la sensibilidad de la solución a los errores cometidos en las medidas. En la figura III.5 se ilustra el intervalo temporal (y de temperaturas) que se utiliza en el caso de ser $\mathrm{z}=6 \mathrm{y} \mathrm{r}=7$; es decir, en el proceso de estimación se habrían ya obtenido los valores $\zeta_{0}, \zeta_{1}, \ldots, \zeta_{5}$ y se trataría de determinar $\zeta_{6}$.

Intervalos de estimación

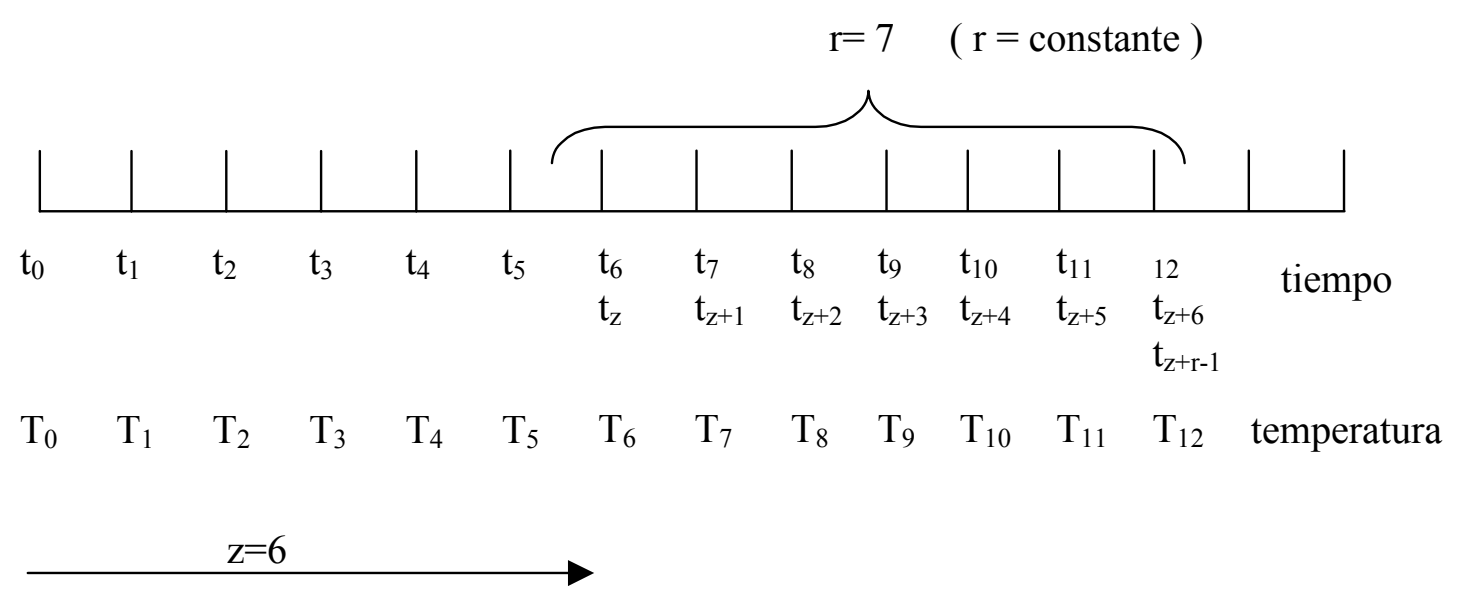

Figura III.5. Ilustración en la estimación de funciones dependientes del tiempo

Concretando más, fijémonos en la figura III.6, en la que se ilustra el caso de la estimación de una función dependiente del tiempo, el flujo de calor $\zeta(\mathrm{t})$, en donde se observa que se han realizado ya tres estimaciones: $\left(\zeta_{0}, \mathrm{t}_{0}\right),\left(\zeta_{1}, \mathrm{t}_{1}\right)$ y $\left(\zeta_{2}, \mathrm{t}_{2}\right)$. Actualmente se está estimando $\zeta_{3}(\mathrm{z}=3)$. En el caso de estimación de funciones dependientes del tiempo, el número de temperaturas futuras es un valor constante en todo el dominio de estimación, siendo $r=4$ en el ejemplo analizado. 


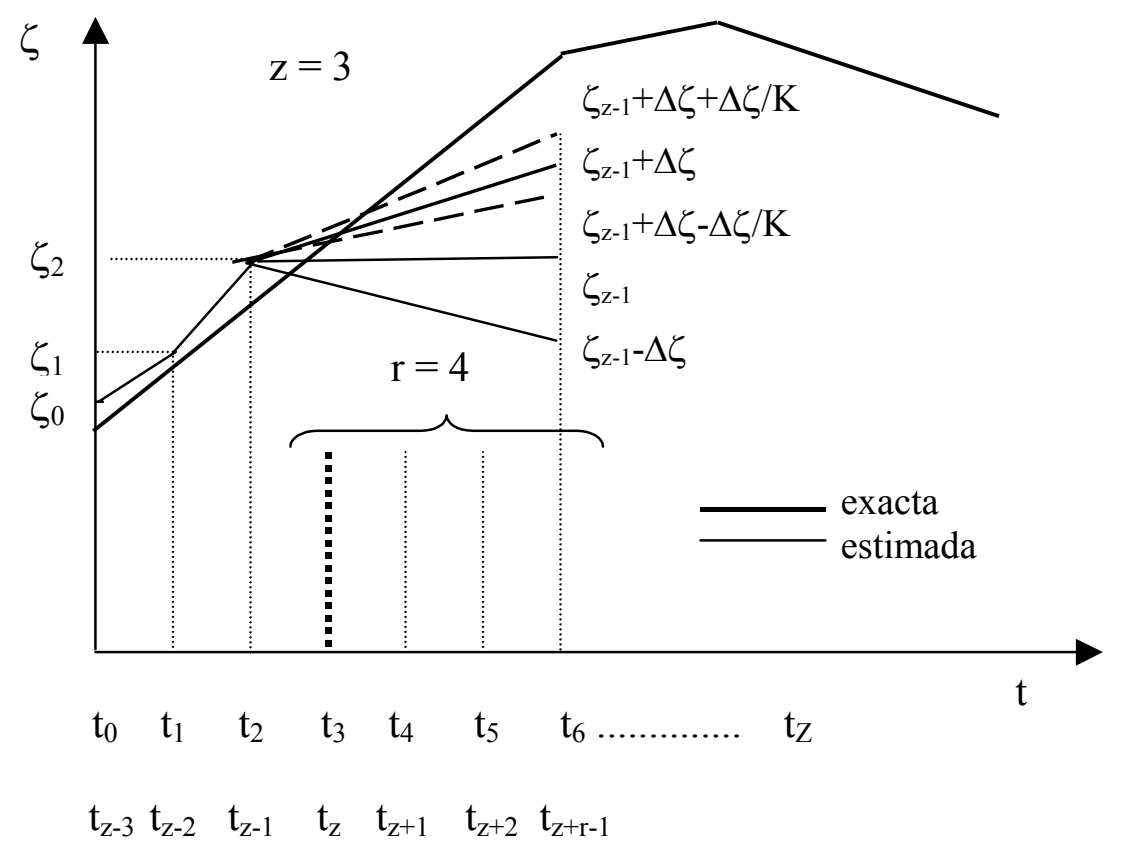

Figura III.6. Detalle de la estimación de una función temporal

A partir de $\zeta_{z-1}$ se trata de determinar $\zeta_{z}$ correspondiente a $t_{z}$, siendo $r=4$. Para ello se establecen los segmentos definidos por los puntos $\left(\zeta_{2}, \mathrm{t}_{2}\right),\left(\zeta_{2}+\Delta \zeta, \mathrm{t}_{6}\right) ;\left(\zeta_{2}, \mathrm{t}_{2}\right),\left(\zeta_{2}, \mathrm{t}_{6}\right)$ y $\left(\zeta_{2}, \mathrm{t}_{2}\right)$, $\left(\zeta_{2}-\Delta \zeta, \mathrm{t}_{6}\right)$; se evalúa el funcional $\mathrm{F}$ en cada segmento, y se elige aquel que corresponde con el mínimo de F. Supongamos que sea el primero. Alrededor de el se establecen los segmentos definidos por los puntos $\left(\zeta_{2}, \mathrm{t}_{2}\right),\left(\zeta_{2}+\Delta \zeta+\Delta \zeta / \mathrm{K}, \mathrm{t}_{6}\right) ;\left(\zeta_{2}, \mathrm{t}_{2}\right),\left(\zeta_{2}+\Delta \zeta, \mathrm{t}_{6}\right)$ y $\left(\zeta_{2}, \mathrm{t}_{2}\right),\left(\zeta_{2}+\Delta \zeta-\right.$ $\Delta / \mathrm{K}, \mathrm{t}_{6}$ ), siendo $\mathrm{K}$ un numero natural mayor que la unidad, disponiéndose así de otros tres nuevos segmentos. Evaluado $\mathrm{F}$ en ellos se elige aquel que sea mínimo. El proceso se repite hasta alcanzar una cota preestablecida en la variación (o incremento) del flujo (criterio de convergencia). Finalmente, seleccionado el último segmento, mediante interpolación se determina el valor $\zeta_{3}$ correspondiente a $t_{3}$.

En la figura III.7 se ilustra gráficamente y de forma aproximada el procedimiento desarrollado por Beck (ejemplos en trabajos Beck [1970] y Beck y col. [1982]), donde se aprecia la diferencia esencial con el método empleado en esta tesis doctoral, consistente en estimación mediante tramos escalón en vez de inclinados. 


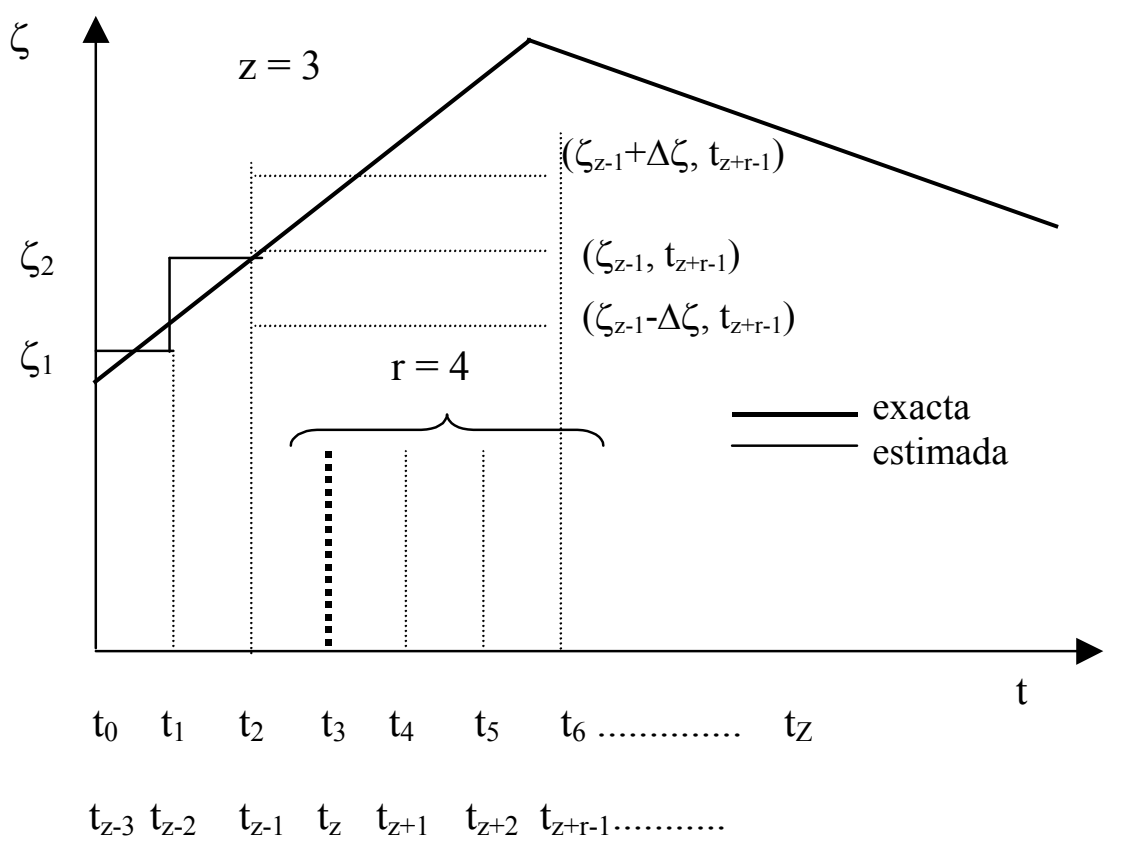

Figura III.7. Procedimiento de Beck para la estimación de una función temporal

Los valores que se van obteniendo, $\zeta_{0}, \zeta_{1}, \zeta_{2}$, se emplean en la salida de la fuente de corriente, que simula el flujo entrante del proceso, para la determinación de las nuevas temperaturas T(inv) para la obtención de $\zeta_{3}$.

Para el caso de estimación simultánea de funciones, diferentes valores de $\zeta$ y $\psi$ proporcionan diferentes valores de $\mathrm{T}\left(\mathrm{inv}, \mathrm{x}_{\mathrm{f}}, \mathrm{t}_{\mathrm{j}}, \zeta, \psi\right) \mathrm{y}$, en consecuencia del funcional, siendo los valores óptimos $\zeta_{z, \min } \mathrm{y} \psi_{z, \min }$, los correspondientes al valor mínimo del funcional. Los valores del error, $\varepsilon$, del número medidas, $\mathrm{n}$ y de la posición, $\mathrm{x}_{\mathrm{f}}$ permanecen constantes en cada uno de los cálculos de los valores mínimos en todos los tipos de estimaciones. Así,

$$
\zeta_{\mathrm{z}, \min } \mathrm{y} \psi_{\mathrm{z}, \min }=\operatorname{mín}\left[\mathrm{F}\left(\mathrm{x}_{\mathrm{f}}, \zeta, \psi, \varepsilon, \mathrm{z}\right)\right], \zeta \mathrm{y} \psi \text { variables } \mathrm{y} \Delta \mathrm{t}, \varepsilon, \mathrm{n} \mathrm{y} \mathrm{x}_{\mathrm{f}} \text { fijos } \quad \text { (III.8) }
$$

A continuación, se dan los procedimientos computacionales específicos para el caso de estimación de funciones, que es el más general y complejo, distinguiendo entre estimación de funciones dependientes de la temperatura, ídem dependientes del tiempo y estimación simultánea de funciones dependientes de la temperatura; en cada caso se específica la forma que adquiere el funcional, para finalmente exponer el algoritmo general que se sigue para todos los problemas tratados en esta Memoria. 


\section{III.2. ESTIMACIÓN DE FUNCIONES DEPENDIENTES DEL TIEMPO}

\section{III.2.1. Especificación del funcional}

Para el caso de estimación de una sola función dependiente del tiempo, y suponiendo que existe un sólo punto de medida, el funcional viene dado por:

$$
\mathrm{F} \quad\left[\mathrm{x}_{\mathrm{f}}, \zeta, \mathrm{t}, \varepsilon_{\mathrm{j}}, \Delta \mathrm{t}, \mathrm{z}\right]=\sum_{\mathrm{j}=\mathrm{z}}^{\mathrm{z} \mathrm{r}-1}\left[\mathrm{~T}\left(\operatorname{inv}, \mathrm{x}_{\mathrm{f}}, \mathrm{t}_{\mathrm{j}}, \zeta\right)-\mathrm{T}\left(\operatorname{med}, \mathrm{x}_{\mathrm{f}}, \mathrm{t}_{\mathrm{j}}, \varepsilon\right)\right]^{2}
$$

donde:

$\mathrm{x}_{\mathrm{f}} \quad$ es la posición concreta donde se realizan las medidas,

$\Delta \mathrm{t} \quad$ es el intervalo de tiempo entre medidas,

$\mathrm{r} \quad$ es el número de temperaturas que entran dentro del funcional en cada intervalo de estimación, siendo en este caso un valor constante definido por el programador,

$\mathrm{z}=1,2, \ldots, \mathrm{Z}$, identifica el tramo de la función piece-wise continua que cubre el rango de la función a estimar, $\mathrm{y}$

$\zeta \quad$ es el parámetro a estimar.

El valor de $\mathrm{j}=\mathrm{z}$ se corresponde con el extremo inicial de tiempo (dentro del intervalo de tiempo de estimación en el que se calcula el funcional), siendo ( $\mathrm{z}+\mathrm{r}-1)$ el extremo final del intervalo, que se corresponde con el ultimo valor de la medida de temperatura dentro de dicho intervalo. En el apartado siguiente se numeran todos los pasos seguidos para la estimación de este tipo de funciones.

\section{III.2.2. Procedimiento computacional}

Algunos ejemplos de aplicación de este procedimiento computacional son: la estimación de flujos de calor o temperaturas en la frontera, la estimación del coeficiente de convección, etc. En este caso el parámetro " $r$ " es de valor constante. El siguiente algoritmo resume el procedimiento computacional empleado:

Paso 1: Solución del problema directo (DHCP) por medio del MESIR. Obtención de los datos de entrada de temperaturas ("medidas experimentales"), 
Paso 2: Determinación del primer punto (del primer tramo) de la solución, $\left(\zeta_{0}, \mathrm{t}_{0}\right)$. Esta determinación se realiza de forma independiente al resto del procedimiento, definiendo tres segmentos horizontales de la función de estimación que contengan un cierto número de medidas, y calculando el funcional para estos segmentos. Para el segmento asociado al mínimo funcional y otros dos nuevos definidos mediante un criterio de aproximación se repite el procedimiento iterativamente hasta una cierta cota predefinida del parámetro de estimación, figura 8 ,

Paso 3: Fijar los valores de los parámetros: $\delta$ (criterio de convergencia), $\Delta \zeta$ (intervalo de paso de la función de estimación) y $K(K>1$, factor de reducción del parámetro de estimación). $\mathrm{z}=0$,

Paso 4: $\mathrm{z}=\mathrm{z}+1$,

Paso 5: Para cada tramo a determinar, cuyo punto inicial es $\left(\zeta_{\mathrm{z}-1}, \mathrm{t}_{\mathrm{z}-1}\right)$, se evalúa el funcional para los tres segmentos definidos por los puntos: $\left(\zeta_{\mathrm{z}-1}, \mathrm{t}_{\mathrm{z}-1}\right)$ y $\left(\zeta_{\mathrm{z}-1}+\Delta \zeta, \mathrm{t}_{\mathrm{z}+\mathrm{r}-1}\right),\left(\zeta_{\mathrm{z}-1}, \mathrm{t}_{\mathrm{z}-1}\right)$ $\mathrm{y}\left(\zeta_{\mathrm{z}-1}, \mathrm{t}_{\mathrm{z}+\mathrm{r}-1}\right)$, y finalmente $\left(\zeta_{\mathrm{z}-1}, \mathrm{t}_{\mathrm{z}-1}\right)$ y $\left(\zeta_{\mathrm{z}-1}-\Delta \zeta, \mathrm{t}_{\mathrm{z}+\mathrm{r}-1}\right)$. Previamente se ha ejecutado Pspice para cada uno de los tres segmentos, obteniéndose la serie de valores de temperaturas $\mathrm{T}\left(\mathrm{inv}, \mathrm{x}_{\mathrm{f}}, \mathrm{t}_{\mathrm{j}}, \zeta\right.$ ) que intervienen en el funcional. De estos tres segmentos se selecciona el que está asociado al mínimo valor del funcional; el extremo de este segmento que llamaremos $\left(\zeta_{\mathrm{z}-1, \min }, \mathrm{t}_{\mathrm{z}+\mathrm{r}-1}\right)$ es el punto retenido para la siguiente iteración,

Paso 6: $\Delta \zeta=\Delta \zeta / \mathrm{K}$

Paso 7: Repetir el paso 5 para tres nuevos segmentos definidos por los puntos: $\left(\zeta_{\mathrm{z}-1}, \mathrm{t}_{\mathrm{z}-1}\right)$ y $\left(\zeta_{\mathrm{z}-}\right.$ $\left.1, \min +\Delta \zeta, \mathrm{t}_{\mathrm{z}+\mathrm{r}-1}\right),\left(\zeta_{\mathrm{z}-1}, \mathrm{t}_{\mathrm{z}-1}\right)$ y $\left(\zeta_{\mathrm{z}-1, \min }, \mathrm{t}_{\mathrm{z}+\mathrm{r}-1}\right)$, y el último $\left(\zeta_{\mathrm{z}-1}, \mathrm{t}_{\mathrm{z}-1}\right)$ y $\left(\zeta_{\mathrm{z}-1, \min }-\Delta \zeta, \mathrm{t}_{\mathrm{z}+\mathrm{r}-1}\right)$, determinar un nuevo valor $\left(\zeta_{z-1, \min }, \mathrm{t}_{\mathrm{z}+\mathrm{r}-1}\right)$,

Paso 8: Si se cumple que $\Delta \zeta>\delta$, repetir los pasos 6 y 7. Si se cumple que $\Delta \zeta<\delta$, del último segmento seleccionado retener (mediante interpolación lineal) el punto $\left(\zeta_{\mathrm{z}}, \mathrm{t}_{\mathrm{z}}\right)$,

Paso 9: Volver al paso 4 para evaluar un nuevo tramo. 


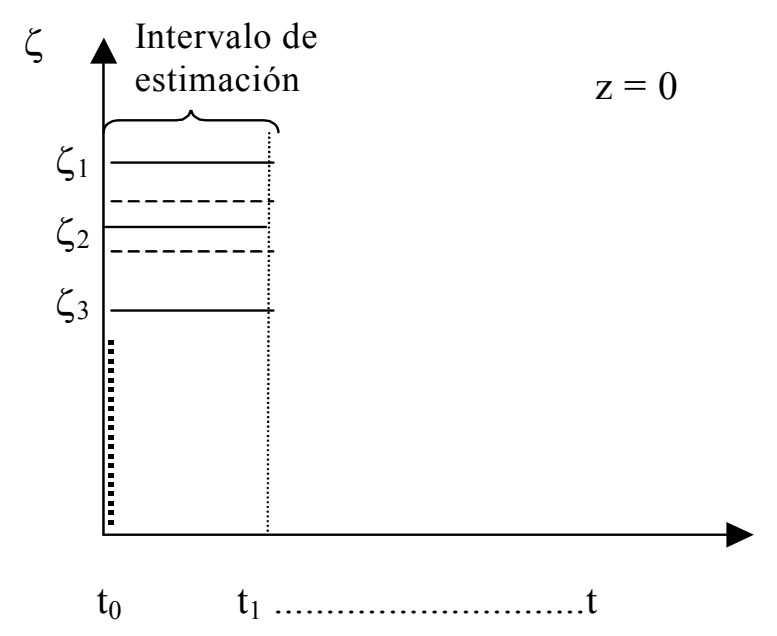

Figura III.8. Estimación del punto inicial

\section{III.3. ESTIMACIÓN DE FUNCIONES DEPENDIENTES DE LA TEMPERATURA}

\section{III.3.1. Especificación del funcional}

La expresión del funcional para el caso de estimación de una función dependiente de la temperatura, considerando un solo punto de medida es:

$$
\mathrm{F}\left[\mathrm{x}_{\mathrm{f}}, \zeta, \mathrm{T}, \varepsilon, \Delta \mathrm{t}, \Delta \mathrm{T}_{\mathrm{a}}, \mathrm{z}\right]=\sum_{\mathrm{j}=0}^{\mathrm{z}}\left[\mathrm{T}\left(\operatorname{inv}, \mathrm{x}_{\mathrm{f}}, \mathrm{t}_{\mathrm{j}}, \zeta\right)-\mathrm{T}\left(\operatorname{med}, \mathrm{x}_{\mathrm{f}}, \mathrm{t}_{\mathrm{j}}, \varepsilon\right)\right]^{2}
$$

$\zeta, \mathrm{x}_{\mathrm{f}}, \Delta \mathrm{t} \mathrm{y} \mathrm{z}=1,2, \ldots, \mathrm{Z}$ tienen el mismo significado que en el caso anterior. Se incorpora un nuevo parámetro que representa el intervalo de temperatura que posee cada tramo de estimación, $\Delta \mathrm{T}_{\mathrm{a}}$. Este parámetro ha de definirse antes de iniciar la estimación.

En este caso, el número de términos que integran el funcional no es constante, como ocurría en el funcional definido para la estimación de funciones dependientes del tiempo (ecuación III.8), ya que el número de términos que integran el funcional se corresponde con el número de temperaturas (medidas) en el intervalo de temperatura en el que se está realizando la estimación, por ello se define el parámetro $\mathrm{r}_{\mathrm{z}}$, número de temperaturas que entran dentro del funcional para el intervalo de estimación $z$. 
Los intervalos de estimación serán: $\mathrm{T}_{0} \rightarrow \mathrm{T}_{0}+\Delta \mathrm{T}_{\mathrm{a}}, \mathrm{T}_{0}+0.5 \Delta \mathrm{T}_{\mathrm{a}} \rightarrow \mathrm{T}_{0}+1.5 \Delta \mathrm{T}_{\mathrm{a}}, \mathrm{T}_{0}+\Delta \mathrm{T}_{\mathrm{a}} \rightarrow$ $\mathrm{T}_{0}+2 \Delta \mathrm{T}_{\mathrm{a}}, \quad \mathrm{T}_{0}+1.5 \Delta \mathrm{T}_{\mathrm{a}} \rightarrow \mathrm{T}_{0}+2.5 \Delta \mathrm{T}_{\mathrm{a}} \quad \ldots \ldots . \mathrm{T}_{0}+(\mathrm{Z}-1) / 2 \quad \Delta \mathrm{T}_{\mathrm{a}} \rightarrow \mathrm{T}_{0}+(\mathrm{Z}+1) / 2 \quad \Delta \mathrm{T}_{\mathrm{a}}$, siendo $\mathrm{T}_{0}$ la temperatura inicial de la estimación. En la figura III.9 se muestra un ejemplo, observándose que en este caso los valores de $r_{z}$ (estos valores dependen únicamente de las temperaturas medidas, del intervalo de tiempo entre medidas, $\Delta \mathrm{t}$, y del intervalo de temperatura en la estimación $\Delta \mathrm{T}_{\mathrm{a}}$ ) aumentan conforme se avanza en la estimación, de la función. Por ejemplo para $\mathrm{z}=4$, de la expresión, $\left.\mathrm{T}_{0}+(\mathrm{z}-1) / 2 \Delta \mathrm{T}_{\mathrm{a}} \rightarrow \mathrm{T}_{0}+(\mathrm{z}+1) / 2 \Delta \mathrm{T}_{\mathrm{a}}\right)$, se obtiene el intervalo de estimación $\mathrm{T}_{0}+$ $1.5 \Delta \mathrm{T}_{\mathrm{a}} \rightarrow \mathrm{T}_{0}+2.5 \Delta \mathrm{T}_{\mathrm{a}}$. Los extremos de dicho intervalo se corresponden con las temperaturas mínima y máxima del mismo, y con los tiempos $t_{\mathrm{r} 3}$ y $t_{\mathrm{r} 5}$ respectivamente, siendo $t_{\mathrm{r} 3}=\mathrm{r}_{3} \Delta t$ y $\mathrm{t}_{\mathrm{r} 5}=\mathrm{r}_{5} \Delta \mathrm{t}$. Si los parámetros toman los valores, $\Delta \mathrm{T}_{\mathrm{a}}=2{ }^{\circ} \mathrm{C}, \mathrm{T}_{0}=0{ }^{\circ} \mathrm{C}$, el intervalo de temperaturas a estimar será, $3{ }^{\circ} \mathrm{C} \rightarrow 5^{\circ} \mathrm{C}$. Las temperaturas que integran el funcional poseen los extremos siguientes:

i) medidas por el sensor: $\mathrm{T}\left(\right.$ med, $\left.\mathrm{x}_{\mathrm{f}}, \mathrm{t}_{0}, \varepsilon\right) \rightarrow \mathrm{T}\left(\right.$ med, $\left.\mathrm{x}_{\mathrm{f}}, \mathrm{t}_{\mathrm{r} 4}, \varepsilon\right)$

ii) obtenidas por simulación: $\mathrm{T}\left(\mathrm{inv}, \mathrm{x}_{\mathrm{f}}, \mathrm{t}_{0}, \Delta \mathrm{t}, \zeta\right) \rightarrow \mathrm{T}\left(\mathrm{inv}, \mathrm{x}_{\mathrm{f}}, \mathrm{t}_{\mathrm{r} 4}, \Delta \mathrm{t}, \zeta\right)$.

Intervalos de estimación

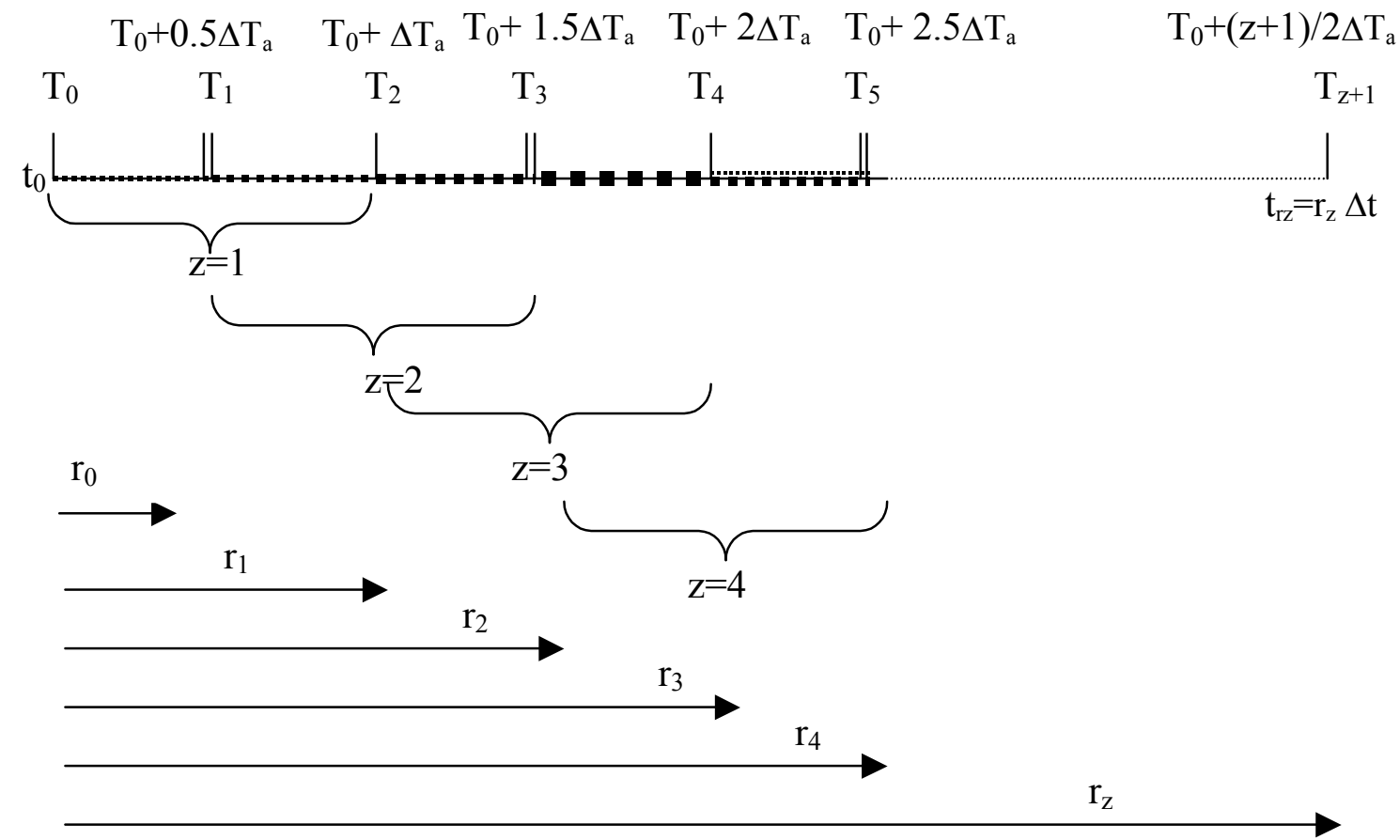

Figura III.9. Ilustración del valor de los distintos $r_{z}$ en la estimación de funciones dependientes de la temperatura 
En el apartado siguiente se numeran todos los pasos seguidos para la estimación de este tipo de funciones.

\section{III.3.2. Procedimiento computacional}

Ejemplos de aplicación de este procedimiento computacional son la estimación de las propiedades termofísicas (calor especifico y conductividad), estimación de la generación interna de calor, del coeficiente de convección, etc. $\mathrm{r}_{\mathrm{z}}$, número de términos del funcional (variable en cada tramo), es el número de medidas (temperaturas) desde la inicial hasta la correspondiente al extremo del intervalo de estimación (figura III.9). El siguiente algoritmo resume el procedimiento computacional de resolución:

Paso 1: Solución del problema directo (DHCP) por medio del MESIR. Obtención de los datos de entrada de temperaturas ("medidas experimentales"),

Paso 2: Determinación del primer punto, $\left(\zeta_{0}, \mathrm{~T}_{0}\right)$, del primer tramo de la solución. En la figura III.10, se ilustra el procedimiento seguido en la estimación del punto inicial $\left(\zeta_{0}, \mathrm{~T}_{0}\right)$, donde se definen tramos rectos $\zeta_{1}, \zeta_{2}, \ldots$, hasta que se verifique el criterio de convergencia (de forma similar a como se ha descrito en el apartado III.2.2),

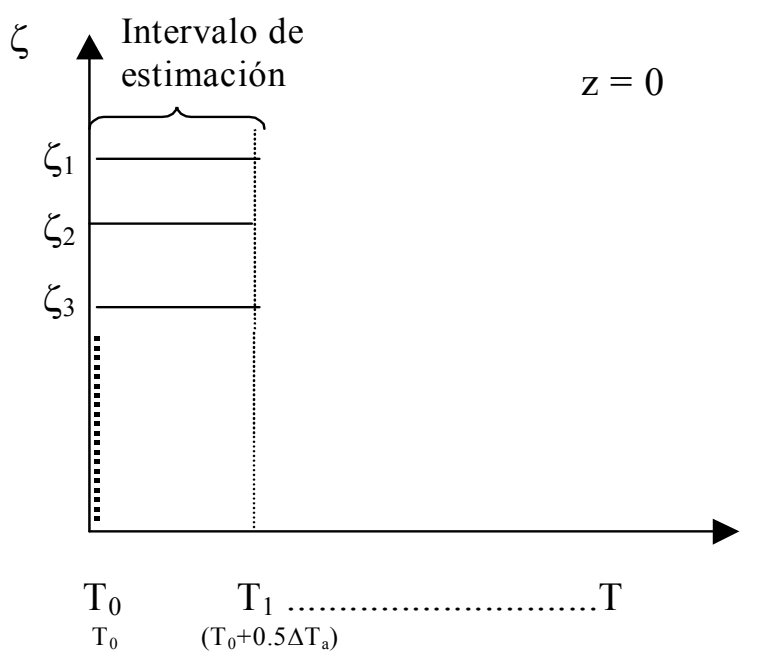

Figura III.10. Estimación del punto inicial

Paso 3: Fijar los valores de los parámetros: $\delta$ (criterio de convergencia), $\mathrm{K}(\mathrm{K}>1)$, factor de reducción del parámetro de estimación para obtener el intervalo de paso, $\Delta \zeta$, y el primer valor de $\Delta \zeta . \mathrm{z}=0$, 
Paso 4: $\mathrm{z}=\mathrm{z}+1 . \mathrm{T}_{\mathrm{z}}=\mathrm{T}_{\mathrm{z}-1}+0.5 \Delta \mathrm{T}_{\mathrm{a}}, \mathrm{T}_{\mathrm{z}+1}=\mathrm{T}_{\mathrm{z}-1}+\Delta \mathrm{T}_{\mathrm{a}}$,

Paso 5: Evaluación de $\mathrm{r}_{\mathrm{z}}$,

Paso 6: Para cada tramo que va a ser determinado, cuyo punto inicial es $\left(\zeta_{z-1}, T_{z-1}\right)$, se evalúa el funcional para los tres segmentos definidos por los puntos: $\left(\zeta_{z-1}, T_{z-1}\right)$ y $\left(\zeta_{z-1}+\Delta \zeta, T_{z+1}\right)$, $\left(\zeta_{z-1}, T_{z-1}\right)$ y $\left(\zeta_{z-1}, T_{z+1}\right)$, y el tercero $\left(\zeta_{z-1}, T_{z-1}\right)$ y $\left(\zeta_{z-1}-\Delta \zeta, T_{z+1}\right)$. Previamente se ejecuta Pspice para cada uno de los tres segmentos, obteniéndose la serie de valores de temperaturas $\mathrm{T}\left(\mathrm{inv}, \mathrm{x}_{\mathrm{f}}, \mathrm{t}_{\mathrm{j}}, \zeta\right)$ que forman parte del funcional. Se retiene el segmento cuyo punto final es el correspondiente al valor mínimo del funcional, $\left(\zeta_{\mathrm{z}+1, \min }, \mathrm{T}_{\mathrm{z}+1}\right)$,

Paso 7: $\Delta \zeta=\Delta \zeta / \mathrm{K}$

Paso 8: Repetir el paso 6 para tres nuevos segmentos definidos por los puntos: $\left(\zeta_{\mathrm{z}-1}, \mathrm{~T}_{\mathrm{z}-1}\right) \mathrm{y}$ $\left(\zeta_{z+1, \min }+\Delta \zeta, T_{z+1}\right),\left(\zeta_{z-1}, T_{z-1}\right)$ y $\left(\zeta_{z+1, \min }, T_{z+1}\right)$, y el último $\left(\zeta_{z-1}, T_{z-1}\right)$ y $\left(\zeta_{z+1, \min }-\Delta \zeta, T_{z+1}\right)$. Determinar el nuevo $\left(\zeta_{z+1, \min }, \mathrm{T}_{z+1}\right)$,

Paso 9: Si se cumple que $\Delta \zeta>\delta$, repetir los pasos 7 y 8 . Si se cumple que $\Delta \zeta<\delta$, retener el último punto del segmento, $\left(\zeta_{z+1, \min }, T_{z+1}\right)$,

Paso 10: Retener el valor del punto $\left(\zeta_{z}, T_{z}\right)$ como el valor medio entre los puntos $\left(\zeta_{z-1}, T_{z-1}\right)$ y $\left(\zeta_{z+1, \min }, T_{z+1}\right)$,

Paso 11: Volver al paso 4 para evaluar un nuevo tramo.

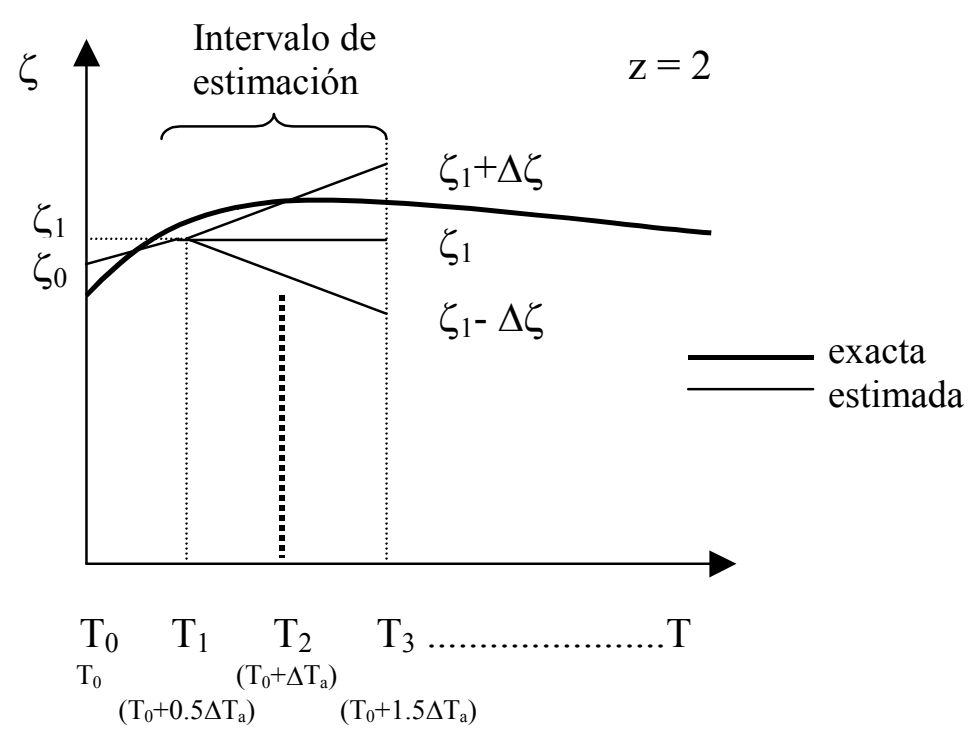

Figura III.11. Ilustración de la estimación de una función dependiente de la temperatura 
En la figura III.11 se muestra un ejemplo para la obtención del tramo z=2.

\section{III.4. ESTIMACIÓN SIMULTÁNEA DE DOS FUNCIONES AMBAS DEPENDIENTES DE LA TEMPERATURA}

La estimación simultánea de dos funciones es un problema de mayor complejidad que requiere una expresión más complicada del funcional. La técnica más frecuente [Yang, 2000] exige tomar datos de temperatura en varios puntos de medida (un único punto de medida puede conducir con frecuencia a estimaciones poco fiables).

\section{III.4.1. Especificación del funcional}

Para el caso de estimación simultánea de dos funciones dependientes del tiempo $(\zeta(t)$, $\psi(\mathrm{t}))$ se define el siguiente funcional,

$\mathrm{F}\left[\mathrm{x}_{\mathrm{f}}, \zeta(\mathrm{t}), \psi(\mathrm{t}), \varepsilon, \Delta \mathrm{t}, \mathrm{z}\right]=\sum_{\mathrm{f}=1}^{\mathrm{P}}\left[\sum_{\mathrm{j}=\mathrm{z}}^{\mathrm{z}+\mathrm{r}-1}\left[\mathrm{~T}\left(\mathrm{inv}_{\mathrm{f}}, \mathrm{x}_{\mathrm{f}}, \mathrm{t}_{\mathrm{j}}, \zeta, \psi\right)-\mathrm{T}\left(\operatorname{med}, \mathrm{x}_{\mathrm{f}}, \mathrm{t}_{\mathrm{j}}, \varepsilon\right)\right]^{2}\right]$

Si se trata de dos funciones dependientes de la temperatura $(\zeta(T), \psi(T))$, el funcional tiene la forma,

$\mathrm{F}\left[\mathrm{x}_{\mathrm{f}}, \zeta(\mathrm{T}), \psi(\mathrm{T}), \varepsilon, \Delta \mathrm{t}, \Delta \mathrm{T}_{\mathrm{a}}, \mathrm{z}\right]=\sum_{\mathrm{f}=1}^{\mathrm{P}}\left[\sum_{\mathrm{j}=0}^{\mathrm{r}, \mathrm{f}}\left[\mathrm{T}\left(\text { inv, }_{\mathrm{f}}, \mathrm{t}_{\mathrm{j}}, \zeta, \psi\right)-\mathrm{T}\left(\text { med, }_{\mathrm{f}}, \mathrm{t}_{\mathrm{j}}, \varepsilon\right)\right]^{2}\right]$

En estas expresiones,

$\mathrm{P} \quad$ es el número total de sensores de medida de las temperaturas,

$\mathrm{x}_{\mathrm{f}} \quad$ es la localización particular de cada sensor, $\mathrm{y}$

$\mathrm{r}_{\mathrm{z}, \mathrm{f}} \quad$ número de temperaturas dentro del funcional para el tramo $\mathrm{z}$ y sensor $\mathrm{f}$

En la figura III.12 se ilustra mediante un ejemplo el uso de $r_{z, f}$ para tres puntos de medida, $\mathrm{x}_{1}, \mathrm{x}_{2}, \mathrm{y} \mathrm{x}_{3}$ para $\mathrm{z}=3$. 


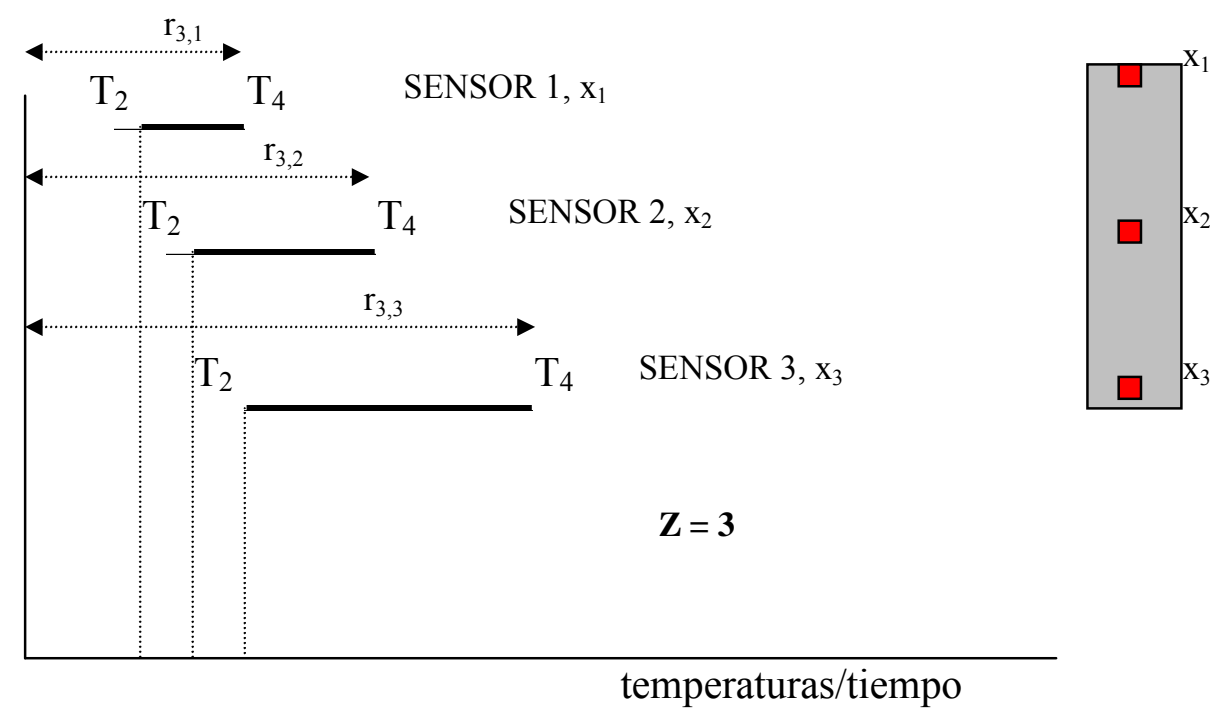

Figura III.12. Ilustración de distintos puntos de medida en un medio

\section{III.4.2. Procedimiento computacional}

El ejemplo de aplicación de este procedimiento computacional más frecuente en la bibliografía es la estimación simultánea de las propiedades térmicas, calor especifico y conductividad, dependientes ambas de la temperatura [Huang y Özisik, 1991; Huang y Yang, 1995; Yang, 2000]. Aunque también es posible combinar otro par de parámetros, por ejemplo el coeficiente de convección y la conductividad térmica o el calor especifico.

El algoritmo computacional de resolución es el siguiente:

Paso 1: Solución del problema directo (DHCP) por medio del MESIR. Obtención de los datos de entrada de temperaturas,

Paso 2: Determinación de los primeros puntos del primer tramo de la solución, $\left(\zeta_{0}, \mathrm{~T}_{0}\right)$ y $\left(\psi_{0}, \mathrm{~T}_{0}\right)$, por un procedimiento similar al mencionado para los casos anteriores,

Paso 3: Fijar los valores de los parámetros: $\delta_{1}$ y $\delta_{2}$ (criterio de convergencia), de las constantes $\mathrm{K}_{1}\left(\mathrm{~K}_{1}>1\right)$ y $\mathrm{K}_{2}\left(\mathrm{~K}_{2}>1\right)$, factores de reducción de los parámetros de estimación $\zeta$ y $\psi$ para obtener los intervalos de paso $\Delta \zeta$ y $\Delta \psi$, respectivamente, y del primer valor de $\Delta \zeta$ $\mathrm{y} \Delta \psi \cdot \mathrm{z}=0$,

Paso 4: $\mathrm{z}=\mathrm{z}+1 . \mathrm{T}_{\mathrm{z}}=\mathrm{T}_{\mathrm{z}-1}+0.5 \Delta \mathrm{T}_{\mathrm{a}}, \mathrm{T}_{\mathrm{z}+1}=\mathrm{T}_{\mathrm{z}-1}+\Delta \mathrm{T}_{\mathrm{a}}$

Paso 5: Evaluación del término $r_{z, f}$,

Paso 6: Fijar las nueve posibles combinaciones de pares de segmentos, uno de ellos tomado de entre los tres siguientes definidos por los pares de puntos $\left(\zeta_{z-1}, T_{z-1}\right)$ y $\left(\zeta_{z-1}+\Delta \zeta, T_{z+1}\right)$, 
$\left(\zeta_{z-1}, T_{z-1}\right)$ y $\left(\zeta_{z-1}, T_{z+1}\right),\left(\zeta_{z-1}, T_{z-1}\right)$ y $\left(\zeta_{z-1}-\Delta \zeta, T_{z+1}\right)$, y el otro tomado de entre los tres siguientes definidos por los pares de puntos $\left(\psi_{z-1}, T_{z-1}\right)$ y $\left(\psi_{z-1}+\Delta \psi, T_{z+1}\right),\left(\psi_{z-1}, T_{z-1}\right)$ y $\left(\psi_{z-1}, T_{z+1}\right)$, y $\left(\psi_{z-1}, T_{z-1}\right)$ y $\left(\psi_{z-1}-\Delta \psi, T_{z+1}\right)$. Se ejecuta Pspice para estas combinaciones obteniéndose nueve series de valores de temperaturas $\mathrm{T}\left(\mathrm{inv}, \mathrm{x}_{\mathrm{f}}, \mathrm{t}_{\mathrm{j}}, \zeta, \psi\right)$. Se evalúa el funcional para cada serie reteniendo el par de segmentos que corresponden al valor mínimo de este. Se retienen los extremos de este par de segmentos, que llamaremos $\left(\zeta_{z+1, \min }, T_{z+1}\right)$ y $\left(\psi_{z+1, \min }, T_{z+1}\right)$,

Paso 7: $\Delta \zeta=\Delta \zeta / \mathrm{K}_{1}, \Delta \psi=\Delta \psi / \mathrm{K}_{2}$,

Paso 8: Repetir el paso 6 para los segmentos asociados a la variable $\zeta$ definidos por los puntos $\left(\zeta_{z-1}, T_{z-1}\right)$ y $\left(\zeta_{z+1, \min }+\Delta \zeta, T_{z+1}\right),\left(\zeta_{z-1}, T_{z-1}\right)$ y $\left(\zeta_{z+1, \min }, T_{z+1}\right)$, y $\left(\zeta_{z-1}, T_{z-1}\right)$ y $\left(\zeta_{z+1, \min }-\Delta \zeta\right.$, $\left.\mathrm{T}_{\mathrm{z}+1}\right)$ y los segmentos asociados a la variable $\psi$ definidos por los puntos $\left(\psi_{\mathrm{z}-1}, \mathrm{~T}_{\mathrm{z}-1}\right)$ y $\left(\psi_{z+1, \min }+\Delta \zeta, T_{z+1}\right),\left(\psi_{z-1}, T_{z-1}\right)$ y $\left(\psi_{z+1, \min }, T_{z+1}\right)$, y $\left(\psi_{z-1}, T_{z-1}\right)$ y $\left(\psi_{z+1, \min }-\Delta \zeta, T_{z+1}\right) . S e$ retienen los nuevos valores de $\left(\zeta_{z+1, \min }, T_{z+1}\right)$ y $\left(\psi_{z+1, \min }, T_{z+1}\right)$,

Paso 9: Si se cumple $\Delta \zeta>\delta_{1}$ o $\Delta \psi>\delta_{2}$, repetir los pasos 7 y 8 . Si $\Delta \zeta<\delta_{1}$ y $\Delta \psi<\delta_{2}$ retener los últimos puntos del segmento, $\left(\zeta_{z+1, \min }, T_{z+1}\right)$ y $\left(\psi_{z+1, \text { min }}, T_{z+1}\right)$,

Paso 10: Retener el valor $\left(\zeta_{z}, T_{z}\right)$ como el valor medio entre los puntos $\left(\zeta_{z-1}, T_{z-1}\right)$ y $\left(\zeta_{z+1, m i n}\right.$, $\left.\mathrm{T}_{\mathrm{z}+1}\right)$. Retener el valor $\left(\psi_{z}, \mathrm{~T}_{\mathrm{z}}\right)$ como el valor medio entre los puntos $\left(\psi_{\mathrm{z}-1}, \mathrm{~T}_{\mathrm{z}-1}\right) \mathrm{y}$ $\left(\psi_{z+1, \min }, T_{z+1}\right)$,

Paso 11: Volver al paso 4 para evaluar un nuevo tramo.
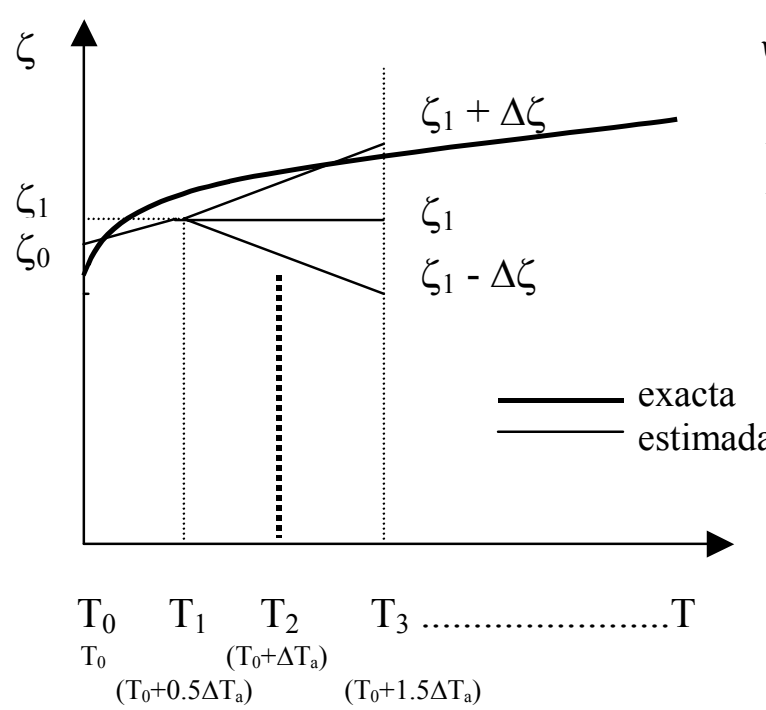

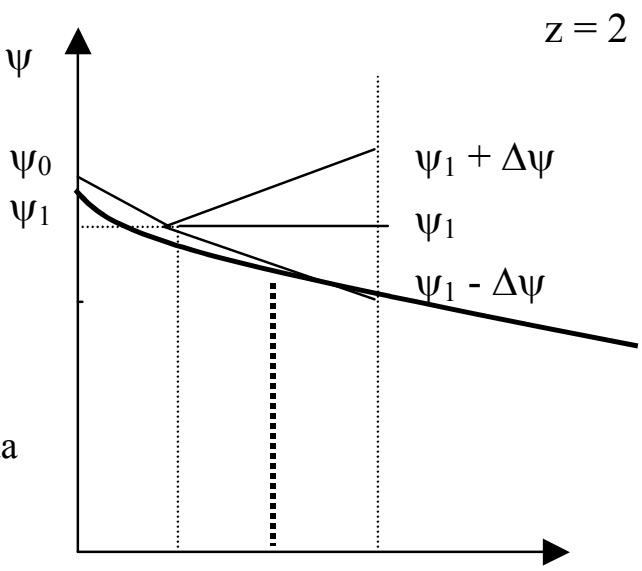

$\begin{array}{cccc}\mathrm{T}_{0} & \mathrm{~T}_{1} \underset{\substack{\left(\mathrm{T}_{0}+\Delta \mathrm{T}_{\mathrm{a}}\right) \\ \mathrm{T}_{0}}}{\mathrm{~T}_{2}} \mathrm{~T}_{3} \ldots \ldots . \mathrm{T} \\ & \left(\mathrm{T}_{0}+0.5 \Delta \mathrm{T}_{\mathrm{a}}\right) & \left(\mathrm{T}_{0}+1.5 \Delta \mathrm{T}_{\mathrm{a}}\right)\end{array}$

Figura III.13. Estimación simultánea de dos funciones dependientes de la temperatura 
En la figura III.13, se esquematiza un ejemplo para $\mathrm{z}=2$.

En problemas estacionarios la expresión del funcional toma la forma,

$$
\mathrm{F}\left(\mathrm{x}_{\mathrm{f}}, \zeta, \psi, \varepsilon, \mathrm{z}\right)=\sum_{\mathrm{f}=1}^{\mathrm{P}}\left[\mathrm{T}\left(\operatorname{inv}, \mathrm{x}_{\mathrm{f}}, \zeta, \psi\right)-\mathrm{T}\left(\operatorname{med}, \mathrm{x}_{\mathrm{f}}, \varepsilon\right)\right]^{2}
$$

\section{III.5. ESTIMACIÓN DE PARÁMETROS DE FUNCIONES LINEALES}

\section{III.5.1. Especificación del funcional}

El funcional, para el caso de una función dependiente de la temperatura de la forma $\rho=\rho_{0}+\rho_{1}(T)$ viene dado por la expresión

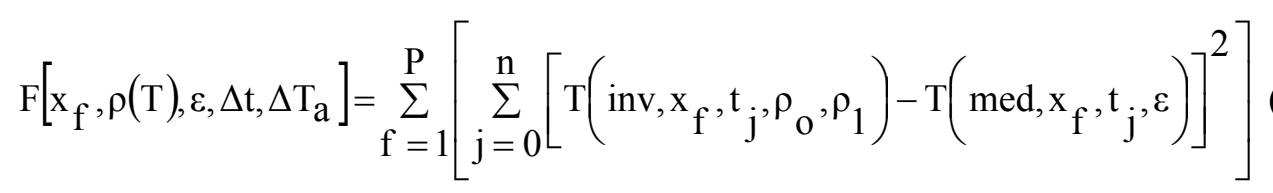

\section{III.5.2. Procedimiento computacional}

El rango de temperaturas del problema está limitado por los valores $\mathrm{T}_{0}$ y $\mathrm{T}_{1}$. El procedimiento propuesto consta de los siguientes pasos:

Paso1: Solución del problema directo (DHCP) por medio del MESIR. Obtención de los datos de entrada de temperaturas,

Paso 2: Fijar los valores de los parámetros: $\delta$ (criterio de convergencia) y de la constante $\mathrm{K}_{1}$ $\left(\mathrm{K}_{1}>1\right), \Delta \rho>1$ (intervalo de paso para obtener el tramo recto),

Paso 3: Estimación inicial: Obtención de un segmento horizontal. Se ejecuta Pspice para las dependencias definidas por los segmentos $\left(\rho_{0}, T_{0}\right)$ y $\left(\rho_{0}, T_{1}\right),\left(\rho_{0}+\Delta \rho, T_{0}\right)$ y $\left(\rho_{0}+\Delta \rho, T_{1}\right)$, y $\left(\rho_{0}-\Delta \rho, T_{0}\right)$ y $\left(\rho_{0}-\Delta \rho, T_{1}\right)$, obteniéndose tres series de valores de temperatura $T\left(\right.$ inv $, x_{f}, t_{j}$, $\rho)$. Se evalúa el funcional para cada serie y se retiene el segmento asociado al mínimo valor del funcional que llamaremos $\left(\rho_{0, \min }+\Delta \rho, \mathrm{T}_{0}\right)$,

Paso 4: $\Delta \rho=\Delta \rho / K_{1}$, 
Paso 5: Repetir el paso 3 para los nuevos segmentos segmentos definidos por los puntos: $\left(\rho_{0, \min }\right.$, $\left.T_{0}\right)$ y $\left(\rho_{0, \min }, T_{1}\right),\left(\rho_{0, \min }+\Delta \rho, T_{0}\right)$ y $\left(\rho_{0, \min }+\Delta \rho, T_{1}\right)$ y $\left(\rho_{0, \min }-\Delta \rho, T_{0}\right)$ y $\left(\rho_{0, \min }-\Delta \rho, T_{1}\right)$ Determinar el nuevo segmento que hace mínimo el funcional,

Paso 6: Si se cumple que $\Delta \rho>\delta$, repetir los pasos 4 y 5 . Si se cumple que $\Delta \rho<\delta$, retener el punto medio del segmento que une los puntos, $\left(\rho_{0, \min }, \mathrm{T}_{0}\right)$ y $\left(\rho_{0, \mathrm{~min}}, \mathrm{~T}_{1}\right)$, definido como, $\left(\rho_{\text {mean }}\right.$, $\mathrm{T}_{\text {mean }}$ ),

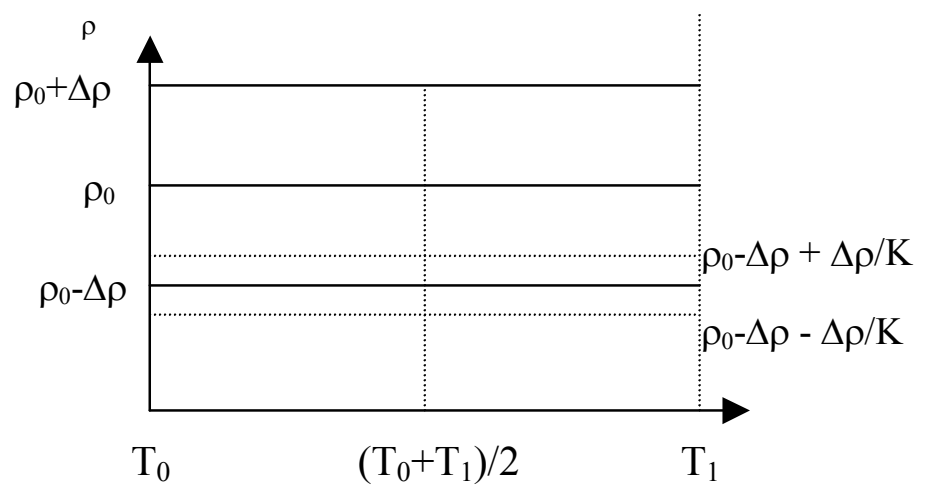

Figura III.14. Determinación del segmento horizontal

Paso 7: Girar el segmento anterior, pivotando sobre su punto central hasta obtener por sucesivas iteraciones un segmento asociado con el funcional mínimo, obtenido a partir de la solución Pspice de cada inclinación y de acuerdo con criterios de acercamiento y convergencia previamente establecidos. Dicho segmento, que pasa por el punto ( $\rho_{\text {mean }}$, $\mathrm{T}_{\text {mean }}$ ) está caracterizado por la inclinación $\mathrm{v}_{\min }$,

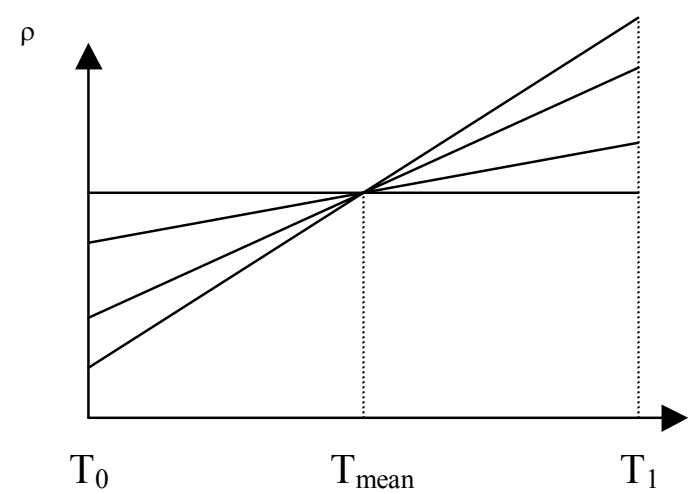

Figura III.15. Giro sobre el punto medio 
Paso 8: Desplazar paralelamente el segmento anterior hasta obtener por sucesivas iteraciones el segmento paralelo asociado con el funcional mínimo, obtenido nuevamente a partir de la solución Pspice de cada caso y de acuerdo con criterios de acercamiento y convergencia previamente establecidos. Los valores de $\left(\rho_{\text {mean }}, \mathrm{T}_{\text {mean }}\right)$ del segmento retenido (de inclinación $v_{\min }$ ) sustituyen a los anteriores,

Paso 9: Repetir los pasos 7 y 8 hasta obtener la estimación final de acuerdo con los criterios de aproximación establecidos.

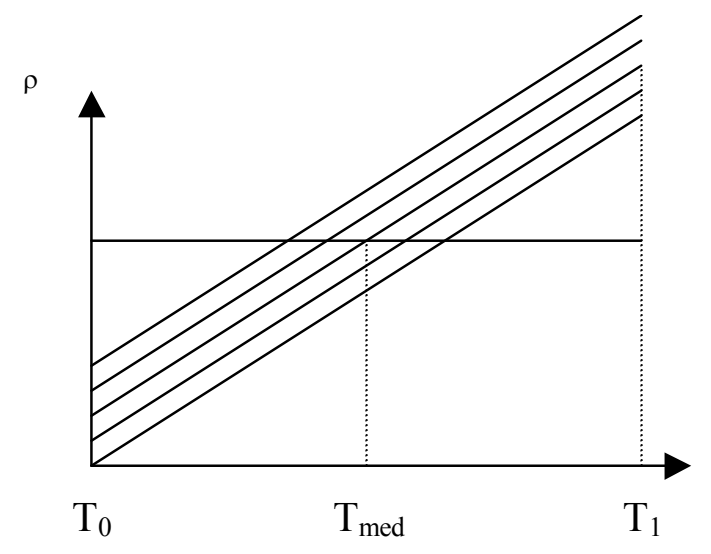

Figura III.16. Desplazamiento paralelo

\section{III.6. ALGORITMO GENERAL DE RESOLUCIÓN}

La filosofía general de resolución se muestra en la figura III.17, donde se describen las operaciones comunes empleadas en los distintos procedimientos inversos analizados; el apartado IV.2 detalla los tipos de ficheros utilizados, su función y los componentes de librería de Pspice que integran los modelos en red.

El primer paso consiste en generar un programa o fichero fuente (en esta Memoria se ha utilizado un programa en Visual $\mathrm{C}++$ ), que contenga todos los datos del problema a resolver, mediante una información completa del tipo de coordenadas, condiciones iniciales y de contorno, propiedades físicas, número de celdas, etc. Además hay que informar del intervalo de temperatura de las medidas de los sensores, $\Delta \mathrm{t}$, y del intervalo de temperatura de cada tramo de estimación, $\Delta \mathrm{T}_{\mathrm{a}}$. 


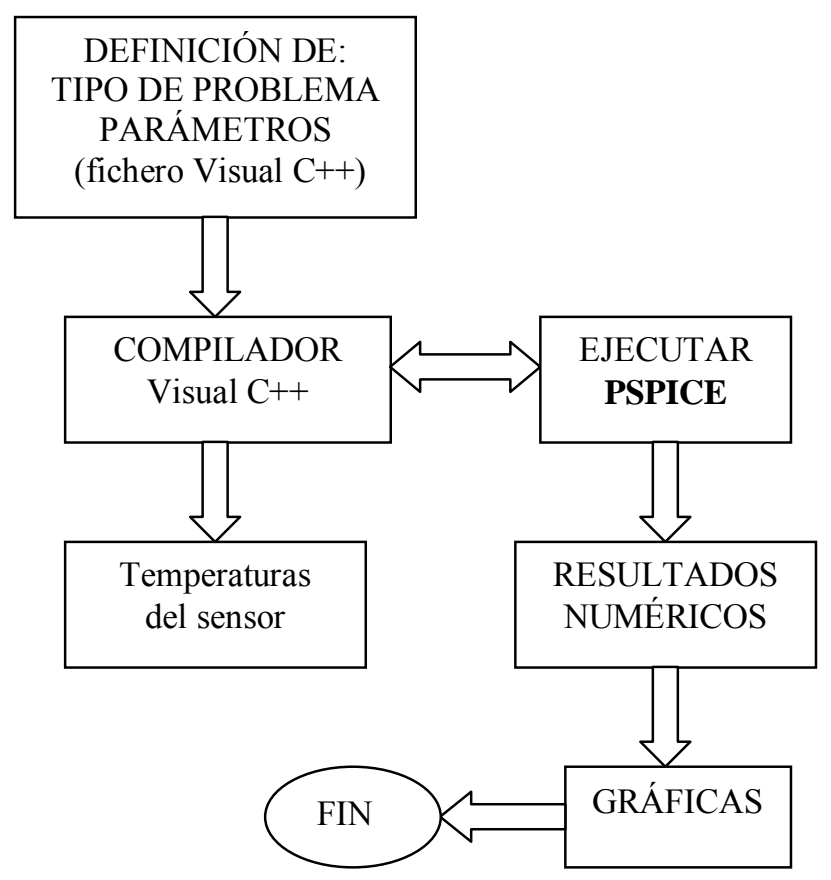

Figura III.17. Diagrama del algoritmo general de resolución

El compilador de Visual $\mathrm{C}++$ se encargará de ejecutar las órdenes del fichero fuente anterior, leer las temperaturas medidas por el sensor (definidas en un fichero de texto), generar el fichero de extensión ".cir", de acuerdo con las órdenes de control previamente establecidas, que posteriormente se ejecutará en un simulador de circuitos eléctricos [Pspice, 1994], y obtener el funcional y compararlo con funcionales previamente obtenidos.

En la figura III.18 se explica con mayor detalle el caso de estimación de funciones. Es posible ejecutar todas las operaciones anteriores de manera automática, mediante programación (programa en Visual $\mathrm{C}++$ ). En este sentido se ha elaborado un programa que incluye: ejecutar la simulación, obtener los datos solicitados de ella, compararlos con las medidas de temperatura para determinar el funcional, modificar adecuadamente la estimación, volver a ejecutar la simulación, ... todo ello hasta conseguir, mediante un criterio límite establecido, el valor $\zeta_{\text {óptimo }}$ para cada segmento de la función estimada. Una vez estimado un segmento se pasa a la estimación del siguiente aplicando el mismo procedimiento iterativo, hasta llegar a la estimación del último tramo. 


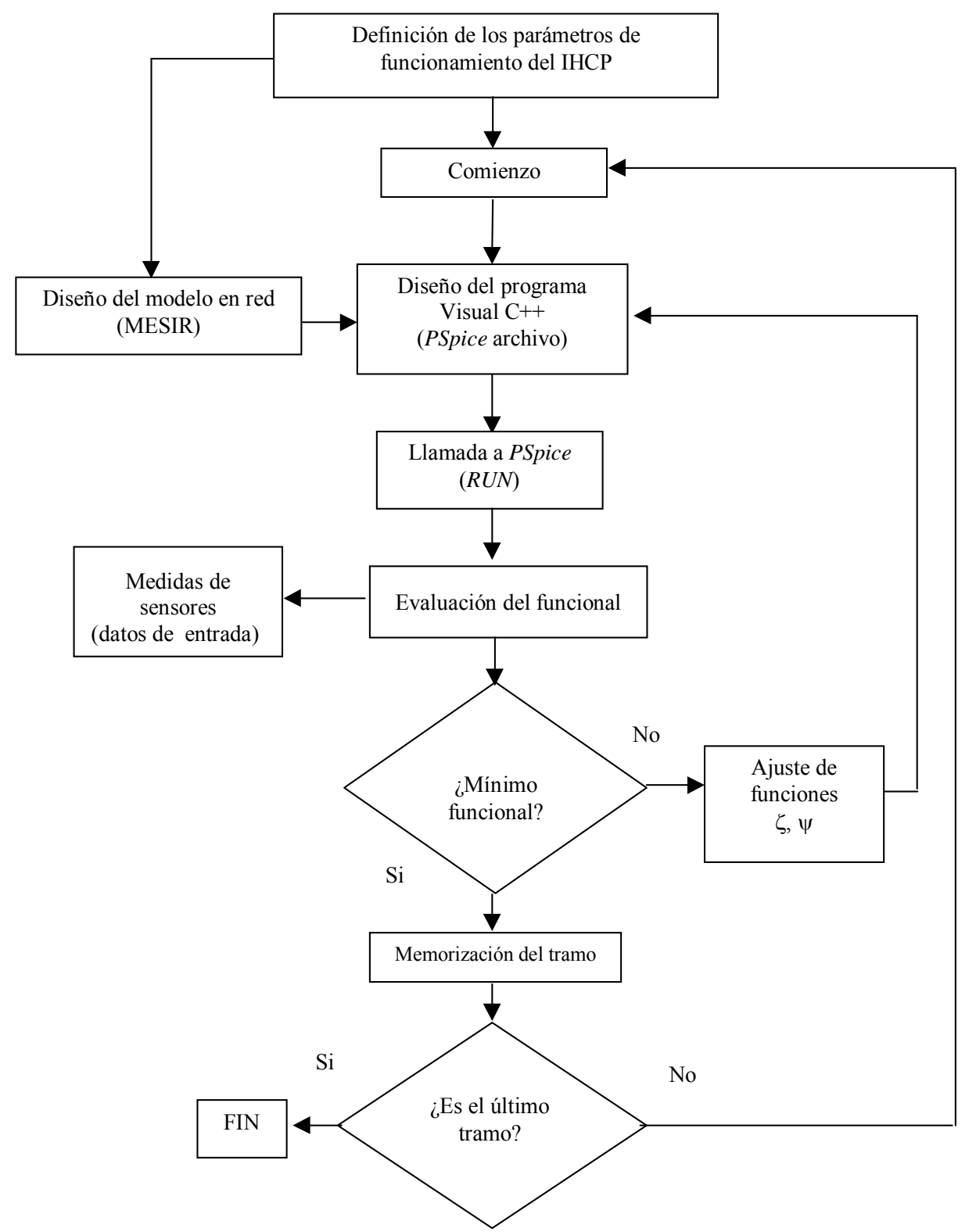

Figura III.18. Procedimiento computacional desarrollado para la estimación de funciones

Para encontrar el valor mínimo del funcional, es necesario usar un algoritmo de cálculo. En esta Memoria y con el objetivo de disminuir al máximo el tiempo de computación, se ha empleado el método de aproximaciones sucesivas mediante mínimos cuadrados. Se trata de un método de búsqueda aleatoria, donde es necesario incluir el intervalo de paso $\Delta \zeta$ (de un $\zeta_{z}$ al $\left.\zeta_{z+2}\right)$. Al disminuir el valor de $\Delta \zeta$ aumenta la precisión de la estimación efectuada, pero en contrapartida el tiempo de computación se incrementa ya que el número de iteraciones aumenta considerablemente. 


\section{III.7. REFERENCIAS}

Alhama López, F., "Estudio de respuestas térmicas transitorias en procesos no lineales de conducción de calor mediante el Método de simulación por redes". Tesis Doctoral. Universidad de Murcia (1999)

Beck, J. V., "Nonlinear estimation applied to the nonlinear inverse heat conduction problem", Int. J. Heat Mass Transf., 13, 703-716 (1970)

Beck, J. V., Litkouhi, B., y St.Clair.Jr. C.R., "Efficient sequential solution of the nonlinear inverse heat conduction problem", Numer. Heat Transfer, 5, 275-286 (1982)

Beck, J. V., Blackwell, B. y St.Clair.Jr. C.R., "Inverse heat conduction”, New York, John Wiley \& Sons. Inc. (1985)

Huang C.H. y M.N. Özisik, "Direct integration approach for simultaneously estimating temperature dependent thermal conductivity and heat capacity", Numer. Heat Transfer-A, 20, 95-110 (1991)

Huang, C.H. y Yan, J.Y., "An inverse problem in simultaneously measuring temperaturedependent thermal conductivity and heat capacity”, Int. J. Heat Mass Transf., 38, 18, 3433$3441(1995)$

PSpice 6.0, Microsim Corporation, 20 Fairbanks, Irvine, California 92718 (1994)

Statistics for Windows, ver. 2 (1998)

Yang, Ching-Yu, "Determination of the temperature dependent thermophysical properties from temperature responses measured at medium's boundaries", Int. J. Heat Mass Transf., 43, 1261-1270 (2000)

\section{CONTRIBUCIONES Y CONCLUSIONES}

Se establece un procedimiento de estimación de funciones mediante tramos de pendiente variable, lo que lo diferencia de los empleados hasta la fecha. Dicho procedimiento se particulariza en protocolos concretos para los casos de i) estimación de funciones dependientes del tiempo, ii) ídem de la temperatura y iii) estimación simultánea de dos funciones diferentes 
dependientes de la temperatura. Por otra parte, los protocolos propuestos pueden abordar también problemas de estimación de parámetros, lo que aumenta su generalidad.

La similitud y generalidad de los protocolos establecidos permitirá, en el capítulo siguiente, diseñar modelos en red sencillos, y prácticamente idénticos, en los tres tipos de problemas.

Se ha desarrollado un procedimiento computacional para la automatización de todo el proceso de estimación de funciones.

Por último se establece un protocolo para la estimación de funciones lineales o no lineales que contienen dos parámetros. 


\section{Capítulo IV}

\section{Modelos en red}

IV.1. Problema directo. Modelos en red

IV.2. Problema inverso. Modelos en red

IV.2.1. Estimación de magnitudes constantes

IV.2.1.1. Estimación de propiedades termofísicas (conductividad y calor específico)

IV.2.1.2. Estimación de condiciones de contorno de primera y segunda clase

IV.2.1.3. Estimación de otros parámetros

IV.2.2. Estimación de funciones

IV.2.2.1. Estimación del calor específico dependiente de la temperatura

IV.2.2.2. Estimación de la conductividad térmica variable con la temperatura

IV.2.2.3. Estimación de flujos incidentes (condición de contorno)

IV.2.2.4. Estimación de focos térmicos (condición de contorno de temperatura en función del tiempo)

IV.2.2.5. Estimación de otras funciones

IV.2.2.6. Estimación simultánea del calor especifico y de la conductividad térmica

IV.2.3. Estimación de funciones lineales

IV.3. Referencias

Contribuciones y conclusiones 


\section{Capitulo IV}

\section{Modelos en red}

En el presente capitulo se presentan los modelos en red propuestos para la resolución de los diferentes problemas inversos estudiados en esta memoria: estimación de flujos de calor incidentes, de propiedades termofísicas, de generación interna de calor, etc. El diseño del modelo en red parte de la discretización o reticulación del medio continuo en elementos de volumen (o celdas), de modo que cada término de la ecuación diferencial en diferencias finitas (resultante de la discretización espacial del modelo matemático), se implementa mediante un dispositivo que implica una relación entre las variables potencial y corriente eléctrica, formalmente equivalente a la existente entre las variables temperatura y densidad de flujo de calor (respectivamente), en el modelo matemático.

\section{IV.1. PROBLEMA DIRECTO. MODELOS EN RED}

La nomenclatura correspondiente al modelo en red de una celda elemental o elemento de volumen, común para los problemas directo e inverso, se muestra en la figura IV.1. Las celdas, de tamaño $\Delta \mathrm{x}$ o $\Delta \mathrm{r}$, se han diseñado en forma simétrica. $\mathrm{N}$ es el número total de éstas.

Con carácter general se consideran tres posiciones para la variable dependiente temperatura, las de los extremos de la celda y la central $\mathrm{T}_{\mathrm{i}-\Delta}, \mathrm{T}_{\mathrm{i}+\Delta}$ y $\mathrm{T}_{\mathrm{i}}$, respectivamente. De esta forma existen, al menos, tres posibilidades para formular las dependencias de cualquiera de las características termofísicas, conductividad y calor específico, con la temperatura. La asociación en serie de las $\mathrm{N}$ celdas reproduce el modelo en red de todo el medio, figura IV.2. Los requisitos de la variable temperatura y de conservación de la variable flujo térmico se satisfacen de manera automática, sin ningún requisito añadido, ya que estas condiciones son equivalentes a los teoremas de circuitos (de Kirchhoff), de voltajes y corrientes eléctricas, respectivamente. 


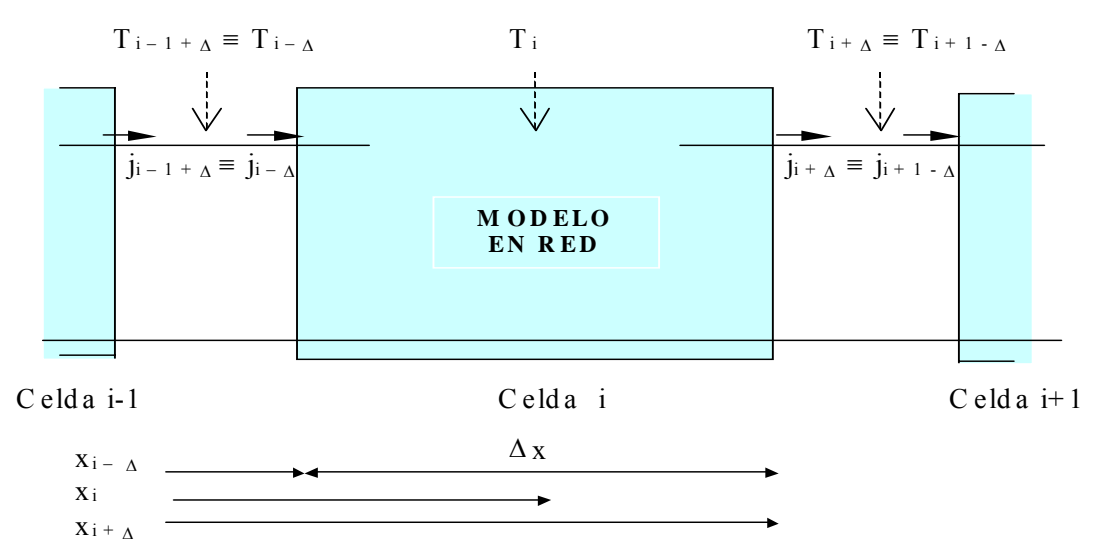

Figura IV.1. Geometría y nomenclatura para la celda elemental, i

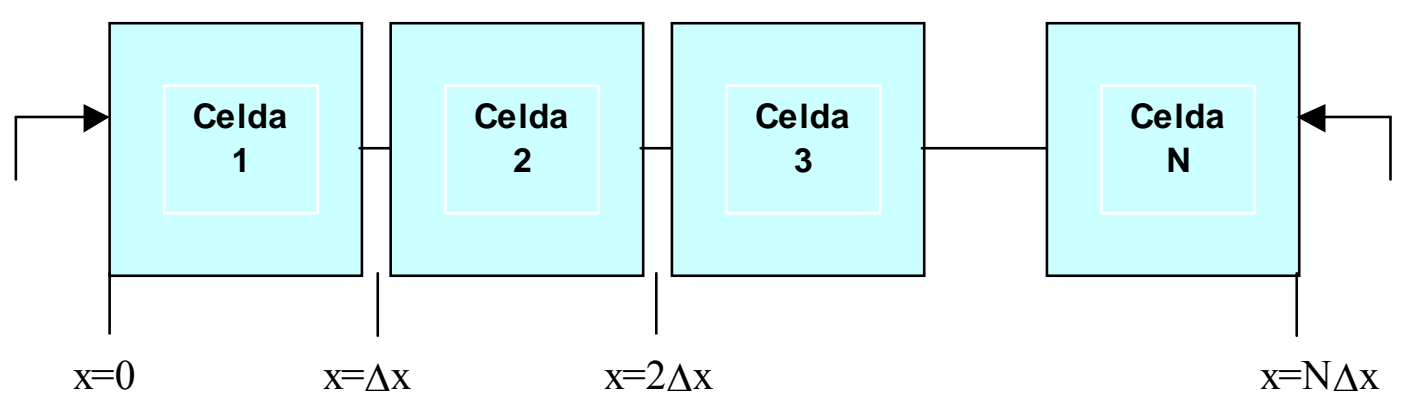

Figura IV.2. Asociación de N celdas

\section{Modelos en red de las diferentes condiciones de contorno}

La figura IV.3 muestra la implementación en el modelo de algunas de las condiciones de contorno más generales, ya especificadas en el Capítulo II.

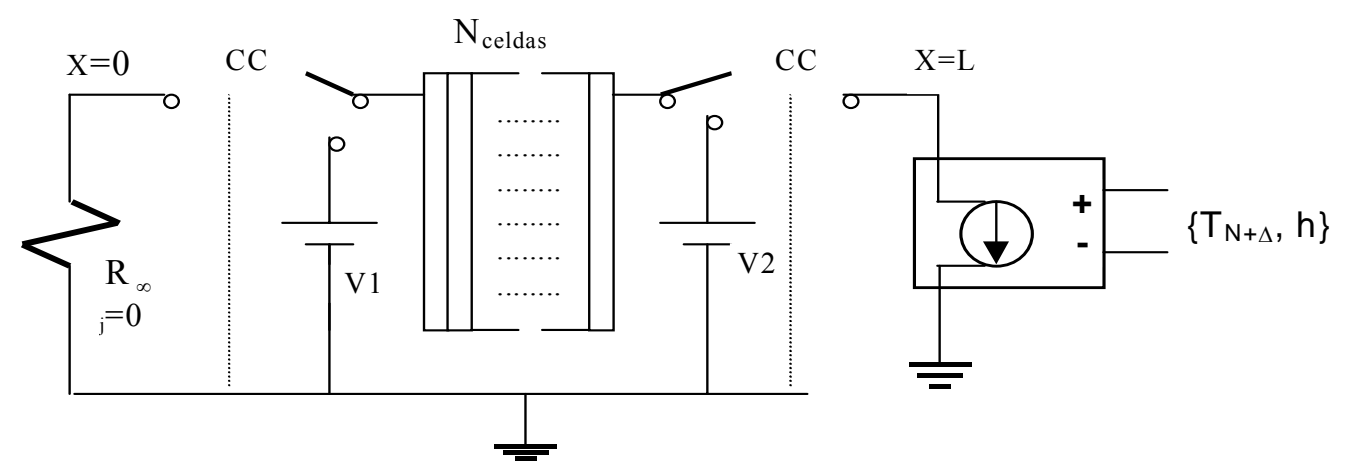

Figura IV.3. Implementación de diferentes tipos de condiciones de contorno

- Especificación de la temperatura (condición de frontera de primera clase). Se implementa mediante una fuente de tensión dependiente o independiente del tiempo, Figura IV.4. 


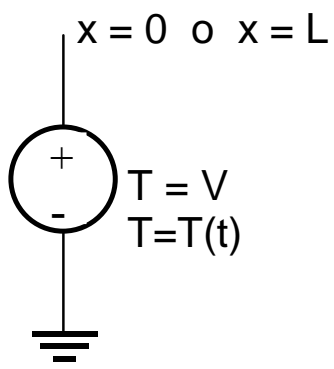

Figura IV.4. Condición de contorno de primera clase

- Especificación del flujo de calor (condición de frontera de segunda clase). Se implementa mediante una fuente de corriente dependiente o independiente del tiempo, Figura IV.5. La condición adiabática (flujo nulo) es una caso particular que puede implementarse alternativamente mediante una resistencia de valor infinito entre el extremo del medio y el nudo de referencia, figura IV.5.

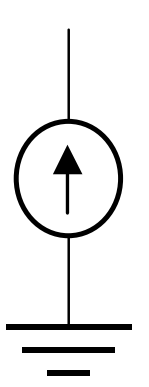

(a) $\mathrm{x}=0 \quad \mathrm{o} \quad \mathrm{x}=\mathrm{L}$

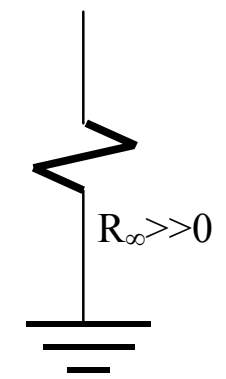

(b) Caso adiabático

Figura IV.5. Condición de contorno de segunda clase

- Condición de contorno convectiva (condición de frontera de tercera clase). El caso más general de dependencia del coeficiente de convección con la temperatura en la frontera del medio (convección natural, p. ej.) se implementa mediante una fuente de corriente controlada por tensión, figura IV.6; la acción de control viene dada por la expresión del flujo de calor por convección obtenida a partir de las correlaciones adimensionales conocidas. La convección forzada (ley de Newton) puede implementarse, alternativamente, de forma más sencilla mediante una simple resistencia ohmica. 


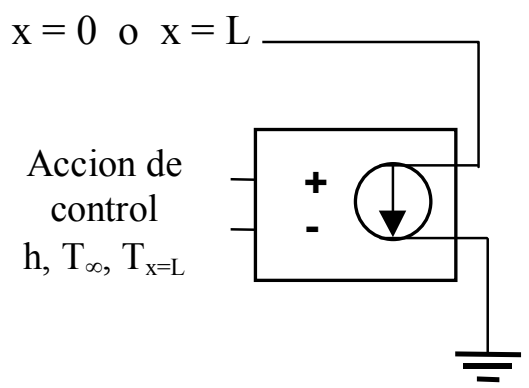

Figura IV.6. Condición de contorno de tercera clase

- Condición de contorno de radiación. Es una condición fuertemente no lineal cuya implementación se lleva a cabo, también, mediante un generador de corriente controlado por tensión, figura IV.6. La acción de control viene dada por la ley de Stefan-Boltzmann de la radiación.

- Condición de contacto entre medios. Se da cuando dos medios de igual o diferente naturaleza se someten a un contacto térmico imperfecto; se implementa mediante una simple resistencia ohmica y cuando el efecto es independiente de la temperatura.

\section{Modelo en red de la celda elemental. Propiedades térmicas constantes}

Sea un medio finito y unidimensional con conductividad térmica $\mathrm{k}\left(\mathrm{Wm}^{-1}{ }^{\circ} \mathrm{C}^{-1}\right)$, densidad $\rho\left(\mathrm{Kgm}^{-3}\right)$ y calor especifico $\mathrm{c}_{\mathrm{e}}\left(\mathrm{JKg}^{-1}{ }^{\circ} \mathrm{C}^{-1}\right)$, características independientes de la temperatura y de la posición (medio lineal y homogéneo), cuya temperatura inicial constante en todo el medio es $\mathrm{T}_{\mathrm{o}}\left({ }^{\circ} \mathrm{C}\right)$, figura IV.7. En el instante $\mathrm{t}=0$ segundos, ambos extremos se someten a condiciones de contorno conocidas.

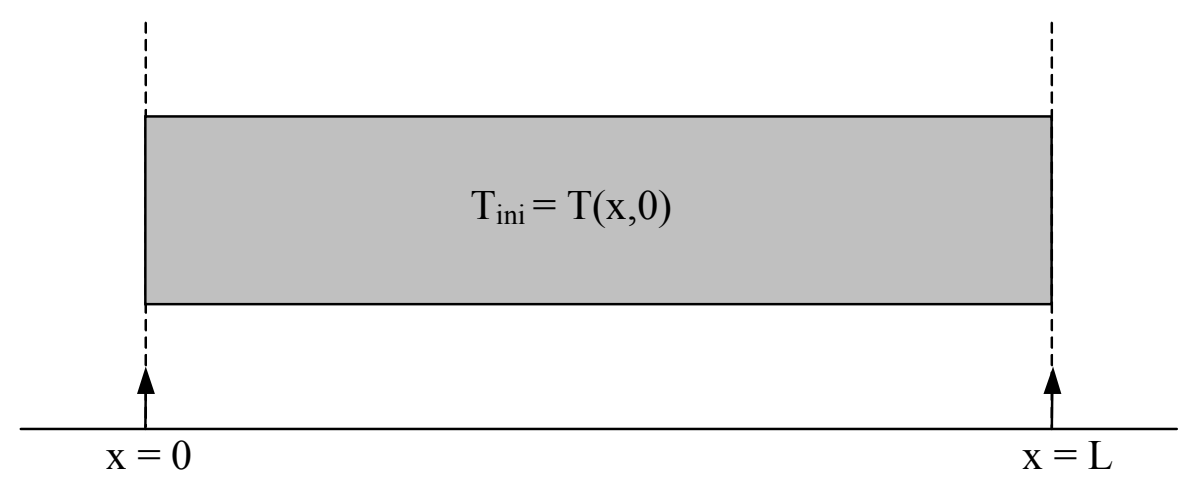

Figura IV.7. Modelo físico del problema (propiedades físicas constantes)

A partir de la ley de Fourier " $\mathrm{j}_{\mathrm{x}}=-\mathrm{k} \partial \mathrm{T} / \partial \mathrm{x}$ ”, y discretizando espacialmente la ecuación de la transferencia de calor en coordenadas cartesianas (por simplificar) 


$$
\rho c_{\mathrm{e}} \partial \mathrm{T} / \partial \mathrm{t}-\mathrm{k}\left(\partial^{2} \mathrm{~T} / \partial \mathrm{x}^{2}\right)=0
$$

podemos escribir

$$
\rho c_{e} d T / d t-(\Delta j / \Delta x)=0
$$

De acuerdo con la nomenclatura de la figura IV.1 los flujos de calor entrante y saliente en la celda i son

$$
\mathrm{j}_{\mathrm{i} \pm \Delta \mathrm{x}}= \pm\left(\mathrm{T}_{\mathrm{i}}-\mathrm{T}_{\mathrm{i} \pm \Delta \mathrm{x}}\right) /(\Delta \mathrm{x} / 2 \mathrm{k})
$$

De las ecuaciones (IV.2) y (IV.3) es inmediato deducir el modelo en red de la figura IV.8, donde los valores del condensador y las resistencias eléctricas son [González-Fernández y Alhama, 2002]:

$$
\mathrm{C}_{\mathrm{i}}=\rho \mathrm{c}_{\mathrm{e}} \Delta \mathrm{x}, \quad \mathrm{R}_{\mathrm{i} \pm \Delta \mathrm{x}}=\Delta \mathrm{x} / 2 \mathrm{k},
$$

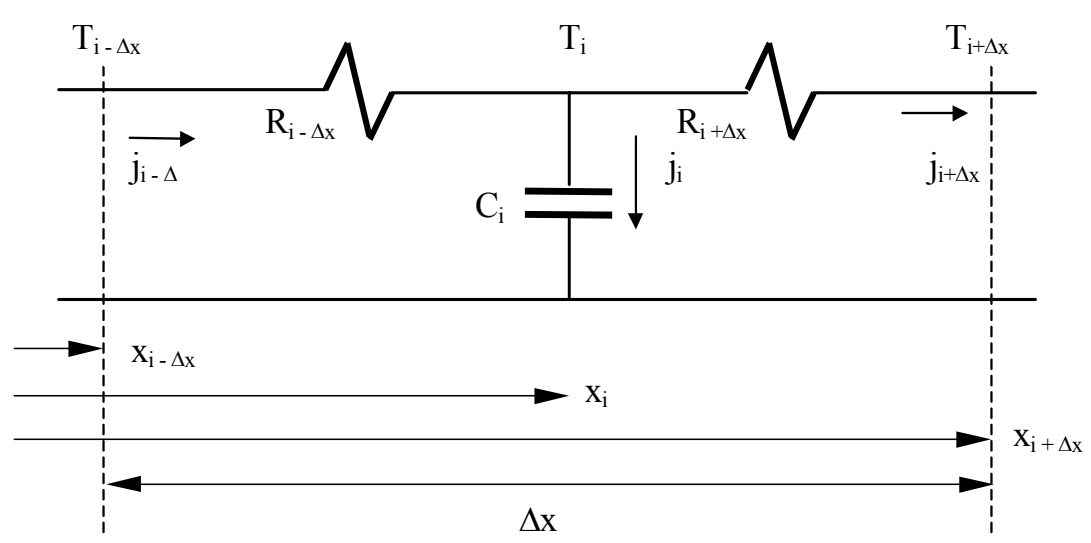

Figura IV.8. Modelo en red de la celda elemental

(conductividad y calor específico constante)

\section{Modelo en red de la celda elemental. Propiedades térmicas dependientes de la}

\section{temperatura}

Sea un medio finito y unidimensional de densidad constante $\rho\left(\mathrm{Kgm}^{-3}\right)$ cuya temperatura inicial constante en todo el medio es $\mathrm{T}_{\mathrm{o}}\left({ }^{\circ} \mathrm{C}\right)$, figura IV.9. Cualquiera de las dos características térmicas $\mathrm{k} \mathrm{o} \mathrm{c}_{\mathrm{e}}$, (o ambas simultáneamente) depende de la temperatura. La forma 
de dependencia puede ser arbitraria, funciones continuas definidas mediante expresiones matemáticas o funciones discontinuas; en este último caso la dependencia con la temperatura puede aproximarse, con la precisión que se requiera, por medio de una función continua a tramos (piece-wise function). Los nuevos componentes de las librerías pspice permiten implementar estas dependencias mediante un dispositivo denominado "etable", a partir de los pares de valores $k-T$ o $\left(c_{e}-T\right)$. En el instante inicial, $t=0$, ambos extremos se someten a condiciones de contorno conocidas.

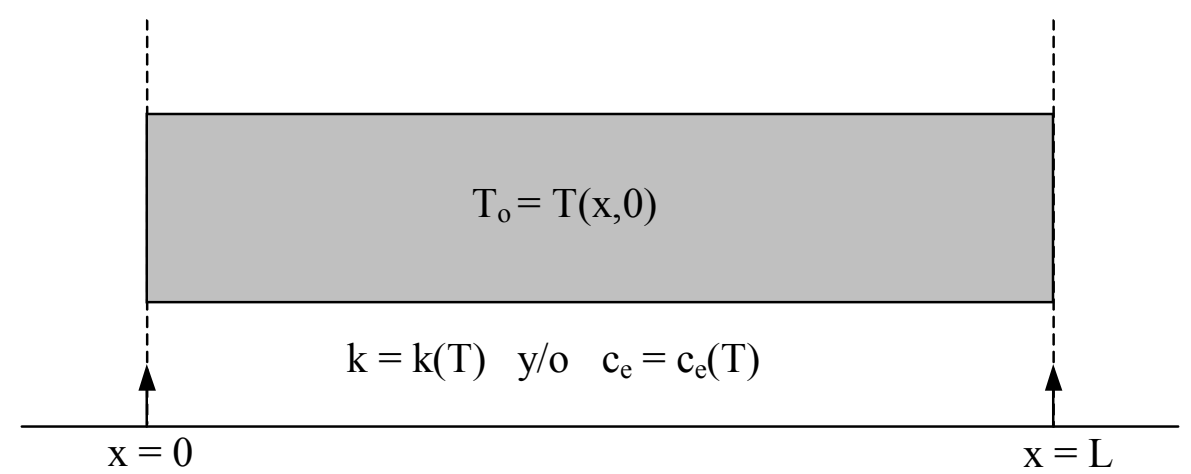

Figura IV.9. Modelo físico del problema (propiedades físicas variables)

\section{Calor especifico dependiente de la temperatura}

Escribiendo el calor específico en la forma $\mathrm{c}_{\mathrm{e}}(\mathrm{T})=\mathrm{c}_{\text {mean }}+\Delta \mathrm{c}_{\mathrm{e}}(\mathrm{T})$, donde $\mathrm{c}_{\text {mean }}$ es el valor medio (o cualquier otro valor constante diferente al medio) de la función $c_{e}(T), y \quad \Delta c_{e}\left(T_{i}\right)$ es la desviación de la función respecto de su valor medio a la temperatura $\mathrm{T}_{\mathrm{i}}$, $\mathrm{y}$ sustituyendo en la ecuación (IV.1), tenemos

$\rho \mathrm{c}_{\text {mean }} \partial \mathrm{T} / \partial \mathrm{t}+\rho \Delta \mathrm{c}_{\mathrm{e}}(\mathrm{T}) \partial \mathrm{T} / \partial \mathrm{t}=\rho \mathrm{c}_{\text {mean }} \partial \mathrm{T} / \partial \mathrm{t}+\rho\left[\mathrm{c}_{\mathrm{e}}(\mathrm{T})-\mathrm{c}_{\text {mean }}\right] \partial \mathrm{T} / \partial \mathrm{t}=\mathrm{k}\left(\partial^{2} \mathrm{~T} / \partial \mathrm{x}^{2}\right)$

ecuación que, discretizada en la variable espacial, conduce a

$$
\rho c_{\text {mean }} d T_{i} / d t+\rho\left[c_{e}\left(T_{i}\right)-c_{\text {mean }}\right] d T_{i} / d t=-\Delta j_{i} / \Delta x
$$

donde, $\Delta \mathrm{j}_{\mathrm{i}}=\mathrm{j}_{\mathrm{i}+\Delta \mathrm{x}}-\mathrm{j}_{\mathrm{i}-\Delta \mathrm{x}}, \mathrm{y} \mathrm{j}_{\mathrm{i} \pm \Delta \mathrm{x}}= \pm\left(\mathrm{T}_{\mathrm{i}}-\mathrm{T}_{\mathrm{i} \pm \Delta \mathrm{x}}\right)(\mathrm{k} / 2 \Delta \mathrm{x})$. Ahora, si llamamos

$$
\begin{aligned}
& \mathrm{j}_{\mathrm{i}}=\Delta \mathrm{x} \rho \mathrm{c}_{\text {mean }} \mathrm{dT}_{\mathrm{i}} / \mathrm{dt} \\
& \mathrm{j}_{1}=\Delta \mathrm{x} \rho\left[\mathrm{c}_{\mathrm{e}}\left(\mathrm{T}_{\mathrm{i}}\right)-\mathrm{c}_{\text {mean }}\right] \mathrm{dT}_{\mathrm{i}} / \mathrm{dt} \\
& \mathrm{j}_{\mathrm{i}+\Delta \mathrm{x}}=\left(\mathrm{T}_{\mathrm{i}}-\mathrm{T}_{\mathrm{i}+\Delta \mathrm{x}}\right)(\mathrm{k} / 2 \Delta \mathrm{x})
\end{aligned}
$$




$$
\mathrm{j}_{\mathrm{i}-\Delta \mathrm{x}}=\left(\mathrm{T}_{\mathrm{i}-\Delta \mathrm{x}}-\mathrm{T}_{\mathrm{i}}\right)(\mathrm{k} / 2 \Delta \mathrm{x})
$$

la ecuación $\Delta x \rho c_{\text {mean }} \mathrm{dT}_{\mathrm{i}} / \mathrm{dt}+\Delta \mathrm{x} \rho\left[\mathrm{c}_{\mathrm{e}}\left(\mathrm{T}_{\mathrm{i}}\right)-\mathrm{c}_{\text {mean }}\right] \mathrm{dT}_{\mathrm{i}} / \mathrm{dt}=\mathrm{j}_{\mathrm{i}+\Delta \mathrm{x}}-\mathrm{j}_{\mathrm{i}-\Delta \mathrm{x}}$ es el balance del flujo de calor en la celda (ley de Kirchhoff de las corrientes, $j_{i}+j_{1}+j_{i-\Delta x}-j_{i+\Delta x}=0$ ). La corriente $j_{i}=\Delta x$ $\rho \mathrm{c}_{\text {mean }} \mathrm{dT}_{\mathrm{i}} / \mathrm{dt}$ se implementa mediante un condensador de capacidad $\mathrm{C}_{\mathrm{i}}=\Delta \mathrm{x} \rho \mathrm{c}_{\text {mean }}$; y las corrientes $\mathrm{j}_{\mathrm{i}+\Delta \mathrm{x}}=\left(\mathrm{T}_{\mathrm{i}}-\mathrm{T}_{\mathrm{i}+\Delta \mathrm{x}}\right)(\mathrm{k} / 2 \Delta \mathrm{x}) \mathrm{yj}_{\mathrm{i}-\Delta \mathrm{x}}=\left(\mathrm{T}_{\mathrm{i}-\Delta \mathrm{x}}-\mathrm{T}_{\mathrm{i}}\right)(\mathrm{k} / 2 \Delta \mathrm{x})$ mediante las resistencias $\mathrm{R}_{\mathrm{i}+\Delta \mathrm{x}}=$ $\mathrm{R}_{\mathrm{i}-\Delta \mathrm{x}}=2 \Delta \mathrm{x} / \mathrm{k}$. Por último, la corriente $\mathrm{j}_{1}=\Delta \mathrm{x} \rho\left[\mathrm{c}_{\mathrm{e}}\left(\mathrm{T}_{\mathrm{i}}\right)-\mathrm{c}_{\text {mean }}\right] \mathrm{dT}_{\mathrm{i}} / \mathrm{dt}$ se implementa por medio de un generador de corriente controlado, cuya salida es el producto de la corriente del condensador " $\Delta \mathrm{x} \rho \mathrm{c}_{\text {mean }} \mathrm{dT}_{\mathrm{i}} / \mathrm{dt}$ ” por la función “ $\left(\mathrm{c}_{\mathrm{e}}\left(\mathrm{T}_{\mathrm{i}}\right)-\mathrm{c}_{\text {mean }}\right) / c_{\text {mean }}$ ” $\mathrm{c}_{\mathrm{e}}\left(\mathrm{T}_{\mathrm{i}}\right)$ se genera en los extremos del generador que constituye el circuito auxiliar (figura IV.10) de la fuente $\mathrm{E}_{\mathrm{i}}$.

El modelo en red de la celda elemental, que ya fue diseñado con anterioridad de forma más compleja al no existir las librerías actuales, [Alhama, 1999], se muestra en la figura IV.10.

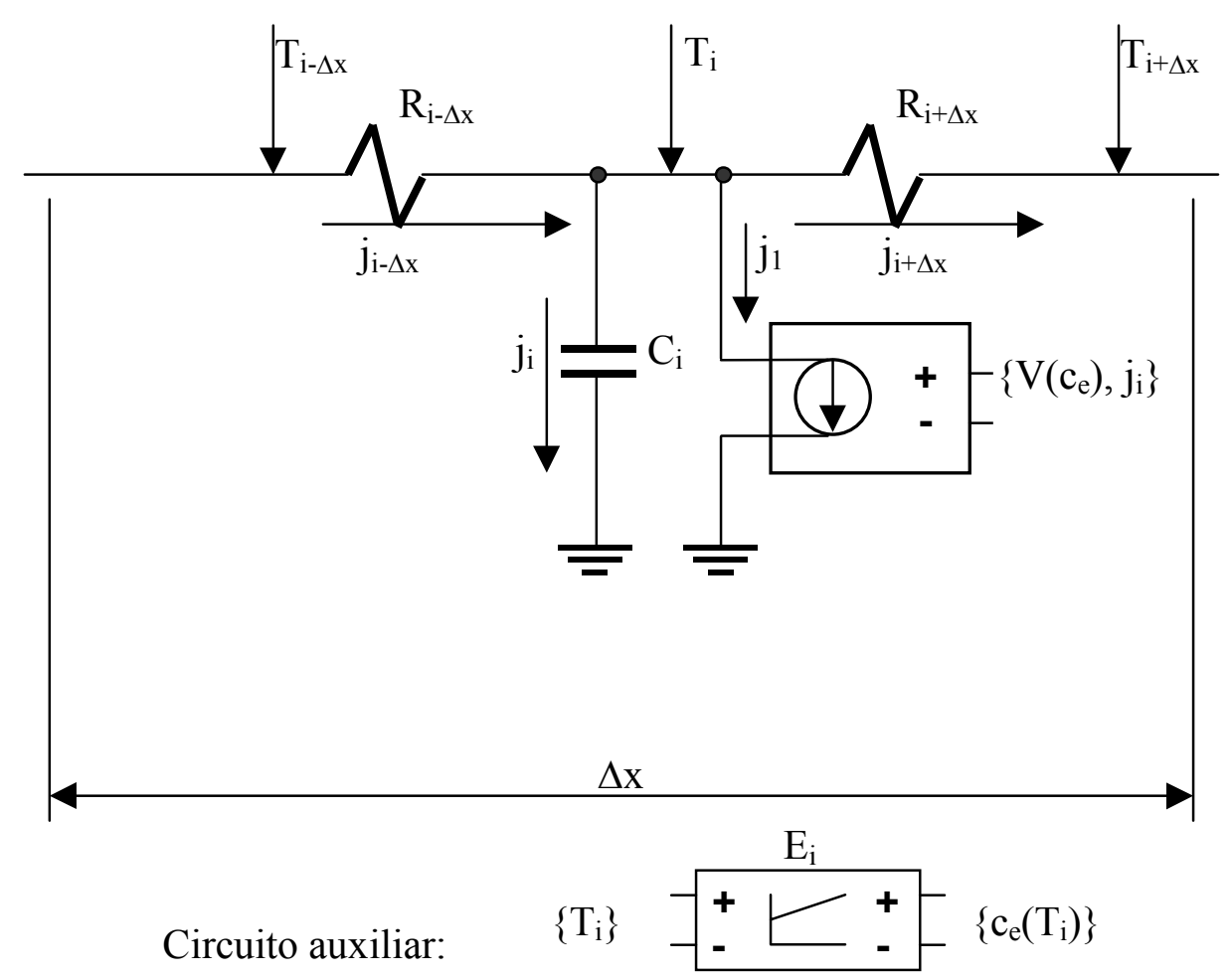

Figura IV.10. Modelo en red para calor específico dependiente de la temperatura

\section{Conductividad térmica variable con la temperatura}

El modelo admite cualquier tipo de dependencia $\mathrm{k}=\mathrm{k}(\mathrm{T})$, una función continua o una función lineal a tramos que aproxime la dependencia tanto como se quiera a los valores dados por los manuales de características térmicas. De nuevo, la discretización espacial de la ecuación (IV.1) conduce a 


$$
\Delta \mathrm{x} \rho \mathrm{c}_{\mathrm{e}} \mathrm{dT}_{\mathrm{i}} / \mathrm{dt}=-\Delta \mathrm{j}_{\mathrm{i}}
$$

donde $\Delta \mathrm{j}_{\mathrm{i}}=\mathrm{j}_{\mathrm{i}+\Delta \mathrm{x}}-\mathrm{j}_{\mathrm{i}-\Delta \mathrm{x}}, \mathrm{y} \mathrm{j}_{\mathrm{i} \pm \Delta \mathrm{x}}$ las corrientes que abandonan y entran a la celda. Es decir, $\mathrm{j}_{\mathrm{i} \pm \Delta \mathrm{x}}= \pm$ $\left(\mathrm{T}_{\mathrm{i}}-\mathrm{T}_{\mathrm{i} \pm \Delta \mathrm{x}}\right)\left(\mathrm{k}_{\mathrm{i} \pm \Delta \mathrm{x}} / 2 \Delta \mathrm{x}\right)$, donde $\mathrm{k}_{\mathrm{i} \pm \Delta \mathrm{x}}$ son las conductividades a ambos lados de la celda $\mathrm{i}$, que son dependientes de las temperaturas en los extremos de la celda, $\mathrm{T}_{\mathrm{i}-\Delta x}$ y $\mathrm{T}_{\mathrm{i}+\Delta \mathrm{x}}$.

$$
\Delta \mathrm{x} \rho \mathrm{c}_{\mathrm{e}} \mathrm{dT}_{\mathrm{i}} / \mathrm{dt}=2 \mathrm{k}_{\mathrm{i}-\Delta \mathrm{x}}\left(\mathrm{T}_{\mathrm{i}-\Delta \mathrm{x}}-\mathrm{T}_{\mathrm{i}}\right) / \Delta \mathrm{x}-2 \mathrm{k}_{\mathrm{i}+\Delta \mathrm{x}}\left(\mathrm{T}_{\mathrm{i}}-\mathrm{T}_{\mathrm{i}+\Delta \mathrm{x}}\right) / \Delta \mathrm{x}
$$

que se puede expresar en forma de ley de Kirchoff, $\mathrm{j}_{\mathrm{i}}-\mathrm{j}_{\mathrm{i}-\Delta \mathrm{x}}+\mathrm{j}_{\mathrm{i}+\Delta \mathrm{x}}=0$, donde

$$
\begin{aligned}
& \mathrm{j}_{\mathrm{i}}=\Delta \mathrm{x} \rho \mathrm{c}_{\mathrm{e}} \mathrm{dT}_{\mathrm{i}} / \mathrm{dt} \\
& \mathrm{j}_{\mathrm{i}+\Delta \mathrm{x}}=2\left(\mathrm{~T}_{\mathrm{i}}-\mathrm{T}_{\mathrm{i}+\Delta \mathrm{x}}\right) /\left(\Delta \mathrm{x} / \mathrm{k}_{\mathrm{i}+\Delta \mathrm{x}}\right) \\
& \mathrm{j}_{\mathrm{i}-\Delta \mathrm{x}}=2\left(\mathrm{~T}_{\mathrm{i}-\Delta \mathrm{x}}-\mathrm{T}_{\mathrm{i}}\right) /\left(\Delta \mathrm{x} /\left(\mathrm{k}_{\mathrm{i}-\Delta \mathrm{x}}\right)\right.
\end{aligned}
$$

Los valores de las conductividades $\mathrm{k}_{\mathrm{i}-\Delta \mathrm{x}}$ y $\mathrm{k}_{\mathrm{i}+\Delta \mathrm{x}}$ se determinan a partir de las temperaturas $T_{i-\Delta x}$ y $T_{i+\Delta x}$, respectivamente; los circuitos auxiliares de los generadores aislados $E_{1 \mathrm{i}}$ y $E_{2 \mathrm{i}}$ de la figura IV.11, que se especifican por medio de la expresión $\mathrm{k}=\mathrm{k}(\mathrm{T})$, proporcionan en sus extremos dos tensiones eléctricas cuyos valores numéricos coinciden con los de $\mathrm{k}_{\mathrm{i}-\Delta \mathrm{x}} \mathrm{y}$ $\mathrm{k}_{\mathrm{i}+\Delta \mathrm{x}}$; esto es " $\mathrm{E}_{1 \mathrm{i}}=\mathrm{k}_{\mathrm{i}-\Delta \mathrm{x}}=\mathrm{k}\left(\mathrm{T}_{\mathrm{i}-\Delta \mathrm{x}}\right)$ " $\mathrm{y}$ " $\mathrm{E}_{2 \mathrm{i}}=\mathrm{k}_{\mathrm{i}+\Delta \mathrm{x}}=\mathrm{k}\left(\mathrm{T}_{\mathrm{i}+\Delta \mathrm{x}}\right)$ "

Los flujos de calor $j_{i-\Delta x} y j_{i+\Delta x}$ se implementan en el modelo por medio de sendas fuentes de corriente controladas por tensiones. $\mathrm{j}_{\mathrm{i}-\Delta \mathrm{x}}$ está controlado por las tensiones $\mathrm{T}_{\mathrm{i}-\Delta \mathrm{x}}, \mathrm{T}_{\mathrm{i}} \mathrm{y} \mathrm{E}_{1 \mathrm{i}}$, de acuerdo con la función de control $2\left(\mathrm{~T}_{\mathrm{i}}-\mathrm{T}_{\mathrm{i}+\Delta \mathrm{x}}\right) /\left(\Delta \mathrm{x} / \mathrm{k}_{\mathrm{i}+\Delta \mathrm{x}}\right)$ mientras que $\mathrm{j}_{\mathrm{i}+\Delta \mathrm{x}}$ está controlado por las tensiones $\mathrm{T}_{\mathrm{i}+\Delta \mathrm{x}}, \mathrm{T}_{\mathrm{i}} \mathrm{y} \mathrm{E}_{2 \mathrm{i}}$, de acuerdo con la función de control $2\left(\mathrm{~T}_{\mathrm{i}-\Delta \mathrm{x}}-\mathrm{T}_{\mathrm{i}}\right) /\left(\Delta \mathrm{x} /\left(\mathrm{k}_{\mathrm{i}-\Delta \mathrm{x}}\right)\right.$.

La figura IV.11 muestra el modelo en red de la celda elemental $i$, las resistencias $\mathrm{R}_{\infty}$ se incluyen por requisitos de trabajo de Pspice, en relación con estas fuentes controladas. 


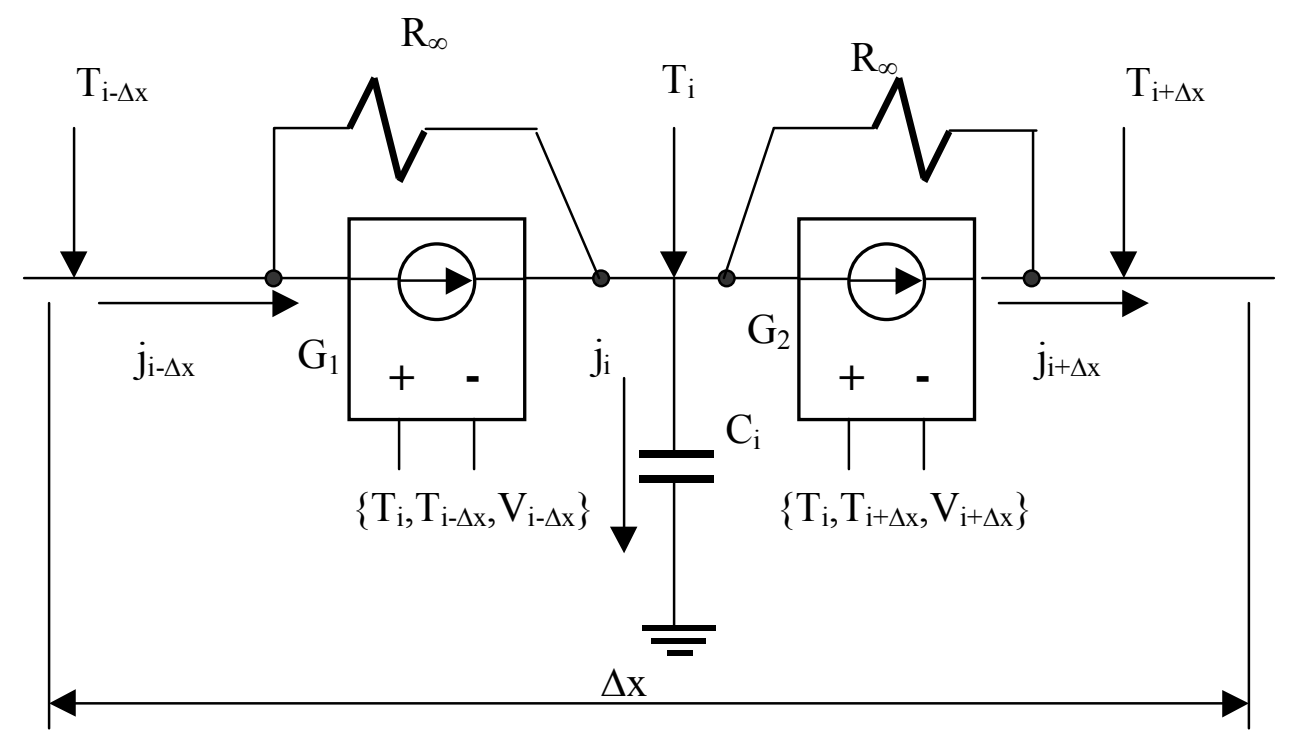

Circuitos auxiliares:

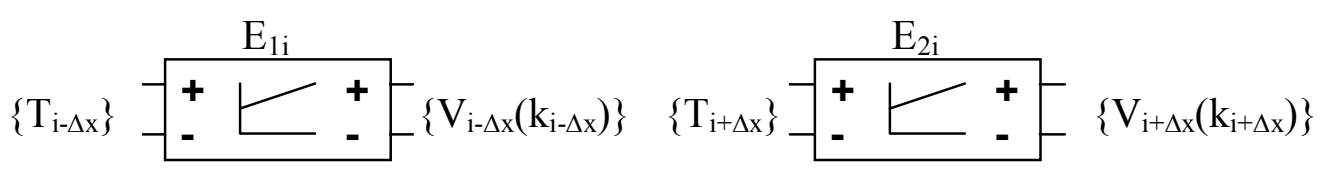

Figura IV.11. Modelo en red para conductividad variable de la temperatura

\section{Conductividad y calor específico dependientes con la temperatura (simultáneamente)}

$\mathrm{Al}$ igual que en los casos anteriores, el modelo propuesto admite cualquier tipo de dependencias $\mathrm{k}=\mathrm{k}(\mathrm{T}) \mathrm{y}_{\mathrm{e}}(\mathrm{T})$. De nuevo, discretizando en la variable espacial la ecuación (1) y definiendo el calor específico en la forma $c_{e}(T)=c_{\text {mean }}+\Delta c_{e}(T)$, resulta

$\Delta \mathrm{x} \rho \mathrm{c}_{\text {mean }} \mathrm{dT}_{\mathrm{i}} / \mathrm{dt}+\Delta \mathrm{x} \rho\left[\mathrm{c}_{\mathrm{e}}\left(\mathrm{T}_{\mathrm{i}}\right)-\mathrm{c}_{\text {mean }}\right] \mathrm{d} \mathrm{T}_{\mathrm{i}} / \mathrm{dt}=2 \mathrm{k}_{\mathrm{i}-\Delta \mathrm{x}}\left(\mathrm{T}_{\mathrm{i}-\Delta \mathrm{x}}-\mathrm{T}_{\mathrm{i}}\right) / \Delta \mathrm{x}-2 \mathrm{k}_{\mathrm{i}+\Delta \mathrm{x}}\left(\mathrm{T}_{\mathrm{i}}-\mathrm{T}_{\mathrm{i}+\Delta \mathrm{x}}\right) / \Delta \mathrm{x} \quad(\mathrm{IV} .10)$ definiendo los flujos de calor

$$
\begin{aligned}
& \mathrm{j}_{\mathrm{i}}=\Delta \mathrm{x} \rho \mathrm{c}_{\text {mean }} \mathrm{dT}_{\mathrm{i}} / \mathrm{dt} \\
& \mathrm{j}_{1}=\Delta \mathrm{x} \rho\left[\mathrm{c}_{\mathrm{e}}\left(\mathrm{T}_{\mathrm{i}}\right)-\mathrm{c}_{\text {mean }}\right] \mathrm{dT}_{\mathrm{i}} / \mathrm{dt} \\
& \mathrm{j}_{\mathrm{i}+\Delta \mathrm{x}}=\left(\mathrm{T}_{\mathrm{i}}-\mathrm{T}_{\mathrm{i}+\Delta \mathrm{x}}\right) /\left(\Delta \mathrm{x} / 2 \cdot \mathrm{k}_{\mathrm{i}+\Delta \mathrm{x}}\right) \\
& \mathrm{j}_{\mathrm{i}-\Delta \mathrm{x}}=\left(\mathrm{T}_{\mathrm{i}-\Delta \mathrm{x}}-\mathrm{T}_{\mathrm{i}}\right) /\left(\Delta \mathrm{x} / 2 \cdot \mathrm{k}_{\mathrm{i}-\Delta \mathrm{x}}\right)
\end{aligned}
$$

la ecuación anterior es la ecuación de Kirchoff “ $\mathrm{j}_{\mathrm{i}}+\mathrm{j}_{1}-\mathrm{j}_{\mathrm{i}-\Delta \mathrm{x}}+\mathrm{j}_{\mathrm{i}+\Delta \mathrm{x}}=0$ ". Las explicaciones anteriores permiten diseñar el modelo en red de la figura IV.12. 


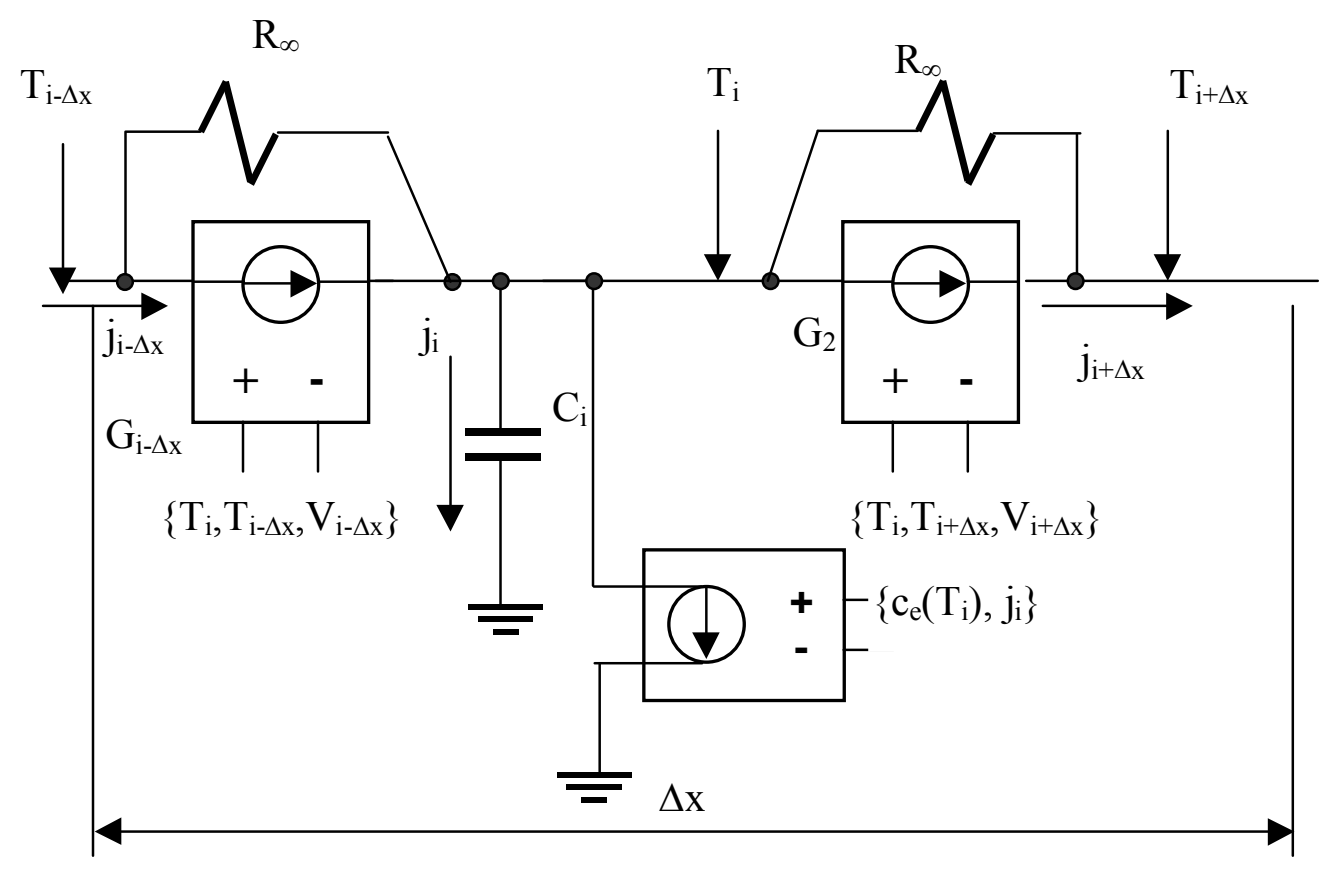

Circuitos auxiliares:

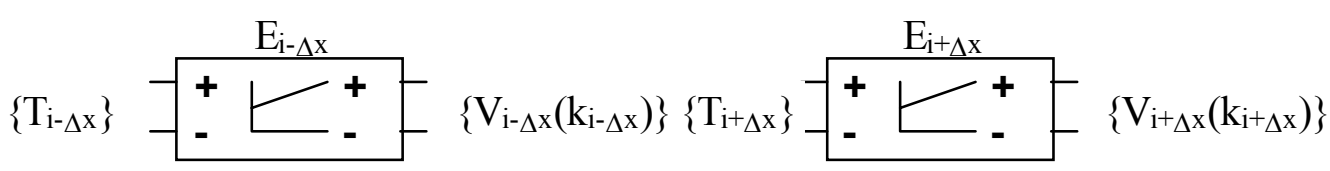

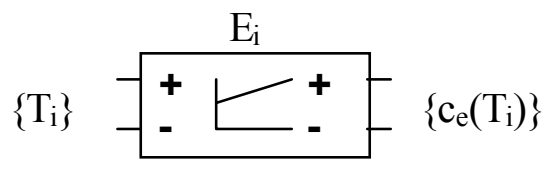

Figura IV.12. Modelo en red para conductividad y calor específico dependientes de la temperatura

Cuando existe una condición de contorno de flujo de calor o convectiva (implementada mediante una fuente de corriente), la celda del mismo extremo contiene una fuente de corriente controlada (caso por ejemplo de la conductividad térmica dependiente de la temperatura). Esta fuente es necesario eliminarla (sólo para esta celda) a fin de hacer compatible la continuidad de la corriente en la frontera. Las temperaturas en los nodos se ajustan, sin necesidad de hipótesis alguna, para cumplir las leyes de los circuitos, Alhama [1999]. 


\section{IV.2. PROBLEMA INVERSO. MODELOS EN RED}

Se elabora el modelo en red partiendo de los datos conocidos del problema (parámetros de propiedades físicas, condiciones de contorno, condiciones iniciales, parámetros geométricos, ....). El modelo en red, recogido en "fichero.cir", se genera por las funciones “salva_datos_en_fichero" y "graba_datos". Una vez definido el "fichero.cir", el valor inicial se va modificando (ver capítulo III) automáticamente en cada iteración, a la vez que se ejecuta Pspice. Para ello se emplea la línea de programación "pspice fichero.cir /r/e", donde la opción / $\mathrm{r}$ inicia la ejecución de la simulación especificada en el archivo actual "fichero.cir", mientras la opción /e cierra la ventana de Pspice una vez finalizada la simulación. Esta opción es necesaria para poder realizar la estimación de funciones de forma automática.

El paso siguiente, en cada simulación, es la lectura de los datos de temperaturas de la misma contenidos en el "fichero.out" que elabora Pspìce. Para ello se emplea la función "Busca_fichero". A continuación, siempre dentro de la iteración, se evalúa el funcional, comparando las medidas de temperatura suministradas por los sensores (datos de partida contenidos en el "fichero.cfg" y leídos mediante la función "Carga_datos") y las temperaturas proporcionadas por la simulación. Se emplean las funciones "Calcula_r" y "Calcula_temp_futuras" para obtener el número de términos que integran el funcional (para el caso de estimación de funciones dependientes de la temperatura), la función "Calculo_Sm" para obtener el valor del funcional, y la función "Calculo_Minima_Sm" para obtener el funcional mínimo cada tres iteraciones.

Una vez obtenido el mínimo funcional, caso de verificar el criterio de convergencia, se utiliza la función "Calcula_Minimo_Final" para obtener el valor de función de estimación buscada en un punto intermedio del tramo estimado. Conseguido esto se pasa a estimar otro punto de un tramo posterior. En caso de no cumplirse el criterio de convergencia, se repite el mismo proceso dentro del mismo tramo. La estimación de la función o funciones termina en el momento en que se obtiene el número de tramos especificado al comienzo de la simulación.

En caso de verse interrumpida la simulación por cualquier anomalía (producida, por ejemplo, por un corte del suministro eléctrico), la función “Lee_datos_ejecutados” permite continuar por el último tramo estimado tras la lectura previa de los datos ejecutados hasta el momento en el fichero "ficherod.cfg".

Al definir el modelo en red se utilizan dispositivos auxiliares en los que se genera la función piece-wise (o dos funciones para el caso de la estimación simultánea de dos funciones); 
se trata de fuentes de tensión o de corriente lineales a tramos en los que se genera, paso a paso, la función estimación buscada. La función "Generacion_rectas" es la encargada de generar está solución por medio de tramos lineales continuos. Existen diversos tipos de dispositivos auxiliares:

i) Fuente de tensión lineal a tramos temporales, para el caso de funciones dependientes del tiempo (se aplica en la estimación de cualquier propiedad térmica que varía con el tiempo)

ii) Fuente de corriente lineal a tramos temporales, también para el caso de funciones dependientes del tiempo (por ejemplo para la estimación de flujos de calor superficiales, generación interna de calor, etc.)

iii) Fuente de tensión, lineal a tramos, controlada por tensión. Se emplea para la obtención de funciones dependientes de la temperatura (por ejemplo para la estimación de propiedades térmicas dependientes de la temperatura)

iv) Fuente de corriente, lineal a tramos, controlada por tensión. También se emplea, alternativamente, para el caso de funciones dependientes de la temperatura

En definitiva, todos estos dispositivos auxiliares, contenidos en las librerías Pspice, generan estimaciones en la forma de funciones lineales a tramos que se especifican mediante pares de puntos "parámetro a estimar-tiempo" o "parámetro a estimar-temperatura". En la figura IV.13 se observan ejemplos de los dispositivos citados y se muestra la forma de especificarlos en el "fichero.cir".

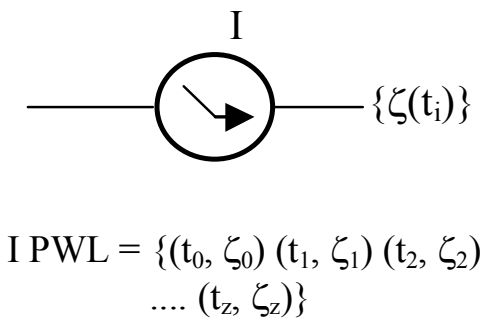

(a)

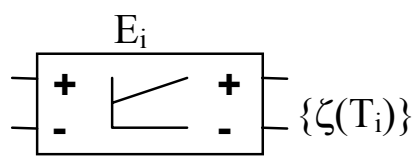

$\begin{aligned} \text { E TABLE }= & \left\{\left(\zeta_{0}, \mathrm{~T}_{0}\right)\left(\zeta_{1}, \mathrm{~T}_{1}\right)\left(\zeta_{2}, \mathrm{~T}_{2}\right) \ldots \ldots\right. \\ & \left.\left(\zeta_{\mathrm{z}}, \mathrm{T}_{\mathrm{z}}\right)\right\}\end{aligned}$

IV.13. Ejemplos de dispositivos auxiliares, (a) Estimación de una función dependiente del tiempo, (b) Estimación de una función dependiente de la temperatura

Para el caso de la estimación de una función dependiente del tiempo se emplea una fuente de corriente PWL (fuente lineal a tramos), en la que es posible definir más de 4000 pares de puntos tiempo-corriente. La figura IV.14a muestra un ejemplo de una fuente de este tipo. Para el caso de estimación de una función dependiente de la temperatura se emplea una fuente 
de tensión controlada por tensión, de forma que la tensión de salida sea función de la tensión de entrada. La salida de la fuente se obtiene entre los nudos $\mathrm{N}^{\text {out }} \mathrm{y} \mathrm{N}_{\text {out, }}$, mientras que la tensión de entrada se aplica a los nudos $\mathrm{N}+_{\text {in }} \mathrm{y} \mathrm{N}-_{\text {in. }}$ La figura IV.14b se muestra la operación señalada.

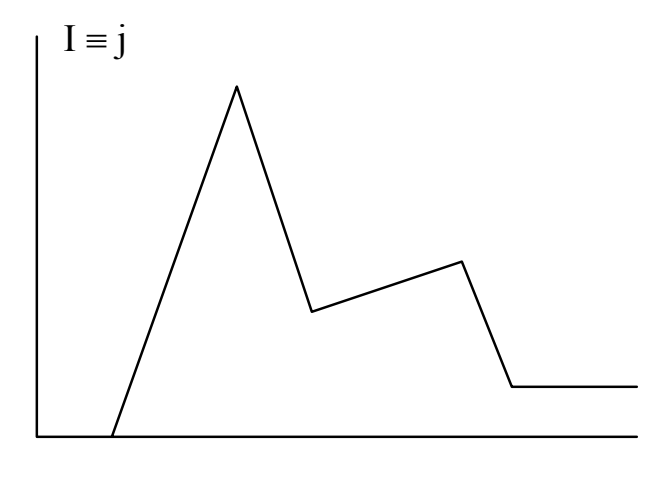

$\begin{array}{llllll}t_{0} & t_{1} & t_{2} & t_{3} & t_{4} & t_{5}\end{array}$

(a)

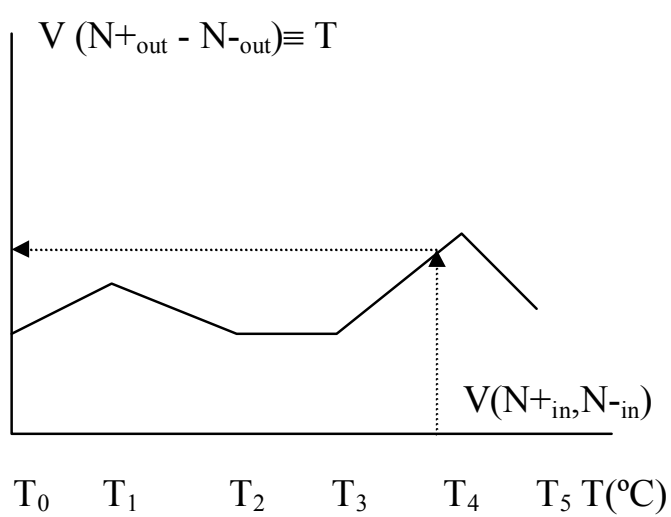

(b)

IV.14. Respuesta de salida de los dispositivos auxiliares, (a) Estimación de una función dependiente del tiempo, (b) Estimación de una función dependiente de la temperatura

\section{IV.2.1. Estimación de magnitudes constantes}

Se incluye en este apartado los casos más sencillos de problema inverso encuadrados dentro del tipo de problemas de estimación de un solo parámetro. La solución por medio del método de redes combina el uso de rutinas auxiliares de programación del tipo de las explicadas en el epígrafe anterior con la posibilidad inherente a Pspice de poder definir paramétricamente la magnitud o coeficiente que se desea estimar. Este parámetro puede estar asociado tanto a dispositivos lineales, tales como resistencias eléctricas, condensadores y fuentes de c.c., como a dispositivos no lineales tales como fuentes controladas.

\section{IV.2.1.1. Estimación de propiedades termofísicas (conductividad y calor específico) constantes}

El modelo en red para la celda elemental es el de la figura IV.8. La resistencia o el condensador, según se trate de la estimación de la conductividad o del calor específico respectivamente, se definen paramétricamente y mediante una rutina de programación se ajusta su valor hasta una cierta cota de error prefijada. Las condiciones de contorno pueden ser arbitrarias, lineales o no. 
La misma técnica puede adoptarse en otros problemas tales como la determinación de la resistencia térmica de contacto entre dos medios, implementada en el modelo por una resistencia ohmica.

\section{IV.2.1.2. Estimación de condiciones de contorno de primera y segunda clase}

El medio puede ser lineal o no por lo que el modelo general de la celda elemental es el de la figura IV.12 el cual puede simplificarse según los casos particulares. El parámetro es el valor de la temperatura (condición isoterma) o de la densidad de flujo de calor, aplicado en el contorno. Al igual que en caso anterior, una rutina de programación aproxima la solución hasta el límite fijado.

\section{IV.2.1.3. Estimación de otros parámetros}

Se incluye en este apartado la estimación de magnitudes o coeficientes que aparecen dentro de ciertas expresiones que definen condiciones de contorno $\mathrm{u}$ otras condiciones especiales tales como el coeficiente de convección, la emisividad superficial, calores latentes de cambio de estado, etc. Tanto el medio como el resto de las condiciones de contorno del problema pueden ser lineales o no. El modelo en red de la celda elemental, depende de las dependencias de las propiedades térmicas (constantes, figura IV.8, o variables, figuras IV.10, IV.11 ó IV.12). La solución más sencilla (aunque no la única) consiste en implementar un circuito auxiliar, un simple generador de tensión (c.c.) cuyo valor, modificado en cada iteración mediante una rutina de programación, es el parámetro a determinar. La condición de frontera que contiene la acción del parámetro a estimar se implementa generalmente mediante una fuente de corriente controlada por la tensión del generador anterior. En la figura IV.15 se muestra el modelo para la determinación del coeficiente de convección forzada.

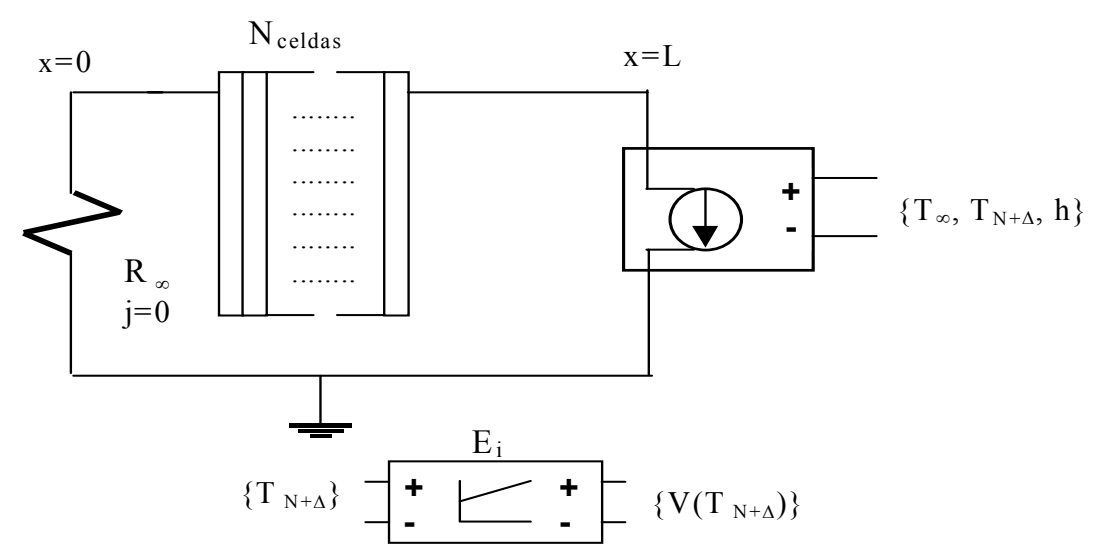

Figura IV.15. Modelo en red para la determinación del coeficiente de convección forzada, $\mathrm{h}$ 


\section{IV.2.2. Estimación de funciones}

\section{IV.2.2.1. Estimación del calor específico dependiente de la temperatura}

El modelo en red para la estimación del calor específico dependiente de la temperatura es básicamente el mismo que el del problema directo (figura IV.10), con la diferencia de que en el problema inverso la fuente de tensión controlada por tensión, $\mathrm{E}_{\mathrm{i}}$, se va especificando, tramo a tramo, conforme avanza la estimación. Se utiliza la función "Generacion_rectas" para cada especificación parcial $\mathrm{c}_{\mathrm{e}, \mathrm{i}}\left(\mathrm{T}_{\mathrm{i}}\right)$.

\section{IV.2.2.2. Estimación de la conductividad térmica variable con la temperatura}

Como en el caso anterior, el modelo en red es básicamente el mismo que el del problema directo análogo (figura IV.11). Las fuentes de tensión controladas por tensión, $\mathrm{E}_{\mathrm{i} \pm \Delta}$, se especifican progresivamente tramo a tramo. También se utiliza la función "Generacion_rectas" para cada especificación parcial $\mathrm{k}_{\mathrm{i}}\left(\mathrm{T}_{\mathrm{i}}\right)$.

\section{IV.2.2.3. Estimación de flujos incidentes (condición de contorno)}

En este caso la acción de control es aplicada a la condición de contorno, de modo que lo primero es elegir el modelo en red para el medio, figura IV.8 para un medio con propiedades térmicas constantes o figuras IV.10, IV.11 o IV.12 para medios con propiedades térmicas función de la temperatura. La figura IV.16 muestra la configuración global para la estimación de un flujo de calor en la superficie, en $\mathrm{x}=0$; la condición de contorno en $\mathrm{x}=\mathrm{L}$ puede ser cualquiera.

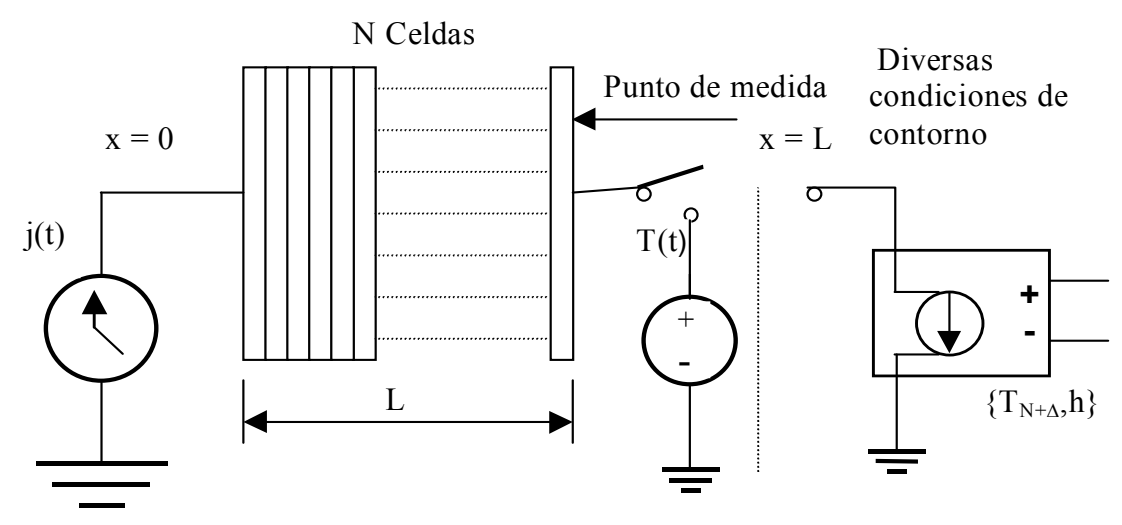

Figura IV.16. Modelo en red para la determinación del flujo de calor incidente 
La solución del problema inverso consiste en ir modificando la fuente de corriente en función del tiempo $I(t)$, que se corresponde con la función buscada $j(t)$. Se trata de una fuente de corriente lineal a tramos, obtenida mediante la función "Generacion_rectas". En la figura IV.17 se muestra un ejemplo (se dibujan las soluciones exacta y estimada hasta el momento).

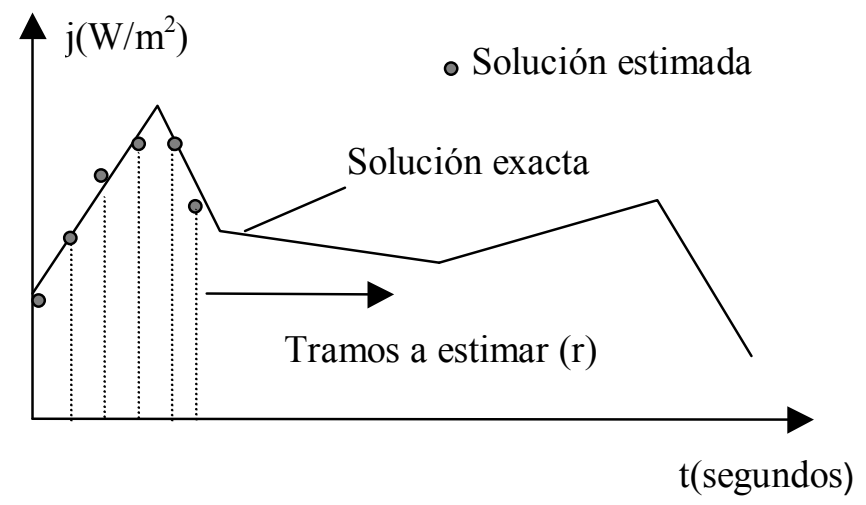

Figura IV.17. Ilustración de la estimación de un flujo de calor incidente

\section{IV.2.2.4. Estimación de focos térmicos (condición de contorno de temperatura en función del tiempo)}

El modelo en red para la celda elemental es el de las configuraciones ya conocidas para medios con propiedades térmicas constantes o dependientes de la temperatura. La figura IV.18, de propósito general, muestra la configuración global para la implementación en Pspice. El punto de medida de temperatura para obtener los datos de entrada puede ser el extremo opuesto al foco, $\mathrm{x}=\mathrm{L}$, o un punto interior del medio. La estimación de temperatura se hace para la posición $\mathrm{x}=0$ donde la condición de contorno previsiblemente debería ser un foco térmico de temperatura $\mathrm{T}(\mathrm{t})$. Para la solución del problema se emplea una fuente de tensión lineal a tramos.

Eventualmente, podría estimarse $\mathrm{T}(\mathrm{t})$ en $\mathrm{x}=0$ para otras condiciones de contorno en esa posición, tales como una condición de flujo variable o una condición adiabática. Este sería un procedimiento alternativo e indirecto a la estimación de flujos de calor discutida en el apartado anterior. La estimación se consigue por idénticos procedimientos a los empleados en los casos anteriores. 


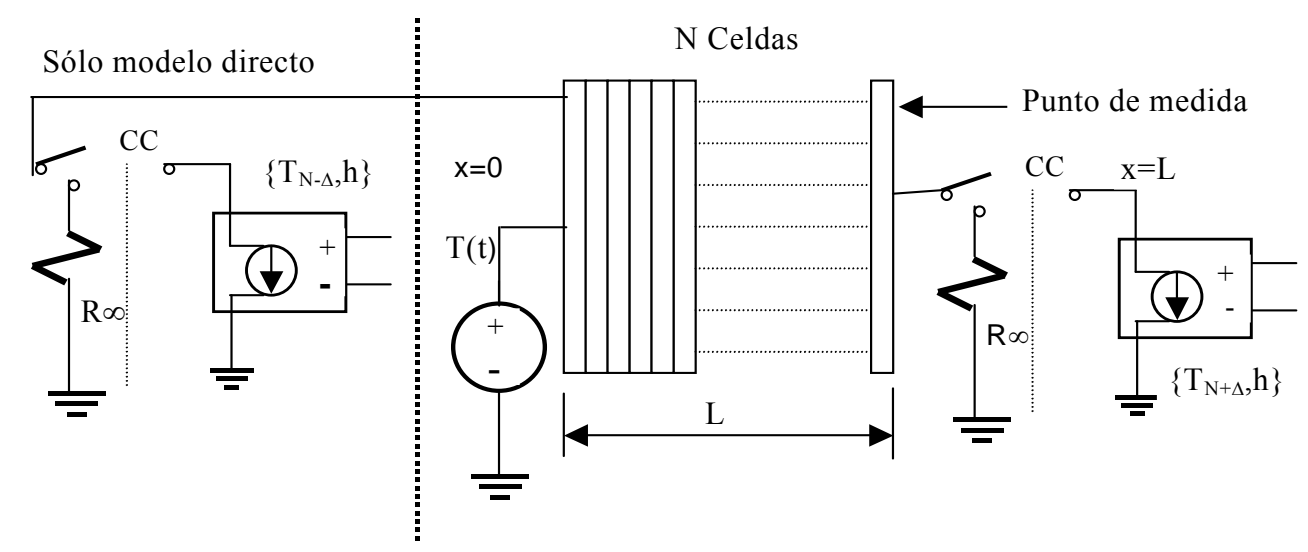

Figura IV.18. Configuración global del sistema para la obtención de un foco térmico

\section{IV.2.2.5. Estimación de otras funciones}

Estimación del coeficiente convectivo dependiente de la temperatura

Como se trata de la estimación de una condición de contorno el modelo en red para la celda elemental para el medio conductor se elige de entre las configuraciones ya conocidas, según éste tenga propiedades térmicas constantes o dependientes de la temperatura. La figura IV.19 muestra el modelo a implementar en Pspice. La dependencia temporal de este coeficiente se resuelve, como en los casos anteriores, utilizando una fuente de tensión a tramos $\mathrm{T}(\mathrm{t})$.

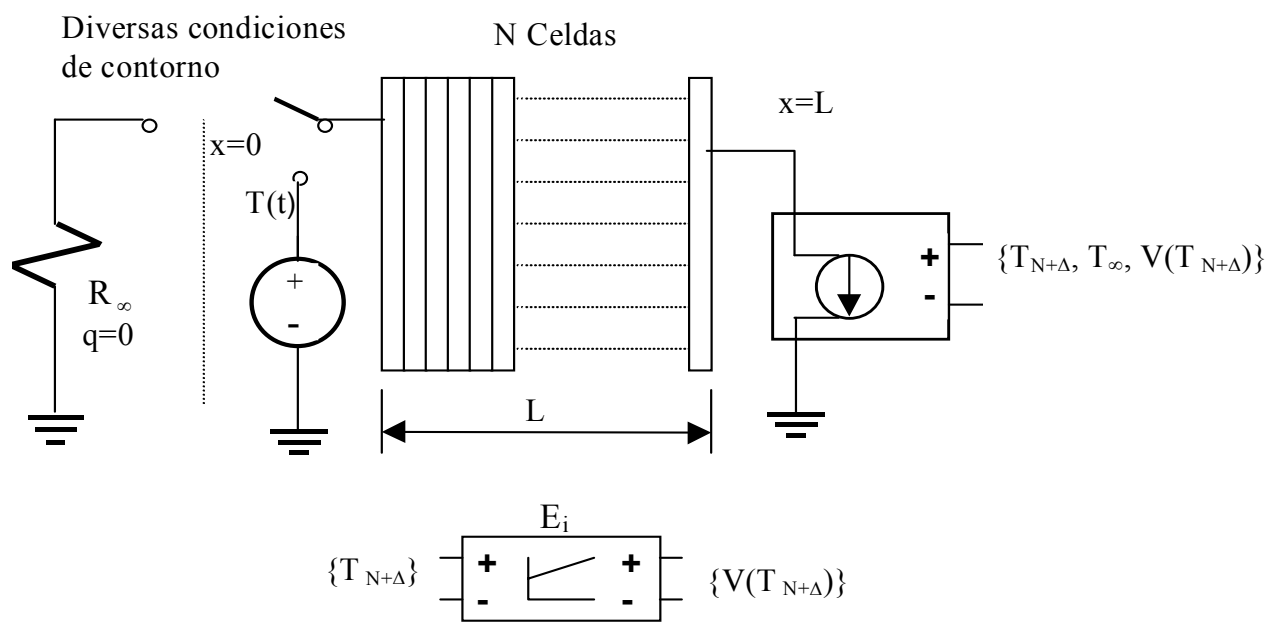

Figura IV.19. Modelo en red para la estimación del coeficiente convectivo dependiente de la temperatura

Estimación de la resistencia térmica de contacto

En los sistemas multicapa los contactos entre paredes no son perfectos desde el punto de vista térmico; existe una resistencia térmica entre capas, que puede depender de la presión y de 
la temperatura en la superficie de contacto debido principalmente a la rugosidad superficial, que da lugar a la aparición de intersticios generalmente llenos de aire o gas. En consecuencia, el calor fluye a través de esta superficie de contacto no sólo por el clásico mecanismo de conducción sino por convección debido al gas aprisionado, e incluso por radiación. Es posible caracterizar esta superficie mediante una resistencia térmica constante o variable con la temperatura o el tiempo. El problema inverso es la estimación de esta resistencia.

El modelo matemático, suponiendo propiedades físicas constantes en ambos medios, viene dado por:

$$
\begin{aligned}
& \partial \mathrm{T}_{1} / \partial \mathrm{t}=\alpha_{1}\left(\partial^{2} \mathrm{~T}_{1} / \partial \mathrm{x}^{2}\right) \\
& \partial \mathrm{T}_{2} / \partial \mathrm{t}=\alpha_{2}\left(\partial^{2} \mathrm{~T}_{2} / \partial \mathrm{x}^{2}\right) \\
& -\mathrm{k}_{1} \partial \mathrm{T}_{1} / \partial \mathrm{x}=-\mathrm{k}_{2} \partial \mathrm{T}_{2} / \partial \mathrm{x}=\left(\mathrm{T}_{1}-\mathrm{T}_{2}\right) / \mathrm{R}(\mathrm{t})=\mathrm{I}(\mathrm{t})
\end{aligned}
$$

La ecuación (IV.13) es la condición de contorno en la interfase. El modelo en red para la estimación de la resistencia térmica de contacto se presenta en la figura IV.20 donde, si el parámetro a estimar se considera constante es necesario colocar una resistencia $R_{c}$ de valor constante en $\mathrm{x}=\mathrm{L}_{1}$. Si por el contrario se considera variable con la temperatura o el tiempo hay que utilizar sendas fuentes de corriente a tramos $\mathrm{I}(\mathrm{T})$ o $\mathrm{I}(\mathrm{t})$, respectivamente.

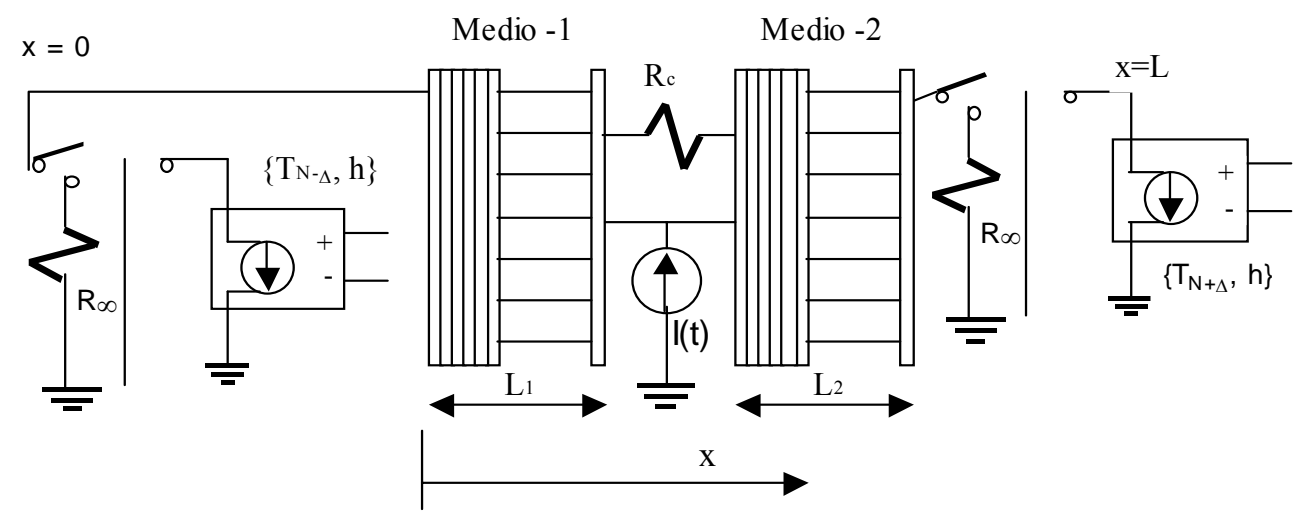

Figura IV.20. Modelo en red para la estimación de la resistencia térmica de contacto

En Beck [1967 y 1968] y Orlande y Özisik [1993] se realizan estimaciones de este parámetro.

\section{Estimación de la generación interna de calor}

Si se considera el término de generación interna de calor, la ecuación de la transferencia del calor, adopta la forma, 


$$
\rho c_{\mathrm{e}} \frac{\partial \mathrm{T}}{\partial \mathrm{t}}=\mathrm{k} \frac{\partial^{2} \mathrm{~T}}{\partial \mathrm{x}^{2}}+g_{\text {gen }}
$$

Introduciendo la densidad de flujo de calor " $\mathrm{j}_{\mathrm{x}}=\mathrm{-} \mathrm{k} \partial \mathrm{T} / \partial \mathrm{x}$ " en la ecuación diferencial resultante de la discretización espacial de IV.14 se obtiene la ecuación,

$$
\rho c_{e} d T / d t-k(\Delta j / \Delta x)=g_{g e n}
$$

donde aparece el término de generación de calor aplicado a cada celda. La figura IV.21 muestra el modelo en red, para el caso de propiedades térmicas constantes, con generación interna variable con el tiempo. Incluye una fuente de corriente $\mathrm{g}_{\mathrm{gen}}$, que inyecta calor en el centro de cada celda. El caso de propiedades térmicas dependientes de la temperatura sigue un procedimiento similar (figura IV.12).

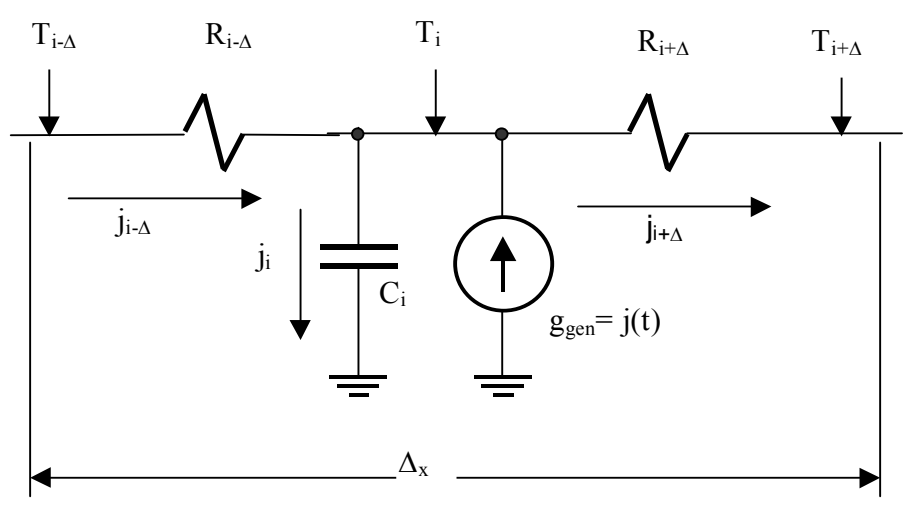

Figura IV.21. Modelo en red de la celda para la estimación de una dependencia temporal de la generación interna de calor

Como en casos anteriores la acción de control es similar al caso de estimación del flujo de calor aplicado como condición de contorno, existiendo una función de control j(t) o j(T) en cada celda elemental.

La figura IV.22 muestra la configuración local para el caso de propiedades térmicas constantes, con generación interna dependiente de la temperatura en cada punto. Se ha incluido una fuente de corriente controlada por tensión. ggen que aporta el calor generado por unidad de volumen; $\mathrm{V}\left(\mathrm{T}_{\mathrm{i}}\right)$ es la acción de control. Es aplicable, lo dicho en párrafos anteriores respecto de la aplicación a medios con propiedades térmicas dependientes de la temperatura. 


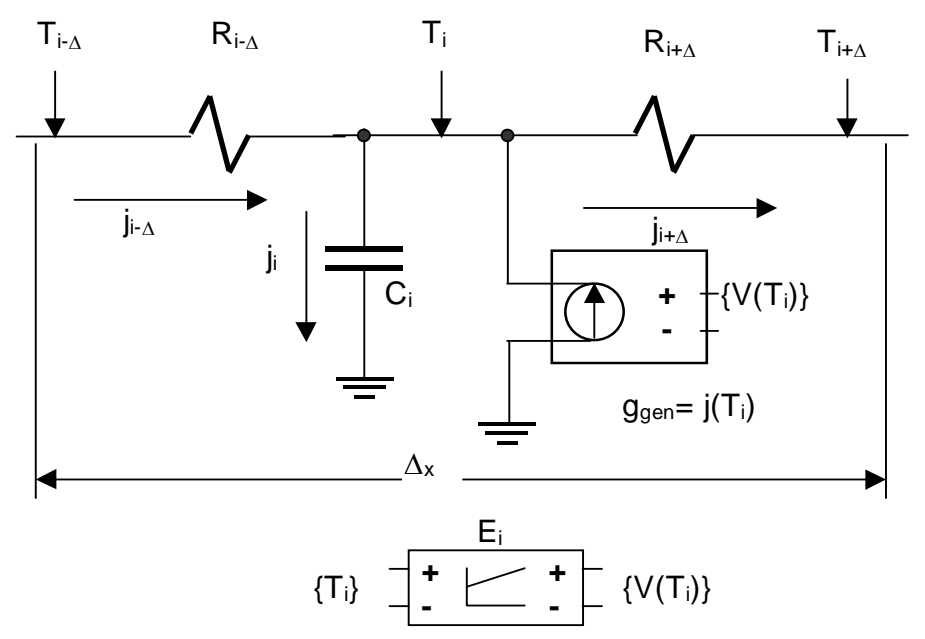

Figura IV.22. Modelo en red para la estimación de la generación interna de calor dependiente de la temperatura

Los trabajos de Silva y Neto [1992] y Yang [1997 y 1998] están relacionados con este tipo de estimaciones.

\section{IV.2.2.6. Estimación simultánea del calor especifico y de la conductividad térmica}

El modelo en red para la estimación simultánea del calor específico y de la conductividad térmica, ambas propiedades dependientes de la temperatura, es básicamente el del problema directo (figura IV.12), con la diferencia de que en el problema inverso las fuentes de tensión controladas por tensión $\mathrm{E}_{\mathrm{i}}, \mathrm{E}_{\mathrm{i}-\Delta} \mathrm{y} \mathrm{E}_{\mathrm{i}+\Delta}$ son funciones inicialmente desconocidas que se van estimando por tramos de manera secuencial. La fuente de tensión $\mathrm{E}_{\mathrm{i}}$ genera el valor del calor específico en función de la temperatura $\mathrm{T}_{\mathrm{i}}, \mathrm{c}_{\mathrm{e}}\left(\mathrm{T}_{\mathrm{i}}\right)$ mediante la función "Generacion_rectas", mientras que las fuentes de tensión $\mathrm{E}_{\mathrm{i}-\Delta} \mathrm{y} \mathrm{E}_{\mathrm{i}+\Delta}$ generan la conductividad térmica en los extremos de cada celda en función de la temperatura en dichos extremos, mediante la función "Generacion_rectas1". Es decir en cada iteración se realizan dos alteraciones de los parámetros, uno para el calor específico y otro para la conductividad térmica (ver capítulo III).

El programa fuente para la estimación simultánea de la dependencia con la temperatura de las propiedades térmicas, por ser el más complejo, se adjunta en el Anexo 1 de esta Memoria.

\section{IV.2.3. Estimación de funciones lineales}

Los problemas inversos de estimación de más de un parámetro no se han incluido en esta Memoria debido a la extensión que ello supondría. Además, no se trata en realidad de un 
problema práctico puesto que conocer a priori el tipo de aproximación funcional de cualquier magnitud no es habitual; más bien se trataría de una suposición que, a menos que esté suficientemente justificada, no aportaría soluciones aceptables al problema inverso por su inherente dificultad de convergencia. Por otro lado, puede considerarse que los modelos propuestos con anterioridad, que incluyen la solución de cualquier tipo de función desconocida, son capaces a priori de abordar los problemas de estimación de parámetros.

No obstante y por su simplicidad y generalidad se presenta a continuación el problema de estimación de funciones "lineales", cuya aplicación es adecuada para la estimación de conductividades y calores específicos en numerosos medios sometidos a amplios rangos de temperaturas de trabajo.

Suponiendo que se quiere estimar la dependencia de la conductividad térmica con la temperatura de un sólido, sabiendo a priori que dicha dependencia es, $\mathrm{k}=\mathrm{k}_{0}+\mathrm{k}_{1} \mathrm{~T}$, el objetivo es estimar los parámetros $\mathrm{k}_{0} \mathrm{y} \mathrm{k}_{1}$, o bien los valores de $\zeta_{0} \mathrm{y} \zeta_{1}$ asociados a los puntos $\left(\zeta_{0}, \mathrm{~T}_{0}\right)$ y $\left(\zeta_{1}\right.$, $\mathrm{T}_{1}$ ). Para ello se utilizan dos fuentes de tensión controladas por tensión idénticas a las empleadas en el apartado IV.2.3.2.

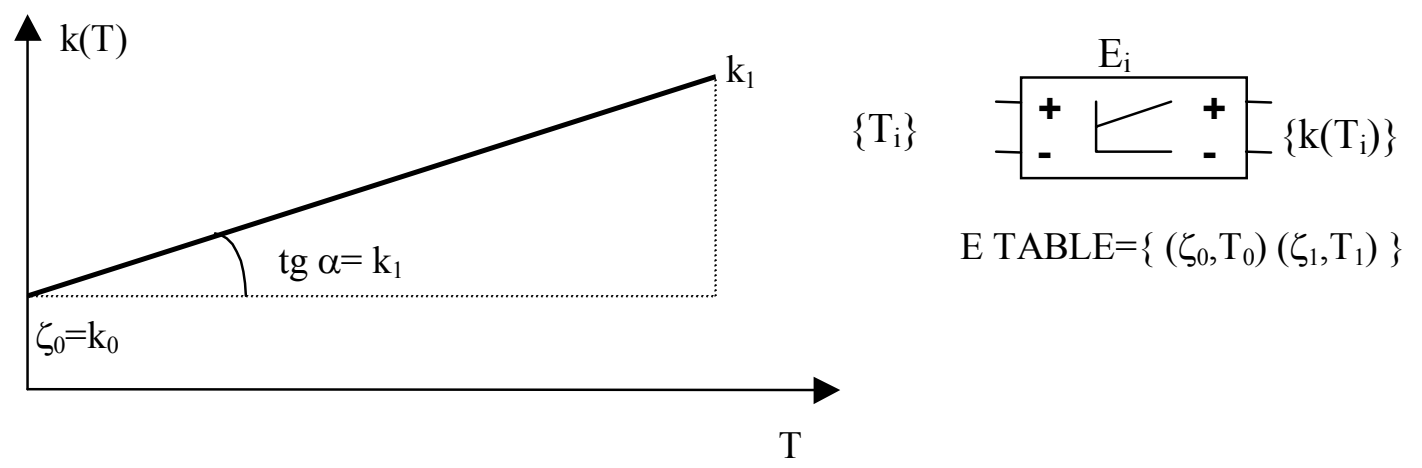

Figura IV.23. Ilustración para la estimación de parámetros

En la figura IV.23 se ilustra la estimación de una función "lineal". Se trata de ajustar los parámetros $\mathrm{k}_{0} \mathrm{y} \mathrm{k}_{1}$, mediante un proceso iterativo. En este caso no se trata de una estimación secuencial (empleada en los casos anteriores de estimación de funciones), sino que se trata de actuar sobre el dispositivo $E_{\mathrm{i}}$ alterando únicamente los puntos buscados $\left(\zeta_{0}, \mathrm{~T}_{0}\right)$ y $\left(\zeta_{1}, \mathrm{~T}_{1}\right)$, donde se suponen conocidas las temperaturas $\mathrm{T}_{0} \mathrm{y} \mathrm{T}_{1}$, según el protocolo explicado en el capítulo III, apartado III.3.5.

El programa fuente para la estimación de funciones lineales, se adjunta en el Anexo 1 de esta Memoria. 


\section{IV.3. REFERENCIAS}

Alhama López, F., "Estudio de respuestas térmicas transitorias en procesos no lineales de conducción de calor mediante el Método de simulación por redes”. Tesis Doctoral. Universidad de Murcia (1999)

Beck, J.V., "Transient Sensitivity Coefficients for the Thermal Contact Conductance", Int. J. Heat Mass Transf., 10, 1615-1616 (1967)

Beck, J.V., "Determination of optimun, transient experiments for thermal contact conductance", Int. J. Heat Mass Transf., 12, 621-633 (1968)

González-Fernández, C.F. y Alhama, F., "Heat Transfer and the Network Simulation Method", en "Network Simulation Method", Ed. J. Horno, Research Singpost, Trivandrum, India (2002)

Orlande, H.R.B, and Özisik, M.N., "Inverse problem of estimating interface conductance between periodically contacting surfaces", J. Therm. Heat Transf., 7, 2, 319-325 (1993)

Silva Neto A.J. and Özisik, M.N., "Two-dimensional inverse heat conduction problem of estimating the time-varying strength of a line heat source", J. Appl. Phys., 71 (11), 53575362 (1992)

Yang, Ching-Yu, "Non-iterative solution of inverse heat conduction problems in one dimension", Commun. Numer. Meth. Eng., 13, 419-427 (1997)

Yang, Ching-Yu, "Solving the two-dimensional inverse heat source problem through the linear least-squares error method", Int. J. Heat Mass Transf., 43, 2, 393-398 (1998)

\section{CONTRIBUCIONES Y CONCLUSIONES}

En base a los modelos en red de problemas directos lineales y no lineales de conducción, ya conocidos anteriormente en otros trabajos, y utilizando las modernas librerías de dispositivos eléctricos especiales (no lineales) contenidos en los programas de resolución de circuitos, tales como fuentes controladas y generadores tipo "stimulus", se proponen modelos en red que permiten la solución de problemas inversos 1-D de geometría regular en transmisión de 
calor. La extensión de estos modelos a otros sistemas de coordenadas y grados de libertad es inmediata.

Los modelos propuestos se extienden a problemas de estimación de:

flujos de calor incidentes (estimación directa e indirecta)

temperaturas de contorno (estimación directa e indirecta)

características térmicas

condiciones de contorno

otros parámetros tales como el coeficiente de convección, generación interna, resistencia de contacto

En todos los casos la estimación puede ser una función constante o una función variable dependiente de la temperatura o del tiempo, y el medio (de acuerdo con el tipo de problema) puede asumir características térmicas constantes o dependientes de la temperatura. Los modelos propuestos estiman la solución mediante una función lineal a tramos cuya precisión puede ser ajustada convenientemente.

Por otro lado, se proponen modelos para la determinación de funciones "lineales" (de dos parámetros). Por último se propone un modelo para la determinación simultánea de dos funciones arbitrarias.

Se aportan las rutinas de programación que permiten las tareas necesarias para la solución completa y de forma automática del problema inverso. Estas rutinas han sido desarrolladas en lenguaje $\mathrm{C}++$. 


\section{Capítulo V}

\section{Estimación de flujos incidentes. Estimación de temperaturas de contorno}

V.1. Estimación del flujo de calor en la superficie de un sólido

V.1.1. Introducción

V.1.2. Estimación del flujo de calor en medios con características térmicas constantes

V.1.3. Estimación del flujo de calor en medios con características térmicas dependientes de la temperatura

V.2. Estimación de la temperatura

V.2.1. Introducción

V.2.2. Estimación de temperatura en un medio sometido a convección forzada

V.3. Referencias

Contribuciones y conclusiones 


\section{Capitulo V}

\section{Estimación de flujos incidentes. Estimación de temperaturas de contorno}

Dedicamos la primera parte de este capítulo a la estimación del flujo de calor incidente (como condición de contorno) por tratarse de un problema fundamental (capítulo II) dentro de este campo y al que numerosos autores han dedicado su atención buscando soluciones mediante diferentes técnicas numéricas o técnicas analíticas aproximadas.

Se aborda la estimación de flujos incidentes que responden a un espectro de funciones dependientes del tiempo suficientemente amplio, tales como funciones constantes, trigonométricas, en escalón, triangulares y rectangulares, y se utilizan indistintamente medios con características térmicas tanto independientes como dependientes de la temperatura, no existiendo problema alguno en asumir otras dependencias funcionales continuas de cualquier tipo. En todos los casos se utiliza la técnica de estimación de funciones, construyendo la solución final en la forma de una función lineal a tramos.

Las técnicas básicas de estimación inherentes a la solución del problema inverso y los modelos en red que se utilizan en este capítulo han sido explicados en los capítulos anteriores III y IV, respectivamente. El planteamiento de los problemas, que entraña primero la solución del problema directo también por medio del MESIR, tiene la ventaja de poder comparar las estimaciones obtenidas con las soluciones exactas, verificando de esta forma la fiabilidad de las soluciones propuestas.

La estimación de la temperatura en el contorno, a la que se dedica la segunda parte del capítulo, puede obtenerse a partir de los datos del flujo de calor incidente. El MESIR proporciona esta estimación directamente sin necesidad de cálculo adicional ni modificación alguna del modelo. La estimación directa de la temperatura en el contorno en función del tiempo, que proporciona a su vez una estimación indirecta del flujo incidente de calor, se incluye al final del capítulo. Se utiliza para ello la misma técnica de estimación de función. 


\section{V.1. ESTIMACIÓN DEL FLUJO DE CALOR EN LA SUPERFICIE DE UN SÓLIDO}

\section{V.1.1. Introducción}

El conocimiento del flujo de calor en la superficie de un sólido es importante y necesario en numerosas aplicaciones. Esta variable es fundamental para determinar coeficientes de transferencia de calor o temperaturas de fluidos convectivos, para la calibración de fuentes de calor, etc. En muchos procesos termodinámicos (reales) tales como los que ocurren en el interior de un cilindro de un motor de combustión interna alternativo y en su sistema de refrigeración, en las superficies de las toberas de ensayo de cohetes, en las superficies exteriores de los vehículos espaciales durante el despegue y en esas mismas superficies a la reentrada en la atmósfera terrestre, en determinados procesos de fabricación de metales y cristales, etc., no es posible la medida directa de temperatura en la superficie del sólido (capítulo III) debido a su inaccesibilidad o a los límites mecánicos de resistencia de los aparatos de medida. En estos casos se procede a obtener los datos de temperatura en otros puntos interiores del medio o en otras superficies no expuestas a condiciones tan severas de flujo incidente (figura V.1).

La determinación o estimación del flujo incidente es un problema de solución sensiblemente más difícil que la del problema directo clásico por doble motivo: no existe una única solución y ésta es enormemente sensible a los errores en las medidas de la temperatura. Es, por excelencia, el problema inverso en conducción de calor (capítulo III).

En la práctica, en los algoritmos desarrollados para estimar el flujo de calor incidente está implícita la estimación de la temperatura superficial y viceversa, en tanto que ambas variables están relacionadas a través de la ley de conducción de Fourier. En este trabajo, gracias a la metodología empleada (MESIR), por la aplicación directa de la ley de Fourier, la relación entre flujos y temperatura queda implícita en las leyes de circuitos del modelo.

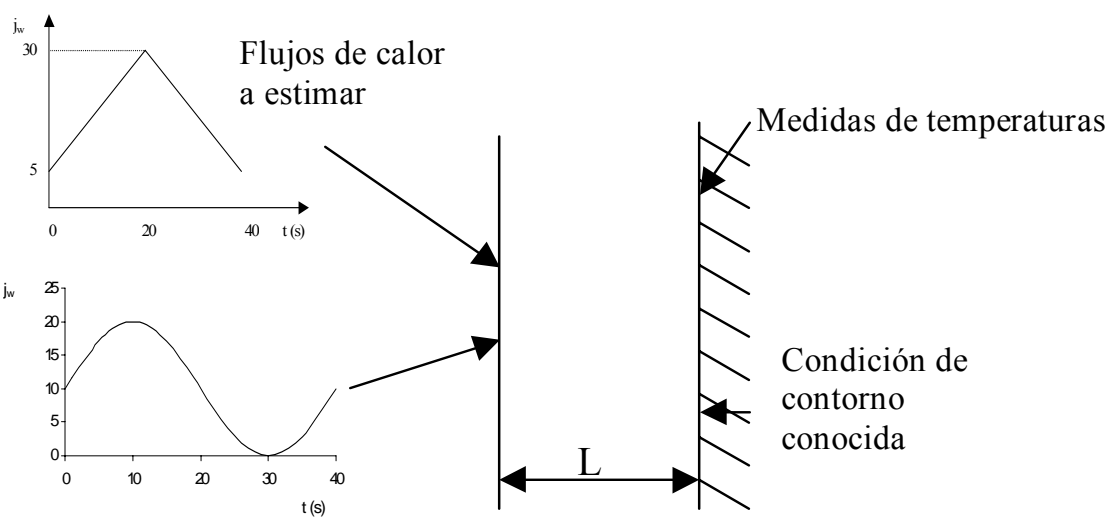

Figura V.1 Geometría del problema 
Para la estimación del flujo incidente se emplea una fuente de corriente variable, especificada a tramos en intervalos de tiempo consecutivos. Para la estimación de temperaturas se emplea una fuente de tensión de características similares.

A continuación se presenta una breve revisión bibliográfica de los trabajos publicados hasta la fecha sobre estimación de flujos incidentes. Stolz [1960] utilizando un método integral de inversión numérica (basado en la integral de Duhamel) estimó el flujo de calor y la temperatura en la superficie de un sólido en función del tiempo a partir de la medida de temperaturas en una posición interior del sólido. Este trabajo está limitado a situaciones en las que la temperatura inicial del cuerpo es uniforme. Por debajo de un cierto límite en el intervalo de tiempo de estimación se producen oscilaciones en los resultados. Se trata de un planteamiento lineal que permite aplicar el principio de superposición.

Más tarde Burggraf [1964] encontró la solución exacta del problema inverso lineal de la estimación de flujos de calor o temperaturas, en geometrías plana, cilíndrica y esférica, a partir del conocimiento de forma continua de la historia de temperatura o flujos de calor en un punto interno del medio. Cuando los datos experimentales son discretos la solución es aproximada.

Beck [1963 y 1968] estimó el flujo de calor en una superficie de un sólido midiendo la temperatura en una o varias posiciones en el interior del mismo y utilizando métodos de cálculo basados en la integral de Duhamel (método de la convolución). Aplicó el concepto de temperaturas futuras para estabilizar la solución y asumió propiedades térmicas constantes. Redujo el problema de las oscilaciones de Stolz. Otro trabajo pionero en la estimación de flujos de calor fue realizado por Shumakov [1957].

Sparrow y col. [1964] presentaron un procedimiento de cálculo, basado también en técnicas de inversión de la integral general de Duhamel, para la estimación del flujo de calor o de la temperatura en la superficie del medio, en geometrías plana, cilíndrica y esférica, partiendo de medidas de temperaturas en su interior. Admite una distribución no uniforme de la temperatura inicial pero las propiedades térmicas del medio han de ser constantes.

Beck [1970] empleó una aproximación discreta por diferencias finitas, para resolver el problema inverso con propiedades térmicas dependientes de la temperatura. Utilizó dos variantes, una muy potente para la estimación paso a paso (con temperaturas futuras), en función del tiempo, de los componentes del flujo de calor y otra de menor interés para estimar todos los componentes del flujo de manera simultánea. Beck, en un libro posterior [1985], desarrolla este método que, en parte, se integra en el protocolo de estimación y cálculo propuesto en esta tesis. 
Alifanov y Artyukhin [1975] estiman el flujo de calor con propiedades térmicas dependientes de la temperatura, en medios 1-D, mediante un procedimiento de minimización iterativa basado en el método de gradientes conjugados.

France y Chiang [1980] estiman la temperatura en la superficie interior de un medio cilíndrico hueco (conducción radial) estando la superficie exterior sometida a convección; realizaron ensayos para diferentes puntos de medida de temperatura, uno tomando medidas en un punto interior del cilindro y otro tomando medidas en la superficie convectiva. Weber [1981] estimó el flujo de calor y temperatura en una superficie de un sólido, para ello desarrolla un procedimiento para el caso unidimensional que aproxima la ecuación de la conducción del calor mediante una ecuación hiperbólica que es resuelta numéricamente.

Beck y col. [1982] utilizando diferencias finitas y con ayuda de los coeficientes de sensibilidad desarrollan un caso de estimación no-lineal (propiedades térmicas dependientes de la temperatura) que reducía los tiempos de computación respecto a los trabajos anteriores de Beck, [1970]. Por otro lado, Hills y Hensel [1986] estiman temperaturas y flujos de calor en problemas 1-D, con propiedades dependientes de la temperatura, usando diferencias finitas y temperaturas pasadas y futuras, e introduciendo estabilizadores (filtros digitales) para reducir el efecto del error en las medidas. Así mismo, Kurpisz [1991] encontró una solución en términos de series, parecida a la solución exacta de Burggraf [1964], para la estimación de flujos de calor y temperaturas a partir de la evolución transitoria de la temperatura en diversos puntos.

Pasquetti y Le Niliot [1991] emplearon el método aproximado de elementos de contorno para resolver problemas inversos de conducción del calor multidimensionales y no lineales. Estudiaron los casos de situación estacionaria y transitoria para la estimación del flujo de calor en función del tiempo y de la coordenada angular. Realizaron una aplicación al caso bidimensional en situación transitoria.

Huang y $\mathrm{Wu}$ [1994] resolvieron, mediante el método de gradiente conjugado para el proceso de minimización, un problema inverso bidimensional para la estimación simultánea de flujos de calor que actúan sobre el interior de las paredes del cilindro de un motor de combustión interna alternativo, desde las medidas de temperaturas tomadas a su alrededor. Recientemente, Le Niliot [2000] hace un estudio detallado de las dificultades de la estimación inversa de flujos y fuentes de calor en procesos de difusión del calor.

Por último, Alhama y col. [2001, 2003] determinaron diferentes flujos de calor (constante, sinusoidal, triangular, función escalón) aplicados a una superficie, empleando el MESIR. 


\section{V.1.2. Estimación del flujo de calor en medios con características térmicas constantes}

El sistema considerado es una placa plana 1-D, con condición adiabática en una de sus caras y de flujo incidente (a estimar) en la otra, Alhama y col. [2001]. El modelo matemático se muestra en el cuadro V.1.

\begin{tabular}{|c|c||}
\hline \multicolumn{2}{|c|}{ Modelo matemático } \\
\hline Ec. diferencial & $\rho \mathrm{c}_{\mathrm{e}} \partial \mathrm{T} / \partial \mathrm{t}-\mathrm{k}\left(\partial^{2} \mathrm{~T} / \partial \mathrm{x}^{2}\right)=0$ \\
\hline $\begin{array}{c}\text { Condiciones } \\
\text { iniciales }\end{array}$ & $\mathrm{T}(\mathrm{x}, \mathrm{t}=0)=\mathrm{T}_{0}$ \\
\hline $\begin{array}{c}\text { Condiciones de } \\
\text { contorno }\end{array}$ & $\mathrm{j}(\mathrm{L}, \mathrm{t})=\mathrm{j}_{\text {in }}(\mathrm{t})$ \\
\hline $\begin{array}{c}\text { Condición } \\
\text { adicional }\end{array}$ & $\mathrm{j}(0, \mathrm{t})=0($ punto de medida $)$ \\
\hline
\end{tabular}

Cuadro V.1. Modelo matemático del problema

donde $\mathrm{x}, \mathrm{t}$ y $\mathrm{T}$ son las variables posición, tiempo y temperatura. Se estudian cuatro problemas distintos, asociados con las cuatro formas de onda del flujo de calor incidente recogidas en el cuadro V.2.

\begin{tabular}{|c|c|}
\hline \multicolumn{2}{|c|}{ Aplicaciones de flujos de calor } \\
\hline Flujo de calor constante & $\mathrm{j}(\mathrm{L}, \mathrm{t})=\mathrm{j}_{1}$ \\
\hline Flujo de calor triangular & $\begin{array}{l}\mathrm{j}\left(\mathrm{L}, 0<\mathrm{t}<\mathrm{t}_{1}\right)=\mathrm{j}_{2}+\left[\left(\mathrm{j}_{3}-\mathrm{j}_{2}\right) / \mathrm{t}_{1}\right] \mathrm{t} \\
\mathrm{j}\left(\mathrm{L}, \mathrm{t}_{1}<\mathrm{t}<2 \mathrm{t}_{1}\right)=\mathrm{j}_{3}-\left[\left(\mathrm{j}_{2}-\mathrm{j}_{3}\right) / \mathrm{t}_{1}\right]\left(\mathrm{t}-\mathrm{t}_{1}\right)\end{array}$ \\
\hline Flujo de calor sinusoidal & $\mathrm{j}(\mathrm{L}, \mathrm{t})=\mathrm{j}_{4}+\mathrm{j}_{5} \operatorname{sen}(\omega \mathrm{t})$ \\
\hline Flujo de calor escalón & $\begin{array}{l}\mathrm{j}\left(\mathrm{L}, 0<\mathrm{t}<\mathrm{t}_{1}\right)=\mathrm{j}_{6} \\
\mathrm{j}\left(\mathrm{L}, \mathrm{t}_{1}<\mathrm{t}\right)=\mathrm{j}_{7}\end{array}$ \\
\hline
\end{tabular}

Cuadro V.2. Tipos estimados de flujo de calor incidente 
Por sencillez, se han tomado valores numéricos sencillos para los diferentas parámetros (geometría, características térmicas, condiciones iniciales y parámetros que definen los flujos incidentes) empleados en los problemas. Estos, se muestran en el cuadro V.3.

$$
\begin{aligned}
& \mathrm{c}_{\mathrm{e}}=1 \mathrm{~J} / \mathrm{kg}{ }^{\circ} \mathrm{C}, \quad \rho=1 \mathrm{~kg} / \mathrm{m}^{3}, \quad \mathrm{k}=1 \mathrm{~W} / \mathrm{m}^{2}{ }^{\circ} \mathrm{C}, \quad \mathrm{T}_{\mathrm{o}}=100^{\circ} \mathrm{C}, \quad \mathrm{L}=1 \mathrm{~m} \\
& \mathrm{j}_{1}=30 \mathrm{~W} / \mathrm{m}^{2}, \quad \mathrm{j}_{2}=5 \mathrm{~W} / \mathrm{m}^{2}, \quad \mathrm{j}_{3}=30 \mathrm{~W} / \mathrm{m}^{2}, \quad \mathrm{j}_{4}=10 \mathrm{~W} / \mathrm{m}^{2}, \quad j_{5}=10 \mathrm{~W} / \mathrm{m}^{2} \\
& \mathrm{j}_{6}=5 \mathrm{~W} / \mathrm{m}^{2}, \quad \mathrm{j}_{7}=10 \mathrm{~W} / \mathrm{m}^{2}
\end{aligned}
$$

\section{Cuadro V.3. Valores numéricos}

Los modelos en red son los indicados y discutidos en el capítulo IV, figuras IV.5 y IV.8. En las figuras V.2a-d se muestran las series de medidas (temperaturas afectadas de error) correspondientes a errores del $\pm 0.5, \pm 1, \pm 2 \%$ o $\pm 5 \%$. El error cuadrático medio de las estimaciones, $s$ (ecuación V.1), que pueden obtenerse al disponer de las soluciones exactas, se mostrará junto con las estimaciones. Las desviaciones típicas de los errores asociados a las medidas de temperatura (conjunto de datos para el problema inverso) no se han incluido debido a que estos errores son estrictamente aleatorios y, en consecuencia, su distribución no es gaussiana. Esta circunstancia impone condiciones más severas al problema inverso que, sin embargo, se acercan más a la realidad cuando se trata de un pequeño conjunto de medidas. Más tarde se aportan soluciones (generalmente más rápidas y precisas) a problemas en los que las aludidas medidas se obtienen por medio de una distribución normal de errores aleatorios.

El programa fuente para la estimación de flujos y temperaturas, se adjunta en el Anexo de esta Memoria.

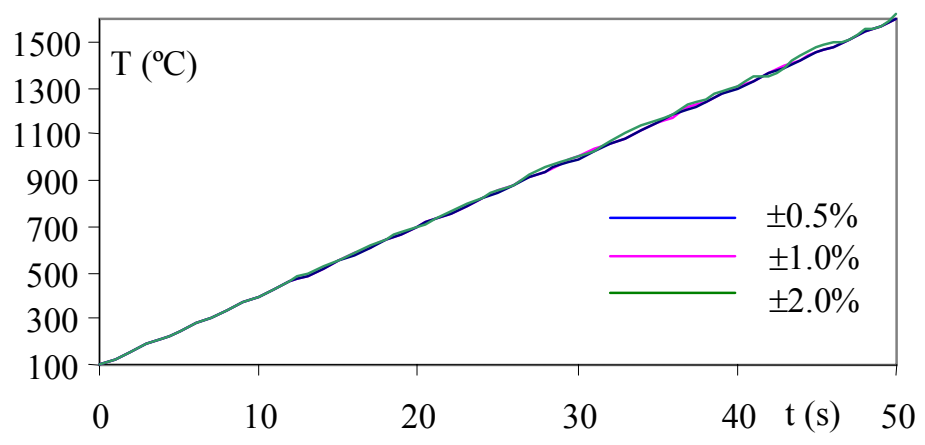

Figura V.2a. Solución del problema directo. Flujo de calor constante

$$
\mathrm{T}_{\mathrm{IHCP}}\left(\mathrm{x}_{\mathrm{f}}, \mathrm{t}_{\mathrm{j}}\right), \varepsilon= \pm 0.5, \pm 1 \% \mathrm{y} \pm 2 \%
$$




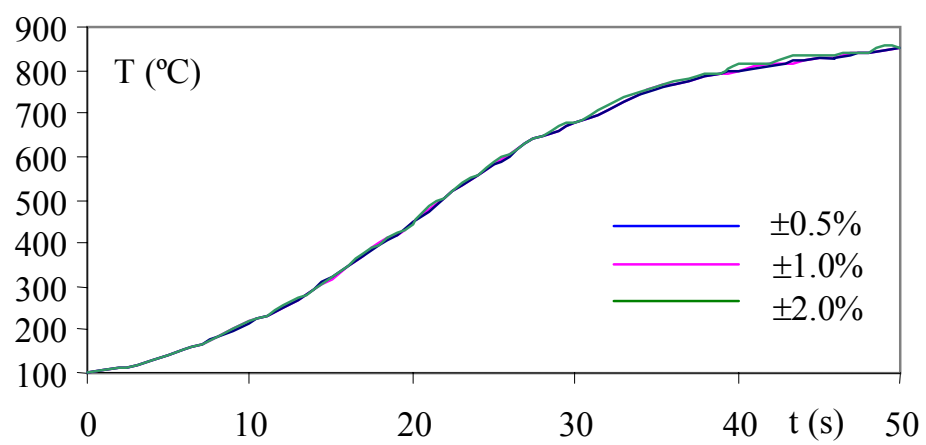

Figura V.2b. Solución del problema directo. Flujo de calor triangular $\mathrm{T}_{\mathrm{IHCP}}\left(\mathrm{x}_{\mathrm{f}}, \mathrm{t}_{\mathrm{j}}\right), \varepsilon= \pm 0.5, \pm 1 \% \mathrm{y} \pm 2 \%$

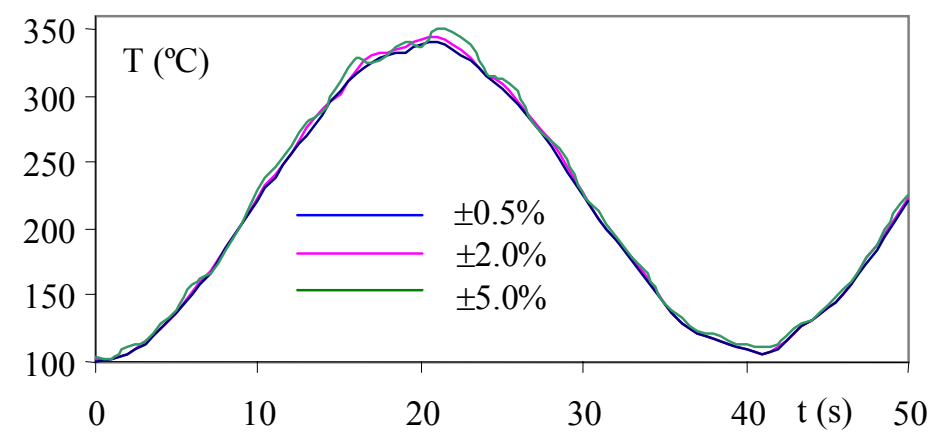

Figura V.2c. Solución del problema directo. Flujo de calor sinusoidal $\mathrm{T}_{\text {IHCP }}\left(\mathrm{x}_{\mathrm{f}}, \mathrm{t}_{\mathrm{j}}\right), \varepsilon= \pm 0.5, \pm 2 \% \mathrm{y} \pm 5 \%$

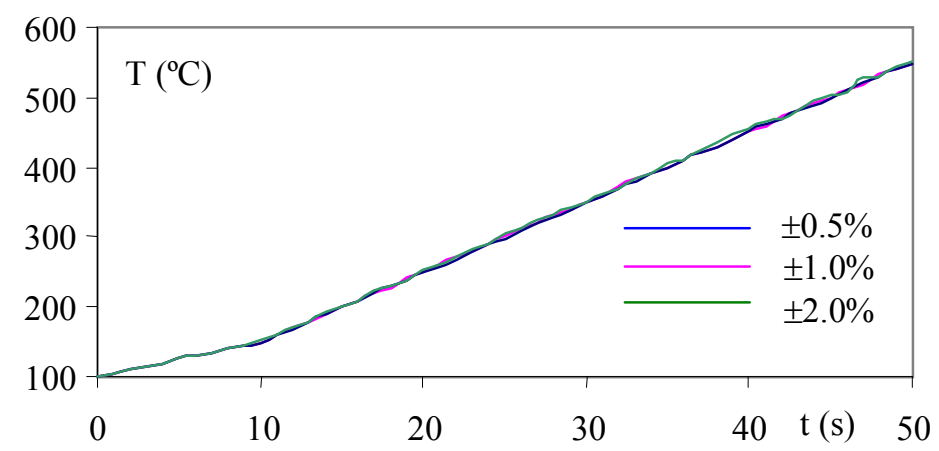

Figura V.2d. Solución del problema directo. Flujo de calor escalón $\mathrm{T}_{\mathrm{IHCP}}\left(\mathrm{x}_{\mathrm{f}}, \mathrm{t}_{\mathrm{j}}\right), \varepsilon= \pm 0.5, \pm 1 \% \mathrm{y} \pm 2 \%$ 


\section{Flujo de calor constante}

En la figura V.3 se muestran estimaciones (funciones lineales a tramos suavizadas) de un flujo de calor constante, $j(L, t)=j_{1}=30 \mathrm{Wm}^{-2}$, para un error en las medidas del $\pm 0.5 \%$, mientras que en la figura V.4 se muestra estas estimaciones para un error en las medidas del $\pm 1 \%$. En todos los casos el punto inicial se ha obtenido siguiendo el procedimiento explicado en el apartado III.3.3. En las estimaciones se han considerado distintos valores de r, (número de términos del funcional en cada intervalo de estimación) para analizar su influencia en los resultados.

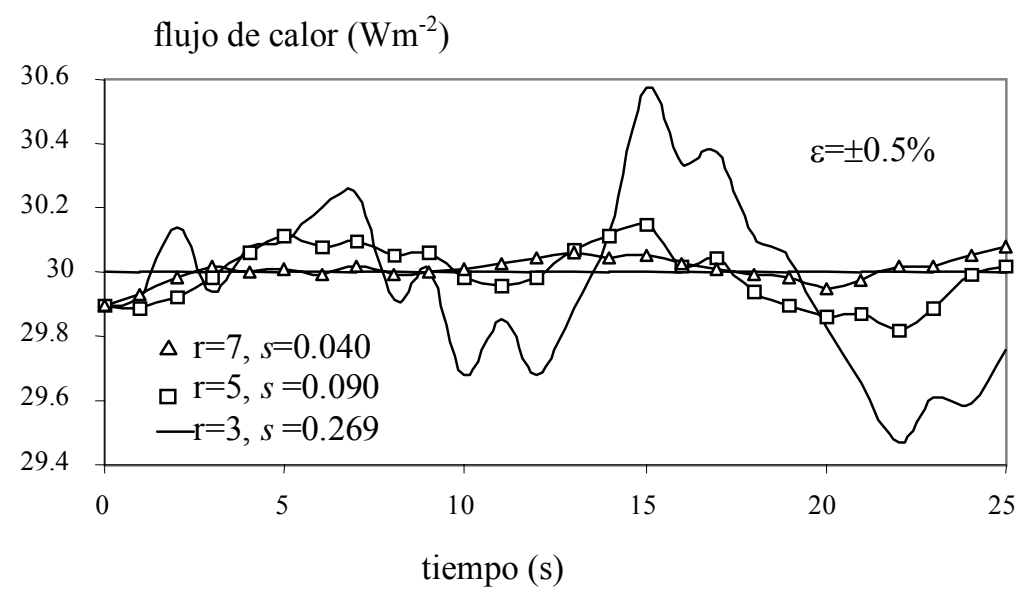

Figura V.3. IHCP solución para flujo de calor constante

$$
\varepsilon= \pm 0.5 \% ; \mathrm{r}=3,5 \text { y } 7 ; \mathrm{n}=25
$$

Para poder comparar los resultados obtenidos en las estimaciones se ha empleado la siguiente expresión, denominada error cuadrático medio $s$,

$$
s=\left[\sum_{i=0}^{Z}\left(\zeta_{\text {est }, \mathrm{i}}-\zeta_{\text {exa, } \mathrm{i}}\right)^{2} / Z\right]^{1 / 2}
$$

donde $\zeta_{\text {est,i }}$ es la función a estimar, $\zeta_{\text {exa,i }}$ la función buscada y $\mathrm{Z}$ el número total de tramos en la estimación.

De ambas figuras puede observarse que para el valor $\mathrm{r}=7$ (mayor número de términos del funcional) se obtiene la mejor aproximación. El valor del flujo incidente está confinado en el intervalo $\left[j(L, t)-0.1 \leq j_{\text {est }} \leq j(L, t)+0.1\right]$ para un error del $\pm 0.5 \%$, y en el intervalo $\left[j(L, t)-0.3 \leq j_{\text {est }}\right.$ $\leq \mathrm{j}(\mathrm{L}, \mathrm{t})+0.3]$ para un error del $\pm 1 \%$. En consecuencia puede deducirse que, en el caso de flujos 
constantes, con medidas de temperaturas afectadas de error, al aumentar el número de términos del funcional los resultados de la estimación son más precisos.

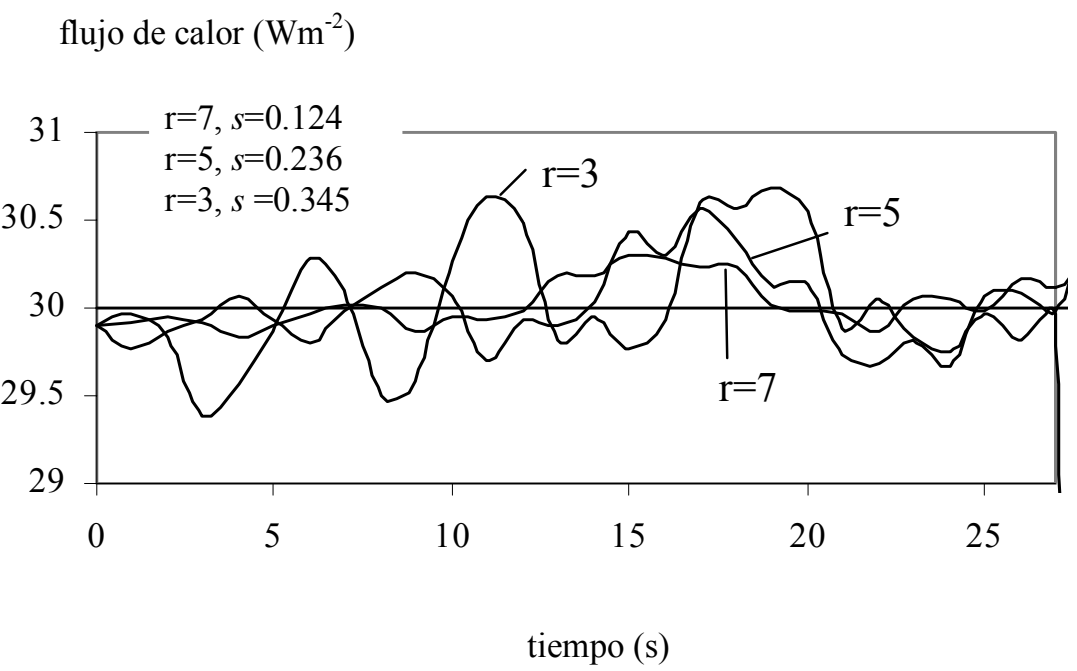

Figura V.4. IHCP solución para flujo de calor constante

$$
\varepsilon= \pm 1 \% ; \mathrm{r}=3,5 \text { y } 7 ; \mathrm{n}=25
$$

La figura V.5 muestra las estimaciones obtenidas para un mismo número de términos del funcional, $\mathrm{r}=5$, utilizando como parámetro el error en las medidas $( \pm 0.5,1$ y $2 \%)$. Al aumentar este error las estimaciones se alejan de las soluciones exactas.

La figura V.6 muestra los resultados de la simulación cuando las medidas no están afectadas de error. Para $\mathrm{r}=3$ se reproduce prácticamente el valor exacto antes de 8 segundos. De la observación de las figuras V.3 a V.6 se deduce, en concordancia con lo afirmado por Beck [1985], que para errores crecientes en las medidas la estimación mejora al aumentar el número de términos del funcional (y viceversa).

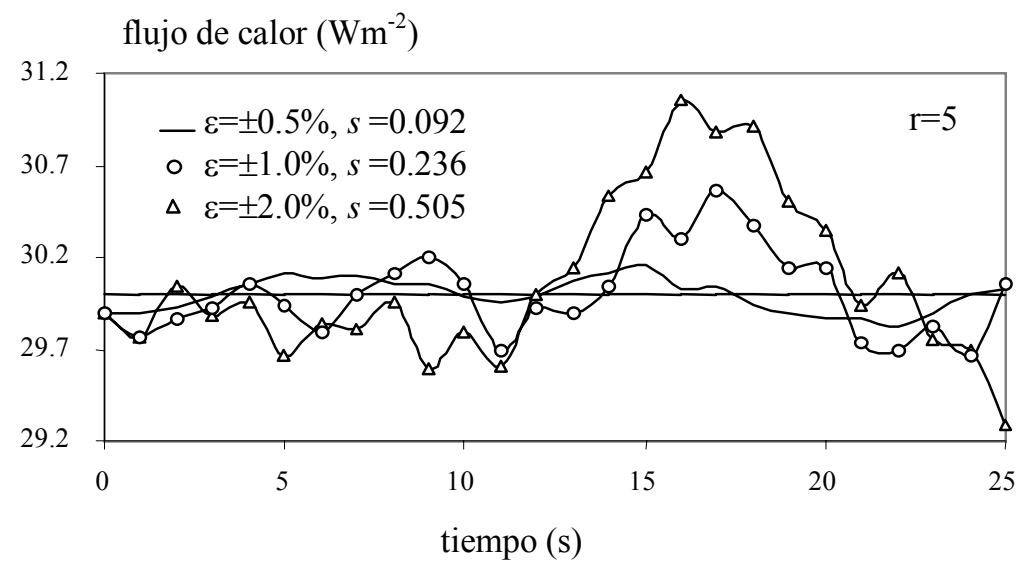

Figura V.5. IHCP solución para flujo de calor constante

$$
\varepsilon= \pm 0.5,1 \text { у } 2 \% ; \mathrm{r}=5 ; \mathrm{n}=25
$$


flujo de calor $\left(\mathrm{Wm}^{-2}\right)$

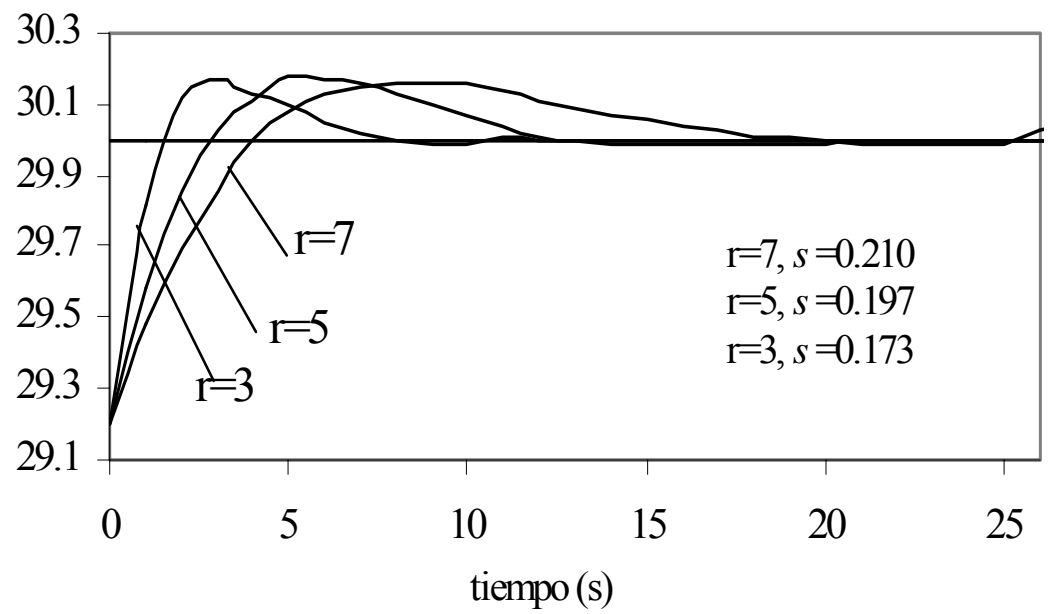

Figura V.6. IHCP solución para flujo de calor constante

Sin error, $\mathrm{r}=3,5$ y $7 ; \mathrm{n}=25$

flujo de calor $\left(\mathrm{Wm}^{-2}\right)$

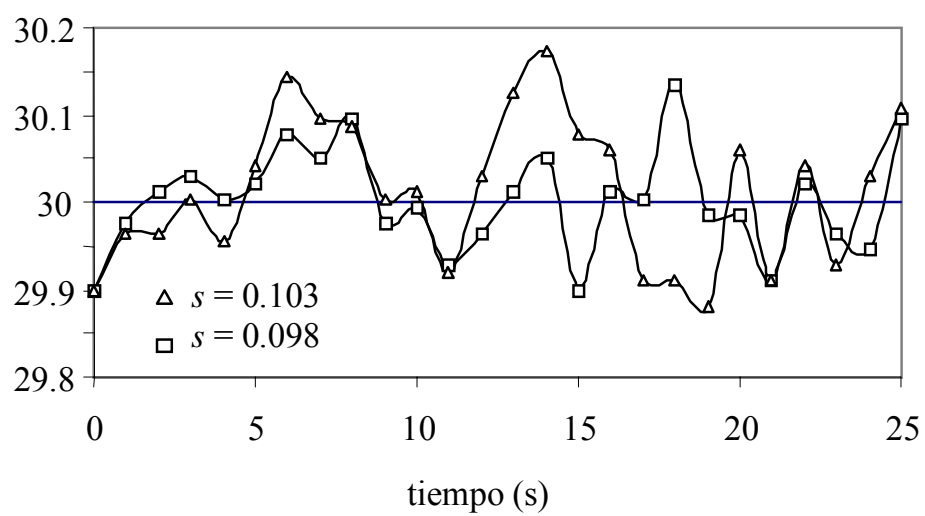

Figura V.7. Estimación del flujo de calor constante para dos series de medidas

$$
\text { Error }= \pm 0.5 \% ; r=5 ; n=25
$$

flujo de calor $\left(\mathrm{Wm}^{-2}\right)$

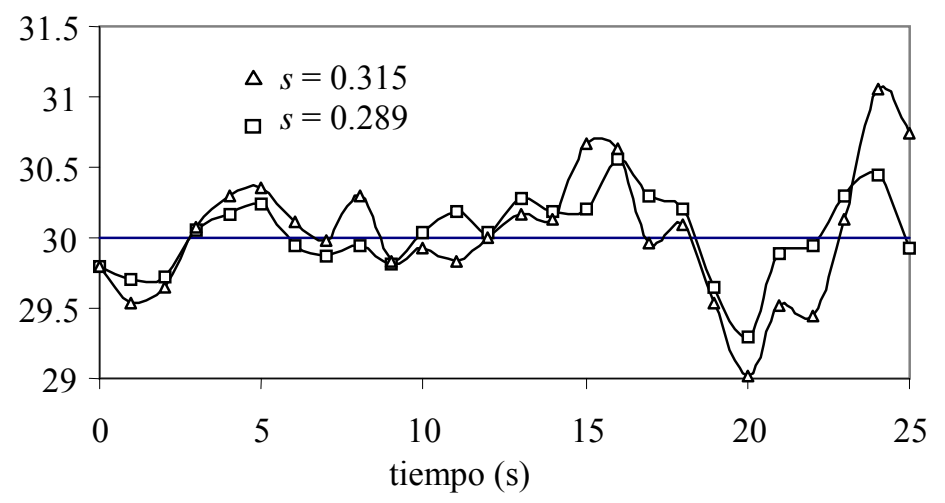

Figura V.8. Estimación del flujo de calor constante para dos series de medidas

$$
\text { Error }= \pm 1 \% ; r=5 ; n=25
$$


Para apreciar los efectos de la aleatoriedad en las medidas dentro de un mismo error con $\mathrm{r}=5$, las figuras V.7-9 muestran los resultados de la estimación para dos series (distintas) de medidas "experimentales" correspondientes a un error de $0.5,1$ y $2 \%$. Resultados similares para errores del 1 y $2 \%$ se muestran en las figuras V.8a-c y V.9a-c.

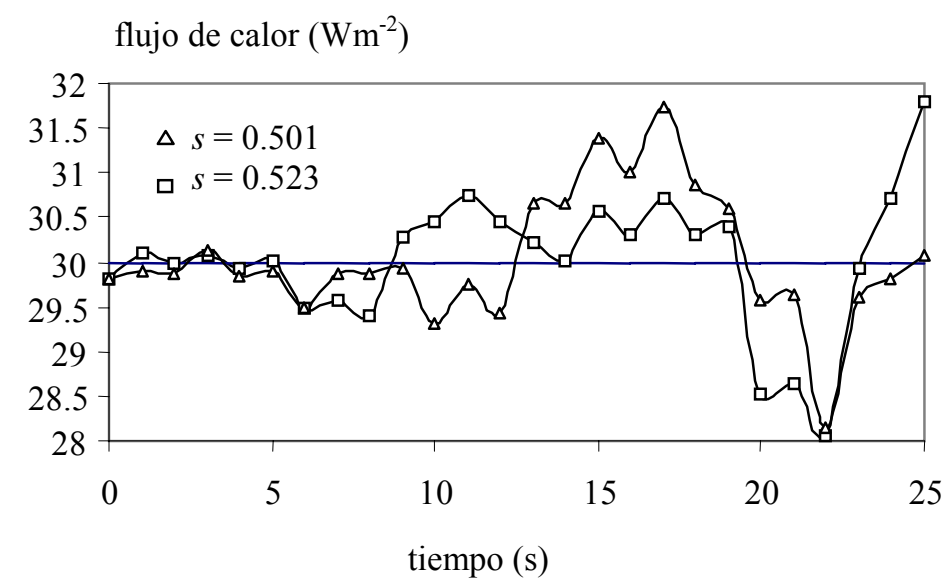

Figura V.9. Estimación del flujo de calor constante para dos series de medidas

$$
\text { Error }= \pm 2 \% ; r=5 ; n=25
$$

La influencia del punto inicial en la estimación se muestra en la figura V.10 para un error en las medidas del $1 \%$ y r=5 (este punto se ha seleccionado directamente sin cálculo alguno). Se observa que cuanto más cercano se tome $\mathrm{j}_{\text {ini }}$ respecto del valor exacto $\left(30 \mathrm{Wm}^{-2}\right)$, la estimación mejora. Se aportan los valores de desviación típica de las tres estimaciones.

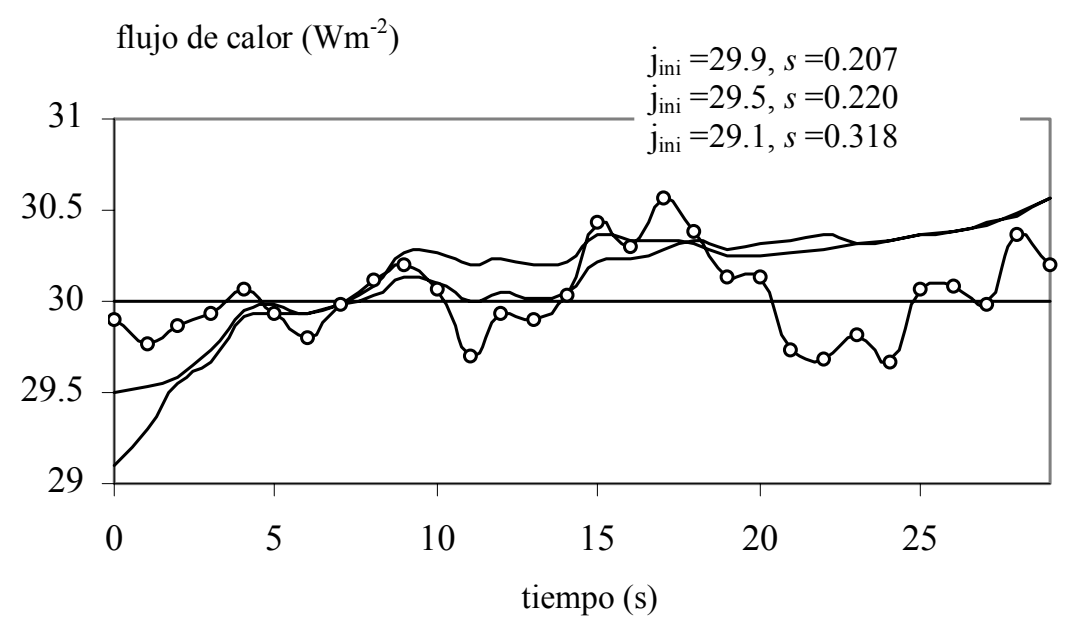

Figura V.10. Estimaciones para diferentes valores iniciales

$$
\text { Error }= \pm 1 \% ; r=5 ; n=25
$$


La tabla V.1 recoge los errores cuadráticos medios $(s)$ de las estimaciones en función del número de términos del funcional $(\mathrm{r}) \mathrm{y}$ del error en las medidas $(\varepsilon)$, para diferentes casos. Puede observarse que el error en la estimación aumenta, para un mismo r, al aumentar $\varepsilon$, y que, para un mismo valor de $\varepsilon$, el error en la estimación disminuye al aumentar r. También la variación relativa de $s$ para distintas series de medidas del mismo valor de $\varepsilon$ y $r$ es inferior al $10 \%$ de promedio, en los casos ensayados.

\begin{tabular}{|l|l|l|l|}
\hline & \multicolumn{3}{|c|}{ Valores de $s$} \\
\hline $\boldsymbol{\varepsilon ( \pm \% )}$ & $\mathrm{r}=3$ & $\mathrm{r}=5$ & $\mathrm{r}=7$ \\
\hline 0 & 0.173 & 0.197 & 0.210 \\
& 0.169 & 0.191 & 0.223 \\
\hline 0.5 & 0.240 & 0.103 & 0.042 \\
& 0.234 & 0.098 & 0.037 \\
\hline 1 & 0.412 & 0.315 & 0.096 \\
& 0.396 & 0.289 & 0.113 \\
\hline 2 & 0.726 & 0.501 & 0.298 \\
& 0.789 & 0.523 & 0.332 \\
\hline
\end{tabular}

Tabla V.1. Valores del error cuadrático medio $s$

En cualquier caso, la variación relativa de $s$ debido a la serie de medidas es mucho menor que la debida al cambio de r o del error.

\section{Flujo de calor triangular}

La figura V.11 muestra la estimación del flujo incidente de calor para un error en las medidas del $\pm 2 \%, r=3,5$ y 7, y $n=40$. De los resultados se desprende que la mejor estimación se obtiene para $\mathrm{r}=5$. ¿Cómo interpretar este resultado en aparente contradicción con el obtenido para flujo de calor constante? La explicación reside en la propia forma de la onda de flujo de calor. Cuando el flujo de calor no es constante, la elección de un número alto de términos para el funcional, siempre asociada a una estimación más exacta, induce más error en la estimación ya que los últimos valores comparados dentro del funcional son sensiblemente diferentes en las cercanías de las zonas de cambio de flujo incidente. Es decir hay una tendencia distinta entre los valores de medida experimentales (que sí tienen en cuenta el cambio en el flujo incidente) y los que resultan de la solución del problema inverso (que no pueden tener en cuenta dicha tendencia por la propia técnica de solución del problema inverso). 


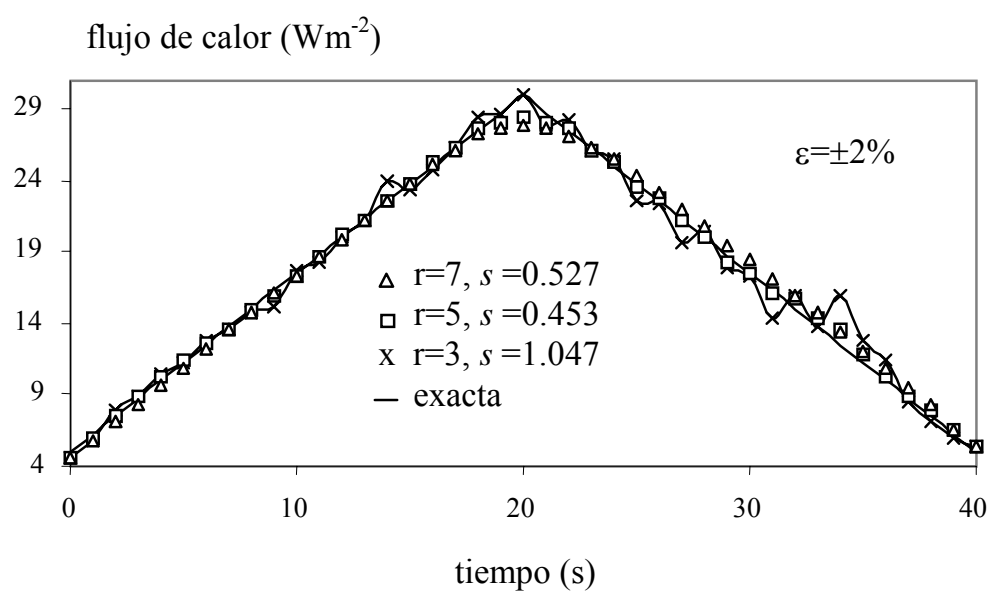

Figura V.11. IHCP solución para un flujo de calor triangular

$$
\text { Error }= \pm 2 \% ; r=3,5 \text { y } 7 ; n=40
$$

En la figura V.12 se presenta la estimación del flujo de calor triangular para $\mathrm{r}=5 \mathrm{y}$ valores de errores en las medidas de $\pm 0.2,2$ y $5 \%$, con $n=40$. Los resultados de la estimación son bastante precisos como en el caso de flujo de calor constante; el error en la estimación aumenta al aumentar el error en las medidas de temperatura, así para un error del $\pm 5 \%$ se obtiene $s=0.768$, mientras que para un error del $\pm 0.5 \%, s=0.401$.

\section{flujo de calor $\left(\mathrm{Wm}^{-2}\right)$}

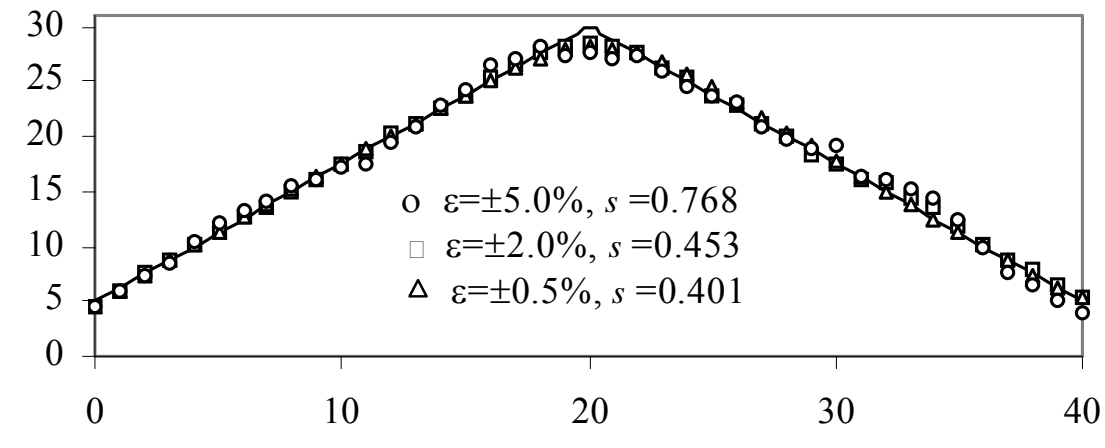

tiempo (s)

Figura V.12. IHCP solución para un flujo de calor triangular

$$
\text { Errores }=0.5,2 \text { y } 5 \% ; \mathrm{r}=5 ; \mathrm{n}=40
$$

Las figuras V.13 y V.14 muestra la estimación del flujo de calor triangular para unas medidas sin error, con $n=40, r=5$ (figura V.13) y $r=3$ (figura V.14). La ausencia de error en las medidas proporciona estimaciones muy precisas, tanto más cuanto menor se $\mathrm{r}$. 
flujo de calor $\left(\mathrm{Wm}^{-2}\right)$

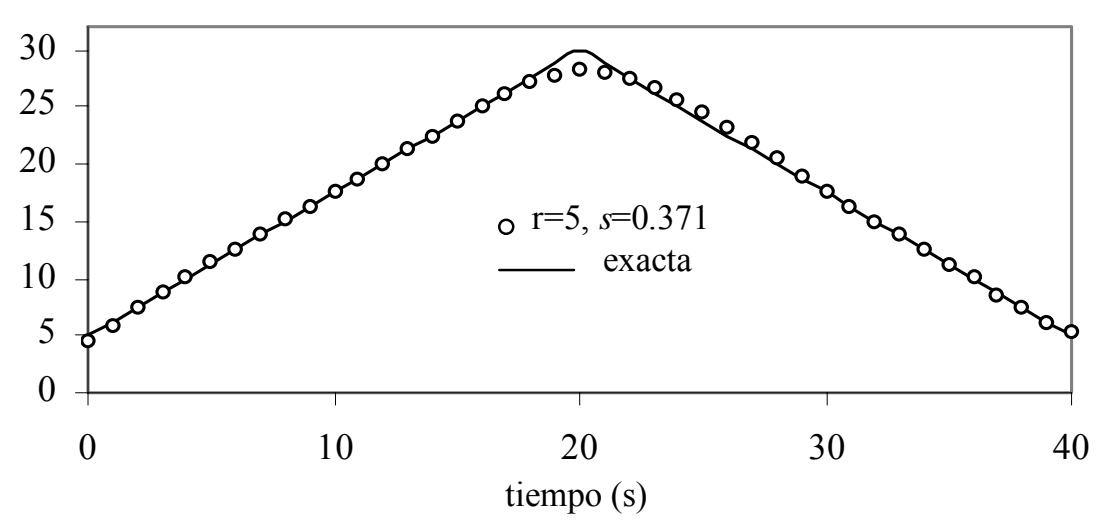

Figura V.13. IHCP solución para un flujo de calor triangular

Sin error, $r=5 ; n=40$

flujo de calor $\left(\mathrm{Wm}^{-2}\right)$

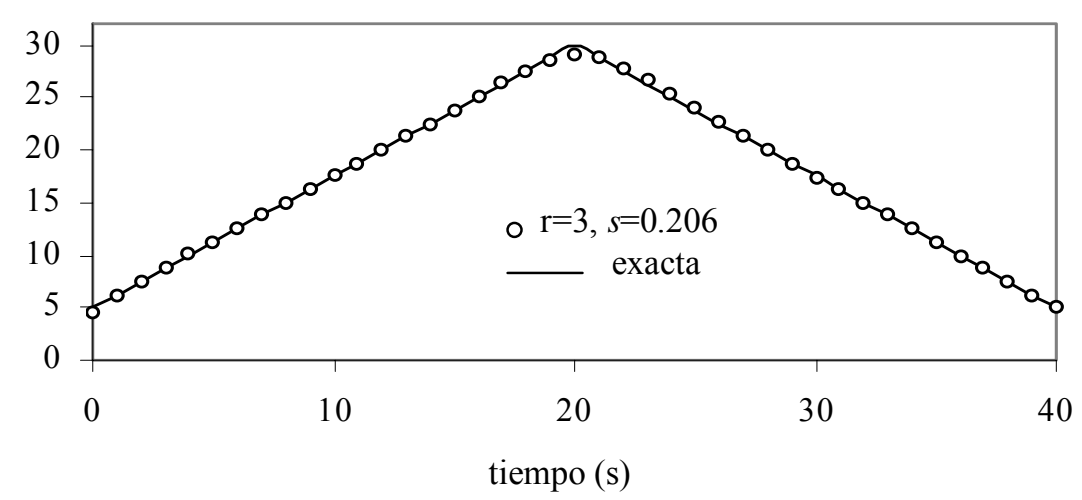

Figura V.14. IHCP solución para un flujo de calor triangular

Sin error; $r=3 ; n=40$

Como en el caso de flujo incidente constante, a continuación se introducen gráficas (figuras V.15 a V.17) que demuestran que series de medidas con el mismo error aleatorio dan estimaciones muy cercanas entre sí. En la tabla V.2 se recogen los valores obtenidos para $s$ en función de los de $\mathrm{r}$ y $\varepsilon$ anteriores.

Como conclusión puede significarse que, para un mismo $\mathrm{r}$, el error en la estimación aumenta al crecer $\varepsilon$. En cuanto al error en la estimación al mantener $\varepsilon$, existe un valor óptimo de $\mathrm{r}(\mathrm{r}=5$ para los valores numéricos de nuestro problema) que da la mejor estimación; ésta es una diferencia sustancial con el caso de flujo constante. 
flujo de calor $\left(\mathrm{Wm}^{-2}\right)$

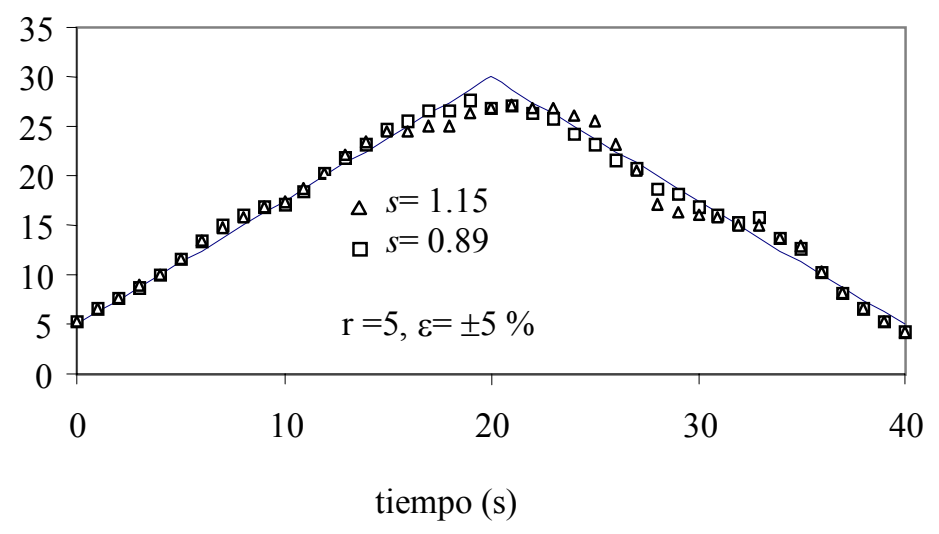

Figura V.15. IHCP solución para un flujo de calor triangular

$$
\text { Error }= \pm 5 \% ; r=5 ; n=40
$$

flujo de calor $\left(\mathrm{Wm}^{-2}\right)$

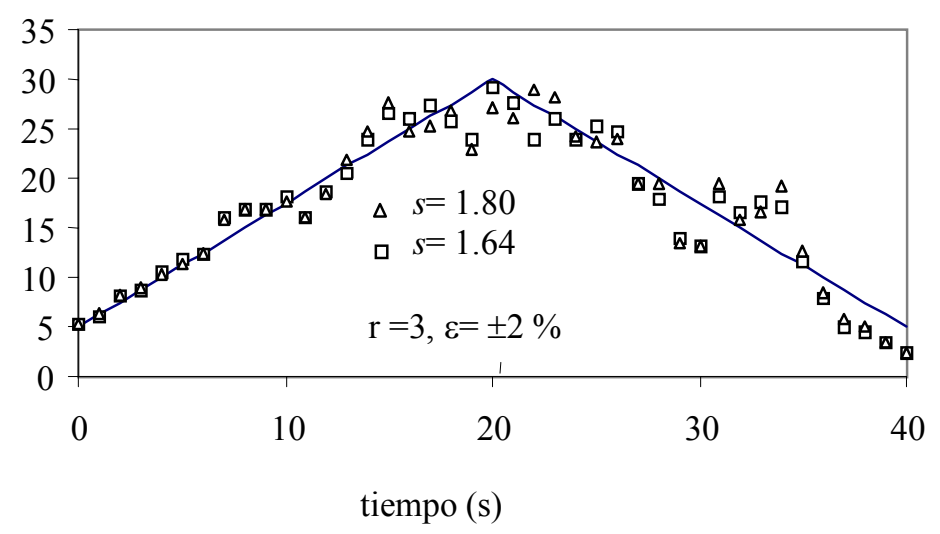

Figura V.16. IHCP solución para un flujo de calor triangular

$$
\text { Error }= \pm 2 \% ; r=3 ; n=40
$$

flujo de calor $\left(\mathrm{Wm}^{-2}\right)$

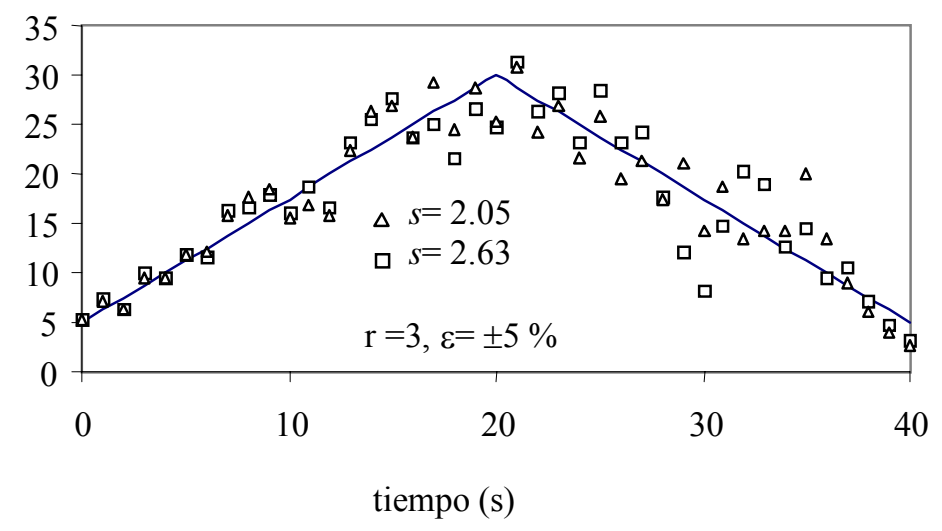

Figura V.17. IHCP solución para un flujo de calor triangular

$$
\text { Error }= \pm 5 \% ; r=3 ; n=40
$$




\begin{tabular}{|l|l|l|l|}
\hline & \multicolumn{3}{|c|}{ Valores de $s$} \\
\hline $\boldsymbol{\varepsilon ( \pm \% )}$ & $\mathrm{r}=3$ & $\mathrm{r}=5$ & $\mathrm{r}=7$ \\
\hline 0 & 0.20 & 0.37 & 0.49 \\
& 0.21 & 0.40 & 0.51 \\
\hline 2 & 1.80 & 0.45 & 0.52 \\
& 1.64 & 0.48 & 0.55 \\
\hline 5 & 2.05 & 1.15 & 1.66 \\
& 2.63 & 0.89 & 2.07 \\
\hline
\end{tabular}

Tabla V.2. Valores del error en la estimación $s$

\section{Flujo de calor sinusoidal}

Los valores de los parámetros que definen el flujo son $\omega=2 \pi(0.025) \mathrm{rad} / \mathrm{s}_{\mathrm{j}} \mathrm{j}_{1}=\mathrm{j}_{2}=$ $10 \mathrm{Wm}^{-2}$. La figura V.18 muestra la estimación para un error aleatorio en la medida (sin especificación previa de su desviación típica) del $\pm 2 \%$ y para medidas sin error, con $r=3$ y $n=40$. Puede observarse que la estimación para medidas sin error reproduce fielmente el flujo incidente. La figura V.19 presenta los resultados de la estimación para un error del $\pm 5 \%, \mathrm{r}=3 \mathrm{y}$ 5 , y $n=40$. La estimación más precisa, consecuencia siempre de un compromiso entre la forma de onda del flujo incidente y el número de términos del funcional, se obtiene para $\mathrm{r}=5$.

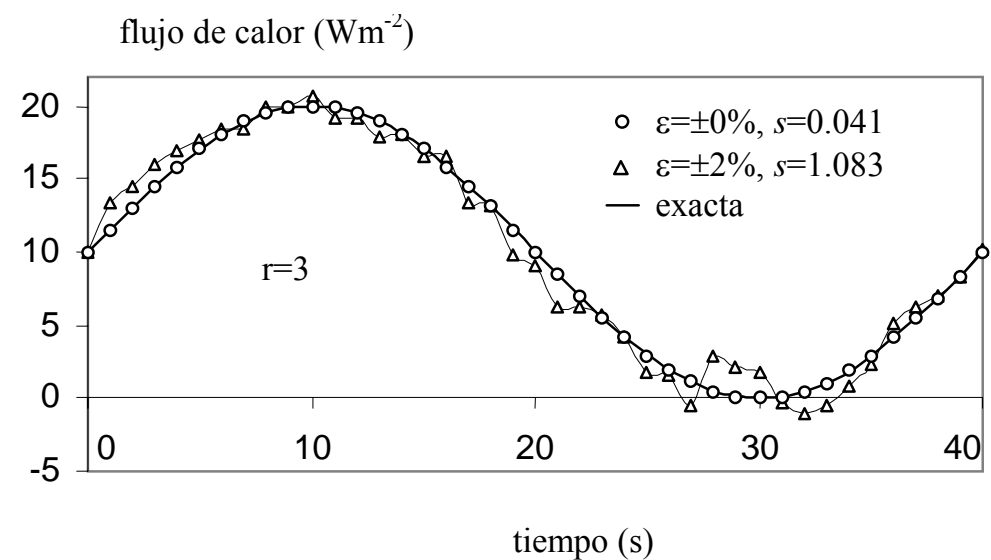

Figura V.18. IHCP solución para un flujo de calor incidente sinusoidal Error $=0$ y $\pm 2 \% ; r=3 ; n=40$ 


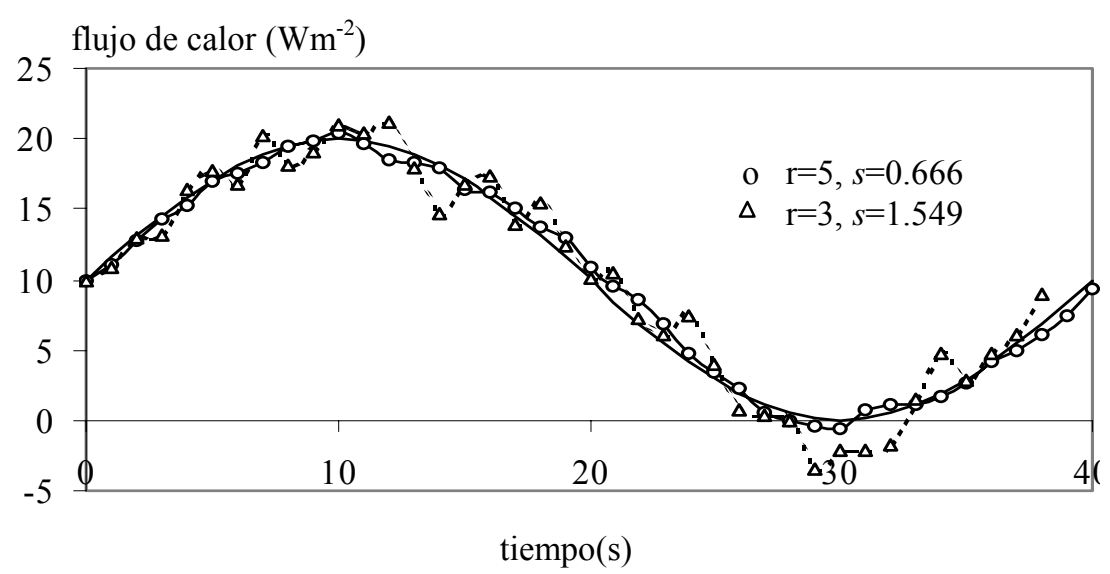

Figura V.19. IHCP solución para un flujo de calor incidente sinusoidal

$$
\text { Error }= \pm 5 \% ; r=3 \text { y } 5 ; n=40
$$

En la figura V.20 se muestran las estimaciones sin error en las medidas, para $\mathrm{r}=3,5$ y 7 y $n=40$. Puede apreciarse que la sinusoide se reproduce casi fielmente para $r=3$. En general puede establecerse que, como en el caso de flujo constante, cuando aumenta el error en las medidas es necesario incrementar el número de términos del funcional para una mejor estimación, mientras que cuando el error en las medidas es nulo o despreciable el valor óptimo de $\mathrm{r}$ disminuye.

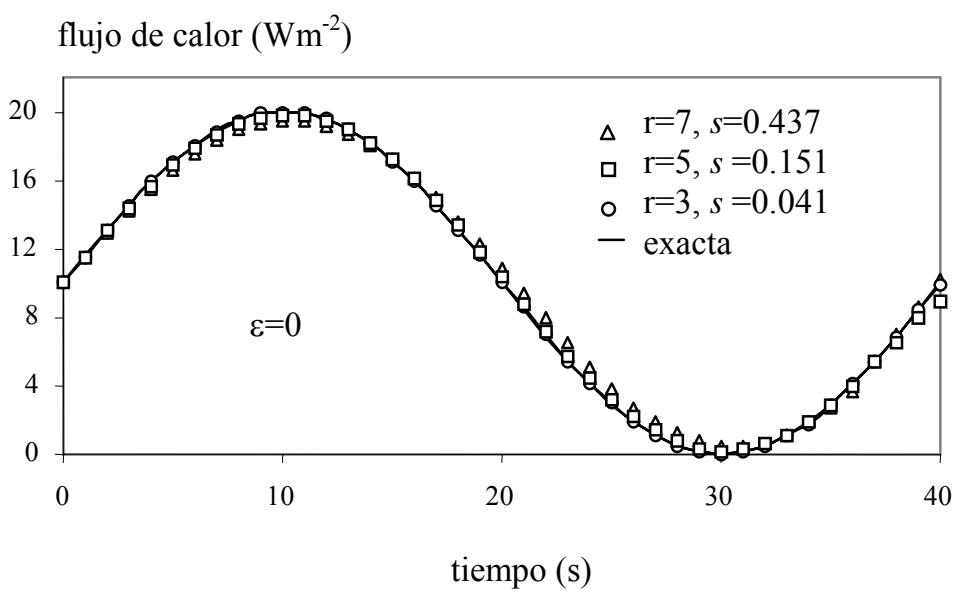

Figura V.20. IHCP solución para un flujo de calor incidente sinusoidal

$$
\text { Error }= \pm 0.1 \%, r=3,5 \text { y } 7 ; n=40
$$

Las gráficas de las figuras siguientes, V.21 a V.23, inciden sobre la influencia en la estimación de diferentes series de medidas con un mismo error. Por último, la tabla V.3 recoge los valores de $s$ de las distintas estimaciones dentro de este apartado. 
flujo de calor $\left(\mathrm{Wm}^{-2}\right)$

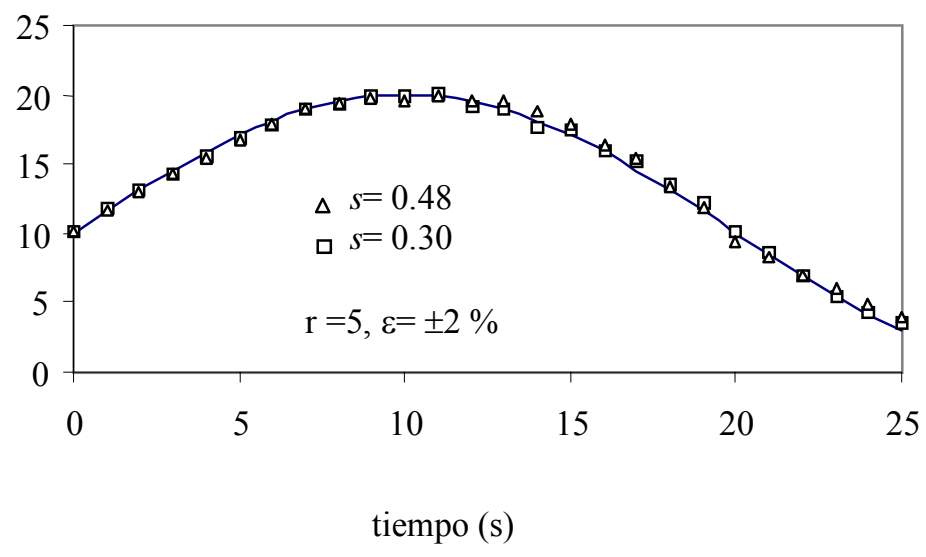

Figura V.21. IHCP solución para un flujo de calor incidente sinusoidal Error $= \pm 2 \% ; r=5 ; n=25$

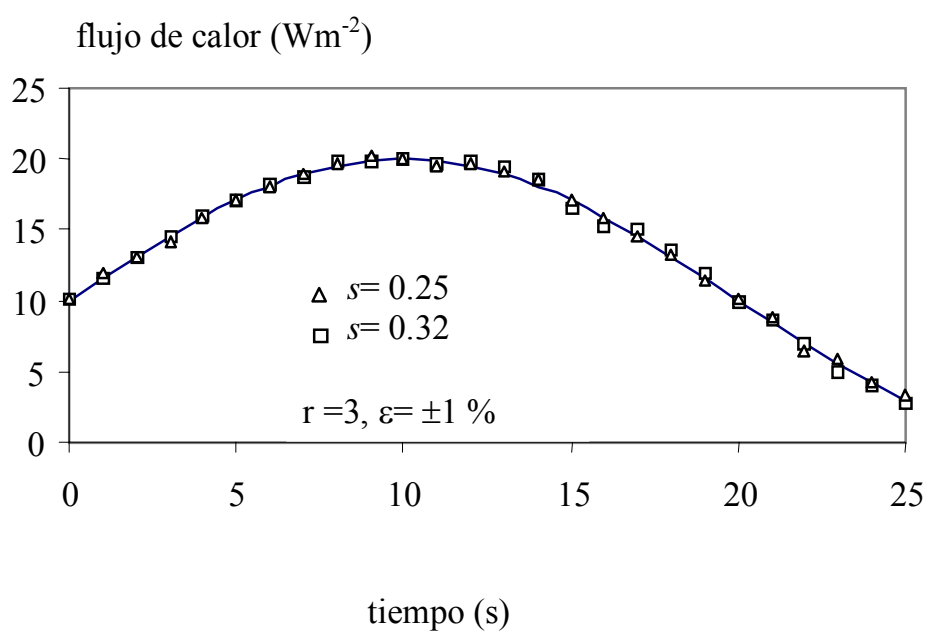

Figura V.22. IHCP solución para un flujo de calor incidente sinusoidal Error $= \pm 1 \% ; r=3 ; n=25$ flujo de calor $\left(\mathrm{Wm}^{-2}\right)$

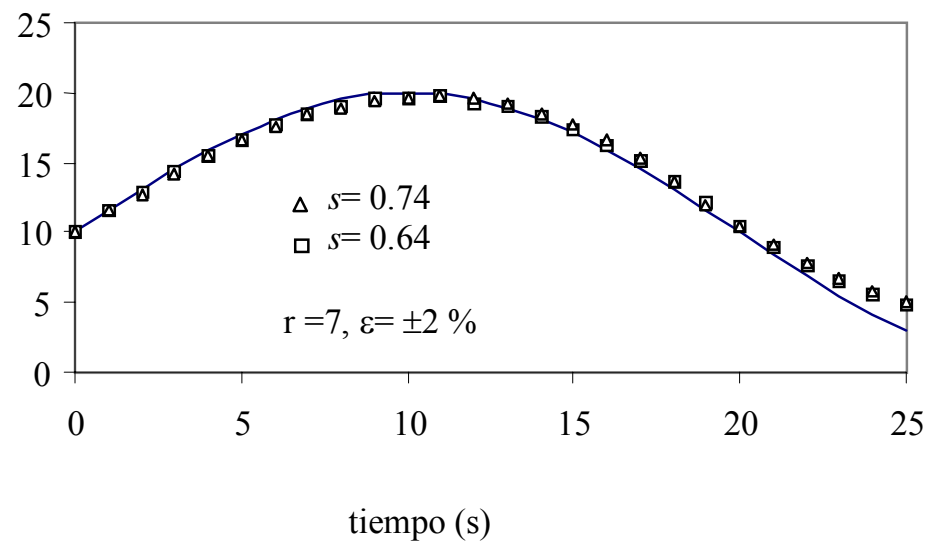

Figura V.23. IHCP solución para un tlujo de calor incidente sinusoidal Error $= \pm 2 \% ; r=7 ; n=25$ 


\begin{tabular}{|l|l|l|l|}
\hline & \multicolumn{3}{|c|}{ Valores de $s$} \\
\hline $\boldsymbol{\varepsilon ( \pm \% )}$ & $\mathrm{r}=3$ & $\mathrm{r}=5$ & $\mathrm{r}=7$ \\
\hline 0 & 0.04 & 0.15 & 0.43 \\
& 0.05 & 0.12 & 0.39 \\
\hline 1 & 0.25 & 0.22 & 0.45 \\
& 0.32 & 0.21 & 0.47 \\
\hline 2 & 1.08 & 0.48 & 0.74 \\
& 0.97 & 0.30 & 0.64 \\
\hline 5 & 1.54 & 0.66 & 1.18 \\
& 1.71 & 0.74 & 1.24 \\
\hline
\end{tabular}

Tabla V.3. Valores del error en la estimación $s$

Como conclusión podemos decir que para un mismo valor de r, la estimación mejora al disminuir el error. También, como en el caso de flujo triangular, para un mismo valor de $\varepsilon$, se obtiene una estimación óptima para un valor particular de r (5 en nuestro problema).

\section{Flujo de calor escalón}

Los valores de los parámetros que definen este flujo son $\mathrm{j}_{6}=5 \mathrm{Wm}^{-2}$ y $\mathrm{j}_{7}=10 \mathrm{Wm}^{-2}$. La figura V.24 muestra la estimación del flujo para $r=3,5,7$ y $n=100$ (intervalo de tiempo entre medidas de $0.2 \mathrm{~s}$ ). Debido a la no linealidad inherente a la enorme pendiente del flujo incidente, las estimaciones resultan menos aproximadas a la solución exacta que en los casos anteriores; de ahí que se haya trabajado con un error nulo, $\varepsilon=0$. Los resultados de la estimación son más precisos al disminuir $\mathrm{r}$.

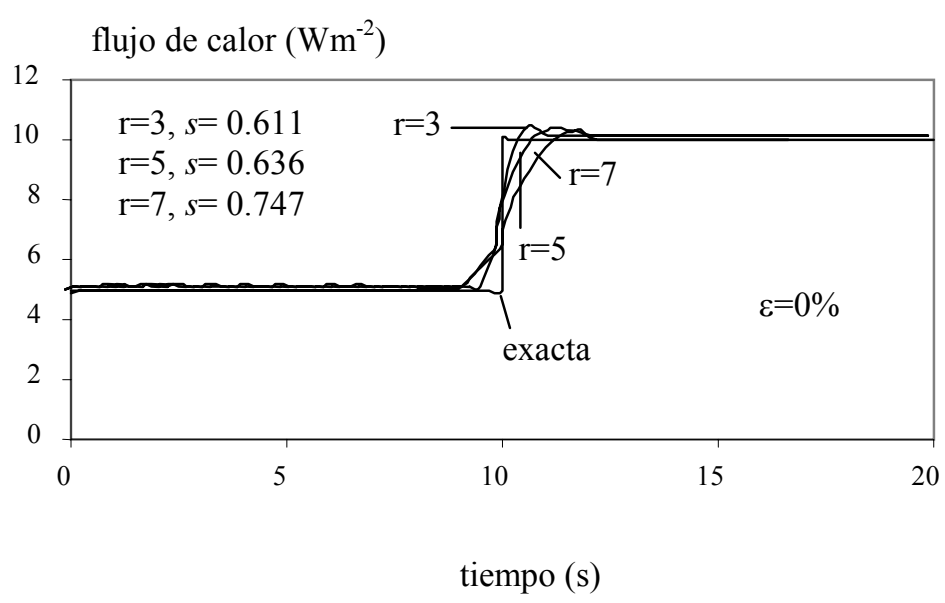

Figura V.24. IHCP solución para un flujo de calor escalón Sin error; $r=3,5$ y $7 ; n=100$ 


\section{V.1.3. Estimación de diferentes flujos de calor con características térmicas dependientes de la temperatura}

Estos problemas tienen una no linealidad añadida asociada con estas dependencias. Su planteamiento es similar: placa 1-D, con condición adiabática en una de sus caras y de flujo incidente, objeto de la estimación, en la otra. Las características térmicas de la placa en función de la temperatura pueden definirse mediante funciones continuas arbitrarias, especificadas mediante expresiones matemáticas, o aproximarse por funciones continuas lineales a tramos (piece-wise functions), obtenidas a partir de datos experimentales tabulados (http:Imetalcasting.auburn.edu).

El modelo matemático para el primer caso se muestra en el cuadro V.4, donde $f_{c}$ y $f_{k}$ representan las mencionadas expresiones matemáticas. Como aplicación, se ha adoptado funciones de tipo polinómico. Se estudian cuatro problemas asociados con las cuatro formas de onda para el flujo incidente mostradas en el cuadro V.5, Alhama y col. [2001].

Los valores numéricos de los parámetros que definen estos flujos se muestran en el cuadro V.6. Para el rango completo de temperaturas a que queda sometida la placa los valores extremos de $\mathrm{k}$ y c $\mathrm{c}_{\mathrm{e}}$ son: $\mathrm{k}_{\min }=3 \mathrm{Wm}^{-1}{ }^{\mathrm{o}} \mathrm{C}^{-1}, \mathrm{c}_{\mathrm{e}, \min }=3 \mathrm{~kJ} \mathrm{~kg}^{-1} \mathrm{C}^{-1}, \mathrm{k}_{\max }=1641 \mathrm{Wm}^{-1}{ }^{\mathrm{o}} \mathrm{C}^{-1} \mathrm{y} \mathrm{c}_{\mathrm{e}, \max }=$ $1641 \mathrm{~kJ} \mathrm{~kg}^{-1}{ }^{\circ} \mathrm{C}^{-1}$; este amplio intervalo de valores para ambas características puede no ser real pero asegura una fuerte no linealidad en todos los casos.

Los modelos en red son los indicados y discutidos en el capítulo IV, figura IV.12.

\begin{tabular}{|c|c||}
\hline \multicolumn{2}{|c|}{ Modelo matemático } \\
\hline Ec. diferencial & $\begin{array}{c}\rho \mathrm{c}_{\mathrm{e}}(\mathrm{T}) \partial \mathrm{T} / \partial \mathrm{t}-\mathrm{k}(\mathrm{T})\left(\partial^{2} \mathrm{~T} / \partial \mathrm{x}^{2}\right)=0 \\
\mathrm{c}_{\mathrm{e}}(\mathrm{T})=\mathrm{f}_{\mathrm{c}}(\mathrm{T})=\mathrm{c}_{0}+\mathrm{c}_{1} \mathrm{~T}+\mathrm{c}_{2} \mathrm{~T}^{2} \\
\mathrm{k}(\mathrm{T})=\mathrm{f}_{\mathrm{k}}(\mathrm{T})=\mathrm{k}_{0}+\mathrm{k}_{1} \mathrm{~T}+\mathrm{k}_{2} \mathrm{~T}^{2}\end{array}$ \\
\hline $\begin{array}{c}\text { Condiciones } \\
\text { iniciales }\end{array}$ & $\mathrm{T}(\mathrm{x},=0)=\mathrm{T}_{0}$ \\
\hline $\begin{array}{c}\text { Condiciones de } \\
\text { contorno }\end{array}$ & $\mathrm{j}(\mathrm{L}, \mathrm{t})=\mathrm{j}_{\mathrm{in}}(\mathrm{t})$ \\
\hline $\begin{array}{c}\text { Condición } \\
\text { adicional }\end{array}$ & $\mathrm{j}(0, \mathrm{t})=0($ punto de medida $)$ \\
\hline
\end{tabular}

Cuadro V.4. Modelo matemático del problema 


\begin{tabular}{|l|l|}
\hline \multicolumn{2}{|c|}{ Aplicaciones de flujos de calor } \\
\hline Flujo de calor constante & $j(\mathrm{~L}, \mathrm{t})=\mathrm{j}_{1}$ \\
\hline Flujo de calor triangular & $\begin{array}{l}\mathrm{j}\left(\mathrm{L}, 0<\mathrm{t}<\mathrm{t}_{1}\right)=\mathrm{j}_{2}+\left[\left(\mathrm{j}_{3}-\mathrm{j}_{2}\right) /\left(\mathrm{t}_{1}\right)\right] \mathrm{t} \\
\mathrm{j}\left(\mathrm{L}, \mathrm{t}_{1}<\mathrm{t}<2 \mathrm{t}_{1}\right)=\mathrm{j}_{3}-\left[\left(\mathrm{j}_{2}-\mathrm{j}_{3}\right) / \mathrm{t}_{1}\right]\left(\mathrm{t}-\mathrm{t}_{1}\right)\end{array}$ \\
\hline Flujo de calor sinusoidal & $\mathrm{j}(\mathrm{L}, \mathrm{t})=\mathrm{j}_{4}+\mathrm{j}_{5} \operatorname{sen}(\omega \mathrm{t})$ \\
\hline Flujo de calor rectangular & $\begin{array}{l}\mathrm{j}\left(\mathrm{L}, 0<\mathrm{t}<\mathrm{t}_{2}\right)=\mathrm{j}_{6}, \mathrm{j}\left(\mathrm{L}, \mathrm{t}_{2}<\mathrm{t}<\mathrm{t}_{3}\right)=\mathrm{j}_{7}, \\
\mathrm{j}\left(\mathrm{L}, \mathrm{t}_{3}<\mathrm{t}<\mathrm{t}_{4}\right)=\mathrm{j}_{8}\end{array}$ \\
\hline
\end{tabular}

Cuadro V.5. Aplicaciones de flujos de calor. Propiedades térmicas variables

$$
\begin{aligned}
& \mathrm{L}=1 \mathrm{~m}, \rho=1 \mathrm{~kg} \mathrm{~m}^{-3}, \\
& \mathrm{c}_{0}=1 \mathrm{~kJ} \mathrm{~kg}^{-1} \mathrm{C}^{-1}, \mathrm{c}_{1}=1 \mathrm{~kJ} \mathrm{~kg}^{-1}{ }^{\circ} \mathrm{C}^{-2}, \mathrm{c}_{2}=1 \mathrm{~kJ} \mathrm{~kg}^{-1} \mathrm{o}^{-3}, \\
& \mathrm{k}_{0}=1 \mathrm{Wm}^{-1} \mathrm{o}^{-1}, \mathrm{k}_{1}=1 \mathrm{Wm}^{-1} \mathrm{o}^{-2}, \mathrm{k}_{2}=1 \mathrm{Wm}^{-1} \mathrm{o}^{-3}, \\
& \mathrm{~T}_{0}=1^{\circ} \mathrm{C}, \mathrm{n}=40, \Delta \mathrm{t}=1 \mathrm{~s}, \\
& \mathrm{j}_{1}=100 \mathrm{Wm}^{-2}, \mathrm{j}_{2}=0 \mathrm{Wm}^{-2}, \mathrm{t}_{1}=20 \mathrm{~s}, \mathrm{j}_{3}=1000 \mathrm{Wm}^{-2}, \mathrm{j}_{4}=100 \mathrm{Wm}^{-2}, \mathrm{j}_{5}=50 \mathrm{Wm}^{-2}, \\
& \omega=2 \pi(0.025) \mathrm{rad} / \mathrm{s}, \mathrm{j}_{6}=50 \mathrm{Wm}^{-2}, \mathrm{t}_{2}=10 \mathrm{~s}, \mathrm{j}_{7}=500 \mathrm{Wm}^{-2}, \mathrm{t}_{3}=30 \mathrm{~s}, \mathrm{j}_{8}=50 \mathrm{Wm}^{-2}, \mathrm{t}_{4}=40 \mathrm{~s} .
\end{aligned}
$$

Cuadro V.6. Valores de los parámetros que definen los flujos

La solución exacta del campo térmico transitorio del problema directo (medidas realizadas en la frontera adiabática) se muestra en la figura V.25. De estas gráficas puede apreciarse que la influencia del tipo de flujo incidente en el aspecto de estas curvas no es muy grande, en particular cuando ha transcurrido un tiempo apreciable desde el inicio del transitorio. Así, puede observarse, por ejemplo, la inflexión en el campo térmico debido al escalón ascendente del flujo incidente rectangular, inflexión prácticamente inexistente para el escalón descendente del flujo. Algo parecido puede apuntarse para el flujo sinusoidal y, en menor grado, para el flujo triangular.

Naturalmente, en los resultados de la estimación influye tanto la geometría como las propiedades térmicas de la placa y el punto en el que se realizan las medidas, siendo a veces necesario realizar tanteos previos hasta conseguir coeficientes de sensibilidad apreciables.

Hay que mencionar que en este caso, las medidas de temperaturas reales se obtienen a partir de la distribución normal de errores (apartado III.1.2). 


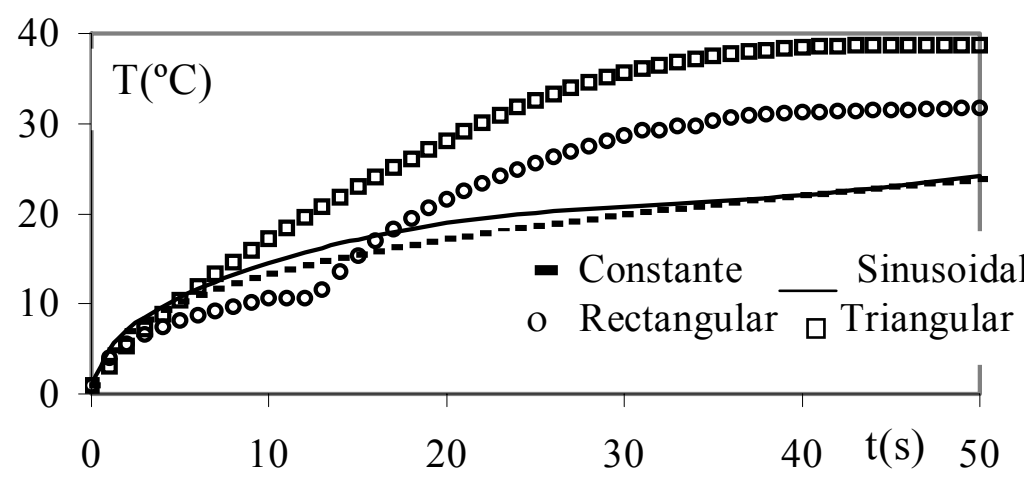

Figura V.25. Solución exacta del campo térmico para distintos flujos incidentes

\section{Flujo de calor constante}

Para la estimación del punto inicial se ha seguido el procedimiento explicado en III.3.3. En la figura V.26 se muestra para un error nulo en las medidas, el efecto del número de términos del funcional en la estimación del flujo constante cuyo valor exacto es $\mathrm{j}(\mathrm{L}, \mathrm{t})=\mathrm{j}_{1}=100 \mathrm{Wm}^{-2}$. Las máximas desviaciones observadas son del orden del 1, 2 y 4\% para valores de $\mathrm{r}$ de 7, 5 y 3 , respectivamente; estos son errores ciertamente aceptables en ingeniería. Los valores $s$ de cada estimación se muestran en la misma figura.

Para una desviación típica de $\sigma=0.1$ en el error, los resultados de la estimación, junto con los valores de $s$, se muestran en figura V.27. Este valor de $\sigma$ da lugar a un error máximo en las temperaturas medidas, del orden del $25 \%$, al principio del transitorio donde las temperaturas son del orden de la unidad (fig. V.25); valor que disminuye sensiblemente hasta el $1 \%$ al final del transitorio donde las temperaturas son del orden de $24{ }^{\circ} \mathrm{C}$. Los errores cuadráticos medios en la estimación son apreciables (como era de esperar), del orden del 10 y 20\% para valores de $\mathrm{r}$ de 7 y 5 , respectivamente.

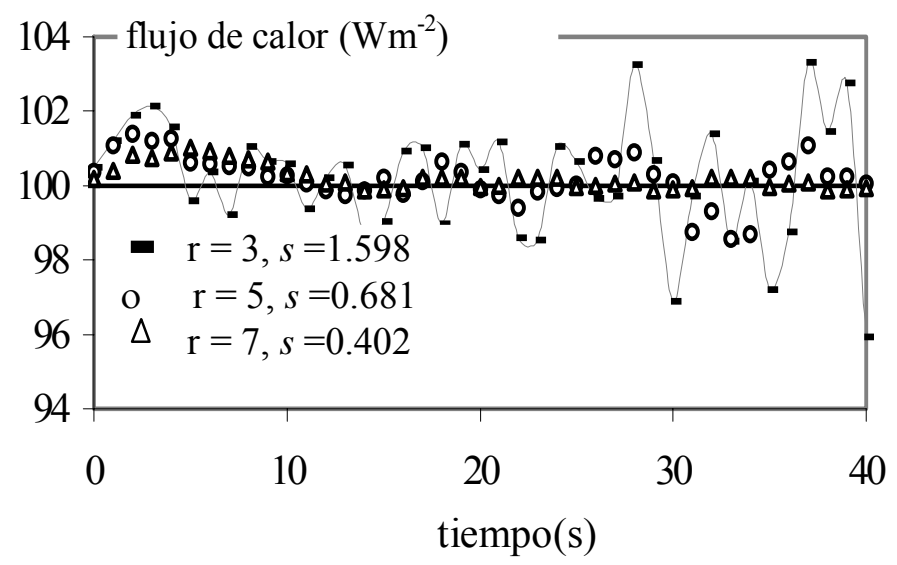

Figura V.26. Estimación de un flujo constante, $\mathrm{r}=3,5 \mathrm{y}=7 ; \sigma=0$ 


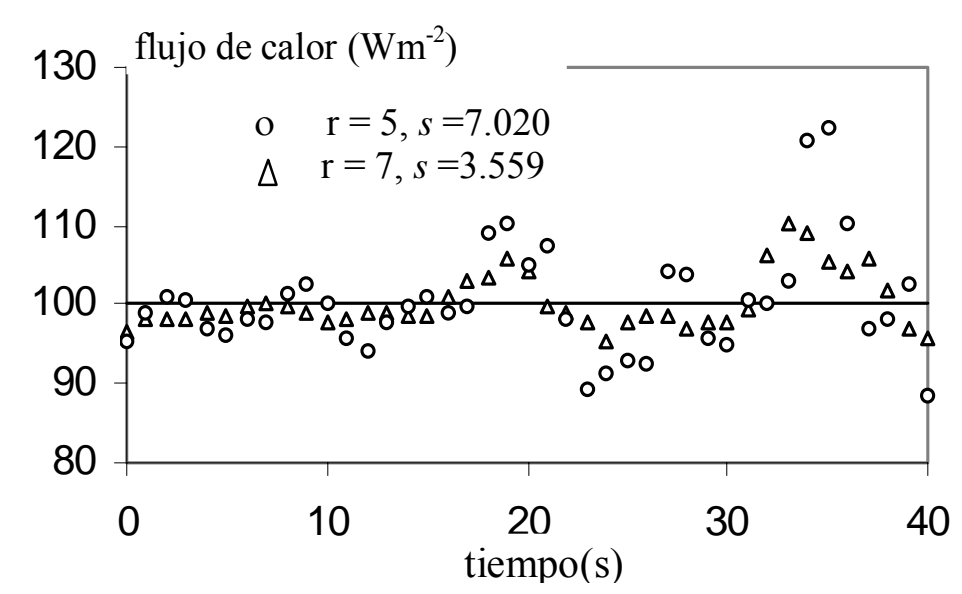

Figura V.27. Estimación de un flujo constante, $r=5$ y $7 ; \sigma=0.1$

\section{Flujo de calor triangular}

La figura V.28 muestra la estimación de este flujo incidente para medidas no afectadas de error, valores de 3, 5 y 7 para $r$, y $n=40$. El valor $r=3$ es el que proporciona la mejor estimación. Para medidas afectadas de error, con desviación $\sigma=0.1$, y r $=4,5$ y 7, los resultados se muestran en la figura V.29. En este caso la mejor estimación se obtiene para r=7.

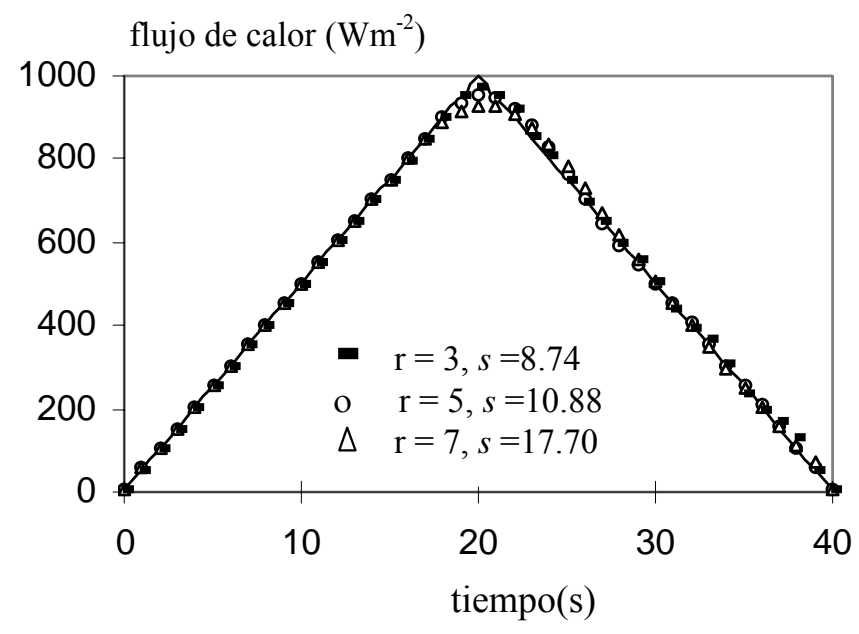

Figura V.28. Estimación de flujo triangular, $\mathrm{r}=3,5$ y $7 ; \sigma=0$ 


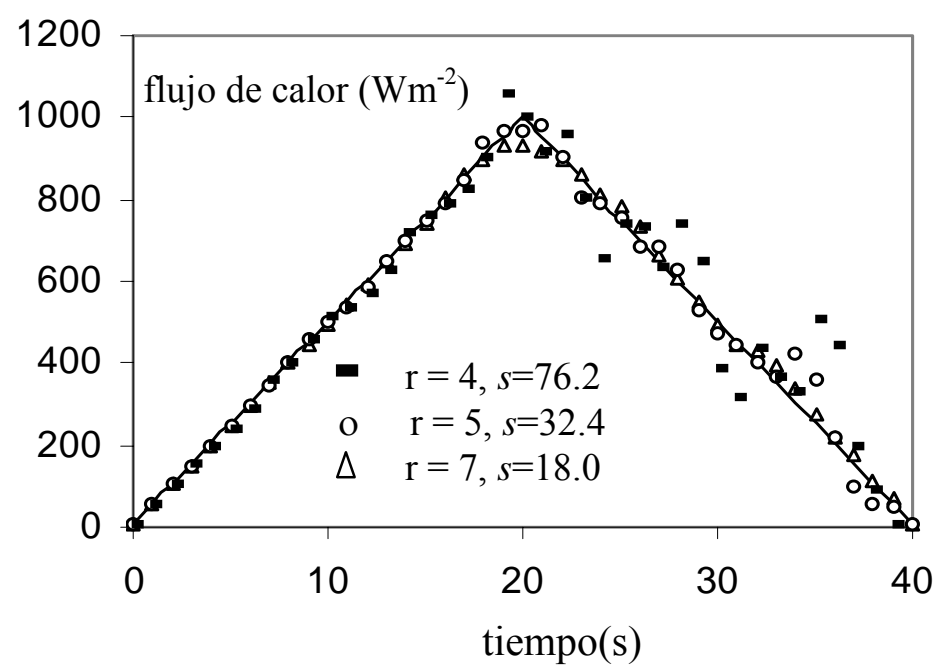

Figura V.29. Estimación de flujo triangular, $\mathrm{r}=4,5$ y $6 ; \sigma=0.1$

\section{Flujo de calor sinusoidal}

Los resultados de las estimaciones con $\sigma=0.1$, junto con los valores de $s$ de las mismas, se muestran en las figuras V.30 y V.31. A pesar de que las tendencias en las curvas estimadas son más suaves que en el caso anterior, debido a la ausencia de picos en la onda incidente, pueden apuntarse conclusiones parecidas. En ausencia de error la estimación más precisa se obtiene para $\mathrm{r}=5$, mientras que con $\sigma=0.1$ la mejor estimación se obtiene para $\mathrm{r}=7$.

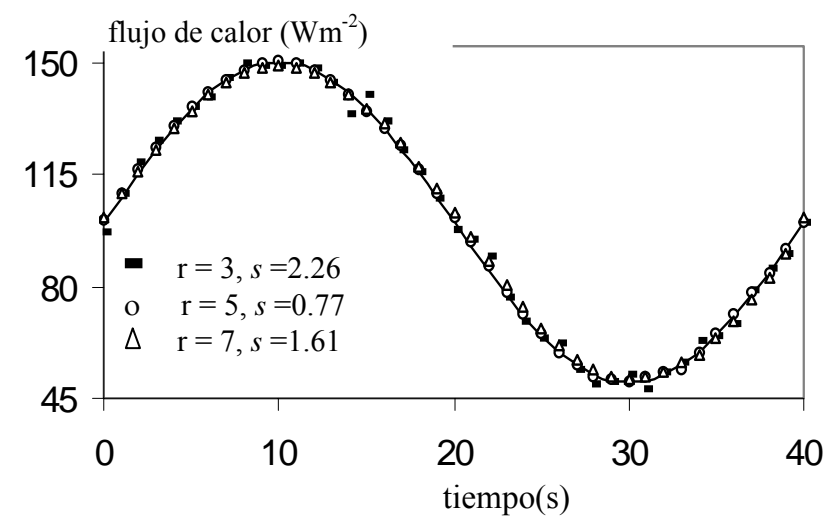

Figura V.30. Estimación de flujo de calor sinusoidal, $r=3,5$ y $7 ; \sigma=0$ 


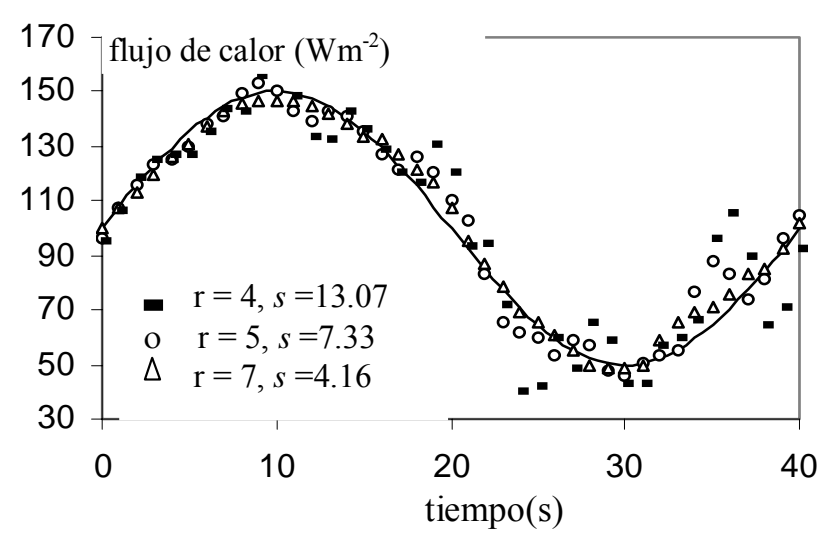

Figura V.31. Estimación de flujo de calor sinusoidal, $\mathrm{r}=4,5$ y $7 ; \sigma=0.1$

\section{Flujo de calor rectangular}

Se trata de una forma de onda muy compleja por la existencia de dos discontinuidades. Para medidas sin error, y $\mathrm{r}=3$ y 5 , las estimaciones obtenidas se presentan en la figura V.32, junto con los valores de $s$, muy altos. La mejor estimación se obtiene para $r=3$. La introducción de errores en las medidas, aunque sean pequeños, proporcionan estimaciones aproximadas. En la figura V.33 se presentan los resultados para $\sigma=0.01$, con $\mathrm{r}=5$.

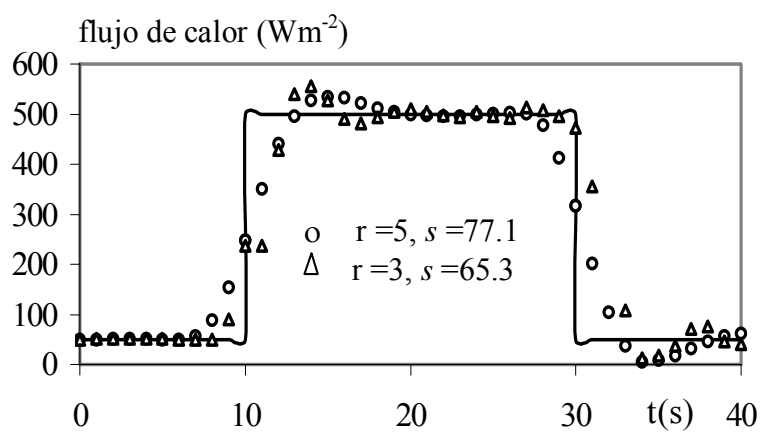

Figura V.32. Estimación de flujo de calor rectangular, $r=3$ y $r=5, \sigma=0$

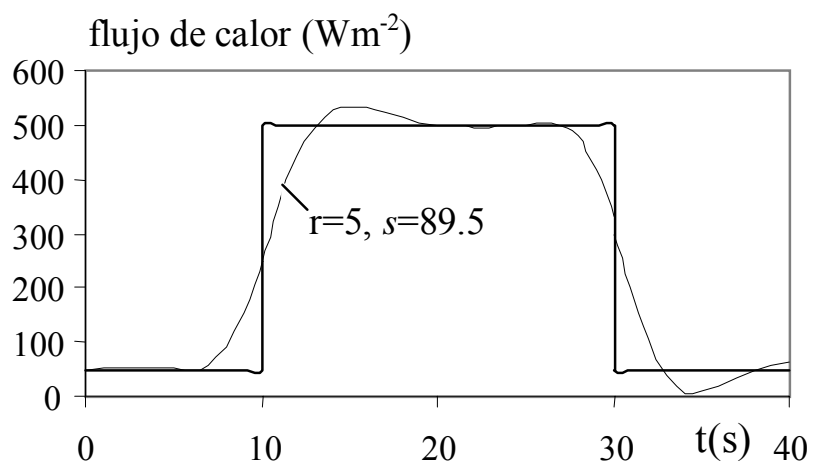

Figura V.33. Estimación de flujo de calor sinusoidal, $\mathrm{r}=5 ; \sigma=0.01$ 


\section{V.2. ESTIMACIÓN DE LA TEMPERATURA}

\section{V.2.1. Introducción}

En relación con esta estimación, ampliaremos la bibliografía mencionada en el apartado V.1.1. Reinhardt [1991] desarrolló un método numérico para obtener la solución de problemas en medios bidimensionales con generación interna de calor y propiedades térmicas constantes. El procedimiento seguido está basado en el método de Beck [1982]. Contiene realizan aplicaciones numéricas para la obtención de flujos de calor y temperaturas en la superficie del medio.

Haji-Sheikh y Yan [1992] emplean las funciones de Green para resolver un problema inverso de conducción del calor multidimensional, aportando un ejemplo numérico para el caso de la estimación de la temperatura superficial.

Yang [1997] desarrolló un método basado en diferencias finitas y resuelto por mínimos cuadrados para determinar las condiciones de contorno en problemas inversos de conducción del calor bidimensionales. Estudia el efecto del error en las medidas de temperaturas.

Al-Najem y col [1998] estimaron la temperatura en la superficie de un medio bidimensional que trabaja en situación estacionaria mediante dos métodos, método de elementos de contorno y un método integral con mínimos cuadrados. Estudiaron el efecto de la sensibilidad al cambiar el punto de medida.

Al-Khalidy [1998] empleó una formulación numérica parabólica e hiperbólica, y el método de volúmenes de control (CVM), junto con filtros digitales, para resolver problemas inversos en conducción del calor. Estimó las temperaturas y flujos de calor aplicados en la superficie de un medio con propiedades térmicas dependientes de la temperatura, a partir de temperaturas realizadas en el interior de él, analizando el efecto del error en las medidas.

Monde y col. [2000] desarrollaron un método analítico 1-D usando la transformación de Laplace para determinar la temperatura o el flujo de calor en la superficie de un sólido, a partir de las medidas de temperatura en dos puntos del medio.

Jarny y col. [2001] utilizando el método de gradientes conjugados resolvieron un problema inverso consistente en la estimación de temperaturas o flujos de calor a partir de medidas de temperaturas realizadas en el interior del medio bidimensional o en los extremos del medio, en un proceso de convección natural. 


\section{V.2.2. Estimación de la temperatura en un medio sometido a convección forzada}

En este apartado se aborda la estimación directa de temperatura en una de las caras exteriores de un sólido 1-D de geometría plana sometida a convección. Las propiedades térmicas, por sencillez, se asumen constantes. El punto de medida de temperaturas estará en la superficie opuesta, bajo condición adiabática, o en el interior del sólido.

El modelo matemático se recoge en el cuadro V.7.

\begin{tabular}{|l|l|}
\hline \multicolumn{2}{|c|}{ Modelo matemático } \\
\hline Ecuaciones diferenciales & $\rho \mathrm{c}_{\mathrm{e}} \partial \mathrm{T} / \partial \mathrm{t}-\mathrm{k}\left(\partial^{2} \mathrm{~T} / \partial \mathrm{x}^{2}\right)=0$ \\
\hline Condiciones iniciales & $\mathrm{T}_{(\mathrm{x}, \mathrm{t}=0),}=\mathrm{T}_{\mathrm{o}}$ \\
\hline Condiciones de contorno & $(\partial \mathrm{T} / \partial \mathrm{x})_{(\mathrm{x}=0, \mathrm{t})}=\mathrm{h}(\mathrm{T}) \Delta \mathrm{T}$ \\
& $(\partial \mathrm{T} / \partial \mathrm{x})_{(\mathrm{x}=\mathrm{L}, \mathrm{t})}=0$ (punto de medida) \\
\hline Condición adicional & $\mathrm{T}_{\mathrm{IHCP}}\left(\mathrm{x}_{\mathrm{f}}, \mathrm{t}_{\mathrm{j}}, \varepsilon_{\mathrm{j}}\right)=\mathrm{T}_{\mathrm{DHCP}}\left(\mathrm{x}_{\mathrm{f}}, \mathrm{t}_{\mathrm{j}}\right)+\varepsilon_{\mathrm{j}}$ \\
\hline
\end{tabular}

Cuadro V.7. Modelo matemático

Los datos numéricos se muestran en el cuadro V.8

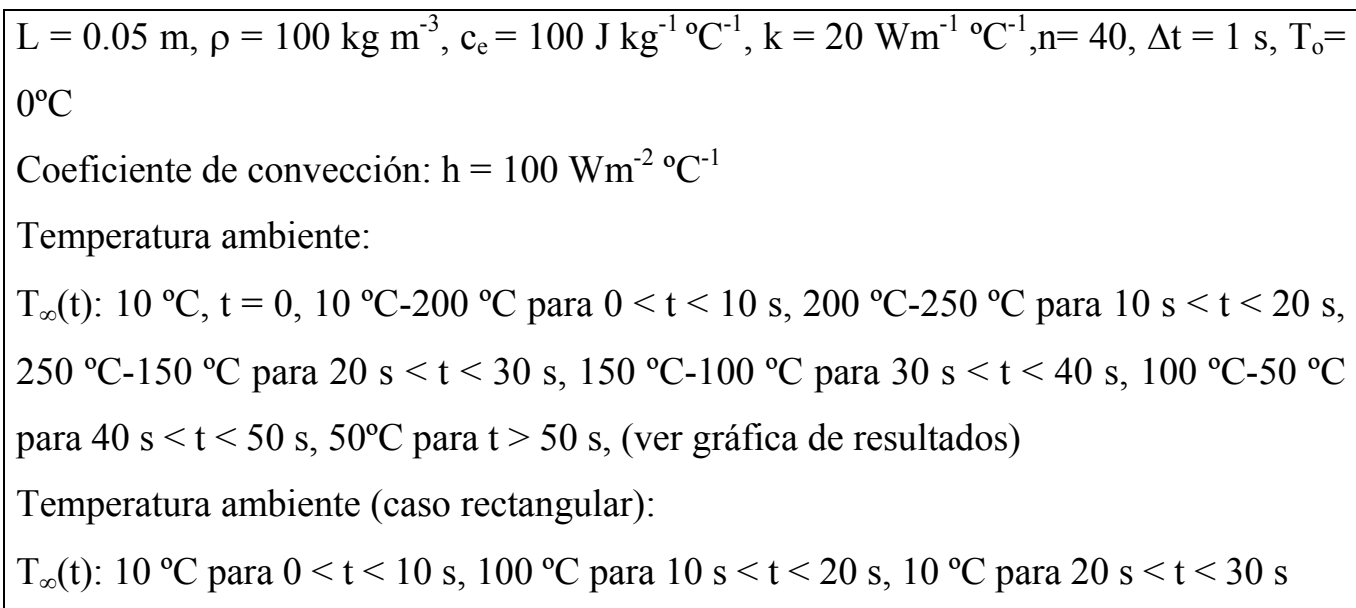

Cuadro V.8. Datos numéricos del problema

La tabla V.4 se muestra el valor estimado inicial de temperatura (según protocolo del capítulo III), en función de r y del intervalo de tiempo de medidas, $\Delta$ t. El valor exacto (1.39) es, ciertamente, muy distinto del valor estimado pero como inmediatamente se verá no influye apenas en los resultados de la estimación para las formas de onda propuestas. El valor inicial más próximo (5.27) se obtiene para $\mathrm{r}=3, \Delta \mathrm{t}=0.1 \mathrm{~s}$. Un refinamiento del cálculo del valor inicial 
podría conseguirse mediante protocolos similares a los aquí propuestos usando tramos de estimación con pendiente variable. Este aspecto se deja para trabajos posteriores.

\begin{tabular}{|c|c|c|}
\hline $\mathrm{r}$ & $\Delta \mathrm{t}=0.1 \mathrm{~s}$ & $\Delta \mathrm{t}=0.2 \mathrm{~s}$ \\
\hline 3 & 5.27 & 6.45 \\
\hline 5 & 6.25 & 9.57 \\
\hline
\end{tabular}

Tabla V.4. Estimación inicial

En problemas donde el parámetro a estimar no varíe demasiado con el tiempo, será fácil obtener una buena estimación inicial. Esta información no es conocida a priori, por lo que la elección de los parámetros $\mathrm{r}$ y $\Delta \mathrm{t}$ es meramente orientativa; de aquí que sea necesario, a veces, por la aparición de oscilaciones en la estimación proceder a nuevos tanteos del valor inicial.

En la figura V.34 se muestra la estimación de la temperatura en la superficie (cara sometida a convección), sin error en las medidas y con $r=5$. El valor inicial adoptado es $5.27 \mathrm{y}$ error en la estimación, $s=3.43$.

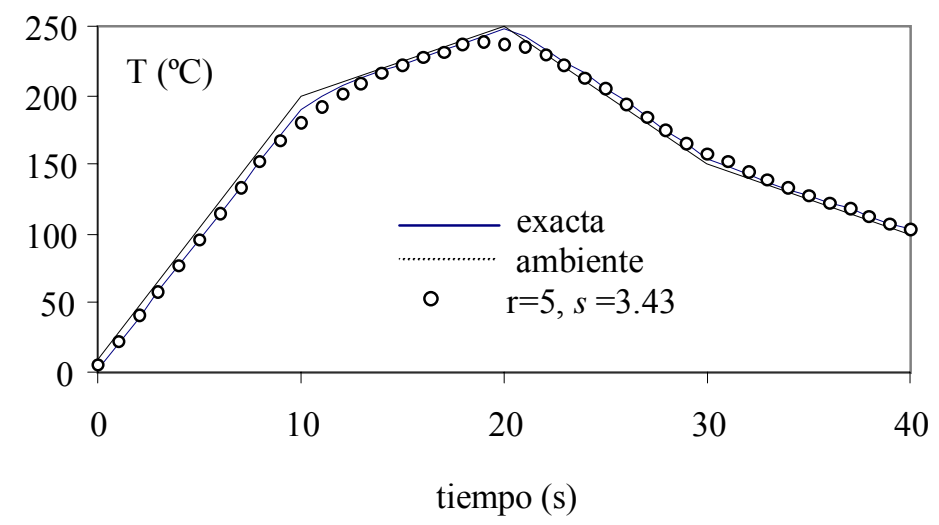

Figura V.34. Estimación de la temperatura en la superficie convectiva $\mathrm{r}=5$, temperatura estimada inicial $=5.27, \mathrm{~h}=100 \mathrm{~W} / \mathrm{m}^{2}{ }^{\circ} \mathrm{C}$

La figura V.35 muestra la estimación de la temperatura superficial, sin error en las medidas y con $\mathrm{r}=3$ y 7 . El valor inicial de la estimación es 5.27 y los errorres resultantes 1.17 y 7.29, respectivamente. La mejor estimación para este caso corresponde a $r=3$. La figura V.36 muestra la misma estimación, para $r=3$ y 5 , tomando un valor inicial mayor, 9.57. Para estas estimaciones $s$ es 1.64 y 3.75, respectivamente. Puede observarse que la mejor estimación sigue siendo para $\mathrm{r}=3$, sin embargo ambos valores son (aunque por muy poco) superiores a los obtenidos con el valor inicial de estimación de 5.27. 


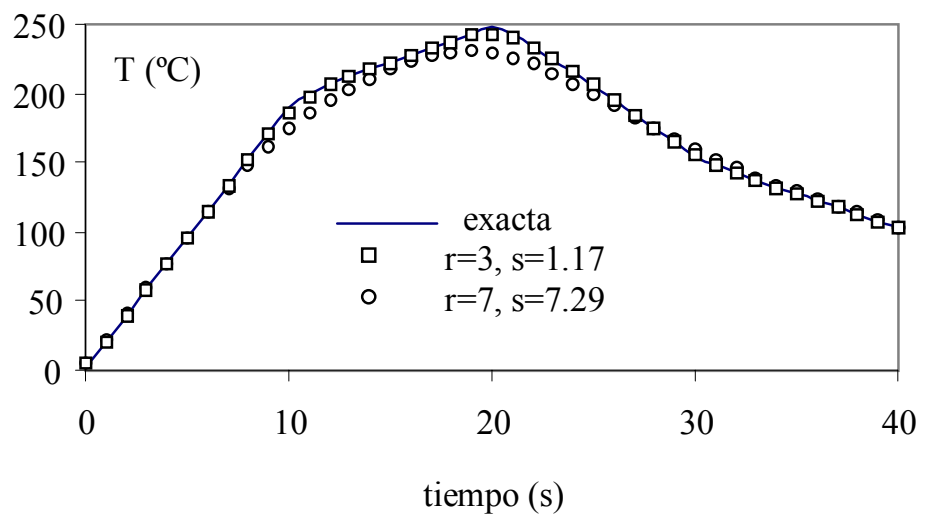

Figura V.35. Estimación de la temperatura en la superficie convectiva $r=3$, y r $=7$, temperatura estimada inicial $=5.27, \mathrm{~h}=100 \mathrm{~W} / \mathrm{m}^{2}{ }^{\circ} \mathrm{C}$

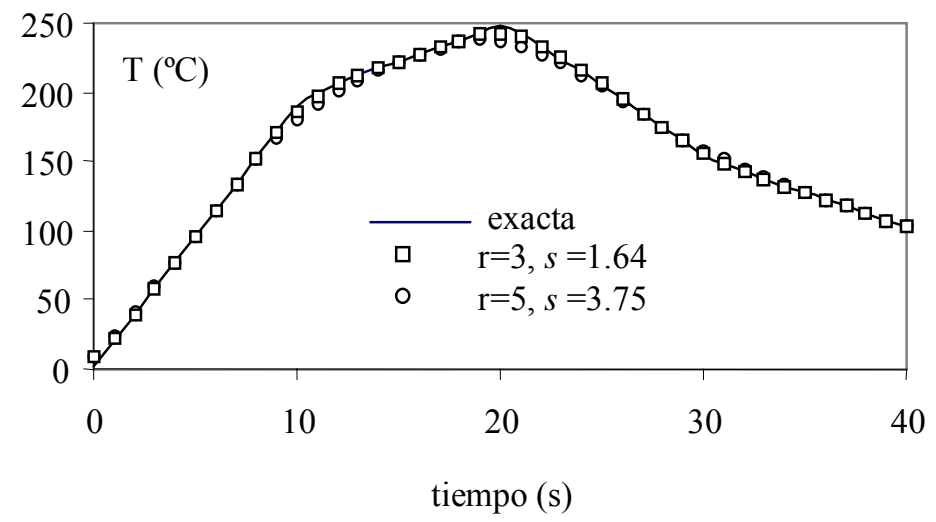

Figura V.36. Estimación de la temperatura en la superficie convectiva $\mathrm{r}=3$, y r $=5$, temperatura estimada inicial $=9.57, \mathrm{~h}=100 \mathrm{~W} / \mathrm{m}^{2}{ }^{\circ} \mathrm{C}$

En las figuras V.37 y V.38 se muestran las estimaciones de la temperatura, para $\mathrm{r}=5 \mathrm{y}$ $\mathrm{r}=3$, respectivamente, y para los puntos iniciales de 5.27 y 9.57, sin error en las medidas. En la figura V.37 se aprecia que sólo los tres primeros puntos (para $\mathrm{t}=1,2$ y 3 segundos) difieren; a partir del cuarto punto ( $\mathrm{t}=4$ segundos) los puntos estimados son prácticamente iguales, mientras que en la figura V.38, para $\mathrm{r}=3$, la estimación se ajusta a partir del segundo punto $(\mathrm{t}=2$ segundos). Aunque la estimación de temperaturas es un problema más sencillo que el de la determinación de flujos incidentes, debido a la menor sensibilidad de las estimaciones anteriores sobre las futuras en el primer caso que en segundo, con estos ejemplos queda probado la idoneidad del método numérico propuesto. 


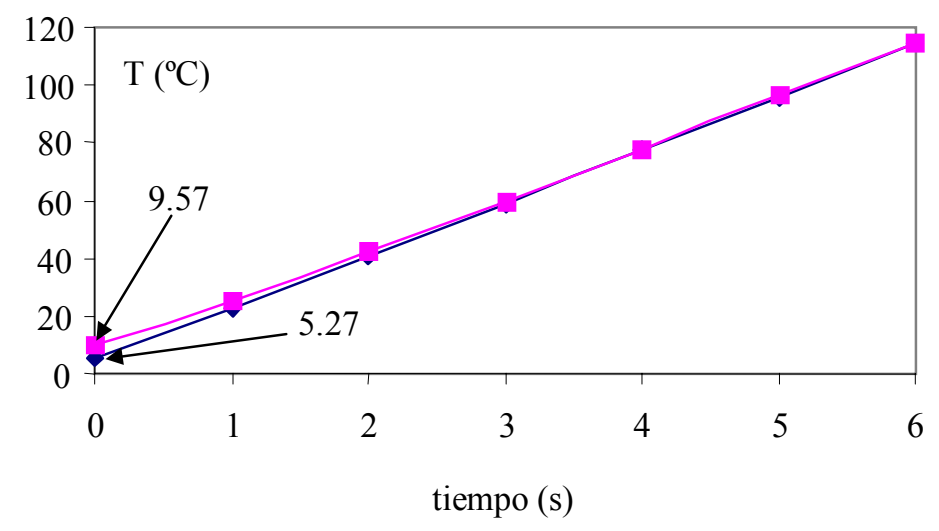

Figura V.37. Estimación de la temperatura en la superficie convectiva $\mathrm{r}=5$, temperatura estimada inicial $=5.27$ y $9.57, \mathrm{~h}=100 \mathrm{~W} / \mathrm{m}^{2}{ }^{\circ} \mathrm{C}$

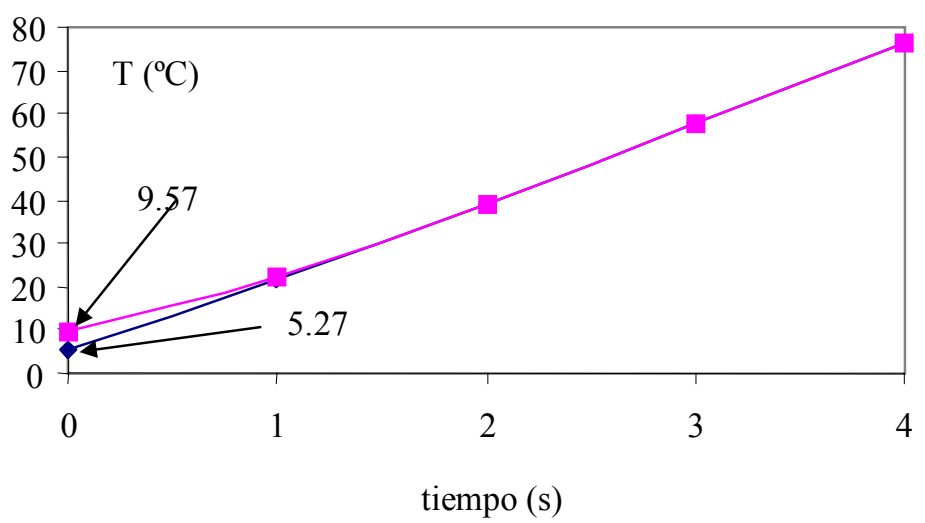

Figura V.38. Estimación de la temperatura en la superficie convectiva $r=3$, temperatura estimada inicial $=5.27$ y $9.57, \mathrm{~h}=100 \mathrm{~W} / \mathrm{m}^{2}{ }^{\circ} \mathrm{C}$

En relación con el punto de medida, se han realizado estimaciones en $\mathrm{x}=\mathrm{L} / 2$, con $\mathrm{r}=3 \mathrm{y}$ 5 , para un valor inicial de $5.27 \mathrm{y}$ sin errores en las medidas. Los valores de $s$ son similares a los anteriores, en concreto de 1.56 y 1.67 , para $r=3$ y 5 respectivamente.

Los resultados de la inclusión de error en las medidas, con desviaciones típicas de $\sigma=0.1$ y 0.5 , se muestran en la figura V.39, tomando $\mathrm{r}=3 \mathrm{y}$ un valor inicial de 5.27. Los valores de $s$ para ambos casos son 2.16 y 3.70 . 


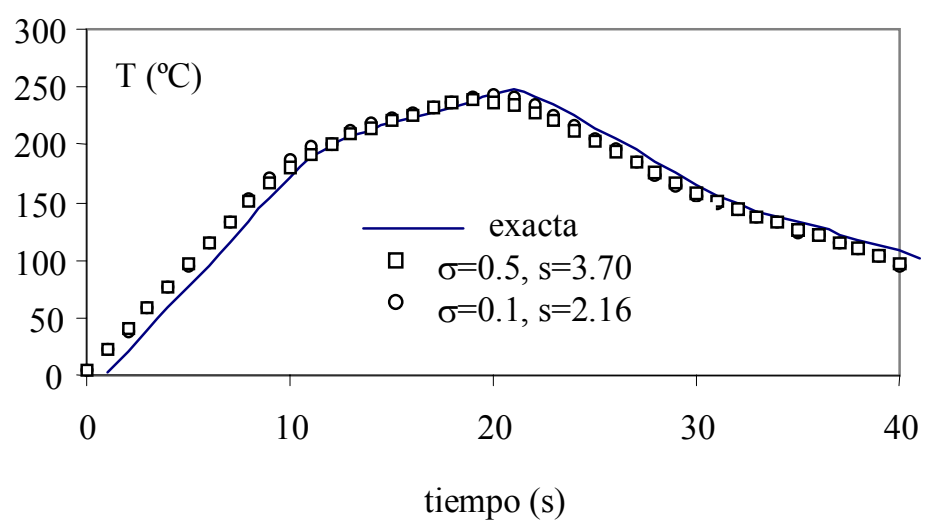

Figura V.39. Estimación de la temperatura en la superficie convectiva $r=3$, temperatura estimada inicial $=5.27, \sigma=0.1$ y $0.5, \mathrm{~h}=100 \mathrm{~W} / \mathrm{m}^{2}{ }^{\circ} \mathrm{C}$.

La figura V.40 muestra los resultados de la estimación de una temperatura de contorno compleja en forma de onda rectangular, sin error en las medidas y para 2, 3 y 4 términos en el funcional. El valor inicial de la estimación es 3.03. Los valores de $s$ de estas estimaciones son $11.02,7.91$ y 10.04, respectivamente. Para r=3 y 4 desaparecen las oscilaciones de la estimación que se producen para $\mathrm{r}=2$, en la zona de ascenso de la onda (en torno a los $100{ }^{\circ} \mathrm{C}$ ). La mejor estimación se obtiene para $\mathrm{r}=3$.

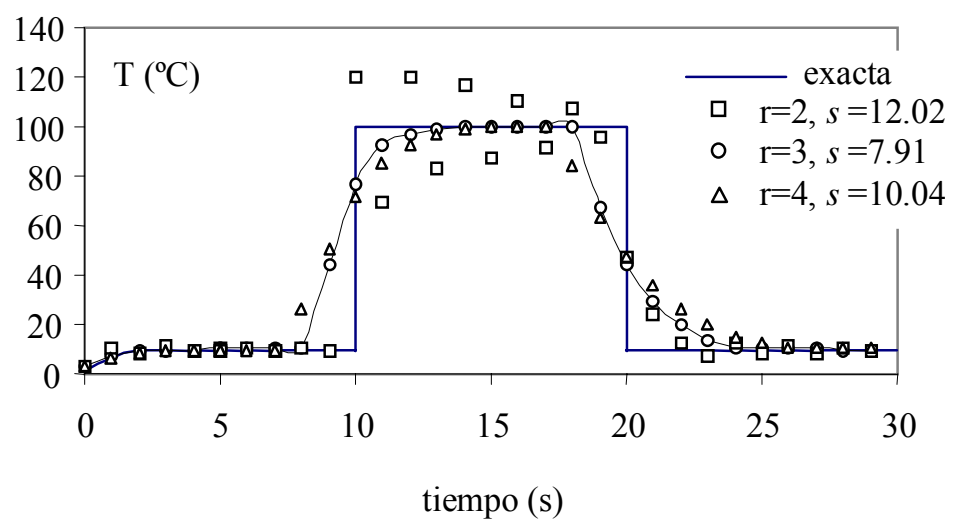

Figura V.40. Estimación de la temperatura en la superficie convectiva $r=2,3$ y 4 , temperatura estimada inicial $=3.03, \sigma=0, \mathrm{~h}=100 \mathrm{~W} / \mathrm{m}^{2}{ }^{\circ} \mathrm{C}$

Por último, en la figura V.41 se muestran resultados de las estimaciones de la misma forma de onda, con desviación típica $\sigma=0.5$. Temperatura estimada inicial 3.03 y r=3 y 4 . Los 
valores de $s$ resultantes son ligeramente superiores a los del caso anterior para los mismos valores de $r$. La mejor estimación se obtiene también para $r=3$.

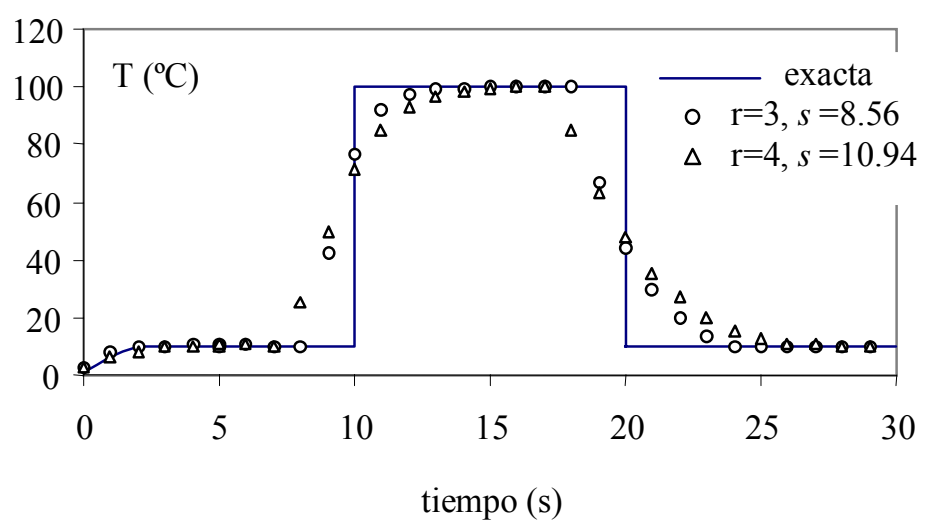

Figura V.41. Estimación de la temperatura en la superficie convectiva $r=3$ y 4 , temperatura estimada inicial $=3.03, \sigma=0.5, \mathrm{~h}=100 \mathrm{~W} / \mathrm{m}^{2}{ }^{\circ} \mathrm{C}$.

\section{V.3. REFERENCIAS}

Alhama, F., Zueco, J., Campo, A. y González-Fernández, C.F., "Determination of the different incident heat fluxes using the network simulation method", Proc. Eurotherm Seminar 68, (Poitiers), 145-149 (2001)

Alhama, F., Zueco, J. y González-Fernández, C.F., "An inverse determination of unsteady heat fluxes using a network simulation method“", J. Heat Transfer (en prensa)

Al-Khalidy, N.,"On the solution of parabolic and hyperbolic inverse heat conduction problems", Heat Mass Transfer, 41, 3731-3740 (1998)

Al-Najem, N.M., Osman, A.M., El- Refaee, M.M. y Khanafer, K.M., "Two dimensional steadystate inverse heat conduction problems", Int. Commun. Heat Mass Transf., 25, 4, 541-550 (1998)

Alifanov O.M., y Artyukhin, F.A. "Regularized numerical solution of nonlinear inverse heatconduction problem", J. Eng. Phy. 29, 934-938 (1975)

Beck, J.V., "Calculation of surface heat flux from an internal temperature history", ASME Paper 62-HT-46 (1963) 
Beck, J.V., "Surface Heat Flux Determination Using an Integral Method", Nucl. Eng. Des., 7, 170-178 (1968)

Beck, J.V., "Nonlinear Estimation Applied to the Nonlinear Heat Conduction Problem", Int. J. Heat Mass Transf., 13, 703-716 (1970)

Beck, J. V., Litkouhi B. y St.Clair.Jr. C.R., "Effective sequential solution of the nonlinear inverse heat conduction problem", Numer. Heat Transfer, 5, 275-286 (1982)

Beck, J. V., Blackwell, B. y St.Clair.Jr. C.R., "Inverse heat conduction”, New York, John Wiley \& Sons. Inc. (1985)

Burggraf, O.R. "An exact solution of the inverse problem in heat conduction theory and pubblications", J. Heat Transfer, 86C, 373-382 (1964)

France, D.M. y Chiang, T., "Analytic solution to inverse heat conduction problems with periodicity", J. Heat Transfer, 102, 579-581 (1980)

Haji-Sheikh, A. y Yan, L., "Multidimensional inverse heat conduction using alternative Green's function solution", Int. Commun. Heat Mass Transf., 19, 661-672 (1992)

Hills, R.G. y Hensel, E.C. Jr., "One-dimensional nonlinear inverse heat conduction technique", Numer. Heat Transfer, 10, 369-393 (1986)

Huang, C.H. y Wu, J.Y., "Two-dimensional inverse problem in estimating heat fluxes of an enclosure with unknown internal heat sources", J. Appl. Phys., 76,133-141 (1994)

Jarny, Y., Lecointe, Y. y Ahssaï ni, A., "Resolution of an inverse natural convection problem by using a gradient method”, Proc. Eurotherm Seminar 68, (Poitiers), 151-157 (2001)

Kurpisz K., "Numerical solution of one case inverse heat conduction problems", J. Heat Transfer, 113, 280-286 (1991)

Le Niliot, C., "Inverse problems in heat diffusion: Fluxes and sources identification", $3^{\text {rd }}$ European Thermal Sciences Conference, 5-16 (2000)

Monde, M., Arima, H. y Mitsutake, Y., "Analytical method in inverse heat transfer problem using Laplace transform technique (second and third boundary conditions)", $3^{\text {rd }}$ European Thermal Sciences Conference, 115-120 (2000)

Pasquetti, R. y Le Niliot C., "Boundary element approach for inverse heat conduction problems: Application to a bidimensional transient numerical experiment", Numer. Heat Transfer-B, 20, 169-189 (1991) 
Reinhardt, H.J., "A numerical method for the solution of two-dimensional inverse heat conduction problems", Int. J. Numer. Meth. Eng., 32, 363-383 (1991)

Shumakov, N. V. "A method for the experimental study of the process of heating a solid body". Soviet-Technical Physics (translated by American Institute of Physics), 2, 771 (1957)

Sparrow, E.M., Haji-sheikn, A. y Lundgren, T.S., "The inverse problem in transient heat conduction", J. Appl. Mech., 369-375 (1964)

Stolz, G. Jr., "Numerical solutions to an inverse problem of heat conduction for simple shapes", J. Heat Transfer, 82C, 20-60 (1960)

Weber, C.F., "Analysis and solution of ill-posed inverse heat conduction problem", Int. J. Heat Mass Transf., 24, 11, 783-1792 (1981)

Yang, Ching-Yu, "Symbolic computation to estimate two-sided boundary conditions in twodimensional conduction problems", J. Thermophys. Heat Transf., 11, 3, 472-476 (1997)

\section{CONTRIBUCIONES Y CONCLUSIONES}

Se han estimado flujos incidente, tanto en medios con propiedades térmicas constantes como en medios con propiedades térmicas dependientes de la temperatura. El espectro de estos flujos abarca las siguientes formas de onda: constante, triangular, sinusoidal, escalón y rectangular. Las "medidas experimentales", obtenidas numéricamente, han sido afectadas de error aleatorio con y sin distribución normal. También se han obtenido estimaciones para medidas no afectadas de error; en todos los casos éstas pueden considerarse suficientemente precisas.

Se ha estudiado la influencia de los diferentes parámetros en las estimaciones. En particular, el número de términos del funcional, el error en las medidas y los valores iniciales de la estimación (que a su vez han sido estimados). Mientras que al aumentar el error en las medidas se obtienen invariablemente estimaciones menos precisas, no hay una correlación clara entre el número de términos del funcional y la precisión de la estimación ya que ésta viene también determinada por la suavidad (cambio de pendiente) de la curva que representa el flujo incidente. En flujos incidentes quebrados hay un valor de compromiso para el número de términos del funcional que permite obtener la estimación más precisa. En el caso de error nulo en las medidas la estimación del flujo no mejora al aumentar el número de términos del funcional. 
Las tendencias anteriores no se ven alteradas al tomar diferentes series de medidas ni diferentes valores de error en las mismas.

Se han obtenido los valores del error cuadrático medio de todas las estimaciones y comprobado que, en todos los casos, caen dentro de los límites aceptables en el campo de la ingeniería térmica.

Se ha estimado directamente la temperatura superficial como condición de contorno, para formas de onda de tipo piece-wise y rectangular, estudiando la influencia del valor inicial de la estimación, del número de términos del funcional y del error en las medidas. El valor inicial en la estimación apenas ejerce influencia en los resultados de la estimación. También aquí, la estimación mejora al disminuir tanto el error como el número de términos del funcional. 


\section{Capítulo VI}

\section{Generación interna de calor, coeficiente de convección, emisividad y resistencia térmica de contacto; su estimación}

VI.1. Generación interna de calor

VI.1.1. Introducción

VI.1.2. Aplicaciones

VI.2. Coeficiente de convección

VI.2.1. Introducción

VI.2.2. Aplicaciones

VI.3. Emisividad

VI.4. Resistencia térmica de contacto

VI.5. Referencias

Contribuciones y conclusiones 


\section{Capitulo VI}

\section{Generación interna de calor, coeficiente de convección, emisividad y resistencia térmica de contacto; su estimación}

Dedicamos este capítulo a la estimación de otros parámetros de interés en ingeniería térmica: el coeficiente de convección asociado a la condición de contorno del mismo nombre, la resistencia térmica de contacto entre medios asociada a esta condición límite, la emisividad superficial y la generación interna de calor. Se usa indistintamente el método de estimación de funciones o el de estimación de parámetros, según la naturaleza o el tipo de dependencia del parámetro a estimar.

\section{VI.1. GENERACIÓN INTERNA DE CALOR}

\section{VI.1.1. Introducción}

El consumo o producción de energía de un dispositivo se puede alterar si no se mantiene su temperatura de operación constante o dentro de cierto margen generalmente pequeño. Tal ocurre, por ejemplo, en numerosos reactores de tipo químico y en plantas de almacenamiento de residuos orgánicos y nucleares. La generación interna de calor da lugar a sobrecalentamientos locales que derivan en serios fallos de los equipos eléctricos y mecánicos que a su vez, eventualmente, pueden traer consecuencias desastrosas para el medio ambiente.

La generación interna de calor en un sólido, generalmente función de la temperatura, es una variable de interés cuya medida suele ser difícil bien por la propia naturaleza de los sensores de medida, por el espacio disponible para su ubicación y su accesibilidad, por la existencia de ambientes corrosivos o explosivos, etc. Es frecuente, en estos casos, resolver el problema inverso en sus diferentes versiones a partir del conocimiento de las medidas locales de temperatura en algunos puntos del medio. 
Muchos autores que integran en sus trabajos términos de generación interna no abordan el problema inverso de su estimación. Entre los que tratan este tema cabe citar a Silva y Özisik [1992] que emplean el método de gradiente conjugado para estimar con distintos tipos de dependencias (rectangular, triangular y sinusoidal), en un medio bidimensional sometido a condiciones de contorno adiabáticas. Se trata de un problema de estimación de función, ya que no se dispone de información previa de la forma de variación temporal de las funciones a estimar.

Martin y Dulikravich [1996] resolvieron el problema estacionario, en geometría bidimensional (problema de Poisson), usando el método de elementos de contorno (BEM) y las funciones de Green.

Yang [1997, 1998] estudia en trabajos separados las geometrías uni y bidimensional, mediante un modelo inverso lineal basado en una aproximación por diferencias finitas, para obtener la fuente de calor de forma explícita. Obtiene estimaciones para distintas formas de onda, función escalón, triangular y sinusoidal, y analiza los efectos del error en la medición y de la ubicación del punto de medida.

\section{VI.1.2. Aplicaciones}

El problema a estudiar responde al modelo matemático mostrado en el cuadro VI.1. Para la generación interna se ha tomado una dependencia triangular con la temperatura con objeto de asegurar la versatilidad del modelo para otro tipo de dependencias comunes más suaves (dependencias lineales o polinómicas). El punto de medida de temperaturas está situado en la superficie adiabática, siguiendo a Silva y Özisik [1992].

La generación interna de calor, para la solución del problema directo, es:

$$
\begin{aligned}
& \mathrm{g}_{\text {gen }}\left(\mathrm{L}, 0<\mathrm{t}<\mathrm{t}_{1}\right)=\mathrm{g}_{1}+\left[\left(\mathrm{g}_{2}-\mathrm{g}_{1}\right) /\left(\mathrm{t}_{1}\right)\right] \mathrm{t} \\
& \mathrm{g}_{\mathrm{gen}}\left(\mathrm{L}, \mathrm{t}_{1}<\mathrm{t}<2 \mathrm{t}_{1}\right)=\mathrm{g}_{2}-\left[\left(\mathrm{g}_{1}-\mathrm{g}_{2}\right) / \mathrm{t}_{1}\right]\left(\mathrm{t}-\mathrm{t}_{1}\right)
\end{aligned}
$$

con $\mathrm{g}_{1}=5, \mathrm{Wm}^{-3}, \mathrm{~g}_{2}=30 \mathrm{Wm}^{-3}, \mathrm{t}_{1}=20 \mathrm{~s}$, para la dependencia temporal, $\mathrm{y}$

$$
\begin{aligned}
& \mathrm{g}_{\mathrm{gen}}\left(\mathrm{L}, 0<\mathrm{T}<\mathrm{T}_{1}\right)=\mathrm{g}_{1}+\left[\left(\mathrm{g}_{2}-\mathrm{g}_{1}\right) /\left(\mathrm{T}_{1}\right)\right] \mathrm{T} \\
& \mathrm{g}_{\mathrm{gen}}\left(\mathrm{L}, \mathrm{T}_{1}<\mathrm{t}<2 \mathrm{~T}_{1}\right)=\mathrm{g}_{2}-\left[\left(\mathrm{g}_{1}-\mathrm{g}_{2}\right) / \mathrm{T}_{1}\right]\left(\mathrm{T}-\mathrm{T}_{1}\right)
\end{aligned}
$$

con $\mathrm{g}_{1}=50, \mathrm{Wm}^{-3}, \mathrm{~g}_{2}=200 \mathrm{Wm}^{-3}, \mathrm{~T}_{1}=30^{\circ} \mathrm{C}$, para la dependencia con la temperatura. 


\begin{tabular}{|c|c|}
\hline \multicolumn{2}{|c|}{ Modelo matemático } \\
\hline Ecuación diferencial & $\begin{array}{l}\rho c_{e} \partial T / \partial t=j_{\text {gen }}+k\left(\partial^{2} T / \partial x^{2}\right) \\
g_{g e n}=g_{g e n}(t) o g_{g e n}(T)\end{array}$ \\
\hline Condición inicial & $\mathrm{T}(\mathrm{x},=0)=\mathrm{T}_{0}$ \\
\hline Condiciones de contorno & $\begin{array}{l}\mathrm{J}(\mathrm{L}, \mathrm{t})=\mathrm{h} \Delta \mathrm{T} \\
\mathrm{j}(0, \mathrm{t})=0 \text { (punto de medida) }\end{array}$ \\
\hline Condición adicional & $\mathrm{T}_{\mathrm{IHCP}}\left(\mathrm{x}_{\mathrm{f}}, \mathrm{t}_{\mathrm{j}}, \varepsilon_{\mathrm{j}}\right)=\mathrm{T}_{\mathrm{DHCP}}\left(\mathrm{x}_{\mathrm{f}}, \mathrm{t}_{\mathrm{j}}\right)+\varepsilon_{\mathrm{j}}$ \\
\hline
\end{tabular}

Cuadro VI.1. Modelo matemático

Los valores numéricos del problema se indican el cuadro VI.2.

Densidad: $\rho=1000 \mathrm{~kg} \mathrm{~m}^{-3}$, calor específico: $\mathrm{c}_{\mathrm{e}}=1 \mathrm{~kJ} \mathrm{~kg}^{-1}{ }^{\circ} \mathrm{C}^{-1}$, conductividad: $\mathrm{k}=2 \mathrm{~W}$ $\mathrm{m}^{-1}{ }^{\circ} \mathrm{C}^{-1}$, longitud: $\mathrm{L}=1 \mathrm{~m}$, temperatura inicial: $\mathrm{T}_{\mathrm{o}}=0{ }^{\circ} \mathrm{C}$, temperatura ambiente: $\mathrm{T}_{\infty}=$ $60{ }^{\circ} \mathrm{C}$, coeficiente convectivo: $\mathrm{h}=5 \mathrm{~W} \mathrm{~m}^{-2}{ }^{\circ} \mathrm{C}^{-1}, \Delta \mathrm{t}=1 \mathrm{~s} \mathrm{y} \Delta \mathrm{T}_{\mathrm{a}}=4{ }^{\circ} \mathrm{C}$

Cuadro VI.2. Valores numéricos

El modelo en red del problema ya se estudió en el Capítulo IV (figura IV.20). En la figura VI.1 se muestra la estimación de la generación interna en función del tiempo. Para la estimación del punto inicial se ha seguido el mismo procedimiento que en los casos estudiados hasta el momento; se estima un pequeño tramo horizontal del cual se toma el primer punto. El efecto en la estimación de la elección del punto inicial puede apreciarse en la figura VI.1, donde se han tomado valores iniciales de 3.5, 5.2 y 6.2 , un funcional de cinco términos y $\sigma=0.01$. La influencia de $\mathrm{r}(\mathrm{r}=3$ y 5), para $\sigma=0.01$ y un valor de 5.2 para el punto inicial, puede verse en la figura VI.2.

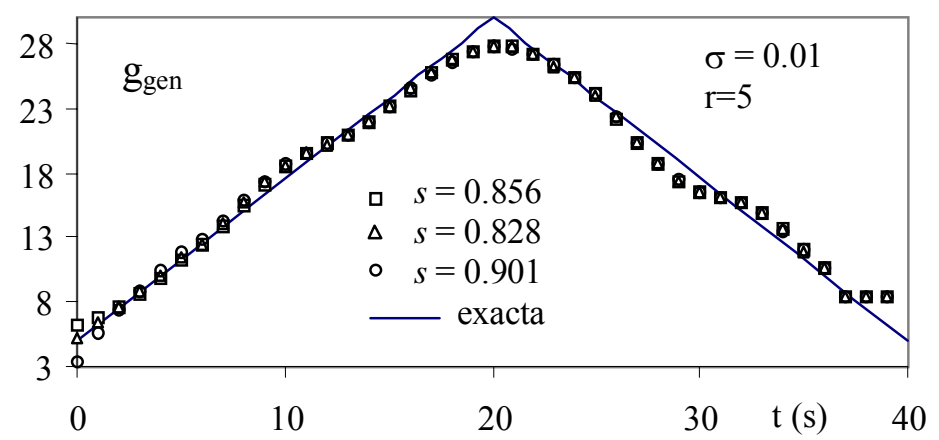

Figura VI.1. Estimación de la generación interna de calor $(\sigma=0.01)$

para diferentes valores iniciales 


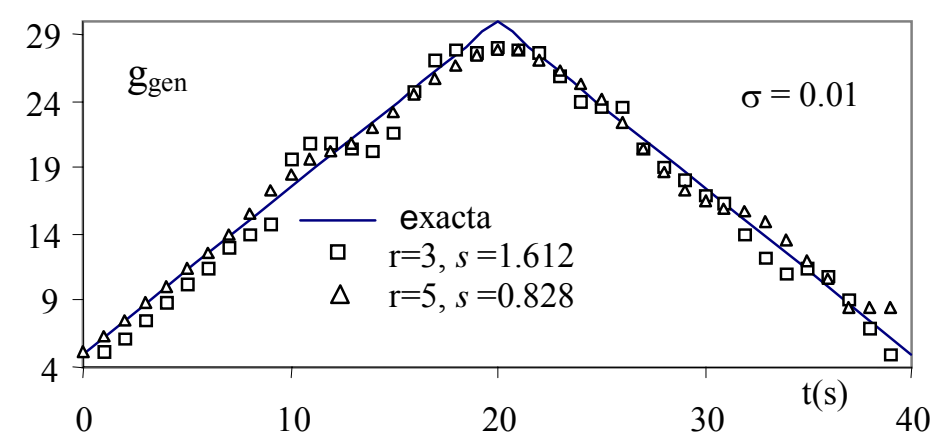

Figura VI.2. Estimación de la generación interna de calor, $\sigma=0.01, \mathrm{r}=3$ y 5

De estas figuras se desprende que la elección del punto inicial (para un mismo valor de r) apenas influye en la estimación ya que los valores de error, $s$, de ésta son relativamente iguales. La elección de $r$ depende del número total de medidas o del intervalo de tiempo de separación entre las mismas; para el problema estudiado el valor $\mathrm{r}=5$ proporciona la mejor estimación. Un valor mayor de $r$ resultaría en mayores desviaciones de la estimación tanto en el tramo final de la misma como en el vértice del triángulo, y ello debido a la influencia negativa en el funcional de los puntos de medida finales y de los puntos en la cercanía del tramo quebrado (vértice del triángulo). En todo caso este efecto es ineludible en la zona final de estimación al disminuir los términos del funcional debido a la ausencia de medidas en ese tramo, inconveniente que puede subsanarse ampliando el rango de medidas más alla del extremo del tiempo de estimación.

En la figura VI.3 se muestra la estimación de la generación interna de calor en función de la temperatura (ver Figura IV.21 para el modelo en red), para $r=5$ y errores en las medidas de $\sigma=0.01$ y 0.05 . Los valores de los puntos iniciales de estimados son 54.08 y 56.64 , respectivamente. Puede apreciarse cómo las estimaciones se acercan sensiblemente a la solución exacta al disminuir $\sigma$, y coincide prácticamente con ella para $\sigma=0.01$.

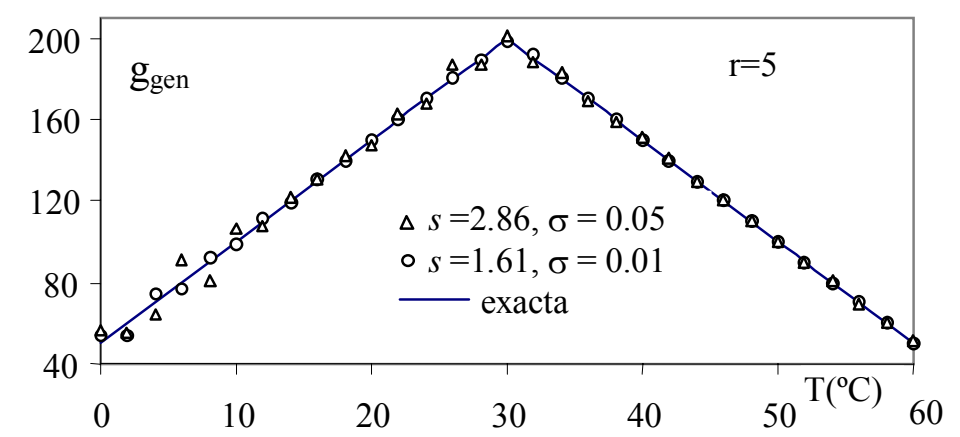

Figura VI.3. Estimación de la generación interna de calor en función de la temperatura del medio 


\section{VI.2. COEFICIENTE DE CONVECCIÓN}

\section{VI.2.1. Introducción}

El coeficiente de transferencia de calor por convección o coeficiente de película es un parámetro fundamental en multitud de problemas de transmisión de calor y son muchos los trabajos que se han dedicado y dedican a su determinación por vía experimental, y a establecer correlaciones empíricas aproximadas para su uso en diferentes aplicaciones [Mc Adams, 1954; Churchill, 1983 y Yuge, 1960]. En cuanto a su estimación vía problema inverso también existen algunos trabajos entre los que cabe citar a Osman y Beck [1990] que estudiaron la dependencia temporal del coeficiente convectivo durante el enfriamiento de una esfera 1-D como un problema no-lineal de estimación de parámetros; emplearon el método de especificación de función secuencial. Maillet y col. [1991], en un problema estacionario, utilizaron el método de elementos de contorno y obtuvieron la dependencia del coeficiente de transferencia de calor con la temperatura para un cilindro 2-D cuya conductividad depende linealmente de la temperatura estudiando, en particular, la influencia de esta dependencia en los resultados.

Hernández-Morales y col. [1992] mediante la aplicación de una técnica inversa, basada en el método de especificación de función secuencial, determinaron inicialmente el flujo de calor y la distribución de temperatura en la superficie de un acero inoxidable sometido a un proceso de enfriamiento mediante agua. A partir de los resultados determinaron el coeficiente de convección en función de la temperatura en la superficie del acero.

Haji-Shelkh y Buckingham [1993] estimaron la dependencia del coeficiente de convección con el tiempo y la posición, mediante el método de Monte Carlo, en un problema inverso de conducción del calor multidimensional. Chantasiriwan [1999], mediante el método de especificación de función secuencial junto con funciones del tipo piece-wise y condiciones de contorno lineales, desarrolló un algoritmo para estimar la dependencia temporal del coeficiente de convección en un problema 1-D.

Oosthuizen y Naylor [1999] emplearon medidas de temperaturas transitorias en distintas localizaciones de la superficie exterior de una pieza de sección cuadrada, sometida a convección forzada, y de una de sección circular, sometida a convección natural, para estimar el coeficiente de convección bajo la forma de una función piece-wise.

Alhama y col. [1999] obtuvieron el coeficiente de convección medio en flujos forzados normales a tubos cilíndricos. Se trata de la determinación de un parámetro constante para el que proponen protocolos sencillos que resuelven con auxilio de Método de simulación por redes. 
Guzik y Nowarski [2001] estimaron el coeficiente de transferencia de calor local durante la ebullición en película, para una selección de fluidos refrigerantes, resolviendo la ecuación de transferencia de calor bidimensional en coordenadas cilíndricas y con propiedades térmicas dependientes de la temperatura. El método de resolución es el llamado método de filtración de mínimos.

Le Masson y col [2001] determinaron el coeficiente convectivo en tratamientos térmicos metalúrgicos rápidos. El algoritmo está basado en el método de regularización iterativa y el método de gradientes conjugados. El procedimiento fue aplicado a dos materiales diferentes, el níquel sin transformación metalúrgica y el acero 16MND5 que sí la tiene, implicando en este último caso fuertes no linealidades en el material.

Woodbury y $\mathrm{Ke}$ [2000] determinaron el coeficiente convectivo en un problema sometido a unas condiciones que hacían posible conocer su solución exacta del problema directo, con lo que fue posible obtener los parámetros de sensibilidad y estudiar como se alteran éstos en función de otros parámetros del problema.

\section{VI.2.2. Aplicaciones}

El sistema físico bajo estudio (Zueco y col. [2001]) es una placa 1-D, de espesor L, densidad $\rho$, conductividad térmica $\mathrm{k}$ y calor específico $\mathrm{c}_{\mathrm{e}}$, sometida a convección libre en una de sus caras, manteniendo la otra bajo condición adiabática, figura VI.4. El modelo matemático completo se recoge en el cuadro VI.3.

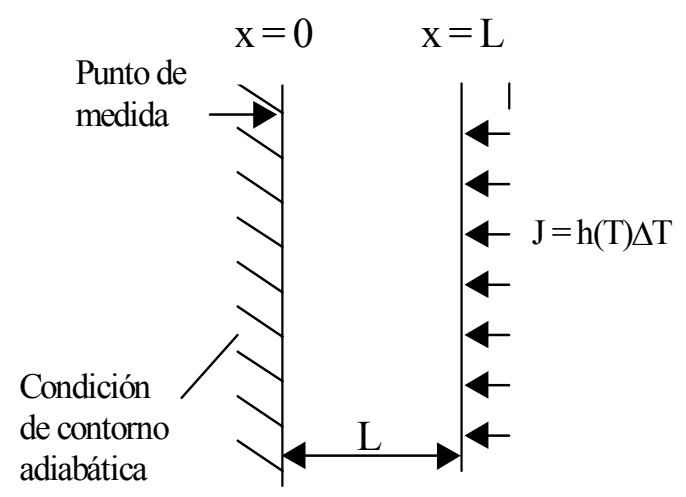

Figura VI.4. Esquema del problema 


\begin{tabular}{|l|l|}
\hline \multicolumn{2}{|c|}{ Modelo matemático } \\
\hline Ecuación diferencial & $\rho \mathrm{c}_{\mathrm{e}}(\mathrm{T}) \partial \mathrm{T} / \partial \mathrm{t}-\mathrm{k}\left(\partial^{2} \mathrm{~T} / \partial \mathrm{x}^{2}\right)=0$ \\
\hline Condición inicial & $\mathrm{T}_{(\mathrm{x}, \mathrm{t}=0),}=\mathrm{T}_{\mathrm{o}}$ \\
\hline Condiciones de contorno & $(\partial \mathrm{T} / \partial \mathrm{x})=\mathrm{h}(\mathrm{T})\left(\mathrm{T}_{\infty}-\mathrm{T}\right)=1.42\left(\mathrm{~T}_{\infty}-\mathrm{T}\right)^{5 / 4} \mathrm{~L}^{-1 / 4}, 10^{9}>\mathrm{G}_{\mathrm{r}} \mathrm{P}_{\mathrm{r}}>10^{4}$ \\
& $(\partial \mathrm{T} / \partial \mathrm{x})=0$ para $\mathrm{x}=\mathrm{L} \quad($ punto de medida $)$ \\
\hline Condición adicional & $\mathrm{T}_{\mathrm{IHCP}}\left(\mathrm{x}_{\mathrm{f}}, \mathrm{t}_{\mathrm{j}}, \varepsilon_{\mathrm{j}}\right)=\mathrm{T}_{\mathrm{DHCP}}\left(\mathrm{x}_{\mathrm{f}}, \mathrm{t}_{\mathrm{j}}\right)+\varepsilon_{\mathrm{j}}$ \\
\hline
\end{tabular}

Cuadro VI.3. Modelo matemático

Se trata de estimar el número de Nusselt, número adimensional que caracteriza la convección libre, $\mathrm{N}_{\mathrm{u}}=\mathrm{hL} / \mathrm{k}$, donde $\mathrm{h}$ es el coeficiente de convección. Se han adoptado los valores numéricos sencillos para las propiedades térmicas y condiciones iniciales y de contorno que se reflejan en el cuadro VI.4.

Densidad: $\rho=10 \mathrm{~kg} \mathrm{~m}^{-3}$, calor específico: $\mathrm{c}_{\mathrm{e}}=1 \mathrm{~J} \mathrm{~kg}^{-1}{ }^{\circ} \mathrm{C}^{-1}$, conductividad: $\mathrm{k}=1 \mathrm{~W} \mathrm{~m}^{-1}{ }^{\circ} \mathrm{C}^{-1}$, longitud: $\mathrm{L}=1 \mathrm{~m}$, temperatura inicial: $\mathrm{T}_{\mathrm{o}}=10^{\circ} \mathrm{C}$, temperatura ambiente: $\mathrm{T}_{\infty}=0{ }^{\circ} \mathrm{C}, \Delta \mathrm{t}=1 \mathrm{~s}$

\section{Cuadro VI.4. Valores numéricos}

Una correlación para un proceso de convección natural es, $\mathrm{N}_{\mathrm{u}}=\mathrm{D}\left(\mathrm{R}_{\mathrm{a}}\right)^{\mathrm{d}}$, donde $\mathrm{R}_{\mathrm{a}}$ es el número Rayleigh. Para régimen laminar $\left(10^{4}<\mathrm{R}_{\mathrm{a}}<10^{9}\right)$, $\mathrm{D}=0.59 \mathrm{y} \mathrm{d}=1 / 4$ (Mc Adams [1954]).

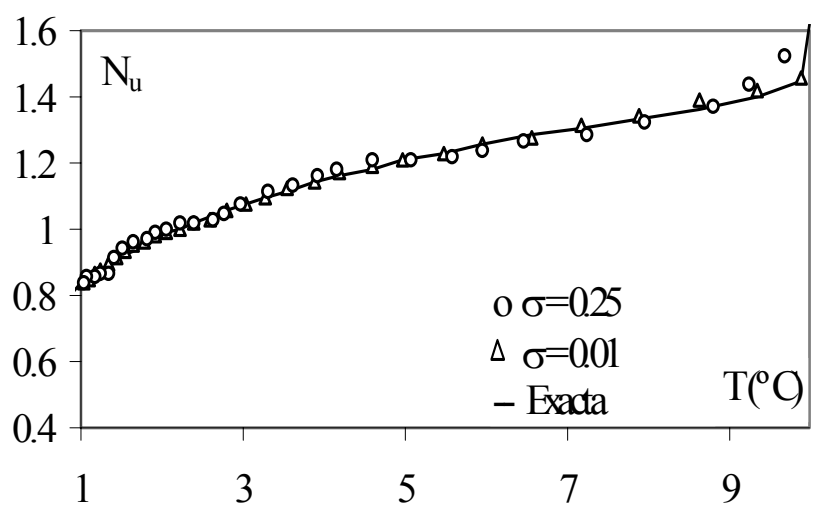

Figura VI.5. Evolución del Número de Nusselt con la temperatura, $\mathrm{r}=7$ 
En la Figura VI.5 se muestra la dependencia del número de Nusselt con la temperatura para la mejor aproximación $(r=7)$ y $\sigma=0.01$ y 0.25 . Las estimaciones de $\mathrm{N}_{\mathrm{u}}(\mathrm{T})$ pueden considerarse bastante aproximadas a la solución exacta.

La influencia del error en la medida y del número de términos del funcional en $s$, se muestra en la tabla VI.1. El valor $\mathrm{r}=7$ proporciona el menor error en ambos casos y, como se esperaba, los errores relativos son menores para el menor error en la medida.

\begin{tabular}{|l|l|l|l|}
\hline \multirow{2}{*}{$\sigma$} & \multicolumn{3}{|c|}{$r$} \\
\cline { 2 - 4 } & 5 & 6 & 7 \\
\hline 0.01 & 1.44 & 1.05 & 0.78 \\
\hline 0.25 & 3.32 & 3.01 & 2.67 \\
\hline
\end{tabular}

Tabla VI.1. Errores $s$ de las estimaciones

\section{VI.3. EMISIVIDAD}

El conocimiento de la emisividad superficial de sólidos es un problema de enorme interés en tanto que la radiación térmica ocurre en numerosas aplicaciones industriales, por ejemplo en las tecnologías avanzadas de solidificación rápida por medio de sprays térmicos y radiadores de gota líquida para aeronaves [Siegel y Howell 1992; Apelian, 1987; Dimitriev, 1993]. A pesar de la no linealidad del proceso, debido a la cuarta potencia de la temperatura en la expresión del flujo de radiación (ley de Stefan-Boltzmann), su solución numérica en la forma de problema inverso puede ser fácil en tanto que se trata de un coeficiente prácticamente independiente de la temperatura [Beck, 1985]. Sin embargo, en procesos en los que se dan simultáneamente los fenómenos de convección y radiación, la primera puede enmascarar el cálculo de la emisividad para determinados valores del coeficiente de transferencia de calor y ciertos intervalos de temperatura, ya que los flujos de radiación pueden ser muy inferiores a los de convección. En convección natural y rangos de temperatura en torno a $300 \mathrm{~K}$ ambos flujos son del mismo orden [Mills, 1992].

Sacadura y Osman [1989] desarrollaron un método para estimar la emisividad en función de la temperatura en superficies opacas. Realizando medidas de temperatura en dos puntos del material es posible estimar la temperatura superficial y el flujo neto de calor de radiación, y deducir posteriormente la emisividad. 
Para obtener esta propiedad en un medio 1-D, se emplea la técnica de estimación de parámetros y se asume la compatibilidad de existencia de radiación más convección. La superficie opuesta es adiabática. Debido, como se ha mencionado, a que los flujos de radiación y convección pueden ser del mismo orden para valores del coeficiente de convección próximos a la convección natural, se estudia la influencia de dicho coeficiente en los resultados, así como la influencia del punto de medida y otros parámetros de interés.

El modelo matemático (Zueco y col. [2002]) se muestra en el cuadro VI.6. $\varepsilon$ la emisividad superficial y $\sigma_{\mathrm{b}}$ la constante de Boltzmann, $\mathrm{T}_{\mathrm{o}}$ la temperatura inicial y $\mathrm{T}_{\infty}$ la temperatura de referencia exterior para la convección y para la radiación (pueden emplearse distintas temperaturas de referencia utilizando el mismo modelo). El resto de los símbolos ya son conocidos.

El modelo en red de las condiciones de contorno se muestra en la figura VI.6.

\begin{tabular}{|l|l|}
\hline \multicolumn{2}{|c|}{ Modelo matemático } \\
\hline Ecuación diferencial & $\rho \mathrm{c}_{\mathrm{e}} \partial \mathrm{T} / \partial \mathrm{t}-\mathrm{k}\left(\partial^{2} \mathrm{~T} / \partial \mathrm{x}^{2}\right)=0$ \\
\hline Condición inicial & $\mathrm{T}_{(\mathrm{x}, \mathrm{t}=0),}=\mathrm{T}_{\mathrm{o}}$ \\
\hline Condiciones de contorno & $-\mathrm{k}(\partial \mathrm{T} / \partial \mathrm{x})_{(\mathrm{x}=0, \mathrm{t})}=\mathrm{h}\left(\mathrm{T}_{\mathrm{x}=0}-\mathrm{T}_{\infty}\right)+\varepsilon \sigma_{\mathrm{b}}\left(\mathrm{T}_{\mathrm{x}=0}{ }^{4}-\mathrm{T}_{\infty}{ }^{4}\right)$ \\
& $(\partial \mathrm{T} / \partial \mathrm{x})_{(\mathrm{x}=\mathrm{L}, \mathrm{t})}=0, \quad$ (punto de medida) \\
\hline Condición adicional & $\mathrm{T}_{\mathrm{IHCP}}\left(\mathrm{x}_{\mathrm{f}}, \mathrm{t}_{\mathrm{j}}, \varepsilon_{\mathrm{j}}\right)=\mathrm{T}_{\mathrm{DHCP}}\left(\mathrm{x}_{\mathrm{f}}, \mathrm{t}_{\mathrm{j}}\right)+\varepsilon_{\mathrm{j}}$ \\
\hline
\end{tabular}

Cuadro VI.5. Modelo matemático

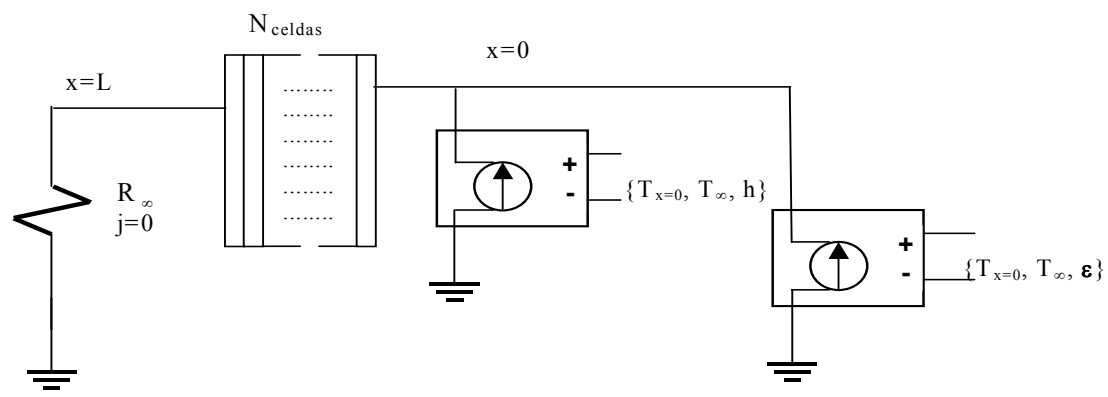

Figura VI.6. Modelo en red de las condiciones de contorno

Se adoptan valores numéricos sencillos para los datos del problema; en unidades S.I., estos son: $\rho=100, \mathrm{c}_{\mathrm{e}}=\mathrm{k}=1, \mathrm{~L}=1, \mathrm{~T}_{\mathrm{o}}=10^{\circ} \mathrm{C}, \mathrm{T}_{\infty}=25^{\circ} \mathrm{C}, \varepsilon=0.8, \mathrm{~h}=0,5,20$ y 100 . Las figuras VI.7 y VI.8 muestran los flujos de calor de radiación y convección, respectivamente, solución del problema directo para diferentes valores de h. Puede observarse que al disminuir h, los flujos de 
calor por radiación son comparables a los de convección, mientras que para valores elevados de h, el fenómeno de radiación queda oscurecido por el de convección.

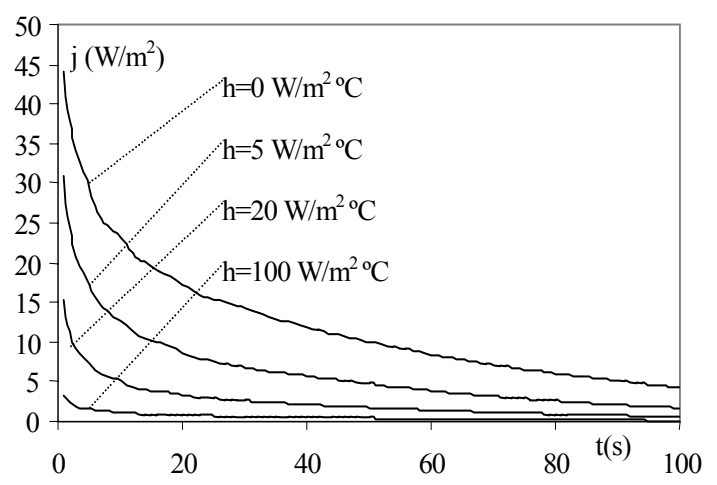

Figura VI.7. Flujos de radiación. h $=0,5,20$ y 100

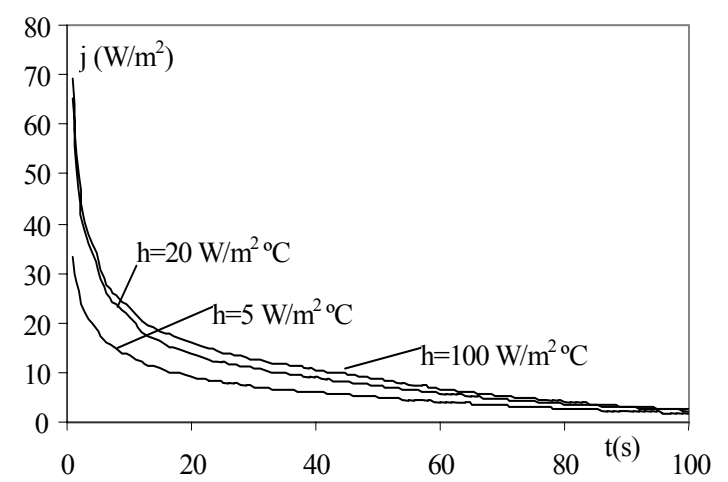

Figura VI.8. Flujos de convección. h = 5, 20 y 100

La tabla VI.2 muestra los intervalos en los que está confinada la estimación de la emisividad para diferentes posiciones del punto de medida; $\mathrm{n}=200, \Delta \mathrm{t}=1 \mathrm{~s}$ y $\sin$ error en las medidas experimentales $(\sigma=0)$ en todos los casos. Puede apreciarse que las soluciones son tanto más precisas (menor intervalo de emisividades) cuanto menor es el coeficiente de convección, es decir cuando los flujos de convección y radiación son comparables o el primero es despreciable frente al segundo. En cuanto a la posición del punto podemos afirmar que no tiene una clara influencia en los resultados. 


\begin{tabular}{|c|c|c|c|c|c|}
\hline & $\mathrm{x}=0$ & $\mathrm{x}=\mathrm{L} / 4$ & $\mathrm{x}=\mathrm{L} / 2$ & $\mathrm{x}=3 \mathrm{~L} / 4$ & $\mathrm{x}=\mathrm{L}$ \\
\hline $\mathrm{h}=0$ & $\varepsilon=0.80$ & $\varepsilon=0.80$ & $\varepsilon=0.80$ & $\varepsilon=0.80$ & $\varepsilon=0.80$ \\
\hline $\mathrm{h}=5$ & $\varepsilon=0.80$ & $\varepsilon=0.80$ & $\varepsilon=0.80$ & $\varepsilon=0.80$ & $\varepsilon=0.80$ \\
\hline $\mathrm{h}=20$ & $0.77 \leq \varepsilon \leq 0.80$ & $0.78 \leq \varepsilon \leq 0.80$ & $\varepsilon=0.80$ & $\varepsilon=0.80$ & $\varepsilon=0.80$ \\
\hline $\mathrm{h}=50$ & $0.76 \leq \varepsilon \leq 0.83$ & $0.74 \leq \varepsilon \leq 0.80$ & $0.78 \leq \varepsilon \leq 0.85$ & $0.75 \leq \varepsilon \leq 0.81$ & $0.78 \leq \varepsilon \leq 0.86$ \\
\hline $\mathrm{h}=100$ & $0.69 \leq \varepsilon \leq 1.0$ & $0.75 \leq \varepsilon \leq 0.90$ & $0.77 \leq \varepsilon \leq 0.9$ & $0.80 \leq \varepsilon \leq 0.86$ & $0.73 \leq \varepsilon \leq 0.80$ \\
\hline
\end{tabular}

Tabla VI.2. Resultados de la estimación para error cero en las medidas

Midiendo en $\mathrm{x}_{\mathrm{f}}=(3 / 4) \mathrm{L}$, para $\mathrm{h}=5, \mathrm{n}=200, \mathrm{y}$ aplicando un error a las medidas experimentales, con diferentes desviaciones típicas. Los resultados de la estimación se muestran en la tabla VI.3. Se observa, como era previsible, que al aumentar $\sigma$ la estimación es menos precisa. Para valores $\sigma<0.1$ la estimación es buena, mientras que para valores $\sigma>0.2$ se cometen errores por encima del $10 \%$. Como información añadida, en la misma tabla se muestran los valores del funcional, $\mathrm{F}$.

\begin{tabular}{|c|c|c|c|c|c|}
\hline $\begin{array}{c}\mathrm{X}=3 \mathrm{~L} / 4 \\
\mathrm{~h}=5\end{array}$ & $\sigma=0$ & $\sigma=0.01$ & $\sigma=0.05$ & $\sigma=0.1$ & $\sigma=0.2$ \\
\hline$\varepsilon$ & 0.8 & 0.801 & 0.797 & 0.795 & 0.893 \\
\hline $\mathrm{F}$ & 0 & $8.8721 \mathrm{E}-02$ & $6.246 \mathrm{E}-01$ & $2.874 \mathrm{E}+00$ & $1.045 \mathrm{E}+01$ \\
\hline
\end{tabular}

Tabla VI.3. Resultados de la estimación con error en las medidas

La tabla VI.4 recoge la influencia del número de medidas para $\mathrm{x}_{\mathrm{f}}=\mathrm{L} / 4$ (próximo a la pared sometida a radiación) con $\mathrm{h}=20 \mathrm{y} \sigma=0$. Al aumentar el número de medidas se estrecha el intervalo de estimación que llega a ser de 0.02 para 200 medidas de temperatura.

\begin{tabular}{|l|c|c|c|c|c|}
\hline $\begin{array}{c}\mathrm{x}=\mathrm{L} / 4 \\
\mathrm{~h}=20\end{array}$ & $\mathrm{~m}=200$ & $\mathrm{~m}=100$ & $\mathrm{~m}=50$ & $\mathrm{~m}=25$ & $\mathrm{~m}=15$ \\
\hline$\varepsilon$ & $0.78 \leq \varepsilon \leq 0.80$ & $0.78 \leq \varepsilon \leq 0.80$ & $0.77 \leq \varepsilon \leq 0.80$ & $0.74 \leq \varepsilon \leq 0.80$ & $0.70 \leq \varepsilon \leq 0.80$ \\
\hline
\end{tabular}

Tabla VI.4. Influencia del número de medidas en la estimación

Se han realizado estimaciones para distintos valores de la temperatura de referencia $\left(\mathrm{T}_{\infty}\right)$ comprobándose que estos apenas influyen en la estimación. 


\section{VI.4 RESISTENCIA TÉRMICA DE CONTACTO}

Se trata de un parámetro fundamental en numerosos problemas de ingeniería térmica cuya determinación es muy complicada debido a los numerosos factores de los que depende; entre estos cabe mencionar la naturaleza de las superficies en contacto, sus rugosidades, el estado de limpieza, la presión de contacto, los gases confinados entre las superficies, etc. Así, es frecuente recurrir a la experimentación para disponer de datos fiables de este parámetro mediante mediciones que, a su vez, entrañan inevitablemente errores de medida. Existen pocos trabajos en la literatura que traten este tema como problema inverso. Beck [1967] propone un método para obtener este parámetro en función del tiempo, analizando los efectos de los coeficientes de sensibilidad en la solución del problema. Este mismo autor, más tarde [1969] insiste en la estimación de la resistencia de contacto proporcionando un criterio de comparación. El mismo autor [1977] desarrolla un método mediante un procedimiento secuencial para la estimación de parámetros característicos en transmisión del calor, coeficientes de transmisión del calor, flujos de calor, emisividad, etc.

Flach y Özisik [1988]; Huang y col. [1989] y finalmente Orlande y Özisik [1993] resuelven el problema por el método de gradientes conjugados.

Milosevic y col [2001] desarrollaron un procedimiento experimental basado en el método denominado "destellos con laser" (laser flash method) para obtener la resistencia de contacto de una muestra de doble capa.

Huang y col [2001] empleando el método de gradientes conjugados estimaron la variación de este parámetro con el tiempo en un intercambiador de calor de placas, con propiedades térmicas dependientes de la temperatura en intercambiador y placas a partir de la medida de temperaturas mediante termopares alojados en la placa.

El modelo matemático del problema directo 1-D (Zueco y col. [2000]), cuyo esquema se muestra en la figura VI.9, es el conjunto de ecuaciones del cuadro VI.6. Dos láminas metálicas iguales de cobre y aluminio están en contacto; la pared externa del aluminio se enfría por convección mientras que la del cobre se mantiene bajo condición adiabática (figura VI.9). 


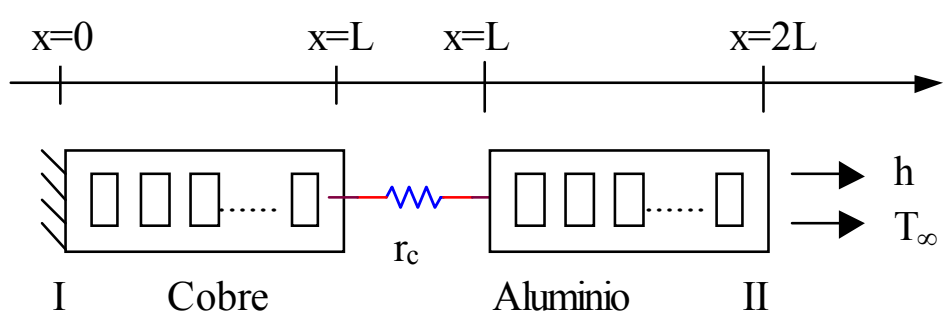

Figura VI.9. Representación esquemática del problema

\begin{tabular}{|l|l|}
\hline \multicolumn{2}{|c|}{ Modelo matemático } \\
\hline Ecuación diferencial & $\rho \mathrm{c}_{\mathrm{e}}(\mathrm{T}) \partial \mathrm{T} / \partial \mathrm{t}-\mathrm{k}\left(\partial^{2} \mathrm{~T} / \partial \mathrm{x}^{2}\right)=0$ \\
\hline Condiciones iniciales & $\mathrm{T}_{(\mathrm{x}, \mathrm{t}=0), \mathrm{Al}}=\mathrm{T}_{\mathrm{o}, \mathrm{Al}}, \quad \mathrm{T}_{(\mathrm{x}, \mathrm{t}=0), \mathrm{Cu}}=\mathrm{T}_{\mathrm{o}, \mathrm{Cu}}$ \\
\hline Condiciones de contorno & $\mathrm{k}_{\mathrm{Al}}(\partial \mathrm{T} / \partial \mathrm{x})_{(\mathrm{x}=2 \mathrm{~L}, \mathrm{t})}=-\mathrm{h}\left(\mathrm{T}_{(\mathrm{x}=2 \mathrm{~L}, \mathrm{t})}-\mathrm{T}_{\infty}\right)$, \\
& $\mathrm{k}_{\mathrm{Cu}}(\partial \mathrm{T} / \partial \mathrm{x})_{(\mathrm{x}=0, \mathrm{t})}=0$ \\
& $\mathrm{k}_{\mathrm{Cu}}(\partial \mathrm{T} / \partial \mathrm{x})_{(\mathrm{x}=\mathrm{L}, \mathrm{t}), \mathrm{Cu}}=\mathrm{k}_{\mathrm{Al}}(\partial \mathrm{T} / \partial \mathrm{x})_{(\mathrm{x}=\mathrm{L}, \mathrm{t}), \mathrm{Al}}$ \\
\hline Condición adicional & $\mathrm{T}_{\mathrm{IHCP}}\left(\mathrm{x}_{\mathrm{f}}, \mathrm{t}_{\mathrm{j}}, \varepsilon_{\mathrm{j}}\right)=\mathrm{T}_{\mathrm{DHCP}}\left(\mathrm{x}_{\mathrm{f}}, \mathrm{t}_{\mathrm{j}}\right)+\varepsilon_{\mathrm{j}}$ \\
\hline
\end{tabular}

Cuadro VI.6. Modelo matemático

Los valores numéricos se muestran en el cuadro VI.7.

$$
\begin{aligned}
& \text { Cu: } \rho=8.954 \mathrm{~kg} \mathrm{~m}^{-3}, \mathrm{c}_{\mathrm{e}}=383.1 \mathrm{~J} \mathrm{~kg}^{-1}{ }^{\circ} \mathrm{C}^{-1}, \mathrm{k}=386 \mathrm{~W} \mathrm{~m}^{-1}{ }^{\circ} \mathrm{C}^{-1}, \mathrm{~L}=1 \mathrm{~m}, \mathrm{~T}_{\mathrm{o}}=25^{\circ} \mathrm{C} \\
& \text { Al: } \rho=2.707 \mathrm{~kg} \mathrm{~m}^{-3}, \mathrm{c}_{\mathrm{e}}=896 \mathrm{~J} \mathrm{~kg}^{-1}{ }^{\circ} \mathrm{C}^{-1}, \mathrm{k}=204 \mathrm{~W} \mathrm{~m}^{-1}{ }^{\circ} \mathrm{C}^{-1}, \mathrm{~L}=1 \mathrm{~m}, \mathrm{~T}_{\mathrm{o}}=25^{\circ} \mathrm{C} \\
& \mathrm{h}=10,100 \text { y } 1000 \mathrm{Wm}^{-2} \mathrm{o}^{-1}, \mathrm{~T}_{\infty}=15^{\circ} \mathrm{C} \\
& \mathrm{r}_{\mathrm{c}} \text { (resistencia térmica de contacto) }=5 \cdot 10^{-3} \mathrm{~m}^{2}{ }^{\circ} \mathrm{C} \mathrm{W}^{-1}
\end{aligned}
$$

\section{Cuadro VI.7. Valores numéricos}

La solución numérica del problema directo, obtenida mediante el Método de simulación por redes con un número total de celdas de 80 (40 para cada lámina metálica) se muestra en la figura VI.10 para distintos valores del coeficiente de convección. Por otro lado, la figura VI.11 representa el campo térmico transitorio de la solución del problema directo, en $\mathrm{x}=0$, junto con las 30 medidas afectadas de error $(\sigma=0.1$ y 0.25$)$ tomadas cada $15 \mathrm{~s}$ para cubrir la totalidad del transitorio $(\mathrm{h}=100)$. En la figura VI.12 es un perfil de temperatura en el medio, donde puede apreciarse el efecto de la resistencia de contacto, en el instante $t=100 \mathrm{~s}(\mathrm{~h}=100)$. 
$\mathrm{T}\left({ }^{\circ} \mathrm{C}\right)$

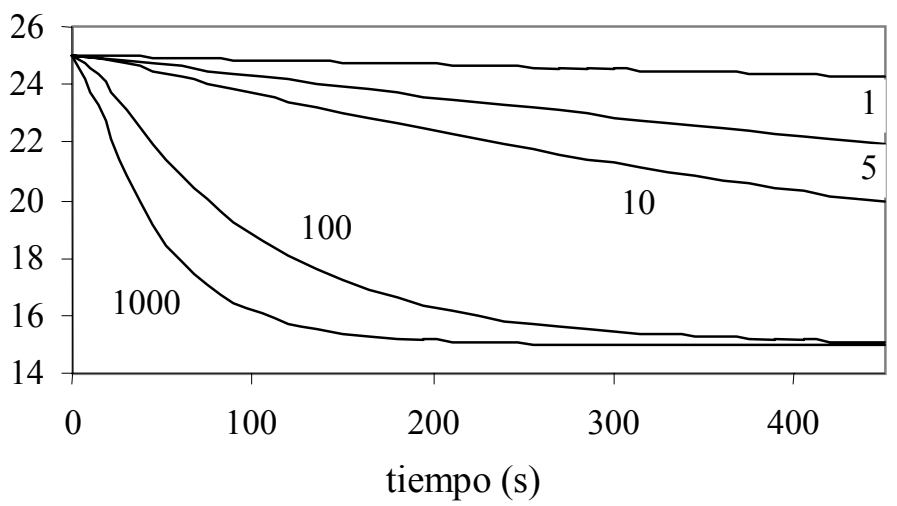

Figura VI.10. Temperaturas exactas en la superficie adiabática cada $15 \mathrm{~s}$, para distintos valores de $\mathrm{h}$

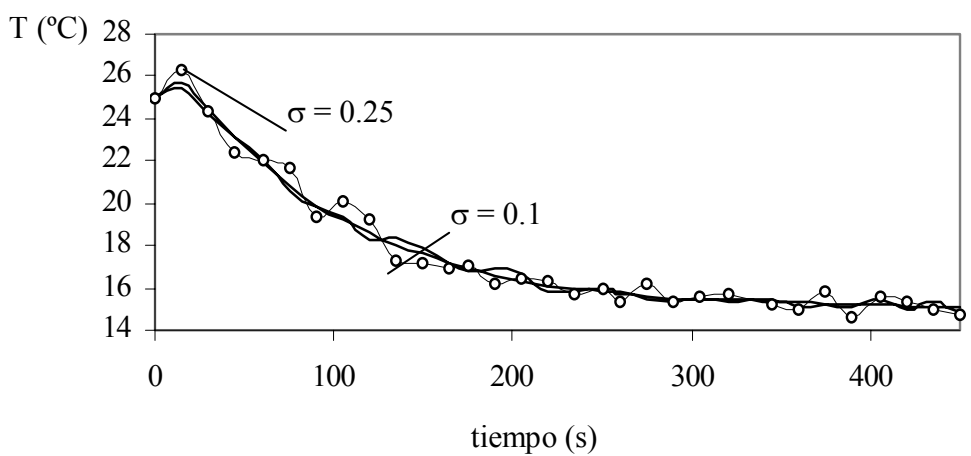

Figura VI.11. Temperaturas exacta y afectada de error $(\sigma=0.1$ y 0.25$)$, en la superficie adiabática cada $15 \mathrm{~s}$, para $\mathrm{h}=100$

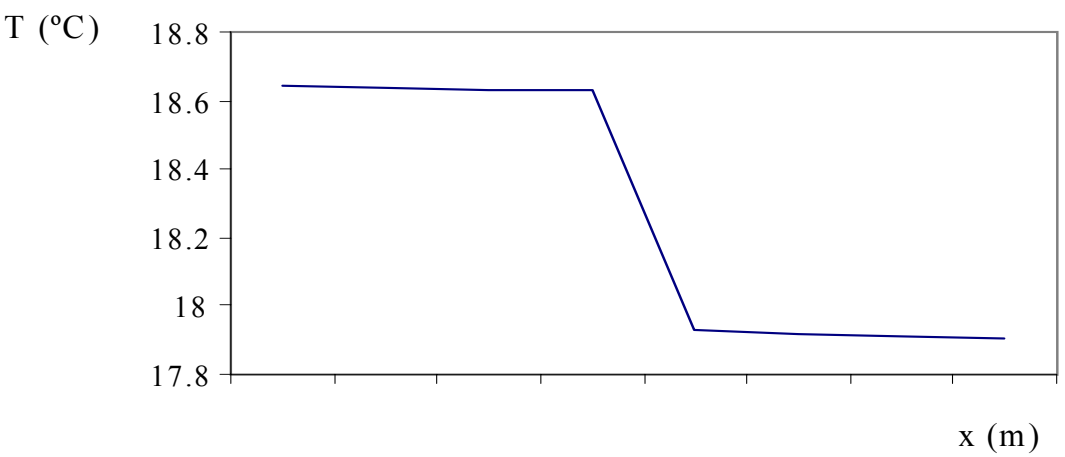

Figura VI.12. Perfil de temperaturas a lo largo del medio, $\mathrm{h}=100, \mathrm{t}=100 \mathrm{~s}$ 


\section{Problema inverso}

La tabla VI.5 muestra los valores estimados de la resistencia térmica de contacto, junto con los valores del funcional, para distintos valores de $h$, sin errores en las medidas y $n=30$. $\Delta \mathrm{t}=30 \mathrm{~s}$, lo supone cubrir los $450 \mathrm{~s}$ que dura aproximadamente el transitorio.

\begin{tabular}{|c|l|l|l|}
\hline $\mathrm{h}$ & \multicolumn{3}{|c|}{$\mathrm{r}_{\mathrm{c}}\left(\mathrm{m}^{2}{ }^{\circ} \mathrm{C} / \mathrm{W}\right)$} \\
\cline { 2 - 4 }$\left(\mathrm{Wm}^{-2}{ }^{{ }^{\circ}} \mathrm{C}^{-1}\right)$ & $\mathbf{4 . 9 \cdot 1 0}^{-3}$ & \multicolumn{1}{|c|}{$\mathbf{5 \cdot 1 0}^{-\mathbf{3}}$} & \multicolumn{1}{|c|}{$\mathbf{5 . 1 \cdot 1 0}$} \\
\hline 1000 & $1.10 \mathrm{E}-02$ & 0 & $6.0 \mathrm{E}-03$ \\
\hline 100 & $2.9 \mathrm{E}-03$ & 0 & $2.7 \mathrm{E}-03$ \\
\hline 10 & $7.0 \mathrm{E}-04$ & 0 & $9.0 \mathrm{E}-04$ \\
\hline 5 & $2.0 \mathrm{E}-04$ & 0 & $6.99 \mathrm{E}-04$ \\
\hline 2 & 0 & 0 & $2.99 \mathrm{E}-04$ \\
\hline 1 & 0 & 0 & 0 \\
\hline
\end{tabular}

Tabla VI.5. Resistencia térmica de contacto y valores del funcional en función de $\mathrm{h}$ ( $\sin$ errores en las medidas, $\Delta \mathrm{t}=30 \mathrm{~s}$ y $\mathrm{n}=30$ )

Al disminuir el valor de $\mathrm{h}$ (proceso más lento) disminuye la sensibilidad de la estimación y ésta se hace más imprecisa. Los pequeños y muy similares valores del funcional, asociados a los valores más precisos de la resistencia térmica de contacto demuestran esta falta de sensibilidad. Se ha comprobado que para el intervalo $5 \leq \mathrm{h} \leq 1000$, el método propuesto da una estimación exacta del parámetro buscado mientras que, por ejemplo, para $\mathrm{h}=2$ se obtienen dos puntos (resistencias de contacto de valores $5 \cdot 10^{-3}$ y $4.9 \cdot 10^{-3}$ ) con funcionales mínimos relativos, y para $\mathrm{h}=1$ se obtienen tres puntos (resistencias de contacto de valores $5 \cdot 10^{-3}, 5.1 \cdot 10^{-3}$ y $4.9 \cdot 10^{-3}$ ) con mínimos relativos del funcional. Para otros valores de $r_{c}$ el funcional siempre es mayor.

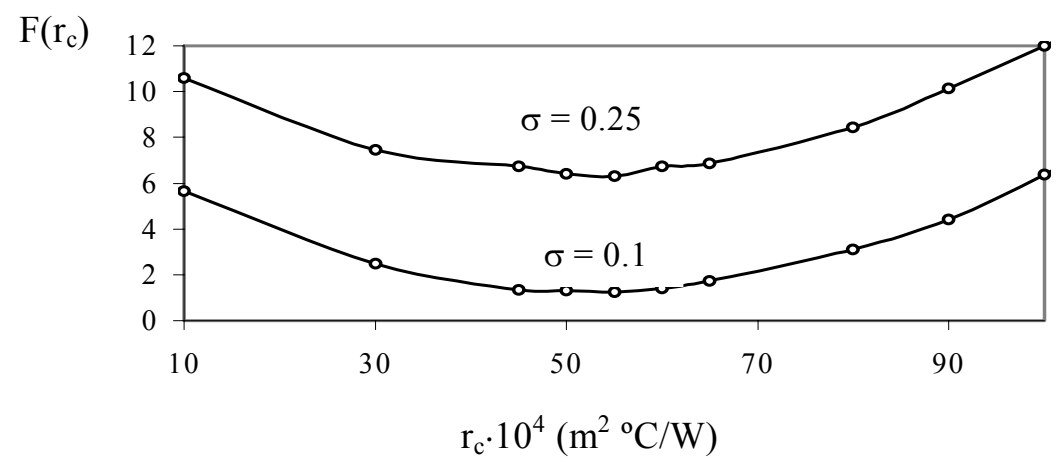

Figura VI.13. Curva de sensibilidad $\mathrm{F}\left(\mathrm{r}_{\mathrm{c}}\right), \sigma=0.1$ y $0.25, \mathrm{~h}=100 \mathrm{Wm}^{-2}{ }^{\circ} \mathrm{C}^{-1}, \Delta \mathrm{t}=15 \mathrm{~s}$ 
Para el proceso más lento y el mismo $\Delta$ t, es preciso aumentar el número de medidas hasta cubrir la práctica totalidad del transitorio, o bien aumentar $\Delta \mathrm{t}$ (para un mismo número de medidas) con el mismo objetivo. Los valores $\Delta \mathrm{t}=15 \mathrm{y} \mathrm{n}=30$ no satisfacen estos requisitos por los que la estimación no es buena. Volviendo a estimar $r_{c}$, $\sin$ errores en las medidas, $\Delta \mathrm{t}=30 \mathrm{~s} \mathrm{y}$ $\mathrm{n}=300$ (a fin de que se ocupe prácticamente la totalidad del transitorio) se obtiene una estimación exacta. Los valores del funcional para $r_{c}=4.9 \cdot 10^{-3}$ y $5.1 \cdot 10^{-3}$ son $7.99 \mathrm{E}-04$ y $8.0 \mathrm{E}-$ 04, respectivamente, cantidades muy pequeñas que indican la dificultad en la estimación con coeficientes de convección pequeños, aún con valores grandes de $\mathrm{m}$. Esta dificultad se incrementa más cuando se introducen errores en las medidas.

La figura VI.13 muestra dos curvas del funcional para la misma serie de valores de $r_{c} y$ desviaciones típicas de los errores de 0.1 y 0.25 . Puede apreciarse que son prácticamente idénticas en su tendencia a excepción del valor medio del funcional que obviamente crece con el error. De las estimaciones se desprende que la solución del problema inverso es $r_{c}=5.2 \cdot 10^{-3}$ ${ }^{\circ} \mathrm{C} / \mathrm{W}$ para $\sigma=0.1 \mathrm{y} \mathrm{r}_{\mathrm{c}}=5.5 \cdot 10^{-3}{ }^{\circ} \mathrm{C} / \mathrm{W}$ para $\sigma=0.25$. Esto supone un error del $4 \%$ en la primera estimación y de $10 \%$ en la segunda, respecto del valor exacto.

La influencia de h, que determina sensiblemente la duración del transitorio, se muestra en las gráficas de las figuras VI.14 y VI.15. Para un enfriamiento rápido, $\mathrm{h}=1000 \mathrm{Wm}^{-2} \mathrm{o}^{-1}$, $\sigma=0.1$ y $n=25$, la expresión del funcional frente a $r_{c}$ se muestra en la figura VI.14. Puede apreciarse mayor sensibilidad en la determinación de la resistencia de contacto (mayor curvatura) que ahora vale $5.1 \cdot 10^{-3} \mathrm{~m}^{20} \mathrm{C} / \mathrm{W}$ (error del $0.2 \%$ ).

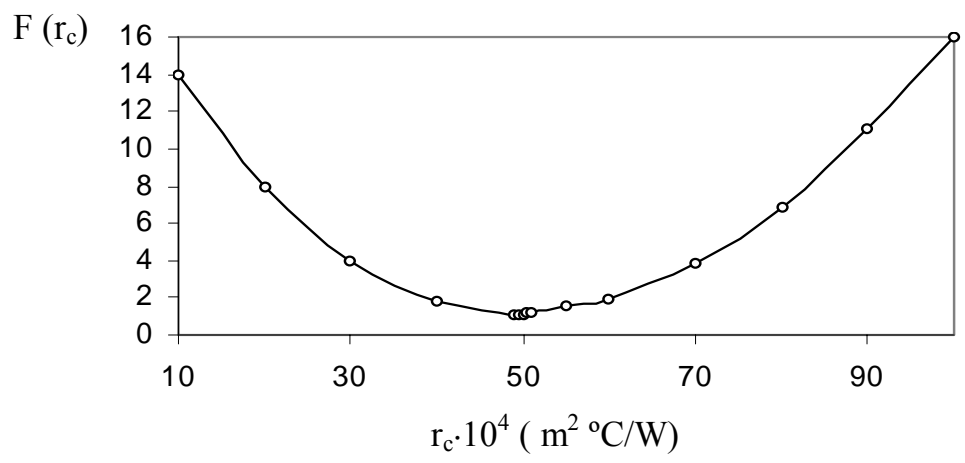

Figura VI.14. Curva de sensibilidad del funcional $\mathrm{F}\left(\mathrm{r}_{\mathrm{c}}\right)$ para $\sigma=0.1$.

$$
\mathrm{h}=1000 \mathrm{Wm}^{-2} \mathrm{C}^{-1}
$$

Por otro lado, un enfriamiento muy lento $(\mathrm{h}=10)$ y un número relativamente pequeño de medidas $(n=30)$ proporciona una curva del funcional F poco sensible (gráfica inferior de la figura VI.17) hasta el punto que es imposible fijar un valor de $r_{c}$. Como se afirmó anteriormente, 
para obtener una solución con coeficientes de transferencia de calor bajos es precio aumentar en número de medidas. Con $\mathrm{n}=200, \Delta \mathrm{t}=30 \mathrm{~s}$ (gráfica superior de la figura VI.15) se aprecia ya cierta sensibilidad en la curva y se obtiene el resultado $r_{c}=4.8 \cdot 10^{-3} \mathrm{~m}^{2}{ }^{\circ} \mathrm{C} / \mathrm{W}$ (error de $4 \%$ respecto del valor exacto).

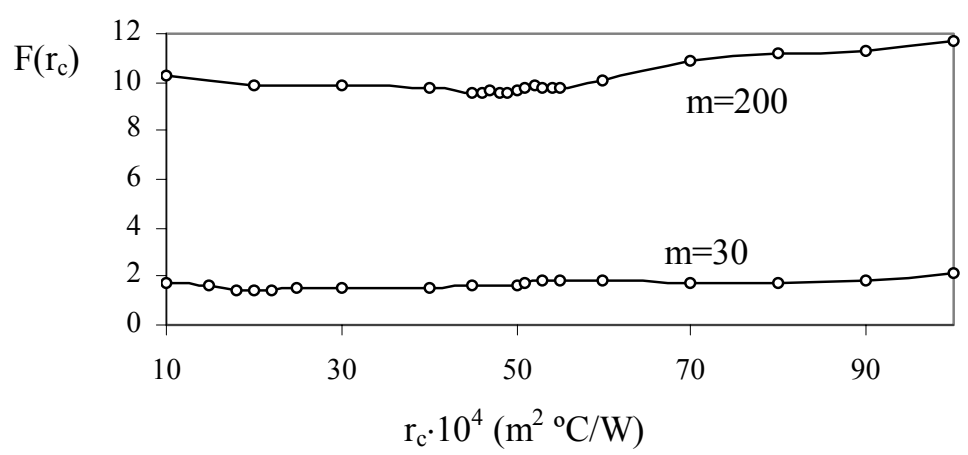

Figura VI.15. Curva de sensibilidad del funcional $F\left(r_{c}\right)$ para $\sigma=0.1$ y $n=30$ y 200 .

$$
\mathrm{h}=10 \mathrm{Wm}^{-2}{ }^{\circ} \mathrm{C}^{-1}
$$

\section{VI.5 REFERENCIAS}

Alhama, F., Campo, A. y González-Fernández, C.F., "Determination of the average convection coefficients in forced flows normal to solid cylinders via an inverse heat conduction problem and the network simulation method", $3^{\text {rd }}$ European Thermal Sciences Conference, 1, 109-114 Heidelberg, Germany (1999)

Apelian, D., In processing of structural metals by rapid solidification, Eds. Freos y Savage, ASM Metals Park, OH (1987)

Beck, J.V., "Transient Sensitivity Coefficients for the Thermal Contact Conductance", Int. J. Heat Mass Transf., 10, 1615-1616 (1967)

Beck, J.V., "Determination of optimum, transient experiments for thermal contact conductance", Int. J. Heat Mass Transf., 12, 621-633 (1969)

Beck, J.V., "Sequential estimation of thermal parameters", J. Heat Transfer, 19, 314-321 (1977)

Beck, J. V., Blackwell, B. y St.Clair.Jr. C.R., "Inverse heat conduction”, New York, John Wiley \& Sons. Inc. (1985)

Chantasiriwan, S., "Inverse heat conduction problem of determining time-dependent heat transfer coefficient", Int. J. Heat Mass Transf., 42, 4275-4285 (1999) 
Churchill, S.W. "Free convection around inmerser bodies”. E.U. Schlünder, Ed.-in-Chief, Heat Exchange Design Handbook, Section 2.5.7, Hemisphere Publishimng, New York (1983)

Dimitriev, A.S., "In Aerospace Heat Exchanger Technology”, Eds. Shah, Elsevier, Amsterdam, The Netherlands (1993)

Flach, G.P. y Özisik, M.N., "Inverse heat conduction problem of periodically contacting surfaces", J. Heat Transfer, 110, 821-829 (1988)

Guzik, A. y Nowarski, A., "Estimation of the local heat transfer coefficients during film boiling for selected cooling fluids", Proc. Eurotherm- Seminar 68. (2001)

Haji-Sheikh, A. y Buckingham, F.P., "Multidimensional inverse heat conduction using the Monte Carlo method", J. Heat Transfer, 115, 26-33 (1993)

Hernández-Morales B., Brimacombe J.K. y Hawbolt E.B., "Application of inverse techniques to determine heat-transfer coefficients in heat-treating operations", J. Mater. Eng. Perform., (1), 763-772 (1992)

Huang, C.H., Özisik, M.N. y Sawaf, B.,"Conjugate gradient method for determining the unknown contact conductance during metal casting", Int. J. Heat Mass Transf., 35, 17791789 (1989)

Huang, C.H., Hsu, G.-C. y Jang, J.-Y.,"A nonlinear inverse problem for the prediction of local thermal contact conductance in plate finned-tube heat exchangers", Heat Mass Tranfer, 37, $351-359(2001)$

Le Masson, P., Loulou, T., Artioukhine, E., Rogeon, P., Carron, D. y Quemener, J.J., “A numerical study for the estimation of a convection heat transfer coefficient during a metallurgical <<jominy end-quench $>>$ test", Proc. Eurotherm Seminar 68, Poitiers, 183$190(2001)$

Maillet, D., Degiovanni, A. y Pasquetti R., "Inverse heat conduction applied to the measurement of heat transfer coefficient on a cylinder: Comparison between an analytical and a boundary element technique", J. Heat Transfer, 113, 549-557 (1991)

Mc Adams, W. H., Heat transmission. 3rd ed., New York, McGraw Hill (1954)

Martin, T.J. y Dulikravich, G.S., "Inverse determination of boundary conditions and sources in steady heat conduction with heat generation", J. Heat Transfer, 118, 546-554 (1996)

Mills, A.F., Heat Transfer, Ed. Richard Irwin, Boston, MA (1992) 
Milosevic, N.D., Raynaud, M. y Maglic, K.D., "Estimation procedure in thermal diffusivity measurement of double-layer sample using the laser flash method", Proc. Eurotherm Seminar 68, Poitiers, 311-318 (2001)

Oosthuizen P. y Naylor D., "A numerical evaluation of a simple procedure for using transient surface temperature measurements to determine local convective heat transfer rates", HTD, 364 (4) Proc. of the ASME (1999)

Orlande, H.R.B. y Özisik, M.N., "Inverse problem of estimating interface conductance between periodically contacting surfaces", J. Thermophys Heat Tr., 7, 2, 319-325 (1993)

Osman A.M. y Beck, J.V., "Investigation of transient heat transfer coefficients in quenching experiments", J. Heat Transfer, 112, 843-848 (1990)

Sacadura, J.F. y Osman, T.T., "Emissivity estimation through the solution of an inverse heatconduction problem". J. Thermophysics, 4, 1, 86-91 (1989)

Siegel, R. y Howell, J.R., "Thermal radiation heat transfer". Hemisphere Publishing Corporation, $3^{\mathrm{a}}$ ed., New York (1992)

Silva A.J. y Özisik, M.N., "Two-dimensional inverse heat conduction problem of estimating the time-varying strength of a line heat source”, J. Appl. Phys., 71 (11) 5357-5362 (1992)

Yang, Ching-Yu, "Non-iterative solution of inverse heat conduction problems in one dimension", Comm. Num. Meth. Eng., 13, 419-427 (1997)

Yang, Ching-Yu, "Solving the two-dimensional inverse heat source problem through the linear least-squares error method", Int. J. Heat Mass Transf., 41, 2, 393-398 (1998)

Yuge T. "Experiments on heat transfer from spheres including combined natural and forced convection”. J. Heat Transfer, 82, 214-220 (1960)

Woodbury Keith, A. y Ke Quanpeng, "An inverse algorithm for direct determination of heat transfer coefficients", Proc. NHTC'00, Pittsburgh, 1-7 (2000)

Zueco, J., Alhama, F., y González-Fernández, C.F., "El problema inverso de la determinación de la resistencia térmica de contacto entre cuerpos y su solución mediante el método de simulación por redes”, Anales de Ingeniería Mecánica, 13 (4), 2567-2572 (2000)

Zueco, J., Alhama, F., y del Cerro, F., "Problema inverso de la determinación del número de Nusselt en convección natural “, Ciencia e Ingeniería, 23 (3) (2002) 
Zueco, J., Alhama, F. y González Fernández, C.F., "El problema inverso de la determinación de emisividades: Solución mediante el método de simulación por redes”, Anales de Ingeniería Mecánica (2002) (en prensa)

\section{CONTRIBUCIONES Y CONCLUSIONES}

Se han obtenido estimaciones precisas de la generación interna de calor para dependencias triangulares de la misma respecto al tiempo y a la temperatura. Se estudia la influencia de la elección del punto inicial de estimación (cuyo efecto sobre ésta es pequeño) y la del error en la medida. En cuanto a r, su valor no puede aumentarse caprichosamente por las desviaciones (que aparecen) en la estimación tanto al final de la misma como en la zona quebrada de la dependencia (vértice del triángulo). Su elección deberá hacerse teniendo en cuenta el número total de medidas dentro del transitorio.

También se han obtenido estimaciones precisas para el coeficiente de convección (o su adimensional, el número de Nusselt) en convección natural, para valores extremos del error en las medidas y funcionales con distinto número de términos, estudiando la influencia de estas variables. Los errores relativos de los Nusselt obtenidos son muy pequeños y, en cualquier caso, aceptables para este tipo de problemas de ingeniería. Comentarios similares a los del párrafo anterior cabe mencionar en relación con los valores de r.

La estimación de emisividades se lleva a cabo considerando la posible simultaneidad de esta condición con la condición de convección, por ser compatibles, estudiándose tanto el caso en que el flujo de convección obscurece el de radiación (valores elevados de h) como el caso en que ambos son del mismo orden de magnitud. Se estudia la influencia, en la estimación, de los siguientes parámetros: posición del punto de medida, error en las medidas, número de éstas y temperatura de referencia para la radiación.

Por último se ha estimado la resistencia térmica de contacto entre cuerpos con la técnica sencilla de estimación de parámetros. Se determina el importante papel que juega, en los resultados de la estimación, el coeficiente de convección de la condición de contorno de uno de los cuerpos en contacto. Para valores muy pequeños de este coeficiente (del orden de la convección natural) da estimaciones muy poco sensibles incluso para un número de medidas relativamente elevado. Por otro lado se comprueba que el rango de medidas debe cubrir la totalidad del transitorio si se quieren estimaciones precisas. Los errores en las medidas también 
juegan un papel importante en estos problemas ya que errores relativamente pequeños producen un error proporcionalmente mayor en la estimación. 


\section{Capítulo VII}

\section{Estimación del calor específico y de la conductividad térmica dependientes de la temperatura}

VII.1. Introducción

VII.2. Estimación del calor especifico

VII.2.1. Dependencia de la temperatura. Convección forzada débil

VII.2.2. Dependencia de la temperatura. Convección natural

VII.2.3. Aplicación

VII.3. Estimación de la conductividad térmica

VII.3.1. Estimación de función

VII.3.2. Estimación de parámetros

VII.4. Estimación simultánea del calor especifico y de la conductividad térmica

VII.4.1. Aplicación

VII.5. Referencias

Contribuciones y conclusiones 


\section{Capitulo VII}

\section{Estimación del calor específico y de la conductividad térmica dependientes de la temperatura}

En este capitulo se resuelven tres tipos de problemas, estimación de la conductividad térmica dependiente de la temperatura, ídem del calor específico y estimación simultánea de ambas características térmicas en medios sólidos 1-D. Se presentan ejemplos de interés técnico en los campos de materiales (elementos puros o aleaciones) y de la industria agro-alimentaria (alimentos). Se aplican técnicas complejas de estimación de función (las más potentes) capaces de aportar estimaciones para cualquier tipo de dependencia, y de estimación de parámetros (más sencillas) con tiempos de computación sensiblemente inferiores. Los modelos en red para la resolución de estos problemas y los protocolos de estimación se expusieron en los capítulos III y IV, respectivamente.

\section{VII.1. INTRODUCCIÓN}

El transporte de calor en medios sólidos está controlado por las propiedades termofísicas conductividad térmica y capacidad calorífica o calor específico (capitulo II). Cuando la conductividad térmica es constante, ambos coeficientes, junto con la densidad, pueden agruparse en una única característica llamada difusividad térmica. Estas propiedades tienen una influencia determinante en la distribución de temperaturas y densidades de flujo de calor durante los procesos transitorios de calentamiento o enfriamiento, distribución que es preciso conocer en numerosas aplicaciones como, por ejemplo, para el diseño de un sistema óptimo de control de estos procesos.

En la mayoría de problemas prácticos de ingeniería, las propiedades termofísicas son dependientes de la temperatura y, en consecuencia, la ecuación de conducción es una ecuación en derivadas parciales no lineal cuya solución, en general, se obtiene por medio de técnicas numéricas, [Carslaw y Jaeger, 1959]. La estimación, pues, de una cualquiera de las propiedades termofísicas de un medio sólido es un problema inverso no lineal de enorme complejidad, en 
cualquier caso mucho más difícil que la estimación de propiedades constantes o, incluso, que la estimación de propiedades con dependencia temporal o espacial. Ni que decir tiene que el interesante problema de la estimación simultánea de ambas propiedades térmicas a partir de las medidas tomadas en un único experimento, estudiado recientemente por algunos autores [Yang, 2000], es aún de mayor complejidad, requiriendo ajustes más finos (por medio de un funcional adecuadamente definido) para conseguir una solución convergente y válida.

En la literatura científica existen numerosas publicaciones que, mediante diferentes técnicas numéricas, estiman las propiedades térmicas dependientes de la temperatura en la forma de problema inverso. Así, Tervola [1988] estima la conductividad térmica dependiente de la temperatura; investiga el uso de diferentes localizaciones para el punto de medida de las temperaturas y la influencia del número de medidas. Ambas elecciones influyen notablemente en la estimación. Por otro lado, Flach y Özisik [1989] y Lam y Yeung [1995] obtienen estimaciones espaciales de esta propiedad térmica.

Bayazitoglu y col. [1989] utilizan técnicas analíticas aproximadas basadas en la transformada de Laplace para estimar la difusividad térmica de materiales a alta temperatura en un medio unidimensional. Se contrasta el método en procesos de enfriamiento para tres materiales, níquel, niobio y paladio, y se estudia el efecto del error en las medidas.

Huang y Ozisik [1991] mediante un método aproximado de integración directa realizan la estimación simultánea de la conductividad térmica y capacidad calorífica, ambas propiedades funciones lineales de la temperatura.

Sawaf y col. [1995], haciendo uso de varios puntos de medida, estiman la dependencia térmica de la conductividad y de la capacidad calorífica en un medio 2-D ortotrópico, empleando el procedimiento iterativo de Levenberg-Marquardt basado en la minimización de un funcional característico.

Huang y col. [1995] utilizan el método de gradiente conjugado, con una ecuación adjunta, para obtener la conductividad térmica dependiente de la temperatura (sinusoidal y exponencialmente); se trata de un problema unidimensional de estimación de función. Los resultados obtenidos son muy precisos y el tiempo de computación pequeño. Estudian los efectos del error en la medida de temperaturas y el número de puntos de medida (dos puntos de medida situados cada uno en una frontera y nueve puntos más de medida en el interior del cuerpo). Huang y Özisik [1991], con el mismo método, obtienen estimaciones simultáneas de la conductividad térmica y la capacidad calorífica en medios 1-D con dependencias sinusoidal y lineal, respectivamente. Estudian la influencia de los errores en las mediciones y, como en el caso anterior, obtienen estimaciones muy precisas con tiempos de computación pequeños. 
Huang y Yan [1995] obtienen también estimaciones simultáneas de las propiedades térmicas en función de la temperatura.

Lam y Yeung [1995] determinan la conductividad térmica en un medio 1-D con generación interna de calor. Emplean dos métodos de resolución basados en diferencias finitas; en el primero parten de un conjunto continuo de medidas de temperatura mientras que en el segundo utilizan un conjunto discreto. Ambos métodos se pueden aplicar a problemas lineales y no lineales, sin conocimiento a priori del tipo de dependencia de la conductividad. Presentan tres aplicaciones: conductividad constante, conductividad dependiente de la posición y conductividad dependiente de la temperatura.

Dantas y Orlande [1996] estimaron las propiedades termofísicas mediante el procedimiento de estimación de función, aplicando el método de gradiente conjugado, y estudiando los efectos en la estimación al modificarse la posición del sensor de medidas.

Yang [1998] desarrolló un modelo inverso lineal para estimar la dependencia con la temperatura de la conductividad térmica de forma explícita en problemas unidimensionales. Se obtiene un sistema de ecuaciones lineales (sistema matricial), cuya resolución se hace de una vez, evitando así el procedimiento iterativo típico en este tipo de problemas. El problema es que es necesario realizar muchas medidas de temperaturas y en puntos interiores del medio. Los resultados muestran que es posible obtener prácticamente la solución exacta si los errores en las medidas son despreciables. Se estudian tres tipos de dependencias, lineal, exponencial y sinosuidal.

Yang [1999] mediante una aproximación iterativa estimó la conductividad térmica dependiente de la temperatura en un medio unidimensional, a partir de medidas realizadas en un punto del contorno del medio, obteniéndose un conjunto de ecuaciones no lineales que es preciso linealizar para obtener la solución. Se consiguen estimaciones muy cercanas a la solución exacta mediante unas pocas iteraciones.

Yang [2000] estima simultáneamente la conductividad térmica (con dependencia exponencial y senoidal) y la capacidad calorífica (con dependencia lineal) en función de la temperatura con un método similar al del trabajo anterior. Son necesarios dos sensores para la medida de temperatura en el medio. Al igual que en el caso anterior, se consigue una solución con pocas iteraciones muy cercana a la solución exacta.

Chantasiriwan [2002] propuso un algoritmo inverso para la estimación de la conductividad térmica dependiente polinómicamente de la temperatura, en procesos estacionarios de conducción del calor en medios homogéneos. El autor propone un método indirecto, donde es necesario resolver inversamente la ecuación de transmisión del calor en un 
medio 2-D. Huang y Chin [2000], que trabajaron con medios no homogéneos, 2-D, emplearon un método indirecto parecido al anterior. Otros trabajos en medios 1-D son los de Jurkowski y col. [1997] que determinaron las conductividades térmicas de termoplásticos durante el cambio de fase; Chen y Lin [1998] que hicieron una estimación simultánea de las propiedades térmicas, y Lesnic y col. [1999] que obtuvieron la conductividad térmica de diferentes materiales conductores. Martin y Dulikravich [2000] determinaron la conductividad térmica en el cobre considerando el medio multidimensional.

Milosevic y col [2001] desarrollaron un procedimiento experimental basado en el método denominado "laser flash method" para obtener la difusividad térmica de una muestra de doble capa con resistencia térmica de contacto. Está basado en la ecuación iterativa de Gauss para la minimización del funcional característico de los problemas inversos.

Kim y col [2002] estimaron de manera directa las propiedades térmicas dependientes de la temperatura en un medio unidimensional en situación transitoria, asumiendo que tiene difusividad térmica constante. Aplicando la transformación de Kirchhoff, el problema de conducción del calor no lineal se convierte en lineal, cuya solución analítica es conocida. Este procedimiento no requiere la realización de inversiones numéricas ni cálculos iterativos. Kim y Lee [2002] obtuvieron la dependencia con la temperatura de las propiedades térmicas de fluidos (gases y aceites) mediante la resolución de un problema de estimación de parámetros.

Zueco y col. [2001 y 2002c] determinan el calor especifico en función de la temperatura, en un medio unidimensional sometido a condiciones adiabática y de convección natural utilizando el Método de simulación por redes y los protocolos de resolución propuestos y explicados en esta tesis doctoral. Con el mismo método, estos autores [2002a y 2002b] han determinado el calor especifico en función de la temperatura (y a partir de él la entalpía) durante el calentamiento por convección forzada en productos de naturaleza orgánica (alimentos), en geometrías cartesiana y esférica, que cambian de fase durante el proceso. Por último, estos autores [2002d] determinan de manera simultánea las propiedades térmicas en medios unidimensionales y aplican los resultados a metales y aleaciones metálicas.

De todo lo expuesto se desprende el interés que suscita este tipo de problemas a cuya solución contribuye el Método de simulación por redes con las estimaciones obtenidas en esta tesis. Todos los casos estimados en este capítulo emplean el método de estimación de función, que no requiere, a priori, información sobre el tipo de dependencia buscada, a excepción del caso de estimación de parámetros (conductividad térmica con dependencia lineal de la temperatura) del apartado VII.3.2. 


\section{VII.2. ESTIMACIÓN DEL CALOR ESPECÍFICO}

El sistema considerado es una placa unidimensional de espesor L, sometida a condición de contorno adiabática en una de sus caras y a convección libre o forzada en la otra. El calor específico o capacidad calorífica es dependiente de la temperatura, mientras que la conductividad térmica se considera constante. El protocolo seguido, que no precisa información alguna sobre la función a estimar, se describe en el capítulo III de esta tesis. El modelo matemático se muestra en el cuadro VII.1.

\begin{tabular}{|c|c|}
\hline \multicolumn{2}{|c|}{ Modelo matemático } \\
\hline $\begin{array}{c}\text { Ecuación } \\
\text { diferencial }\end{array}$ & $\mathrm{C}(\mathrm{T}) \partial \mathrm{T} / \partial \mathrm{t}-\mathrm{k}\left(\partial^{2} \mathrm{~T} / \partial \mathrm{x}^{2}\right)=0$ \\
\hline $\begin{array}{c}\text { Condición } \\
\text { inicial }\end{array}$ & $\mathrm{T}(\mathrm{t}=0)=\mathrm{T}_{0}$ \\
\hline $\begin{array}{c}\text { Condiciones de } \\
\text { contorno }\end{array}$ & $\mathrm{j}(\mathrm{L}, \mathrm{t})=\mathrm{j}_{\text {con-forzada }}$ o $\mathrm{j}_{\text {con-libre }}$ \\
\hline $\begin{array}{c}\text { Condición } \\
\text { adicional }\end{array}$ & $\mathrm{j}(0, \mathrm{t})=0$ \\
\hline \hline
\end{tabular}

Cuadro VII.1. Modelo matemático

$\mathrm{C}$ el calor específico por unidad de volumen, $\mathrm{C}=\mathrm{c}_{\mathrm{e}} \cdot \rho$. Se estudian cuatro aplicaciones correspondientes a los tipos de dependencias mostrados en el cuadro VII.2.

\begin{tabular}{|l|l|}
\hline \multicolumn{2}{|c|}{ Aplicaciones: Dependencias de la capacidad calorífica } \\
\hline Dependencia lineal & $\mathrm{C}=\mathrm{C}_{0}+\mathrm{C}_{1} \mathrm{~T}$ \\
\hline Dependencia sinusoidal & $\mathrm{C}=\mathrm{C}_{0}+\mathrm{C}_{1} \mathrm{sen}(\omega \mathrm{T})$ \\
\hline Dependencia piece-wise & $\mathrm{C}\left(\mathrm{T}_{1}\right)=\mathrm{C}_{1}, \mathrm{C}\left(\mathrm{T}_{2}\right)=\mathrm{C}_{2}, \mathrm{C}\left(\mathrm{T}_{3}\right)=\mathrm{C}_{3}$, \\
& $\left.\mathrm{C}\left(\mathrm{T}_{4}\right)=\mathrm{C}_{4} \mathrm{y} \mathrm{C}_{5}\right)=\mathrm{C}_{5}$ \\
\hline Dependencia rectangular & $\mathrm{C}\left(\mathrm{T}_{1}\right)=\mathrm{C}_{1}, \mathrm{C}\left(\mathrm{T}_{2}\right)=\mathrm{C}_{2}, \mathrm{C}\left(\mathrm{T}_{3}\right)=\mathrm{C}_{3}$, \\
& $\mathrm{C}\left(\mathrm{T}_{4}\right)=\mathrm{C}_{4}, \mathrm{C}\left(\mathrm{T}_{5}\right)=\mathrm{C}_{5} \mathrm{y} \mathrm{C}\left(\mathrm{T}_{6}\right)=\mathrm{C}_{6}$ \\
\hline
\end{tabular}

Cuadro VII.2. Tipos de dependencias de la capacidad calorífica 


\section{VII.2.1. Dependencia de la temperatura. Convección forzada débil}

Se impone la condición de convección forzada en $\mathrm{x}=\mathrm{L}$. Por simplicidad, se han adoptado valores sencillos para los diferentes parámetros y coeficientes del problema. Estos se indican en el siguiente cuadro:

\begin{tabular}{|c|}
\hline $\begin{array}{cc}\text { Convección forzada } \\
\text { Dependencia lineal: } \mathrm{C}_{0}=1 \mathrm{~kJ} \mathrm{~m}^{-3}{ }^{\circ} \mathrm{C}^{-1}, \mathrm{C}_{1}=5 \text { y } 11 \mathrm{~kJ} \mathrm{~m}^{-3}{ }^{\circ} \mathrm{C}^{-2}\end{array}$ \\
\hline Dependencia sinusoidal: $\mathrm{C}_{0}=4 \mathrm{~kJ} \mathrm{~m}^{-3}{ }^{\circ} \mathrm{C}^{-1}, \mathrm{C}_{1}=2 \mathrm{~kJ} \mathrm{~m}^{-3}{ }^{\circ} \mathrm{C}^{-2}, \omega=2 \pi \mathrm{rad} \mathrm{s}^{-1}$ \\
\hline Dependencia piece-wise: $\mathrm{C}_{1}\left(\mathrm{~T}_{1}=0{ }^{\circ} \mathrm{C}\right)=1 \mathrm{~kJ} \mathrm{~m}^{-3}{ }^{\circ} \mathrm{C}^{-1}, \mathrm{C}_{2}\left(\mathrm{~T}_{2}=0.2^{\circ} \mathrm{C}\right)=1 \mathrm{~kJ} \mathrm{~m}^{-3}{ }^{\circ} \mathrm{C}^{-1}, \mathrm{C}_{3}\left(\mathrm{~T}_{3}=0.5\right.$ \\
\hline$\left.{ }^{\circ} \mathrm{C}\right)=6 \mathrm{~kJ} \mathrm{~m}^{-3}{ }^{\circ} \mathrm{C}^{-1}, \mathrm{C}_{4}\left(\mathrm{~T}_{4}=0.8^{\circ} \mathrm{C}\right)=1 \mathrm{~kJ} \mathrm{~m}^{-3}{ }^{\circ} \mathrm{C}^{-1} \mathrm{y} \mathrm{C}_{5}\left(\mathrm{~T}_{5}=1^{\circ} \mathrm{C}\right)=1 \mathrm{~kJ} \mathrm{~m}^{-3}{ }^{\circ} \mathrm{C}^{-1}$ \\
\hline Dependencia rectangular: $\mathrm{C}_{1}\left(\mathrm{~T}_{1}=0^{\circ} \mathrm{C}\right)=1 \mathrm{~kJ} \mathrm{~m}^{-3}{ }^{\circ} \mathrm{C}^{-1}, \mathrm{C}_{2}\left(\mathrm{~T}_{2}=0.299^{\circ} \mathrm{C}\right)=1 \mathrm{~kJ} \mathrm{~m}^{-3}{ }^{\circ} \mathrm{C}^{-1}, \mathrm{C}_{3}\left(\mathrm{~T}_{3}=0.3\right.$ \\
\hline$\left.{ }^{\circ} \mathrm{C}\right)=3 \mathrm{~kJ} \mathrm{~m}^{-3}{ }^{\circ} \mathrm{C}^{-1}, \mathrm{C}_{4}\left(\mathrm{~T}_{4}=0.699^{\circ} \mathrm{C}\right)=3 \mathrm{~kJ} \mathrm{~m}^{-3}{ }^{\circ} \mathrm{C}^{-1}, \mathrm{C}_{5}\left(\mathrm{~T}_{5}=0.7^{\circ} \mathrm{C}\right)=1 \mathrm{~kJ} \mathrm{~m}^{-3}{ }^{\circ} \mathrm{C}^{-1} \mathrm{y} \mathrm{C}_{6}\left(\mathrm{~T}_{6}=1^{\circ} \mathrm{C}\right)=1 \mathrm{~J}$ \\
\hline $\mathrm{m}^{-3}{ }^{o} \mathrm{C}^{-1}$ \\
\hline Otros parámetros: $\mathrm{k}=10 \mathrm{~W} \mathrm{~m}^{-1}{ }^{\circ} \mathrm{C}^{-1}, \mathrm{~L}=0.5 \mathrm{~m}, \mathrm{~T}_{\mathrm{o}}=0{ }^{\circ} \mathrm{C}, \mathrm{T}_{\infty}=1{ }^{\circ} \mathrm{C}, \mathrm{h}=10 \mathrm{~W} \mathrm{~m}^{-2}{ }^{\circ} \mathrm{C}^{-1}$ \\
\hline
\end{tabular}

Cuadro VII.3. Datos numéricos de la convección forzada débil

\section{Dependencia lineal}

Como primer caso de estimación del calor específico se plantea una dependencia lineal con la temperatura, en un sólido 1-D bajo la hipótesis de convección forzada, de acuerdo con el modelo y los datos numéricos expuestos anteriormente.

La solución de temperaturas del problema directo en la superficie adiabática $\left(\mathrm{x}_{\mathrm{f}}=0\right)$, cuya solución se ha obtenido por el MESIR, se muestra en la figura VII.1. La gráfica de la evolución de la temperatura (junto con el intervalo de temperaturas) del transitorio nos permite orientar los valores tanto del intervalo de tiempo entre medidas, $\Delta \mathrm{t}$, como del intervalo de estimación de temperaturas, $\Delta \mathrm{T}_{\mathrm{a}}$, de forma que haya un número aceptable de medidas y que éstas sean sensiblemente diferentes entre sí a lo largo del transitorio. De acuerdo con las condiciones impuestas los valores de $\mathrm{C}(\mathrm{T})$ están comprendidos dentro de un amplio rango (de aproximadamente un orden de magnitud) para asegurar una apreciable no linealidad.

La figura VII.2 muestra las estimaciones para $\Delta \mathrm{t}=1 \mathrm{~s} \mathrm{y} \Delta \mathrm{T}_{\mathrm{a}}=0.1{ }^{\circ} \mathrm{C}$. El número de términos del funcional, de acuerdo con lo indicado en el capítulo III (apartado III.2.1) no es un parámetro que pueda establecerse independientemente de $\Delta \mathrm{t}$ y $\Delta \mathrm{T}_{\mathrm{a}}$ sino que depende de estos valores y se ajusta automáticamente en el propio algoritmo de solución del problema inverso. Las medidas no se han afectado de error. Puede observarse que en ambos casos la estimación es prácticamente coincidente con la solución exacta del problema. 


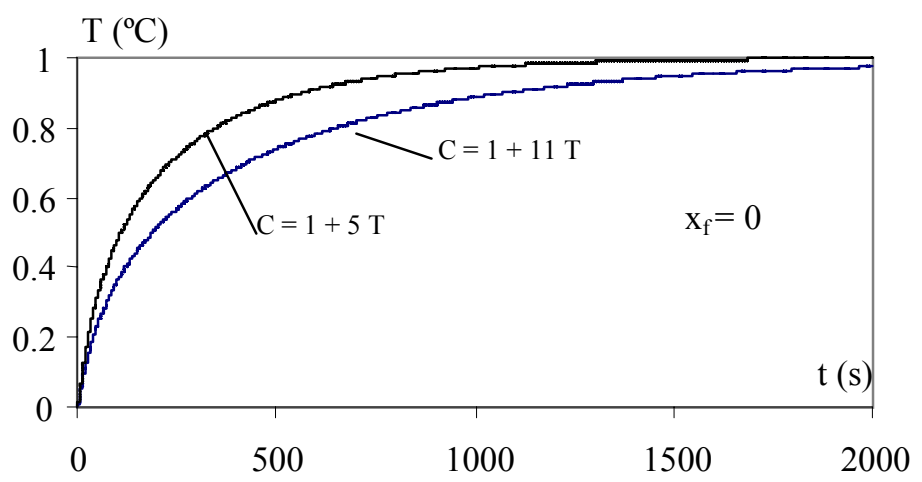

Figura VII.1. Solución del problema directo $\mathrm{T}\left(\mathrm{x}_{\mathrm{f}}=0, \mathrm{t}\right)$.

$$
\mathrm{c}_{\mathrm{e}}=1+11 \mathrm{~T} \text { y } \mathrm{c}_{\mathrm{e}}=1+5 \mathrm{~T}
$$

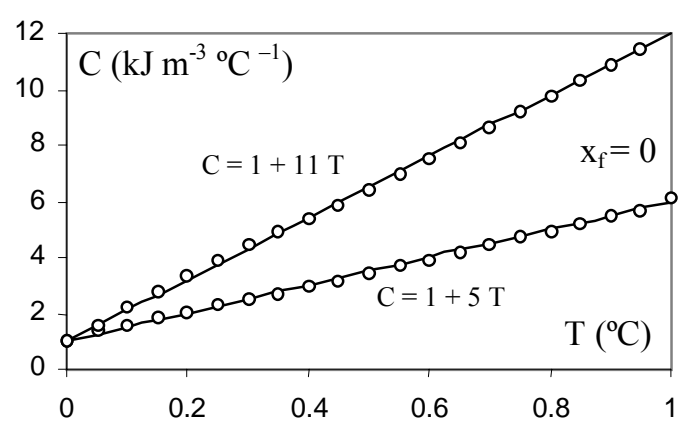

Figura VII.2. Estimación de la capacidad calorífica para dependencias lineales.

Sin error, $\Delta \mathrm{T}_{\mathrm{a}}=0.1{ }^{\circ} \mathrm{C}, \Delta \mathrm{t}=0.1 \mathrm{~s}$

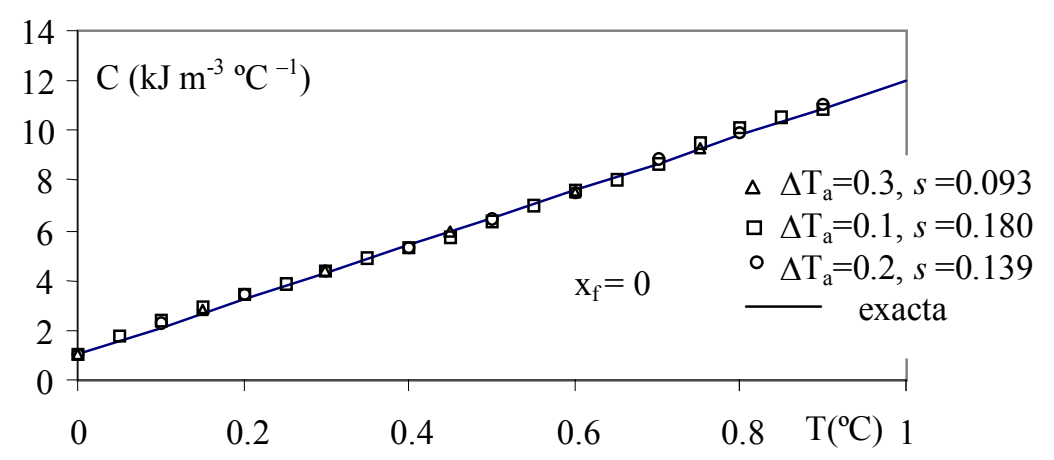

Figura VII.3. Influencia de $\Delta \mathrm{T}_{\mathrm{a}}$ en la estimación de la capacidad calorífica para dependencia lineal. $\mathrm{c}_{\mathrm{e}}=1+11 \mathrm{~T}$, sin error, $\Delta \mathrm{T}_{\mathrm{a}}=0.1,0.2$ y $0.3,{ }^{\circ} \mathrm{C}, \Delta \mathrm{t}=1 \mathrm{~s}$

La figura VII.3 muestra la influencia del intervalo de estimación de temperaturas, $\Delta \mathrm{T}_{\mathrm{a}}$ $\left(0.1,0.2\right.$ y $\left.0.3^{\circ} \mathrm{C}\right)$, para un intervalo de tiempo de medidas $\Delta \mathrm{t}=1 \mathrm{~s} \mathrm{y} \mathrm{c}_{\mathrm{e}}=1+11 \mathrm{~T}$. En este caso 
se observa que la mejor estimación se consigue para $\Delta \mathrm{T}_{\mathrm{a}}=0.3{ }^{\circ} \mathrm{C}$, con un error de 0.093 . El error $s$, muy pequeño, disminuye con el aumento de $\Delta \mathrm{T}_{\mathrm{a}}$.

Por otro lado la influencia del intervalo de tiempo entre medidas se muestra en la figura VII.4, para $\mathrm{c}_{\mathrm{e}}=1+11 \mathrm{~T}$ y $\Delta \mathrm{T}_{\mathrm{a}}=0.1^{\circ} \mathrm{C}$. Se observa que la mejor estimación se consigue para $\Delta \mathrm{t}=$ $0.5 \mathrm{~s}$, con $\mathrm{s}=0.144$, mientras que para los valores superior $(\Delta \mathrm{t}=1.0 \mathrm{~s})$ e inferior $(\Delta \mathrm{t}=0.1 \mathrm{~s}) \mathrm{s}$ es, respectivamente, 0.180 y 0.206 . Se deduce que en este problema existe un valor idóneo para el intervalo de tiempo entre medidas, por encima o por debajo del cual las estimaciones empeoran.

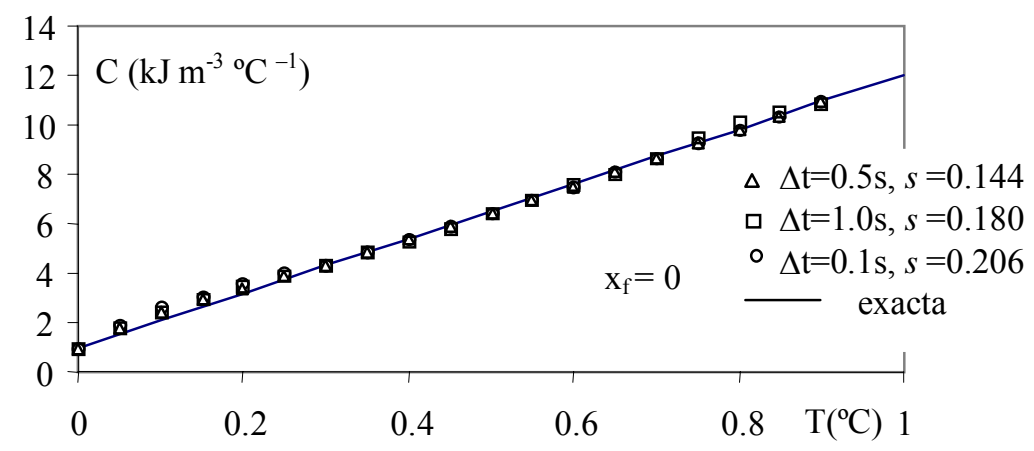

Figura VII.4. Influencia de $\Delta t$ en la estimación de la capacidad calorífica para dependencia lineal. $\mathrm{c}_{\mathrm{e}}=1+11 \mathrm{~T}$, sin error, $\Delta \mathrm{T}_{\mathrm{a}}=0.1{ }^{\circ} \mathrm{C}, \Delta \mathrm{t}=1,0.5,0.1 \mathrm{~s}$

La solución de temperaturas del problema directo para mediciones de temperatura en la posición $\mathrm{x}=\mathrm{L}$ (superficie convectiva), se muestra en la figura VII.5.

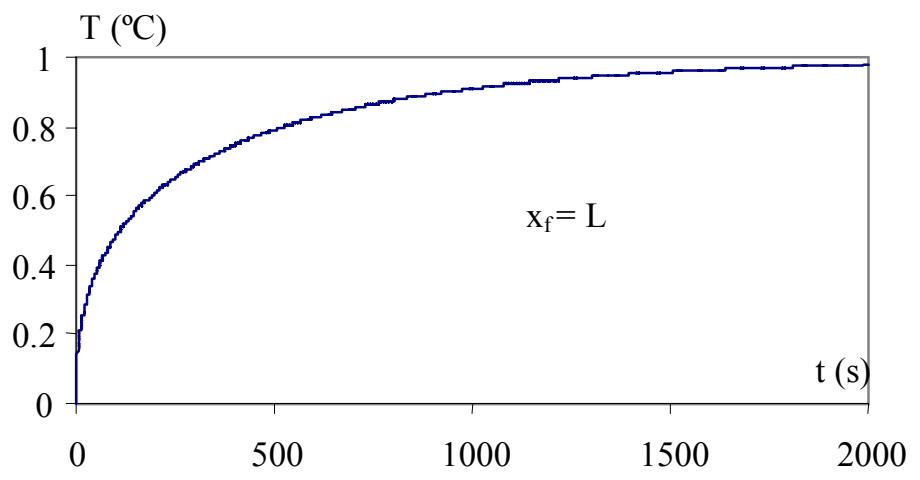

Figura VII.5. Solución del problema directo $\mathrm{T}\left(\mathrm{x}_{\mathrm{f}}=\mathrm{L}, \mathrm{t}\right)$.

$$
\mathrm{c}_{\mathrm{e}}=1+11 \mathrm{~T}
$$

La figura VII.6 muestra la influencia de $\Delta \mathrm{T}_{\mathrm{a}}$ en este caso, para un intervalo de tiempo de medidas $\Delta \mathrm{t}=0.1 \mathrm{~s}$. Los resultados de la estimación mejoran claramente respecto de los 
obtenidos en el caso anterior. La mejor estimación sigue siendo para $\Delta \mathrm{T}_{\mathrm{a}}=0.3{ }^{\circ} \mathrm{C}$, con $s=0.018$ (frente a 0.093 en el caso anterior), siendo la peor estimación también para $\Delta \mathrm{T}_{\mathrm{a}}=0.1{ }^{\circ} \mathrm{C}$ con $s=0.112$ (frente a 0.180 en el caso anterior). También, las estimaciones para $\Delta \mathrm{T}_{\mathrm{a}}=0.2$ y $0.3{ }^{\circ} \mathrm{C}$ están muy próximas cuando el punto de medida está en la superficie convectiva.

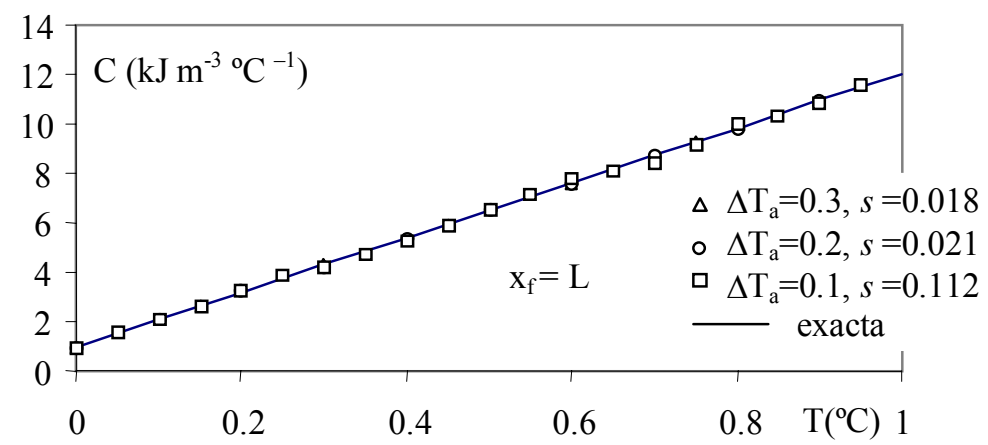

Figura VII.6. Influencia de $\Delta \mathrm{T}_{\mathrm{a}}$ en la estimación de la capacidad calorífica para dependencia lineal. $\mathrm{c}_{\mathrm{e}}=1+11 \mathrm{~T}$, sin error, $\Delta \mathrm{T}_{\mathrm{a}}=0.2$ y $0.3{ }^{\circ} \mathrm{C}, \Delta \mathrm{t}=0.1 \mathrm{~s}$

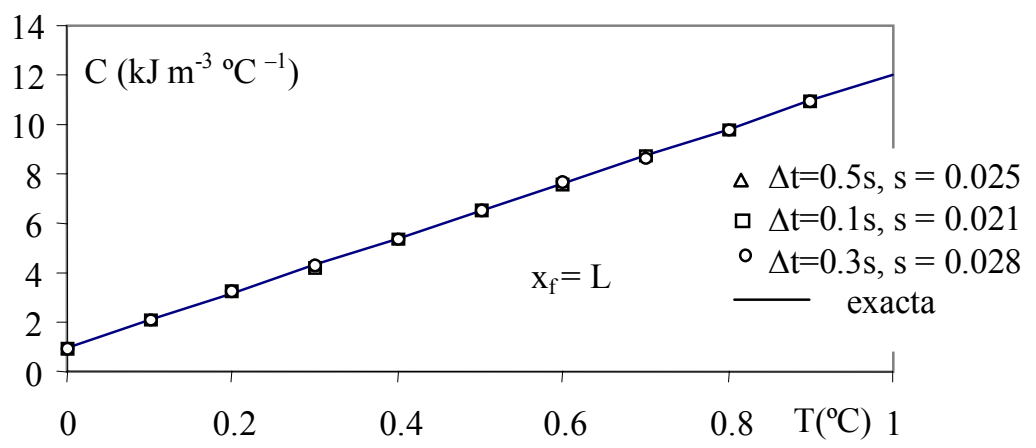

Figura VII.7. Influencia de $\Delta \mathrm{t}$ en la estimación de la capacidad calorífica para dependencia lineal. $\mathrm{c}_{\mathrm{e}}=1+11 \mathrm{~T}$, sin error, $\Delta \mathrm{T}_{\mathrm{a}}=0.2^{\circ} \mathrm{C}, \Delta \mathrm{t}=0.1,0.3$ y $0.5 \mathrm{~s}$

La figura VII.7 muestra la influencia de $\Delta \mathrm{t}$ para el caso de medidas realizadas en el extremo convectivo $\left(\mathrm{x}_{\mathrm{f}}=\mathrm{L}\right)$, con $\Delta \mathrm{T}_{\mathrm{a}}=0.2^{\circ} \mathrm{C}$. Al igual que en la figura VII.6, se observa que los resultados obtenidos mejoran sensiblemente respecto de los obtenidos midiendo en el extremo adiabático $\left(\mathrm{x}_{\mathrm{f}}=0\right)$. Por otro lado, los errores cometidos en las tres estimaciones $(\Delta \mathrm{t}=$ $0.1,0.3$ y $0.5 \mathrm{~s}$ ) son prácticamente idénticos, aunque se aprecia que la mejor estimación en este caso se da para $\Delta \mathrm{t}=0.3 \mathrm{~s}$, con $\mathrm{s}=0.021$ (frente a $s=0.206$ en el caso anterior).

En las figuras VII.8 y VII.9 se analiza la influencia en la estimación del punto inicial. En ambas figuras las medidas de temperatura han sido tomadas en $\mathrm{x}_{\mathrm{f}}=\mathrm{L}$, el intervalo de tiempo 
entre medidas es $\Delta \mathrm{t}=0.5 \mathrm{~s}$, y los valores iniciales 0.1 y 2.0. Puede apreciarse que las oscilaciones de la estimación en torno a la solución exacta tienen lugar para valores iniciales de la estimación por encima y por debajo de su valor exacto a fin de corregir el error que este valor inicial induce en los resultados. En la figura VII.8 $\Delta \mathrm{T}_{\mathrm{a}}=0.3{ }^{\circ} \mathrm{C}$, mientras en la figura VII.9 $\Delta \mathrm{T}_{\mathrm{a}}$ $=0.2{ }^{\circ} \mathrm{C}$. El error máximo de la estimación tiene lugar para $\Delta \mathrm{T}_{\mathrm{a}}=0.3{ }^{\circ} \mathrm{C}$; por otro lado, para $\Delta \mathrm{T}_{\mathrm{a}}=0.2{ }^{\circ} \mathrm{C}$ los errores inducidos por los valores iniciales anteriores son coincidentes.

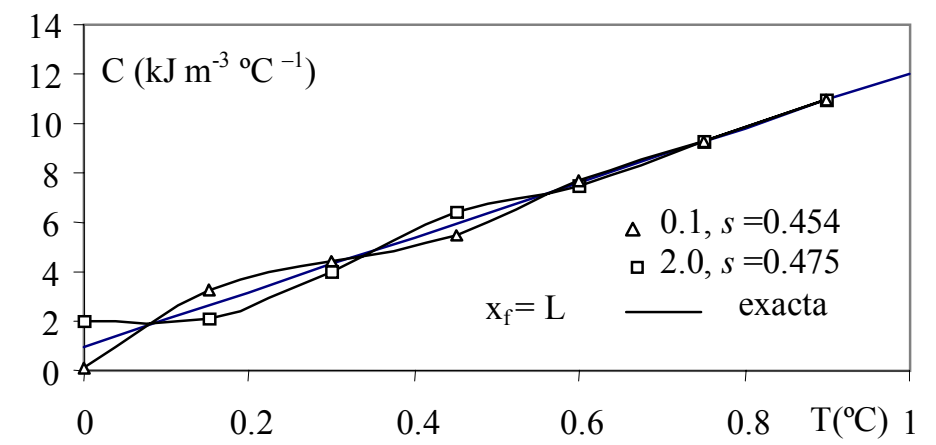

Figura VII.8. Influencia de la estimación del punto inicial para dependencia lineal.

$$
\mathrm{c}_{\mathrm{e}}=1+11 \mathrm{~T} \text {, sin error, } \Delta \mathrm{T}_{\mathrm{a}}=0.3^{\circ} \mathrm{C}, \Delta \mathrm{t}=0.5 \mathrm{~s}
$$

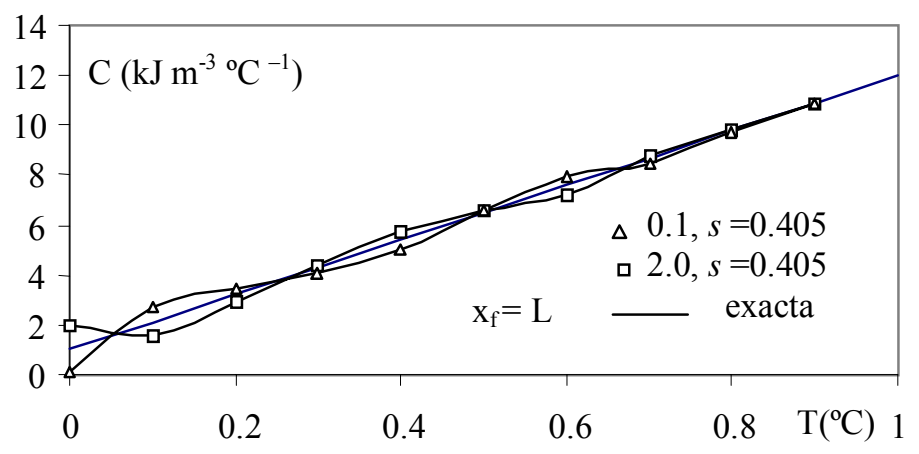

Figura VII.9. Influencia de la estimación del punto inicial para dependencia lineal.

$$
\mathrm{c}_{\mathrm{e}}=1+11 \mathrm{~T} \text {, sin error, } \Delta \mathrm{T}_{\mathrm{a}}=0.2^{\circ} \mathrm{C}, \Delta \mathrm{t}=0.5 \mathrm{~s}
$$

Los valores de los errores finales de las estimaciones de todos los casos anteriores para $\mathrm{c}_{\mathrm{e}}=1+11 \mathrm{~T}$, que permiten comparar la exactitud relativa de las mismas, se muestra en el cuadro VII.4. 


\begin{tabular}{|l|l|l|l|l|l|l|l|}
\hline \multicolumn{5}{|c|}{ Medidas, $\mathrm{x}_{\mathrm{f}}=0$} & \multicolumn{5}{c|}{ Medidas, $\mathrm{x}_{\mathrm{f}}=\mathrm{L}$} \\
\hline$\Delta \mathrm{T}_{\mathrm{a}}(\Delta \mathrm{t}=1 \mathrm{~s})$ & 0.1 & 0.2 & 0.3 & $\Delta \mathrm{T}_{\mathrm{a}}(\Delta \mathrm{t}=0.1 \mathrm{~s})$ & 0.1 & 0.2 & 0.3 \\
\hline$s$ & 0.180 & 0.139 & 0.093 & $s$ & 0.112 & 0.021 & 0.018 \\
\hline$\Delta \mathrm{t}\left(\Delta \mathrm{T}_{\mathrm{a}}=0.1^{\circ} \mathrm{C}\right)$ & 0.1 & 0.5 & 1.0 & $\Delta \mathrm{t}\left(\Delta \mathrm{T}_{\mathrm{a}}=0.2^{\circ} \mathrm{C}\right)$ & 0.1 & 0.3 & 0.5 \\
\hline$s$ & 0.206 & 0.144 & 0.180 & $s$ & 0.021 & 0.028 & 0.025 \\
\hline
\end{tabular}

Cuadro VII.4. Valores de s para dependencias C(T) lineales

\section{Dependencia triangular (o del tipo “piece-wise function”)}

Se trata de un tipo de dependencia cuya estimación tiene, obviamente, mayor dificultad debido a los cambios bruscos de pendiente en varios puntos de la función $\mathrm{C}(\mathrm{T})$ o $\mathrm{c}_{\mathrm{e}}(\mathrm{T})$.

La figura VII.10 muestra el campo de temperaturas solución del problema directo (obtenido mediante el método de simulación por redes), para puntos de medida en $\mathrm{x}_{\mathrm{f}}=0$ y $\mathrm{x}_{\mathrm{f}}=$ L.

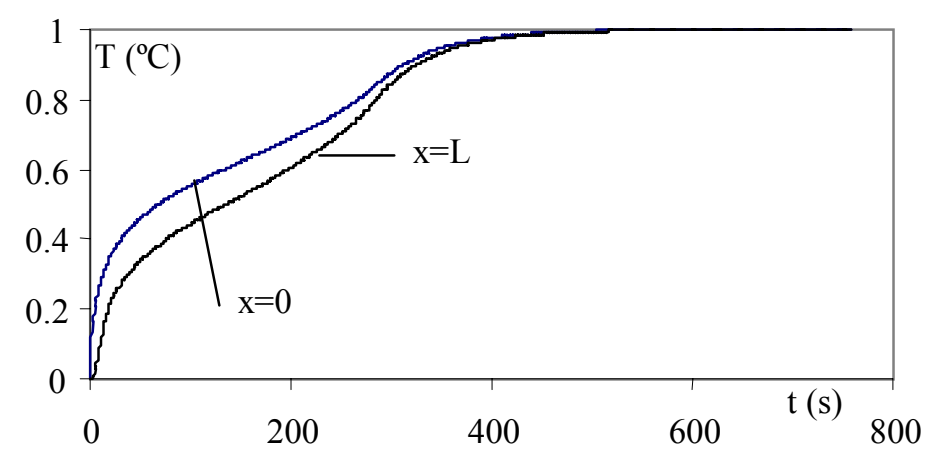

Figura VII.10. Solución del problema directo $\mathrm{T}(\mathrm{x}=0, \mathrm{t})$ y $\mathrm{T}(\mathrm{x}=\mathrm{L}, \mathrm{t})$

La figura VII.11 muestra la influencia del intervalo de estimación de temperaturas, $\Delta \mathrm{T}_{\mathrm{a}}$, para un intervalo de tiempo entre medidas $\Delta \mathrm{t}=0.1 \mathrm{~s}$, con medidas realizadas en $\mathrm{x}_{\mathrm{f}}=\mathrm{L}$. Para $\Delta \mathrm{T}_{\mathrm{a}}$ $=0.2{ }^{\circ} \mathrm{C}$ se obtiene la mejor estimación; con $s=0.237$. Se observa que el error en la estimación crece al aumentar $\Delta \mathrm{T}_{\mathrm{a}}$.

Por otro lado la influencia del intervalo de tiempo entre medidas se muestra en las figuras VII.12 (con medidas realizadas en $\mathrm{x}_{\mathrm{f}}=0$ ) y VII.13 (con medidas realizadas en $\mathrm{x}_{\mathrm{f}}=\mathrm{L}$ ), con $\Delta \mathrm{T}_{\mathrm{a}}=0.2^{\circ} \mathrm{C}$. En el primer caso los errores son superiores (siguiendo la tónica anterior de la estimación de funciones lineales), siendo la mejor estimación para $\Delta \mathrm{t}=1.0 \mathrm{~s}$, con $\mathrm{s}=0.578$; mientras en el segundo caso (figura VII.13), la estimación es mucho más precisa, alcanzándose prácticamente el mismo error para los valores, $\Delta \mathrm{t}=0.1,0.2$ y $0.3 \mathrm{~s}$. En cualquier caso, el 
intervalo temporal entre medidas no afecta significativamente a la dispersión de las estimaciones (como ocurría con la dependencia anterior).

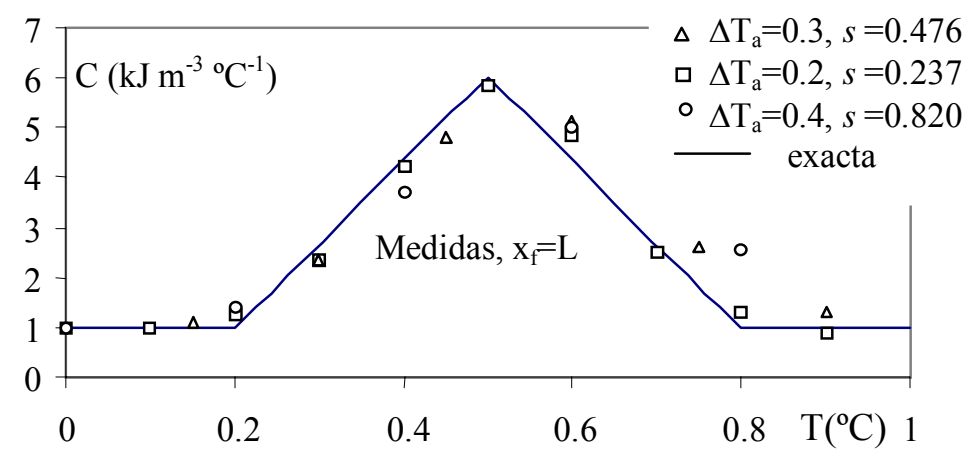

Figura VII.11. Influencia de $\Delta \mathrm{T}_{\mathrm{a}}$ en la estimación, dependencia triangular.

Sin error, $\Delta \mathrm{t}=0.1 \mathrm{~s}, \Delta \mathrm{T}_{\mathrm{a}}=0.2,0.3$ y $0.4^{\circ} \mathrm{C}, \mathrm{x}_{\mathrm{f}}=\mathrm{L}$

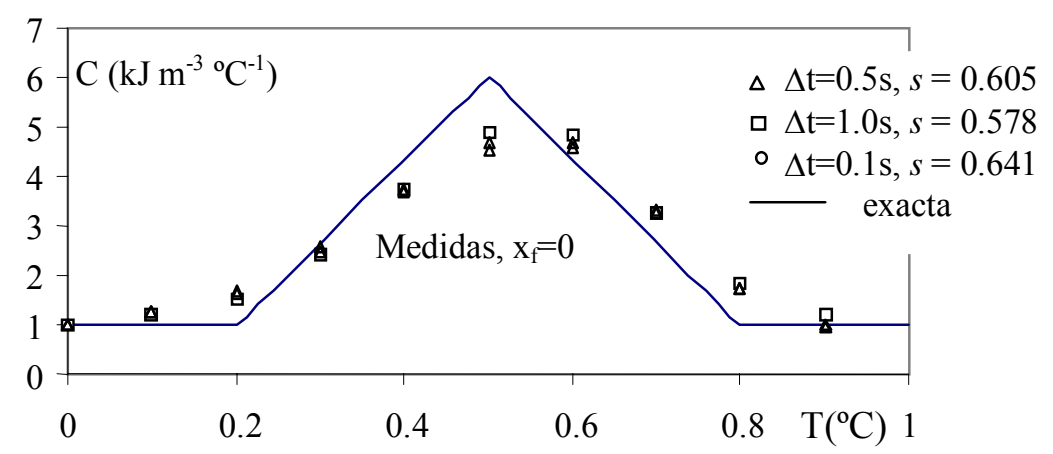

Figura VII.12. Influencia de $\Delta \mathrm{t}$ en la estimación, dependencia triangular.

Sin error, $\Delta \mathrm{t}=0.1,0.5$ y $1 \mathrm{~s}, \Delta \mathrm{T}_{\mathrm{a}}=0.2^{\circ} \mathrm{C}, \mathrm{x}_{\mathrm{f}}=0$

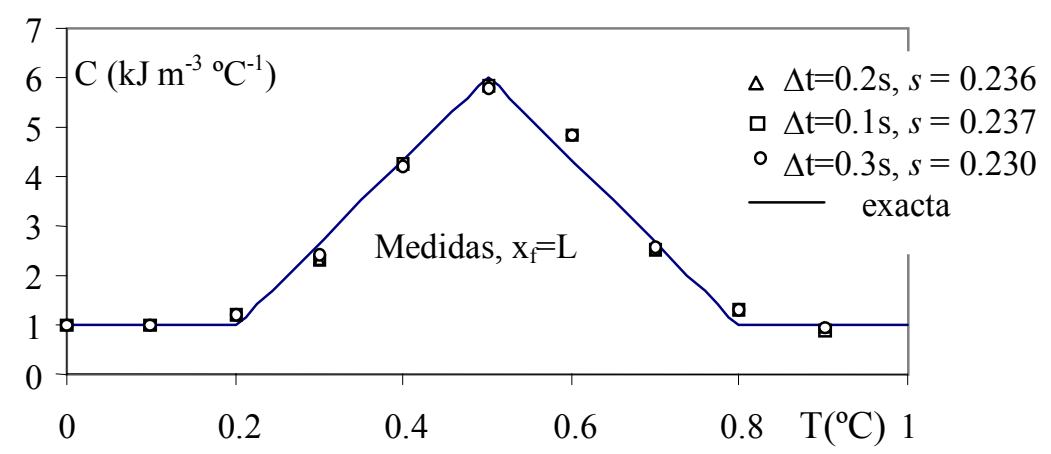

Figura VII.13. Influencia de $\Delta t$ en la estimación, dependencia triangular.

Sin error, $\Delta \mathrm{t}=0.1,0.2$ y $0.3 \mathrm{~s}, \Delta \mathrm{T}_{\mathrm{a}}=0.2{ }^{\circ} \mathrm{C}, \mathrm{x}_{\mathrm{f}}=\mathrm{L}$ 
Los valores de los errores finales de estas estimaciones, que resumen las influencias de las diferentes variables en la estimación, se muestran en los cuadros VII.5 y VII.6.

\begin{tabular}{|l|c|c|c|}
\hline$\Delta \mathrm{T}_{\mathrm{a}}(\Delta \mathrm{t}=0.1 \mathrm{~s})$ & 0.2 & 0.3 & 0.4 \\
\hline$S$ & 0.237 & 0.476 & 0.820 \\
\hline$\Delta \mathrm{t}\left(\Delta \mathrm{T}_{\mathrm{a}}=0.2^{\circ} \mathrm{C}\right)$ & 0.1 & 0.2 & 0.3 \\
\hline$S$ & 0.237 & 0.236 & 0.230 \\
\hline
\end{tabular}

Cuadro VII.5. Errores $s$ de las estimaciones para dependencias $\mathrm{C}(\mathrm{T})$ triangulares, $\mathrm{x}_{\mathrm{f}}=\mathrm{L}$

\begin{tabular}{|l|l|l|l|}
\hline$\Delta \mathrm{t}\left(\Delta \mathrm{T}_{\mathrm{a}}=0.2\right)$ & 0.1 & 0.5 & 1.0 \\
\hline$S$ & 0.641 & 0.605 & 0.578 \\
\hline
\end{tabular}

Cuadro VII.6. Errores $s$ de las estimaciones para dependencias $\mathrm{C}(\mathrm{T})$ triangulares, $\mathrm{x}_{\mathrm{f}}=0$

\section{Dependencia sinusoidal}

Se trata de una dependencia más suave que la triangular por lo que las estimaciones se obtienen con relativa mayor facilidad. La figura VII.14 muestra la solución (MESIR) del problema directo, para medidas realizadas en los puntos $\mathrm{x}_{\mathrm{f}}=0 \mathrm{y} \mathrm{x}_{\mathrm{f}}=\mathrm{L}$.

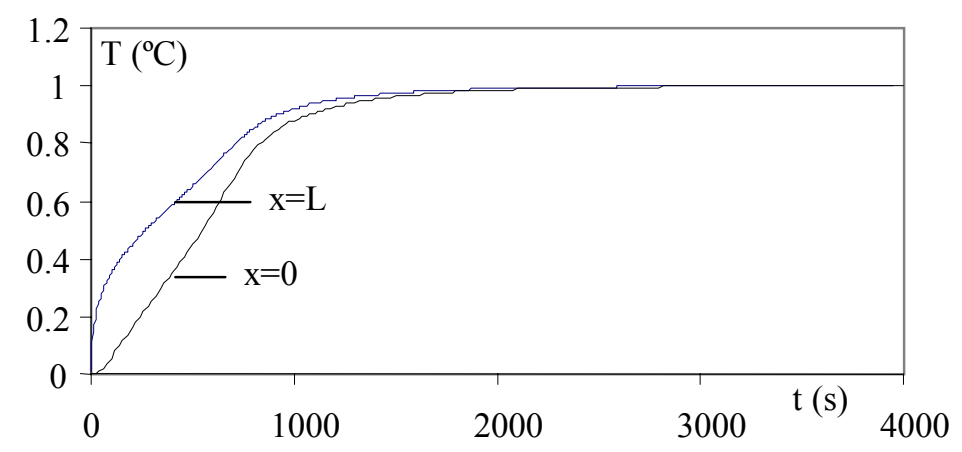

Figura VII.14. Solución del problema directo $\mathrm{T}(\mathrm{x}=0, \mathrm{t})$ y $\mathrm{T}(\mathrm{x}=\mathrm{L}, \mathrm{t})$

Dependencia sinusoidal

La figura VII.15 muestra la influencia del intervalo de temperaturas, $\Delta \mathrm{T}_{\mathrm{a}}$, en las estimaciones, con $\mathrm{x}_{\mathrm{f}}=\mathrm{L}$ y $\Delta \mathrm{t}=0.1 \mathrm{~s}$. Para $\Delta \mathrm{T}_{\mathrm{a}}=0.2^{\circ} \mathrm{C}$ se obtiene la mejor estimación aunque para $\Delta \mathrm{T}_{\mathrm{a}}=0.3^{\circ} \mathrm{C} \mathrm{s}$ es muy similar. Para el caso de $\Delta \mathrm{T}_{\mathrm{a}}=0.4{ }^{\circ} \mathrm{C}$ el error obtenido, es prácticamente el doble del obtenido en la mejor estimación. 
Por otro lado la influencia del intervalo de tiempo entre medidas se muestra en la figura VII.16, $\mathrm{x}_{\mathrm{f}}=\mathrm{L}$ y $\Delta \mathrm{T}_{\mathrm{a}}=0.2^{\circ} \mathrm{C}$. Para $\Delta \mathrm{t}=0.3 \mathrm{~s}$ se obtiene $s=0.039$, mientras que para $\Delta \mathrm{t}=0.1 \mathrm{y}$ $0.5 \mathrm{~s}$ se obtienen valores de $s$ de 0.109 y 0.052 , respectivamente. Al igual que en las dependencias anteriores, no es posible determinar a priori, cuál es el valor idóneo de $\Delta \mathrm{t}$.

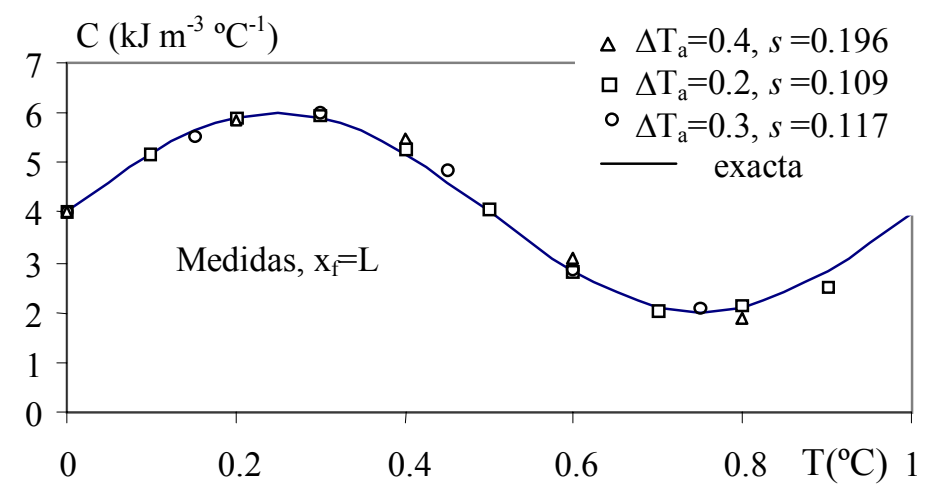

Figura VII.15. Influencia de $\Delta \mathrm{T}_{\mathrm{a}}$ en la estimación sinusoidal.

Sin error, $\Delta \mathrm{t}=0.1 \mathrm{~s}, \Delta \mathrm{T}_{\mathrm{a}}=0.1,0.3$ y $0.4^{\circ} \mathrm{C}, \mathrm{x}_{\mathrm{f}}=\mathrm{L}$

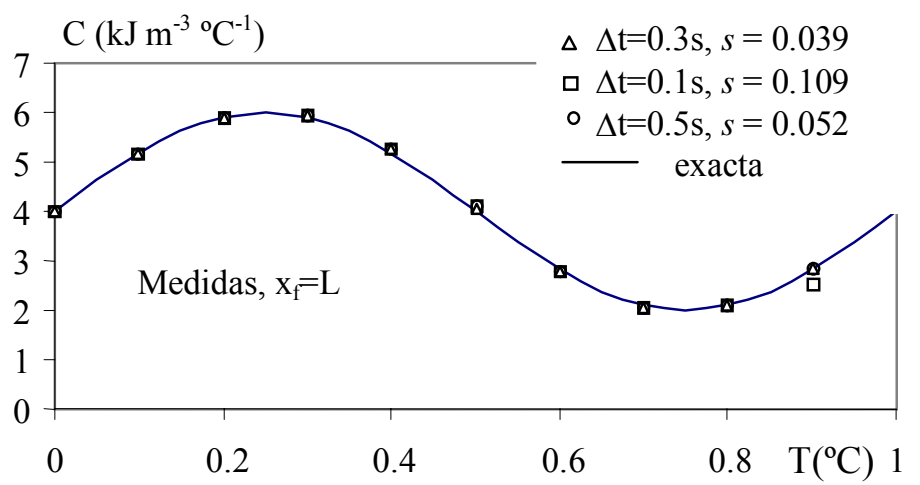

Figura VII.16. Influencia de $\Delta t$ en la estimación sinusoidal.

Sin error, $\Delta \mathrm{t}=0.1,0.3$ y $0.5 \mathrm{~s}, \Delta \mathrm{T}_{\mathrm{a}}=0.2{ }^{\circ} \mathrm{C}, \mathrm{x}_{\mathrm{f}}=\mathrm{L}$

La figura VII.17 muestra la influencia del intervalo de tiempo entre medidas de temperaturas, $\Delta t$, en las estimaciones, en el caso de medidas de temperaturas efectuadas en el extremo adiabático, con $\Delta \mathrm{T}_{\mathrm{a}}=0.2^{\circ} \mathrm{C}$. Se observa que los errores cometidos son mayores que en el caso de medir en $\mathrm{x}=\mathrm{L}$. Para $\Delta \mathrm{t}=2.0 \mathrm{~s}$ se obtiene la mejor aproximación; también, para $\Delta \mathrm{t}=$ 0.5 y $1.0 \mathrm{~s}$ los errores son muy próximos al anterior. 
La influencia en la estimación del valor inicial se muestra en la figura VII.18, en el caso de medidas realizadas en el extremo adiabático, con $\Delta \mathrm{t}=1.0$ s y $\Delta \mathrm{T}_{\mathrm{a}}=0.1{ }^{\circ} \mathrm{C}$. Como era de esperar los errores obtenidos para puntos iniciales alejados del valor exacto aumentan; en concreto para los puntos 3.5 y 4.5 se obtienen errores de 0.125 y 0.139 respectivamente, frente al valor de 0.064 para el punto inicial 4.0. Sin embargo se puede observar claramente que una pequeña variación en la estimación del punto inicial apenas al proceso global de estimación.

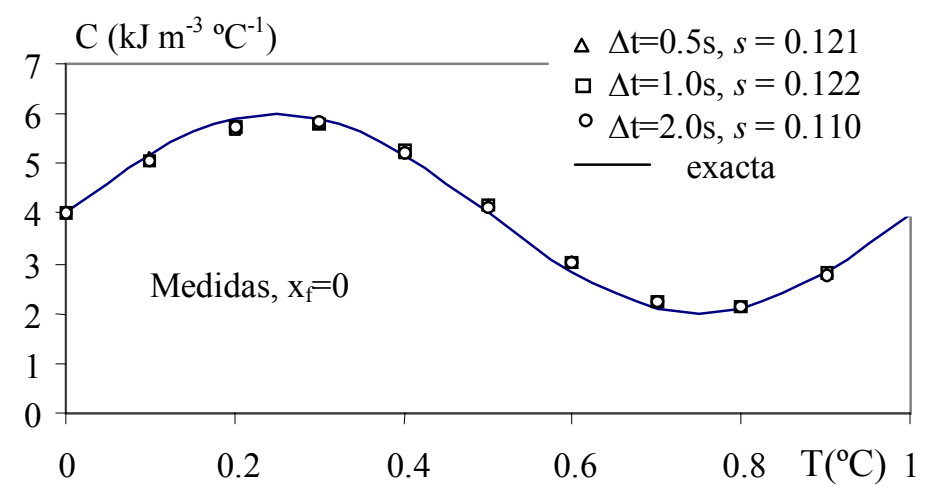

Figura VII.17. Influencia de $\Delta \mathrm{t}$ en la estimación sinusoidal.

Sin error, $\Delta \mathrm{t}=0.5,1.0$ y $2.0 \mathrm{~s}, \Delta \mathrm{T}_{\mathrm{a}}=0.2^{\circ} \mathrm{C}, \mathrm{x}_{\mathrm{f}}=0$

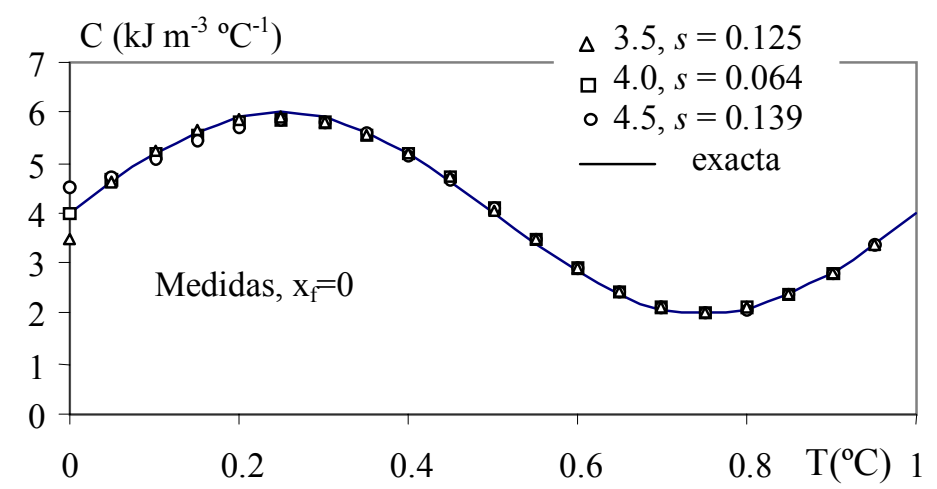

Figura VII.18. Influencia del punto inicial en la estimación sinusoidal.

Sin error, $\Delta \mathrm{t}=1.0 \mathrm{~s}, \Delta \mathrm{T}_{\mathrm{a}}=0.1^{\circ} \mathrm{C}, \mathrm{x}_{\mathrm{f}}=0$

Los valores finales de $s$ para esta dependencia, que resumen las influencias de las diferentes variables en la estimación, se muestran en los cuadros VII.7 y VII.8. 


\begin{tabular}{|l|c|l|l|}
\hline$\Delta \mathrm{T}_{\mathrm{a}}(\Delta \mathrm{t}=0.1)$ & 0.2 & 0.3 & 0.4 \\
\hline$s$ & 0.109 & 0.117 & 0.196 \\
\hline$\Delta \mathrm{t}\left(\Delta \mathrm{T}_{\mathrm{a}}=0.2\right)$ & 0.1 & 0.2 & 0.3 \\
\hline$s$ & 0.109 & 0.039 & 0.052 \\
\hline
\end{tabular}

Cuadro VII.7. Errores $s$ de las estimaciones para dependencias $\mathrm{C}(\mathrm{T})$ sinusoidales, $\mathrm{x}_{\mathrm{f}}=\mathrm{L}$

\begin{tabular}{|l|c|c|c|}
\hline$\Delta \mathrm{t}\left(\Delta \mathrm{T}_{\mathrm{a}}=0.2\right)$ & 0.5 & 1.0 & 2.0 \\
\hline$S$ & 0.121 & 0.122 & 0.110 \\
\hline
\end{tabular}

Cuadro VII.8. Errores $s$ de las estimaciones para dependencias $C(T)$ sinusoidales, $x_{f}=0$

\section{Dependencia rectangular}

La figura VII.19 muestra la solución mediante el MESIR del problema directo. El intervalo de temperaturas y el intervalo de tiempo entre medidas, $\Delta \mathrm{T}_{\mathrm{a}}$ y $\Delta \mathrm{t}$, influyen notablemente en la estimación para este tipo de señal con discontinuidades pronunciadas. La elección de valores altos para $\Delta \mathrm{T}_{\mathrm{a}} \mathrm{y} \Delta \mathrm{t}$ conduce a estimaciones muy distorsionadas.

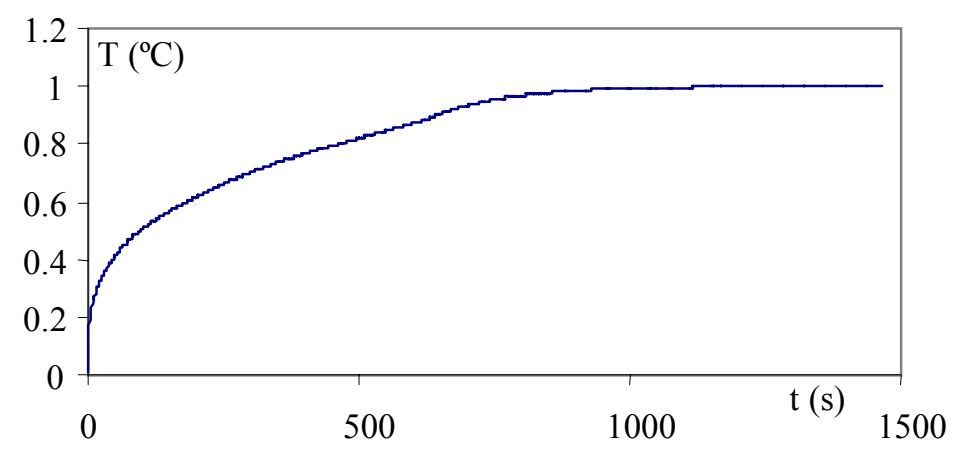

Figura VII.19. Solución del problema directo, $\mathrm{T}\left(\mathrm{x}_{\mathrm{f}}=\mathrm{L}, \mathrm{t}\right)$.

Dependencia rectangular

En las figuras VII.20, VII.21 y VII.22 se muestran estimaciones, para distintos valores de $\Delta \mathrm{T}_{\mathrm{a}}$ y $\Delta \mathrm{t}$. En la primera, $\Delta \mathrm{T}_{\mathrm{a}}=0.3$ y $0.2^{\circ} \mathrm{C}$, con $\Delta \mathrm{t}=0.1 \mathrm{~s}$, mientras que en la segunda $\Delta \mathrm{T}_{\mathrm{a}}=$ 0.4 y $0.2{ }^{\circ} \mathrm{C}$, con $\Delta \mathrm{t}=0.2 \mathrm{~s}$. Puede apreciarse (para $\Delta \mathrm{T}_{\mathrm{a}}=0.2{ }^{\circ} \mathrm{C}$ ) que el error obtenido en la estimación es prácticamente el mismo. Finalmente en la figura VII.22 $\Delta \mathrm{T}_{\mathrm{a}}=0.06,0.1$ y $0.2 \mathrm{y} \Delta \mathrm{t}$ $=0.01 \mathrm{~s}$, obteniéndose resultados mejores. En todos los casos el punto de medida se realiza en el 
extremo convectivo, que es el que muestra mayor sensibilidad a la estimación. En general, se trata de estimaciones menos precisas que para los otros tipos de dependencias a causa de las fuertes discontinuidades como se ha mencionado.

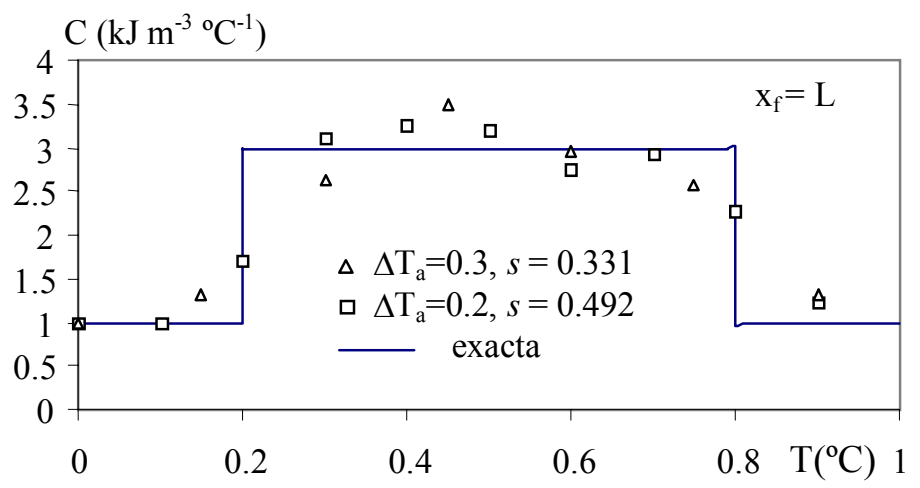

Figura VII.20. Influencia de $\Delta \mathrm{T}_{\mathrm{a}}$ en la estimación rectangular.

Sin error, $\Delta \mathrm{t}=0.1 \mathrm{~s}, \Delta \mathrm{T}_{\mathrm{a}}=0.2$ y $0.3^{\circ} \mathrm{C}, \mathrm{x}_{\mathrm{f}}=\mathrm{L}$

Para conseguir mejores estimaciones de esta dependencia es preciso mejorar el protocolo o la técnica de estimación, tarea que puede conseguirse mediante un refinamiento de dicha técnica por medio de una detección de las temperaturas que causan desviaciones puntuales grandes del valor del funcional; en torno a estas pueden refinarse tanto los valores de $\Delta \mathrm{T}_{\mathrm{a}}$ como los de $\Delta \mathrm{t}$ lo que daría lugar a una estimación más precisa. Esta labor se deja para futuras investigaciones dentro de este campo.

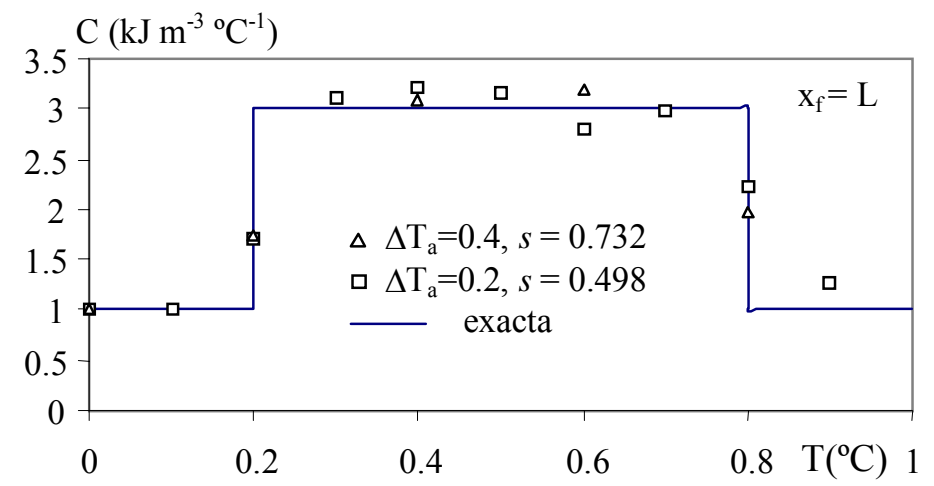

Figura VII.21. Influencia de $\Delta \mathrm{T}_{\mathrm{a}}$ en la estimación rectangular.

Sin error, $\Delta \mathrm{t}=0.2 \mathrm{~s}, \Delta \mathrm{T}_{\mathrm{a}}=0.2$ y $0.4^{\circ} \mathrm{C}, \mathrm{x}_{\mathrm{f}}=\mathrm{L}$ 


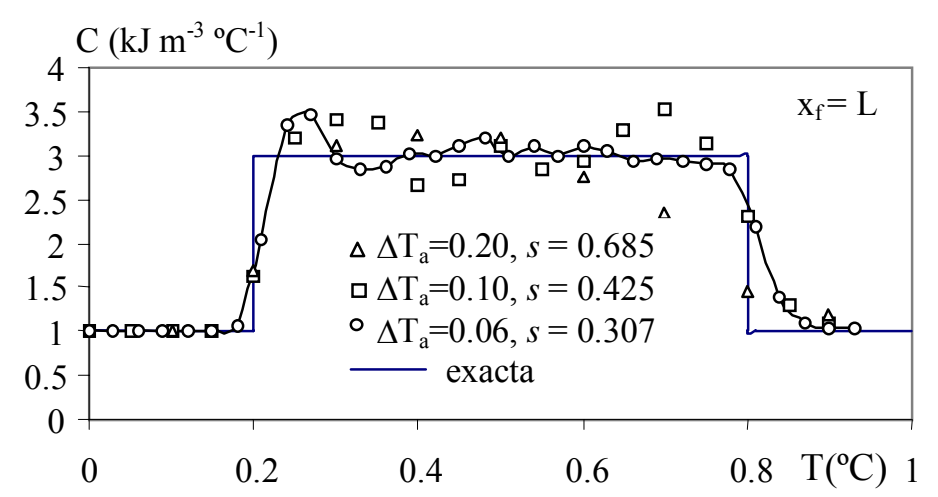

Figura VII.22. Influencia de $\Delta \mathrm{T}_{\mathrm{a}}$ en la estimación rectangular.

Sin error, $\Delta \mathrm{t}=0.01 \mathrm{~s}, \Delta \mathrm{T}_{\mathrm{a}}=0.06,0.1$ y $0.2^{\circ} \mathrm{C}, \mathrm{x}_{\mathrm{f}}=\mathrm{L}$

Los valores de $s$ finales de estas estimaciones, que resumen las influencias de las diferentes variables en la estimación, se muestran los cuadros VII.9 y VII.10.

\begin{tabular}{|l|l|l|l|}
\hline \multirow{2}{*}{$\Delta \mathrm{t}$} & \multicolumn{3}{|c|}{$\Delta \mathrm{T}_{\mathrm{a}}$} \\
\cline { 2 - 4 } & 0.2 & 0.3 & 0.4 \\
\hline 0.1 & 0.492 & 0.331 & ----- \\
\hline 0.2 & 0.498 & ----- & 0.732 \\
\hline
\end{tabular}

Cuadro VII.9. Errores $s$ de las estimaciones para dependencias $\mathrm{C}(\mathrm{T})$ rectangulares

\begin{tabular}{|l|l|l|l|}
\hline \multirow{2}{*}{$\Delta \mathrm{t}$} & \multicolumn{3}{|c|}{$\Delta \mathrm{T}_{\mathrm{a}}$} \\
\cline { 2 - 4 } & 0.06 & 0.1 & 0.2 \\
\hline 0.01 & 0.307 & 0.425 & 0.685 \\
\hline
\end{tabular}

Cuadro VII.10. Errores $s$ de las estimaciones para dependencias $C(T)$ rectangulares

\section{VII.2.2. Dependencia de la temperatura. Convección natural}

Se estudia ahora el uso de convección natural como condición de contorno junto con la introducción de errores en las medidas. Como en el epígrafe anterior, por simplicidad, se han tomado valores numéricos sencillos para los distintos parámetros y coeficientes del problema. Estos son los mostrados a continuación: 


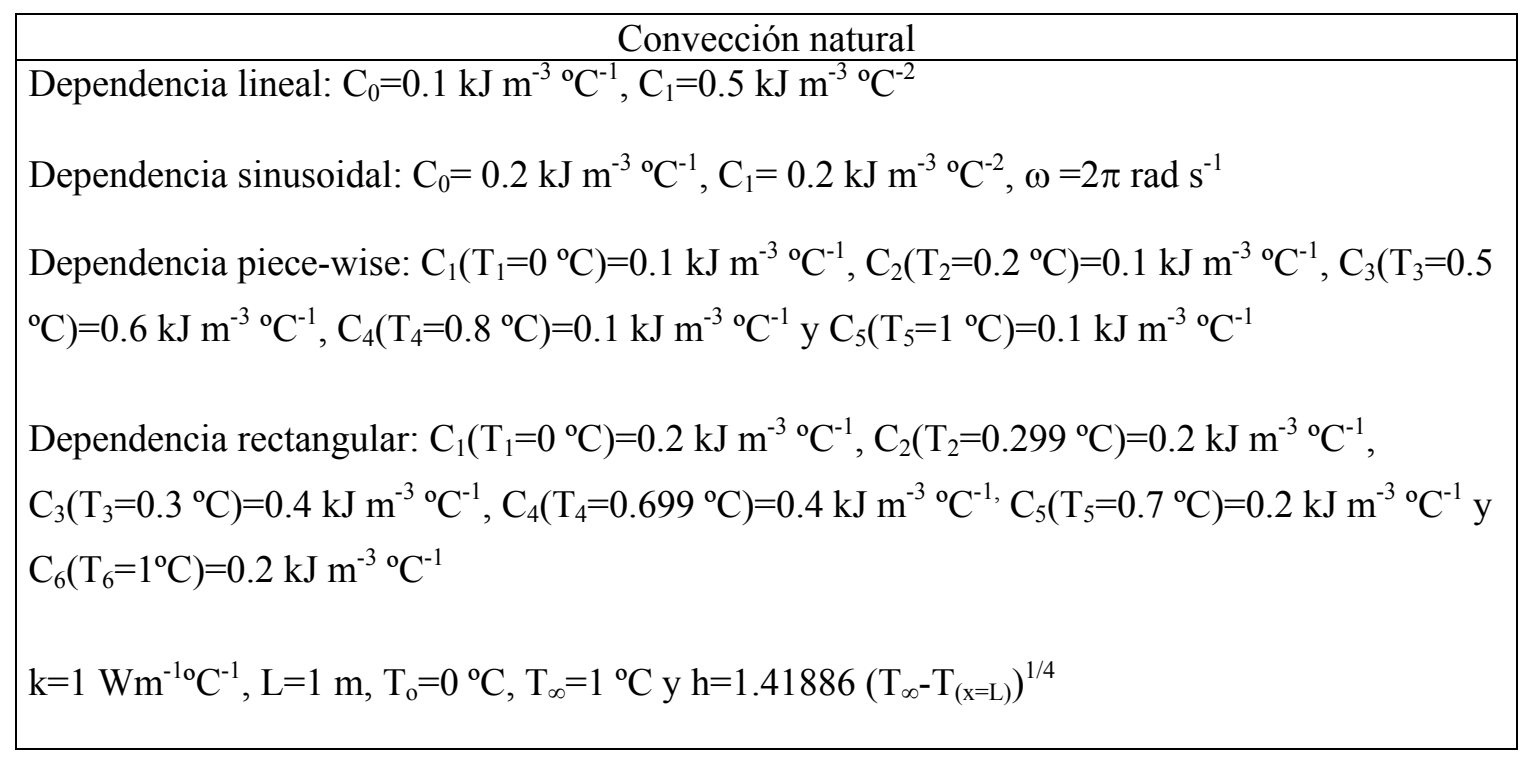

Cuadro VII.11. Datos numéricos de los tipos de dependencias y otros parámetros

La convección natural aumenta sensiblemente la no linealidad del problema por la dependencia del flujo de calor en la superficie con una potencia no entera de la diferencia de temperaturas. La figura VII.23 muestra las soluciones del campo térmico transitorio en $\mathrm{x}_{\mathrm{f}}=\mathrm{L}$, posición donde se toman las medidas, para las distintas dependencias. La correlación a emplear para el coeficiente de convección natural se expresa en la ecuación VII.1 (Mc Adams [1954]), donde $R_{a}$ es el número adimensional de Rayleigh, $R_{a}=P_{r} G_{r, L}$, producto de los números adimensionales Prandtl y Grashof. El coeficiente D y el exponente d dependen del rango en el que se encuentre $\mathrm{R}_{\mathrm{a}}$.

$$
\mathrm{N}_{\mathrm{u}}=\mathrm{D}\left(\mathrm{R}_{\mathrm{a}}\right)^{\mathrm{d}}, \quad \text { para } 10^{4}<\mathrm{R}_{\mathrm{a}}<10^{9}
$$

Las estimaciones, para la dependencia $\mathrm{c}_{\mathrm{e}}=1+5 \mathrm{~T}$, para $, \mathrm{x}_{\mathrm{f}}=\mathrm{L}, \mathrm{y}$ errores aleatorios en las medidas de $\pm 0.2, \mathrm{y} \pm 2.5 \%$ (valores límites que pueden considerarse cubren la mayor parte de los casos prácticos de interés en ingeniería), se muestran en la figura VII.24. Para $\Delta \mathrm{T}_{\mathrm{a}} \mathrm{y} \Delta \mathrm{t}$ se han adoptado los valores $0.08{ }^{\circ} \mathrm{C}$ y $0.2 \mathrm{~s}$, respectivamente. Es inmediato apreciar cómo la estimación, que oscila (al aumentar la temperatura) en torno a su valor exacto, es aún bastante precisa para el error de $\pm 2.5 \%$. 


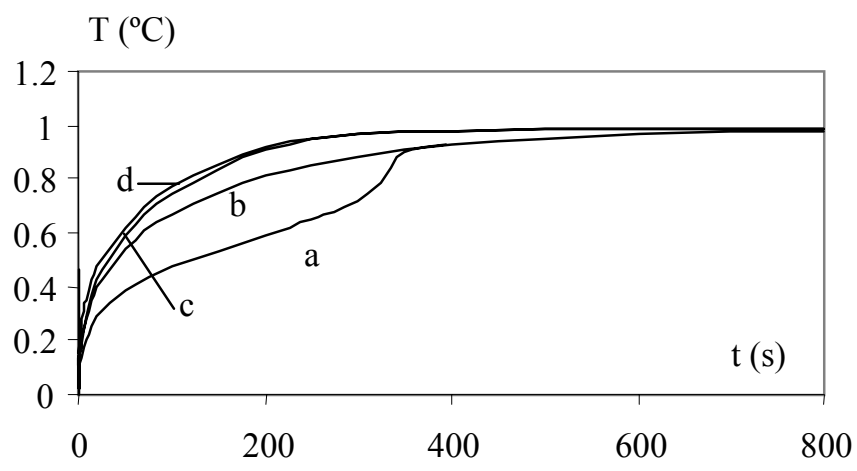

Figura VII.23. Solución del problema directo, $\mathrm{T}\left(\mathrm{x}_{\mathrm{f}}=\mathrm{L}, \mathrm{t}\right)$.

(a) Sinusoidal, (b) Lineal, (c) Rectangular, (d) Piece-wise

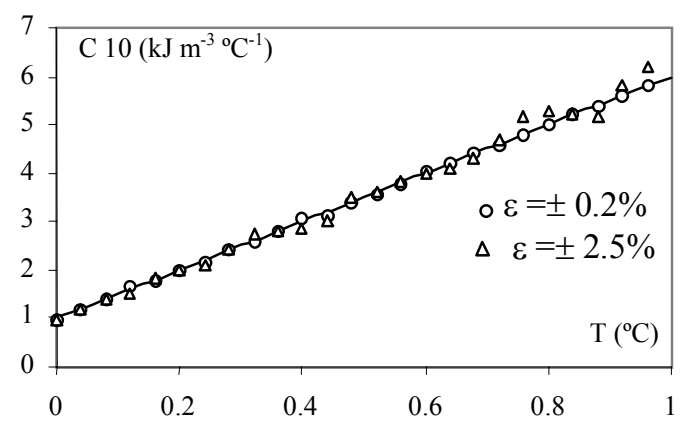

Figura VII.24. Estimación para dependencia lineal.

Errores: \pm 0.2 y $\pm 2.5 \%, \Delta \mathrm{t}=0.2 \mathrm{~s}, \Delta \mathrm{T}_{\mathrm{a}}=0.08^{\circ} \mathrm{C}, \mathrm{x}_{\mathrm{f}}=\mathrm{L}$

Para dependencias tipo piece-wise, con errores en las medidas de \pm 0.2 y $\pm 2.5 \%, \mathrm{x}_{\mathrm{f}}=$ $\mathrm{L}, \Delta \mathrm{T}_{\mathrm{a}}=0.06^{\circ} \mathrm{C}$ y $\Delta \mathrm{t}=0.1 \mathrm{~s}$, las estimaciones se muestran en la figura VII.25. Puede apreciarse que errores pequeños (del orden del $0.2 \%$ ) dan estimaciones muy aceptables mientras que para errores del orden de $2.5 \%$ (ciertamente elevados en el campo de la medición de temperaturas) las estimaciones son menos precisas.

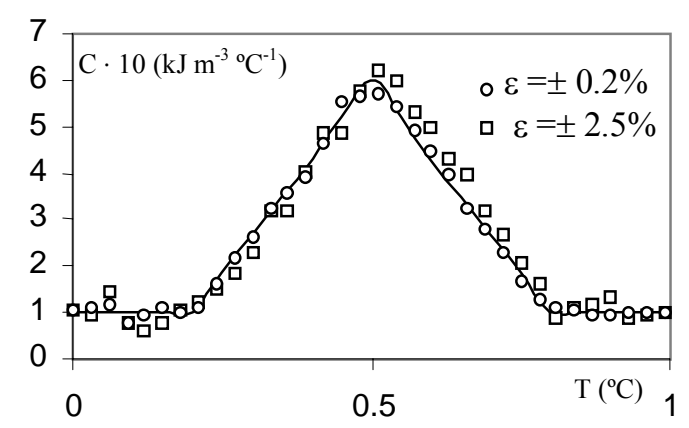

Figura VII.25. IHCP solución para una dependencia triangular.

Errores: \pm 0.2 y $\pm 2.5 \%, \Delta \mathrm{t}=0.1 \mathrm{~s}, \Delta \mathrm{T}_{\mathrm{a}}=0.06^{\circ} \mathrm{C}, \mathrm{x}_{\mathrm{f}}=\mathrm{L}$ 
En cuanto a dependencias sinusoidales, con errores en las medidas de \pm 0.2 y $\pm 2.5 \%$, $\mathrm{x}_{\mathrm{f}}$ $=\mathrm{L}, \Delta \mathrm{t}=0.2 \mathrm{~s}$ y $\Delta \mathrm{T}_{\mathrm{a}}=0.08{ }^{\circ} \mathrm{C}$, las estimaciones se muestran en la figura VII.26. Como en el caso anterior, la estimación correspondiente al error de $0.2 \%$ es, prácticamente, una reproducción de la solución exacta, mientras que para el error de $2.5 \%$ la desviación de la estimación respecto del valor exacto es ya sensible.

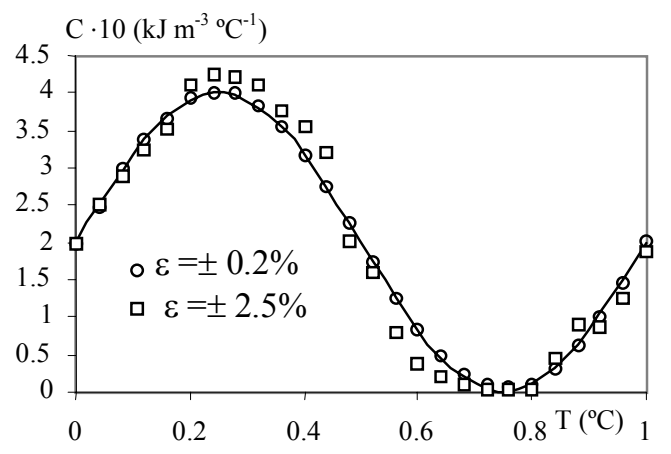

Figura VII.26. IHCP solución para una dependencia sinusoidal.

Errores: \pm 0.2 y $\pm 2.5 \%, \Delta \mathrm{t}=0.2 \mathrm{~s}, \Delta \mathrm{T}_{\mathrm{a}}=0.08^{\circ} \mathrm{C}, \mathrm{x}_{\mathrm{f}}=\mathrm{L}$

Por último, las estimaciones para dependencia rectangular, con errores en las medidas de \pm 0.2 y $\pm 2.5 \%, \Delta \mathrm{t}=0.1 \mathrm{~s}$ y $\Delta \mathrm{T}_{\mathrm{a}}=0.08^{\circ} \mathrm{C}$ se muestran en la figura VII.27. Puede apreciarse la dificultad en obtener estimaciones precisas. Aunque no puede decirse estrictamente que para errores del orden de $0.2 \%$ las estimaciones sean malas para este tipo de dependencia de enorme complejidad. Pueden apuntarse las conclusiones ya mencionadas en el epígrafe de convección forzada en cuanto a refinamiento del protocolo de estimación.

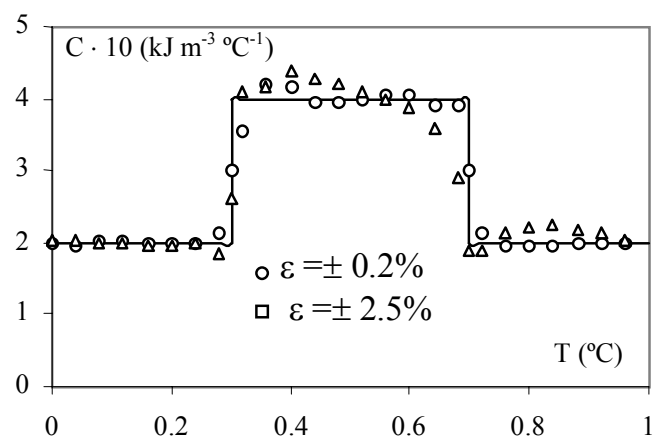

Figura VII.27. Estimación de C(T) para dependencia rectangular.

Errores: $\pm 0.2 \mathrm{y} \pm 2.5 \%, \Delta \mathrm{t}=0.1 \mathrm{~s}, \Delta \mathrm{T}_{\mathrm{a}}=0.08^{\circ} \mathrm{C}, \mathrm{x}_{\mathrm{f}}=\mathrm{L}$ 


\section{VII.2.3. Aplicación}

Como aplicación de lo anterior se procede a la estimación del calor específico de algunos alimentos, sustancias en las que esta propiedad depende fuertemente de la temperatura, particularmente en el intervalo de cambio de fase (congelación-descongelación). A partir del conocimiento de estas dependencias, datos proporcionados por la literatura científica en forma tabulada $\left(\mathrm{c}_{\mathrm{e}}-\mathrm{T}\right)$, se resuelve el problema directo para obtener "las medidas experimentales". A partir de ellas se procede a la solución del problema inverso (estimación de $\mathrm{c}_{\mathrm{e}}$ ), contrastándose los resultados con el valor exacto.

Las tablas VII.1 y VII.2 (Polley y col., [1980]; Rao y Rizyi [1986]) reproducen los valores numéricos del calor específico, conductividad térmica y entalpía específica de cuatro tipos de productos alimenticios comunes: la fresa, la mantequilla, el pescado blanco y la carne de ternera.

\begin{tabular}{|c|c|c|c|c|c|c|c|c|c|}
\hline \multicolumn{10}{|c|}{ Fresas } \\
\hline $\mathrm{T}\left({ }^{\circ} \mathrm{C}\right)$ & 50 & 40 & 30 & 20 & 10 & 0 & -5 & -10 & -20 \\
\hline $\mathrm{k}$ & 0.3778 & 0.3722 & 0.3628 & 0.3533 & 0.3439 & 0.3345 & 1.183 & 1.255 & 1.338 \\
\hline $\mathrm{c}_{\mathrm{e}}$ & 3.978 & 3.978 & 3.978 & 3.978 & 3.978 & 3.978 & 7.744 & 3.427 & 2.322 \\
\hline$h$ & 198.88 & 159.1 & 119.3 & 79.56 & 39.78 & 0 & -29.30 & -57.23 & -85.97 \\
\hline \multicolumn{10}{|c|}{ Mantequilla } \\
\hline $\mathrm{T}\left({ }^{\circ} \mathrm{C}\right)$ & 50 & 40 & 30 & 20 & 10 & 0 & -5 & -10 & -20 \\
\hline $\mathrm{c}_{\mathrm{e}}$ & 2.010 & 2.090 & 3.340 & 5.350 & 4.390 & 3.340 & 2.650 & 2.010 & 1.840 \\
\hline
\end{tabular}

Tabla VII.1 Propiedades térmicas de las fresas y la mantequilla,

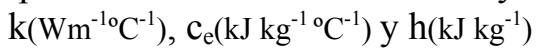

\begin{tabular}{|l|c|c|c|c|c|c|c|c|c|c|c|}
\hline \multicolumn{10}{|c|}{ Pescado blanco } \\
\begin{tabular}{|c|c|c|c|c|c|c|c|c|c|c|c|}
\hline $\mathrm{T}\left({ }^{\circ} \mathrm{C}\right)$ & -30 & -20 & -10 & -6 & -4 & -3 & -2 & -1 & 0 & 5 & 10 \\
\hline $\mathrm{k}$ & 1.872 & 1.675 & 1.479 & 1.400 & 1.361 & 1.341 & 1.322 & 1.302 & 0.430 & 0.430 & 0.430 \\
\cline { 2 - 12 } $\mathrm{c}_{\mathrm{e}}$ & 2.055 & 2.595 & 4.227 & 7.744 & 15.111 & 26.539 & 65.636 & 102.72 & 4.144 & 3.641 & 3.683 \\
\hline \multicolumn{10}{|c|}{ Carne de ternera } \\
\hline $\mathrm{T}\left({ }^{\circ} \mathrm{C}\right)$ & -40 & -30 & -20 & -15 & -13 & -11 & -10 & -7.5 & -5 & 0 & 10 \\
\hline $\mathrm{c}_{\mathrm{e}}$ & 2.008 & 2.092 & 2.720 & 4.185 & 5.400 & 6.486 & 6.905 & 7.323 & 2.929 & 2.720 & 2.870 \\
\hline
\end{tabular}
\end{tabular}

Tabla VII.2. Propiedades térmicas del pescado blanco $\mathrm{k}\left(\mathrm{Wm}^{-10} \mathrm{C}^{-1}\right), \mathrm{c}_{\mathrm{e}}\left(\mathrm{kJ} \mathrm{kg}^{-10} \mathrm{C}^{-1}\right)$ y de la carne de ternera, $\mathrm{c}_{\mathrm{e}}\left(\mathrm{kJ} \mathrm{kg}^{-10} \mathrm{C}^{-1}\right)$

Los datos relativos a la geometría del problema y otros parámetros necesarios se muestran en el cuadro VII.12. 


\begin{tabular}{|l|c|c|c|c|}
\hline \multicolumn{1}{|c|}{ Propiedad } & Mantequilla & Fresas & Pescado blanco & Carne de ternera \\
\hline $\mathrm{L}(\mathrm{cm})$ & 3 & 2 & 5 & 10 \\
\hline$\rho\left(\mathrm{kg} \mathrm{m}^{-3}\right)$ & 964 & 870 & 1054 & 980 \\
\hline $\mathrm{k}\left(\mathrm{W} \mathrm{m}^{-1}{ }^{\circ} \mathrm{C}^{-1}\right)$ & 1 & (ver tabla VII.1) & (ver tabla VII.2) & 0.476 \\
\hline $\mathrm{h}\left(\mathrm{W} \mathrm{m}^{-2} \mathrm{C}^{-1}\right)$ & 10 & 20 & 10 & 10 \\
\hline $\mathrm{T}_{\mathrm{o}}\left({ }^{\circ} \mathrm{C}\right)$ & -20 & -20 & -20 & -40 \\
\hline $\mathrm{T}_{\infty}\left({ }^{\circ} \mathrm{C}\right)$ & 60 & 30 & 40 & 20 \\
\hline$\Delta \mathrm{t}(\mathrm{s})$ & 1 & 0.2 & $1,5 \mathrm{y} 30$ & 10 \\
\hline$\Delta \mathrm{T}_{\mathrm{a}}\left({ }^{\circ} \mathrm{C}\right)$ & 4 & 4 & $1,2 \mathrm{y} 5$ & $2 \mathrm{y} 4$ \\
\hline
\end{tabular}

Cuadro VII.12. Datos numéricos para la mantequilla y las fresas

Para la mantequilla (de conductividad constante) y pescado blanco (conductividad dependiente de la temperatura) se usa coordenadas planas y para las fresas (conductividad dependiente de la temperatura) se adopta la geometría esférica 1-D.

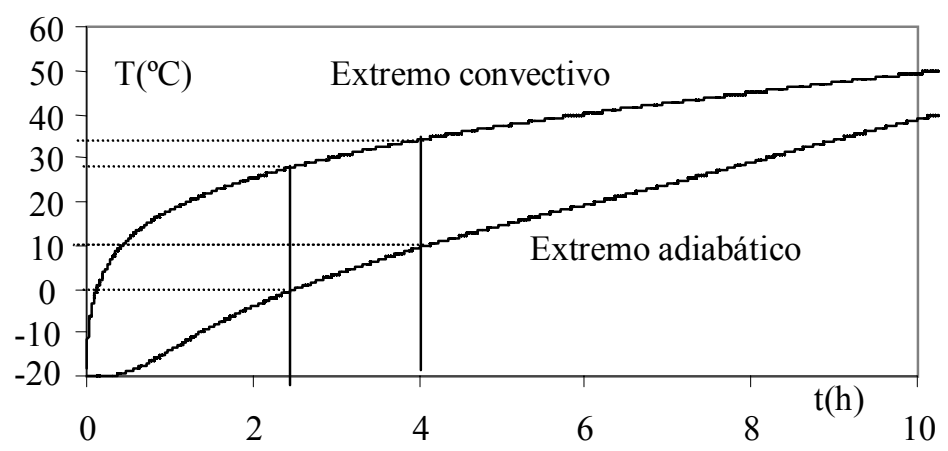

Figura VII.28. Evolución de la temperatura con el tiempo en la descongelación de mantequilla, Posiciones $\mathrm{x}_{\mathrm{f}}=0$ (superficie adiabática) y $\mathrm{x}_{\mathrm{f}}=\mathrm{L}$ (superficie convectiva)

En la figura VII.28 se muestra la evolución temporal de la temperatura en ambas fronteras, adiabática y convectiva, obtenida de la solución del problema directo (MESIR) en un problema de descongelación de la mantequilla.

La figura VII.29 muestra las estimaciones del calor especifico, para errores con desviaciones típicas en las medidas de $\sigma=0$ y $\sigma=0.07$ (supone un error que oscila entre el 0.5 y el $2 \%$ ), tomando el punto de medida en el extremo convectivo. La estimación en ausencia de error reproduce prácticamente la solución exacta mientras que para el error $\sigma=0.07$ el resultado empeora. 


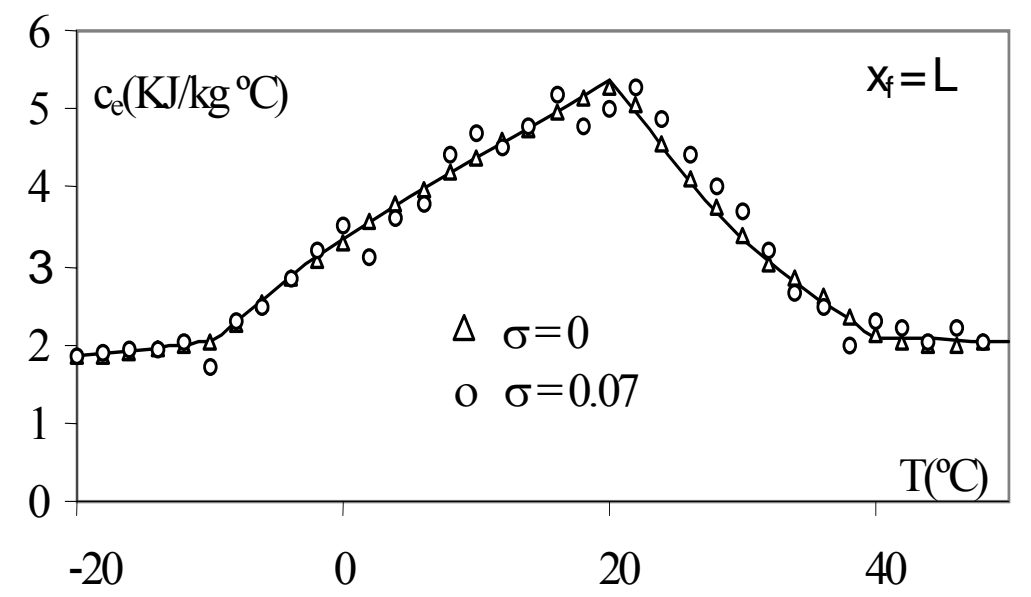

Figura VII.29. Estimación del calor especifico, para $\sigma=0$ y $\sigma=0.07$.

Punto de medida en el extremo convectivo

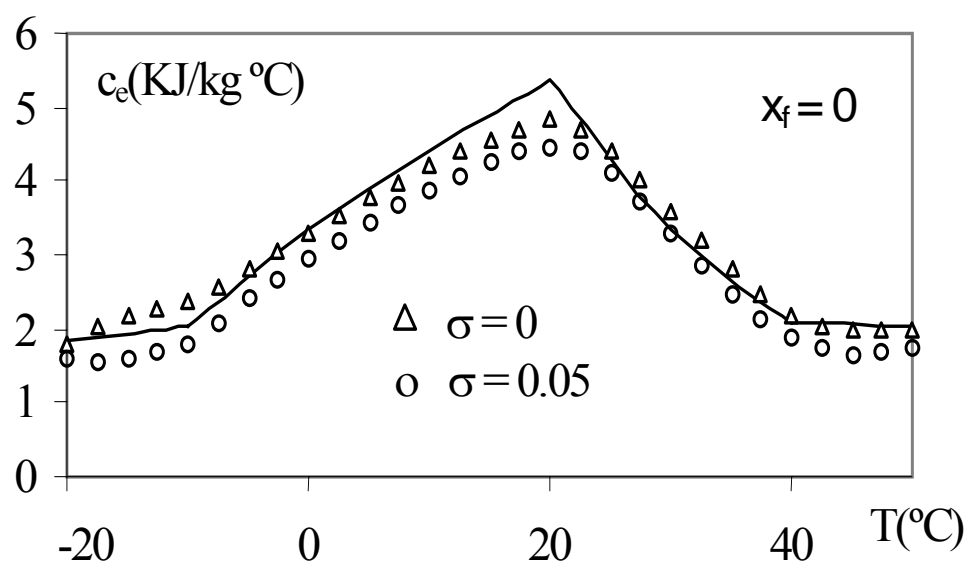

Figura VII.30. Estimación del calor especifico, para $\sigma=0$ y $\sigma=0.07$ Punto de medida en el extremo adiabático

En la figura VII.30 se muestran las estimaciones del calor especifico, para $\sigma=0$ y $\sigma=$ 0.07 , tomando el punto de medida en el extremo adiabático. Se obtienen estimaciones peores, resultado concordante con lo estudiado hasta ahora en relación a la elección más adecuada para el punto de medida.

Una posible explicación de porqué midiendo en el extremo adiabático se obtienen peores estimaciones, es la siguiente: La variación de temperatura en el punto adiabático es más lenta que en el extremo convectivo, por ello cuando se está realizando una estimación entre un determinado intervalo de temperaturas, por ejemplo entre las temperaturas $0^{\circ} \mathrm{C} \mathrm{y} 10^{\circ} \mathrm{C}$ (ver figura VII.28), que se corresponde con un intervalo aproximado de tiempo de 2.3 y 4 horas, existen puntos cercanos a $\mathrm{x}=\mathrm{L}$ que pueden estar trabajando en ese intervalo de tiempo a 
temperaturas superiores a $30^{\circ} \mathrm{C}$. La acción de control (mediante la fuente de corriente controlada por tensión, o sea por temperatura), estará limitada a $10^{\circ} \mathrm{C}$, por ello ocurre que hay fuentes de corriente (situadas en las cercanías de la superficie convectiva) trabajando con temperaturas de control superiores a la acción de control, con lo que la estimación se hace más imprecisa.

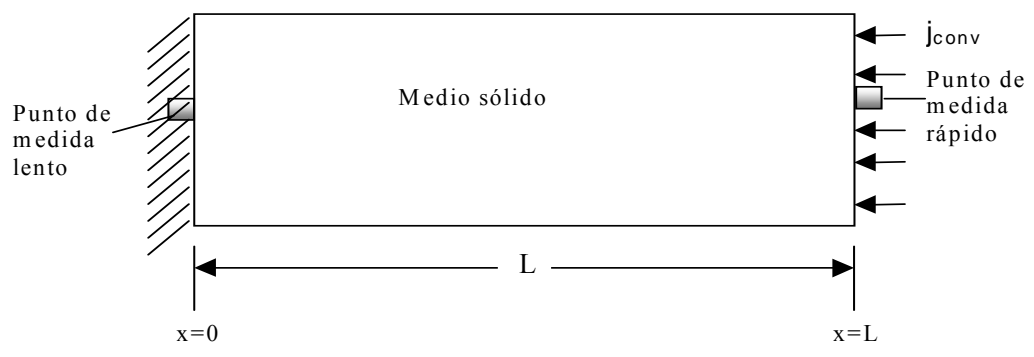

Figura VII.31. Ilustración de los puntos de medida

Midiendo en el extremo convectivo no hay fuentes de corriente controladas con temperaturas de control superiores al rango de temperaturas que se pretende estimar. Los errores cometidos en la estimación al medir en el extremo adiabático serán mayores conforme aumente el desfase de las medidas de temperatura con el tiempo, ya que las acciones de control sobre las fuentes de corriente serán más significativas. También es posible encontrar casos donde las estimaciones no difieran mucho de medir en un punto o en otro.

En el problema de descongelación de fresas, la figura VII.32 muestra el campo transitorio de temperaturas de la superficie convectiva (superficie exterior de la esfera) procedente de la solución del problema directo resuelto por medio del Método de simulación por redes.

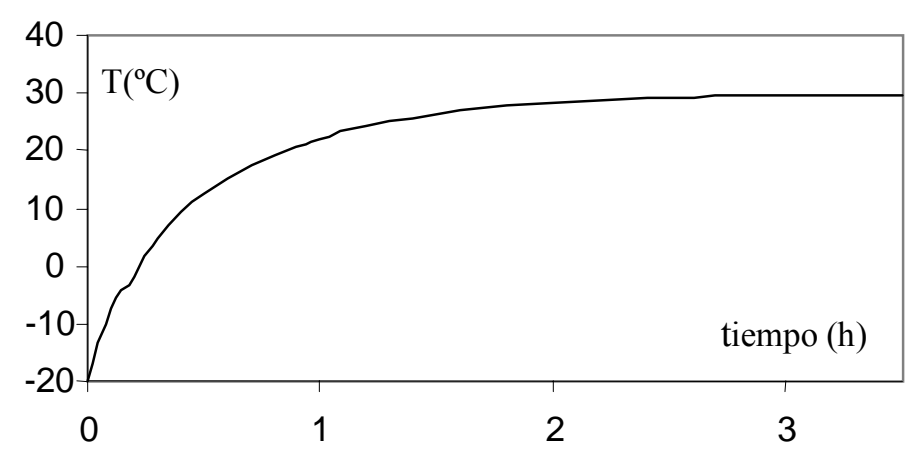

Figura VII.32. Fresas. Evolución de la temperatura con el tiempo, para medidas realizadas en $\mathrm{x}_{\mathrm{f}}=\mathrm{L}$

En la figura VII.33 se muestra la estimación del calor especifico (punto de medida en el extremo convectivo) para los casos $\sigma=0$ y 0.1 (supone un error que oscila entre el 1 y el 3\%), 
utilizando las medidas anteriores. Mediante sencillas operaciones es posible obtener el valor de la entalpía específica cuya estimación se refleja en la figura VII.34.

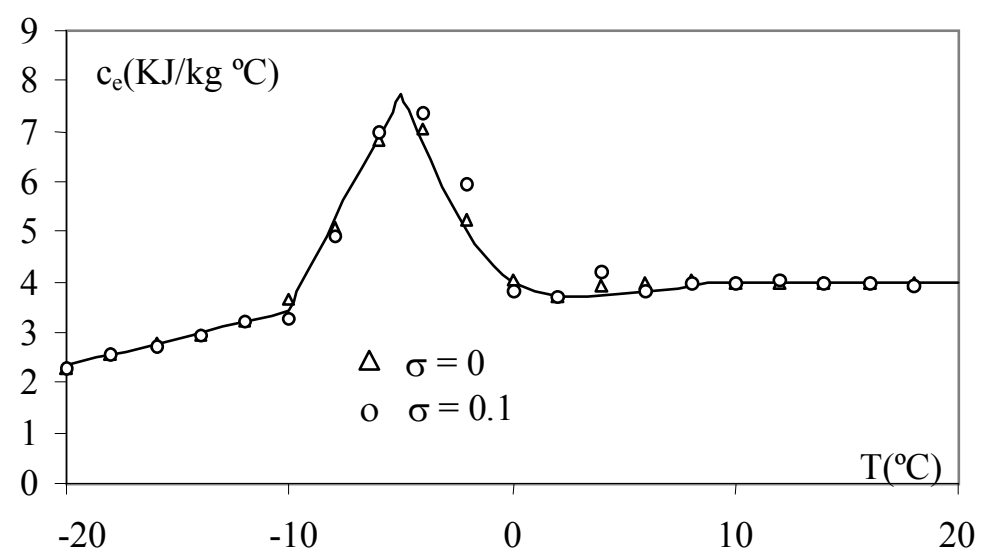

Figura VII.33. Estimación del calor especifico, para $\sigma=0$ y $\sigma=0.1$

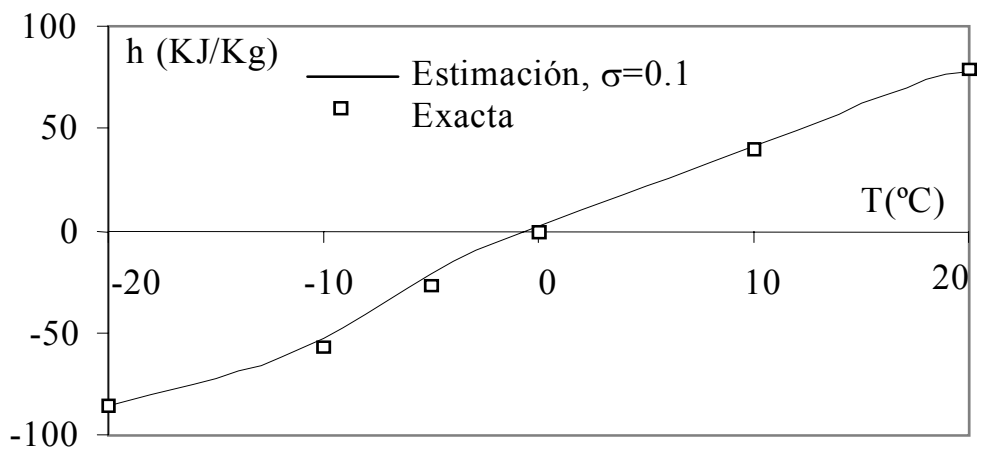

Figura VII.34. Estimación de la entalpía específica, para $\sigma=0.1$

La figura VII.35 se muestra el campo transitorio de temperaturas de la superficie convectiva para el pescado blanco y la carne de ternera. La figura VII.36 muestra las estimaciones del calor específico para intervalos de estimación de temperatura de 1 y $2^{\circ} \mathrm{C}$, observándose que para $\Delta \mathrm{T}_{\mathrm{a}}=1{ }^{\circ} \mathrm{C}$ se obtiene una mejor aproximación que para $\Delta \mathrm{T}_{\mathrm{a}}=2{ }^{\circ} \mathrm{C}$, sin embargo el tiempo de computación es aproximadamente el doble. La figura VII.37 analiza el caso de $\Delta \mathrm{T}_{\mathrm{a}}=5{ }^{\circ} \mathrm{C}$, en donde se observa que la estimación no es buena. En consecuencia, es preciso disminuir el valor del intervalo de estimación de temperaturas para poder detectar cambios bruscos de la función a estimar. 


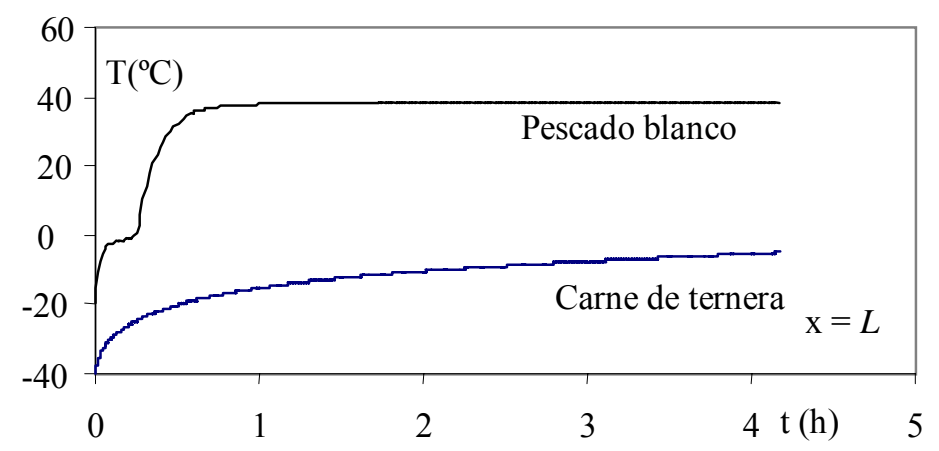

Figura VII.35. Evolución de la temperatura con el tiempo, para el pescado blanco y la carne de ternera

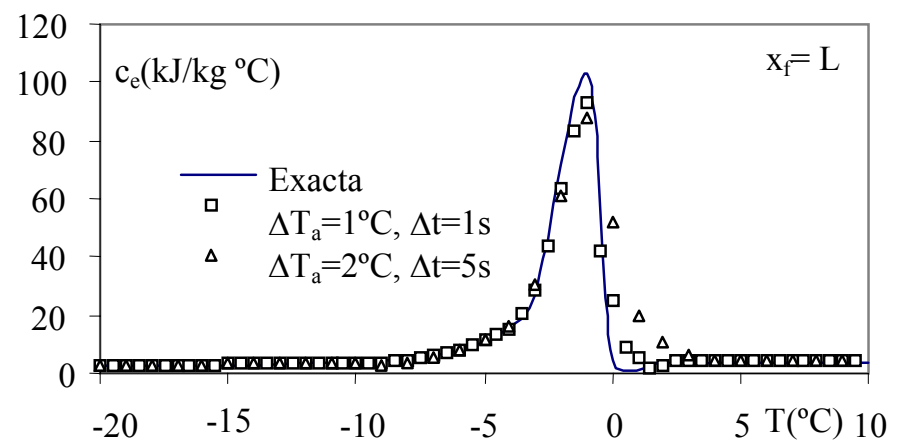

Figura VII.36. Estimación del $\mathrm{c}_{\mathrm{e}}$ del pescado blanco. $\Delta \mathrm{T}_{\mathrm{a}}=1$ y $2{ }^{\circ} \mathrm{C}$

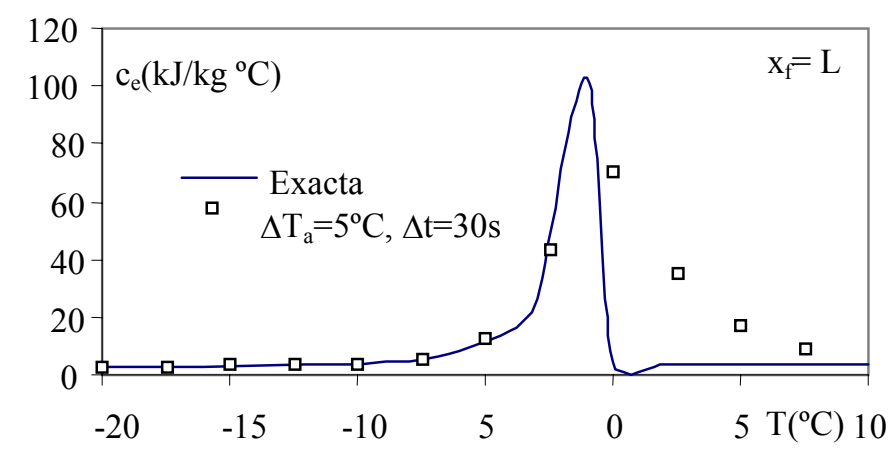

Figura VII.37. Estimación del $\mathrm{c}_{\mathrm{e}}$ del pescado blanco. $\Delta \mathrm{t}=30 \mathrm{~s}, \Delta \mathrm{T}_{\mathrm{a}}=5^{\circ} \mathrm{C}$

La figura VII.38 muestra el efecto del intervalo de tiempo de medida no observándose grandes diferencias en la estimación, $\Delta \mathrm{t}=5$ y $30 \mathrm{~s}$. Este parámetro apenas afecta los resultados. 


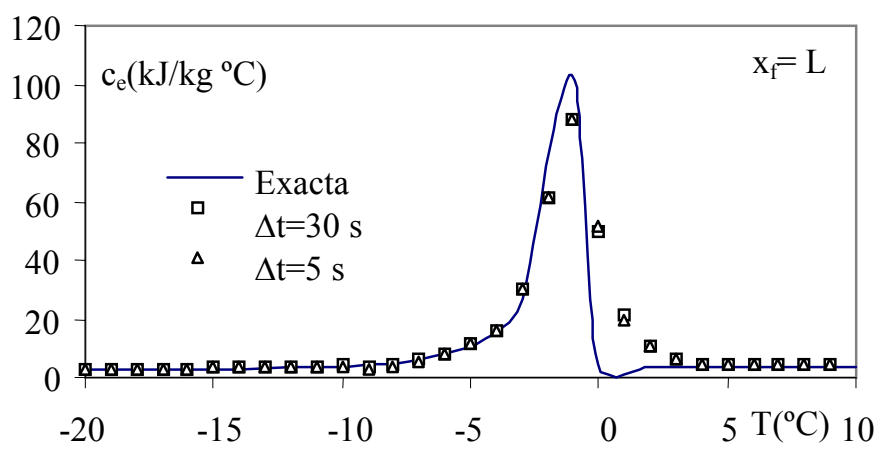

Figura VII.38. Estimación del $\mathrm{c}_{\mathrm{e}}$ del pescado blanco. $\Delta \mathrm{T}_{\mathrm{a}}=2{ }^{\circ} \mathrm{C}, \Delta \mathrm{t}=5$ y $30 \mathrm{~s}$

La figura VII.39 muestra la estimación del calor específico de la carne de ternera en un proceso de descongelación, con medidas realizadas en $\mathrm{x}=\mathrm{L}$, para $\Delta \mathrm{T}_{\mathrm{a}}=2$ y $4^{\circ} \mathrm{C}$, y con un error en las medidas pequeño $(\sigma=0.02)$, observándose que para $\Delta \mathrm{T}_{\mathrm{a}}=2$ se obtiene una mejor estimación. En la figura VII.40 se aplican errores más importantes en las medidas de temperaturas $(\sigma=0.1$ y 0.5$)$, apreciándose el efecto de estos en las estimaciones.

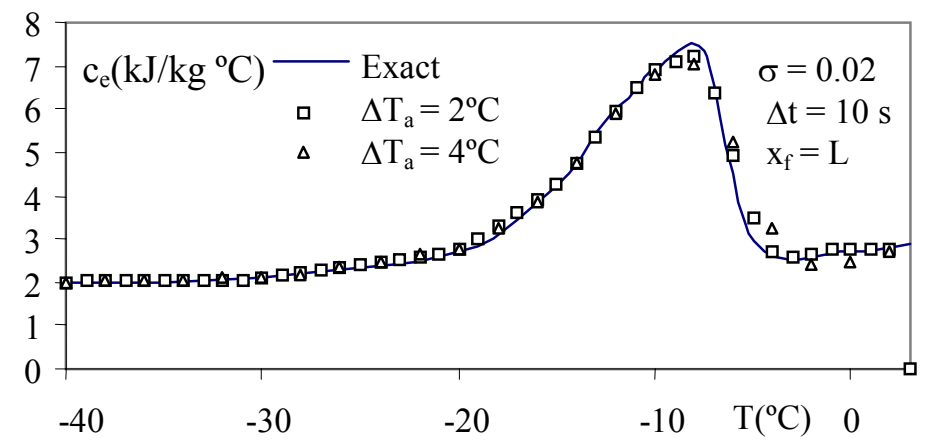

Figura VII.39. Estimación del $\mathrm{c}_{\mathrm{e}}$ de la carne de ternera.

$$
\Delta \mathrm{T}_{\mathrm{a}}=2 \mathrm{y} 4^{\circ} \mathrm{C}, \Delta \mathrm{t}=10 \mathrm{~s}, \sigma=0.02
$$

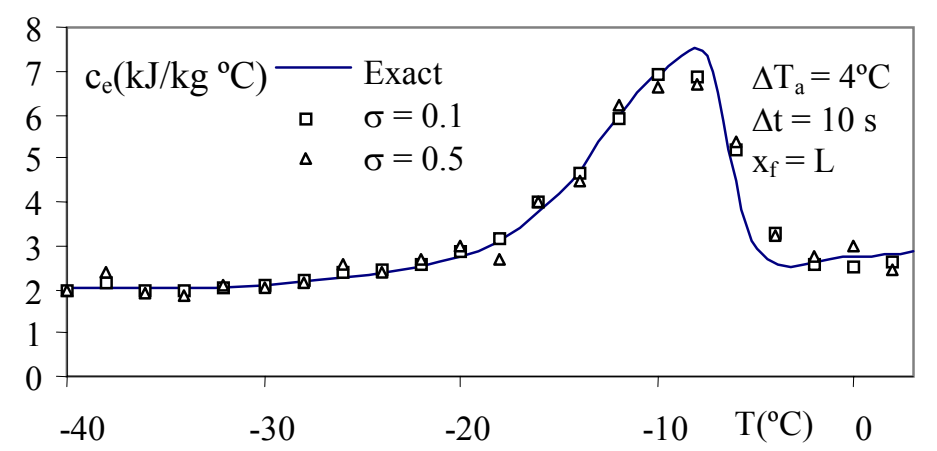

Figura VII.40. Estimación del $\mathrm{c}_{\mathrm{e}}$ de la carne de ternera.

$$
\Delta \mathrm{T}_{\mathrm{a}}=4^{\circ} \mathrm{C}, \Delta \mathrm{t}=10 \mathrm{~s}, \sigma=0.1 \text { y } 0.5
$$


Con objeto de contrastar estos resultados, las figuras VII.41 y VII.42 muestran la solución del campo térmico del problema directo (que se utilizó para obtener los datos de entrada del problema inverso) y la solución del problema directo usando para el calor específico los valores estimados en el problema inverso para el caso de las fresas. En la primera figura para medidas $\sin$ error y en la segunda para el caso $\sigma=0.1$. Ambos campos corresponden a mediciones en la pared convectiva. Los errores $s$ de comparar las curvas dentro de cada figura son de 0.101 y 0.539 .

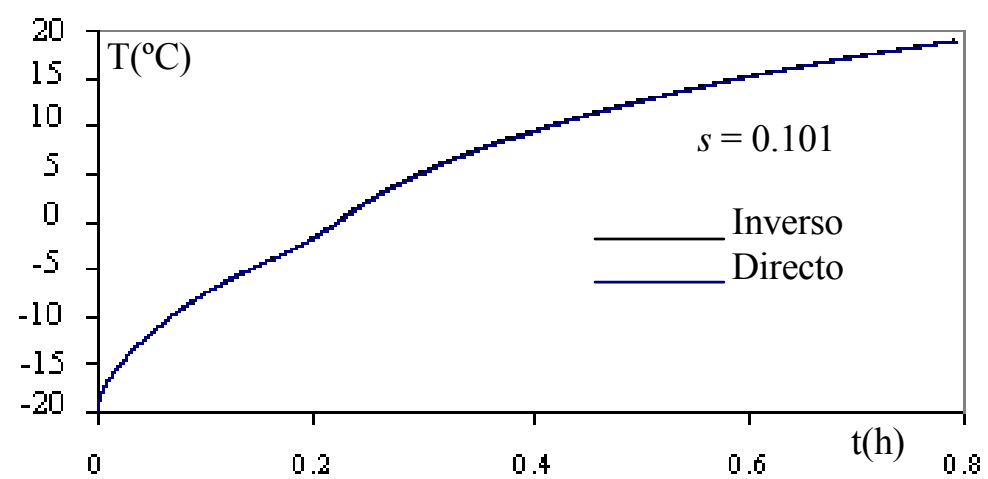

Figura VII.41. Evolución de la temperatura para el caso $\sigma=0$

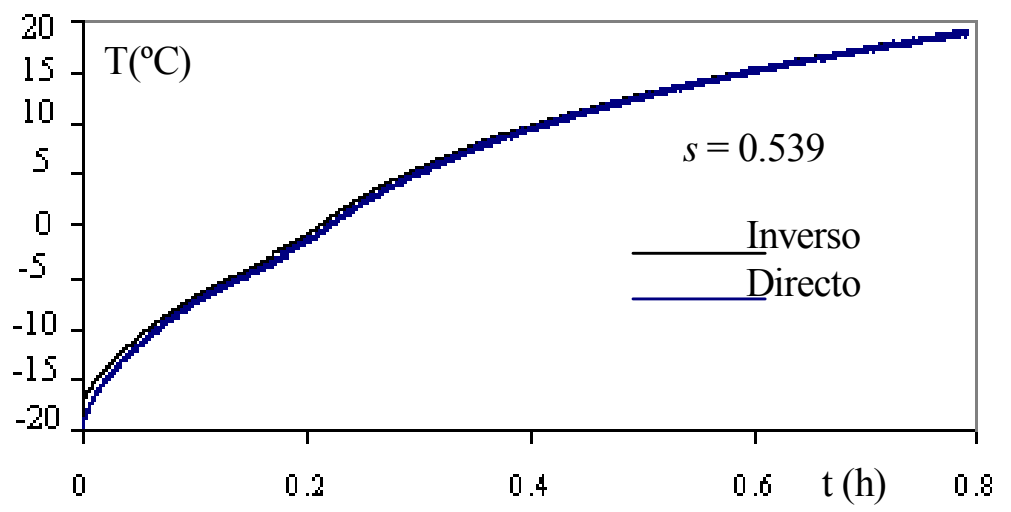

Figura VII.42. Evolución de la temperatura para el caso $\sigma=0.1$

\section{VII.3. ESTIMACIÓN DE LA CONDUCTIVIDAD TÉRMICA}

\section{VII.3.1. Estimación de función}

El sistema considerado es una placa unidimensional con condiciones de contorno adiabática y de flujo de calor por convección en sus superficies exteriores. Los protocolos aplicados para la solución de este problema inverso se exponen en el capítulo III de esta tesis. No es necesario conocimiento a priori de la forma de dependencia de esta propiedad. El modelo 
matemático, las distintas dependencias y los valores numéricos del resto de problemas se muestran en los cuadros VII.13, VII.14 y VII.15, respectivamente.

\begin{tabular}{|c|c|}
\hline \multicolumn{2}{|c|}{ Modelo matemático } \\
\hline $\begin{array}{c}\text { Ecuación } \\
\text { diferencial }\end{array}$ & $\mathrm{c}_{\mathrm{e}} \rho \partial \mathrm{T} / \partial \mathrm{t}-\mathrm{k}(\mathrm{T})\left(\partial^{2} \mathrm{~T} / \partial \mathrm{x}^{2}\right)=0$ \\
\hline $\begin{array}{c}\text { Condición } \\
\text { inicial }\end{array}$ & $\mathrm{T}(\mathrm{x},=0)=\mathrm{T}_{0}$ \\
\hline $\begin{array}{c}\text { Condiciones de } \\
\text { contorno }\end{array}$ & $\mathrm{j}(\mathrm{L}, \mathrm{t})=\mathrm{j}_{\text {con-forzada }}$ \\
\hline $\begin{array}{c}\text { Condición } \\
\text { adicional }\end{array}$ & $\mathrm{j}(0, \mathrm{t})=0$ \\
\hline
\end{tabular}

Cuadro VII.13. Modelo matemático

\begin{tabular}{|l|l|}
\hline \multicolumn{2}{|c|}{ Dependencias de la conductividad térmica } \\
\hline Dependencia lineal & $\mathrm{k}=\mathrm{k}_{0}+\mathrm{k}_{1} \mathrm{~T}$ \\
\hline Dependencia sinusoidal & $\mathrm{k}=\mathrm{k}_{0}+\mathrm{k}_{1} \operatorname{sen}(\omega \mathrm{T})$ \\
\hline Dependencia piece-wise & $\begin{array}{l}\mathrm{k}\left(\mathrm{T}_{1}\right)=\mathrm{k}_{1}, \mathrm{k}\left(\mathrm{T}_{2}\right)=\mathrm{k}_{2}, \mathrm{k}\left(\mathrm{T}_{3}\right)=\mathrm{k}_{3}, \\
\mathrm{k}\left(\mathrm{T}_{4}\right)=\mathrm{k}_{4} \text { and } \mathrm{k}\left(\mathrm{T}_{5}\right)=\mathrm{k}_{5}\end{array}$ \\
\hline Dependencia rectangular & $\mathrm{k}\left(\mathrm{T}_{1}\right)=\mathrm{k}_{1}, \mathrm{k}\left(\mathrm{T}_{2}\right)=\mathrm{k}_{2}, \mathrm{k}\left(\mathrm{T}_{3}\right)=\mathrm{k}_{3}$, \\
& $\mathrm{k}\left(\mathrm{T}_{4}\right)=\mathrm{k}_{4}, \mathrm{k}\left(\mathrm{T}_{5}\right)=\mathrm{k}_{5} \mathrm{y}\left(\mathrm{T}_{6}\right)=\mathrm{k}_{6}$ \\
\hline
\end{tabular}

Cuadro VII.14. Formas de dependencias k(T)

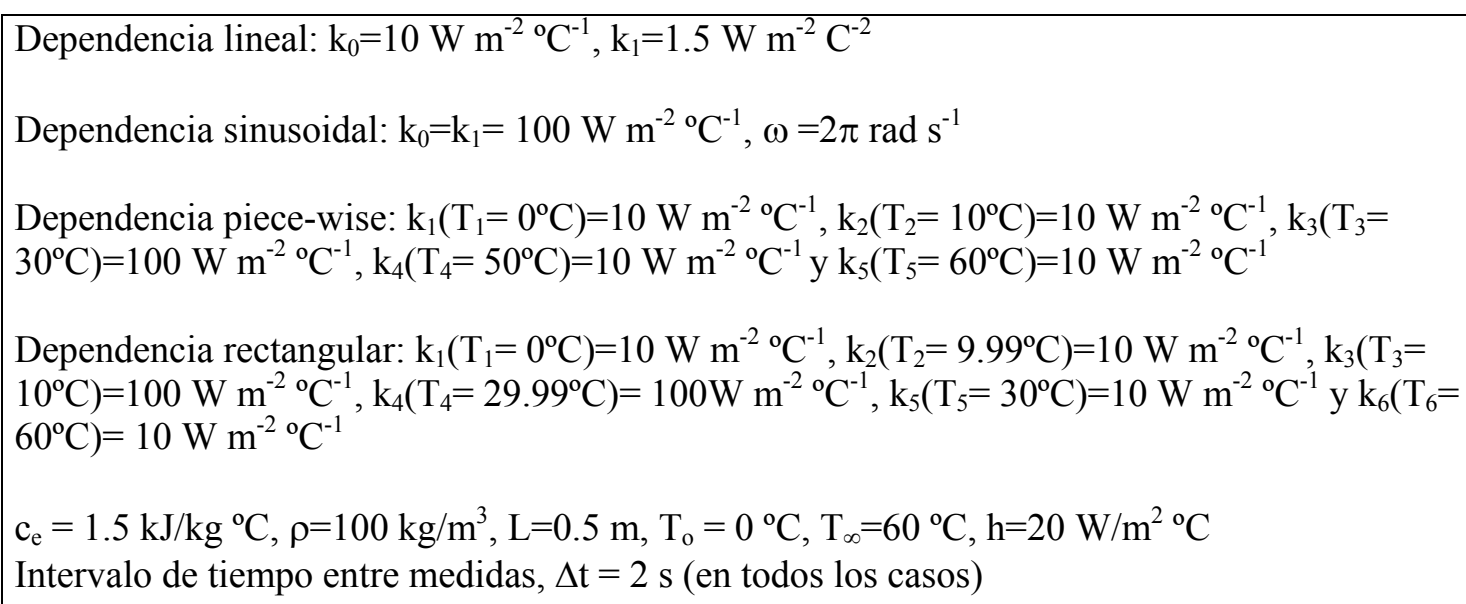




\section{Dependencia lineal}

En la figura VII.43 se muestra el campo térmico solución del problema directo en las superficies adiabática y de convección, para el caso de dependencia lineal. La figura VII.44 muestra la estimación de la conductividad térmica a partir de los datos de temperatura en la superficie adiabática, en función de varios puntos iniciales de estimación de valores 2, 0.94 y 0.5. El error $s$ de la estimación, indicado en la misma figura, aumenta conforme el punto inicial se aleja más de su valor exacto, que es 1 . Puede apreciarse, no obstante, que los resultados son muy similares en los tres casos, lo que permite concluir que el método no es demasiado sensible a la adopción de diferentes valores iniciales dentro de cierto rango.

Las estimaciones correspondientes a puntos de medida en la superficie convectiva se muestran en la figura VII.45, también para diferentes valores del punto inicial de estimación, 2, 1.5 y 0.5 . La figura VII.46 compara los resultados anteriores para un mismo punto inicial de valor 2. De nuevo se obtienen estimaciones más precisas para medidas realizadas en el extremo convectivo.

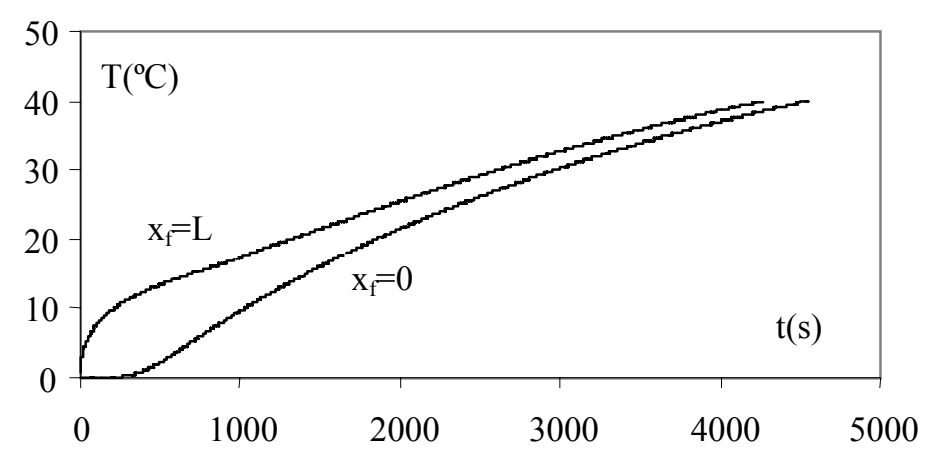

Figura VII.43. Solución del problema directo en dos puntos de medida, adiabático $\left(\mathrm{x}_{\mathrm{f}}=0\right)$ y convectivo $\left(\mathrm{x}_{\mathrm{f}}=\mathrm{L}\right)$

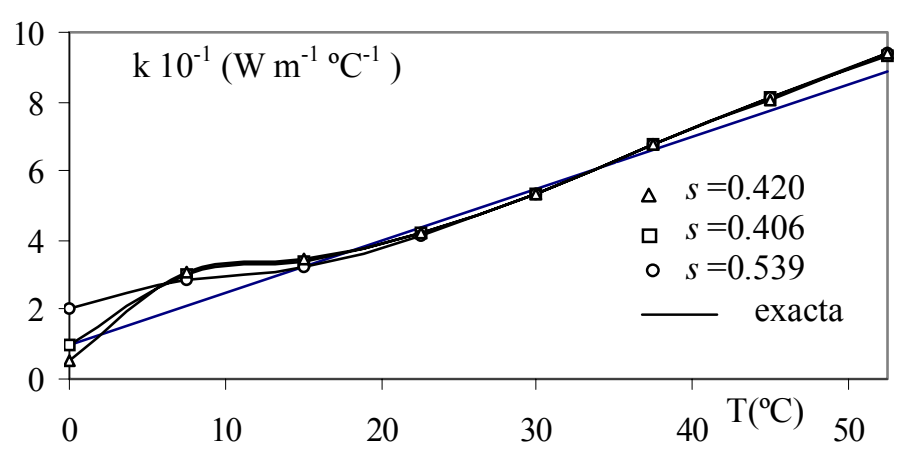

Figura VII.44. Estimación de la conductividad térmica para el caso lineal, en función de varios puntos iniciales, $\Delta \mathrm{T}_{\mathrm{a}}=15^{\circ} \mathrm{C}, \mathrm{x}_{\mathrm{f}}=0$ 


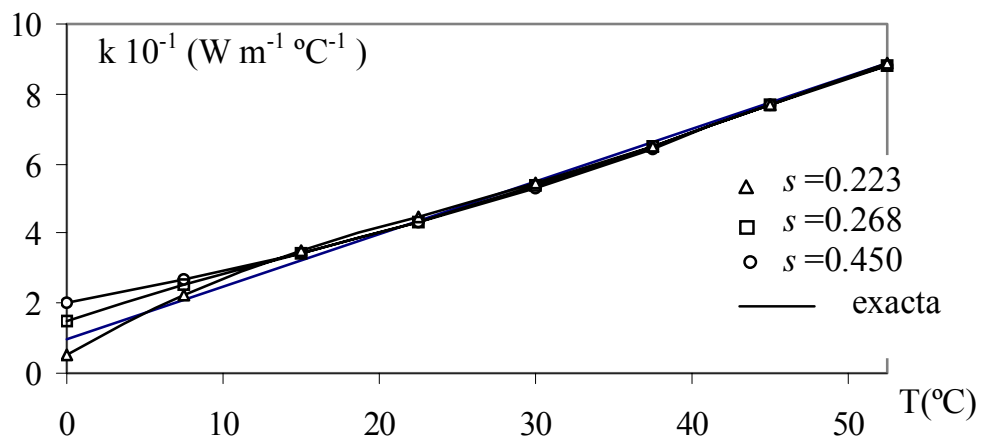

Figura VII.45. Estimación de la conductividad térmica para el caso lineal, en función de varios puntos iniciales, $\Delta \mathrm{T}_{\mathrm{a}}=15^{\circ} \mathrm{C}, \mathrm{x}_{\mathrm{f}}=\mathrm{L}$

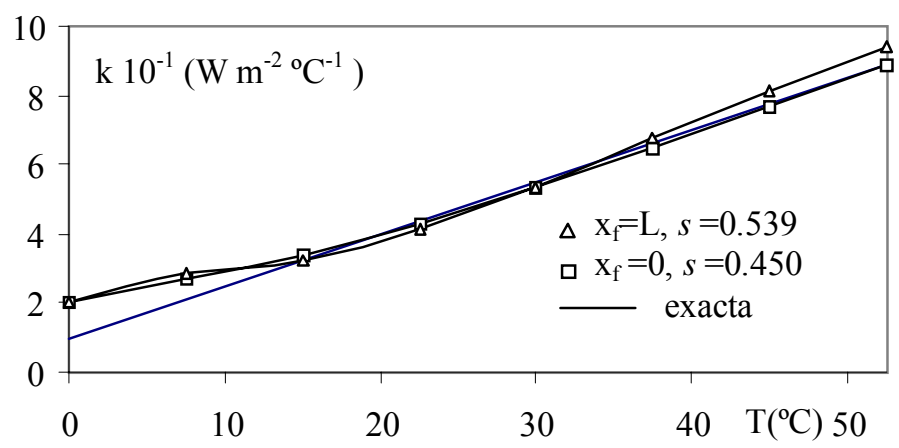

Figura VII.46. Comparación de puntos de medida, $\mathrm{x}_{\mathrm{f}}=0 \mathrm{y} \mathrm{x}_{\mathrm{f}}=\mathrm{L}$

\section{Dependencia triangular}

La figura VII.47 muestra la solución del problema directo para medidas realizadas en el extremo adiabático y en el extremo convectivo. En la figura VII.48 se presenta la estimación para diferentes valores del punto inicial de la estimación, a saber 1.5, 0.94 y 0.5, y con las medidas en el extremo adiabático. El mayor error $s$ es de 0.613 y corresponde al valor inicial de 1.5. Al igual que en el caso anterior, a simple vista puede apreciarse que los resultados son muy similares en los tres casos. El valor de $\Delta \mathrm{T}_{\mathrm{a}}$ es de $7.5{ }^{\circ} \mathrm{C}$ ya que, observando la respuesta $\mathrm{T}\left(\mathrm{x}_{\mathrm{f}}=\mathrm{L}, \mathrm{t}\right)$, para un intervalo menor no existen medidas de temperatura. La comparación con el caso de medida en la superficie convectiva se muestra en la figura VII.49, observándose que los mejores resultados se obtienen para mediciones en la superficie convectiva que dan un error $s$ de 0.251 frente a 0.429 , obtenido para mediciones en la superficie adiabática. 


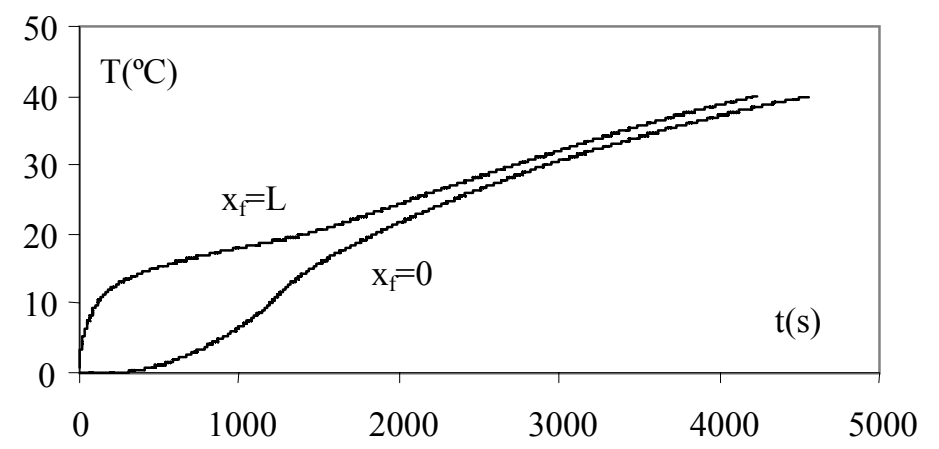

Figura VII.47. Solución del problema directo en dos puntos de medida, adiabático $\left(\mathrm{x}_{\mathrm{f}}=0\right)$ y convectivo $\left(\mathrm{x}_{\mathrm{f}}=\mathrm{L}\right)$

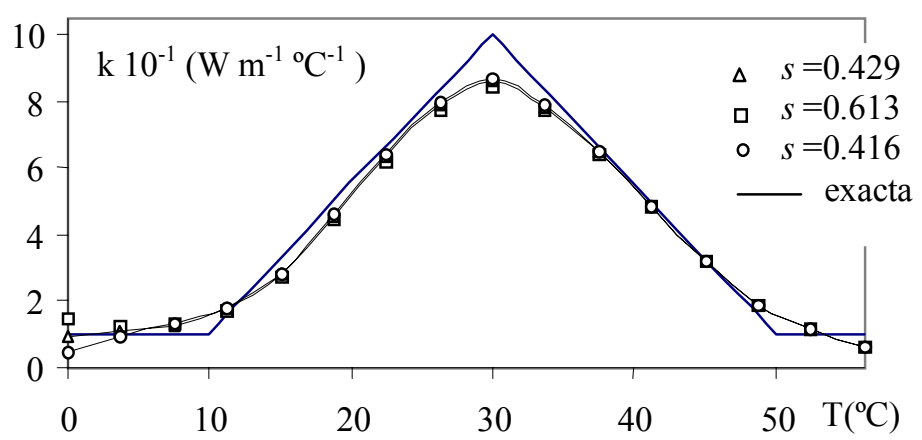

Figura VII.48. Estimación de la conductividad térmica para la dependencia triangular, en función del punto iniciale, $\Delta \mathrm{T}_{\mathrm{a}}=7.5^{\circ} \mathrm{C}, \mathrm{x}_{\mathrm{f}}=0$

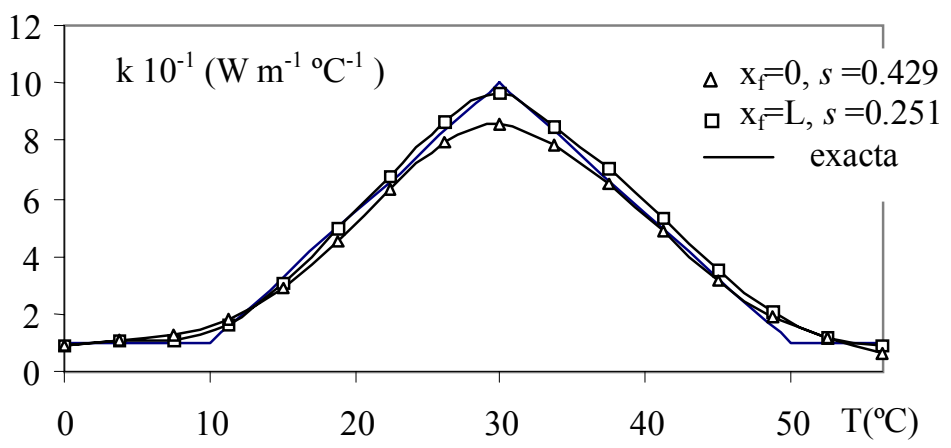

Figura VII.49. Comparación de los distintos extremos de medida,

$$
\Delta \mathrm{T}_{\mathrm{a}}=7.5^{\circ} \mathrm{C}
$$




\section{Dependencia sinusoidal}

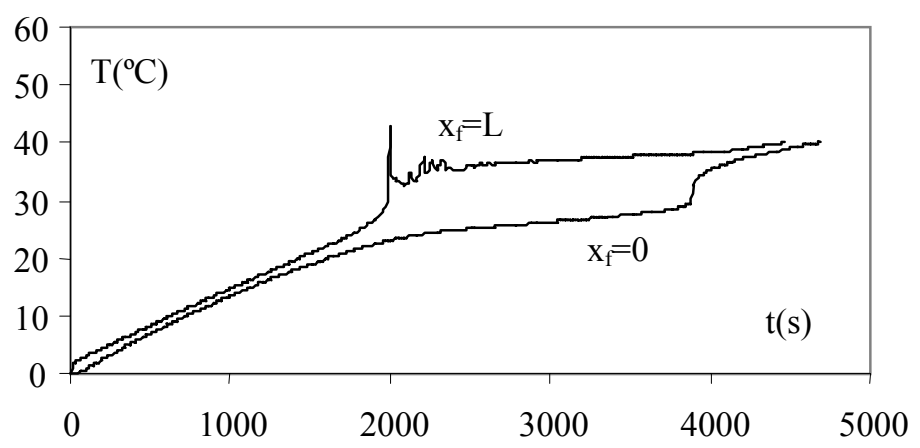

Figura VII.50. Solución del problema directo en dos puntos de medida, adiabático $\left(\mathrm{x}_{\mathrm{f}}=0\right)$ y convectivo $\left(\mathrm{x}_{\mathrm{f}}=\mathrm{L}\right)$

La figura VII.50 muestra la solución del problema directo para medidas realizadas en el extremo adiabático y en el extremo convectivo. En ella se observa que, para ambos casos, en $\mathrm{T}=30^{\circ} \mathrm{C}$ aparece un salto justificado por la singularidad de que a esa temperatura la conductividad se hace nula (singularidad que puede asumir el problema aunque esté alejada de un caso real). En la figura VII.51 se presentan las estimaciones para dos valores iniciales distintos, 12.5 y 10.7, con las medidas realizadas en la superficie convectiva. De acuerdo con los valores del error $s$, mostrados en la figura, la estimación mejora cuando el punto inicial se acerca a su valor exacto, que es $10 . \Delta \mathrm{T}_{\mathrm{a}}=7.5^{\circ} \mathrm{C}$, lo que implica un total de 10 puntos de estimación más el punto inicial, separados por $3.75^{\circ} \mathrm{C}$ cada uno.

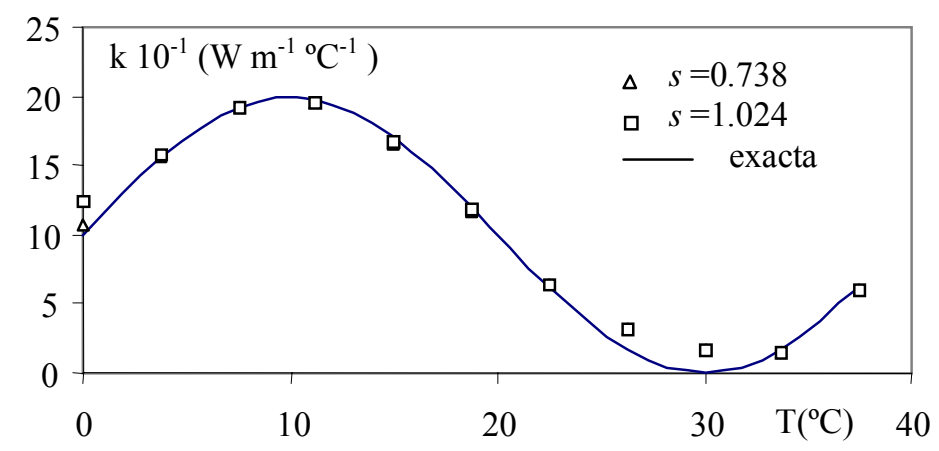

Figura VII.51. Estimación de la conductividad térmica para dependencia sinusoidal, en función de los valores 12.5 y 10.7 para el punto inicial, $\Delta \mathrm{T}_{\mathrm{a}}=7.5^{\circ} \mathrm{C}, \mathrm{x}_{\mathrm{f}}=0$ 


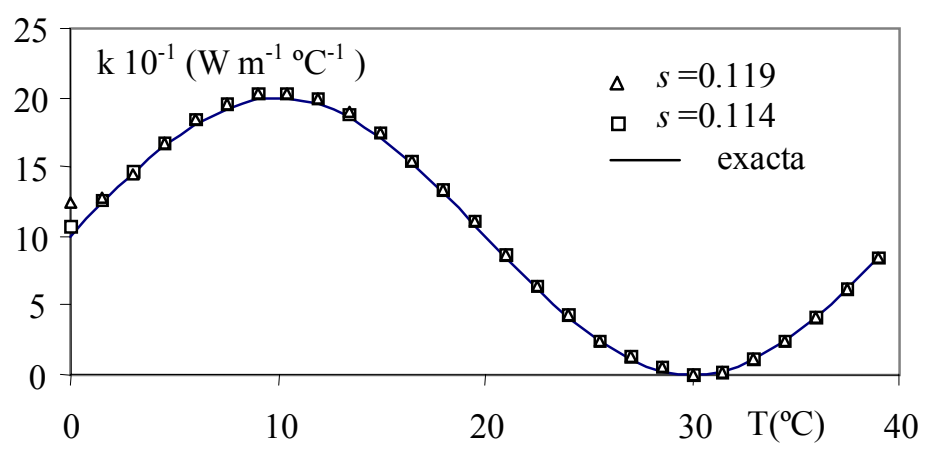

Figura VII.52. Estimación de la conductividad térmica para dependencia sinusoidal, en función de los valores 12.5 y 10.7 para el punto inicial, $\Delta \mathrm{T}_{\mathrm{a}}=3{ }^{\circ} \mathrm{C}, \mathrm{x}_{\mathrm{f}}=\mathrm{L}$

En la figura VII.52 se muestra los resultados de la estimación para el mismo caso excepto que se ha disminuido el intervalo de temperaturas de estimación a $\Delta \mathrm{T}_{\mathrm{a}}=3{ }^{\circ} \mathrm{C}$ (lo que implica un total de 26 puntos de estimación más el punto inicial, separados por $1.5^{\circ} \mathrm{C}$ ). La estimación mejora considerablemente (los errores $s$ disminuyen sensiblemente) si bien con mayores tiempos de computación.

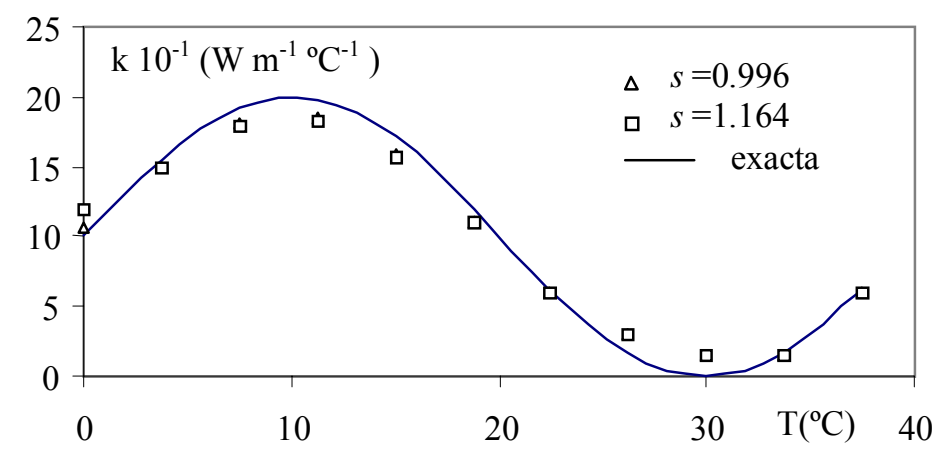

Figura VII.53. Estimación de la conductividad térmica para dependencia sinusoidal, en función de los valores 12.5 y 10.7 para el punto inicial, $\Delta \mathrm{T}_{\mathrm{a}}=7.5^{\circ} \mathrm{C}, \mathrm{x}_{\mathrm{f}}=0$

En las figuras VII.53 y VII.54 se presentan los resultados de la estimación, para el mismo caso de la figura VII.49, excepto que las medidas se toman en la superficie adiabática siendo los valores de $\Delta \mathrm{T}_{\mathrm{a}} 7.5$ y $3{ }^{\circ} \mathrm{C}$, respectivamente. Aunque el método es igualmente insensible al valor inicial de la estimación los resultados globales son peores que los obtenidos para medidas en la superficie convectiva. Puede apreciarse inmediatamente la influencia de $\Delta T_{a}$ que permite una estimación casi exacta para el menor de los valores adoptados. 


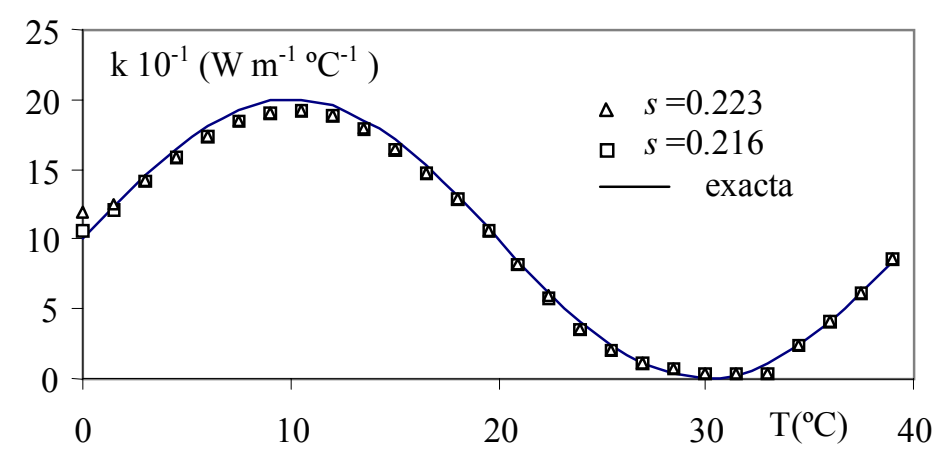

Figura VII.54. Estimación de la conductividad térmica para dependencia sinusoidal, en función de los valores 12.5 y 10.7 para el punto inicial, $\Delta \mathrm{T}_{\mathrm{a}}=3{ }^{\circ} \mathrm{C}, \mathrm{x}_{\mathrm{f}}=0$

\section{Dependencia rectangular}

Las figuras VII.55 y VII.56 muestran estimaciones de la conductividad térmica para esta dependencia, con medidas realizadas en los extremos adiabático y de convección, respectivamente, y $\Delta \mathrm{T}_{\mathrm{a}}, 3$ y $7.5^{\circ} \mathrm{C}$. Las estimaciones más precisas, siguiendo la tónica anterior, se obtienen para el menor $\Delta \mathrm{T}_{\mathrm{a}} \mathrm{y}$ tomando el punto de medida en el extremo convectivo. El cuadro VII.16 recoge los errores $s$ de todas las estimaciones.

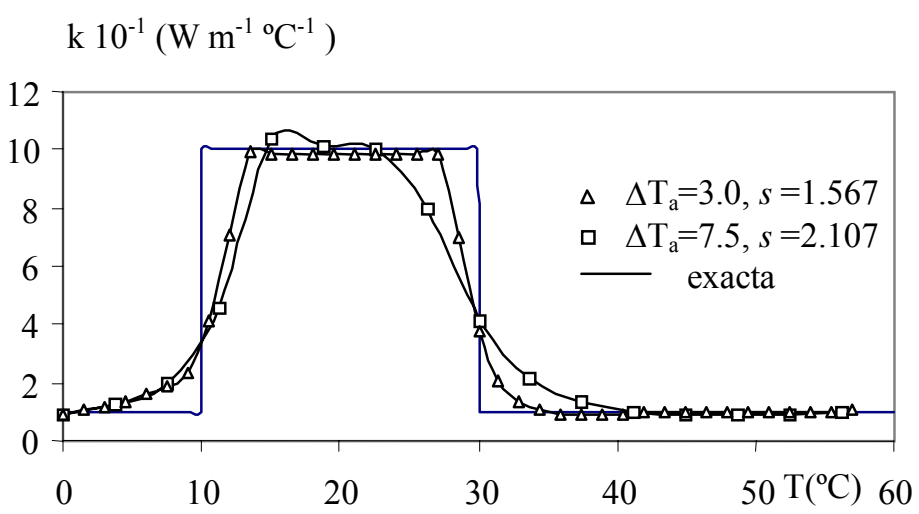

Figura VII.55. Punto de medida en la superficie adiabática, $\Delta \mathrm{T}_{\mathrm{a}}=3$ y $7.5^{\circ} \mathrm{C}$

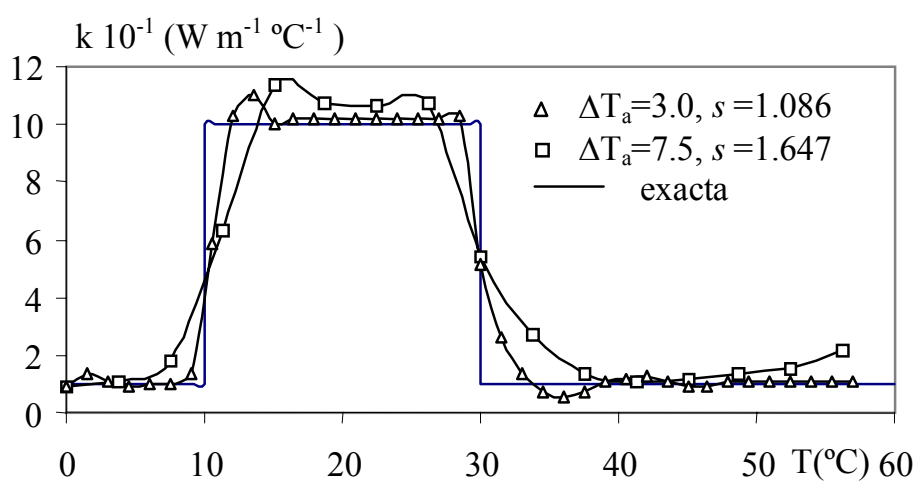

Figura VII.56. Punto de medida condición convectiva, $\Delta \mathrm{T}_{\mathrm{a}}=3$ y $7.5^{\circ} \mathrm{C}$ 


\begin{tabular}{|l|l|l|l|l|l|l|}
\hline \multirow{3}{*}{$\Delta \mathrm{T}_{\mathrm{a}}$} & \multicolumn{6}{|c|}{ Punto de medida } \\
\cline { 2 - 7 } & \multicolumn{7}{|c|}{ Superficie convectiva } & \multicolumn{3}{c|}{ Superficie adiabática } \\
\hline & Triangular & Sinusoidal & Rectangular & Triangular & Sinusoidal & Rectangular \\
\hline 3 & -------- & 0.114 & 1.086 & -------- & 0.216 & 1.567 \\
\hline 7.5 & 0.251 & 0.738 & 1.647 & 0.429 & 0.996 & 2.107 \\
\hline
\end{tabular}

Cuadro VII.16. Valores de $s$ de las diferentes estimaciones

\section{VII.3.2. Estimación de parámetros}

En este apartado se aplica la técnica de estimación de parámetros, de acuerdo con los algoritmos propuestos en el apartado III.3.5, para estimar la conductividad térmica definida como una función lineal de dos parámetros. El sistema considerado es una placa unidimensional, cuyas condiciones de contorno son adiabática e isoterma. El modelo matemático completo se recoge en el cuadro VII.17. Los parámetros a estimar son $\mathrm{k}_{0} \mathrm{y}_{1}$. Los valores numéricos del problema son: $\mathrm{c}_{\mathrm{e}}=1 \mathrm{~kJ} / \mathrm{kg}{ }^{\circ} \mathrm{C}, \rho=1 \mathrm{~kg} / \mathrm{m}^{3}, \mathrm{k}_{0}=1 \mathrm{~W} / \mathrm{m}^{2}{ }^{\circ} \mathrm{C}, \mathrm{k}_{1}=0.9$ $\mathrm{W} / \mathrm{m}^{2}, \mathrm{~T}_{\mathrm{o}}=0^{\circ} \mathrm{C}, \mathrm{T}_{\infty}=10^{\circ} \mathrm{C}, \mathrm{L}=1 \mathrm{~m}, \mathrm{y} \Delta \mathrm{t}=1 \mathrm{~s}$.

\begin{tabular}{|c|c|}
\hline \multicolumn{2}{|c|}{ Modelo matemático } \\
\hline $\begin{array}{c}\text { Ecuación } \\
\text { diferencial }\end{array}$ & $\rho \mathrm{c}_{\mathrm{e}} \partial \mathrm{T} / \partial \mathrm{t}-\mathrm{k}(\mathrm{T})\left(\partial^{2} \mathrm{~T} / \partial \mathrm{x}^{2}\right)=0$ \\
\hline $\begin{array}{c}\text { Condición } \\
\text { inicial }\end{array}$ & $\mathrm{T}(\mathrm{x},=0)=\mathrm{T}_{0}$ \\
\hline $\begin{array}{c}\text { Condiciones de } \\
\text { contorno }\end{array}$ & $\mathrm{T}(\mathrm{L}, \mathrm{t})=\mathrm{T}_{\infty}$ \\
\hline $\begin{array}{c}\text { Condición } \\
\text { adicional }\end{array}$ & $\mathrm{j}(0, \mathrm{t})=0($ punto de medida $)$ \\
\hline
\end{tabular}

Cuadro VII.17. Modelo matemático

El cuadro VII.18 muestra los resultados de la estimación para diferentes desviaciones típicas en las medidas de temperaturas. Como en los casos anteriores las estimaciones, son menos precisas al aumentar el error. Es importante mencionar que los tiempos de computación son bastante inferiores que en los problemas de estimación de función. 


\begin{tabular}{|l|l|l|}
\hline \multicolumn{1}{|c|}{$\sigma$} & \multicolumn{1}{c|}{$\mathrm{k}_{0}$} & \multicolumn{1}{c|}{$\mathrm{k}_{1}$} \\
\hline 0 & 0.950 & 0.908 \\
\hline 0.05 & 1.120 & 0.923 \\
\hline 0.1 & 1.323 & 0.842 \\
\hline
\end{tabular}

Cuadro VII.18. Valores estimados de los parámetros $\mathrm{k}_{0} \mathrm{y}_{1}$

\section{VII.4. ESTIMACIÓN SIMULTÁNEA DEL CALOR ESPECÍFICO Y DE LA CONDUCTIVIDAD TÉRMICA}

En este apartado se aborda un problema inverso de reciente interés en la literatura científica, la estimación simultánea de las dos propiedades termofísicas conductividad térmica y calor específico. Su resolución requiere un protocolo diferente (apartado III.4) que incluye la toma de medidas de temperatura en más de una posición y la evaluación de un funcional de mayor complejidad. El modelo matemático se recoge en el cuadro VII.19.

\begin{tabular}{|l|l|}
\hline \multicolumn{2}{|c|}{ Modelo matemático } \\
\hline Ecuación diferencial & $\rho \mathrm{c}_{\mathrm{e}}(\mathrm{T}) \partial \mathrm{T} / \partial \mathrm{t}-\mathrm{k}\left(\partial^{2} \mathrm{~T} / \partial \mathrm{x}^{2}\right)=0$ \\
\hline Condición inicial & $\mathrm{T}(\mathrm{x}, 0)=\mathrm{T}_{0}$ \\
\hline Condiciones de contorno & $\begin{array}{l}\mathrm{j}(0, \mathrm{t})=0 \\
\mathrm{j}(\mathrm{L}, \mathrm{t})=\mathrm{j}_{\text {con-forzada }}\end{array}$ \\
\hline Condición adicional & $\mathrm{T}_{\mathrm{IHCP}}\left(\mathrm{x}_{\mathrm{f}}, \mathrm{t}_{\mathrm{j}}, \varepsilon_{\mathrm{j}}\right)=\mathrm{T}_{\mathrm{DHCP}}\left(\mathrm{x}_{\mathrm{f}}, \mathrm{t}_{\mathrm{j}}\right)+\varepsilon_{\mathrm{j}}$ \\
\hline
\end{tabular}

Cuadro VII.19. Modelo matemático

Los tipos de dependencias con la temperatura $c_{e}(T)$ y $k(T)$ se muestran en el cuadro VII.20 y los datos numéricos del problema en el cuadro VII.21. Los puntos de medida de temperatura se han tomado en las posiciones $\mathrm{x}=\mathrm{L} / 4, \mathrm{x}=\mathrm{L} / 2$ y $\mathrm{x}=\mathrm{L}$. 


\begin{tabular}{|l|l|l|}
\hline \multicolumn{2}{|c|}{ Dependencias del calor especifico y de la conductividad } \\
\hline \multirow{2}{*}{$\begin{array}{l}\text { Dependencia lineal de } \\
\text { ambas }\end{array}$} & Calor específico & $\mathrm{c}_{\mathrm{e}}=\mathrm{c}_{0}+\mathrm{c}_{1} \mathrm{~T}$ \\
\cline { 2 - 3 } & Conductividad & $\mathrm{k}=\mathrm{k}_{0}+\mathrm{k}_{1} \mathrm{~T}$ \\
\hline $\begin{array}{l}\text { Dependencia senoidal } \\
\text { de ambas }\end{array}$ & Calor específico & $\mathrm{c}_{\mathrm{e}}=\mathrm{c}_{0}+\mathrm{c}_{1} \operatorname{sen}(\omega \mathrm{T})$ \\
\cline { 2 - 3 } & Conductividad & $\mathrm{k}=\mathrm{k}_{0}+\mathrm{k}_{1} \operatorname{sen}(\omega \mathrm{T})$ \\
\hline $\begin{array}{l}\text { Dependencia } \\
\text { triangular del calor } \\
\text { específico y lineal de } \\
\text { la conductividad }\end{array}$ & Calor específico & $\begin{array}{l}\mathrm{c}_{\mathrm{e}}\left(\mathrm{T}_{1}\right)=\mathrm{c}_{1}, \mathrm{c}_{\mathrm{e}}\left(\mathrm{T}_{2}\right)=\mathrm{c}_{2}, \mathrm{c}_{\mathrm{e}}\left(\mathrm{T}_{3}\right)=\mathrm{c}_{3}, \\
\mathrm{c}_{\mathrm{e}}\left(\mathrm{T}_{4}\right)=\mathrm{c}_{4} \mathrm{y} \mathrm{c}_{\mathrm{e}}\left(\mathrm{T}_{5}\right)=\mathrm{c}_{5}\end{array}$ \\
\cline { 2 - 3 } & Conductividad & $\mathrm{k}=\mathrm{k}_{0}+\mathrm{k}_{1} \mathrm{~T}$ \\
\hline
\end{tabular}

Cuadro VII.20. Tipos de dependencias

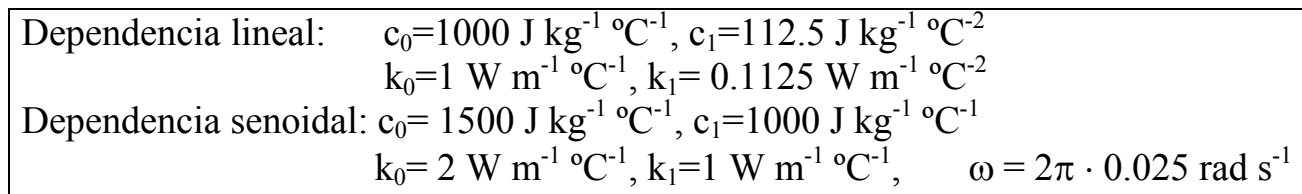

Dependencia triangular del calor específico y lineal de la conductividad:

Calor específico: $\mathrm{c}_{1}\left(\mathrm{~T}_{1}=0{ }^{\circ} \mathrm{C}\right)=1500 \mathrm{~J} \mathrm{~kg}^{-1}{ }^{\circ} \mathrm{C}^{-1}, \mathrm{c}_{2}\left(\mathrm{~T}_{2}=10^{\circ} \mathrm{C}\right)=1500 \mathrm{~J} \mathrm{~kg}^{-1}{ }^{\circ} \mathrm{C}^{-1}, \mathrm{c}_{3}\left(\mathrm{~T}_{3}=20\right.$

$\left.{ }^{\circ} \mathrm{C}\right)=4000 \mathrm{~J} \mathrm{~kg}^{-1}{ }^{\circ} \mathrm{C}^{-1}, \mathrm{c}_{4}\left(\mathrm{~T}_{4}=30^{\circ} \mathrm{C}\right)=2000 \mathrm{~J} \mathrm{~kg}^{-1}{ }^{\circ} \mathrm{C}^{-1} \mathrm{y} \mathrm{c}_{5}\left(\mathrm{~T}_{5}=40^{\circ} \mathrm{C}\right)=2000 \mathrm{~J} \mathrm{~kg}^{-1}{ }^{\circ} \mathrm{C}^{-1}$

Conductividad: $\mathrm{k}_{0}=2 \mathrm{~W} \mathrm{~m}^{-1}{ }^{\circ} \mathrm{C}^{-1}, \mathrm{k}_{1}=0.1375 \mathrm{~W} \mathrm{~m}^{-1}{ }^{\circ} \mathrm{C}^{-2}$

Datos generales:

$\rho=870 \mathrm{~kg} / \mathrm{m}^{3}, \mathrm{~L}=2 \mathrm{~cm}, \mathrm{~T}_{\mathrm{o}}=0{ }^{\circ} \mathrm{C}, \mathrm{T}_{\infty}=80^{\circ} \mathrm{C}$ (caso lineal), $\mathrm{T}_{\infty}=60{ }^{\circ} \mathrm{C}$ (caso senoidal), $\mathrm{h}=10$

$\mathrm{W} / \mathrm{m}^{2}$

Intervalo de tiempo de medida: $\Delta \mathrm{t}=5 \mathrm{~s}$

Intervalo de temperatura en la estimación: $\Delta \mathrm{T}_{\mathrm{a}}=2^{\circ} \mathrm{C}$

\section{Cuadro VII.21. Datos numéricos}

En la figura VII.57 se representa el modelo físico, con las condiciones de contorno y la situación de los sensores en todos los casos analizados.

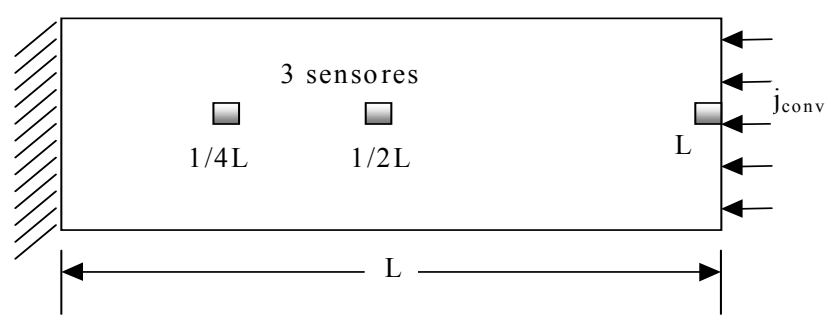

Figura VII.57. Modelo físico 
Las estimaciones obtenidas, para el caso de dependencias lineales, se muestran en las figuras VII.58 y VII.59, para una desviación típica en las medidas de $\sigma=0.01$. En estas gráficas puede apreciarse inmediatamente la bondad de la estimación.

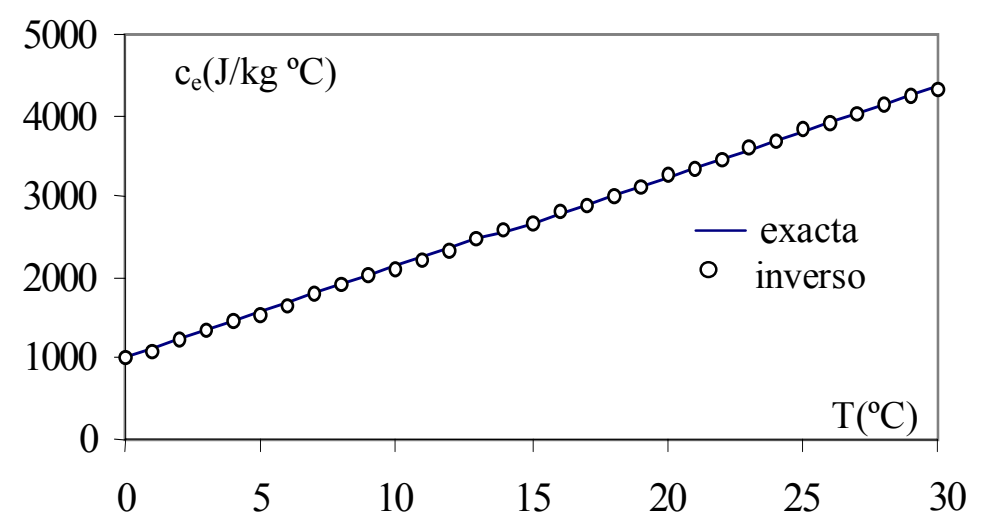

Figura VII.58. Calor específico. Caso lineal, $\sigma=0.01, \Delta \mathrm{T}_{\mathrm{a}}=2^{\circ} \mathrm{C}$ y $\Delta \mathrm{t}=5 \mathrm{~s}$

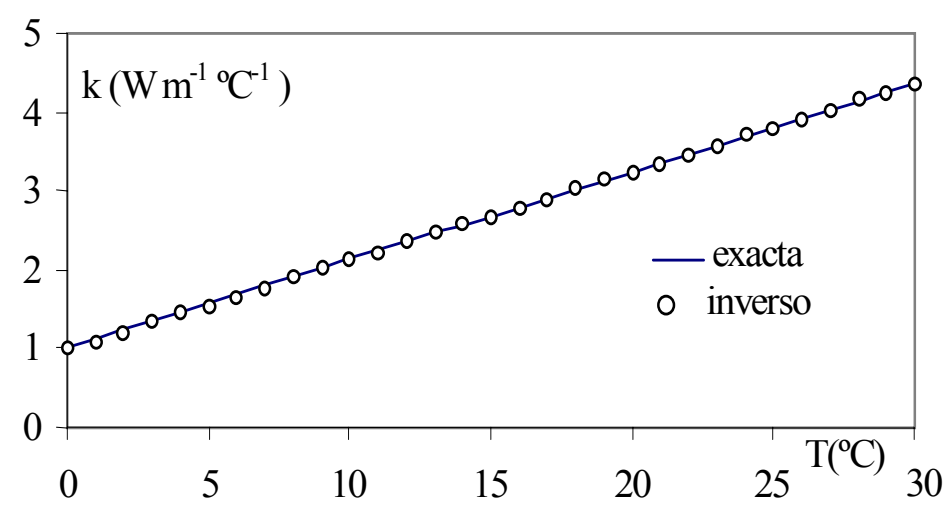

Figura VII.59. Conductividad térmica. Caso lineal, $\sigma=0.01, \Delta \mathrm{T}_{\mathrm{a}}=2^{\circ} \mathrm{C}$ y $\Delta \mathrm{t}=5 \mathrm{~s}$

En cuanto a dependencias tipo sinusoidal, las figuras VII.60 y VII.61 muestran las estimaciones obtenidas para $\sigma=0.05$. Aun a pesar del alto valor de $\sigma$ los resultados pueden considerarse relativamente buenos. 


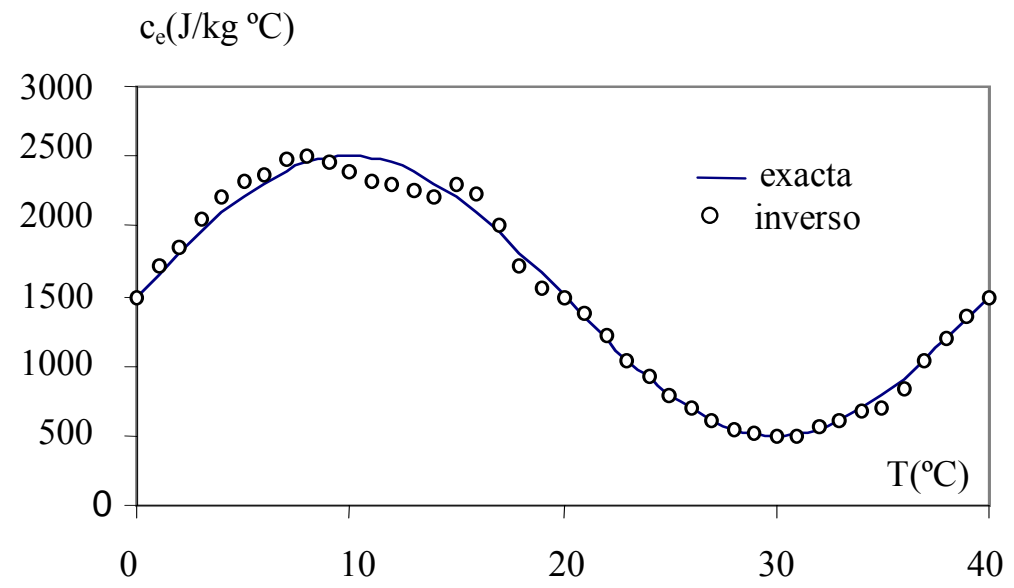

Figura VII.60. Calor específico. Caso sinusoidal, $\sigma=0.05, \Delta \mathrm{T}_{\mathrm{a}}=2^{\circ} \mathrm{C}$ y $\Delta \mathrm{t}=5 \mathrm{~s}$

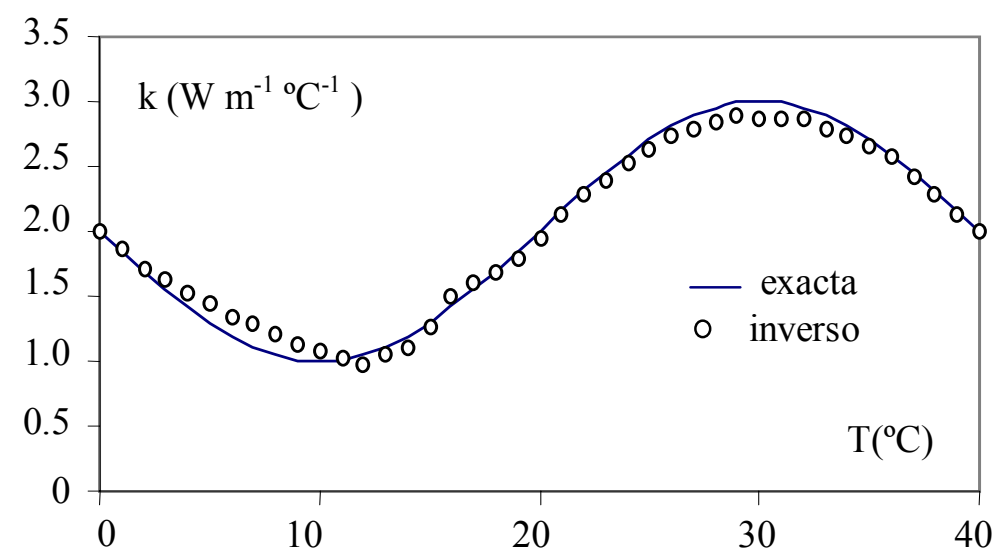

Figura VII.61. Conductividad térmica. Caso sinusoidal, $\sigma=0.05, \Delta \mathrm{T}_{\mathrm{a}}=2^{\circ} \mathrm{C}$ y $\Delta \mathrm{t}=5 \mathrm{~s}$

En las figuras VII.62 y VII.63 se muestran las estimaciones simultáneas para dependencias tipo triangular y lineal para el calor específico y la temperatura, respectivamente, con $\sigma=0$. Como puede apreciarse se trata de estimaciones muy precisas.

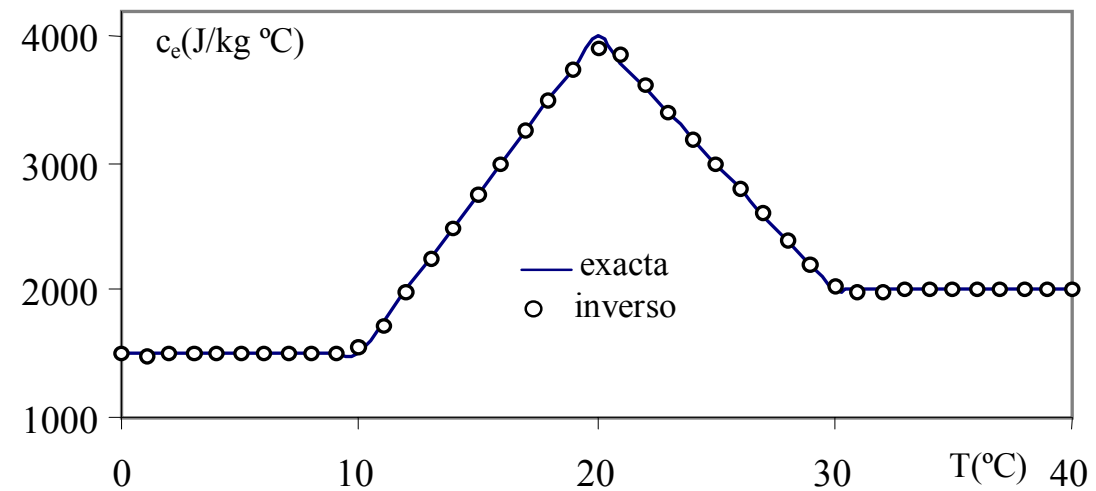

Figura VII.62. Calor específico. Caso combinado, $\sigma=0, \Delta \mathrm{T}_{\mathrm{a}}=2^{\circ} \mathrm{C}$ y $\Delta \mathrm{t}=5 \mathrm{~s}$ 


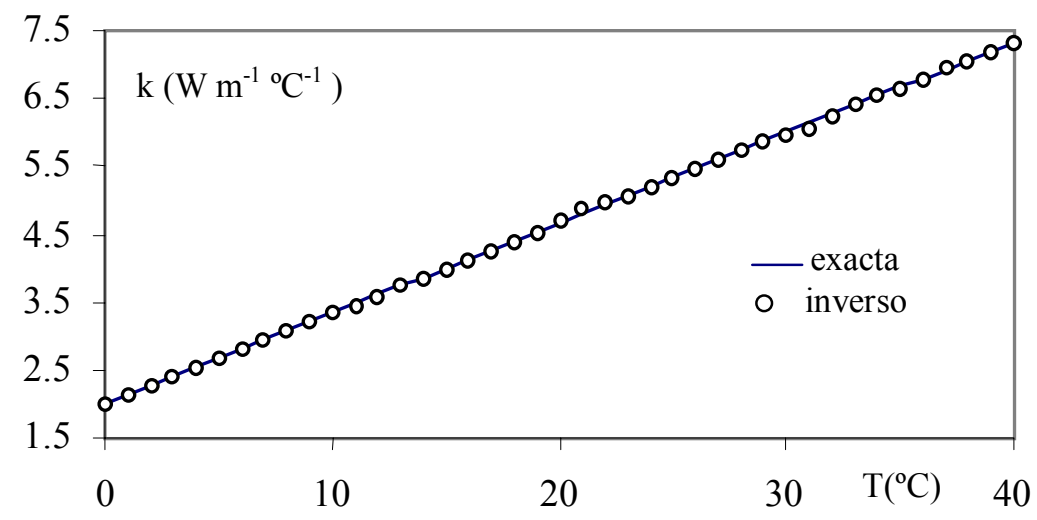

Figura VII.63. Conductividad térmica. Caso combinado, $\sigma=0, \Delta \mathrm{T}_{\mathrm{a}}=2^{\circ} \mathrm{C}$ y $\Delta \mathrm{t}=5 \mathrm{~s}$

\section{VII.4.1. Aplicaciones}

Como primera aplicación práctica se determina, a continuación, la estimación simultánea del calor especifico y la conductividad de las fresas. Los datos y la geometría utilizados son los expuestos en el apartado VII.2.3 (tabla VII.1 y cuadro VII.12), $\Delta \mathrm{T}_{\mathrm{a}}=2^{\circ} \mathrm{C}$ y $\Delta \mathrm{t}=5 \mathrm{~s}$. Las estimaciones obtenidas con $\sigma=0$, muy precisas, se muestran en las figuras VII.64 y VII.65.

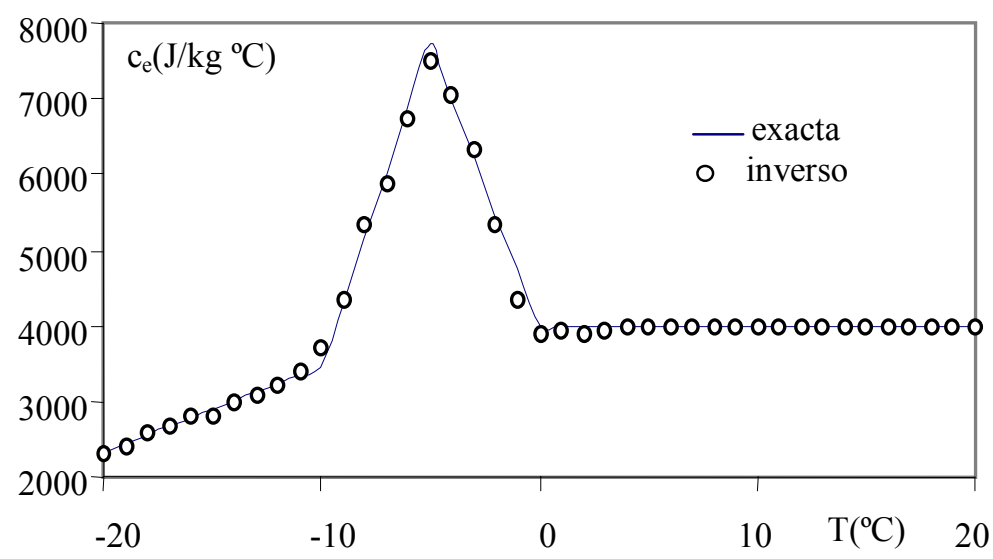

Figura VII.64. Calor específico de las fresas, $\sigma=0, \Delta \mathrm{T}_{\mathrm{a}}=2^{\circ} \mathrm{C}$ y $\Delta \mathrm{t}=5 \mathrm{~s}$ 


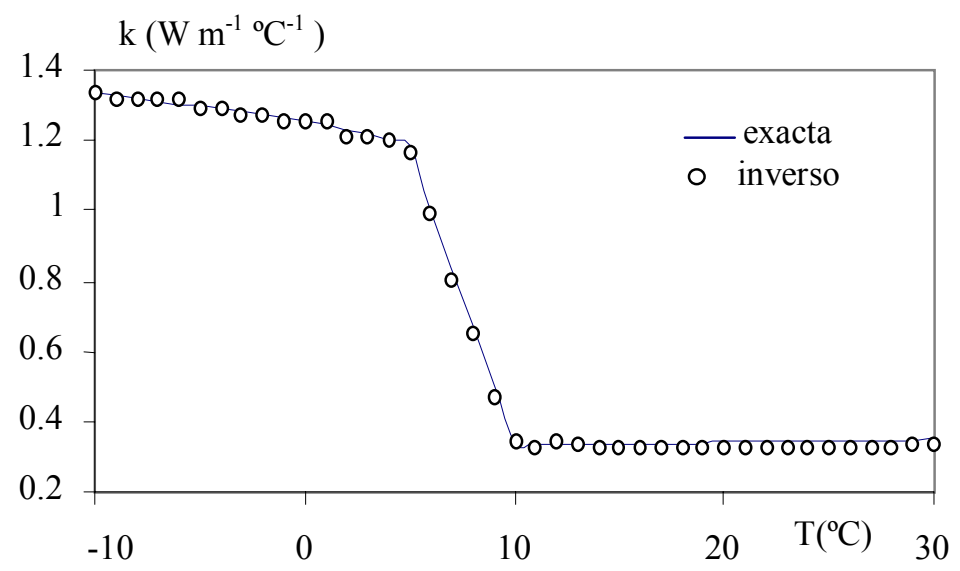

Figura VII.65. Conductividad térmica de las fresas, $\sigma=0, \Delta \mathrm{T}_{\mathrm{a}}=2^{\circ} \mathrm{C}$ y $\Delta \mathrm{t}=5 \mathrm{~s}$

Como segunda aplicación de estimación simultánea, se han estimado las propiedades térmicas del hierro, sometido a condiciones de contorno adiabática y de flujo de calor constante, $\mathrm{j}=25 \mathrm{kWm}^{-2}$. Los datos numéricos se muestran en el cuadro VII.22 y los resultados de la estimación en las figuras VII.66 y VII.67.

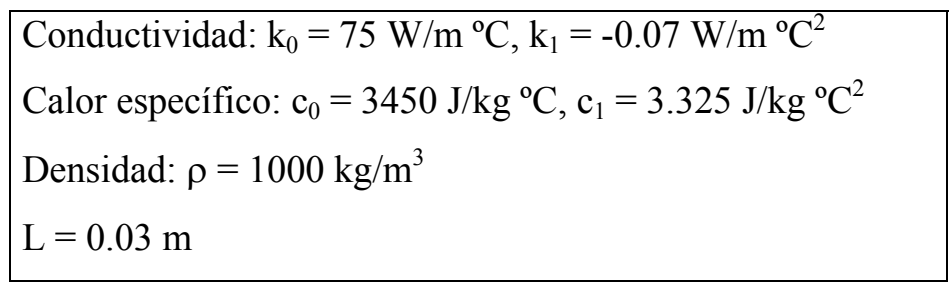

Cuadro VII.22. Datos numéricos

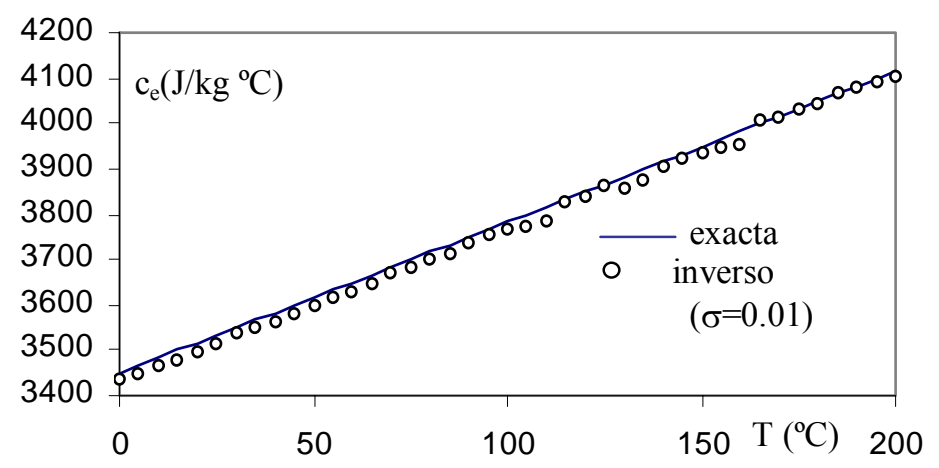

Figura VII.66. Calor específico del hierro, $\sigma=0.01, \Delta \mathrm{T}_{\mathrm{a}}=2^{\circ} \mathrm{C}$ y $\Delta \mathrm{t}=5 \mathrm{~s}$ 


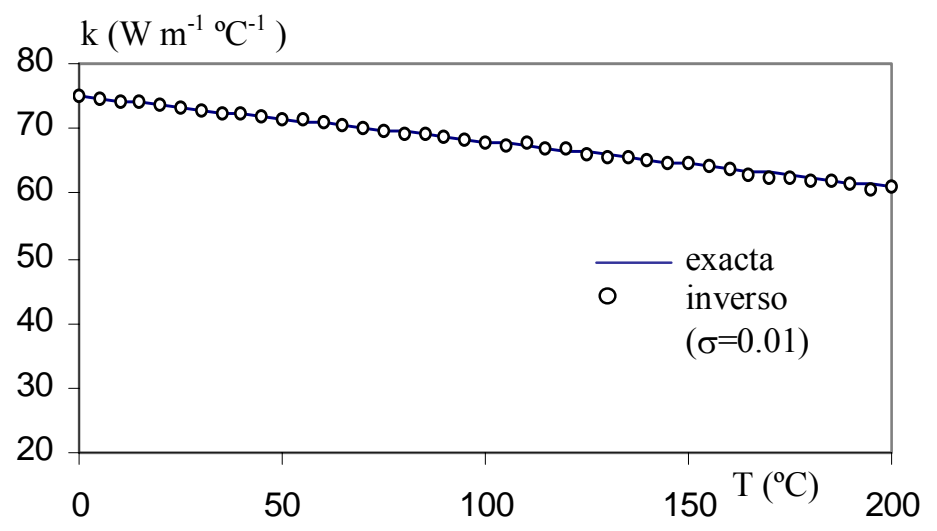

Figura VII.67. Conductividad térmica del hierro, $\sigma=0.01, \Delta \mathrm{T}_{\mathrm{a}}=2^{\circ} \mathrm{C}$ y $\Delta \mathrm{t}=5 \mathrm{~s}$

El problema reproduce las condiciones de ensayo propuestas por Huang y Özisik [1991].

\section{VII.5. REFERENCIAS}

Bayazitoglu, Y., Suryanarayana, P.V.R. y Sathuvalli, U.B., "High-temperature thermal diffusivity determination procedure for solids and liquids", J. Thermophysics, 4 (4), 462468 (1989)

Carslaw, H.S. y Jaeger, J.C., "Conduction of heat in solids”, 2ª ed., Oxford Univ. Press, London y New York, cap. 3 (1959)

Chantasiriwan, S., "Steady-state determination of temperature-dependent thermal conductivity", Int. Commun. Heat Mass Transf., 29, 6, 811-819 (2002)

Chen, H.T. y Lin, J.Y.,"Simultaneous estimations of temperature-dependent thermal conductivity and heat capacity", Int. J. Heat Mass Transf., 41(14), 2237-2244 (1998)

Dantas L.B. y Orlande, H.R.B., "A function estimation approach for determining temperaturedependent thermophysical properties", Inv. Prob. Eng., 3, 261-279 (1996)

Flach G.P. y M.N. Özisik, "Inverse heat conduction problem of simultaneosly estimating spatially varying thermal conductivity and heat capacity per unit volume", Numer. Heat Transfer-A, 16, 249-266 (1989) 
Huang C.H. y Özisik, M.N. "Direct integration approach for simultaneously estimating temperature dependent thermal conductivity and heat capacity", Numer. Heat Transfer-A, 20, 95-110 (1991)

Huang Cheng-Hung, Yan Jan-Yuan y Chen Han-Taw, "Function estimation in predicting temperature-dependent thermal conductivity without internal measurements", J. Thermophys Heat Transf., 9(4), 667-673 (1995)

Huang Cheng-Hung y Yan Jan-Yuan, "An inverse problem in simultaneously measurement temperature-dependent thermal conductivity and heat capacity", Int. J. Heat Mass Transf., 38(18), 3433-3441 (1995)

Huang Cheng-Hung y Chin S.C., "A two-dimensional inverse problem in imaging the thermal conductivity of a non-homogeneous medium", Int. J. Heat Mass Transf., 43(22), 4061-4071 (2000)

Jurkowski, T., Jarny, Y. y Delaunay, D.,"Estimation of thermal conductivity of thermoplastics under moulding conditions: an apparatus and an inverse algorithm", Int. J. Heat Mass Transf., 40(17), 4169-4182 (1997)

Kim, S., Chung B-J, Chan M. y Youn, K. "A note on the direct estimation of thermal properties in a transient nonlinear heat conduction medium", Int. Commun. Heat Mass Transf., 29, 6, 787-795 (2002)

Kim, S., Lee, W., "An inverse method for estimating thermophysical properties of fluid flowing in a circular duct", Int. Commun. Heat Mass Transf., 29, 8, 1029-1036 (2002)

Lam, T.T. y Yeung W.K., "Inverse determination of thermal conductivity for one-dimensional problems", J. Thermophys Heat Transf., 9 (2), 235-344 (1995)

Lesnic, L., Elliot, L., Inghan, D.B., Clennell, B. y Knipe, R.J.,"The identification of the piecewise homogeneous thermal conductivity of conductors subjected to a heat flow test", Int. J. Heat Mass Transf., 42(1), 143-152 (1999)

Martin, T.J. y Dulikravich, G.S., "Inverse determination of temperature-dependent thermal conductivity using steady surface data on arbitrary objects ", J. Heat Transfer, 122, 450459 (2000)

Mc Adams, W. H., “Heat transmission” 3ª ed., New York, McGraw Hill (1954)

Milosevic, N.D., Raynaud, M. y Maglic, K.D., "Estimation procedure in thermal diffusivity measurement of double-layer sample using the laser flash method", Proc. Eurotherm Seminar 68, Poitiers, 311-318 (2001) 
Polley, S.L., Snyder, O.P. y Kotnour, P. “A compilation of thermal properties of foods”. Food Technol, 76-94 (1980)

Rao, M.A. y Rizvi, S.S.H. "Engineering properties of foods. Food-Analysis. Series: Food science and technology”. Marcel Dekker Inc., New York (1986)

Sawaf, B., Özisik, M.N. y Jarny, Y., "An inverse analysis to estimate linearly temperature dependent thermal conductivity components and heat capacity of an orthotropic medium", Int. J. Heat Mass Transf., 28 (16), 3005-3010 (1995)

Tervola, P. "A method to determine the thermal conductivity from measured temperature profiles", Int. J. Heat Mass Transf., 32, 8, 1425-1430 (1989)

Yang, Ching-Yu, "A linear inverse model for the temperature-dependent thermal conductivity determination in one-dimensional problems", Appl. Math. Model., 22, 1-9 (1998)

Yang, Ching-Yu, "Estimation of the temperature dependent thermal conductivity in inverse heat conduction problem", Appl. Math. Model., 23, 469-478 (1999)

Yang, Ching-Yu, "Determination of the temperature dependent thermophysical properties from temperature responses measured at medium's boundaries", Int. J. Heat Mass Transf., 43, 1261-1270 (2000)

Zueco, J., Alhama, F., Pérez, A. y González-Fernández, C.F., "Determination of the specific heat versus temperature under a boundary condition of natural convection using the network simulation method”, Proc. Eurotherm Seminar 68, Poitiers, 319-324 (2001)

Zueco, J., Alhama, F. y González Fernández, C.F., "Estimación de propiedades térmicas en productos agroalimenticios de forma regular”, I CYTEF, Cartagena (2002a) (en prensa)

Zueco, J., Alhama, F. y González-Fernández, C.F., “A simple model for estimating enthalpy versus temperature in the range of phase change. Application to foods products", Proc. 12 Int. Heat Transfer Conference, Grenoble, 15-20 (2002b)

Zueco, J., Alhama, F y González-Fernández, C.F., “An inverse problem to estimate temperature dependent heat capacity under convection processes”, Heat and Mass Transfer (2002c) (en prensa)

Zueco, J., Alhama, F. y González Fernández, C.F., "Estimación inversa simultánea de las propiedades térmicas", Anales de Ingeniería Mecánica (2002d) (en prensa) 


\section{APORTACIONES Y CONCLUSIONES}

Se incluye un estudio bibliográfico de las contribuciones que diferentes autores, con distintas técnicas, han aportado en el campo de la estimación de propiedades térmicas dependientes de la temperatura.

Se han obtenido estimaciones precisas del calor específico dependiente linealmente de la temperatura, para medios 1-D, con condiciones de contorno adiabática en un extremo y de convección forzada débil en el otro extremo, empleando los protocolos propuestos en el capítulo III que asumen el desconocimiento a priori de las formas de estas dependencias.

Se ha estudiado la influencia en el resultado de los siguientes parámetros: (i) intervalo de temperatura de estimación, (ii) intervalo de tiempo entre medidas, (iii) posición del punto de medida, y (iv) valor inicial de la estimación, obteniéndose los errores $s$ de todas las estimaciones. La posición de medida en la superficie convectiva proporciona en todos los casos estimaciones sensiblemente mejores; la influencia del intervalo de tiempo entre medidas no es significativa en la estimación, observándose, sin embargo que valores pequeños del intervalo de temperatura de estimación consiguen estimaciones más precisas.

Igualmente, se han obtenido estimaciones muy aceptables del calor específico dependiente de la temperatura en forma triangular (piece-wise) y en forma sinusoidal, aunque con errores algo superiores a la dependencia lineal, por la dificultad inherente a esta dependencia. Se han estudiado las influencias de los parámetros: (i) intervalo de estimación de temperaturas, (ii) intervalo de tiempo entre medidas, (iii) valor inicial de la estimación y (iv) posición del punto de medida. Este último parámetro tiene mayor incidencia en los resultados, obteniéndose estimaciones más precisas, siempre, cuando el punto de medida se sitúa en la superficie convectiva. Por otro lado, las influencias del intervalo de tiempo entre medidas y del punto inicial de estimación (estudiada en la dependencia sinusoidal), para desviaciones no muy grandes, pueden considerarse despreciables.

Las estimaciones del calor específico para dependencias rectangulares y con medidas sin error son, obviamente, menos precisas con los protocolos propuestos aunque pueden aproximarse, en cierto grado, al bajar el parámetro intervalo de temperatura de estimación. La adopción de errores en las medidas, proporciona también estimaciones aceptables dentro del campo de la ingeniería térmica para dependencias lineales, sinusoidales y del tipo piece-wise, particularmente para errores pequeños. Su análisis se ha realizado para condiciones de contorno 
de convección natural. Las estimaciones se desvían sensiblemente de la solución exacta para dependencias rectangulares o errores elevados en las medidas.

Se presentan aplicaciones del cálculo de $\mathrm{c}_{\mathrm{e}}$ a productos alimenticios (mantequilla, pescado blanco, fresas y carne de ternera) en procesos de cambio de fase (descongelación), obteniéndose estimaciones satisfactorias. Las medidas de temperatura se han afectado de errores de valor típico para la instrumentación propia de este campo.

Se obtienen estimaciones muy precisas de la conductividad térmica para dependencias lineal, triangular y sinusoidal usando los protocolos de estimación del capítulo III que, como siempre, no precisan de información a priori del tipo de función a estimar. Se estudia la influencia del valor de la estimación inicial, que se comprueba es pequeña, y la influencia de intervalo de temperaturas de medida, cuya disminución mejora sensiblemente la estimación. Por otro lado, las mediciones en la superficie convectiva proporcionan mejores estimaciones que las efectuadas en la superficie adiabática.

Se obtienen estimaciones de parámetros usando el protocolo propuesto en el capítulo III para el caso de funciones dos parámetros, aplicado a la obtención de conductividad linealmente dependiente de la temperatura.

Por último, se han realizado estimaciones simultáneas del calor específico y la conductividad térmica de dos sustancias de diferente naturaleza, las fresas y el hierro. En el primer caso se trabaja en la zona de cambio de fase (donde se dan cambios muy acusados de estas propiedades), con geometría esférica y sin errores en las medidas. En el segundo, de dependencias más suaves, se introducen errores en las medidas. 


\section{Capítulo VIII}

\section{Contribuciones y conclusiones}

Se incluye, a continuación, lo que a nuestro juicio constituyen las aportaciones y conclusiones más importantes que se derivan de esta memoria:

\section{Primera}

Se establecen, dentro del campo de la transmisión de calor, nuevos procedimientos de estimación de funciones (que pueden ser extendidos al problema de estimación de parámetros) basados en el uso de funciones continuas del tipo "lineal a tramos" (piece wise functions). Dichos procedimientos, que se aplican tanto para la estimación de condiciones de contorno (particularmente flujos incidentes de calor dependientes del tiempo) como para la estimación de propiedades térmicas (dependientes de la temperatura), proporcionan estimaciones muy aceptables en todos los tipos de dependencia estudiados. También se incluye un procedimiento para la estimación simultánea de las propiedades térmicas conductividad y calor específico.

Estos procedimientos, integrados en un algoritmo de computación en $\mathrm{C}++$ elaborado a tal efecto, se ejecutan proporcionando las soluciones numéricas de las estimaciones con auxilio del método de simulación por redes y el software Pspice.

\section{Segunda}

En base a los modelos conocidos de solución de problemas directos, se han establecido modelos en red para la estimación de diferentes problemas inversos en transferencia de calor. En concreto:

(i) Estimación de variables asociadas a las condiciones de contorno:

- Flujos de calor

- Temperaturas

- Coeficiente de convección 
(ii) Estimación de propiedades térmicas dependientes de la temperatura:

- Calor especifico

- Conductividad

- Calor especifico y conductividad simultáneamente

(iii) Estimación de la generación interna de calor

\section{Tercera}

Se han obtenido estimaciones precisas de flujos incidentes para un amplio espectro de formas de onda (constante, triangular, sinusoidal y en escalón), con discontinuidades en los cambios de flujo (primera derivada), usando medios con propiedades térmicas constantes o dependientes de la temperatura. Las estimaciones se obtiene bajo la forma de función lineal a tramos (piece-wise function) en todos los casos sin conocimiento a priori de la forma de onda a determinar. Para cada forma de onda se estudia la influencia en la estimación de los parámetros más significativos: error en las medidas, número de términos del funcional y valor inicial de la estimación. Se ha trabajado con errores estrictamente aleatorios y con errores sujetos a una distribución normal. La forma de onda rectangular aplicada a medios con características térmicas dependientes de la temperatura y con medidas afectadas de error $(\sigma=0.01)$, proporciona la estimación menos precisa; no obstante en todos los casos, las desviaciones de las estimaciones respecto del valor teórico son aceptables dentro del campo de la ingeniería térmica.

\section{Cuarta}

Del mismo modo, bajo la solución de función lineal a tramos se han obtenido estimaciones directas de temperaturas como condición de contorno para formas de onda lineal a tramos y rectangular. Las estimaciones son muy precisas en el primer caso. La información a priori de la forma de onda es desconocida. La influencia del valor inicial de estimación es despreciable aún en el caso de que esté muy desviado de su valor exacto.

\section{Quinta}

Se han realizado estimaciones precisas de otras magnitudes de interés en transmisión del calor, como, la generación interna de calor, para dependencias triangulares con el tiempo y la temperatura; el coeficiente de convección, (o su valor adimensional el número de Nusselt) para un proceso de convección natural; la emisividad superficial, independiente de la temperatura, en un proceso que combina las condiciones de contorno de convección más radiación; y la resistencia térmica de contacto, independiente de la temperatura. 


\section{Sexta}

Se han obtenido estimaciones precisas para dependencias del calor específico con la temperatura de tipo lineal, lineal a tramos (piece-wise) y sinusoidal. Para ello se han usado condiciones de contorno de convección forzada débil y o convección natural en uno de los extremos del medio y condición adiabática en el otro extremo. La influencia de los errores en las medidas se ha estudiado para el caso de covección natural. Las estimaciones son aceptables para valores de error típicos de los medidores de temperatura propios de este campo de la ingeniería.

Se ha estudiado la influencia en las estimaciones, para todas las dependencias mencionadas, del intervalo de tiempo entre medidas, del intervalo de temperatura de estimación, del punto de medida y del valor inicial de la estimación, no siendo significativa ninguna de ellas, excepto en lo que se refiere al punto de medida, resultando que las mediciones en la superficie convectiva proporcionan estimaciones más precisas en todos los casos que las obtenidas con mediciones en la superficie adiabática. Tampoco el punto inicial tiene un efecto significativo en la estimación.

Para dependencias del calor específico de forma de onda rectangular, los valores del intervalo de temperatura de estimación influyen notablemente en la bondad de la estimación a causa de las fuertes discontinuidades presentes.

\section{Séptima}

Se han obtenido estimaciones precisas de la conductividad térmica para dependencias con la temperatura de tipo lineal, triangular, sinusoidal y rectangular, trabajando en un medio 1$\mathrm{D}$, rectangular y con condiciones convectiva y adiabática en sus superficies exteriores, estudiándose la influencia de la posición del punto de medida (superficie adiabática o convectiva), del valor inicial de la estimación y del intervalo de temperatura de estimación. Los resultados son esencialmente coherentes con los mencionados para la estimación del calor específico. Por otro lado, se han obtenido estimaciones para la dependencia lineal de la conductividad térmica por medio de la técnica de estimación de parámetros, cuyos tiempos de computación son mucho menores que los de la técnica de estimación de función.

\section{Octava}

Se han obtenido estimaciones simultáneas de la conductividad y del calor específico, utilizando los procedimientos y modelos en red propuestos en esta tesis, con medidas en más de una posición, para los casos de dependencia lineal de ambas propiedades con error en las medidas, dependencia sinusoidal de ambas propiedades con error en las medidas y dependencia triangular del calor específico y lineal de la conductividad, sin error en las medidas. 


\section{Novena}

Como aplicación de los protocolos y modelos propuestos en esta memoria, se han obtenido estimaciones muy precisas del calor específico de cambio de fase, de cuatro productos alimenticios (mantequilla, pescado blanco, fresas y carne de ternera), en donde esta propiedad presenta picos de dependencia muy acusados debido a la entalpía de cambio de fase; para ello se han propuesto problemas reales de estimación con geometría cilíndrica en el caso de la fresa. Las medidas de temperatura se han afectado de un error cuyo valor es típico para la instrumentación de este campo. La influencia de los parámetros más importantes del problema sigue las tendencias generales expuestas en la estimación de esta propiedad para diferentes formas de onda.

También se han realizado estimaciones simultáneas de la conductividad y del calor específico para distintos tipos de dependencias, a partir de datos de temperatura en tres puntos del medio (internos y externos). Se ha aplicado este procedimiento a dos sólidos de distinta naturaleza, una fruta (la fresa) y un elemento metálico (el hierro) proporcionando estimaciones muy precisas en ambos casos para diferentes condiciones de contorno. 


\section{Anexo}

\section{Software desarrollado}

A.1. Estimación de flujos incidentes y focos térmicos

A.2. Estimación simultánea del calor específico y de la conductividad térmica 


\section{Anexo}

\section{Software desarrollado}

En el presente anexo se presentan algunos de los programas desarrollados en esta memoria, en concreto, los referentes a la estimación de flujos incidentes y focos térmicos y a la estimación simultánea del calor específico y de la conductividad térmica. Básicamente en cada uno de ellos se ejecutan los pasos comentados en el capítulo III (apartado III.6): ejecución del problema directo, lectura de temperaturas medidas por los sensores, modificación del parámetro a estimar, cálculo del funcional, obtención del mínimo funcional, etc. En el apartado IV.2 (problema inverso-modelos en red), se comentan brevemente las funciones más importantes empleadas.

\section{A.1. ESTIMACIÓN DE FLUJOS INCIDENTES Y FOCOS TÉRMICOS}

//Estimación de flujos de calor y de focos térmicos //(Microsoft Visual C++, versión 6.0)

//Funciones cabecera

\#include "stdafx.h"

\#include "stdio.h"

\#include "stdlib.h"

\#include "process.h"

\#include "string.h"

\#include "time.h"

\#include "math.h"

\#define Nceldas 40

\#define Ntemp 51

/*número total de celdas*/

/*número de temperaturas total medidas por un sensor*/

\#define N_ejecucion 81 /*número máximo de iteraciones por punto,debe multiplo de $3 * /$

\#define BB $10000.0 / / 5.0$ para $r=3$ en flujo cte

\#define BBB 2.0

\#define k 15 
void Salva_resultados(char);

void salva_datos_en_fichero(char activa,char de[500],int);

void graba_datos(char dd[500], int contador);

void Crea_Redes_Inicial(char,int,int);

void Crea_Redes(int,int);

void Lee datos ejecutados(void);

float Calculo_Sm(int,int);

void Busca fichero(int);

void Calculo_Minima_Sm(int,int);

void Calcula_Minimo_Final(int);

void Carga_datos(int,char);

void Generacion_Rectas_corriente_temperaturas(int,int,int,int);

void Espera_Tiempo(int);

void Temperaturas( int, int, int );

//variables externas

int Jini $=2000, a=3, b=1$;

float I_inicial $=2000.0$;

int DatosObt=1;

float Unidad tiempo $=5.0$;

float temp[Ntemp +1$]$;

*estimación inicial del q1*/

float $\mathrm{B}=\mathrm{BB}$;

float tiempo[k+1];

float $[\mathrm{k}+1]\left[\mathrm{N} \_\right.$ejecucion +1$] ; \quad / *$ valores de qi a estimar*/

float $\mathrm{L}=0.05, \mathrm{Ax}$;

float den=2707.0;

float conduct $=204.0$;

FILE *fp2;

float coef pel $=10.0$;

int $\mathrm{r}=4$;

float Temp_sensor[Ntemp +1$]$;

float I_min $[\mathrm{k}+1]$;

float $\operatorname{Min}[\mathrm{k}+1]$;

float $\mathrm{t}[\mathrm{k}+1]$;

float Minimo;

float $\operatorname{Sm}[\mathrm{k}+1]\left[\mathrm{N} \_\right.$ejecucion +1$]$;

/*poner a uno al comenzar desde el principio*/

/*intervalo de tiempo de estimación*/

/*temperaturas leídas del fichero *.out*/

float Minimo_Sm;

int $\mathrm{PP}$ final, $\mathrm{P} P$ inicial, negativo;

float $\overline{\mathrm{Ce}}=896.0, \overline{\mathrm{T}} \mathrm{a}=20.0, \mathrm{ci}=20.0$;

float Ri[ $2 *$ Nceldas];

float emisividad $=0.8$;

char problema $=1$;

char cal $\min =0$;

int caso;

/*número de temperaturas dentro del funcional*/

/*valores de qi estimados previamente*/

/*valores asociado a Sm minimo de I*/

/*variable temporal*/

/*valor mínimo del funcional*/

$/ *$ valor del funcional*/

int main(int argc, char* argv[])

\{

int $\mathrm{i}, \mathrm{d}, \mathrm{l}$;

char clave;

char clave $1=0$;

int Otra $=0$;

FILE *pa;

$\mathrm{Ax}=\mathrm{L} /$ Nceldas;

//Crea_Redes_Inicial $(0,0,0) ; / *$ para obtener la solución del DHCP*/

if $($ DatosObt $>0)$ Lee_datos_ejecutados();/*se parte de datos ya obtenidos*/

for $(1=1 ; 1<6 ; 1++)\{/ /$ para todos los casos a analizar 
if $($ DatosObt $==0)$ Carga_datos $(0,1) ; / /$ carga datos para estimación del punto inicial

caso $=1 ; \quad / *$ Definición de los casos a analizar*/

if $($ caso==1)\{ printf(" --- CASO 1 --- ");//1\%

if $($ DatosObt $>0)\{$ Carga_datos $(1,1) ;$ Unidad_tiempo $=5.0 ; \mathrm{r}=3 ;\}$

\}

$\operatorname{if}($ caso $==2)\{\quad$ printf(" --- CASO 2 --- ");

if $($ DatosObt $>0)\{$ Carga_datos $(1,1) ;$ Unidad_tiempo $=5.0 ; \mathrm{r}=4 ;\}$

\}

if $($ caso $==3)\{\quad$ printf(" --- CASO 3 --- ");

if $($ DatosObt $>0)\{$ Carga_datos $(1,1) ;$ Unidad_tiempo $=5.0 ; \mathrm{a}=1, \mathrm{r}=3 ;\}$

\}

if $($ caso $==4)\{\quad$ printf(" --- CASO 4 --- ");

if $($ DatosObt $>0)\{$ Carga datos $(1,1) ;$ Unidad tiempo $=5.0 ; \mathrm{r}=5 ;\}$

if $($ caso $==5)\{\quad$ printf(" --- CASO 5 --- ");

if(DatosObt $>0)\{$ Carga_datos $(1,1) ;$ Unidad_tiempo $=5.0 ; \mathrm{r}=4 ;\}$

\}

/*INICIO*/

for $(\mathrm{i}=$ DatosObt $; \mathrm{i}<\mathrm{k}+1 ; \mathrm{i}++)\{/ *$ controla el intervalo de tiempo a estimar*/ clave $=1 ; /$ para indicar que se cambia de valor de $i^{*} /$

for $(\mathrm{d}=$ N_ejecucion; $\mathrm{d}>0 ; \mathrm{d}--)\{$

/*controla la ejecución, recta a estimar*/

if $(\mathrm{B}<0.1)$ break; $\quad / *$ criterio de convergencia*/

/*estimación de un punto, excepto el inicial*/

/*al paso siguiente no se ejecuta pspice para el mínimo anterior*/

if $($ clave $==0)\{$

$\operatorname{if}(\mathrm{d} \% 3==0 / * \& \&$ cuen $>0 * /)\{\mathrm{I}[\mathrm{i}][\mathrm{d}]=$ Minimo;

Sm[i][d]=Minimo_Sm;

d--;

printf("\n activa ");

clave $=0$;

\})

system("del ce.out");

system("del cel.out");

Crea_Redes_Inicial $(1, \mathrm{i}, \mathrm{d})$;

if $($ negativo $==0)\{$

system("pspice ce.cir/r/e");

do \{

\}while(rename("ce.out","ce1.out")!=0);

Espera_Tiempo(12); /*espera tiempo de cerrar fichero*/

Busca_fichero(i);/*Busca datos en fichero*/

if $(\mathrm{d}==1) \operatorname{Sm}[\mathrm{i}][0]=1.0 \mathrm{E}+10 ; ; / *$ inicializa $* /$

$\mathrm{Sm}[\mathrm{i}][\mathrm{d}]=$ Calculo_Sm(i,d);

printf("\n\nSm:\%2.10e i=\%d d=\%d",Sm[i][d],i,d);

printf("\nMinimo:\%2.3e Sm_min=\%2.10e ",Minimo,Minimo_Sm);

//while $((\operatorname{getchar}()) !=' \backslash n ')$; exit(0);

\}

else $\quad \operatorname{Sm}[\mathrm{i}][\mathrm{d}]=1.0 \mathrm{E}+10$;

if $(\mathrm{d}<\mathrm{N}$ _ejecucion $\& \& \mathrm{~d} \% 3==1)$ Calculo_Minima_Sm(i,d $)$; 
$\mathrm{B}=\mathrm{BB}$;

\}

Calcula_Minimo_Final(i);

$\mathrm{B}=\mathrm{BB}$;

Salva_resultados(caso);

DatosObt $=1$;

\}

exit(0);

\}

void Espera_Tiempo(int g)

\{

time_t inicio, fin,ds;

inicio=time $($ NULL);

do \{

fin=time $(\mathrm{NULL})$;

//printf("\n \%f ",difftime(fin,inicio));

\} while(difftime(fin,inicio) $<$ g);

printf("TIME");

\}

void Busca fichero(int i)

\{

int 1;

int y;

float nombre $\mathrm{p}$;

char nomb[15];

long int despl;

FILE *fp;

if $($ Nceldas $==30)$ despl $=5470+12+37$;

else if(Nceldas $==20) \mathrm{despl}=3974-30$;

despl=-90;//-85; /*posición final de fichero*/

if $((\mathrm{fp}=$ fopen("ce1.out","rb") $)==\mathrm{NULL})\{$

printf("No puedo abrir fichero"); exit(0);\}

if $(\mathrm{i}==0) \mathrm{y}=\mathrm{r}$;

else $\mathrm{y}=\mathrm{r}+\mathrm{i}$;

for $(1=y ; 1>i-1 ; 1--)\{$

if $(1==y)\{$ if(fseek(fp,despl,2)!=0)\{ $/ *$ final de fichero*/ printf("No puedo mover puntero"); exit( $(0) ;\}$ despl=-35;

else \{

\}

if(fseek(fp,despl,1)!=0)

printf("No puedo mover puntero"); exit(0);

\}

fscanf(fp,"\%e",\&nombre_p);temp[1]=nombre_p;

$/ /$ printf("\n\ntemp[\%d]:\%2.4e",1,temp[1]);

/* fscanf(fp,"\%s",\&nomb);printf("\n\ntemp:\%s",nomb); */

/*while((getchar())!='\n'); *//*espera pulsar INTRO*/

fclose(fp);

\} 


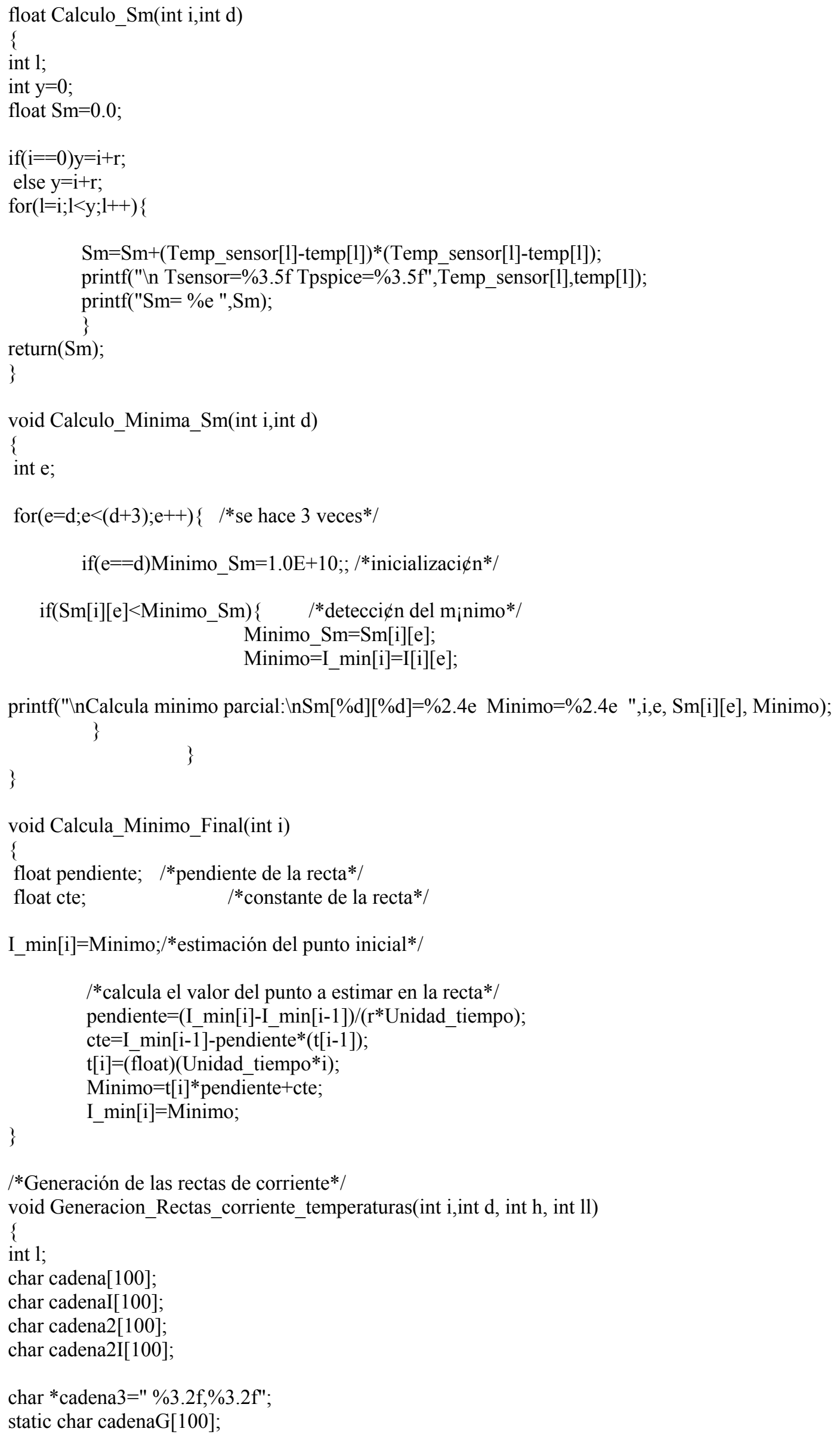

/*Generación de las rectas de corriente*/ void Generacion_Rectas_corriente_temperaturas(int i,int d, int h, int 1l) \{

int 1;

char cadena[100];

char cadenaI[100];

char cadena2[100];

char cadena2I[100];

char *adena3=" \%3.2f, \%3.2f";

static char cadenaG[100]; 
static char cadena5G[100]; static char cadena7G[100]; static char cadena9G[100]; static char cadena11G[100]; static char cadena13G[100]; static char cadena15G[100]; static char cadena17G[100]; static char cadena19G[100]; static char cadena21G[100]; static char cadena23G[100]; static char cadena25G[100]; static char cadena27G[100]; static char cadena29G[100]; static char cadena31G[100]; static char cadena33G[100]; static char cadena35G[100]; static char cadena37G[100]; static char cadena39G[100]; static char cadena41G[100];

char cadena1[100];

char cadena5[100],cadena6[100]; char cadena7[100],cadena8[100]; char cadena9[100],cadena10[100]; char cadena11[100],cadena12[100]; char cadena13[100], cadena14[100]; char cadena15[100],cadena16[100]; char cadena17[100], cadena18[100]; char cadena19[100], cadena20[100]; char cadena21[100], cadena22[100]; char cadena23[100], cadena24[100]; char cadena25[100], cadena26[100]; char cadena27[100], cadena28[100]; char cadena29[100], cadena30[100]; char cadena31[100], cadena32[100]; char cadena33[100], cadena34[100]; char cadena35[100], cadena36[100]; char cadena37[100], cadena38[100]; char cadena39[100], cadena40[100]; char cadena41[100], cadena42[100]; static char paso $=1$; static int cuen $=1$;

sprintf(cadena,"\nR\%d N800 0 1000G $\backslash n$ ",2*Nceldas+1);salva_datos_en_fichero(1,cadena,1); if $($ problema $==2)\{$ sprintf(cadena,"\nV_V1 N800 0 PWL "); //else if(i>0 )strcpy(cadena,"I_I1 0 N0001 PWL 0,\%3.4f"); \}

else if(problema==1)sprintf(cadena,"ไnI_I1 0 N800 PWL ");

strcpy(cadena5,"+");strcpy(cadena8,"+");strcpy(cadena10,"+");strcpy(cadena12,"+"); strcpy(cadena14,"+");strcpy(cadena16,"+");strcpy(cadena18,"+");strcpy(cadena20,"+"); strcpy(cadena22,"+");strcpy(cadena24,"+");strcpy(cadena26,"+");strcpy(cadena28,"+"); strcpy(cadena30,"+");strcpy(cadena32,"+");strcpy(cadena34,"+");strcpy(cadena36,"+"); strcpy(cadena38,"+");strcpy(cadena40,"+");strcpy(cadena42,"+");

$$
\begin{aligned}
& \operatorname{if}(\mathrm{i}==1 \& \& \mathrm{~d}==\mathrm{N} \text { _ejecucion }) \text { cuen }=1 ; \\
& \operatorname{if}(\mathrm{i}==1 \& \& \text { cuen }==1)
\end{aligned}
$$




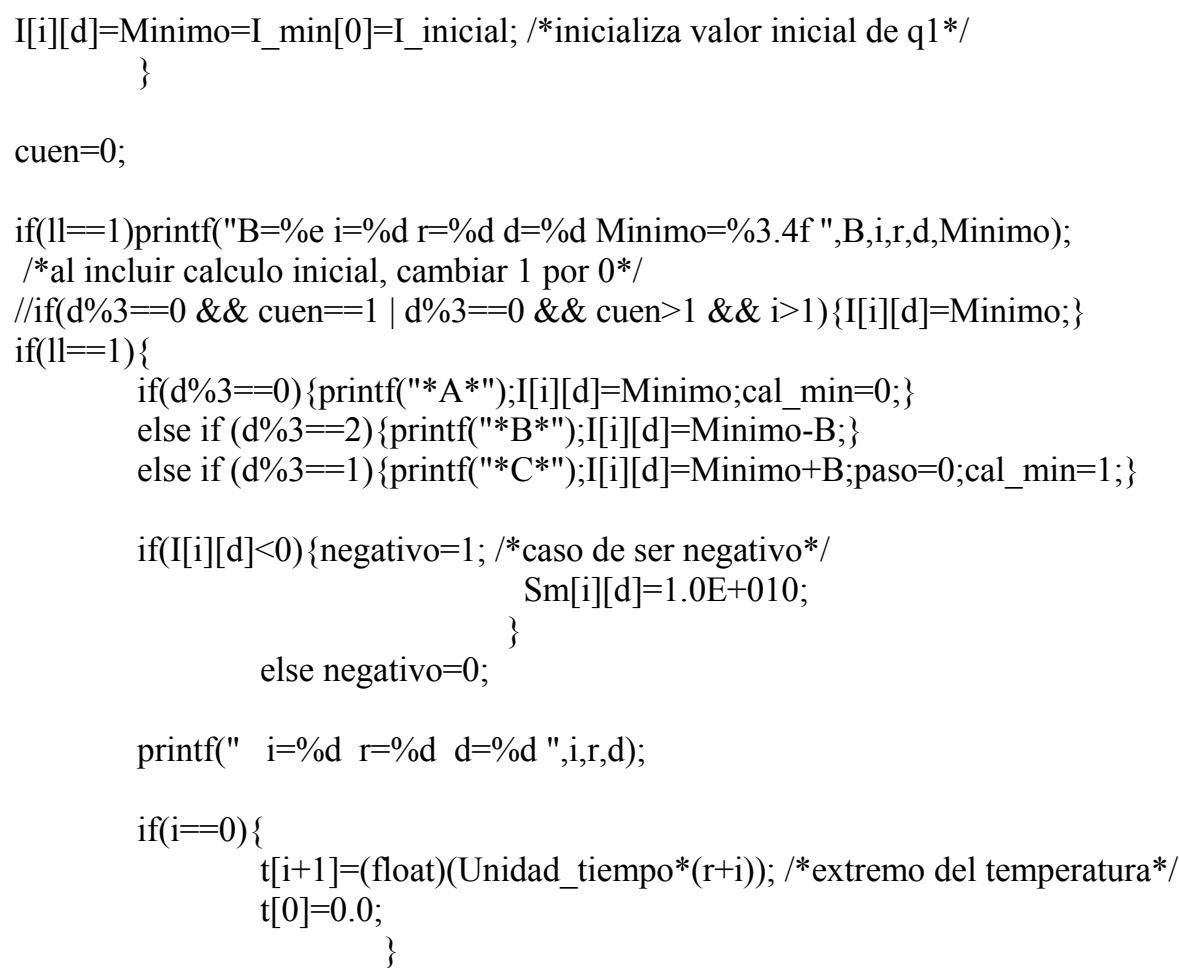
else \{

$\mathrm{t}[\mathrm{i}]=($ float $)($ Unidad_tiempo* $(\mathrm{r}+\mathrm{i}-1)) ; / *$ extremo del temperatura*/ \}

I_min $[i]=I[i][d]$;

if $(\mathrm{i}>0)\{$

for $(1=0 ; 1<\mathrm{i}+1 ; 1++)\{\quad / *$ ajuste de la cadena $* /$

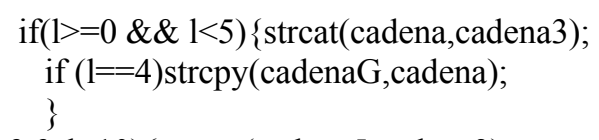

else if $(1>=5 \& \& 1<10)\{$ strcat(cadena5, cadena3);

$$
\}
$$

if $(1==9) \operatorname{strcpy}($ cadena5G,cadena5);

else if $(1>=10 \& \& 1<15)\{\operatorname{strcat}($ cadena8,cadena3); if $(1==14)$ strcpy (cadena $7 G$, cadena 8$)$; \}

else if $(1>=15 \& \& 1<20)\{$ strcat(cadena10,cadena3); if $(1==19)$ strcpy(cadena9G,cadena10);

else if $(1>=20 \& \& 1<25)\{$ strcat(cadena12,cadena3); if $(1==24)$ strcpy(cadena11G,cadena12); \}

else if $(1>=25 \& \& 1<30)\{$ strcat(cadena14,cadena3); \}

else if $(1>=30 \& \& 1<35)\{$ strcat(cadena16, cadena3); if(l==34)strcpy(cadena15G,cadena16); \}

else if $(1>=35 \& \& 1<40)\{$ strcat(cadena18,cadena3); if(l==39)strcpy(cadena17G,cadena18);

else if $(1>=40 \& \& 1<45)\{$ strcat(cadena20,cadena3); if $(1==44) \operatorname{strcpy}($ cadena19G,cadena20); \} 


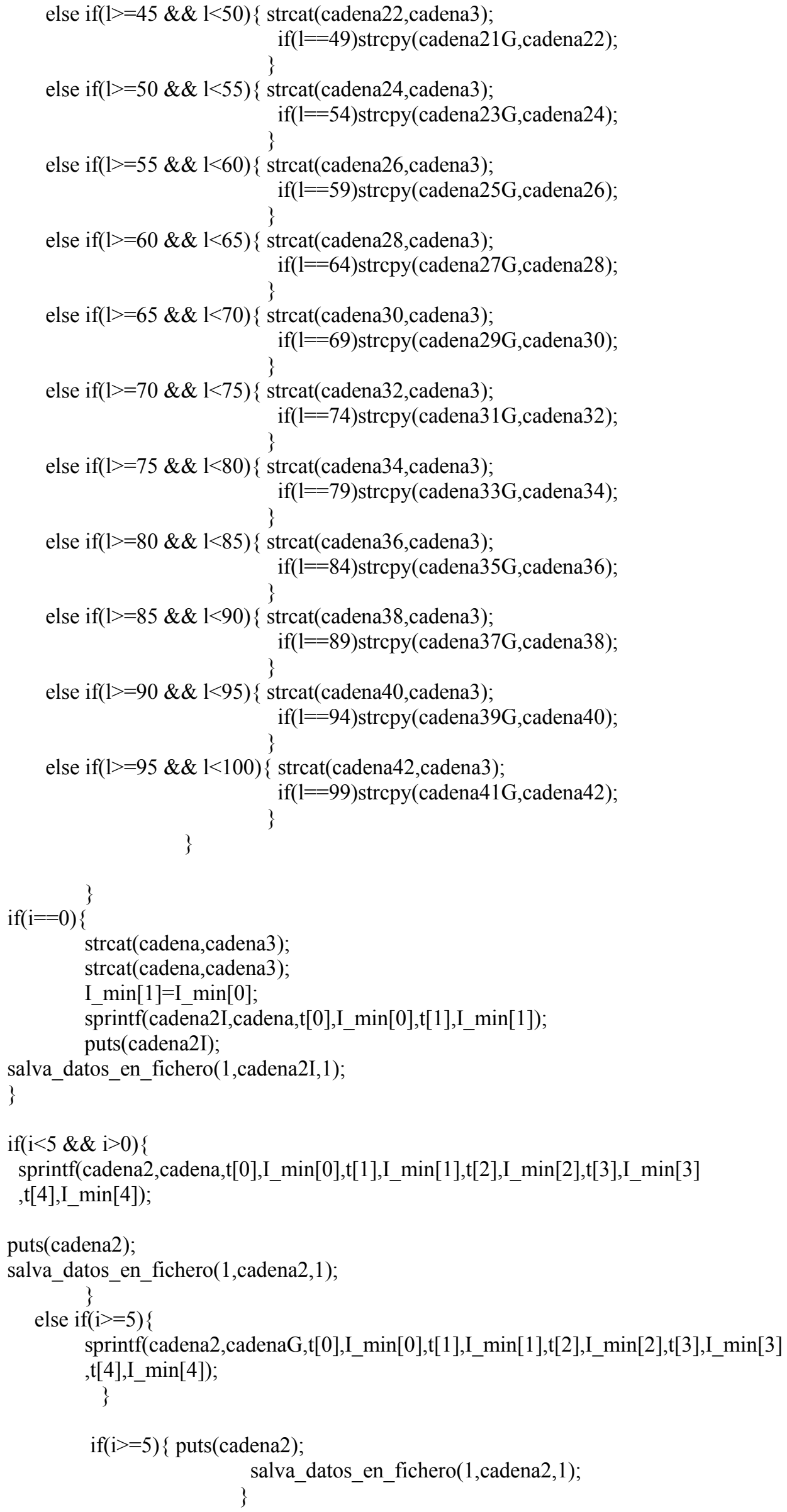




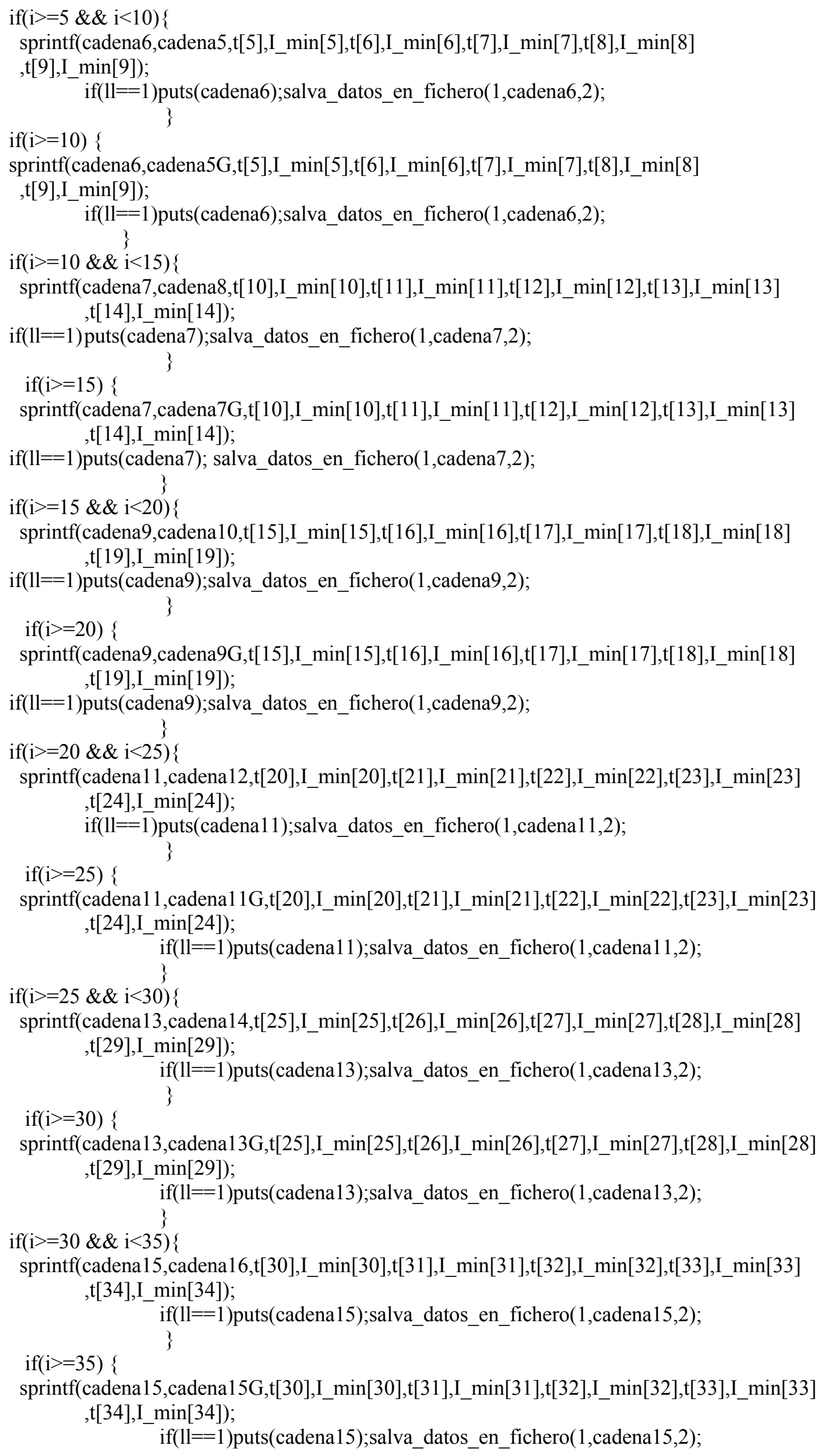




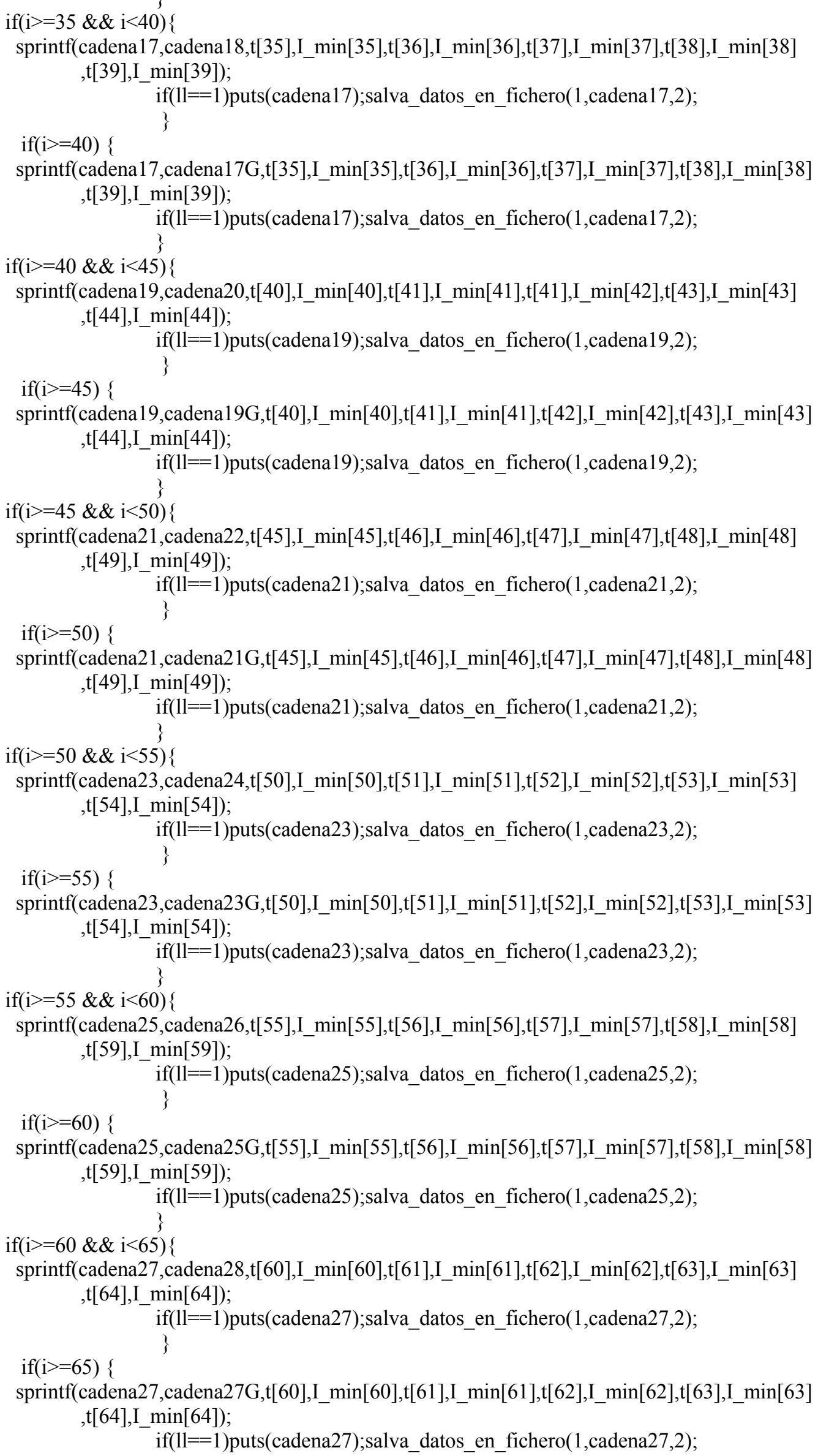




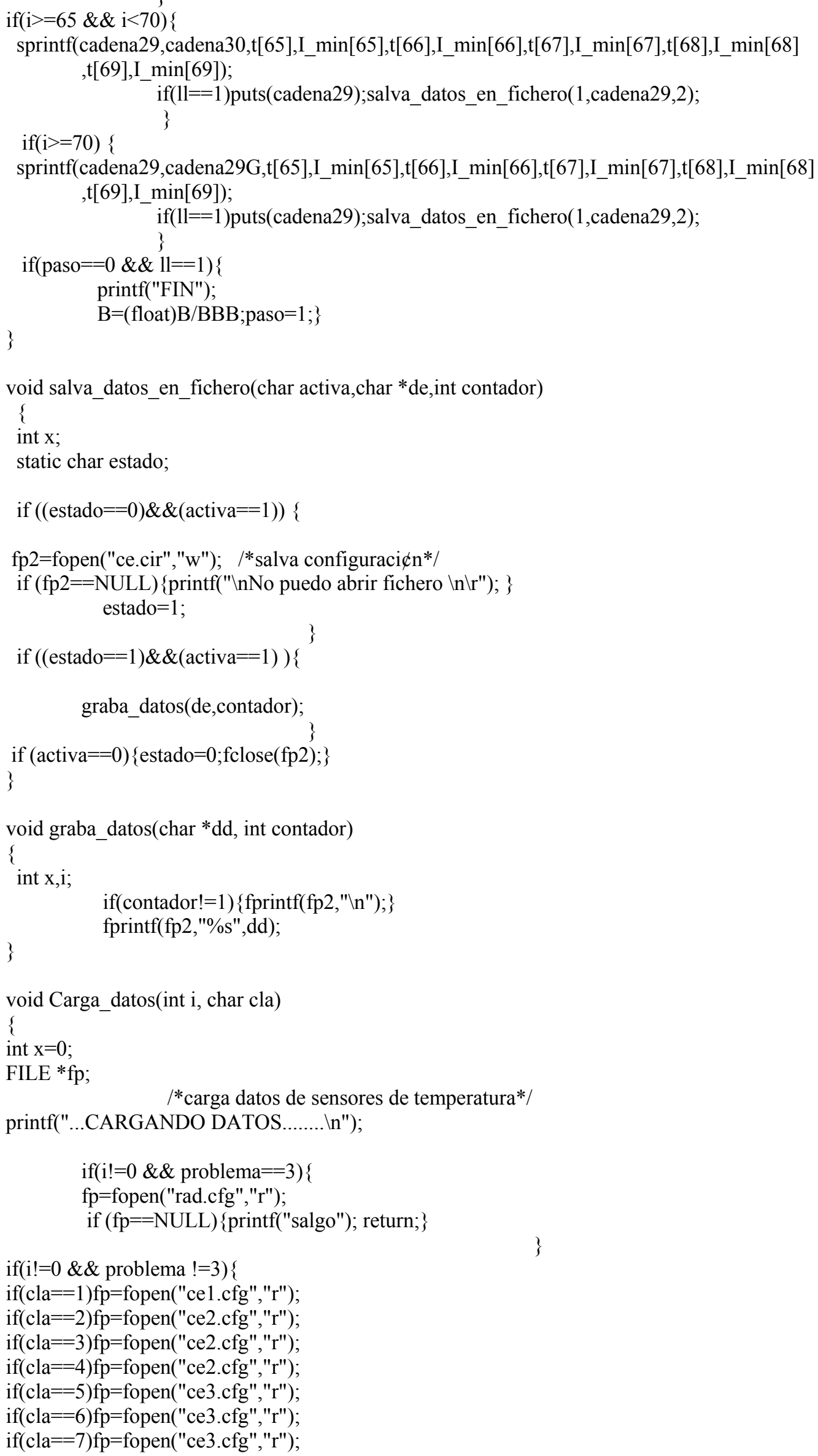




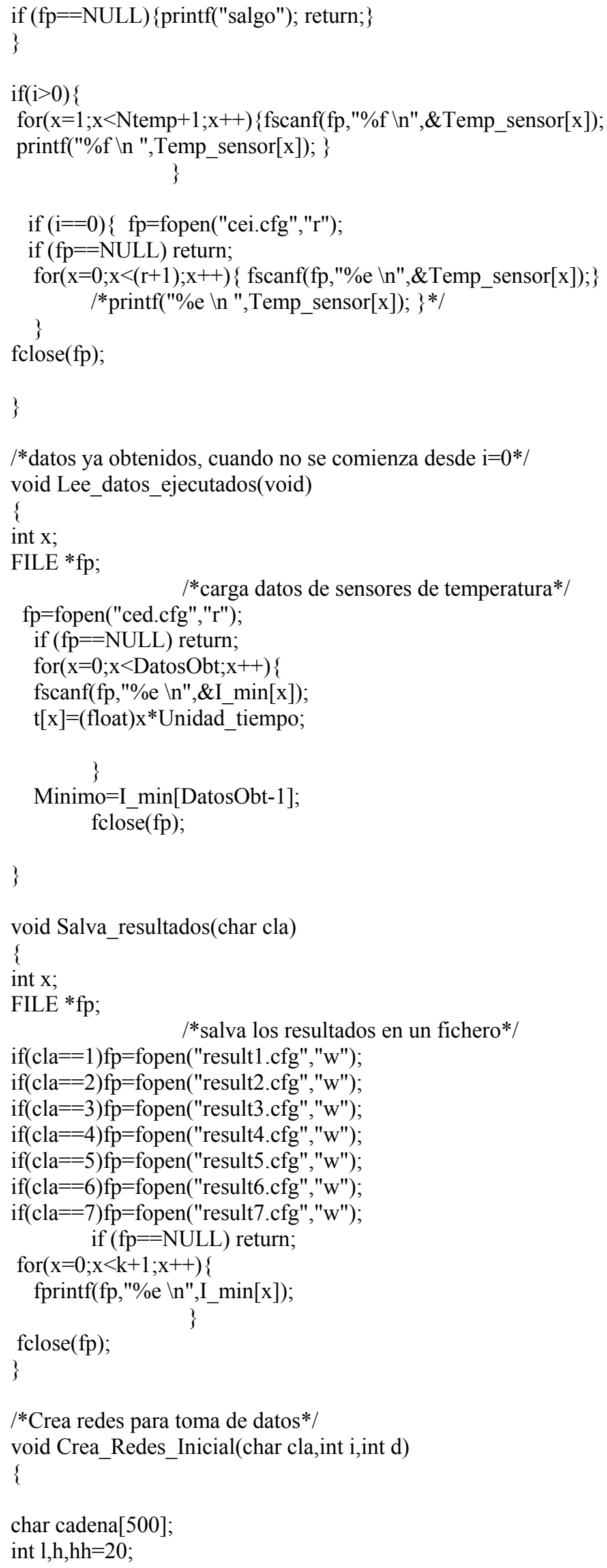


float fcg;

for $(1=1 ; 1<2 *$ Nceldas $+2 ; 1++)\{$

if $(\mathrm{l}==1) \operatorname{Ri}[1]=1.0 \mathrm{E}-06$;

if $(1==2) \operatorname{Ri}[2]=\mathrm{Ax} / 2 ; / / 1.0 \mathrm{E}-06$;

if $(1>2) \operatorname{Ri}[1]=\operatorname{Ri}[1-1]+\mathrm{Ax} / 2$;

\}

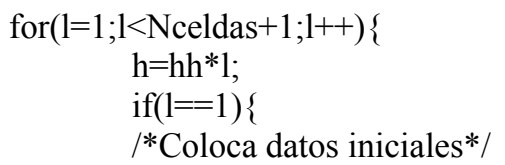

sprintf(cadena,".PROBE \n");salva_datos_en_fichero(1,cadena, 1 );

sprintf(cadena,".OPTIONS NODE\n"); salva_datos_en_fichero(1,cadena, 1$)$;

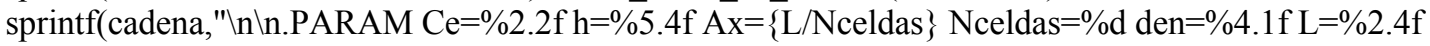

$\mathrm{r}=\{\mathrm{Ax} /(2 * \mathrm{Ko})\} \mathrm{Ko}=\% 1.7 \mathrm{f} \backslash \mathrm{n} "$,Ce,coef_pel,Nceldas,den,L,conduct $)$;

salva_datos_en_fichero(1,cadena, 1$)$;

sprintf(cadena," $+{ }^{-}=\{$den*Ax*Ce $\}$ic $\left.=\% 1.3 \mathrm{f} \mathrm{Ta}=\% 1.3 \mathrm{f} \backslash \mathrm{n} ", \mathrm{ci}, \mathrm{Ta}\right)$;

salva_datos_en_fichero(1,cadena,2);

sprintf(cadena,".OPTIONS RELTOL=0.001 \n"); salva_datos_en_fichero(1,cadena, 1$)$;

sprintf(cadena,".OPTIONS NUMDGT=4 \n"); salva_datos_en_fichero(1,cadena, 1$)$;

sprintf(cadena,".lib nom.lib $\backslash n ")$; salva datos_en_fichero(1,cadena, 1 );

if(i>0)\{sprintf(cadena,".TRAN \%1.4f \%1.4f \%1.4f 10 UIC $\backslash n$ ",Unidad_tiempo,Unidad_tiempo*(i+r-1),

Unidad tiempo*(i-1)); salva datos_en_fichero(1,cadena, 1$) ;\}$

else $\{$ sprintf(cadena,".TRAN \%1.4f \%1.4f \% $1.4 \mathrm{f} 10$ UIC $\backslash n "$ " Unidad_tiempo, Unidad tiempo*(i+r),

Unidad_tiempo*i); salva_datos_en_fichero(1,cadena, 1$) ;\}$

puts(cadena);

sprintf(cadena,"**Celda \%d**",1);

salva_datos_en_fichero(1,cadena,2);

$/ *$ parte central*/

sprintf(cadena,"C\%d N\%d $0\{\mathrm{c}\} \mathrm{IC}=\{\mathrm{ic}\}$ ",1,h-8); salva_datos_en_fichero(1,cadena,2);

sprintf(cadena,"R\%d N\%d N\%d \{r\}",(2*1-1),h-hh,h-8);salva_datos_en_fichero(1,cadena,2);

sprintf(cadena,"R\%d N\%d N\%d \{r\}",2*1,h-8,h);salva_datos_en_fichero(1,cadena,2);

//generación interna de calor

//sprintf(cadena,"G\%d 0 N\%d VALUE $=\{\mathrm{V}(\mathrm{N} \% \mathrm{~d}, 0)\} ", 4 *$ Nceldas $+(2 * 1-1), \mathrm{h}-8, \mathrm{~h}-$

4);salva_datos_en_fichero(1,cadena,2);

/*alor específico*/

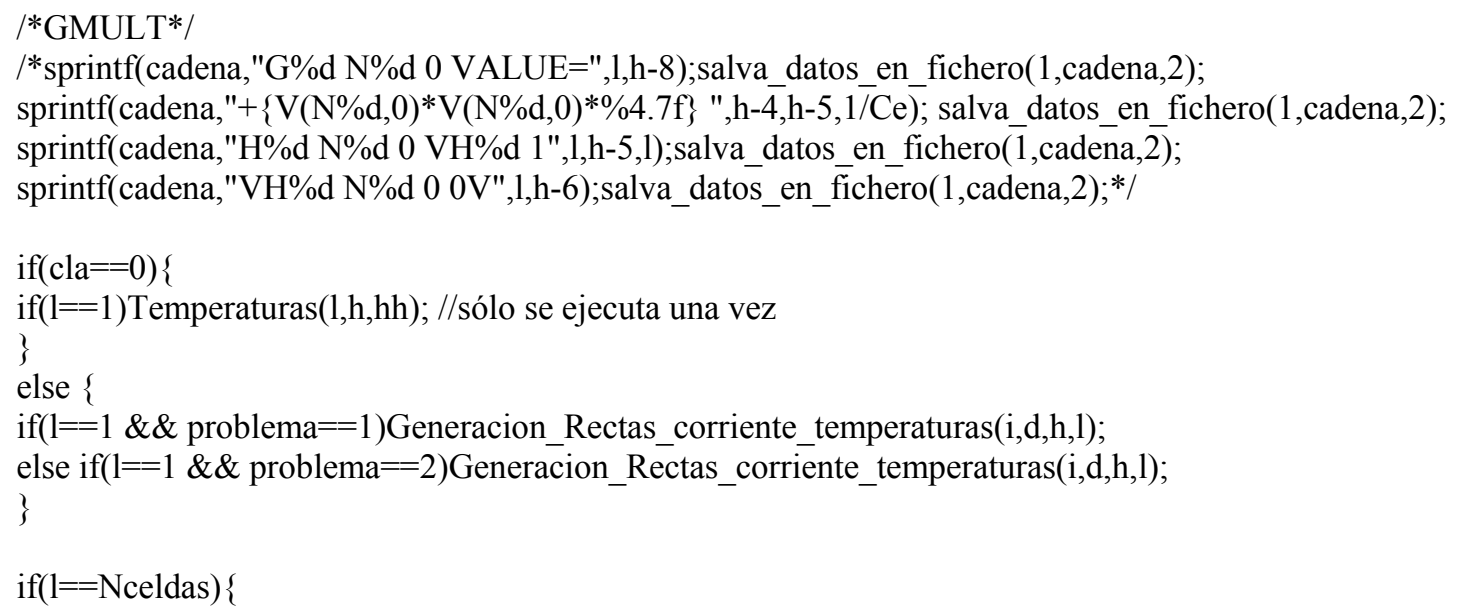


sprintf(cadena,"**CONDICIONES DE CONTORNO**");

salva_datos_en_fichero(1,cadena, 2$)$;

//sprintf(cadena,"ไnR\%d N0 $01 \mathrm{G} \backslash n$ ",2*Nceldas+1);salva_datos_en_fichero(1,cadena,1);

//sprintf(cadena,"'lnV\%d N0 $0 \% 3.3 \mathrm{ftn} ", 2 *$ Nceldas+1,Ta);salva_datos_en_fichero(1,cadena,1);

/*convección forzada*/

/*GMULT*/

sprintf(cadena,"G\%d N0 0 VALUE",Nceldas*20+10);salva_datos_en_fichero(1,cadena,2);

sprintf(cadena,"+\{\%2.4f*(V(N0,0)-\%2.3f) $\} "$, coef_pel,Ta);

salva datos en fichero(1,cadena,2);

//para permitir continuidad

//sprintf(cadena,"\nR\%d N0 0 1000Gไn",2*Nceldas+1);salva_datos_en_fichero(1,cadena,1);

if(caso==1)sprintf(cadena,".PRINT TRAN V([N800]) $\ln ")$;

//else if(caso>1)sprintf(cadena,".PRINT TRAN V([N0]) \n");

else sprintf(cadena,".PRINT TRAN V([NO])\n");

salva_datos_en_fichero(1,cadena,2);

sprintf(cadena,".END");salva_datos_en_fichero(1,cadena,1);

\}

\}

salva_datos_en_fichero( 0 ,cadena, 1$)$;

if $\left(\right.$ cla $\left.^{-}=0\right)\{$ system("pspice ce.cir $\left.") ; \quad \operatorname{exit}(0) ;\right\}$

\}

void Temperaturas( int l,int h,int hh)

\{

char cadena[500];

if (problema $==1)\{$

//flujo de calor dependiente del tiempo

sprintf(cadena,"V\%d 0 N801 PWL 0,0 30,30 100,100

$300,300$ ",Nceldas*20+9); salva datos en fichero(1,cadena, 2$)$;

sprintf(cadena,"E_PWR2 N802 0 VALUE $\{$ PWR(V(N801),\%d)\}",b);

salva_datos_en_fichero(1,cadena,2);

sprintf(cadena,"G\%d 0 N800

VALUE $=\{\% \mathrm{~d}+\% \mathrm{~d} * \mathrm{~V}(\mathrm{~N} 802)\} ", \mathrm{Nceldas} * 20+9$,Jini,a);salva_datos_en_fichero(1,cadena,2);

$/ /$ para permitir continuidad

sprintf(cadena,"ไnR\%d N800 0 1000Gไn",2*Nceldas+1);salva_datos_en_fichero(1,cadena,1);

//flujo de calor triangular

//sprintf(cadena,"I\%d 0 N800 PWL 0,5 20,30 40,5",Nceldas*20+9);salva_datos_en_fichero(1,cadena,2);

//flujo de calor senoidal

//sprintf(cadena,"I\%d 0 N800 SIN(0 200.02500 0) ",Nceldas*20+9);

salva_datos_en_fichero(1,cadena, 2$)$;

//flujo de calor escalón

//sprintf(cadena,"I\%d 0 N800 PWL 0,5 9.999,5 10,20 60,20",Nceldas*20+9);

salva_datos_en_fichero(1,cadena, 2$)$;

\}

if $($ problema $==2)\{$

//Temperatura ambiente

//sprintf(cadena,"\nV_V1 N805 0 PWL 0,10 10,200 20,250 30,150 40,100 50,50 1000,50");

//salva_datos_en_fichero(1,cadena,2);

sprintf(cadena,"'-'nV_V1 N805 0 PWL 0,10 9.99999,10 10,100 20,100 20.0001,10 100,10");

salva datos_en fichero(1,cadena,2);

//convección forzada 
sprintf(cadena,"G\%d 0 N800 VALUE",Nceldas*20+9);salva_datos_en_fichero(1,cadena,2); sprintf(cadena,"+\{\%2.4f*(V(N805,0)-V(N\%d,0) $)\} "$,coef_pel,20*Nceldas); salva_datos_en_fichero(1,cadena,2);

\} \}

\}

\section{A.2. ESTIMACIÓN SIMULTÁNEA DEL CALOR ESPECÍFICO Y DE LA CONDUCTIVIDAD TÉRMICA}

//Estimación simultánea del calor específico y la conductividad térmica

//(Microsoft Visual C++, versión 6.0)

//Funciones cabecera

\#include "stdafx.h"

\#include "stdio.h"

\#include "stdlib.h"

\#include "process.h"

\#include "string.h"

\#include "time.h"

\#include "math.h"

\#define Nceldas 40

\#define F 100

\#define Ntemp 3000

\#define DatosObt 1

\#define Inicial_k 1

\#define Inicial_ce 1000

\#define $\mathrm{N}$ ejecucion 63

\#define s $\overline{3}$

\section{/*número de celdas*/}

/*número de temperaturas máximo, medidas por un sensor*/

/*para activar la estimación en un punto distinto al inicial*/ /*estimación inicial del $\mathrm{k} * /$

/*estimación inicial del Ce*/

/*número de estimaciones por intervalo, debe múltiplo de $9 * /$ /*número de sensores*/

//constantes para del protocolo de resolución

\#define BB 50.0 //para el Ce

\#define BBB 2.0

\#define CC .20 //para la k

\#define CCC 2.0

\#define deltaCe 1E-05 //criterio de convergencia para el calor específico

\#define deltak 1E-05

\#define Int_temp 5.0

//criterio de convergencia para el calor específico

$/ *$ intervalo de temperatura, estimación de temperaturas*/

\#define k 50

/*Número máximo de iteraciones por punto*/

//Funciones

void Salva_resultados(char);

void salva_datos_en_fichero(char activa,char de[F],int);

void graba_datos(chä dd[F], int contador);

void Crea_Redes_Inicial(char,int,int);

void Crea_Redes(int,int);

void Lee_datos_ejecutados(void);

float Calculo_Sm(int,int);

void Busca_fichero(int);

void Calculo_Minima_Sm(int,int);

void Calcula_Minimo_Final(int);

void Carga_datos(int);

void Generacion_Rectas_corriente(int,int,int,int);

void Generacion_Rectas_corriente1(int,int,int,int);

void Calcula_temp_futuras (int, char); 
void Espera_Tiempo(int);

void Calcula r r (void);

void Cartesianas( int, int ,int );

/*variables externas*/

char esfericas;

float Unidad tiempo $=0.5$;

$/ / 0$ cartesianas 1 esfericas

float temp[Ntemp +1$][\mathrm{s}]$;

/*intervalo de tiempo de estimación, en segundos*/

float $\mathrm{B}=\mathrm{BB}$;

float $\mathrm{C}=\mathrm{CC}$;

float tiempo[k+1];

float $\mathrm{I}[\mathrm{k}+1][\mathrm{N}$ ejecucion +1$]$;

$/ *$ valores de $\mathrm{c}_{\mathrm{e}}$ a estimar*/

float II $[\mathrm{k}+1]\left[\mathrm{N} \_\right.$ejecucion +1$]$;

/*valores de k a estimar*/

float $\mathrm{L}=.03$, Ax;

float den $=1000$;

float conduct $=10.0$;

float $\mathrm{A}=1.0$; //area de paso en $\mathrm{m} 2$ (para coordenadas cartesianas)

FILE fp2;

/*fichero para guardar datos*/

float coef_pel $=50.0$;

/*coeficiente convectivo*/

int $\mathrm{r}[\mathrm{k}][\mathrm{s}] ; \quad$ /*"r", número de temperaturas $\mathrm{k}=\mathrm{i}$ intervalo de temp, $\mathrm{s}=\mathrm{n}^{\circ}$ de sensor*/

float Temp_sensor[Ntemp+1][s]; //temperaturas de los "s" sensores

float I_min[k+1];

float II $\min [\mathrm{k}+1]$;

/*valores de Ce estimados previamente*/

float $\operatorname{Min}[\mathrm{k}+1]$;

$/ *$ valores de $\mathrm{k}$ estimados previamente*/

float $\mathrm{t}[\mathrm{k}+1]$;

float Minimo;

*valor asociado a Sm minimo de I(Calor especifico)*/

/* variable temporal*/

/*valor mínimo variable del calor específico*/

float Minimo_k;

int cuen=DatosObt;

/*valor mínimo variable de la conductividad*/

int cuen $\mathrm{k}=1$;

$/ *$ clave para saber si se ejecuto la primera vez, para $\mathrm{Ce} * /$

float $\mathrm{Sm}[\mathrm{k}+1]\left[\mathrm{N} \_\right.$ejecucion +1$]$; /*variable del funcional $*$ /

float Minimo_Sm;

float $P$ os final $=15000$, $P o$ inicial $=0$;

int PP final;

int PP inicial;

float $\overline{\mathrm{Ce}}=$ Inicial_ce;

float $\mathrm{Ta}=60.0, \mathrm{ci}=0.0$;

int clave $=1$;

int negativo;

int negativo_k;

float $\mathrm{Ri}[2 *$ Nceldas $]$;

int $\operatorname{rmax}[\mathrm{k}]$;

int $\operatorname{rmin}[\mathrm{k}]$;

/*inicialización*/

/*posición para la lectura de datos del fichero .out*/

/*posición para la lectura de datos del fichero .out*/

/*valor de Cmedia*/

/*temperatura ambiente y condiciones iniciales*/

$/ *$ para cuando $\mathrm{r}==1 *$ /

//para Ce

//para k

/*para coordenadas esfericas, la fresa*/

$/ /$ de actuales valores de i

$/ /$ de valores de $\mathrm{i}$

int main(int argc, char* argv[])

\{

int i,d;

char clave;

char clave $1=0$;

int Otra $=0$;

FILE *pa;

$\mathrm{Ax}=\mathrm{L} / \mathrm{Nceldas} ;$

/*para ahorrar tiempo, se parte de datos ya obtenidos*/

if(DatosObt $>1)$ Lee_datos_ejecutados();

Carga_datos(1); 


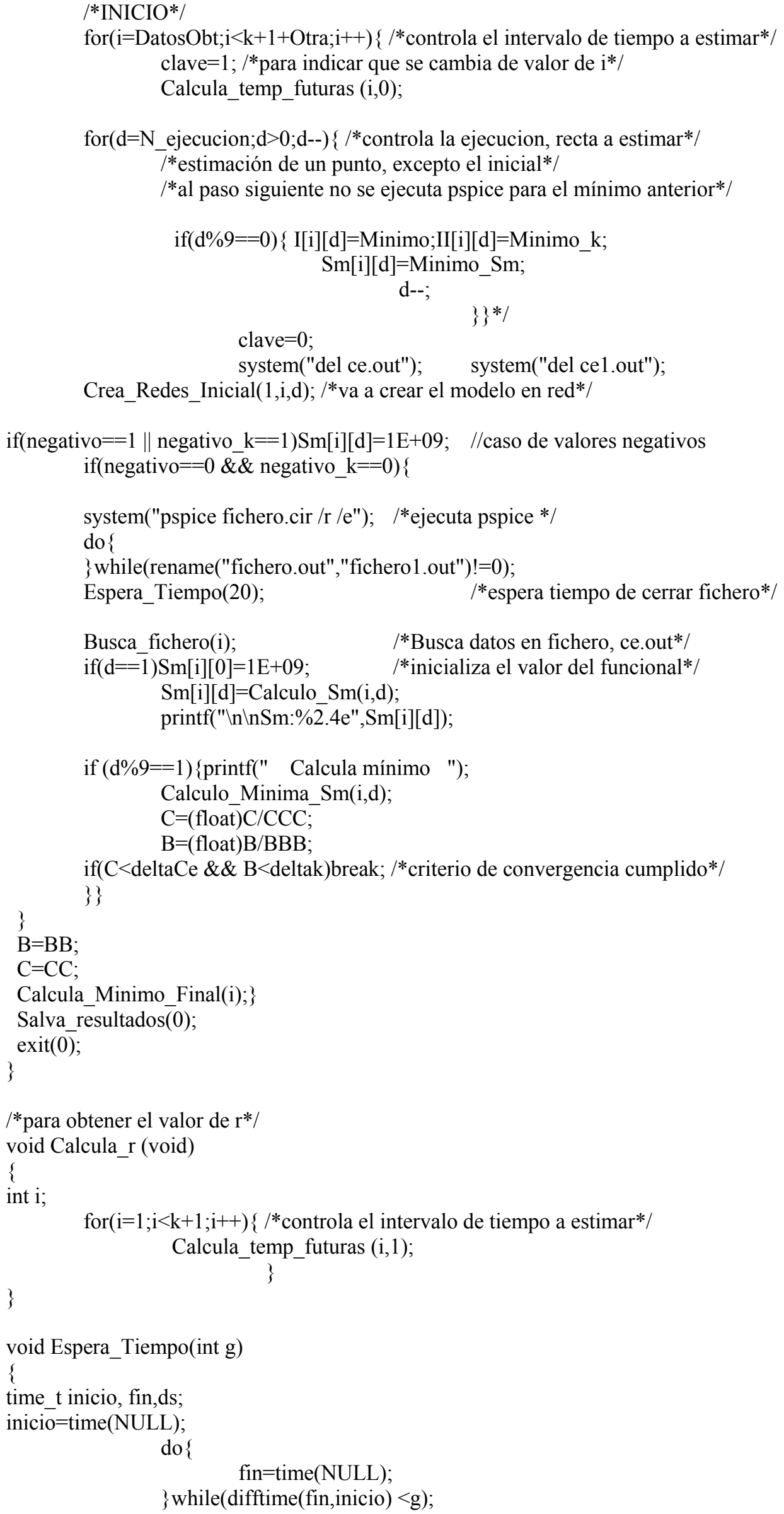


\}

void Calcula temp_futuras (int i, char cla)

\{

int $\mathrm{f}, \mathrm{ss}=0$;

int cuenta $=0$;

for $(\mathrm{ss}=0 ; \mathrm{ss}<\mathrm{s} ; \mathrm{ss}++)\{\mathrm{r}[\mathrm{i}][\mathrm{ss}]=0 ; / *$ pone a cero el valor de $\mathrm{r} * /$

if $(\mathrm{i}==1)\{$

for $(\mathrm{f}=1 ; \mathrm{f}<\mathrm{Ntemp} ; \mathrm{f}++)\{/ *$ leo todos los valores de temp sensor*/

if $(0<$ Temp_sensor[f][ss]

$\& \&$ Temp_sensor[f] [ss] $<((\mathrm{i}+1) *$ Int_temp/4) $)\{$ if $(\mathrm{r}[\mathrm{i}-1][\mathrm{ss}]==0) \mathrm{PP}$ inicial $=\mathrm{f}$; $\mathrm{r}[\mathrm{i}-1][\mathrm{ss}]++;\}$

\}

\}

for $(\mathrm{f}=1 ; \mathrm{f}<\mathrm{Ntemp} ; \mathrm{f}++)\{/ *$ leo todos los valores de temp sensor*/

if $(0<$ Temp_sensor[f][ss] \&\& Temp_sensor[f][ss] $<((i+1) *$ Int_temp/2)) \{

$\overline{\text { if }}(\mathrm{r}[\mathrm{i}][\mathrm{ss}]==0) \mathrm{PP}$ _inicial $=\overline{\mathrm{f}}$; $\mathrm{r}[\mathrm{i}][\mathrm{ss}]++;\}$

\}

$\operatorname{printf}(" \backslash \mathrm{n} r[\% \mathrm{~d}][\% \mathrm{~d}]=\% \mathrm{~d}$,,i,ss,r[i][ss]);

/*inicialización*/

if $(\mathrm{i} !=1 \& \&$ cuenta $==0)\{\operatorname{rmax}[\mathrm{i}]=\mathrm{r}[\mathrm{i}][\mathrm{ss}] ; \mathrm{rmin}[\mathrm{i}-1]=\mathrm{r}[\mathrm{i}-1][\mathrm{ss}] ;\}$

else if $(\mathrm{i}==1 \& \&$ cuenta $==0)\{\operatorname{rmax}[\mathrm{i}]=\mathrm{r}[\mathrm{i}][\mathrm{ss}] ; \mathrm{rmin}[\mathrm{i}-1]=\mathrm{r}[\mathrm{i}-1][\mathrm{ss}] ;\}$

cuenta $=1 ; /$ para que se active una sola vez la inicialización

/*calcula rmax y rmin*/

if(ss $>0$ \&\& r[i][ss] $>\operatorname{rmax}[\mathrm{i}]) \operatorname{rmax}[\mathrm{i}]=\mathrm{r}[\mathrm{i}][\mathrm{ss}]$; //calcula el máximo

if( $\mathrm{ss}>0$ \&\& r[i-1][ss]<rmin[i-1])rmin[i-1] $=\mathrm{r}[\mathrm{i}-1][\mathrm{ss}]$; //calcula el máximo

\}

$P P$ inicial $=0$;

if $(\mathrm{s}==1) \mathrm{PP}$ final $=\mathrm{r}[\mathrm{i}][0]$;

else $P P$ final $=\operatorname{rmax}[\mathrm{i}]+1 ; / / \mathrm{r}[\mathrm{i}][\mathrm{ss}]=\mathrm{r}[\mathrm{i}][\mathrm{ss}] ;\}$

Pos_final=(float)PP_final*Unidad_tiempo;

Pos_inicial=(float) PP_inicial*Unidad_tiempo;

\}

float Calculo_Sm(int i,int d)

\{

int $1, \mathrm{ss}=0$;

float $\mathrm{Sm}=0.0$;

int $P$ inicial, $P$ final;

static int Memo_rmin $=0$;

for $(\mathrm{ss}=0 ; \mathrm{ss}<\mathrm{s} ; \mathrm{ss}++)\{$

$P$ inicial $=0$;

if $(\mathrm{s}==1) \mathrm{P}$ final $=r[\mathrm{i}][0]$;

else $P_{\text {_final }}=\mathrm{r}[\mathrm{i}][\mathrm{ss}]$;

for $(1=\mathrm{P}$ inicial $; 1<(\mathrm{P}$ final +1$) ; 1++)\{$

$\mathrm{Sm}=\mathrm{Sm}+($ Temp_sensor[1][ss]-temp[1][ss])*(Temp_sensor[1][ss]-temp[1][ss]); 
return(Sm);

\section{\}}

\}

void Calculo_Minima_Sm(int i,int d)

\{

int e;

for $(\mathrm{e}=\mathrm{d} ; \mathrm{e}<(\mathrm{d}+9) ; \mathrm{e}++)\{\quad / *$ se hace 3 veces $* /$

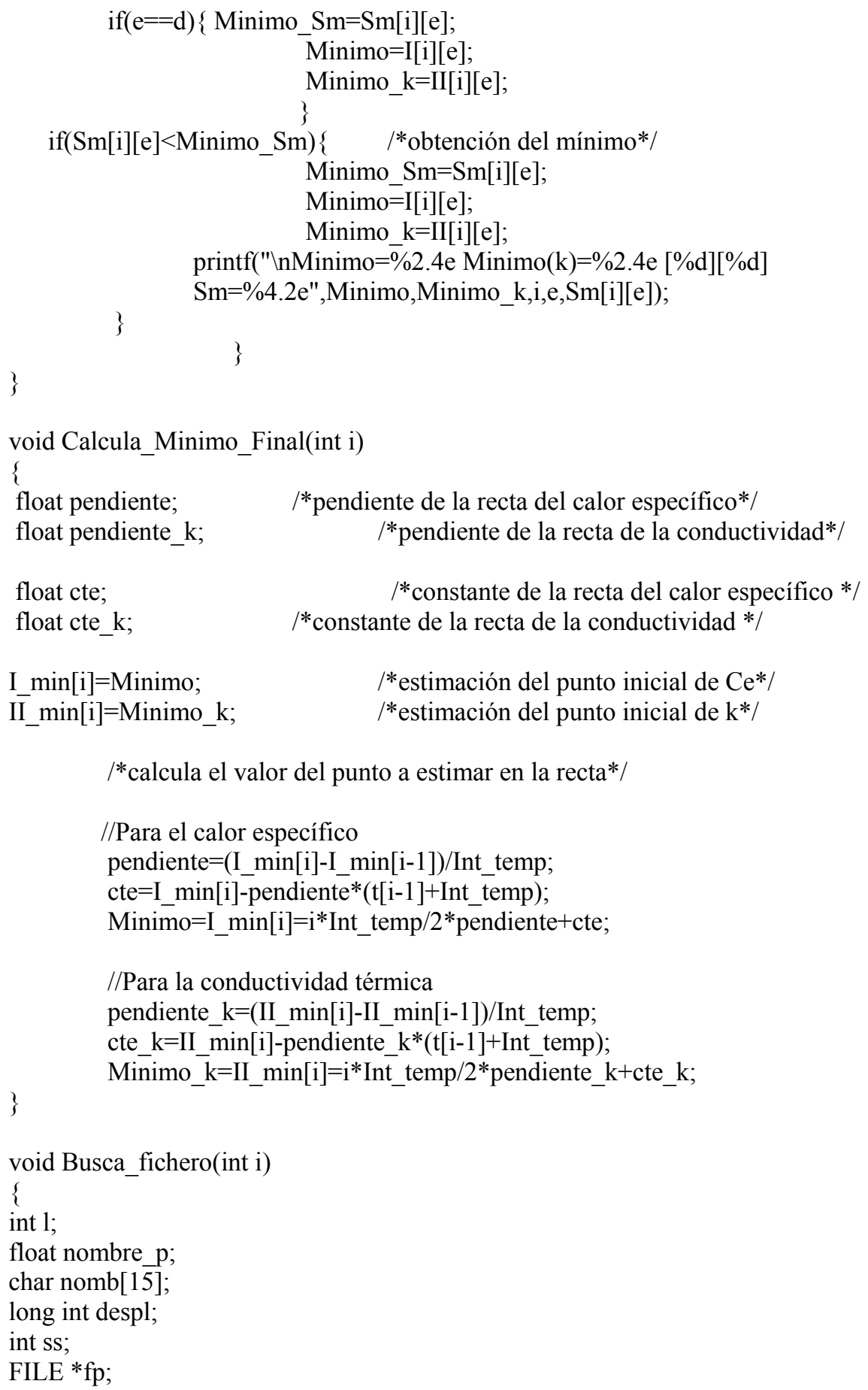

if $($ Nceldas $==30)$ despl $=5470+12+37$; else if(Nceldas==20)despl=3974-30; 


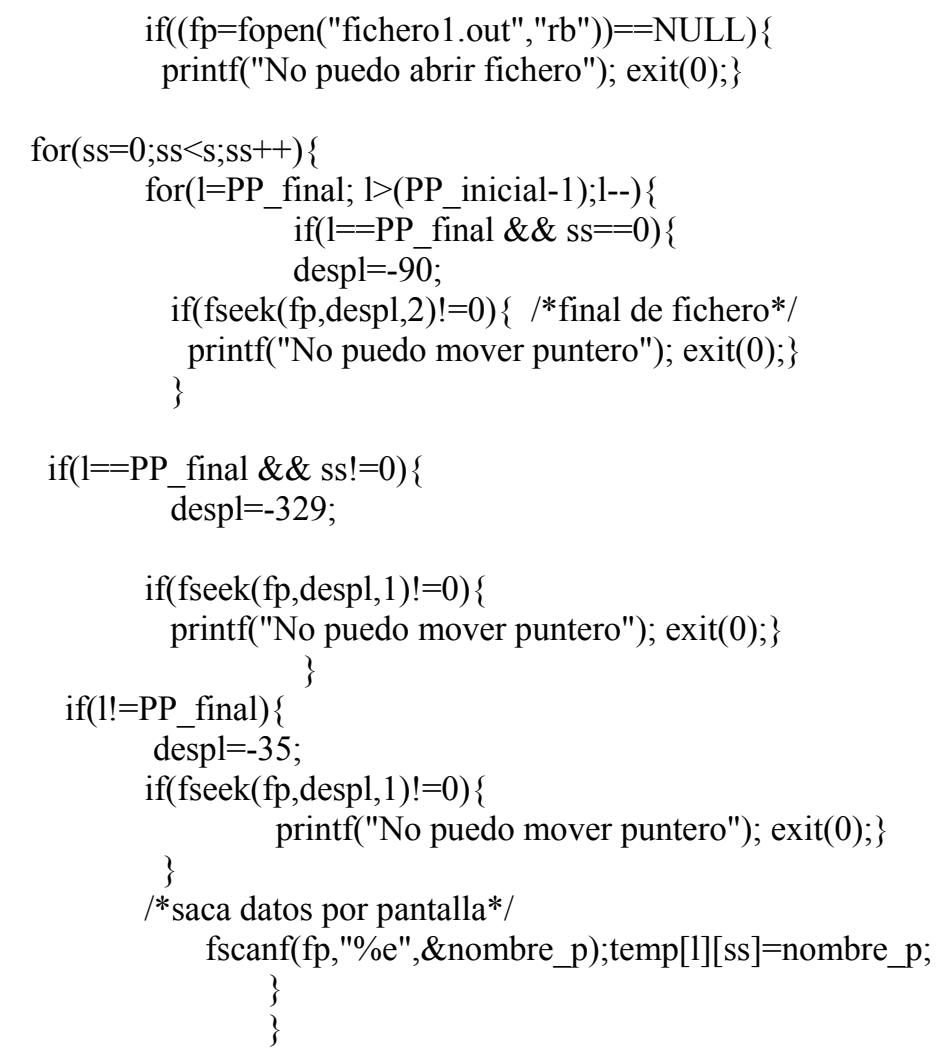

fclose(fp);

\}

/*Generación de las rectas de corriente, calor específico */

void Generacion_Rectas_corriente(int i,int d, int h, int ll)

\{

int 1;

char cadena[F];

char cadena $2[\mathrm{~F}]$;

char *cadena3=" \%3.1f,\%3.1f";

static char cadenaG[F], static char cadena5G[F], static char cadena7G[F];

static char cadena9G[F], static char cadena $11 \mathrm{G}[\mathrm{F}]$, static char cadena $13 \mathrm{G}[\mathrm{F}]$;

static char cadena $15 \mathrm{G}[\mathrm{F}]$, static char cadena17G[F], static char cadena19G[F]; static char cadena21G[F], static char cadena23G[F], static char cadena25G[F]; static char cadena27G[F], static char cadena29G[F], static char cadena31G[F]; static char cadena33G[F], static char cadena35G[F], static char cadena37G[F]; static char cadena39G[F], static char cadena41G[F];

char cadena1 $[\mathrm{F}]$, char cadena5 $[\mathrm{F}]$, cadena6 $[\mathrm{F}]$, char cadena7 $[\mathrm{F}]$, cadena $8[\mathrm{~F}]$; char cadena9[F], cadena10[F], char cadena11[F],cadena12[F]; char cadena13[F], cadena14[F], char cadena15[F], cadena16[F]; char cadena $17[\mathrm{~F}]$, cadena $18[\mathrm{~F}]$, char cadena $19[\mathrm{~F}]$, cadena20 $[\mathrm{F}]$; char cadena21[F], cadena22[F], char cadena23[F], cadena24[F]; char cadena25[F], cadena26[F], char cadena27[F], cadena28[F]; char cadena29[F], cadena30[F], char cadena31[F], cadena32[F]; char cadena33 $[\mathrm{F}]$, cadena34[F], char cadena35[F], cadena36[F]; char cadena37[F], cadena38[F], char cadena39[F], cadena40[F]; char cadena41[F], cadena42[F];

static int contador $=0$;

sprintf(cadena,"\nE_E\%d N\%d 0 TABLE $\{$ V(N\%d,0)\} \%2.1f,\%2.1f"

, 6 *Nceldas $+2 * 11-1$, h-4,h-8,ci,Inicial_ce); 
strcpy(cadena5,"+"); $\operatorname{strcpy}($ cadena8,"+");strcpy(cadena10,"+");strcpy(cadena12,"+"); strcpy(cadena14,"+");strcpy(cadena16,"+");strcpy(cadena18,"+");strcpy(cadena20,"+"); strcpy(cadena22,"+");strcpy(cadena24,"+");strcpy(cadena26,"+");strcpy(cadena28,"+"); strcpy(cadena30,"+");strcpy(cadena32,"+");strcpy(cadena34,"+");strcpy(cadena36,"+"); strcpy(cadena38,"+");strcpy(cadena40,"+");strcpy(cadena42,"+");

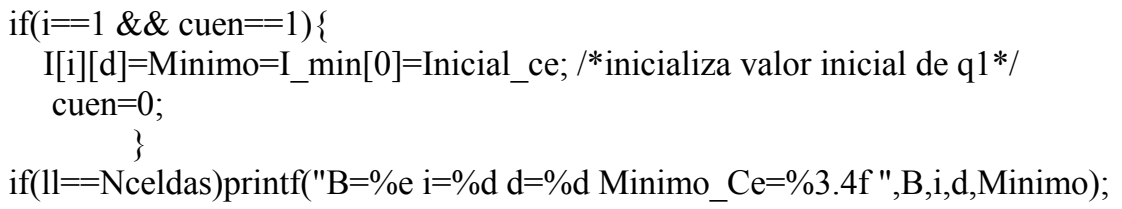




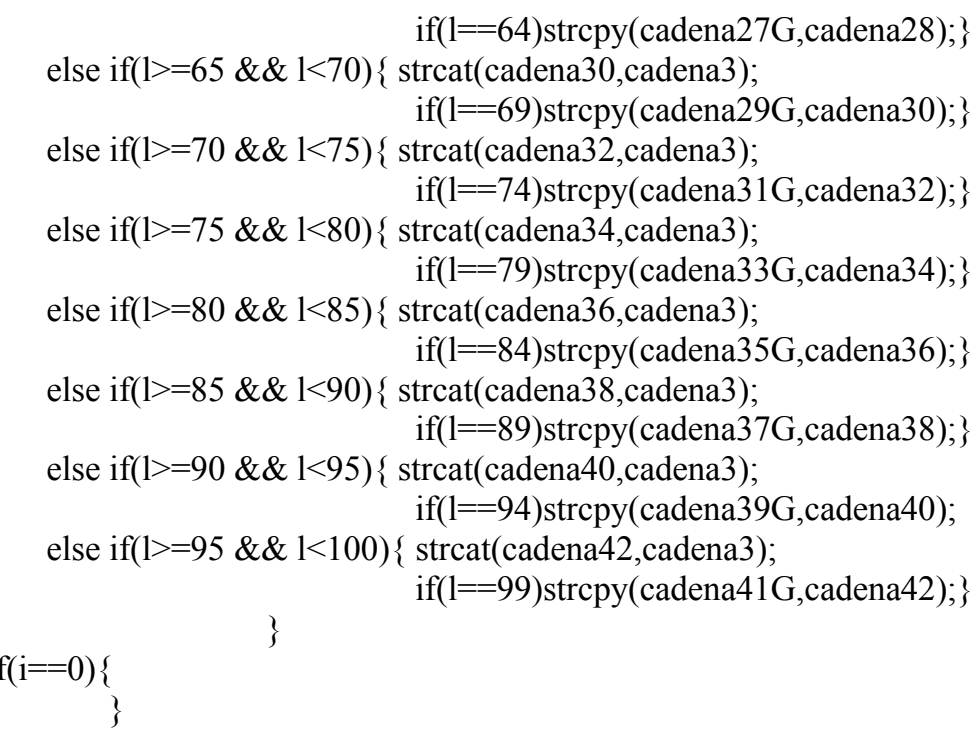

if $(\mathrm{i}<5 \& \& \mathrm{i}>0)\{$

sprintf(cadena2,cadena,t[1],I_min[1],t[2],I_min[2],t[3],I_min[3],t[4],I_min[4]); \}

else if $(i>=5)\{$

sprintf(cadena2,cadenaG,t[1],I_min[1],t[2],I_min[2],t[3],I_min[3],t[4],I_min[4]); \}

if $(11==$ Nceldas $)$ puts(cadena2);

salva_datos_en_fichero(1,cadena2,1);

if $(\mathrm{i}>=5 \& \& \mathrm{i}<10)\{$

sprintf(cadena6,cadena5,t[5],I_min[5],t[6],I_min[6],t[7],I_min[7],t[8],I_min[8]

,t[9],I_min[9]);

if(1l==Nceldas)puts(cadena6);salva_datos_en_fichero(1,cadena6,2);

if $(\mathrm{i}>=10)\{$

sprintf(cadena6,cadena5G,t[5],I_min[5],t[6],I_min[6],t[7],I_min[7],t[8],I_min[8]

,t[9],I_min[9]);

if(ll==Nceldas)puts(cadena6);salva_datos_en_fichero(1,cadena6,2);

\}

if $(\mathrm{i}>=10 \& \& \mathrm{i}<15)\{$

sprintf(cadena7,cadena8,t[10],I_min[10],t[11],I_min[11],t[12],I_min[12],t[13],I_min[13] ,t[14],I_min[14]);

if $(11==$ Nceldas $)$ puts(cadena7);salva_datos_en_fichero(1,cadena7,2);

if $(i>=15)$

sprintf(cadena7,cadena7G,t[10],I_min[10],t[11],I_min[11],t[12],I_min[12],t[13],I_min[13] ,t[14],I_min[14]);

if $(11==$ Nceldas $)$ puts(cadena7); salva_datos_en_fichero(1,cadena7,2);

\}

if $(\mathrm{i}>=15 \& \& \mathrm{i}<20)\{$

sprintf(cadena9,cadena10,t[15],I_min[15],t[16],I_min[16],t[17],I_min[17],t[18],I_min[18] ,t[19],I $\min [19])$;

if $(11==$ Nceldas $)$ puts(cadena9);salva_datos_en_fichero(1,cadena9,2);

if $(\mathrm{i}>=20)\{$

sprintf(cadena9,cadena9G,t[15],I_min[15],t[16],I_min[16],t[17],I_min[17],t[18],I_min[18] ,t[19],__min[19]);

if(ll==Nceldas)puts(cadena9);salva_datos_en_fichero(1,cadena9,2);

if $(\mathrm{i}>=20 \& \& \mathrm{i}<25)$

sprintf(cadena11,cadena12,t[20],I_min[20],t[21],I_min[21],t[22],I_min[22],t[23],I_min[23] ,t[24],I_min[24]); 


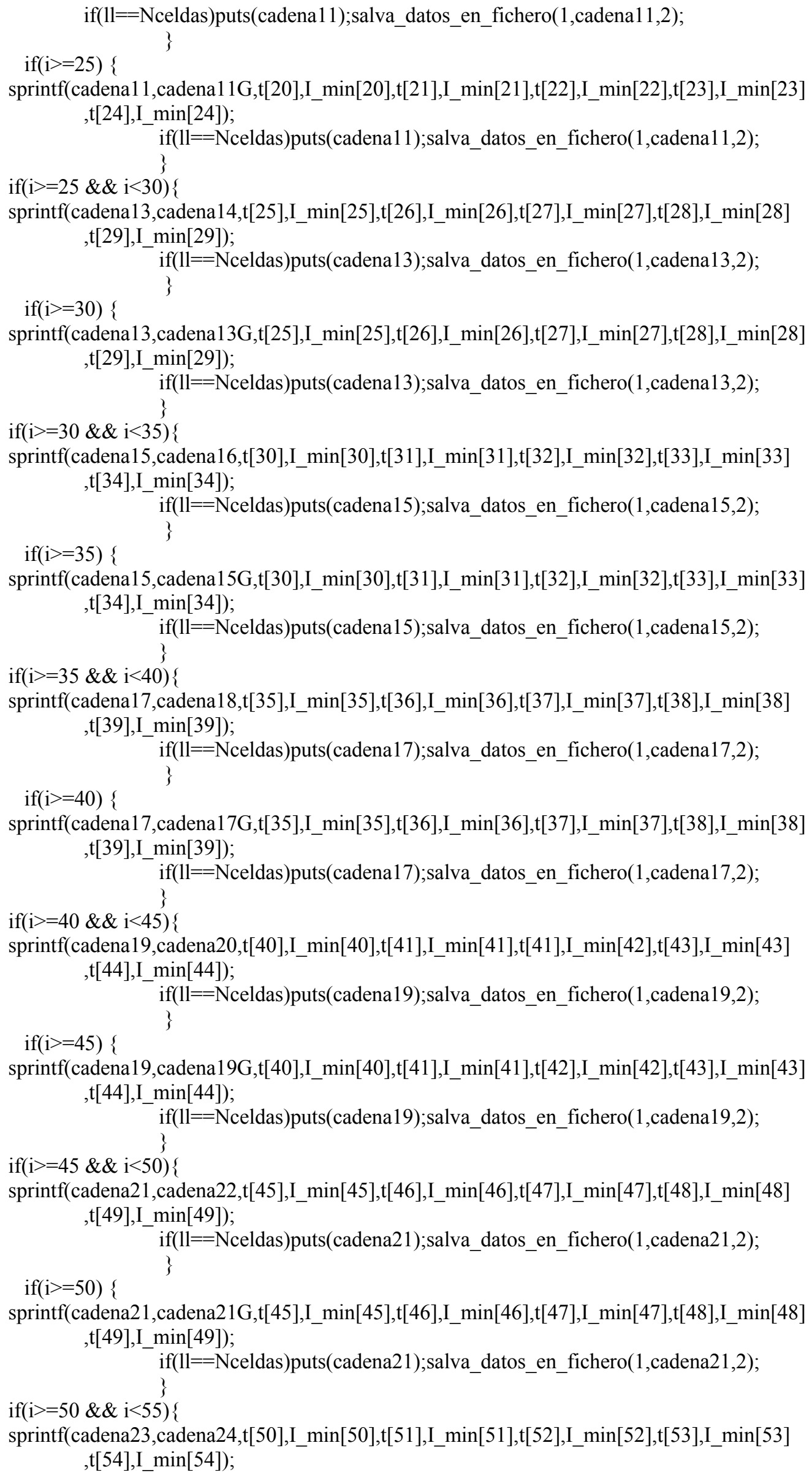




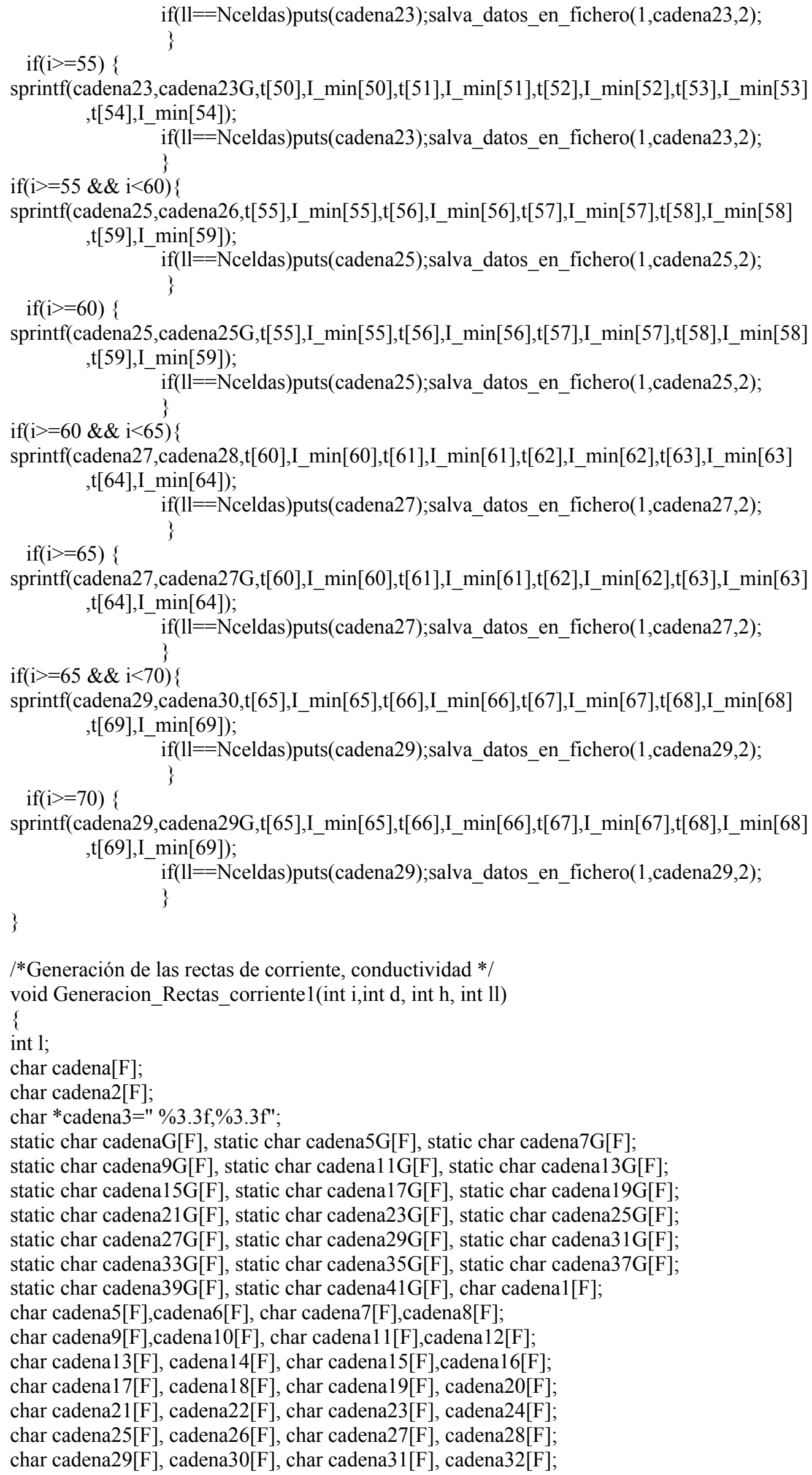


char cadena33[F], cadena34[F], char cadena35[F], cadena36[F];

char cadena37[F], cadena38[F], char cadena39[F], cadena40[F];

char cadena41[F], cadena42[F];

static int cuen_k $=0$;

sprintf(cadena,"'lnE_E\%d N\%d 0 TABLE $=\{\mathrm{V}(\mathrm{N} \% \mathrm{~d}, 0)\}=\% 2.3 \mathrm{f}, \% 2.3 \mathrm{f} ", 10 *$ Nceldas $+11, \mathrm{~h}-11$,h8,ci,Inicial_k);

strcpy(cadena5,"+");strcpy(cadena8,"+");strcpy(cadena10,"+");strcpy(cadena12,"+"); strcpy(cadena14,"+");strcpy(cadena16,"+");strcpy(cadena18,"+");strcpy(cadena20,"+"); strcpy(cadena22,"+"); strcpy(cadena24,"+");strcpy(cadena26,"+");strcpy(cadena28,"+"); strcpy(cadena30,"+");strcpy(cadena32,"+");strcpy(cadena34,"+");strcpy(cadena36,"+"); strcpy(cadena38,"+");strcpy(cadena40,"+");strcpy(cadena42,"+");

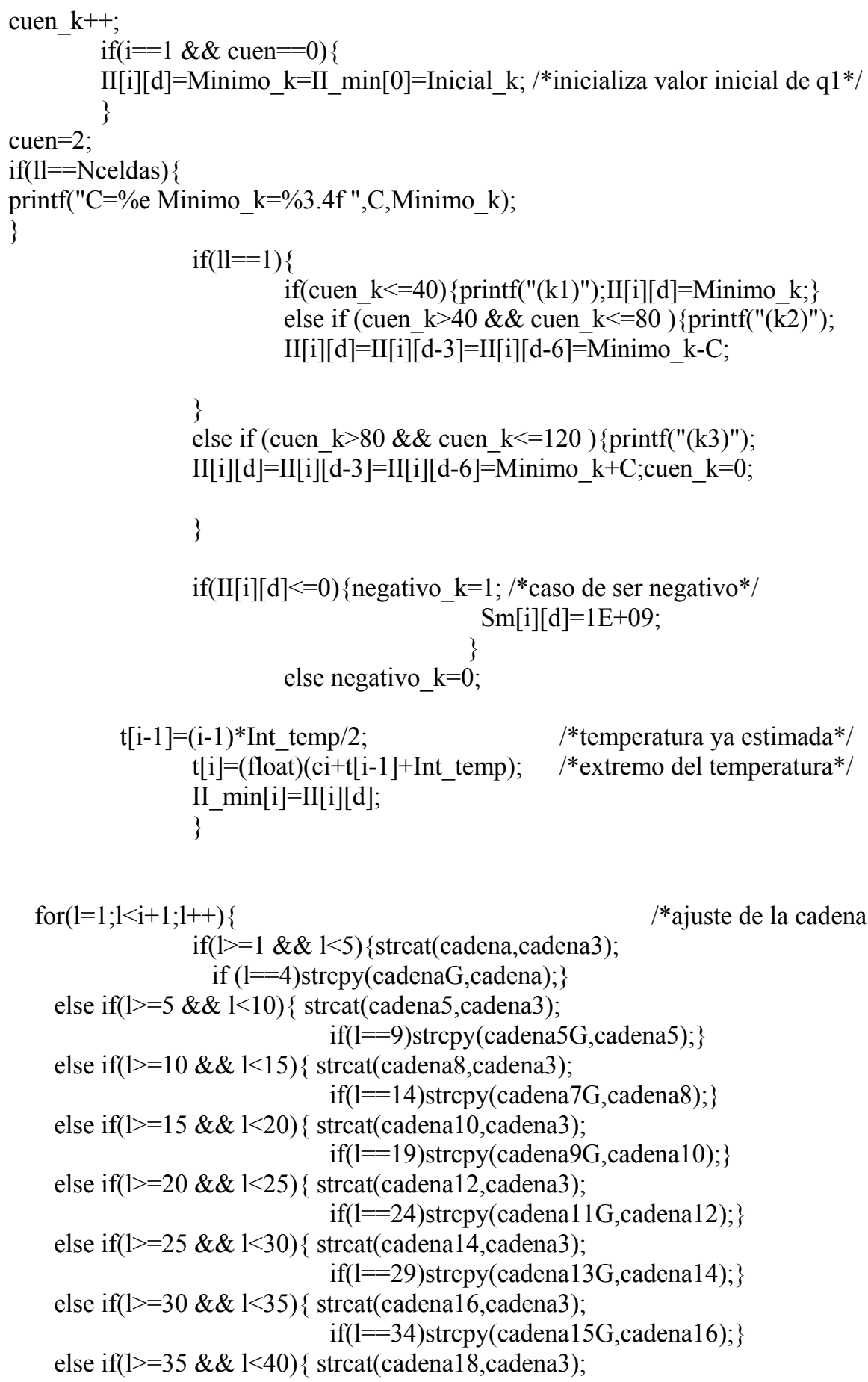




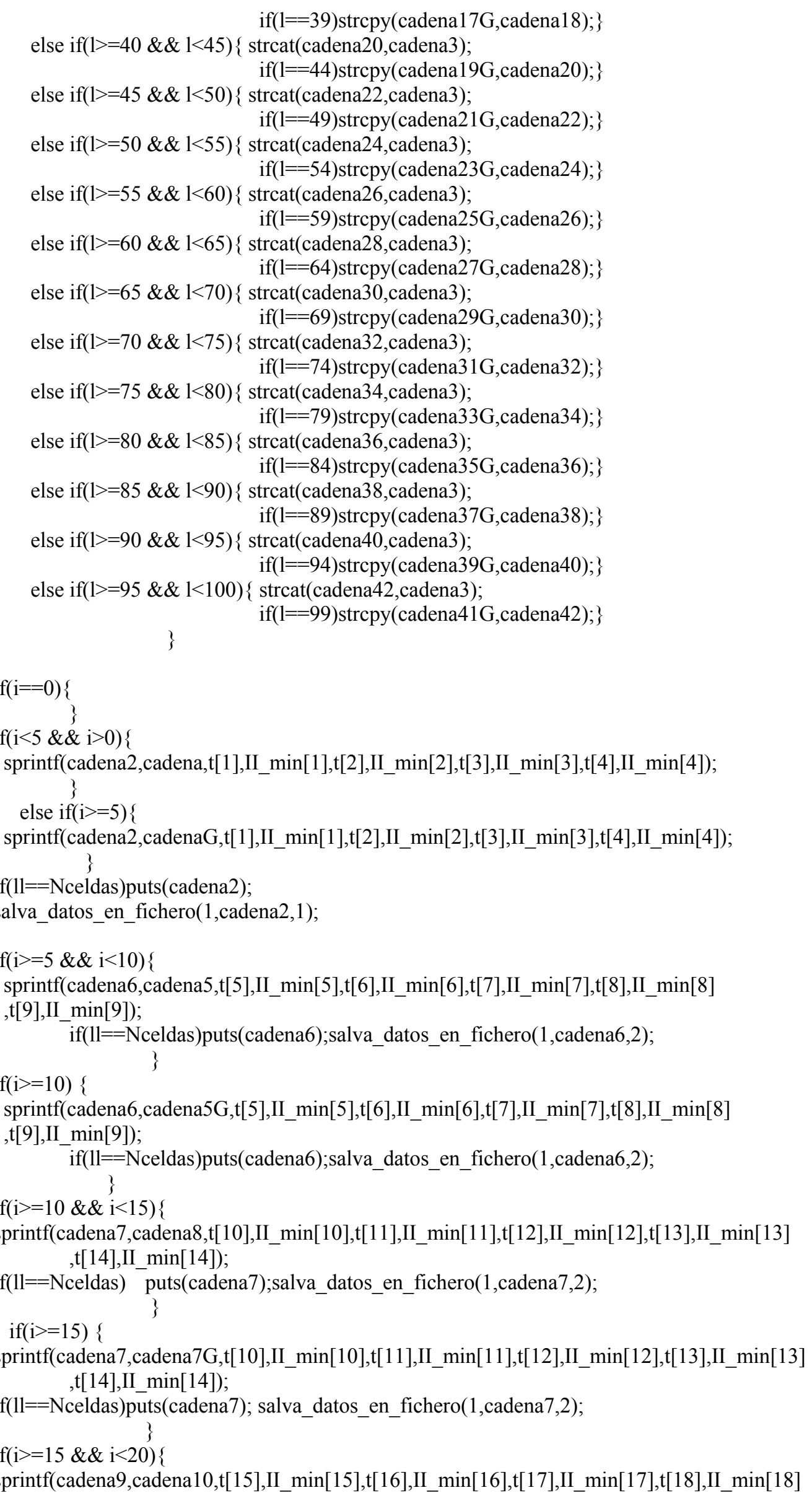


,t[19],II_min[19]);

if(1l==Nceldas)puts(cadena9);salva_datos_en_fichero(1,cadena9,2);

if $(\mathrm{i}>=20)\{$

sprintf(cadena9,cadena9G,t[15],II_min[15],t[16],II_min[16],t[17],II_min[17],t[18],II_min[18] ,t[19],II_min[19]);

if(1l==Nceldas)puts(cadena9);salva_datos_en_fichero(1,cadena9,2);

if $(\mathrm{i}>=20 \& \& \mathrm{i}<25)\{$

sprintf(cadena11,cadena12,t[20],II_min[20],t[21],II_min[21],t[22],II_min[22],t[23],II_min[23] ,t[24],II $\min [24])$;

if(1l==Nceldas)puts(cadena11);salva_datos_en_fichero $(1$, cadena 11,2$)$;

if(i $>=25)\{$

\}

sprintf(cadena11,cadena11G,t[20],II $\min [20], \mathrm{t}[21], \mathrm{II} \min [21], \mathrm{t}[22], \mathrm{II} \min [22], \mathrm{t}[23]$,

II_min[23],t[24],II_min[24]); if $(11==$ Nceldas)puts(cadena11);salva_datos_en_fichero(1,cadena 11,2); \}

if $(\mathrm{i}>=25 \& \& \mathrm{i}<30)\{$

sprintf(cadena13,cadena14,t[25],II_min[25],t[26],II_min[26],t[27],II_min[27],t[28],II_min[28] ,t[29],II_min[29]);

if(1l==Nceldas)puts(cadena13);salva_datos_en_fichero(1,cadena13,2);

if $(\mathrm{i}>=30)\{$

\}

sprintf(cadena13,cadena13G,t[25],II_min[25],t[26],II_min[26],t[27],II_min[27],t[28],

II $\min [28], \mathrm{t}[29]$,II $\min [29])$; if $(11==$ Nceldas $)$ puts(cadena13);salva_datos_en_fichero(1,cadena13,2);

if $(\mathrm{i}>=30 \& \& \mathrm{i}<35)\{$ \}

sprintf(cadena15,cadena16,t[30],II_min[30],t[31],II_min[31],t[32],II_min[32],t[33],II_min[33] ,t[34],II_min[34]);

if(1l==Nceldas)puts(cadena15);salva_datos_en_fichero(1,cadena15,2);

if $(\mathrm{i}>=35)\{$

sprintf(cadena15,cadena15G,t[30],II_min[30],t[31],II_min[31],t[32],II_min[32],t[33],

II_min[33],t[34],II_min[34]); if(1l==Nceldas)puts(cadena15);salva_datos_en_fichero(1,cadena15,2); \}

if $(\mathrm{i}>=35 \& \& \mathrm{i}<40)\{$

sprintf(cadena17,cadena18,t[35],II_min[35],t[36],II_min[36],t[37],II_min[37],t[38],II_min[38] ,t[39],II_min[39]); if(1l==Nceldas)puts(cadena17);salva_datos_en_fichero(1,cadena17,2);

if $(\mathrm{i}>=40)\{$ \}

sprintf(cadena17,cadena17G,t[35],II_min[35],t[36],II_min[36],t[37],II_min[37],t[38],

II $\min [38], \mathrm{t}[39]$,II $\min [39])$; if(1l==Nceldas)puts(cadena17);salva_datos_en_fichero(1,cadena17,2);

if $(\mathrm{i}>=40 \& \& \mathrm{i}<45)\{$ \}

sprintf(cadena19,cadena20,t[40],II_min[40],t[41],II_min[41],t[41],II_min[42],t[43],II_min[43] ,t[44],II_min[44]);

if(1l==Nceldas)puts(cadena19);salva_datos_en_fichero(1,cadena19,2);

if $(\mathrm{i}>=45)\{$

sprintf(cadena19,cadena19G,t[40],II_min[40],t[41],II_min[41],t[42],II_min[42],t[43],

II_min[43],t[44],II_min[44]); if( $(11==$ Nceldas $)$ puts(cadena19);salva_datos_en_fichero(1,cadena19,2);

if $(\mathrm{i}>=45 \& \& \mathrm{i}<50)\{$

sprintf(cadena21,cadena22,t[45],II_min[45],t[46],II_min[46],t[47],II_min[47],t[48],II_min[48] 


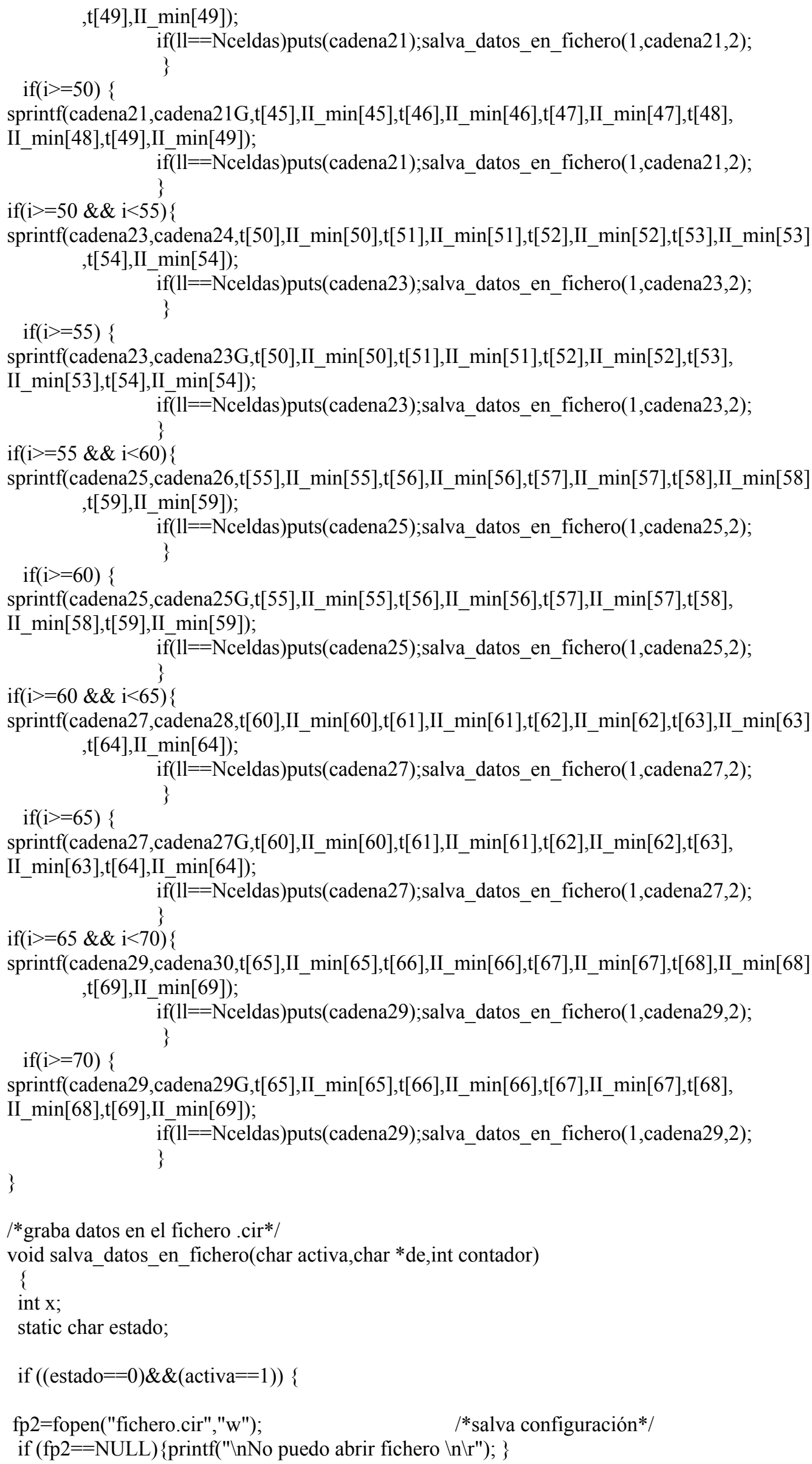




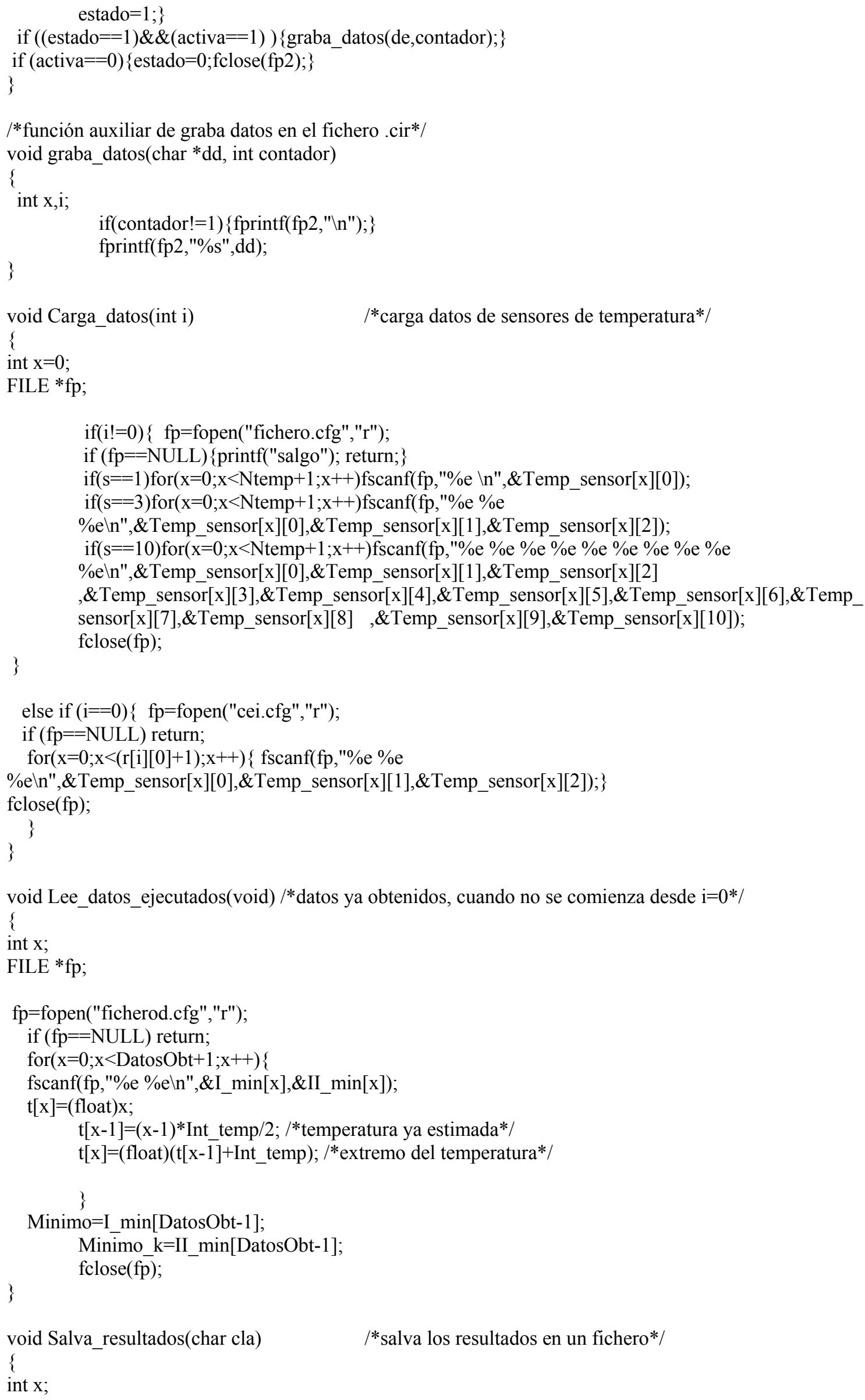




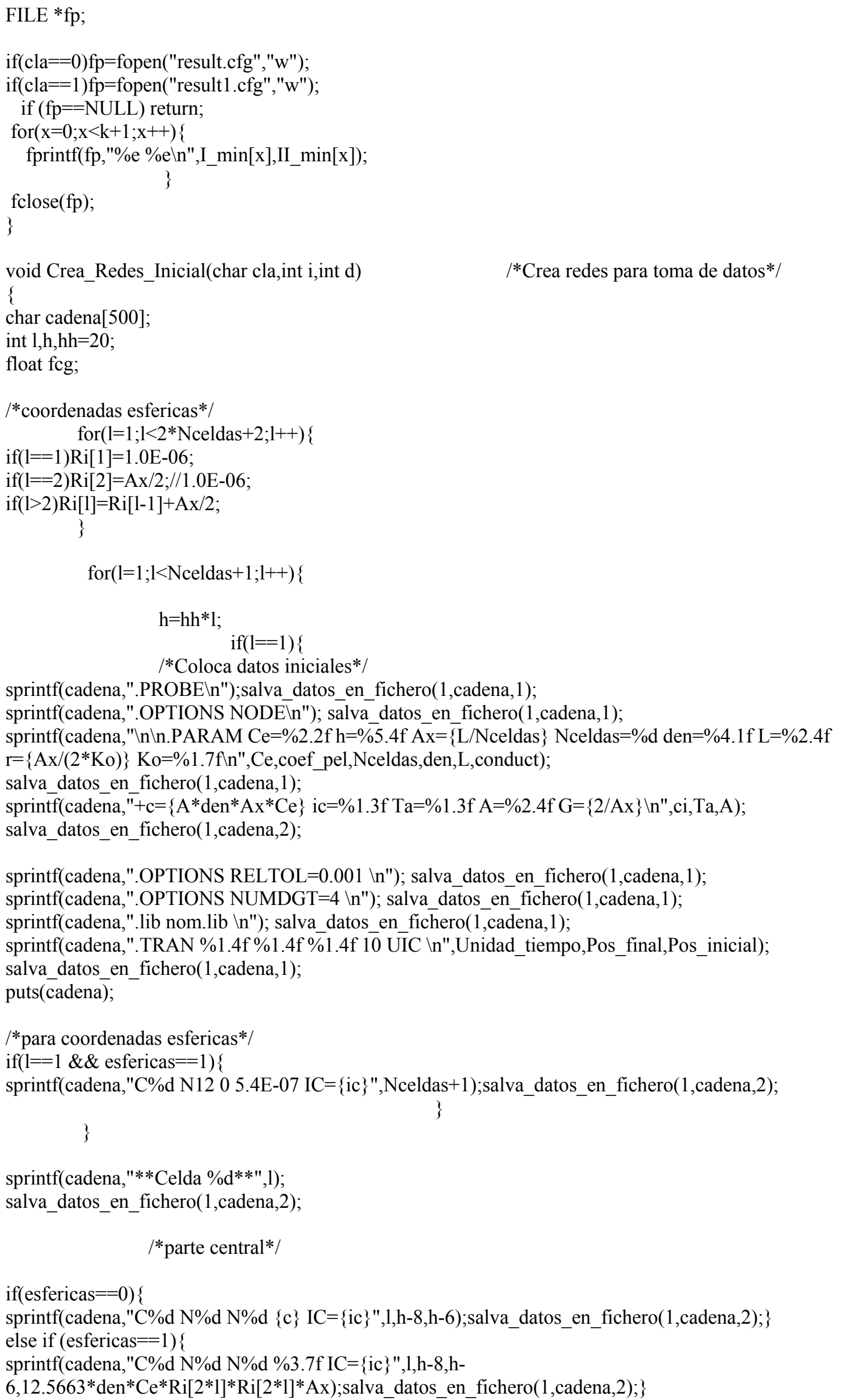


if $($ esfericas $==0)\{$

if(l!=1) \{sprintf(cadena,"R\%d N\%d N\%d 1G",(2*1-1),h-hh,h-8);

salva_datos_en_fichero(1,cadena, 2$)$;

sprintf(cadena,"R\%d N\%d N\%d 1G",2*1,h-8,h);salva_datos_en_fichero(1,cadena,2);

if $(1 !=1)\{$

sprintf(cadena,"G\%d N\%d N\%d VALUE=",Nceldas+(2*1-1),h-hh,h-8);

salva_datos_en_fichero(1,cadena, 2$)$;

sprintf(cadena," $+\{(\mathrm{V}(\mathrm{N} \% \mathrm{~d}, 0)-\mathrm{V}(\mathrm{N} \% \mathrm{~d}, 0)) * \mathrm{~V}(\mathrm{~N} \% \mathrm{~d}, 0) * \mathrm{G}\}$ ",h-hh,h-8,h-11);

salva_datos_en_fichero(1,cadena,2);

\}

sprintf(cadena,"G\%d N\%d N\%d VALUE=",Nceldas $+2 * 1, \mathrm{~h}-8$,h); salva datos en fichero(1,cadena,2);

if $(\mathrm{l}==1)$ sprintf(cadena, $+\{(\mathrm{V}(\mathrm{N} \% \mathrm{~d}, 0)-\mathrm{V}(\mathrm{N} \% \mathrm{~d}, 0)) * \mathrm{~V}(\mathrm{~N} \% \mathrm{~d}, 0) * \mathrm{G}\}$ ",h- $-\overline{8}, \mathrm{~h}, \mathrm{~h}-\overline{1} 1)$;

else sprintf(cadena,"+ $\{(\mathrm{V}(\mathrm{N} \% \mathrm{~d}, 0)-\mathrm{V}(\mathrm{N} \% \mathrm{~d}, 0)) * \mathrm{~V}(\mathrm{~N} \% \mathrm{~d}, 0) * \mathrm{G}\}$ ",h-8,h,h-11);

salva_datos_en_fichero(1,cadena,2);

else \{

if $(1 !=1 \& \&$ esfericas $==0 \||| 1 !=1 \& \&$ esfericas $==1)\{$

sprintf(cadena,"R\%d N\%d N\%d 1G",(2*1-1),h-hh,h-8);

salva_datos_en_fichero(1,cadena, 2$)$;

sprintf(cadena,"R\%d N\%d N\%d 1G",2*1,h-8,h);

salva_datos_en_fichero(1,cadena,2);

if $(1 !=1 \& \&$ esfericas $==1)\{\quad$ /*GMULT, para conductividad*/

sprintf(cadena,"G\%d N\%d N\%d VALUE=",Nceldas+(2*1-1),h-hh,h-8);

salva datos en fichero(1,cadena, 2$)$;

sprintf(cadena," $+\{(\mathrm{V}(\mathrm{N} \% \mathrm{~d}, 0)-\mathrm{V}(\mathrm{N} \% \mathrm{~d}, 0)) * \mathrm{~V}(\mathrm{~N} \% \mathrm{~d}, 0) * \% 4.7 f\}$ ",h-hh,h-8,h-11,22.1327*Ri[2*1-1]*Ri[2*1-

$1] / \mathrm{Ax})$

salva_datos_en_fichero(1,cadena,2);

\}

if $($ esfericas $==1)\{$

sprintf(cadena,"G\%d N\%d N\%d VALUE=",Nceldas $+2 * 1, \mathrm{~h}-8$,h);salva datos en fichero(1,cadena,2);

if $(\mathrm{l}==1)$ sprintf(cadena," $+\{(\mathrm{V}(\mathrm{N} \% \mathrm{~d}, 0)-\mathrm{V}(\mathrm{N} \% \mathrm{~d}, 0)) * \mathrm{~V}(\mathrm{~N} \% \mathrm{~d}, 0) * \% 4.7 \mathrm{f}\}$ ",h-8, h, h-

$11,22.1327 * \operatorname{Ri}[2 * 1+1] * \operatorname{Ri}[2 * 1+1] /(2 * A x))$;

else sprintf(cadena,"+\{(V(N\%d,0)-V(N\%d,0))*V(N\%d,0)*\%4.7f\} ",h-8,h,h-

$11,22.1327 * \operatorname{Ri}[2 * 1+1] * \operatorname{Ri}[2 * 1+1] /$ Ax);

salva_datos_en_fichero(1,cadena,2);

\}

/*GMULT, calor especifico */

sprintf(cadena,"G\%d N\%d 0 VALUE=",1,h-8);salva_datos_en_fichero(1,cadena,2);

sprintf(cadena,"+\{V(N\%d,0)*V(N\%d,0)*\%4.7f\} ",h-4,h-5,1/Ce);

salva_datos_en_fichero(1,cadena,2);

/*onversión I- $\mathrm{V} * /$

sprintf(cadena,"H\%d N\%d $0 \mathrm{VH} \% \mathrm{~d}$ 1",1,h-5,1);salva_datos_en_fichero(1,cadena,2);

sprintf(cadena,"VH\%d N\%d 0 0V",1,h-6);salva_datos_en_fichero(1,cadena,2);

\section{/*ETABLE,DESACTIVAR PARA RESOLUCION*/}

if $(1==$ Nceldas $)\{$

Generacion_Rectas_corriente(i,d,h,l);

Generacion Rectas corrientel(i,d,h,l);

sprintf(cadena,"**CONDICIONES DE CONTORNO**");

salva_datos_en_fichero(1,cadena, 2 );

sprintf(cadena,"'"nR\%d N12 $01 \mathrm{Gln} ", 2 *$ Nceldas +1$)$;salva_datos_en_fichero(1,cadena,1); 
if $($ esfericas $==0)\{$

/*onvección forzada*/

/*flujo de calor*/

sprintf(cadena,"I\%d 0 N\%d 25000",Nceldas*20+10,20*1);salva_datos_en_fichero(1,cadena,2);

else if $($ esfericas $==1)\{$

/*convección forzada*/

sprintf(cadena,"G\%d 0 N\%d

VALUE",Nceldas*20+10,20*1);salva datos_en fichero(1,cadena,2);

sprintf(cadena,"+\{\%2.4f*(\%2.3f-V(N\%d,0))\}",coef_pel* ( $\left.^{*} * 3.1416^{*} \mathrm{~L} * \mathrm{~L}\right), \mathrm{Ta}, 20 *$ Nceldas $)$;

salva_datos_en_fichero(1,cadena,2);

\}

$\mathrm{V}([\mathrm{N} 800]) \backslash \mathrm{n} ") ;$ salva_datos_en_fichero(1,cadena,2);\}

if(s==1) \{sprintf(cadena,".PRINT TRAN V([N800])\n");salva_datos_en_fichero(1,cadena,2);

if $(\mathrm{s}==3 \& \&$ esfericas $==0)\{$ sprintf(cadena,".PRINT TRAN V([N800]) $\backslash \bar{n} \overline{ })$;

salva_datos_en_fichero(1,cadena, 2$)$;

sprintf(cadena,".PRINT TRAN V([N400]) \n");salva_datos_en_fichero(1,cadena,2);

sprintf(cadena,".PRINT TRAN V([N200]) \n");salva_datos_en_fichero(1,cadena,2);

sprintf(cadena,".END");salva_datos_en_fichero(1,cadena, 1 );

\}

if( $(\mathrm{s}==3$ \&\& esfericas==1) \{sprintf(cadena,".PRINT TRAN V([N800])\n");

salva_datos_en_fichero(1,cadena, 2$)$;

sprintf(cadena,".PRINT TRAN V([N600]) \n");salva_datos_en_fichero(1,cadena,2);

sprintf(cadena,".PRINT TRAN V([N400]) \n");salva_datos_en_fichero(1,cadena,2);

sprintf(cadena,".END");salva_datos_en_fichero( 1 ,cadena, $\overline{1}$ );

\}

\}

salva datos en fichero( 0 ,cadena, 1$)$;

if $\left(\mathrm{cla}^{-}=0\right)\{$ system("pspice ce.cir "); $\quad \operatorname{exit}(0) ;\}$

\} 